\title{
ABSTRACTS FOR POSTER PRESENTATION
}

\author{
Student Competition Finalists
}

\section{ABERRANT DNA METHYLATION IN PORCINE IN VITRO-, PARTHENOGENETIC-, AND NUCLEAR TRANSFER-PRODUCED BLASTOCYSTS}

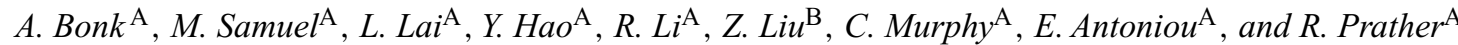 \\ ${ }^{A}$ Department of Animal Sciences, University of Missouri-Columbia, Columbia, MO 65201, USA; \\ ${ }^{\mathrm{B}}$ North-East Agricultural University, Harbin, Heilongiiang Province, China
}

\begin{abstract}
Aberrant DNA methylation of in vitro-, parthenogenetic-, and nuclear transfer-derived embryos has been implicated in the low developmental competence of early embryos. Demethylation of the genome occurs immediately after fertilization and continues through the blastocyst stage. Remethylation or reprogramming of the genome occurs around the time of implantation and is maintained in somatic tissues. The aim of this study was to analyze DNA methylation in porcine gametes and blastocysts. Differential DNA methylation hybridization was conducted to analyze the methylation status of the Bstu I site (CGCG) in the gamete and blastocyst epigenomes. Germinal vesicle oocytes were aspirated from ovaries collected at an abattoir, sperm was isolated from a fresh ejaculate, and blastocysts were derived and collected from in vivo, in vitro, nuclear transfer, and parthenogenetic sources. Genomic clones were selected from a porcine CpG Island library based on the presence of a Bstu I site. The inserts from these clones were PCR amplified and spotted on glass slides. DNA was digested with Mse I, ligated to linkers, and digested with Bstu I. Fragments with methylated Bstu I sites remained intact whereas fragments with unmethylated Bstu I sites were cut. Intact fragments were amplified by PCR and labeled with amino allyl-dUTP. Liver DNA served as the reference and was labeled with Cy5; the other samples were labeled with Cy3. An Axon Genepix 4000B scanner (Axon Instruments, Inc., Union City, CA, USA) was used to scan the slides. Initial analysis of the microarray image was performed with GenePix Pro 4.0 software. Additional analysis, performed by using Genespring 7.0 ANOVA $(P<0.05)$, identified 221 clones as being significantly different in at least one of the biological conditions of the gametes or the blastocysts. Forty-six clones were sequenced and BLAST analysis identified 18 clones that were unique, 16 clones that had no similarity, and 12 clones that had similarity to multiple genes. Ribosomal (RPS20, RPL18) and protoporphyrinogen oxidase (PPOX) genes were identified in several clones. Components of the immune system (CCRs, TLRs), a transcription factor (ATF2), and an embryo-specific gene (WNT8B) were also identified. A condition tree was created according to the standard correlation similarity measure for the spots identified as significantly different. The condition tree shows that the methylation profiles are most similar in the germinal vesicle oocyte, parthenogenetic blastocyst, nuclear transfer blastocyst, in vitro-produced blastocyst, and sperm. In vivo-produced blastocysts grouped separately from the other samples. These results are consistent with previous studies that have shown that gametes undergo demethylation after fertilization on through the blastocyst stage when the genome is remethylated. Additionally, these results suggest that the reprogramming events that occur during the development of the in vivo-produced blastocysts are less likely to occur in in vitro-, nuclear transfer-, and parthenogenetic-produced blastocysts.
\end{abstract}

This work was funded by a grant from the NIH (RR13438) and Food for the 21st Century.

\section{IDENTIFICATION OF GENES INDUCED BY THE CONCEPTUS IN THE BOVINE ENDOMETRIUM DURING THE PRE-IMPLANTATION PERIOD}

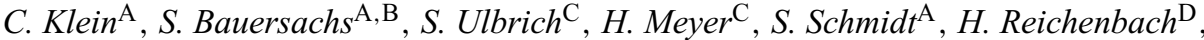 \\ M. Vermehren ${ }^{\mathrm{E}}, H$. Blum ${ }^{\mathrm{B}}$, F. Sinowatz ${ }^{\mathrm{E}}$, and E. Wolf . $^{\mathrm{A}, \mathrm{B}}$
}

\footnotetext{
${ }^{A}$ Institute of Molecular Animal Breeding of the Ludwig-Maximilians University, Feodor-Lynen-Str. 25, 81377 Munich, Germany;

${ }^{B}$ Laboratory for Functional Genome Analysis (LAFUGA) of the Ludwig-Maximilians University, Feodor-Lynen-Str. 25, 81377 Munich, Germany;

${ }^{\mathrm{C}}$ Physiology-Weihenstephan, Technical University of Munich, Weihenstephaner Berg 3, 85354 Freising, Germany;

${ }^{\mathrm{D}}$ Bavarian Research Station for Animal Breeding, 85586 Grub, Germany;

${ }^{\mathrm{E}}$ Institute of Veterinary Anatomy, Histology and Embryology, Ludwig-Maximilians University, Veterinaerstr. 13, 80539 Munich, Germany
}

Early embryonic development, implantation, and maintenance of a pregnancy are critically dependent on an intact embryo-maternal communication So far, only few signals involved in this dialogue have been identified. In ruminants, interferon tau (IFN $\tau$ ) plays a key role in the process of maternal recognition of pregnancy by exhibiting antiluteolytic activity. Even though many experimental findings indicate a pivotal role of IFN $\tau$ in the context of embryo-maternal communication in ruminants, a number of other systems may be involved. To identify genes induced in the bovine endometrium by the signaling of the embryo, a combination of subtracted cDNA libraries and cDNA array hybridization was applied. Monozygotic twin pairs $(n=5)$ were used as the biological model. Pregnancy was created in one twin by transferring two in vitro-produced embryos on Day 7 of the estrous cycle; the other twin received a sham embryo transfer. Pregnant and nonpregnant twins were slaughtered at Day 18; endometrial tissue samples were recovered and processed for transcriptome analysis as described (Bauersachs et al. $2005 \mathrm{~J}$. Mol. Endocrinol. 34, 889-908). Screening of 4608 clones of two subtracted libraries revealed 90 different up-regulated genes and mRNAs, of which almost $50 \%$ are known to be stimulated by type I interferons. Among these interferon-stimulated genes, the ISG15 system is assumed to be of particular interest, and several components were studied in more detail using in situ hybridization. The pattern of mRNA expression suggests that modification of endometrial proteins through ISG15ylation plays a fundamental role during the pre-implantation period. A classification of the identified genes based on Gene Ontologies revealed the prevalence of genes involved in regulation of gene expression, cell communication, cell growth, cell differentiation, cell proliferation, and cell adhesion, and also the prevalence of genes with immune-related functions. These results underline the intense response of the endometrium to the presence of a 
conceptus, culminating in the preparation of the maternal environment for embryonic implantation. Further, for eleven selected genes the expression in the endometrium was quantified by the use of real-time RT-PCR. Overall, the results of quantitative RT-PCR and array hybridization correlated very well. To our knowledge this study provides the first holistic gene expression analysis of the bovine endometrium during the pre-implantation period. The results underline the importance of IFN $\tau$ as an embryo-derived pregnancy recognition signal and depict the molecular mechanisms at the mRNA level underlying the intense embryo-maternal dialog taking place at Day 18 of gestation.

\title{
3 NUCLEAR REPROGRAMMING OF PORCINE FIBROBLAST CELLS BY XENOPUS EGG EXTRACTS
}

\author{
K. Miyamoto $^{\mathrm{A}}$, Y. Nagao $^{\mathrm{A}}, N$. Minami $^{\mathrm{A}}$, M. Yamada $^{\mathrm{A}}, K$. Ohsumi $^{\mathrm{B}}$, and H. Imai ${ }^{\mathrm{A}}$ \\ ${ }^{A}$ Laboratory of Reproductive Biology, Graduate School of Agriculture, Kyoto University, Kyoto, Japan; \\ ${ }^{\mathrm{B}}$ Laboratory of Cell and Developmental Biology, Graduate School of Bioscience and Biotechnology, \\ Tokyo Institute of Technology, Yokohama, Kanagawa, Japan
}

\begin{abstract}
Much evidence indicates that somatic cells can be reprogrammed in an oocyte cytoplasm. The nuclear reprogramming consists of many unknown processes, and mechanisms underlying these processes still remain to be elucidated. Recently some reports noted that Xenopus oocytes or eggs can induce some of the reprogramming events in mammalian cells. We investigated the processes of nuclear reprogramming of porcine fibroblast cells by Xenopus egg extracts to understand how egg extracts trigger the reprogramming and/or dedifferentiation of cells. Unfertilized Xenopus eggs were collected from mature females. After removal of the jelly coat, activation was routinely achieved by calcium ionophore A23187. The eggs were immediately centrifuged and the cytoplasmic fraction was used as egg extracts. Porcine fibroblast cells were permeabilized by streptolysin $\mathrm{O}$ and incubated in the egg extracts under the ATP-generating system ( $1 \mathrm{mM} \mathrm{ATP}, 5 \mathrm{mM}$ phosphocreatine, and $20 \mathrm{U} / \mathrm{mL}$ creatine kinase) for $30 \mathrm{~min}$ at $37^{\circ} \mathrm{C}$ or $2 \mathrm{~h}$ at $23^{\circ} \mathrm{C}$. The incorporation of Xenopus-specific linker histone $\mathrm{B} 4$ into porcine fibroblasts was examined by immunofluorescence and immunobloting analysis. After collection of cells from the extracts, permeabilized membranes of the cells were resealed in culture medium containing $2 \mathrm{mM} \mathrm{CaCl}_{2}$ for $2 \mathrm{~h}$. The cells were then incubated in DMEM with $10 \%$ fetal bovine serum (FBS) or porcine zygote medium-3 (PZM-3: Yoshioka et al. 2002 Biol. Reprod. 66, 112-119) containing $5.55 \mathrm{mM}$ glucose and 5\% FBS. RNAs were extracted from the cells in each culture dish and Oct-4 expression was examined by RT-PCR analysis every day until Day 8 . The primers were designed to span the 99 base-pair intron region of porcine Oct-4 gene for recognizing both spliced and unspliced transcripts. The incorporation of histone B4 from Xenopus egg extracts was observed at the nuclear region of the porcine fibroblasts under both the $37^{\circ} \mathrm{C}$ and the $23^{\circ} \mathrm{C}$ conditions. Because the histone $\mathrm{B} 4$ incorporation was inhibited by addition of Apyrase, an ATPase, a part of reprogramming might be an ATP-dependent process. When treated cells were incubated in DMEM or PZM-3, Oct-4 expression was detected in the cells cultured in DMEM, but not in PZM-3. However, the transcripts of Oct-4 were mainly obtained in unspliced form at the earlier stage of culture (after Day 1 to Day 4 of culture), suggesting that a part of reprogramming processes by the egg extracts involves induction of dedifferention of cells or activation of a pluripotent marker gene such as Oct-4. Xenopus egg extract may provide a system to investigate the processes involving nuclear reprogramming and the pluripotent state of mammalian cells in vitro.
\end{abstract}

\section{GENE EXPRESSION IN CULTURES OF INNER CELL MASSES ISOLATED FROM IN VITRO-PRODUCED AND IN VIVO-DERIVED BOVINE BLASTOCYSTS}

\author{
D. Pant and C. Keefer \\ Department of Animal and Avian Sciences, University of Maryland, College Park, MD 20742, USA
}

Genetic modification of embryonic stem (ES) cells derived from domestic species could be exploited to produce transgenic animals; however, fully validated ES have not been obtained in domestic species. Recent findings regarding key transcription factors and regulation of pluripotency and self-renewal in murine ES cells may provide keys to enable the derivation of ES in domestic species. The aim of this study was to identify and monitor the expression of candidate genes, which are known to be involved in the maintenance of self-renewal and pluripotency in mouse and human ES cells, during the critical first steps in establishment of primary cultures. Inner cell masses (ICMs) were isolated via manual dissection of 25 to 30 commercial in vitro-produced (IVP) blastocysts (Bomed, Inc., Madison, WI, USA) in each of three separate replicates and from 10 in vivo-derived Day 7-8 bovine blastocysts. On the day of ICM isolation (Day 0), 4-5 ICM clumps were collected for RT-PCR analysis. The remaining isolated ICMs were cultured (4-5 ICM clumps per well) on mitomycin C (Sigma-Aldrich, St. Louis, MO, USA)-inactivated mouse embryonic fibroblasts (STO, ATCC, Manassas, VA, USA). The ICM clumps were cultured in 12-well tissue culture dishes in ES medium consisting of Knockout DMEM (Invitrogen, Carlsbad, CA, USA) supplemented with 15\% FCS (Hyclone, Logan, UT, USA), 2 mM L-glutamine (Invitrogen), $0.1 \mathrm{mM} \mathrm{2-mercaptoethanol} \mathrm{(MP}$ Biomedicals, Irvine, CA, USA), and non-essential amino acids (Sigma). Two to four cultured ICM clumps were collected for RT-PCR analysis on Days 1-4 from IVP embryos and on Days 2, 4, and 6 from in vivo-derived embryos. Total RNA was extracted from the collected samples using the Absolutely RNA Nanoprep Kit (Strategene, La Jolla, CA, USA). First-strand DNAs were synthesized using Superscript III (Invitrogen) and cDNAs were amplified with PfuUltra hotstart PCR mastermix (Stratagene). Primers were designed based on homology between human and mouse sequences and were validated using bovine tissues. In experiments spanning these critical first few days of culture, the pluripotency-related genes (Nanog, Oct-4, Sox-2) and components of the LIF (LIFR, Gp130), BMP (Bmpr1a, Id-1), and Wnt (Beta-catenin, Frizzled) pathways were expressed in the ICM cultures over the 4 days of IVP-ICM cultures and the 6 days of in vivo-derived ICM cultures. These results indicate that the markers of pluripotency and the components of signaling pathways implicated in the maintenance of murine embryonic stem cells are present in ICMs of Day 7-8 bovine blastocysts and continue to be expressed at least during the initial days of culture. Genes (NCAM, Lef1) associated with early differentiation, however, were also expressed. Whether their expression is an indicator of ICM differentiation or of residual contamination with trophectoderm remains to be determined. Further studies will determine whether stimulation of these pathways can facilitate efficient derivation and maintenance ruminant ES cells. 


\title{
5 ASSESSMENT OF HSP70.1 TRANSCRIPTION LEVELS IN BOVINE EMBRYOS AFTER CUMULUS REMOVAL BY DIFFERENT TECHNIQUES
}

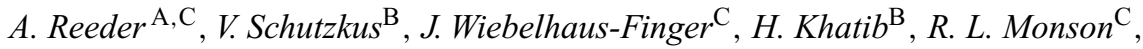 \\ M. B. Wheeler ${ }^{\mathrm{D}}$, D. Beebe ${ }^{\mathrm{E}}$, and J. Rutledge $\mathrm{C}^{\mathrm{C}}$ \\ ${ }^{A}$ Endocrinology and Reproductive Physiology Program, University of Wisconsin-Madison, Madison, WI 53706, USA; \\ ${ }^{B}$ Department of Dairy Science, University of Wisconsin-Madison, Madison, WI 53706, USA; \\ ${ }^{\mathrm{C}}$ Department of Animal Sciences, University of Wisconsin-Madison, Madison, WI 53706, USA; \\ ${ }^{D}$ Department of Animal Sciences, University of Illinois, Urbana, IL 61801, USA; \\ ${ }^{\mathrm{E}}$ Department of Biomedical Engineering, University of Wisconsin-Madison, Madison, WI 53706, USA
}

Previous results indicated that there was a difference in transcriptional activity depending on the method used to remove the cumulus cells after IVF (Zeringue et al. 2005 LabChip 5, 86-90). However, specific gene expression was not examined and therefore was the goal of the present study. The objective of this study was to compare the transcription levels of the HSP70.1 gene in bovine in vitro production (IVP) embryos that underwent cumulus removal by vortexing, hand stripping, or microfluidic treatment. Quantitative real-time PCR was used to estimate transcription levels of the HSP70.1 gene in bovine IVP zygotes and two-cell embryos. Transcription levels were compared between embryos that underwent three methods of cumulus removal. Presumptive zygotes were harvested at 2 or $24 \mathrm{~h}$ after cumulus removal by vortexing, by hand stripping, or by microfluidic means in order to compare the relative embryonic stress of these three treatments. Bovine in vitro embryos were produced by standard means with the only variable being the cumulus removal technique $24 \mathrm{~h}$ after fertilization. At 2 and $24 \mathrm{~h}$ post-cumulus removal, randomly selected presumptive zygotes were taken out of culture, preserved in RNAlater (Ambion, Inc., Austin, TX, USA) and stored at $-20^{\circ} \mathrm{C}$. RNA was extracted from single embryos via Qiagen's RNeasy Micro kit (Valencia, CA, USA). RNA was amplified and PCR products were detected with SYBR Green 1 (Applied Biosystems, Foster City, CA, USA) using an Opticon Monitor 3 real-time PCR machine (Bio-Rad Laboratories, Inc., Waltham, MA, USA). The threshold cycle $\left(\mathrm{C}_{\mathrm{T}}\right)$ numbers were determined for the amplified cDNA of the bovine HSP70.1 mRNA and for the housekeeping gene, acidic ribosomal phosphoprotein (PO), used as a reference. Then the amount of HSP70.1 was divided by the amount of PO to obtain a normalized HSP70.1 value expressed as the ratio of HSP70.1/PO. Ratios were analyzed by the GENMOD procedure in SAS (SAS Institute, Inc., Cary, NC, USA) accounting for replicates and treatments. Transcription levels of the HSP70.1 gene did not differ significantly between the microfluidically treated and vortexed groups of zygotes at $2 \mathrm{~h}$ post-cumulus removal $(P=0.1032)$ or between the hand-stripped and vortexed groups $(P=0.7567)$. In contrast, at $24 \mathrm{~h}$ post-cumulus removal, the embryos in the microfluidically treated group showed significantly higher levels of HSP70.1 transcription than the vortexed group $(P=0.0115)$. The transcription levels did not differ significantly between the hand-stripped and vortexed groups $(P=0.7875)$. This work strongly suggests that there is de novo RNA transcription in the early embryonic stages of the bovine. In addition to previously described improved developmental kinetics, the use of microfluidics in IVP leads to statistically significant differences in RNA transcription levels of HSP70.1.

\section{EXPRESSION PROFILING OF SINGLE BOVINE EMBRYOS REVEALS SIGNIFICANT EFFECTS OF IN VITRO MATURATION, FERTILIZATION AND CULTURE}

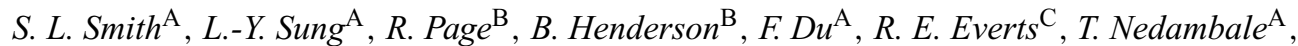 \\ S. Rodriguez-Zas ${ }^{\mathrm{C}}$, J.-P. Renard ${ }^{\mathrm{D}}$, H. A. Lewin ${ }^{\mathrm{C}}, X$. Yang $^{\mathrm{A}}$, and X. C. Tian $^{\mathrm{A}}$ \\ ${ }^{A}$ Department of Animal Science, Center for Regenerative Biology, University of Connecticut, Storrs, CT 06269, USA; \\ ${ }^{B}$ Cyagra, Inc., Elizabethtown, PA 19022, USA; \\ ${ }^{\mathrm{C}}$ Department of Animal Sciences, University of Illinois, Urbana, IL 61801, USA; \\ DUMR Biologie du Developement et Reproduction, Institut National de la Recherche Agronomique, Jouy-en-Josas, France
}

Cattle and sheep embryos transferred after in vitro production are often afflicted by large offspring syndrome (LOS), which has been correlated with the presence of serum and/or cell co-culture. Previous research indicates that post-fertilization culture affects blastocyst quality and gene expression, and in vitro oocyte maturation and fertilization impact developmental competence. To dissect the effects of in vitro maturation, fertilization, and culture, we compared the expression profiles of single bovine blastocysts generated by: (1) in vitro maturation, fertilization and culture (IVF, $n=15$ ); (2) in vivo maturation, in vivo fertilization, and in vitro culture (IVD, $n=14)$; and (3) in vivo maturation, fertilization, and development (AI, $n=14$ ). For in vitro culture, the embryos were cultured for 2 days in CR1 aa medium with bovine serum albumin (BSA) and then transferred to CR1aa with $10 \%$ fetal bovine serum (FBS) with cumulus cells until Day 7, at which time the embryos were vitrified. IVD zygotes were surgically collected from two superovulated Holstein donor cows $24 \mathrm{~h}$ post-insemination and cultured in the same system. To conduct expression profiling, total RNA was isolated from individual thawed embryos. The RNA was subjected to three rounds of amplification utilizing a previously adapted and validated T7 linear amplification protocol. Amplified RNA from each embryo and from a standard reference was indirectly labeled with Cy3 or Cy5 by dye swap and hybridized to a custom bovine cDNA microarray containing $~ 6300$ unique genes. After Loess normalization, an ANOVA model (GeneSpring 6.1 and SAS 9.0) was used to identify differentially expressed genes. The $P$-values were adjusted for multiple comparisons using the false discovery rate approach, and $\mathrm{a} \geq 2$-fold differential criterion was applied. A subset of the differentially expressed genes was verified by real-time RT-PCR. The blastocyst rates for IVF and IVD embryos were $37 \%$ and $75 \%$, respectively. There were 305,365 , and 200 genes differentially expressed between the $\mathrm{AI}$ and IVD, the IVF and IVD, and the AI and IVF comparisons, respectively. Interestingly, 44 differentially expressed genes were identified between the AI embryos and both the IVF and the IVD embryos, making these potential candidates for LOS. There were 61 genes differentially expressed between the IVF embryos and the AI and IVD embryos. The Gene Ontology categories 'RNA processing' and 'RNA binding' were over-represented among the genes that were down-regulated in the IVF embryos, indicating an effect of in vitro oocyte maturation/fertilization on embryonic gene expression. 
Artificial Insemination

\title{
7 PLASMA PROGESTERONE CONCENTRATIONS IN OVARIECTOMIZED COWS WITH INTRAVAGINAL DEVICES CONTAINING DIFFERENT LEVELS OF PROGESTERONE
}

\author{
M. Aviles ${ }^{\mathrm{A}}$, L. Cutaia $^{\mathrm{A}, \mathrm{D}}$, I. Videla Dorna ${ }^{\mathrm{B}, \mathrm{C}}, M \cdot A b a^{\mathrm{B}}$, and G. A. Bo ${ }^{\mathrm{A}, \mathrm{D}}$ \\ A Universidad Catolica de Córdoba, Córdoba, Argentina; \\ ${ }^{B}$ Universidad Nacional del Centro de la Provincia de Buenos Aires, Buenos Aires, Argentina; \\ ${ }^{\mathrm{C}}$ Syntex SA, Buenos Aires, Argentina; \\ DInstituto de Reproducción Animal Córdoba, JL de Cabrera 106 - X5000GVD, Córdoba, Argentina
}

\begin{abstract}
An experiment was designed to determine plasma progesterone (P4) concentrations in ovariectomized cows treated with either CIDR-B devices (Pfizer Animal Health, Hamilton, New Zealand) impregnated with $1.9 \mathrm{~g}$ of P4 or DIB devices (Syntex, Argentina) impregnated with 0.5 or $1.0 \mathrm{~g}$ of P4. Fifteen ovariectomized beef cows, weighing 350 to $450 \mathrm{~kg}$ and with a body condition score (BCS) 2.5 to 3.0 out of 5 were used. All cows were ovariectomized 30 days before the beginning of the trial and had plasma $\mathrm{P} 4$ concentrations $<1 \mathrm{ng} / \mathrm{mL}$ (measured by radioimmunoassay (RIA)) 10 days before the trial. On Day 0 , cows were stratified by weight and randomly assigned to one of three treatment groups to receive intravaginal devices containing $0.5 \mathrm{~g}$ of P4 (DIB 0.5), $1 \mathrm{~g}$ of P4 (DIB 1.0), or $1.9 \mathrm{~g}$ of P4 (CIDR-B). Blood samples were taken 12 and $24 \mathrm{~h}$ after intravaginal device insertion and then every $24 \mathrm{~h}$ until device removal on Day 7. Jugular blood samples were drawn into heparinized tubes and centrifuged within 20 min of collection; the plasma was harvested, frozen, and stored at $-10^{\circ} \mathrm{C}$. Progesterone was measured in plasma samples using a commercial solid-phase RIA (Coat-a-count; Diagnostic Products Corp., Los Angeles, CA, USA). All of the samples were analyzed at one time and the intra-assay coefficient of variation was $13 \%$. Plasma $\mathrm{P} 4$ concentrations were compared among treatments by calculating the area under the curve over time and testing for differences by ANOVA. Plasma P4 profiles were also analyzed by ANOVA for repeated measures. Analysis of plasma P4 profiles revealed a day effect $(P<0.001)$ but not a treatment effect $(P>0.13)$ or a day by treatment interaction $(P>0.16$; Table 1$)$. The area under the curve did not differ $(P=0.95)$ among groups (DIB 0.5: $2236.4 \mathrm{ng}$, DIB 1.0: $2164.6 \mathrm{ng}$, and CIDR-B: $2266.8 \mathrm{ng})$. In all treatments, plasma P4 profiles were characterized by a sudden increase in $\mathrm{P} 4$ concentrations within $12 \mathrm{~h}$ of device insertion and a gradual decrease during the following several days. It was concluded that the use of CIDR-B devices impregnated with $1.9 \mathrm{~g}$ of P4 or DIB devices impregnated with 1.0 or $0.5 \mathrm{~g}$ of P4 did not result in different plasma P4 concentrations in ovariectomized cows. Further studies are needed to confirm these results in lactating and dry intact (not ovariectomized) cattle.
\end{abstract}

Table 1. Mean plasma $P 4$ concentrations $(\mathrm{ng} / \mathrm{mL} \pm \mathrm{SEM})$ in ovariectomized cows treated with intravaginal devices containing $0.5 \mathrm{~g}$ (DIB 0.5), 1.0 g (DIB 1.0), or $1.9 \mathrm{~g}$ (CIDR-B) of P4*

\begin{tabular}{lccccccccc}
\hline Group & $0 \mathrm{~h}$ & $12 \mathrm{~h}$ & $24 \mathrm{~h}$ & $48 \mathrm{~h}$ & $72 \mathrm{~h}$ & $96 \mathrm{~h}$ & $120 \mathrm{~h}$ & $144 \mathrm{~h}$ & $168 \mathrm{~h}$ \\
\hline CIDR-B & $0.8 \pm 0.1$ & $6.4 \pm 0.7$ & $4.6 \pm 0.4$ & $4.7 \pm 0.4$ & $3.3 \pm 0.2$ & $3.1 \pm 0.2$ & $3.5 \pm 0.3$ & $2.6 \pm 0.3$ & $2.4 \pm 0.2$ \\
DIB 0.5 & $0.7 \pm 0.0$ & $6.8 \pm 1.2$ & $5.1 \pm 0.7$ & $4.1 \pm 0.3$ & $3.3 \pm 0.3$ & $2.2 \pm 0.2$ & $2.5 \pm 0.1$ & $1.9 \pm 0.4$ & $2.2 \pm 0.9$ \\
DIB 1.0 & $0.8 \pm 0.2$ & $5.7 \pm 0.8$ & $3.3 \pm 0.4$ & $2.8 \pm 0.1$ & $2.7 \pm 0.7$ & $1.9 \pm 0.1$ & $2.0 \pm 0.2$ & $1.3 \pm 0.1$ & $1.4 \pm 0.1$ \\
\hline
\end{tabular}

${ }^{*}$ Means did not differ significantly $(P>0.13)$.

\section{EFFECT OF DURATION OF TREATMENT WITH PROGESTERONE VAGINAL DEVICES ON PREGNANCY RATES IN CROSS-BRED ZEBU CATTLE ARTIFICIALLY INSEMINATED AT A FIXED TIME}

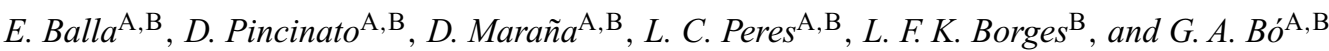 \\ ${ }^{\text {A } U n i v e r s i d a d ~ N a c i o n a l ~ d e ~ C o ́ r d o b a ; ~}$ \\ ${ }^{B}$ Instituto de Reproducción Animal Córdoba, J. Luis de Cabrera 106, X5000GVD, Córdoba, Argentina. \\ E-mail: gabrielbo@iracbiogen.com.ar
}

Three experiments were designed to evaluate the effect of the duration of treatment with progesterone (P4) vaginal devices ( $1 \mathrm{~g}$ of P4, Triu-B; Biogénesis, Buenos Aires, Argentina) on pregnancy rates in crossbred zebu cattle artificially inseminated at a fixed time (FTAI). In Exp. 1, 371 non-lactating multiparous crossbred zebu cows with a body condition score (BCS) between 2.0 and 3.0 out of 5 were randomly allocated to one of nine treatment groups in a $3 \times 3$ factorial design. On Day 0 , all cows received $2 \mathrm{mg}$ of estradiol benzoate (EB, Bioestrogen; Biogénesis) i.m. and were divided into groups to receive a new Triu-B device, a once-previously-used Triu-B (2nd-use), or a Triu-B that was previously used twice (3rd-use) and in which three silicone rings $(2 \mathrm{~cm}$ in length) impregnated with $100 \mathrm{mg}$ P4 each were threaded through the wings of the device. Triu-B devices are currently approved for re-use up to three times. Cows were further subdivided to have their Triu-B devices removed on Days 7, 8, or 9. PGF $(150 \mu \mathrm{g} \mathrm{D}(+)$ cloprostenol, Enzaprost-DC; Biogénesis) was administered i.m. at the time of Triu-B removal, followed by $1 \mathrm{mg}$ EB $24 \mathrm{~h}$ later; all cows were FTAI 52 to $56 \mathrm{~h}$ after Triu-B removal. In Exp. 2, 417 crossbred zebu heifers, 20 to 26 months of age and BCS between 2.5 and 3.5, 
were treated similarly to the cows in Exp. 1, except that only new or 3rd-use Triu-B were used. In Exp. 3, 836 crossbred zebu heifers, similar to those used in Exp. 2, were also treated similarly to the cows in Exp. 1, but in this case, Triu-B devices were inserted for 8, 9, or 10 days. Pregnancy rates were determined by ultrasonography 50 to 60 days after FTAI and were analyzed by logistic regression. In Exp. 1, there were no significant effects of duration of treatment [7 days: 69/137 (50.4\%), 8 days: 68/139 (48.9\%), or 9 days: $41 / 95(43.2 \%) ; P=0.28$ ], or type of Triu-B used $(P=0.91$; new Triu-B: $47.7 \%$, 2nd-use: $48.9 \%$, or 3rd-use: $47.2 \%$ ). In Exp. 2, there were no significant effects of duration of treatment [7 days: 76/139 (54.7\%), 8 days: $63 / 143(44.1 \%)$, or 9 days: $73 / 135(54.1 \%) ; P=0.98$ ] or type of Triu-B used (new Triu-B: $48.6 \%$ and 3rd-use Triu-B: $53.2 \% ; P=0.33$ ). In Exp. 3, there was an interaction between duration of treatment and pregnancy rates $(P<0.05)$. Lower pregnancy rates were obtained in $9-$ and 10-day treatments than in 8-day treatments, but only when previously used Triu-B devices were used. Pregnancy rates were: new Triu-B for 8 days: 42/89 (47.2\%), 9 days: 37/90 (41.1\%), and 10 days: 41/91 (45.1\%); 2nd-use Triu-B for 8 days: 51/103 (49.5\%), 9 days: $39 / 104$ (37.5\%), and 10 days: 39/109 (35.8\%); 3rd-use Triu-B for 8 days: 52/90 (57.4\%), 9 days: 31/90 (34.4\%), and 10 days: $25 / 70$ (35.7\%); $P<0.01$. Results demonstrate that treatment of crossbred zebu cattle with $\mathrm{P} 4$ devices for 7 to 10 days results in comparable pregnancy rates when new Triu-B devices are used. However, pregnancy rates decreased when previously used Triu-B devices were inserted for more than 8 days

\title{
9 USE OF SEXED SEMEN FOR ARTIFICIAL INSEMINATION IN HOLSTEIN HEIFERS IN MEXICO: PRELIMINARY RESULTS
}

\author{
A. Barba ${ }^{\mathrm{A}}$, Y. C. Ducolomb ${ }^{\mathrm{B}}$, and S. Romo ${ }^{\mathrm{C}}$ \\ A Private Practice, Tizayuca, Hidalgo, México; \\ ${ }^{B}$ Departamento de Ciencias de la Salud, UAM-I, México D.F. México; \\ ${ }^{\mathrm{C}}$ Departamento de Posgrado, Facultad de Estudios Superiores-Cuautitlán, UNAM, México D.F. México
}

\begin{abstract}
Nowadays, X- and Y- sperm can be sorted reliably by flow cytometry/cell sorting. However, this new technology is rarely being adopted by dairy farmers in Mexico and in developing countries, mainly due to the higher price of sexed semen straws and to a lack of information and knowledge of the results that can be obtained with its use under field conditions. In 2004, a field experiment started in a dairy farm in Tizayuca, State of Hidalgo, Mexico, aimed to examine fertility and sex ratios after artificial insemination (AI) in heifers under field conditions. Semen from two Holstein bulls was used for the experiment. The semen was sexed and frozen by XY Mexico (subsidiary of XY, Inc., Fort Collins, CO, USA). Only the X fraction of spermatozoa was sorted and frozen in $0.25 \mathrm{cc}$ plastic straws with $2.0 \times 10^{6}$ spermatozoa/straw. Sixty Holstein heifers averaging 14 months of age were artificially inseminated 12 hours after naturally occurring standing heat, using one straw of sexed semen, from June to October 2004. Fertility data of all inseminations were collected by rectal palpation from Days 45 to 60 after AI. A total of 30 heifers were pregnant, indicating a fertility of $50 \%$ froma single service. Sex of the calves was confirmed after calving between March and July 2005. From a total of 30 calves born after normal pregnancy, 27 were females $(90 \%)$ and 3 were males $(10 \%)$. In contrast, the pregnancy rate obtained in 60 heifers inseminated with non-sexed semen from the same two bulls was $68 \%$ (41 pregnant). From this group of heifers, 20 female (49\%) and 21 male $(51 \%)$ calves were born. Chi-square analysis indicated that pregnancy rates and sex ratios between the two groups were significantly different $(P<0.05)$. This preliminary information indicates that there is an advantage in the sex ratio obtained when using sexed semen for AI in Holstein heifers. This is especially important at a time when importation of replacement heifers to Mexico from Canada and the USA is not an option. However, more experiments are needed to confirm the results obtained in this preliminary study.
\end{abstract}

Sexed semen and Holstein heifers were provided by Rancho El Nacimiento, Establo 196, Tizayuca, State of Hidalgo, México.

\section{PREGNANCY RATE IN DAIRY COWS CROSSBREED HOLSTEIN x CARORA, DURING TWO SEASONS OF THE YEAR UNDER TWO ESTRUS SYNCHRONIZATION PROGRAMS AND TROPICAL CONDITIONS IN VENEZUELA}

\author{
M. Cabezas $^{\mathrm{A}}$, J. Cardenas $^{\mathrm{A}}$, T. Diaz ${ }^{\mathrm{B}}$, and A. A. Kowalski ${ }^{\mathrm{A}}$ \\ A Universidad Centroccidental Lisandro Alvarado, Decanato de Agronomia, Cabudare, Lara, Venezuela; \\ ${ }^{\mathrm{B}}$ Universidad Central de Venezuela, Instituto de Reproduccion Animal, Maracay, Aragua, Venezuela
}

Two experiments were performed in a commercial farm located in Lara, Venezuela. The objective was to evaluate the pregnancy rate in postpartum dairy cows with different genetic composition of Holstein $(\mathrm{H})$ and Carora $(\mathrm{C})$ breeds $(1 / 2,3 / 4$, and $7 / 8)$ during two seasons of the year using estrus synchronization programs. The first experiment evaluated the pregnancy rate of three different breeding groups in two seasons of the year. All cows $(n=305)$ were treated with the Heatsynch program (Select Sires, Inc., Miami, FL, USA): Cycling cows between Days 36 and 42 after parturition were injected with two doses of PGF $2 \alpha 14$ days apart. Seven days later, after the last injection of PGF2 $\alpha$, the cows were injected with a GnRH analog (Buserelin). Seven days later cows were injected with PGF $2 \alpha$ and after $24 \mathrm{~h}$ cows were injected with $1 \mathrm{mg}$ of estradiol cypionate (ECP). Cows were inseminated when heat was detected, and after $48 \mathrm{~h}$ all cows that did not present heat were inseminated. During the period from January to May, 152 cows were treated. The average temperature (T) was $25.22^{\circ} \mathrm{C}$ and relative humidity $(\mathrm{RH})$ was $70.16 \%$. During the period from June to October, 153 cows were treated. The $\mathrm{T}$ was $25.26^{\circ} \mathrm{C}$ and $\mathrm{RH}$ was $72.58 \%$. The pregnancy rates for the groups were $1 / 2 \mathrm{H} \times \mathrm{C}, n=89,28.08 \% ; 3 / 4 \mathrm{H} \times \mathrm{C}, n=143$, $25.17 \%$; and $7 / 8 \mathrm{H} \times \mathrm{C}, n=73,26.02 \%$; they were not significantly different $(P<0.14)$. However, the pregnancy rate was higher in January-May $(30.92 \%)$ than in June-October $(22.87 \% ; P<0.05)$. The second experiment evaluated pregnancy rates in the different breeding groups $(\mathrm{H} \times \mathrm{C})$, using two protocols, Heatsynch vs. Ovsynch (Select Sires, Inc.). The Ovsynch program is similar to the Heatsynch program; however, the last injection of ECP in the Heatsynch program is changed to the GnRH analog after $48 \mathrm{~h}$ from the last injection of PGF2 $\alpha$. Cows were inseminated $24 \mathrm{~h}$ 
after the GnRH injection. Pregnancy rate was lower for cows treated with the Heatsynch program $(21.15 \%)$ than for those treated with the Ovsynch program $(45.28 \% ; P<0.01)$. Furthermore, cows in the $1 / 2$ and $3 / 4$ breeding groups had higher pregnancy rates than those in the $7 / 8$ breeding group $(1 / 2=42.05 \%, 3 / 4=38.33 \%$, and $7 / 8=16.72 \% ; P<0.01)$. Data were analyzed using logistic regression analysis. In conclusion, the introduction of the Carora breed in the dairy herd increased the adaptability of the Holstein cows to tropical conditions by increasing their reproductive efficiency. Furthermore, pregnancy rates were increased using synchronization programs that did not require heat detection. This demonstrated the possibility of increasing reproductive performance in the tropics by incorporating estrus synchronization systems that do not require heat detection. These studies also demonstrated that the incorporation of dairy breeds adapted to tropical conditions, such as the Carora breed, aids in improving reproductive performance under high RH conditions.

\title{
11 EFFECTS OF CIDR-BASED PRESYNCHRONIZATION AND ECG ON FERTILITY FOR A GnRH-BASED TIMED-AI PROTOCOL IN BEEF CATTLE
}

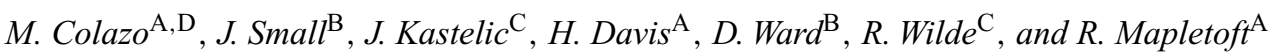 \\ ${ }^{A}$ WCVM, University of Saskatchewan, Saskatoon, SK, Canada; \\ ${ }^{B}$ Agriculture and Agri-Food Canada, Brandon, MB, Canada; \\ ${ }^{\mathrm{C}}$ Agriculture and Agri-Food Canada, Lethbridge, $\mathrm{AB}$, Canada; \\ ${ }^{\mathrm{D}}$ Alberta Agriculture, Food and Rural Development, Edmonton, AB, Canada
}

The objectives were to determine the effects of presynchronization and eCG on fertility for a GnRH-based timed-AI (TAI) protocol in beef cattle. Previously autoclaved once-used CIDR inserts (Colazo et al. 2004 Anim. Reprod. Sci. 81, 25-34) were used for experimental purposes so that all cattle had equivalent exogenous progesterone after Day 7 in Experiment 1, and to induce GnRH-responsive follicles in Experiment 2. In Experiment 1, 12-15 month old beef heifers $(n=447)$ were used (three locations, A, B, and C). On Day 0, half received a new CIDR insert (Pfizer Animal Health, Montreal, Quebec, Canada) and $500 \mu \mathrm{g}$ of cloprostenol i.m. (PGF: Estrumate; Schering-Plough Animal Health, Pointe-Claire, Quebec, Canada). On Day 7, the remainder received an autoclaved once-used CIDR and all received $100 \mu \mathrm{g}$ of GnRH i.m. (Cystorelin; Merial Canada, Inc., Victoriaville, Quebec, Canada). On Day 14, CIDR inserts were removed, PGF was given to all heifers, and heifers were given either $300 \mathrm{IU}$ eCG i.m. (Pregnacol; Bioniche Animal Health, Belleville, Ontario, Canada) or no treatment $(2 \times 2$ factorial design). On Day 16 (54-56h after CIDR removal and PGF administration), heifers were given $100 \mu \mathrm{g}$ of GnRH i.m., concurrent with TAI. Transrectal ultrasonography was done on Day 0 to assess ovarian structures, and on approximately Day 50 (range, 44-69) to confirm pregnancy. Overall, $72.7 \%$ of heifers were puberal on Day $0(150 / 150,5 / 37$, and 170/260 for locations A, B, and C, respectively; $P<0.001)$. Pregnancy rate was affected $(P<0.001)$ by location $(27.3,62.2$, and $51.1 \%$ for the three locations, respectively) but was not affected $(P=0.3)$ by puberal status at locations B and C. Pregnancy rate was not affected $(P=0.5)$ by presynchronization, but tended $(P=0.1)$ to be reduced by eCG treatment ( $40.4 \mathrm{vs} .47 .7 \%$, respectively). In Experiment 2 , lactating crossbred beef cows $(n=411), 2-12$ years of age were randomly allocated to receive either a previously used, autoclaved CIDR for 7 days and $25 \mathrm{mg}$ of dinoprost i.m. (PGF: Lutalyse; Pfizer Animal Health) or no treatment (control; Day 0). At CIDR removal (Day 7), all cows received $100 \mu \mathrm{g}$ of GnRH. On Day 14, all cows received PGF and were allocated to receive either $400 \mathrm{IU}$ of eCG i.m. or no treatment $(2 \times 2$ factorial design). On Day 16 (54-56 h after PGF), cows were given $100 \mu \mathrm{g}$ of GnRH concurrent with TAI. Transrectal ultrasonographic examinations were done on approximately 65 cows in each group on Days 0, 7, and 14 to assess ovarian structures and on all cows on Day 42 to confirm pregnancy. Overall, $88.8 \%$ of cows had a corpus luteum (CL) on Day 0. Presynchronization increased both the proportion of cows that ovulated to GnRH treatment on Day 7 (76.7 vs. $55.0 \%$; $P<0.001)$ and pregnancy rate $(58.2$ vs. $45.4 \% ; P=0.03)$ for cows that had received a CIDR vs. control cows. However, eCG treatment did not affect pregnancy rate $(P=0.3)$ in either group. In summary, presynchronization with a used CIDR and PGF prior to a Cosynch protocol increased ovulation rate to the first GnRH treatment and pregnancy rate in cows but not in heifers. In a GnRH-based TAI protocol, treatment with eCG had no significant effect on fertility in cows, but tended to decrease fertility in heifers.

\section{THE EFFECT OF PROGESTERONE CONTENT IN A VAGINAL INSERT ON PREGNANCY RATES IN BEEF AND DAIRY CATTLE INSEMINATED AT FIXED TIME}

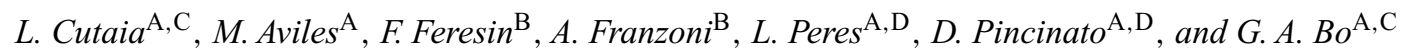 \\ ${ }^{A}$ Universidad Catolica de Cordoba; ${ }^{B}$ Actividad Privada; ${ }^{C}$ Instituto de Reproducción Animal Córdoba (IRAC), Jerónimo L. de Cabrera 110, \\ X5000GVD; ${ }^{D}$ Universidad Nacional de Cordoba, Córdoba, Argentina
}

Three experiments were conducted to compare pregnancy rates in cows and heifers treated with intravaginal progesterone (P4)-releasing devices impregnated with either 0.5 or $1.0 \mathrm{~g}$ of P4 and inseminated at a fixed time (FTAI). In Experiment 1, 102 postpartum beef cows (Hereford and Angus crossbred with Tuli, 60 to $90 \mathrm{~d}$ postpartum) with a body condition score (BCS) of 2.5 to 3.0 ( 1 to 5 scale) were used. On Day 0 , all cows received $2 \mathrm{mg}$ of estradiol benzoate (EB; Syntex, Argentina) i.m. and were randomly assigned to receive an intravaginal progesterone-releasing device with $0.5 \mathrm{~g}$ of P4 (DIB $0.5 \mathrm{~g}$; Syntex) or $1.0 \mathrm{~g}$ of P4 (DIB $1.0 \mathrm{~g}$; Syntex). On Day 7, DIB devices were removed and all cows received $150 \mu \mathrm{g} \mathrm{D}(+)$ cloprostenol (Ciclase; Syntex) i.m. On Day 8, all cows received $1 \mathrm{mg}$ EB and were FTAI 52 to $56 \mathrm{~h}$ after DIB removal. In Experiment 2, 93 Holstein cows that were 90 to 110 days in milk (DIM), producing on average $5000 \mathrm{~kg}$ of milk per lactation and with a BCS of 2.5 to 3.0 received the same treatments as those in Experiment 1. In Experiment 3,100 Holstein heifers, 20 to 22 months old, with a BCS of 2.5 to 3.0, also received the same treatments as those in Experiment 1. Pregnancy rates in all experiments were determined by ultrasonography 35 days after FTAI and compared by chi-square test. No differences in pregnancy rates were detected between lactating beef cows, dairy cows, or dairy heifers treated with DIB devices impregnated 
Table 1. Pregnancy rates in postpartum beef cows, Holstein cows, and Holstein heifers treated with DIB devices impregnated with 0.5 or $1.0 \mathrm{~g}$ of P4 and subjected to FTAI

\begin{tabular}{lccc}
\hline & DIB 1.0 g P4 & DIB 0.5 g P4 & $P$-value \\
\hline Exp. 1 (Beef cows) & $29 / 52(55.7 \%)$ & $26 / 50(52.0 \%)$ & 0.70 \\
Exp. 2 (Holstein cows) & $23 / 47(48.9 \%)$ & $22 / 46(47.8 \%)$ & 0.91 \\
Exp. 3 (Holstein heifers) & $29 / 50(58.0 \%)$ & $27 / 50(54.0 \%)$ & 0.68 \\
\hline
\end{tabular}

with 0.5 or $1.0 \mathrm{~g}$ of $\mathrm{P} 4$ (Table 1). It was concluded that vaginal P4 releasing devices impregnated with $0.5 \mathrm{~g}$ or $1.0 \mathrm{~g}$ of P4 results in comparable pregnancy rates in FTAI protocols in beef and dairy cattle.

\title{
13 EFFECTS OF PLASMA PROGESTERONE CONCENTRATIONS ON LH RELEASE AND OVULATION IN BEEF CATTLE GIVEN GnRH
}

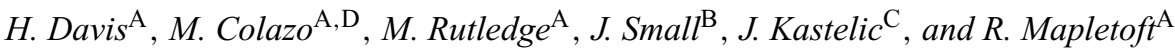 \\ ${ }^{\mathrm{A}}$ WCVM, University of Saskatchewan, Saskatoon, SK, Canada; \\ ${ }^{\mathrm{B}}$ Agriculture and Agri-Food Canada, Brandon, MB, Canada; \\ ${ }^{\mathrm{C}}$ Agriculture and Agri-Food Canada, Lethbridge, $\mathrm{AB}$, Canada; \\ ${ }^{\mathrm{D}}$ Alberta Agriculture, Food and Rural Development, Edmonton, AB, Canada
}

Two experiments were conducted to determine the effects of plasma progesterone (P4) concentrations on LH release and ovulation in beef heifers and lactating beef cows given gonadotropin-releasing hormone (GnRH). Previously autoclaved, once-used CIDR inserts (Colazo et al. 2004 Anim. Reprod. Sci. 81, 25-34) were used for experimental purposes to induce differential plasma progesterone concentrations. In Experiment 1, postpubertal heifers received $25 \mathrm{mg}$ of dinoprost i.m. (prostaglandin F (PGF); Lutalyse; Pfizer Animal Health, Montreal, Quebec, Canada). On Day 4 (estrus $=$ Day 0 ), heifers were randomly assigned (10/group) to receive no treatment (control) or 1 or 2 autoclaved once-used CIDR (Pfizer Animal Health) inserts (1CIDR and 2CIDR, respectively). On Day 5, heifers in the 1CIDR group were given PGF twice $12 \mathrm{~h}$ apart. On Day 6 , all heifers received $100 \mu \mathrm{g}$ of GnRH i.m. (Cystorelin; Merial Canada, Inc., Victoriaville, Quebec, Canada). Once daily on Days 4 to 9, a blood sample was collected and ultrasonography was performed. On Day 6 , heifers in the control $(3.0 \pm 0.4 \mathrm{ng} / \mathrm{mL}$; mean \pm SD) and 1 CIDR groups $(3.0 \pm 0.3 \mathrm{ng} / \mathrm{mL}) \mathrm{had}$ lower $(P<0.01)$ plasma progesterone concentrations than those in the 2CIDR group $(5.7 \pm 0.4 \mathrm{ng} / \mathrm{mL})$. However, the diameter of the dominant follicle was larger $(P<0.001)$ in heifers in the control and 1CIDR groups than in the 2CIDR group $(12.1 \pm 1.0,11.5 \pm 0.7$, and $10.1 \pm 0.7 \mathrm{~mm}$, respectively $)$. More $(P<0.01)$ heifers ovulated in response to GnRH in the control and 1CIDR groups than in the 2CIDR group $(10 / 10,9 / 10$, and 3/10, respectively). In Experiment 2, ultrasound-guided follicular ablation (FA) was performed (to synchronize ovarian follicular wave emergence) 4 to 6 days after estrus in 20 postpubertal heifers and 20 mature lactating cows. Cattle were randomly and equally assigned to receive an autoclaved, once-used CIDR, either with no further treatment (High-P4) or with two PGF treatments $12 \mathrm{~h}$ apart (Low-P4) given after FA. All cattle received $100 \mu \mathrm{g}$ of GnRH either 6 days after FA or the day after the dominant follicle reached $9 \mathrm{~mm}$ in diameter. Ultrasonography was performed daily (from 4 days after FA to ovulation or to 3 days after GnRH treatment). In three cows and three heifers per group, blood samples were collected every $30 \mathrm{~min}$ for $12 \mathrm{~h}$ after GnRH. The dominant follicle at GnRH treatment was larger in cows than heifers $(11.0 \pm 1.1$ vs. $10.3 \pm 0.9 \mathrm{~mm}$, respectively; $P=0.05)$ and tended to be smaller in the High-P4 group vs. the Low-P4 group $(10.3 \pm 1.0$ vs. $11.0 \pm 1.0 \mathrm{~mm} ; P=0.06)$. Ovulatory response was not different $(P=0.9)$ between heifers $(77.7 \%)$ and cows $(78.9 \%)$, but combined for heifers and cows, was lower in High-P4 vs. Low-P4 cattle (61.1 vs. 94.7 ; $P<0.01)$. The GnRH-induced LH surge did not differ $(P=0.23)$ between cows and heifers, but it was lower and of shorter duration $(P<0.001)$ in the High-P4 group than in the Low-P4 group. In summary, higher plasma P4 concentrations resulted in decreased LH release and the proportion of cattle ovulating in response to GnRH treatment. There was no significant difference between heifers and cows in LH release or ovulatory response.

\section{SYNCHRONIZATION OF OVULATION IN ANESTROUS NELORE COWS TREATED WITH HORMONAL PROTOCOL WITHOUT PROGESTERONE OR PROGESTAGENS}

\author{
A. F. Souza ${ }^{\mathrm{A}}$, V. G. Pinheiro ${ }^{\mathrm{A}}$, R. L. Ereno ${ }^{\mathrm{B}}$, and C. M. Barros ${ }^{\mathrm{A}}$ \\ ${ }^{A}$ Department of Pharmacology, Institute of Bioscience, University of São Paulo State (UNESP), Botucatu, São Paulo, Brazil; \\ ${ }^{\mathrm{B}}$ Department of Animal Reproduction, FMVZ - UNESP, Botucatu, São Paulo, Brazil
}

Postpartum anestrous cows are usually treated with progesterone/progestagen protocols in order to induce ovulation and sustain pregnancy. In the present work, the efficiency of a protocol (modified GPE), in which temporary calf removal (TCR) and/or administration of eCG, replaced the use of progesterone/progestagens, was evaluated in anestrous animals. Anestrous Nelore cows (40 to 60 days postpartum, $n=22$ ) were randomly allocated to two groups: GPE/eCG and TCR/GPE/eCG. At a random stage of the estrous cycle (D0), animals from Group GPE/eCG were treated with GnRH $\left(50 \mu \mathrm{g}\right.$, licereline, i.m., Gestran Plus ${ }^{\circledR}$; Tecnopec, Sao Paulo, Brazil) and seven days later (D7) they received PGF2 $\alpha$ (150 mcg, d-cloprostenol, i.m., Prolise ${ }^{\circledR}$ ) and eCG (300 UI, i.m., Novormon ${ }^{\circledR}$ ). On D8, estradiol benzoate (EB, $1 \mathrm{mg}$, Estrogin ${ }^{\circledR}$; Tecnopec) was administered, and 30 to $36 \mathrm{~h}$ afterwards all cows were inseminated at fixed-time (FTAI), without estrus detection. The animals from group TCR/GPE/eCG received the same treatment described above, but with temporary calf removal (during $48 \mathrm{~h}$ ) before beginning hormonal treatments. The ovaries were examined by 
ultrasonography (Aloka SSD 500, 7.5 MHz probe) before (D-12, D-2), during (D0, D7, D8), and after (D9, D10, D11, D17, D60) hormonal treatments. The absence of CL, during the ultrasonography performed before starting the treatments, was the criterion used to classify the cows as in postpartum anestrus. Administration of GnRH (D0) induced ovulation (determined by the presence of CL on D7) in 6 of 11 cows (54.5\%) from Group GPE/eCG and TCR/GPE/eCG. In relation to synchronization of ovulation after EB administration, in Group GPE/eCG, two cows (18.1\%) ovulated until $12 \mathrm{~h}$ after FTAI, and four (36.3\%) $12 \mathrm{~h}$ afterwards ( $24 \mathrm{~h}$ after FTAI). In Group TCR/GPE/eCG, one cow (9\%) ovulated approximately $6 \mathrm{~h}$ before FTAI, two (18.1\%) $12 \mathrm{~h}$ after FTAI, and two (18.1\%) $12 \mathrm{~h}$ afterwards. Preovulatory follicles had a diameter of $11.0 \pm 1.3 \mathrm{and} 11.5 \pm 2.6 \mathrm{~mm}$ in Groups GPE/eCG and TCR/GPE/eCG, respectively. Pregnancy rates, determined by ultrasonograpy 40 to 46 days after FTAI, were $27.2 \%$ (3/11) and $45.4 \%(5 / 11)$, respectively. The results indicate that association of TCR with protocol GPE/eCG did not improve synchronization of ovulation or pregnancy rates $(P>0.005)$. However, increase in pregnancy rates was observed in another experiment with a large number of animals (Pinheiro et al. 2005 Reprod. Fert. Dev. 17, 161, abstr), in which cows from Group TCR/GPE/eCG had higher pregnancy rates $(51.5 \%$; 34/66) when compared to animals from Group GPE/eCG $(28.3 \%, 21 / 74 ; P<0.05)$.

This work was supported by FAPESP and by fellowships for A. F. Souza and V. G. Pinheiro from CNPq, Brazil.

\title{
15 INCREASING ESTRUS DETECTION RATES WITHIN FOUR DAYS IN A PROSTAGLANDIN-BASED ESTRUS SYNCHRONIZATION PROGRAM IN BOS INDICUS BEEF (SISTANI) CATTLE
}

\author{
M. D. Jalinous ${ }^{\mathrm{A}}$, A. Niasari-Naslaji $^{\mathrm{A}} \mathrm{B}$, F. Sarhaddi $^{\mathrm{B}}$, and E. Naghzali ${ }^{\mathrm{C}}$ \\ A Department of Clinical Sciences, Faculty of Veterinary Medicine, University of Tehran, Tehran, Iran; \\ ${ }^{B}$ Animal Science Research Institute, Karaj, Tehran, Iran; \\ ${ }^{\mathrm{C}}$ Research Center for Agriculture and Natural Resources, Ministry of Jihad-e-Agriculture, Zabol, Sistan and Balouchestan, Iran
}

\begin{abstract}
Variation in estrous response is a major concern in estrus synchronization programs using prostaglandin $\mathrm{F}_{2 \alpha}$. The objective of this study was to confine estrous detection within two phases ( $48 \mathrm{~h}$ each) after synchronizing follicular wave emergence using ovarian follicle regressing (first phase) and ovulating (second phase) agents. Cyclic Sistani cattle (Iranian Bos indicus beef breed) at unknown stages of the estrous cycle and $\geq 58$ days open were randomly assigned to two groups in mid summer. Females received a ration comprised of wheat straw (39\%), Lucerne hay ( $28 \%)$ and concentrate $(33 \%)$ that comprised $10 \%$ crude protein and energy at $2.1 \mathrm{Mcal} / \mathrm{kg} \mathrm{DM}$, according to NRC recommendations for beef cattle. In phase one, females in Group 1 ( $n=33$; heifers: 9; lactating cows: 4; dry cows: 20; $59 \pm 5.8$ months of age; $361.2 \pm 11.5 \mathrm{~kg} \mathrm{LW}$; and $2.1 \pm 0.41$ lactations) received estradiol benzoate ( $2 \mathrm{mg}$, i.m., Aburaihan Pharmaceutical Co., Tehran, Iran) and progesterone (100 mg, i.m., Aburaihan, Iran) followed by an injection of prostaglandin analogue (15 mg, i.m., Prosolvin; Intervet, Boxmeer, The Netherlands) and PMSG (400 IU, i.m., Folligon; Intervet) on Day 8. Females in Group $2(n=35$; heifers: 9 ; lactating cows: 5 ; dry cows: $21 ; 59.3 \pm 5.7$ months of age; $356.8 \pm 11.5 \mathrm{~kg} \mathrm{LW}$; and $2 \pm 0.35$ lactations $)$ received two consecutive injections of prostaglandin 14 days apart. In the second phase, starting $72 \mathrm{~h}$ after prostaglandin injection, females in both groups that did not exhibit estrus were given an intra-muscular injection of Gonadorelin (100 $\mu \mathrm{g}$, Fertagyl, Intervet) followed by prostaglandin injection 7 days later. In both phases, standing estrus was recorded for $48 \mathrm{~h}$, after prostaglandin injection, at 4-h intervals, 30 min each time. Within a defined period ( $48 \mathrm{~h}$ after prostaglandin injection) $12 \mathrm{~h}$ after standing estrus, females were inseminated with frozen semen. Pregnancy was diagnosed on Day 30 after insemination using ultrasonography. Data were analyzed using logistic regression and chi-square tests. There was no significant effect of parity and lactation status on the frequency of estrus and conception rates $(P>0.05)$. The total frequency of observed standing estrus throughout two phases for Group 1 and two females were $81.8 \%$ and $80 \%$, respectively $(P>0.05)$. The conception rates from a single AI for Group 1 [1st phase: $70.6 \%(12 / 17)$; 2nd phase: $40 \%(4 / 10)$; total: $59.2 \%(16 / 27)]$ did not differ $(P>0.05)$ to that for Group 2 [1st phase: $66.7 \%(8 / 12)$; 2nd phase: $56.2 \%(9 / 16)$; total: $60.7 \%(17 / 28)]$. The result of this study demonstrated that estrous detection rates of $\geq 80 \%$ can be achieved within 4 day observation period in Bos indicus beef cattle.
\end{abstract}

The authors express their great appreciations to the Intervet Company, Holland and the Aburaihan Company, Iran, for kind provision of pharmaceutical materials to perform this experiment.

\section{OVULATION AND PREGNANCY RATES IN POSTPARTUM BOS INDICUS COWS TREATED WITH PROGESTERONE VAGINAL DEVICES AND ESTRADIOL BENZOATE, WITH OR WITHOUT ECG AND TEMPORARY WEANING}

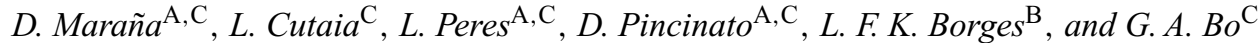 \\ ${ }^{A}$ Universidad Nacional de Córdoba; ${ }^{B}$ Universidad Federal de Santa Maria; ${ }^{C}$ Instituto de Reproducción Animal Córdoba (IRAC), Jerónimo L. de \\ Cabrera 110, X5000GVD, Córdoba, Argentina
}

Two experiments were designed to compare the effects of eCG treatment and temporary weaning on ovulation and pregnancy rates in postpartum cows. In Experiment 1, 39 lactating multiparous crossbred Bos indicus cows, 60 to 80 d postpartum with a body condition score (BCS) between 2.0 to 2.5 out of 5 were randomly allocated to one of four treatment groups in a $2 \times 2$ factorial design. On Day 0 , all cows received a progesterone (P4) intravaginal device (DIB; Syntex, Argentina) and $2 \mathrm{mg}$ estradiol benzoate (EB) i.m. (Syntex). On Day 8, DIB devices were removed and all cows received $150 \mu \mathrm{g} \mathrm{D}(+)$ cloprostenol i.m. (Ciclase; Syntex) and were randomly divided to receive $400 \mathrm{IU}$ eCG i.m. (Novormon 5000; Syntex) at the same time or no treatment. In addition, half of the cows in each group had their calves weaned temporarily (TW) for $56 \mathrm{~h}$ at the time of DIB removal; the other half remained with their calves. All cows received $1 \mathrm{mg}$ EB i.m. on Day 9 and were examined every $8 \mathrm{~h}$ by ultrasonography from the time of 
DIB removal until ovulation. Data were analyzed by ANOVA and chi-square test. The diameter of the dominant follicle on Day 8 (eCG: $7.8 \pm 0.5 \mathrm{~mm}$ vs. no eCG: $8.2 \pm 0.4 \mathrm{~mm}$, and TW: $7.6 \pm 0.4 \mathrm{~mm}$ vs. no TW $8.4 \pm 0.5 \mathrm{~mm}$ ), the proportion of cows that ovulated (eCG: $12 / 20,60 \%$ vs. no eCG: $9 / 19$, $47 \%$; and TW: $13 / 20,65 \%$ vs. no TW: $8 / 19,42 \%$, and the interval to ovulation (eCG: $72.0 \pm 1.4 \mathrm{~h}$ vs. no eCG: $75.6 \pm 2.0 \mathrm{~h}$, and TW: $73.8 \pm 1.6 \mathrm{~h} \mathrm{vs}$. no TW: $73.0 \pm 1.8 \mathrm{~h})$ did not differ among groups $(P>0.05)$. Although there was no effect of eCG treatment or eCG by TW interaction $(P>0.3)$ on the size of the preovulatory follicle (eCG: $11.1 \pm 0.4 \mathrm{~mm}$ vs. no eCG: $10.1 \pm 0.6 \mathrm{~mm}$ ), it was smaller in cows TW $(9.9 \pm 04 \mathrm{~mm})$, compared to those not TW $(11.8 \pm 0.3 \mathrm{~mm} ; P<0.05)$. Nevertheless, the growth rate of the ovulatory follicle was greater $(P<0.02)$ in cows treated with eCG $(1.1 \pm 0.1 \mathrm{~mm} /$ day $)$ than in those not treated with eCG $(0.6 \pm 0.1 \mathrm{~mm} /$ day $)$. In Experiment 2769 lactating multiparous crossbred Bos indicus cows received the same treatments as those in Experiment 1 and were fixed-time inseminated (FTAI) 52 to $56 \mathrm{~h}$ after DIB removal. Cows were examined by ultrasonography on Day 0 (22.5\% with corpora lutea $(\mathrm{CL}), 30.0 \%$ with follicles $>8 \mathrm{~mm}$, and $47.5 \%$ with follicles $<8 \mathrm{~mm})$ and $42 \mathrm{~d}$ after FTAI to determine pregnancy. Data were analyzed by logistic regression. Pregnancy rates were not affected $(P>0.7)$ by TW (TW: $141 / 397,37.2 \%$ vs. no TW: $141 / 390,36.1 \%)$ or the TW by eCG interaction $(P>0.7)$. However, pregnancy rates were higher $(P<0.05)$ in cows treated with eCG $(154 / 377$, $40.8 \%)$ than in those not treated with eCG $(128 / 392,32.6 \%)$. The use of eCG but not TW improved pregnancy rates following FTAI in postpartum Bos indicus cows. Results also suggest that the eCG-related increase in pregnancy rates may be due to the final growth rate of the ovulatory follicle.

\title{
17 FLOW CYTOMETRY ANALYSIS OF THE TURKEY SPERM GLYCOCALYX: EFFECTS OF NEURAMINIDASE TREATMENT
}

\author{
J. Pelaez and J. Long \\ Biotechnology and Germplasm Laboratory, ANRI, ARS, USDA, Beltsville, MD 20705, USA
}

\begin{abstract}
The surface of all eucaryotic cells consists of a carbohydrate-rich zone known as the glycocalyx which mediates a variety of specific cell-recognition processes, including sperm-egg interactions. Several carbohydrate residues are known to be necessary for poultry sperm to traverse the female reproductive tract and for sperm-egg recognition. Alteration of the sperm glycocalyx during semen storage could adversely impact fertility. Accordingly, the aim of this work was to elucidate the sugar residues existing in the surface of turkey spermatozoa as a first step in understanding the deleterious effects of semen storage on sperm physiology and function. Nine turkey toms were randomly assigned to 1 of 3 groups ( 3 males/group) and semen was pooled for each group. Seminal plasma was removed and isolated sperm were resuspended in $\mathrm{Tris}-\mathrm{NaCl}$ buffer at either (1) $\mathrm{pH}$ 7.4 for immediate staining with 1 of 16 FITC-conjugated lectins $\left(100 \mu \mathrm{g} / \mathrm{mL} ; 30 \mathrm{~min} ; 25^{\circ} \mathrm{C} ; 100 \times 10^{6}\right.$ cells $\left./ \mathrm{mL}\right)$, or $(2) \mathrm{pH} 6.0$ for neuraminidase treatment $\left(30 \mathrm{~min} ; 37^{\circ} \mathrm{C} ; 1 \mathrm{IU} / 10^{9}\right.$ cells $)$, followed by washing $(700 \mathrm{~g}, 5 \mathrm{~min} ; 4$ times $)$ and lectin staining. The control consisted of sperm cells incubated only in Tris- $\mathrm{NaCl}$ buffer. Stained and control samples were washed twice $(700 \mathrm{~g}, 5 \mathrm{~min})$ and counterstained with $12 \mu \mathrm{M}$ propidium iodide (PI); the green fluorescence intensity of PI-negative cells was assessed using a Coulter EPICS XL-MCL TM Flow Cytometer (Beckman Coulter, Inc., Fullerton, CA, USA) and single-parameter histogram analysis (6 replicates). Median fluorescence intensity (MdFI) was used to assess differences in the presence of sugar residues among the control, non-neuraminidase-treated, and neuraminidase-treated samples (Kruskal-Wallis test; Tukey's HSD test in post-hoc comparisons). Lectin staining occurred in the PI-positive subpopulation, or membrane-disrupted cells, of all samples. By contrast, only intact cells were labeled by LPA, ConA, RCA-I, and s-WGA lectins in the non-neuraminidase-treated samples, indicating that only residues of sialic acid, $\alpha$-mannose/ $\alpha$-glucose, $\beta$-galactose, and $\mathrm{N}$-acetylglucosamine oligomers were on the surface. As expected, removal of sialic acid significantly $(P<0.05)$ decreased the fluorescence intensity of LPA-labeled cells. This treatment significantly $(P<0.05)$ increased the affinity of ConA, RCA-I, and s-WGA lectins, and exposed sugar residues previously undetected using ECA (N-acetyllactosamine), GNA ( $\alpha-$ mannose), GS-I ( $\alpha$-galactose), GS-II (N-acetylglucosamine monomers), LOTUS ( $\alpha$-L-fucose), PNA ( $\beta$-galactose), SBA ( $\alpha$-N-acetylgalactosamine), and WFA ( $\beta$-N-acetylgalactosamine) lectins. Low levels of MdFI were observed for cells labeled by JAC ( $\alpha$-galactose), PSA ( $\alpha$-mannose/ $\alpha$-glucose), STA $(\mathrm{N}$-acetylglucosamine polymers) and UEA-I ( $\alpha$-L-fucose) irrespective of neuraminidase treatment $(P>0.05)$. These observations suggest that $(1)$ the carbohydrate content of turkey sperm glycocalyx is extensively masked by sialic acid molecules, (2) it contains a diversity of sugar residues, and (3) discrimination between intact and damaged cells needs to be done in order to properly identify surface binding.
\end{abstract}

\section{DO TEMPORARY CALF REMOVAL AND/OR EQUINE CHORIONIC GONADOTROPIN ADMINISTRATION INCREASE PREGNANCY RATES IN LACTATING NELORE COWS TREATED WITH A PROGESTERONE-RELEASE INTRAVAGINAL DEVICE?}

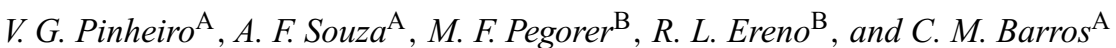 \\ ${ }^{A}$ Department of Pharmacology, Institute of Bioscience, University of São Paulo State (UNESP), Botucatu, São Paulo, Brazil; \\ ${ }^{B}$ Department of Animal Reproduction, FMVZ - UNESP, Botucatu, São Paulo, Brazil
}

Reports indicate that either temporary calf removal or equine chorionic gonadotropin administration can increase the efficiency (pregnancy rate) of hormonal treatments with progestins during postpartum anestrus. This experiment evaluated effects of TCR and/or eCG administration in a protocol with progesterone that is frequently used for fixed-time artificial insemination (FTAI) in cows during postpartum anestrus. The protocols were tested at three farms in lactating Nelore cows (40 to 70 days post-partum, $n=361$ ) with body condition scores from 2.5 to 3.0 ( 0 - to 5 -point scale). At a random stage of the estrous cycle (Day 0), animals received a basic PEPE (progesterone-estrogen-prostaglandin-estrogen) protocol with insertion of an intravaginal device with $1.0 \mathrm{~g}$ progesterone $\left(\mathrm{DIB}^{\circledR}\right.$, Syntex, Buenos Aires, Argentina) and an i.m. injection of $2.5 \mathrm{mg}$ of estradiol benzoate (EB, Estrogin ${ }^{\circledR}$, Farmavet, São Paulo, Brazil). Eight days later (Day 8) cows were treated i.m. with 150 mg D-cloprostenol (PGF2 $\sigma$ Prolise ${ }^{\circledR}$, ARSA S.R.L., Buenos Aires, Argentina), and the DIB was removed. Twenty-four hours after DIB removal, cows received $1.0 \mathrm{mg}$ EB i.m. and 30 to $36 \mathrm{~h}$ later all 
animals were FTAIed without estrus detection. Cows were allocated randomly to four groups: PEPE, PEPE/TCR, PEPE/eCG, and PEPE/TCR/eCG. In Group PEPE/TCR, calves were removed temporarily for $54 \mathrm{~h}$ (from DIB removal until FTAI). In Group PEPE/eCG, animals received PEPE treatment plus one dose i.m. of $300 \mathrm{UI}$ eCG (Novormon $^{\circledR}$, Syntex) following PGF2 $\sigma$ administration (Day 8). In Group PEPE/TCR/eCG, animals were treated as in protocol PEPE/TCR plus eCG on D8. All animals were examined by ultrasonography (Aloka SSD 500, $7.5 \mathrm{MHz}$ probe) 10 days before and at the beginning of hormonal treatment in order to detect anestrous cows (absence of corpus lutcum in both exams). Pregnancy diagnosis was performed by ultrasonography 30 days after FTAI. The data were analyzed by logistic regression. The following variables were considered in the model and did not affect pregnancy rates: farms, inseminators, and semen (sire). A total of $75 \%$ of the animals were in anestrus (absence of $\mathrm{CL})$ and the pregnancy rates were similar $(P>0.05)$ among the four groups: PEPE $(43 / 85=50.6 \%)$, PEPE/TCR $(42 / 98=42.9 \%)$, PEPE/eCG $(41 / 88=46 \%)$, and PEPE/TCR/eCG $(39 / 90=43.3 \%)$. The results indicate that in Nelore cows, in postpartum anestrus and good body condition, TCR and/or eCG administration do not improve the efficiency (pregnancy rate) of the PEPE protocol.

This work was supported by FAPESP. V.G.P. and A.F.S. received fellowships from CNPq, Brazil.

\title{
19 PREGNANCY RATES IN DAIRY COWS TREATED WITH INTRAVAGINAL PROGESTERONE DEVICES AND DIFFERENT FIXED-TIME AI PROTOCOLS
}

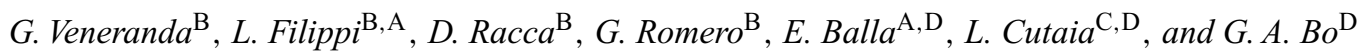

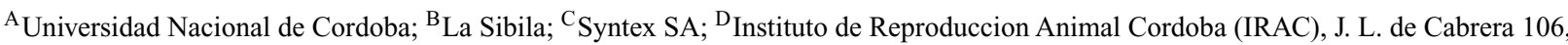 \\ X5000GVD, Cordoba, Argentina
}

Two experiments were designed to evaluate the effect of different treatments for the synchronization of ovulation on pregnancy rates following fixed-time AI (FTAI) of lactating dairy cows. In Experiment 1, 394 Holstein cows that were $61.7 \pm 13.6$ days postpartum (range 35 to 94 days), with a milk yield of $30.7 \pm 6.8$ liters per day (range 12.0 to 52.4 liters) and a body condition score (BCS) between 2.5 to 3.5 out of 5 , were used. Cows were blocked by days postpartum and milk yield and randomly assigned to one of four treatment groups. Cows in P4+EB treatment groups received an intravaginal DIB device ( $1 \mathrm{~g}$ P4; Syntex, Argentina) and $2 \mathrm{mg}$ of estradiol benzoate (EB; Syntex) i.m. on Day 0. On Day 8, DIB devices were removed, and cows received PGF ( $150 \mu \mathrm{g}$ D+cloprostenol: Ciclase; Syntex), and were subdivided to receive $400 \mathrm{IU}$ eCG (Novormon 5000, Syntex) i.m. or no further treatment at that time. On Day 9, all cows received $1 \mathrm{mg}$ of EB and were FTAI $60 \mathrm{~h}$ after DIB removal. Cows in P4-Synch groups received a DIB device and $50 \mu \mathrm{g}$ of GnRH (Lecirelina, Gonasyn; Syntex) i.m. on Day 0. On Day 7, DIB devices were removed; cows received PGF and were divided to receive $400 \mathrm{IU}$ eCG i.m. or no further treatment. On Day 9, all cows received a second GnRH treatment and were FTAI $60 \mathrm{~h}$ after DIB removal. Blood samples were taken on Days -10 and 0 to determine plasma P4 concentrations; $93 \%$ of the cows had $>1 \mathrm{ng} / \mathrm{mL} \mathrm{P4}$ in at least one sample. In Experiment 2, 200 lactating cows from the same farm were treated with the P4+EB+eCG and P4-Synch without eCG treatments with either a DIB or a CIDR-B (1.9g P4; Pfizer Animal Health, Groton, CT, USA) in a $2 \times 2$ factorial design. Cows were examined by rectal palpation 50 days after FTAI to determine pregnancy status, and data were analyzed by chi-square Mantel-Haenszel test. There was an $\mathrm{EB} / \mathrm{GnRH}$ by eCG interaction $(P<0.05)$ which was attributed to a higher pregnancy rate in the P4+EB+eCG group $(44 / 98 ; 44.9 \%)$ than in the P4+EB without eCG group $(30 / 100 ; 30.0 \%)$ and P4-Synch+eCG group $(30 / 98 ; 30.6 \%)$; the P4-Synch without eCG group had an intermediate pregnancy rate that did not differ from the other treatment groups $(37 / 98 ; 38.8 \%)$. In Experiment 2, no significant differences $(P=0.40)$ in pregnancy rates were detected between cows treated with DIB $(51 / 100 ; 51.0 \%)$ or CIDR-B $(42 / 100 ; 42.0 \%)$, and there was no difference $(P=0.18)$ between $\mathrm{P} 4+\mathrm{EB}+\mathrm{eCG}(52 / 100 ; 52.0 \%)$ and $\mathrm{P} 4-$ Synch without eCG $(41 / 100 ; 41.0 \%)$ treated groups. Results suggest that the addition of eCG will improve pregnancy rates following FTAI in lactating dairy cows treated with EB but not in those treated with GnRH at the time of insertion and after removal of a $\mathrm{P} 4$ releasing device. Although treatment with $\mathrm{P} 4+\mathrm{EB}+\mathrm{eCG}$ resulted in numerically higher pregnancy rates, results were not different from those obtained in the GnRH-based treatment, without the addition of eCG.

Cloning/Nuclear Transfer

\section{PRODUCTION OF NUCLEAR TRANSFER EMBRYOS FROM PORCINE FETAL FIBROBLAST CELLS CARRYING A TETRACYCLINE-INDUCIBLE TRANSGENE}

\author{
K. S. Ahn ${ }^{\mathrm{A}}, M . K w o n^{\mathrm{B}}$, B. C. Koo ${ }^{\mathrm{B}}, J . Y . W_{0}{ }^{\mathrm{A}}, S . Y . H e o^{\mathrm{A}}, T . K_{i m}^{\mathrm{B}}$, and H. Shim ${ }^{\mathrm{A}}$ \\ ${ }^{A}$ Department of Physiology, Dankook University School of Medicine, Cheonan, Korea; \\ ${ }^{B}$ Department of Physiology, Catholic University of Daegu School of Medicine, Daegu, Korea
}

Constitutive expression of A transgene often results in serious physiological disturbances in transgenic animals. For instance, systemic overexpression of human growth hormone in transgenic pigs has resulted in detrimental side effects in general health and reproductive performance. One of the solutions to such problem would be inducible expression of a transgene that may restrict production of foreign proteins from transgenic animals only when needed. In this study, a retrovirus vector was designed to express the green fluorescent protein (GFP) gene under the control of the tetracyclineinducible promoter. Transformation of porcine fetal fibroblast cells was achieved by infection of the cells with the vector and subsequent antibiotic selection. To induce transgene expression, transformed porcine fetal fibroblast cells were cultured in medium supplemented with doxycycline for $48 \mathrm{~h}$. Induction of the GFP gene was verified by the emission of fluorescence from transformed cells. Nuclei of transformed cells with or 
without doxycycline treatment were transferred into enucleated oocytes, and the induction efficiency was analyzed by monitoring fluorescent emission during development of reconstituted embryos to the blastocyst stage. In addition, differences in the rates of blastocyst development between experimental groups were analyzed by Student's $t$-test. Blastocyst formation of nuclear transfer embryos using transformed cells with tetracycline-inducible retrovirus vector $(12.0 \%, 128 / 1072)$ was not significantly different $(P>0.05)$ from that with non-inducible control vectors $(13.7 \%, 41 / 300)$, suggesting that an introduction of tetracycline-inducible retrovirus vector was not particularly harmful to the development of nuclear transfer embryos. Also, the blastocyst development rate of nuclear transfer embryos after induction of transgene by doxycycline $(12.1 \%, 99 / 815)$ was not significantly different $(P>0.05)$ from that of the non-induced counterparts $(11.3 \%, 29 / 257)$, suggesting that the induction of transgene did not affect the development of transgenic clone embryos. In a majority of embryos, high expression of the GFP gene was observed in cloned embryos with transgene induction, whereas poor or no GFP expression was detected in non-induced controls. The results from this study suggest that tetracycline-inducible expression of transgenes in nuclear transfer embryos may be used for production of foreign proteins in transgenic animals in a more controlled manner than with conventional procedures. Further experiments on transfer of cloned embryos carrying such an inducible transgene to recipients may enable production of transgenic pigs with fewer side effects from unregulated expression of the transgene.

\title{
21 EFFECT OF SEVERAL PARAMETERS ON PARTHENOGENETIC BOVINE EMBRYO DEVELOPMENT IN VITRO
}

\author{
S. Arat, H. Bagis, A. Tas, and T. Akkoc \\ TUBITAK, Research Institute for Genetic Engineering, Gebze, Kocaeli, Turkey
}

\begin{abstract}
The activation of oocytes is one of the most important steps for a successful cloning and has great importance on embryo development in vitro. The objective of this study was to examine the different parameters affecting parthenogenetic embryo development in vitro. In the first experiment, two activation protocols were compared to examine the effect of electrical pulse on activation. Bovine oocytes isolated from slaughterhouse ovaries were matured in TCM-199 supplemented with fetal bovine serum (FBS), sodium pyruvate, penicillin/streptomycin, rat insulin-like growth factor (rIGF-1), bovine follicle-stimulating hormone (bFSH), and bovine luteinizing hormone (bLH). A group of oocytes was exposed to a DC pulse of $133 \mathrm{~V} / 500 \mu \mathrm{m}$ for $25 \mu \mathrm{s}$, and then activated by calcium ionophore $(5 \mu \mathrm{M})$ for $10 \mathrm{~min}$, cytochalasin $\mathrm{D}(\mathrm{CD})(2.5 \mu \mathrm{g} / \mathrm{mL})+$ cycloheximide $(\mathrm{CHX}, 10 \mu \mathrm{g} / \mathrm{mL})$ for $1 \mathrm{~h}$, and CHX alone for $5 \mathrm{~h}$ (Group 1). Another group of oocytes was activated only by chemicals without electrical pulse. Activated oocytes were cultured for $72 \mathrm{~h}$ in G1-3 and then 4-6 days in G2-3 medium. In the second experiment, oocytes activated by electrical pulse and chemicals were cultured in Barc medium for 7-9 days or 72 h in G1-3 and then 4-6 days in G2-3 medium. In the third experiment, oocytes activated by electrical pulse and chemicals were cultured for $48 \mathrm{~h}$ or $72 \mathrm{~h}$ in G1-3 and then 5-7 days or 4-6 days in G2-3 medium. The differences among groups were analyzed by one-way ANOVA after arcsin square transformation. In the first experiment, cleavage rate (75.6\%), development rate (37.3\%), and blastocyst cell number $(78.4 \pm 3.2)$ of oocytes activated by electrical pulse was higher than for the group without electrical pulse $(28.7 \%, 8.0 \%$, $59.5 \pm 4.3$, respectively; $P<0.05$ ). This result showed that activation was started more effectively by electrical pulse than by chemicals. In the second experiment, there was no significant difference on cleavage rate between the two groups $(66.6 \%, 65.0 \%$, respectively), and the blastocyst development rate of parthenogenetic embryos cultured in G1-3/G2-3 (36.6\%) was higher than in the Barc medium group $(16.6 \% ; P<0.05)$. This result showed that G1-3/G2-3 medium was more effective for parthenogenetic embryo development than Barc medium. In the third experiment, although significant differences could not be found between the two groups in the development rate of parthenogenetic embryos cultured for a total of $7-9$ days $(30.8 \%, 39.2 \%$, respectively), the development rate of embryos cultured for $72 \mathrm{~h}$ in G1-3 was higher (26.4\%) than for the 48-h group $(15 \% ; P<0.05)$ on Day 7 . This result showed that embryos developed more slowly when cultured for a shorter time in G1-3 medium before transfer to G2-3 medium.
\end{abstract}

This study was supported by a grant from TUBITAK, Turkey (VHAG-1022).

\section{EFFECT OF OOCYTE AGE ON THE DEVELOPMENT OF MOUSE PARTHENOGENETIC EMBRYOS AND EFFECT OF OXYGEN CONCENTRATION ON EMBRYO DEVELOPMENT IN VITRO}

\author{
H. Bagis, S. Arat, H. Odaman, and A. Tas \\ TUBITAK, Research Institute for Genetic Engineering and Biotechnology, Gebze, Kocaeli, Turkey
}

The objective of this study was to investigate the effects of two parameters on mouse embryo development in vitro. These parameters were the effect of oocyte age on activation and the effect of $\mathrm{O}_{2}$ concentration in culture. In the first experiment, oocytes were recovered from superovutated mice at $15 \mathrm{~h}$ (group 1) or $20 \mathrm{~h}$ (group 2) after human chorionic gonadotropin (HCG) injection. All oocytes were activated for $6 \mathrm{~h} \mathrm{with} 10 \mathrm{mM} \mathrm{Sr}{ }^{2+}$ in $\mathrm{Ca}^{2+}$ free medium in the presence of $5 \mu \mathrm{g} / \mathrm{mL}$ of cytochalasin B. After activation, embryos were cultured in KSOM.aa medium for 4.5-5.5 days. Zygotes from naturally bred mice were used as control. Differences in blastocyst formation rate and blastocyst cell number among treatments were analyzed by one-way ANOVA after arcsin square transformation. In the first experiment, blastocyst formation rate in the first group was higher than in the second group $(62.6 \%$ vs. $47.1 \% ; P<0.05)$. In addition, blastocyst cell number was also higher in the first group than in the second one $(69.4 \pm 3.2$ vs. $52.4 \pm 2.2 ; P<0.05)$. However, both values were higher in control group $(80 \%, 76.2 \pm 1.2 ; P<0.05)$ than in the experimental groups. These results showed that young oocytes were activated more effectively than aged oocytes. In the second experiment, mouse zygotes were cultured in a humidified atmosphere of $5 \% \mathrm{CO}_{2}$ in air (group 3) or $5 \% \mathrm{CO}_{2}, 5 \% \mathrm{O}_{2}$, and $90 \% \mathrm{~N}_{2}$ (group 4). Blastocyst formation rate and blastocyst cell number of zygotes cultured in low $\mathrm{O}_{2}$ concentration (group 4) for 4.5 days were higher than for group 3 (76.3\% vs. 56.4 and $69.0 \pm 3.4$ vs. 
$52.8 \pm 2.3 ; P<0.05)$. There was a significant difference in blastocyt formation rate of embryos for 5.5 days between the two groups $(25.8 \%$ for group 4 vs. $14.4 \%$ for group $3 ; P<0.05$ ). This suggests that the embryos developed more slowly in high $\mathrm{O}_{2}$ concentration. These results showed that low $\mathrm{O}_{2}$ concentration provided a more suitable environment for mouse embryo development in vitro. The same experiment was repeated with parthenogenetic embryos recently in our laboratory.

This study was supported by a grant from TUBITAK, Turkey (VHAG-1022).

\title{
23 NEONATAL PHYSIOLOGICAL STATUS OF CLONED AND CONTROL CALVES
}

\author{
C. A. Batchelder A , M. B. Whitcomb ${ }^{\mathrm{B}}$, M. Bertolini ${ }^{\mathrm{A}}$, J. B. Mason ${ }^{\mathrm{A}}$, S. G. Petkov ${ }^{\mathrm{A}}$, \\ K. A. Hoffert ${ }^{\mathrm{A}}$, L.W. George ${ }^{\mathrm{B}}$, and G. B. Anderson ${ }^{\mathrm{A}}$ \\ A Department of Animal Science, University of California-Davis, CA 95616, USA; \\ ${ }^{\mathrm{B}}$ School of Veterinary Medicine, University of California-Davis, CA 95616, USA
}

Health problems and mortality rates of cloned calves are major limitations of cloning technology and represent substantial economic losses as well as justifiable animal health and welfare concerns. The objectives of this study were to compare neonatal viability and physiological status of cloned and control calves. Cloned (Holstein, $n=5$; Hereford, $n=3$ ) and control (embryo transfer: Holstein, $n=3$; Hereford, $n=3$ ) calves were carried in the same group of Hereford $\times$ Angus crossbred recipient dams and were delivered by Cesarean section at term (Days 273-280) following induced labor. Additional calves (Holstein, $n=3$; Hereford, $n=2$ ) resulting from AI and delivered vaginally by their natural dams (Days 269-279) following spontaneous initiation of parturition were included as normal controls to evaluate the effects of the induction procedure. Physical evaluations and measurements of blood biochemistry (19 parameters), gases and electrolytes ( 9 parameters), and complete blood counts (18 parameters) were performed within $10 \mathrm{~min}$ of birth and at $1,6,12,18,24,36$, and $48 \mathrm{~h}$ after birth. Cloned calves were observed with increased occurrence of flexural limb deformities $(4 / 8$ clones, $0 / 9$ controls; $P<0.05)$ and developmental delays of physical adjustment parameters such as time to suckle and stand $(5 / 8$ clones requiring $>3 \mathrm{~h} ; P<0.05)$. Cloned calves were more variable than, but not different from, controls for most blood parameters measured. Compared with controls, at birth clones exhibited reduced red blood cell counts $\left(6.8\right.$ and $8.6 \times 10^{9}$ cells $/ \mathrm{mL}$, clones and controls, respectively; $P<0.01$ ), plasma bicarbonate ( $23.1 \mathrm{vs.} 26.2 \mathrm{mmol} / \mathrm{L} ; P<0.05)$, and plasma glucose $(39.4 \mathrm{vs} .73 .6 \mathrm{mg} / \mathrm{dL} ; P<0.05)$. Blood urea nitrogen concentrations in clones tended to be elevated beginning $24 \mathrm{~h}$ after birth and were significantly greater than those in controls by $48 \mathrm{~h}$ $(13.4$ vs. $7.4 \mathrm{mg} / \mathrm{dL} ; P<0.01)$. Echocardiographic measurements at $24 \mathrm{~h}$ of age varied between groups (Table 1$)$ and were indicative of circulatory abnormalities likely originating in utero for clones. The results of this study identified the physiological differences between clones and controls at birth and may be useful in the development of clinical-care protocols to maximize the health and survival of cloned calves.

Table 1. Echocardiographic characteristics of cloned and control calves

\begin{tabular}{lccccc}
\hline Parameter & \multicolumn{2}{c}{ Clones } & $P$ value* & \multicolumn{2}{c}{ Controls } \\
& Mean & Range & & Mean & Range \\
\hline LV free wall $(\mathrm{cm})$ & 1.88 & $1.39-2.48$ & 0.025 & 1.55 & $0.93-1.65$ \\
RV free wall $(\mathrm{cm})$ & 1.48 & $1.31-1.61$ & 0.015 & 1.25 & $1.12-1.28$ \\
RV diameter $(\mathrm{cm})$ & 0.86 & $0.35-1.06$ & 0.028 & 1.27 & $0.65-1.82$ \\
Aorta diameter $(\mathrm{cm})$ & 2.43 & $1.82-2.66$ & 0.09 & 2.65 & $1.88-2.85$ \\
Pulmonary artery diameter $(\mathrm{cm})$ & 2.56 & $2.14-2.9$ & 0.058 & 2.30 & $2.03-2.48$ \\
Aortic outflow velocity $(\mathrm{m} / \mathrm{s})$ & 1.32 & $1.20-1.49$ & 0.019 & 1.02 & $0.96-1.10$ \\
\hline
\end{tabular}

* Student's $t$-test. RV = right ventricle, systolic; $L V=$ left ventricle, systolic.

\section{GLOBAL TRANSCRIPT PROFILING OF CLONED BOVINE BLASTOCYSTS USING AFFYMETRIX GENECHIP TECHNOLOGY}

\author{
Z. Beyhan ${ }^{\mathrm{A}}$, P. Ross $^{\mathrm{A}}$, A. Iager $^{\mathrm{A}}$, A. Kocabas $^{\mathrm{A}}$, K. Cunniff $^{\mathrm{A}}$, M. Maserati $^{\mathrm{B}}$, R. Page $^{\mathrm{B}}$, and J. Cibelli $^{\mathrm{A}}$ \\ ${ }^{\mathrm{A}}$ Cellular Reprogramming Laboratory, Michigan State University, East Lansing, MI 48824, USA; \\ ${ }^{B}$ Cyagra Inc., Elizabethtown, PA 17022, USA
}

Identification of genes implicated in the biological processes of somatic cell nuclear transfer will improve our understanding of reprogramming events, i.e. the transformation of a lineage-committed cell into a pluripotent one. In addition, the gene expression profile of cloned embryos can help explain the widely reported developmental failures in cloned animals. In this study, we investigated global gene expression profiles of bovine in vitro-fertilized and cloned embryos using Gene Chip Bovine Genome Arrays (Affymetrix, Inc., Santa Clara, CA, USA). For the generation of cloned bovine blastocysts from two adult fibroblast lines (C and D), we employed methods previously proven to generate live offspring and compared these offspring to in vitro-produced blastocysts. Total RNA isolated from groups of 10 blastocysts was amplified by a template-switching PCR. Amplified cDNAs were used to synthesize biotin-labeled antisense RNAs (aRNAs) during and in vitro transcription reaction. Labeled aRNAs were 
hybridized to microarrays as described by the manufacturer. Experiments were performed in four replicates. Expression data were analyzed using the Significance Analysis of Microarrays (SAM; Tusher et al. 2001 Proc. Natl. Acad. Sci. 98, 5116-5121) procedure and software. Overall, 48.4\% and $46 \%$ of 23000 bovine transcripts spotted on the arrays were present in cloned and in in vitro-produced control blastocysts, respectively. The SAM procedure identified 43 genes that changed at least 1.5 -fold, with an estimated false discovery rate (FDR) of $20 \%$. Comparison of gene expression between NT embryos produced from two different cell lines and IVF controls with the same criteria revealed 6 (clones from cell line C vs. IVF) and 46 (clones from cell line D vs. IVF) differentially expressed genes. The number of transcripts expressed differentially between the cloned embryos with different donor cell origin was 437. Of the 43 differentially expressed transcripts in cloned blastocysts, 13 have unknown functions and the rest of the genes related to cell structure (tuftelin, desmoplakin), cell cycle/mitosis (Kinesin like 4, katanin, stathmin, PCNA), energy metabolism (lactate dehydrogenase, ATPsynthase, lipid-binding protein, keto acid dehydrogenase E1, metallothionein), and cell signaling (GTP-binding protein1, GTP binding stimulatory protein). Our results indicate that expression profiles of cloned blastocysts could be affected by somatic donor cell.

\title{
25 MATERNAL-TO-EMBRYONIC TRANSITION FOLLOWING NUCLEAR TRANSFER OR PARTHENOGENETIC ACTIVATION
}

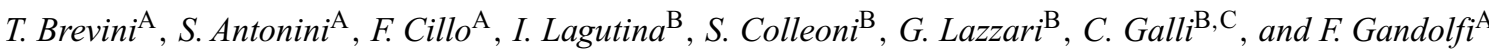 \\ ${ }^{A}$ Instituto di Anatomia degli Animali Domestici, Milano, Italy; \\ ${ }^{B}$ Laboratorio di Tecnologie della Riproduzione, CIZ srl, Instituto Sperimentale Italiano Lazzaro Spallanzani, Cremona, Italy; \\ ${ }^{\mathrm{C}}$ Dipartimente Clinico Veterinario, Universita di Bologna, Bologna, Italy
}

The successful development of embryos generated by somatic cell nuclear transfer (SCNT) requires the ooplasm to reprogram the nucleus. This establishes the gene expression pattern necessary for full development by mechanisms that are currently being clarified. The ooplasm action on somatic nuclei shows many common aspects to the process that leads to the creation of a functional embryonic genome from the differentiated sperm and egg genomes. In order to investigate this aspect we studied a critical phase of early embryonic development: the maternal to embryonic transition (MET). We compared the pattern and level of gene expression between bovine embryos derived from in vitro fertilization (IVF), from nuclear transfer of adult fibroblasts (NT), or from parthenogenetic activation (PG). The study was performed in cattle because MET, in this species, occurs over four cell cycles, making it easier to detect even small deviations. Oocytes, matured for $22 \mathrm{~h}$ and fertilized in vitro or after cumulus removal, were enucleated and fused to fibroblast cells. Nuclear transfer and Met II oocytes were activated at 24-26 h of maturation with ionomycin $(5 \mu \mathrm{M})$ for $5 \mathrm{~min}$ and 6DMAP $(2 \mathrm{mM})$ for $4 \mathrm{~h}$ and then cultured in mSOFaa. Embryos were harvested at the required time for analysis at the 2-, 4-, 8-, and 16-cell; morula; and blastocyst stages and stored snap-frozen in a minimal volume of medium in groups of 5-10 embryos. Semiquantitative RT-PCR was used to study the expression of Nanog, Oct-4, Zar-1, and Par-3, because these genes are directly involved in early embryo development and have a specific expression pattern during MET. Data were analyzed with one-way ANOVA followed by Student-Newman-Keuls All Pairwise Multiple Comparison. No difference in pre-implantation development was observed among the three groups. The Nanog expression pattern was unchanged in all three groups, becoming detectable from the 8-16-cell stage onward. Oct-4 mRNA was detected at all stages in every group, but only in NT embryos did a significant increase occur at the 16-cell stage, suggesting the onset of an anticipated embryonic transcription. the Zar-1 expression pattern, with the characteristic de-novo transcription peak at the 4-cell stage, was observed in both IVF and NT embryos but not in PG embryos. In this group, Zar-1 mRNA levels were significantly higher at the 2- and 4-cell stage than in all of the following stages. The Par-3 gene showed the biggest differences among groups: IVF embryos expressed this gene from the 8-cell stage onward, whereas NT embryos showed high levels of Par-3 mRNA already at the 2-cell stage. Surprisingly, PG embryos showed no detectable Par-3 levels at any stages. The results indicate that, although in vitro development was not affected, gene-specific expression differences during MET occurred among groups. Relating the specific functions exerted by each of these genes in early development to the changes observed following the different manipulations provides useful data toward a better understanding of the role of these genes and of the mechanisms of nuclear reprogramming.

This work was supported by FIRB RBNE01HPMX, FIRST 2004, and ESF-EuroStells.

\section{EFFECTS OF SOMATIC CLONING ON THE IMMUNE RESPONSE IN YOUNG AND ADULT CATTLE}

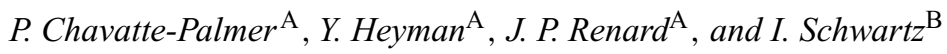 \\ A UMR INRA/CNRS/ENVA 1198, Biologie du Développement et Reproduction, Jouy en Josas, France; \\ ${ }^{B}$ INRA, Virologie et Immunologie Moléculaires, Jouy en Josas, France
}

Somatic cloning in cattle is associated with important gestational abnormalities, including implantation delay during the first 2 months of pregnancy and abnormal fetal and placental growth (known as large offspring syndrome, or LOS) in the third trimester. In our laboratory, between 3 and $25 \%$ of the cloned blastocysts transferred to recipient cows reach term, depending on genotype. About $20 \%$ of the newborns die rapidly due to various causes that appear to be direct consequences of the LOS. We previously reported on a thymic atrophy resulting from nuclear transfer (Renard et al. 1999 Lancet 353, 1459-1491) and have further diagnosed distinct pathological events occurring in the infancy and adult age of clones, including death due to apparently benign infections (despite treatment) and also thymic atrophy in approximately $20 \%$ of the postmortem cases. These observations in clones have led us to investigate the immune function of apparently normal bovine clones. Holstein cows housed in the same farm were used. Circulating lymphocyte populations during the resting state were marked, counted in 17 clones and 17 contemporary controls ranging from 15 days to 5 years of age, and allotted to one of three groups: 1 ( $0.5-2$ months, $n=4$ clones, $n=6$ controls), 2 ( $3-9$ months, $n=7$ clones, $n=5$ controls) and 3 ( $1.5-5$ years, $n=6$ clones, $n=6$ controls). Clones originated from adult fibroblast cells from four different genotypes distributed in the three 
groups. Peripheral mononuclear blood cells (PMBCs) were collected, marked, and counted by flow cytometry. The specific markers were CD2, CD3,

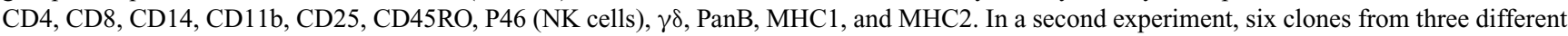
genotypes and six controls aged 8-9 months were vaccinated with $10 \mathrm{mg}$ ovalbumin in alum to evaluate the naïve immune response. The cell subset proportions were not different between clones and controls. There was no difference between groups for antibody response to vaccination. However, T cell restimulation with specific antigens after immunization with evalbumin was significantly lower in clones compared to controls $(P<0.05)$. Furthermore, nonspecific stimulation with phytohemagglutinin (PHA) was also lower in clones $(P<0.05)$. These results show that lymphocyte populations are normally represented in apparently healthy clones. Bovine clones presented, however, a reduced capacity to build up a cellular immune response against a newly encountered antigen, such as ovalbumin. It remains to be determined whether these functional alterations are a result of defective reprogramming of immune functions during the cloning process or the consequence of an abnormal placental development leading to altered feto-placental interactions during pregnancy and fetal programming. Previous work by others has shown that there may be an abnormal expression of MHC1 in the placenta of bovine clones (Hill et al. 2002 Biol. Reprod. 67, 55-63), and this may well be part of the same phenomenon affecting overall immune regulation in clones.

\title{
27 SODIUM CHLORIDE TREATMENT OF CELL MEMBRANE-PERMEABILIZED NUCLEAR DONOR CELLS FACILITATES THE DISPLACEMENT OF THE SOMATIC HISTONE H1 AND HMG-17 PROTEINS IN RECONSTRUCTED PORCINE EMBRYOS
}

\author{
L. Che and V. Bordignon \\ Department of Animal Science, McGill University, Ste-Anne-de-Bellevue, Quebec, Canada
}

Developmental efficiency of somatic cell-reconstructed embryos depends on extensive remodeling of chromatin structural components. Due to their importance for maintaining the high-order chromatin structure and controlling DNA functions, including replication, transcription, repair, and recombination, histones and other chromatin-binding proteins represent leading choice markers to investigate nuclear remodeling in reconstructed embryos. The main objective of this study was to investigate whether or not the exposure of cell membrane permeabilized nuclear donor cells to sodium chloride (salt-extraction) would facilitate the displacement of chromatin-binding proteins in reconstructed porcine embryos. Both linker histone H1 (H1) and high-mobility group (HMG) proteins are known to affect gene expression through the modulation of the high-order chromatin structure. Standard methods of oocyte enucleation and electrofusion were applied for embryo reconstruction using in vitro-matured oocytes and control or salt-extracted fetal fibroblast cells. For salt-extraction, confluent cell cultures were washed with $\mathrm{Ca}^{2+} / \mathrm{Mg}^{2+}$-free Hank's balanced salt solution (HBSS); cells were permeabilized by incubation with $1 \mu \mathrm{g} / \mathrm{mL}$ of streptolysin $\mathrm{O}$ at $37^{\circ} \mathrm{C}$ for $30 \mathrm{~min}$ in $\mathrm{HBSS}$, and then maintained in Tris- $\mathrm{NaCl}$ buffer ( $10 \mathrm{mM}$ Tris- $\mathrm{HCl}, 0.5 \mathrm{mM} \mathrm{MgCl}_{2}, 0.7 \mathrm{M} \mathrm{NaCl}, 1 \mathrm{M}$ sucrose) for $5 \mathrm{~min}$. After salt-extraction, cells were rinsed and cultured in DMEM supplemented with $10 \%$ fetal bovine serum (FBS) and $2 \mathrm{mM} \mathrm{CaCl}_{2}$ for $1 \mathrm{~h}$ at $37.5^{\circ} \mathrm{C}$ for membrane resealing prior to nuclear transfer. Reconstructed embryos were activated using ionomycin $(15 \mu \mathrm{M} / 5 \mathrm{~min})$ and strontium chloride $\left(\mathrm{Sr}^{2+} ; 10 \mathrm{mM} / 4 \mathrm{~h}\right)$, and then cultured in PZM-3 medium. Immunostaining for $\mathrm{H} 1$ and HMG-17 was performed in nuclear donor cells and embryos at different stages after reconstruction. The time required for $\mathrm{H} 1$ displacement in transplanted nuclei was reduced by the salt-extraction treatment (Table 1). Salt-extracted cells showed a stronger HMG-17 cytoplasmic signal compared to control cells. The proportion of HMG-17-positive reconstructed embryos at 1,3 , and $6 \mathrm{~h}$ was $54 \mathrm{vs}$. 19 , 57 vs. 44 , and 75 vs. 62 , for control and salt-extracted cells, respectively. These data suggest that salt-extraction prior to nuclear transplantation enhances the remodeling of chromatin structure in embryos reconstructed with somatic cell nuclei

Table 1. Proportion (n) of H1-positive stained embryos after different times from parthenogenetic activation (PA) and nuclear transfer using control (NT-control) or salt-extracted (NT-extracted) cells

\begin{tabular}{lcccccc}
\hline Treatment & $1 \mathrm{~h}$ & $3 \mathrm{~h}$ & $6 \mathrm{~h}$ & $48 \mathrm{~h}(2$ cells $)$ & $48 \mathrm{~h}(4$ cells $)$ & $72 \mathrm{~h}(8 \mathrm{cells})$ \\
\hline PA & - & - & - & $56 \%(25)$ & $100 \%(13)$ & $100 \%(10)$ \\
NT-control & $100 \%(11)$ & $83 \%(24)$ & $37 \%(32)$ & $20 \%(25)$ & $40 \%(15)$ & $100 \%(2)$ \\
NT-extracted & $90 \%(11)$ & $43 \%(21)$ & $0 \%(16)$ & $0 \%(13)$ & $17 \%(6)$ & $100 \%(6)$ \\
\hline
\end{tabular}

This work was supported by a NSERC Discovery Grant to VB.

\section{EFFECT OF TREATMENT OF OVINE OOCYTES WITH CAFFEINE ON GENE EXPRESSION IN NUCLEAR TRANSFER EMBRYOS}

\author{
I. Choi, J.-H. Lee, and K. Campbell \\ School of Biosciences, University of Nottingham, Loughborough, Leics, LE12 5RD, UK
}

The efficiency of animal production by somatic cell nuclear transfer (SCNT) remains low and this has been linked to incomplete epigenetic reprogramming of the donor somatic cell nucleus. Previous studies have reported that embryos produced by SCNT exhibit abnormal expression patterns for a number of genes, including IL6, FGF4, FGFr2, Hsp, IF-tau, DNMT, and Mash2 (Daniels et al. 2000 Biol. Reprod. 63, 1034-1040; Wrenzycki et al. 2001 Biol. Reprod. 65, 309-317). Recently, we demonstrated that treatment of telophase I or metaphase II ovine oocytes with 
$10 \mathrm{mM}$ caffeine for $6 \mathrm{~h}$ increased the activities of both MPF and MAPK kinases. In NT embryos produced using caffeine treated oocytes as recipient cytoplasts, no increase in the frequency of development to the blastocyst stage was observed; however, blastocyst stage embryos had an increased cell number (Lee and Campbell 2005 Reprod. Fertil. Dev. 16, 125). The objective of the present study was to examine the effects of caffeine treatment on the expression levels of a number of genes involved in early development. Target genes were categorized into seven groups based on their function: (1) transcription factors (Oct-4, Sox-2), (2) growth factors (IGF-1, IGF-1r, IGF-2r, FGF-2, and FGF-4), (3) stress adaptation (Hsp70.1 and Hsp27), (4) metabolism (Glut-1, Glut-3, and Glut-4), (5) compaction/cavitation (DcII), (6) trophoblastic function (IF-tau), and (7) nuclear reprogramming factors (Hist4h4, H2A.Z, and Lmna). To determine the transcript levels semiquantitatively, different PCR cycle parameters were used ( 35 and 40 cycles). Expression levels were compared in blastocyst-stage embryos obtained from five groups produced as previously described (Lee and Campbell 2005; Maalouf et al. 2005 Reproduction 32, 49): (I) IVF embryos, (II) caffeine-treated IVF embryos, (III) parthenotes, (IV) SCNT embryos, and (V) caffeine treated SCNT embryos. No differences in overall expression patterns were observed among groups I, II, and III. In group IV non-treated SCNT-derived embryos, an aberrant gene expression pattern was found with respect to Oct-4 and genes regulated by Oct-4: H2A.Z, IF-tau, and FGF-4, Oct-4, H2A.Z, and FGF-4 were down-regulated and IF-tau was up-regulated. In contrast, the expression patterns of group V caffeine-treated SCNT embryos resembled those of control groups I, II, and III. In comparison to gene expression in group IV embryos, Oct-4, FGF-4, and H2A.Z were up-regulated but IF-tau was down-regulated. Previous studies have demonstrated that FGF-4 and H2A.Z play an important role in early development and implantation, whereas expression of IF-tau is related to embryo quality (Feldman et al. 1995 Science 267, 246-249; Fasst et al. 2001 Curr. Biol. 11, 1183-1187; Wrenzycki et al. 2001). Our results demonstrate that treatment of oocytes with caffeine prior to embryo reconstruction can alter the expression patterns of developmentally regulated genes in SCNT embryos to more closely resemble those of IVF controls.

\title{
29 BIRTH OF A FOAL CLONED BY ADULT SOMATIC CELL NUCLEAR TRANSFER WITH ROSCOVITINE-TREATED DONOR CELLS
}

\author{
Y. H. Choi ${ }^{\mathrm{A}}$, Y. G. Chung ${ }^{\mathrm{B}}$, D. D. Varner ${ }^{\mathrm{A}}$, and K. Hinrichs ${ }^{\mathrm{A}}$ \\ ${ }^{A}$ College of Veterinary Medicine and Biomedical Sciences, Texas A\&M University, College Station, TX 77843, USA; \\ ${ }^{B}$ Advanced Cell Technology, Worcester, MA 01605, USA
}

Only one horse foal produced from adult somatic cell nuclear transfer has been reported in the scientific literature (Galli et al. 2003 Nature 425, 680 ); a second foal from the same laboratory was reported in the popular press in 2005. In these reports, the blastocyst rates were 3 and $17 \%$, and efficiency to birth of a live foal from total reconstructed oocytes was 0.1 and $0.5 \%$, respectively. In cattle, roscovitine treatment of donor cells has been associated with a decrease in blastocyst development, but an increase in live births (Gibbons et al. 2002 Biol. Reprod. 66, 895-900). The present study was performed to determine the effect of roscovitine treatment of donor cells on blastocyst production after equine nuclear transfer and to evaluate the viability of pregnancies established via this treatment. In Experiment 1, fibroblasts were either grown to confluence or treated with $15 \mu \mathrm{g} / \mathrm{mL}$ roscovitine, for $24 \mathrm{~h}$. Enucleated in vitro-matured oocytes were reconstructed by direct injection of fibroblasts using a piezo drill. Recombined oocytes were activated by injection of stallion sperm extract, followed by culture in the presence of $2 \mathrm{mM} 6$-DMAP for $4 \mathrm{~h}$. They were then placed in culture in DMEM/F-12 with 10\% fetal bovine serum (FBS) under mixed gas for 8 days and evaluated for blastocyst development. In Experiment 2, oocytes recombined with either confluent or roscovitine-treated donor cells were activated as above either alone or with the addition of $10 \mu \mathrm{g} / \mathrm{mL}$ cycloheximide at the time of 6-DMAP treatment. Resulting blastocysts from Experiment 2 were transferred transcervically to the uteri of recipient mares. One embryo was transferred per mare. In Experiment 1, there was no difference in rates of cleavage (73-19\%) or blastocyst development between confluence and roscovitine treatments $(2 / 55,3.6 \%$ vs. $2 / 56,3.6 \%$, respectively). In Experiment 2 , there was no significant difference in rates of cleavage (78-18\%) or blastocyst development $(0-1 \% ; 4 / 105,0 / 104,0 / 106,2 / 108)$ among donor cell or activation treatments. Six blastocysts were transferred to mares: two from confluent donor cells and four from roscovitine-treated donor cells. One mare, which received an embryo from the roscovitine donor/6-DMAP treatment, established pregnancy after transfer. The pregnancy continued normally and the mare delivered a colt with minimal assistance on Day 389. Typing for 13 equine microsatellites confirmed that the colt was of the same DNA type as the donor fibroblasts. The colt has grown and developed normally. Results of these studies show that roscovitine treatment of equine donor cells does not negatively affect the proportion of recombined oocytes that progress to the blastocyst stage. A viable colt resulted from an embryo produced with roscovitine-treated donor cells. More work is needed on methods to increase blastocyst rates after nuclear transfer in this species.

This work was supported by the Link Equine Research Endowment Fund, Texas A\&M University.

\section{NUCLEAR AND MICROTUBULE DYNAMICS IN SOMATIC CELL NUCLEAR TRANSFER SHEEP EMBRYOS ACTIVATED WITH T-DIMETHYLAMINOPURINE AND CYCLOHEXIMIDE}

\author{
G. Coppola, B.-G. Jeon, B. Alexander, E. St. John, D. H. Betts, and W. A. King
}

Department of Biomedical Sciences, Ontario Veterinary College, University of Guelph, Guelph, Ontario, Canada N1G 2W1

The early reprogramming events following somatic cell nuclear transfer (SCNT) determine the fate of the cloned embryo and its development to a healthy viable offspring. In the present study, we undertook a detailed immunocytochemical study of the patterns of both microtubules and chromatin during the first cell cycle of sheep nuclear transfer embryos after fusion and artificial activation using either 6-dimethylaminopurine (6-DMAP) or cycloheximede (CHX). Sheep oocytes were collected from abattoir ovaries and matured in vitro for $18-20 \mathrm{~h}$ and enucleated; fetal fibroblasts were transplanted using standard SCNT techniques. Reconstructed cell-cytoplast couplets were fused and activated with ionomycin, followed by 
culture in two separate groups containing 6-DMAP $(2 \mathrm{mM})$ or CHX $(10 \mu \mathrm{g} / \mathrm{mL})$ for $3 \mathrm{~h}$. Following activation, embryos were cultured in in vitro culture (IVC) medium for blastocyst development. Embryos ( $n=15,3$ replicates) were randomly removed from culture at various time points and stained using standard immunocytochemical methods to observe microtubule and nuclear configurations. Images were captured using laser scanning confocal microscopy. Results reveled that at $1 \mathrm{~h}$ post-fusion, $63.3 \%$ of reconstructed embryos underwent nuclear envelope breakdown (NEBD) and premature chromosome condensation (PCC) was apparent as chromosomes were situated on a non-polar spindle. The remaining embryos showed abnormal spindle and DNA configurations including chromosome outliers, congression failure, and non-NEBD. At $1 \mathrm{~h}$ post-activation (hpa), the embryos treated with 6-DMAP had already formed a clearly visible pronucleus (diameter 6-8 $\mu \mathrm{m}$ ), whereas in the CHX-treated group, none of the embryos were at pronuclear stage; instead most of the latter embryos showed two masses of chromatin. At $1 \mathrm{hpa}$, 6-DMAP- and CHX-treated embryos showed one swelled pronucleus with a mean diameter of $8.4 \pm 1.3 \mu \mathrm{m}$ and $25.8 \pm 0.8 \mu \mathrm{m}$, respectively $(P<0.05)$. At $16 \mathrm{hpa}$, embryos from both treatment groups still showed one swelled pronucleus. In the 6-DMAP-treated embryos, most of the embryos showed a metaphase spindle with aligned chromosomes of the first mitotic division as early as $18-10 \mathrm{hpa}$, whereas in the CHX-treated group embryos were still at the pronuclear stage. Typical 2-cell division was seen in most of the 6-DMAP-treated embryos between 24 and $30 \mathrm{hpa}$, but it was slightly delayed in CHX-treated embryos (32-35 hpa). Blastocyst development rates in the 6-DMAP- and CHX-treated groups were $21.4 \pm 5.6 \%$ and $14.0 \pm 6.3 \%$, respectively $(P<0.05)$. In summary, artificial activating agents 6-DMAP and CHX exhibited different effects on chromatin remodeling, cell cycle progression, and the degree of pronuclear swelling which may explain the poor developmental rates and abnormal chromosome complements observed for cloned embryos.

This work was funded by NSERC, OMAF, and International Council for Canadian Studies.

\title{
31 FULL-TERM AND LIVE RABBIT CLONES PRODUCED BY SOMATIC CELL NUCLEAR TRANSFER
}

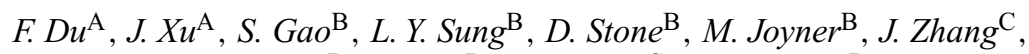

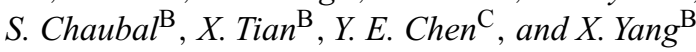 \\ A Evergen Biotechnologies, Inc., Storrs, CT 06269, USA; \\ ${ }^{B}$ Center for Regenerative Biology, University of Connecticut, Storrs, CT 06269, USA \\ ${ }^{\mathrm{C}}$ Cardiovascular Research Institute, Morehouse School of Medicine, Atlanta, GA 30314, USA
}

Transgenic/knockout (KO) rabbits can serve as an excellent animal model for human cardiovascular diseases (CVD) and other diseases. However, the production of transgenic/KO rabbits is hindered by low efficiency of traditional DNA microinjection and the unavailability of embryonic stem cell lines. An alternative approach is to produce transgenic/KO rabbits by somatic cell nuclear transfer (SCNT) using genetically modified somatic cells as nuclear donors. Our initial objective of the study was to prove the feasibility of cloning rabbits by SCNT because rabbit is a difficult species to be cloned. Rabbit oocytes were flushed from the oviducts of superovulated donors treated with the regime of follicle-stimulating hormone (FSH) and human choriani gonadotropin (hCG). Cumulus cells were then denuded from the oocytes by incubation in $0.5 \%$ hyaluronidase and pipetting. Oocyte enucleation was conducted in M199+10\% fetal bovine serum (FBS) and confirmed by fluorescence microscopy. Cumulus cells used for nuclear donors were prepared from fresh cumulus-oocytes complexes. The donor nucleus was transferred into a recipient oocyte by either cell fusion or direct nuclear injection method. In the cell fusion method, a small donor cell with the diameter approximately $15-19 \mu \mathrm{m}$ was transferred into the perivitelline space of an enucleated oocyte; subsequently the somatic cell-cytoplast pair was fused by applying three direct current pulses at $3.2 \mathrm{kV} / \mathrm{cm}$ for a duration of $20 \mu \mathrm{s} /$ pulse. In the direct nuclear injection method, a mechanically lysed donor cell was injected into oocyte cytoplasm with the aid of a piezo-drill system. Fused embryos or injected oocytes were activated by the same electrical stimulation regime described above, and subsequently cultured in M199+ 10\% FBS containing $2.0 \mathrm{mM}$ 6-dimethylaminopurine (DMAP) and $5 \mu \mathrm{g} / \mathrm{mL}$ cycloheximide for $2 \mathrm{~h}$. For the in vitro study, cloned embryos were cultured in B2 medium plus 2.5\% FBS for 5 days (initiation of activation = day 0 ) at $38.5^{\circ} \mathrm{C}$ in $5 \% \mathrm{CO}_{2}$ humidified air. For the in vivo study, cloned embryos were cultured for $20-22 \mathrm{~h}$ in vitro before transfer into pseudopregnant rabbit recipients. Pregnancy was monitored by palpation and/or ultrasound on Days 14-16 post embryo transfer (ET). The results (Table 1) show that the donor nuclei-introducing rate was higher with nuclear direct injection than with the cell fusion method $(P<0.05)$. There were no significant differences among subsequent cleavage and development to morula and blastocysts between both methods, although the development rates of cloned embryos via electrically mediated fusion were higher than those derived from the injection group. One recipient in the injection group $(1 / 6,17 \%)$ and six recipients in the fusion group $(6 / 16,38 \%)$ were diagnosed as pregnant. From the fusion group, one full-term but stillborn and one live and healthy clone rabbit were delivered on Days 33 and 31 post-ET, respectively. To our knowledge, this is the second report of full term development of cloned rabbit by somatic nuclear transfer cloning. Our further study is to clone live rabbit offspring with modified transgenic/KO somatic cell lines.

Table 1. In vitro development of rabbit cloned embryos with cumulus cells as nuclear donors

\begin{tabular}{lccccccr}
\hline Treatment & $\begin{array}{c}\text { Donor } \\
\text { cell type }\end{array}$ & Number & $\begin{array}{c}\text { No. (\%) } \\
\text { nuclei introduced }\end{array}$ & $\begin{array}{c}\text { No. (\%) } \\
\text { cleaved }\end{array}$ & $\begin{array}{l}\text { No. (\%) } \\
\text { 4-celled }\end{array}$ & $\begin{array}{l}\text { No. (\%) } \\
\text { morulae }\end{array}$ & $\begin{array}{r}\text { No. (\%) } \\
\text { blastocysts }\end{array}$ \\
\hline Fusion & Cumulus & 69 & $44(64)$ & $26(59)$ & $20(45)$ & $14(32)$ & $11(25)$ \\
Injection & Cumulus & 71 & $68(96)$ & $22(32)$ & $14(21)$ & $10(15)$ & $7(11)$ \\
\hline
\end{tabular}




\title{
32 CRYOPRESERVATION OF DONOR CELLS FOR NUCLEAR TRANSFER: EFFECT OF CELL FREEZING METHOD ON THE EFFICIENCY OF SOMATIC CELL NUCLEAR TRANSFER IN PIGS
}

\author{
J. Estrada, E. Lee, and J. Piedrahita \\ Molecular Biomedical Sciences, College of Veterinary Medicine, North Carolina State University, Raleigh, NC 27606, USA
}

\begin{abstract}
Donor cell quality is one of the most important factors affecting somatic cell nuclear transfer (SCNT) in mammals. Many studies have been carried out to improve the donor cell characteristics in nuclear transfer, including studies on cell type, cell cycle stage, cell passage, and handling of donor cells before the SCNT. Even though most SCNT work is done with donor cells that have been previously frozen and thawed, no studies have been conducted to evaluate the effect of the cell freezing rate on the SCNT efficiency. The objective of this experiment was to evaluate the effect of the cell freezing method on development of pig SCNT embryos in vitro. Fibroblasts were collected from a 29-day-old female fetus, suspended in DMEM-F $12+40 \%$ fetal bovine serum (FBS) $+10 \%$ dimethyl sulfoxide (DMSO), and placed in 1.6-mL cryovials for freezing. Vials were randomly assigned to two treatments: In treatment 1 , cells were frozen at a controlled rate of $1^{\circ} \mathrm{C} / \mathrm{min}$ in a programmable machine $(\mathrm{P})$ until $-40^{\circ} \mathrm{C}$, and then plunged into liquid nitrogen $\left(\mathrm{LN}_{2} ;-196^{\circ} \mathrm{C}\right)$. In treatment 2 , the traditional system $(\mathrm{T})$, vials were placed in a styrofoam box and left overnight in a freezer at $-80^{\circ} \mathrm{C}$. The next day samples were plunged into $\mathrm{LN}_{2}\left(196^{\circ} \mathrm{C}\right)$. For each treatment, cells were thawed and cultured until confluence before being used for SCNT. Cells were used at passages 2 and 6. Cumulus-oocyte complexes (COCs) were aspirated from slaughterhouse ovaries and cultured for $39 \mathrm{~h}$ in TCM 199 supplemented with $10 \%$ porcine follicular fluid (pFF), $5 \mu \mathrm{g} / \mathrm{mL}$ insulin, $10 \mathrm{ng} / \mathrm{mL}$ epidermal growth factor (EGF), $0.6 \mathrm{mM}$ cysteine, $0.2 \mathrm{mM}$ pyruvate, $25 \mu \mathrm{g} / \mathrm{mL}$ gentamycin and $5 \mu \mathrm{g} / \mathrm{mL}$ each of equine and human chorionic gonadotropin (eCG and hCG). Oocytes were stained with bisbenzimide and enucleated in manipulation media with $7.5 \mu \mathrm{g} / \mathrm{mL}$ cytochalasin B by removing the first polar body and metaphase plate by means of a 16- $\mu \mathrm{m}$ beveled glass pipette. Cells from each treatment were injected into the perivitelline space of recipient enucleated oocytes and fused by two DC pulses of $140 \mathrm{~V}$ for $50 \mu \mathrm{s}$ in fusion media. The fusion rate was evaluated $1 \mathrm{~h}$ later, and reconstructed oocytes were activated by two DC pulses of $120 \mathrm{~V}$ for $60 \mu \mathrm{s}$. After activation, oocytes were placed in bicarbonate-buffered NCSU-13 with $0.4 \%$ BSA and cultured at $38.5^{\circ} \mathrm{C}, 5 \% \mathrm{CO}_{2}$ in a humidified atmosphere. Embryos were observed for cell cleavage at Day 2, and blastocyst development rate and cell number counting were done at Day 7 of culture. Every experiment was repeated three times. The temperature descending rate for $\mathrm{P}$ was slower and more linear $\left(1^{\circ} \mathrm{C} / \mathrm{min}\right.$ vs. $\left.2^{\circ} \mathrm{C} / \mathrm{min}\right)$ than for the T method. Fusion rate was not significantly affected $(P<0.05)$ by the freezing method when they were evaluated either individually at each passage or accumulated regardless the passage $(78.9 \pm 3.6 \% \mathrm{vs} .79 .4 \pm 6.3 \%)$ for $\mathrm{P}$ and T, respectively. The same trends were observed for cleavage $(61.2 \pm 5.2 \%$ vs. $64.3 \pm 5.2 \%)$, blastocyst development $(4.2 \pm 1.8 \%$ vs. $5.0 \pm 2.8 \%)$, and number of cells at the blastocyst stage $(19.4 \pm 3.1$ vs. $19.8 \pm 6.2)$ for $\mathrm{P}$ and T, respectively. The present findings indicate that blastocyst development after SCNT does not differ when fetal fibroblasts donor cells are frozen by the two methods tested.
\end{abstract}

\section{USE OF ADULT MESENCHYMAL STEM CELLS FOR CLONING PIGS}

\author{
R. Faast, L. F. S. Beebe, S. J. Harrison, S. M. McIlfatrick, R. J. Ashman, and M. B. Nottle \\ Research Centre for Reproductive Health and Department of Obstetrics and Gynaecology, \\ University of Adelaide, Adelaide, South Australia, 5005, Australia
}

Current cloning efficiencies are relatively low and there is evidence to suggest that a less differentiated cell type can increase these (Jaenisch et al. 2002 Cloning and Stem Cells 4, 389-396). As part of an ongoing study that aims to develop cloning as a breeding tool for the pig industry, we examined whether mesenchymal stem cells (MSCs) isolated from bone marrow (Experiment 1) and blood (Experiment 2) could increase cloning efficiencies compared with fibroblasts isolated from ear tissue of live animals. MSCs were isolated from the femurs and blood of two pigs over a Histopaque-1077 density gradient. Cells were seeded and cultured in DMEM supplemented with $10 \%$ fetal bovine serum (FBS) and antibiotics/antimycotics. Blood MSCs were plated onto fibronectin-coated dishes (Faast and Nottle, Proc. Australian Health Med. Res. Congress 2004, abst. 1272). Cells from passages 2-4 were used for nuclear transfer by means of a fusion before being subjected to the activation protocol described previously (Harrison et al. 2004 Cloning and Stem Cells 6, 327-331). Embryos were cultured in a modified NCSU23 for 7 days. In Experiment 1 there was no difference in the number of fused embryos that cleaved in the bone marrow MSC and fibroblast groups ( $374 / 447 ; 84 \%$ vs. 370/446; $83 \%$, respectively; 9 replicates). The number of embryos that developed to the blastocyst stage by day 7 was significantly higher in the bone marrow MSC group compared with the fibroblast group (136/447; 30\% vs. 79/446; 18\%, respectively, Chi square test; $P<0.01)$. In Experiment 2 there was no difference in the percentage of embryos that cleaved in the blood MSC and fibroblast groups ( $287 / 375 ; 77 \%$ vs. $275 / 347 ; 79 \%$, respectively; 8 replicates). The number of embryos that developed to the blastocyst stage by day 7 was also similar between the blood MSC and fibroblast groups $(67 / 375 ; 18 \%$ vs. $63 / 347 ; 18 \%$, respectively). In conclusion, our results suggest that bone marrow MSCs may be a more efficient cell type, compared with fibroblasts, for the cloning of live animals.

\section{DEVELOPMENTAL ABILITY OF ZONA-FREE PORCINE CLONED EMBRYOS RECONSTRUCTED BY SOMATIC CELLS AND CENTRIFUGED CYTOPLASTS}

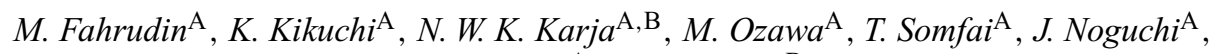 \\ H. Kaneko ${ }^{\mathrm{A}}$, and T. Nagai ${ }^{\mathrm{B}}$ \\ ${ }^{A}$ Genetic Diversity Department, National Institute of Agrobiological Sciences, Tsukuba, Ibaraki 305-1602, Japan; \\ ${ }^{\mathrm{B}}$ Department of Research Planning and Coordination, National Institute of Livestock and Grassland Science, \\ Tsukuba, Ibaraki 305-0901, Japan
}

The combination of bulk enucleation and zona-free cloning will offer simplification of the conventional nuclear transfer technique. A bulk enucleation method such as enucleation by centrifugation could reduce the time of manipulation that is necessary for removing genetic materials from the oocytes. 
The present study was conducted to examine the ability of cytoplasts obtained by centrifugation of zona-free in vitro maturation (IVM) porcine oocytes to support remodeling of the somatic cell nucleus and the subsequent development in vitro of somatic cell nuclear transferred (SCNT) embryos. A primary culture of cumulus cells was used as the source of donor cells, and recipient cytoplasts were derived from IVM oocytes that were cultured for $48 \mathrm{~h}$, denuded of zonae pellucidae, and subjected to gradient centrifugation in Percoll solution to separate the ooplasm into fragments. Fragments were stained with Hoechst-33342 and cytoplasts were selected under an epifluorescence microscope. Then two or three cytoplasts were aggregated with a single somatic cell in phytohemagglutinin solution $(500 \mu \mathrm{g} / \mathrm{mL})$. Fusion between somatic cell and cytoplasts was induced by two DC pulses of $1.5 \mathrm{kV} / \mathrm{cm}$ for $20 \mu \mathrm{s}$, and activation was accomplished by two DC pulses of $0.8 \mathrm{kV} / \mathrm{cm}$ for $30 \mu \mathrm{s}$ at $1 \mathrm{~h}$ after fusion in $0.28 \mathrm{M}$ mannitol solution supplemented with $0.05 \mathrm{mM} \mathrm{CaCl}_{2}$ and $0.1 \mathrm{mM} \mathrm{MgSO}_{4}$. The resultant embryos were transferred to a WOW culture system (Vajta et al. 2000 Mol. Reprod. Dev. 55, 256-264) and cultured in glucose-free NCSU-37 containing $4 \mathrm{mg} / \mathrm{mL}$ BSA supplemented with $0.17 \mathrm{mM}$ sodium pyruvate and $2.73 \mathrm{mM}$ sodium lactate from Days 0 to 2; from Days 2 to 7 they were cultured in NCSU-37 supplemented with $5.55 \mathrm{mM}\{\mathrm{D}\}$-glucose and $5 \%$ FCS. Some of the reconstructed embryos were fixed at 1, 10, and $24 \mathrm{~h}$ after activation and stained with $1 \%(\mathrm{w} / \mathrm{v})$ orcein to display the morphology of the transferred somatic nuclei. The results showed that 53.6\% (30/56) of the SCNT embryos underwent premature chromosome condensation at $1 \mathrm{~h}$, $90.9 \%(50 / 55)$ formed pseudo-pronuclei at $10 \mathrm{~h}$, and $21 \%(19 / 90)$ of them cleaved to the two-cell stage at $24 \mathrm{~h}$ after the activation. The development to the blastocyst stage of the embryos that were reconstructed by quartet cells (three cytoplasts and one somatic cell; $8.9 \%, 10 / 112)$ was significantly higher $(P<0.05)$ than that of the triplet ones $(2.2 \%, 3 / 139)$. However, these blastocyst rates were significantly lower $(P<0.05)$ than the blastocyst development rate of parthenogenetic embryos with the intact zonae pellucidae $(28.3 \%, 17 / 60)$. These results suggest that (1) cytoplasts obtained by gradient centrifugation could support reprogramming of somatic cells and in vitro development of SCNT embryos to the blastocyst stage, and (2) the volume of cytoplasts apparently affects their in vitro development in pigs.

\title{
35 IN VITRO BOVINE EMBRYO DEVELOPMENT AFTER NUCLEAR TRANSFER BY HANDMADE CLONING USING A MODIFIED WOW CULTURE SYSTEM
}

\author{
C. Feltrin, F. Forell, L. dos Santos, and J. L. Rodrigues
}

Experimental Embryology and Biotechnology of Reproduction Laboratory, PO Box 15004 CEP 91501-170, Porto Alegre RS Brazil. Email: cristianofeltrin@terra.com.br

The effect of the microenvironment on embryo development during in vitro culture of zona-free embryos after nuclear transfer is still unclear. The aim of this experiment was to determine the effect of the dimensions of the well (WOW; Vajta et al. 2000 Mol. Reprod. Dev. 55, 256-264) culture system on the in vitro development of handmade cloned bovine embryos to the blastocyst stage. Appropriately ground steel needles were pressed slightly by hand to the bottom of the well of a polystyrene four-well dish (176740, Nunc, Life Technologies AS, Roskilde, Denmark). Embryos were produced by the handmade cloning (HMC) technique (Vajta et al. 2003 Biol. Reprod. 68, 571-578) with modifications, using primary cultures of skin fibroblast cells from an adult cow as nuclear donors. Cumulus-oocyte complexes were in vitro-matured in M-199 supplemented with $10 \%$ estrous cow serum (ECS), FSH, hCG, and estradiol (E2) for $17 \mathrm{~h}$. After maturation, cumulus cells were removed by pipetting. Following zona pellucida removal in $0.5 \%$ protease (Sigma, Brazil), zona-free oocytes were incubated for $15 \mathrm{~min}$ in $5 \mathrm{mg} / \mathrm{mL}$ cytochalasin B (Sigma) and subsequently hand-bisected and screened for nuclear material under UV light after incubation in $10 \mathrm{mg} / \mathrm{mL}$ bisbenzimide (Hoechst 33342). Next, two enucleated halves and one donor cell were aggregated after a quick exposure to phytohemagglutinin (PHA) and subsequently fused by two electrical DC pulses of $1 \mathrm{kV} / \mathrm{cm}$ for $20 \mu \mathrm{s}$, in a BTX 453 chamber coupled to an ECM 2001 Electro Cell Manipulator System (BTX, Inc., San Diego, CA, USA), with additional exposure to brief pre- and post-fusion AC pulses of $15 \mathrm{~V}$. Reconstructed embryos were chemically activated in $5 \mathrm{mM}$ ionomycin (Sigma) for $5 \mathrm{~min}$, followed by 2 mM 6-DMAP (Sigma) for $2.5 \mathrm{~h}$. Finally, activated reconstructed cloned embryos were in vitro-cultured in one of two WOW culture systems (larger vs. smaller micro-wells) in 4-well plates containing $400 \mathrm{~mL}$ modified SOF medium supplemented with $10 \%$ ECS, under mineral oil, at $5 \% \mathrm{CO}_{2}, 5 \% \mathrm{O}_{2}$ and $90 \% \mathrm{~N}_{2}$, and $39^{\circ} \mathrm{C}$ for 7 days. In Group 1 (large-size micro-well), embryos were cultured in individual cylindrical micro-wells with an inner diameter and depth of approximately 280 and $250 \mathrm{~mm}$, respectively, whereas in Group 2 (small size micro-well), embryos were cultured in individual conical micro-wells with approximately $130 \mathrm{~mm}$ inner diameter and $150 \mathrm{~mm}$ depth. Data analysis was performed by the chi-square test. After four replicates, cleavage rates were significantly higher $(P<0.05)$ in Group $2(51 / 63,80.9 \%)$ than in Group $1(43 / 67,64.1 \%)$. Embryo development to the blastocyst stage was also greater $(P<0.05)$ in the small micro-wells $(16 / 63,25.3 \%)$ than in the large ones $(8 / 67,11.9 \%)$. In summary, these results show a significant increase in cleavage and blastocyst developmental rates in handmade cloned embryos cultured in a modified WOW system using individual small size micro-wells, suggesting that a small, tighter micro-well provides favorable in vitro conditions for embryo development.

\section{DEVELOPMENTAL COMPETENCE OF HUMAN AGED OOCYTES AS HOST CELLS FOR NUCLEAR TRANSFER}

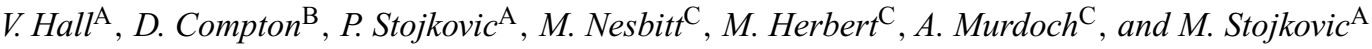 \\ ${ }^{A}$ University of Newcastle upon Tyne, Newcastle upon Tyne, Tyne and Wear, UK; \\ ${ }^{\mathrm{B}}$ Dartmouth Medical School, Hanover, NH 03755, USA; \\ ${ }^{\mathrm{C}}$ Newcastle Fertility Centre at Life, Newcastle upon Tyne, Tyne and Wear, UK
}

The use of aged metaphase II oocytes (cultured in vitro for more than $14 \mathrm{~h}$ ) for somatic cell nuclear transfer (SCNT) in varying species has resulted in lower developmental outcomes compared with non-aged in vitro- or in vivo-matured oocytes. However, due to limited resources of fresh oocytes 
for the derivation of nuclear transfer stem cell lines, further investigation in using spare oocytes is required. Aged spare oocytes (48 h post oocyte retrieval) were consigned for research (under HFEA and local ethics approval) by couples undergoing either in vitro fertilization (failed IVF oocytes, f-IVF) or intracytoplasmic sperm injection (failed-ICSI oocytes, f-ICSI) treatments. Aged oocytes were randomly assigned for double-labeling immunocytochemical analysis (f-IVF, $n=10$; f-ICSI, $n=7$ ) for the microtubule markers, NuMA and $\alpha$-tubulin, or parthenogenetic activation. Immunocytochemical analysis was performed as previously described (Chatzimeletiou et al. 2005 Hum. Reprod. 20, 672-682) using primary antirabbit NuMA (gift from D. Compton, Dartmouth Medical School, Hanover, NH, USA) and anti-mouse DM1- $\alpha$. Secondary antibodies were donkey anti-rabbit and anti-mouse immunoglobulins. Oocytes were counterstained with Hoechst 33342. Negative controls were performed as above with blocking solution substituting for primary antibodies. Parthenogenetic activation was performed for $4 \mathrm{~h}$ using $10 \mu \mathrm{M}$ calcium ionophore (5 min) and $2 \mathrm{mM}$ 6-dimethylaminopurine (Ca-I/DMAP) for f-IVF $(n=10)$ and f-ICSI oocytes $(n=11)$ or $10 \mu \mathrm{g} / \mathrm{mL}$ puromycin $(\mathrm{Ca}-\mathrm{I} / \mathrm{Pur})$ for $\mathrm{f}-\mathrm{IVF}(n=12)$ and f-ICSI oocytes $(n=10)(4 \mathrm{~h})$. Activated oocytes were cultured in a biphasic system, G1.3 ${ }^{\mathrm{TM}}$ and G2.3 ${ }^{\mathrm{TM}}$ (Vitrolife UK, Ltd., Ediburgh, Lothian, UK) for 5 days at $37{ }^{\circ} \mathrm{C}$ in $5 \% \mathrm{CO}_{2}$ in humidified air. NuMA was localized to the metaphase spindle in $6 / 10(60 \%)$ and $7 / 7(100 \%)$ oocytes for f-IVF and f-ICSI, respectively, and/or in cytoplasmic cytasters. One f-IVF oocyte and four f-ICSI oocytes had visible tetrapolar spindles. Unusual patterns of diffuse NuMA staining containing dense foci within these regions, but not associated with the cytasters or metaphase spindle, were also observed in two f-IVF oocytes. The majority of oocytes displayed ring-like staining of DM1- $\alpha$, which was aberrant in two f-ICSI oocytes. Parthenogenetic development was poor for both treatments. Cleavage rates were $17 \%$ and $20 \%$ for $\mathrm{f}-\mathrm{IVF}$ using $\mathrm{Ca}-\mathrm{I} / \mathrm{PUR}$ and Ca-I/DMAP, respectively, and $40 \%$ and $45 \%$ for f-ICSI using Ca-I/PUR and Ca-I/DMAP, respectively. Fragmentation rates were high across all treatments. No parthenogenetic embryos developed beyond the 6-cell stage. Thus, the use of aged human oocytes for SCNT may be difficult due to their incapacity to artificially activate using current activation protocols and, in addition, due to the microtubule abnormalities observed in many of these aged oocytes.

\title{
37 CELL CYCLE ANALYSIS IN IN VITRO-CULTURED ADULT SKIN FIBROBLASTS
} OF THE GORAL (NAEMORHEDUS CAUDATUS)

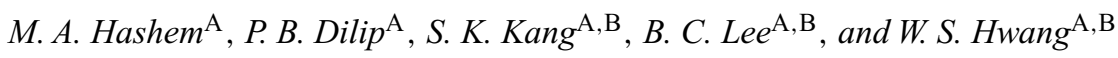 \\ ${ }^{A}$ Department of Theriogenology and Biotechnology, College of Veterinary Medicine, \\ Seoul National University, Seoul 151-742, Korea; \\ B The Xenotransplantation Research Center, Seoul National University Hospital, Seoul, 110-744, Korea; \\ ${ }^{\mathrm{C}}$ School of Agricultural Biotechnology, Seoul National University, Seoul National University, Seoul 151-742, Korea
}

The present study was undertaken to examine the cell cycle characteristics of goral adult skin fibroblasts under a variety of cell cycle-arresting treatments. Gorals are listed as an endangered animal in CITES Appendix I. Experimental findings suggest that G0/G1 stages of the cell cycle give better results in somatic cell nuclear transfer (SCNT) in terms of normal ploidy and development of reconstructed embryos. In this regard, synchronization of the cell cycle stages in $\mathrm{G} 0 / \mathrm{G} 1$ phase is suggested to be one of the key factors for determining the success of nuclear transplantation. Serum deprivation, contact inhibition, chemical inhibitors such as protease, and antioxidant inhibitors are widely used for cell cycle synchronization and inhibition of apoptosis. Four experiments were performed, and each with one-way completely randomized design involving three replicates of all treatments using the general linear models procedure in the statistical analysis system (SAS Institute, Inc., Cary, SC, USA). Least significant difference was used to determine statistical significance $(P<0.05)$ among treatment groups. In Exp. 1, effects of cyclic confluency, serum starvation, or full confluency on goral cells were studied. In Exp. 2, treatments with different antioxidants such as $2 \mathrm{mM}$ beta-mercaptoethanol (B-ME), cysteine, or glutathione for $24 \mathrm{~h}$ were examined in fully confluent cells without serum starvation. In Exp. 3, three protein inhibitors, 2 mM 6-dimethylaminopurine (6-DMAP), $7.5 \mu \mathrm{g} / \mathrm{mL}$ cycloheximide, or $7.5 \mu \mathrm{g} / \mathrm{mL}$ cytochalasin B, were treated as in Exp. 2. In Exp. 4, different concentrations $(0,0.5,1.0$, or $2.5 \%$ ) of dimethyl sulfoxide (DMSO) were treated as in Exp. 2. In all experiments, cell cycle stages were analyzed by fluorescence-activated cell sorting (FACS) analysis. In Exp. 1, more cells (70.4\%) were found in the G0/G1 stage in the serum starved medium compared to cells in the cyclic $(66.1 \%)$ and fully confluent growth phase (65.5\%). In Exp. 2, supplementing with B-ME (61.8\%) or cysteine $(62.0 \%)$ in the culture medium synchronized cell cycle to the G0/G1 stage better than glutathione (53.1\%). In Exp. 3, more cells were synchronized to the G0/G1 stage in media supplemented with cyclohexamide $(62.3 \%)$ than with 6-DMAP $(5.7 \%)$ or cytochalasin B (13.3\%). Incidence of apoptosis was higher in media containing 6-DMAP (91.8\%) or cytochalasin B (76.6\%). In Exp. 4, supplementing the medium with $0.5 \%$ (76.0\%) or $1.0 \%$ (75.9\%) DMSO synchronized the cells effectively to the G0/G1 stage compared to the untreated control medium $(69.3 \%)$ or medium supplemented with $2.5 \%$ of DMSO (8.0\%). In conclusion, cyclohexamide, B-ME, cysteine, or DMSO at the optimum concentration can synchronize the cell cycle effectively, which may have a positive impact on the outcome of SCNT in the goral.

This study was supported by grants from the Korean MOST (Top Scientist Fellowship) and MAF (Biogreen 21 \#20050301-034-443-026-01-00).

\section{FULL TERM DEVELOPMENT IN A COW CARRYING A NUCLEAR TRANSFER EMBRYO DERIVED FROM FIBROBLASTS AND OOCYTES OF ITS OWN CLONE}

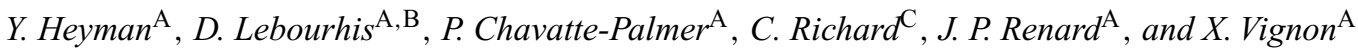 \\ ${ }^{A}$ INRA, UMR INRA/ENVA Biologie du Développement et Reproduction, 78352 Jouy en Josas, France; \\ ${ }^{B}$ UNCEIA, 13 Rue Jouet, 94703 Maisons Alfort, France; \\ ${ }^{\mathrm{C}}$ UCEA INRA, Bressonvilliers, 91630 Leudeville, France
}

Cloned animals are a unique model for studying the role of maternal immune response in failure of pregnancies often reported in bovine somatic cloning. Major histocompatibility complex class 1 (MHC1) has been shown to be down-regulated in placentomes during pregnancy, and abnormal 
MHC1 expression by the fetal membranes has been suggested to be the cause of pregnancy failure in bovine clones. We designed this study to investigate whether pregnancy losses would be decreased in cloned cows transferred with their genetically identical conceptus. Bovine fibroblasts were established from a skin biopsy on an adult Holstein cow from which a clone of 12 females had previously been derived. Five heifers of this set of clones were used for providing oocytes through (OPU) after stimulation, and seven were used as recipient heifers. Fibroblasts from this genotype were thawed, grown to confluency, and then used as the source of donor cells for NT. Recipient oocytes were matured in vitro before enucleation at 20-22 h post maturation (hpm). Embryos were reconstructed by fusion with donor cells at $23-24 \mathrm{hpm}$ and activated in $10 \mu \mathrm{g} / \mathrm{mL}$ cycloheximide and $5 \mu \mathrm{g} / \mathrm{mL}$ cytochalasin B for $5 \mathrm{~h}$ after fusion, and then co-cultured on Vero cells for 7 days in microdrops of B2 medium. A control group of NT embryos was produced simultaneously with the same cell line and unrelated oocytes from slaughterhouse ovaries. Embryos were transferred into synchronous recipients (one blastocyst/recipient). The pregnancies were followed by ultrasonography. The reconstructed embryos fused at the same rate in both groups of oocytes, but the cleavage rate was significantly lower in the clone group (Table 1). The reason for this low rate is unclear. However, pregnancy rates after transfer of NT blastocysts were similar up to term between the related and non-related groups of transplanted embryos. These results suggest that the maintenance of pregnancy in bovine is not linked to genetic disparity between fetus and dam. More investigations are required to elucidate why the in vitro development was so low, and whether pregnancies established in cows with their genetically identical conceptus are less prone to abortion or physiopathological developments.

Table 1. Development of NT embryos with clone or unrelated cell/oocyte/ recipient genotype

\begin{tabular}{lcc}
\hline & Clone & Unrelated \\
\hline In vitro development & & \\
$\quad$ Reconstructed embryos & 113 & 118 \\
Fused (\%) & $97(85.8)$ & $93(78.8)$ \\
Cleaved (\% of fused) & $10(10.3)^{\mathrm{a}}$ & $78(83.9)^{\mathrm{b}}$ \\
Blastocysts (\% of fused) & $7(7 .)^{\mathrm{c}}$ & $46(49.5)^{\mathrm{d}}$ \\
In vivo development & & 10 \\
$\quad$ Blastocysts transferred & 7 & 6 \\
Initiated pregnancies & 4 & 3 \\
Pregnant by Day 35 & 1 & 3 \\
Pregnant by Day 90 & 1 & 1 \\
Calvings & 1 & 1 \\
Live offspring & 1 & \\
\hline a,b,c,d Values in lines with different superscripts are significantly different, \\
$P<0.01$.
\end{tabular}

\title{
39 DEVELOPMENTAL COMPETENCE OF RECONSTRUCTED EMBRYOS PRODUCED BY NUCLEAR TRANSFER BETWEEN CYNOMOLGUS MONKEY FIBROBLAST CELLS AND RABBIT OOCYTES
}

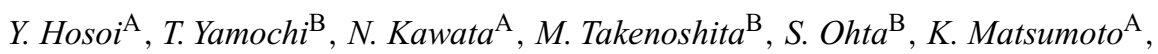 \\ K. Saeki ${ }^{\mathrm{A}}$, and A. Iritani ${ }^{\mathrm{A}}$

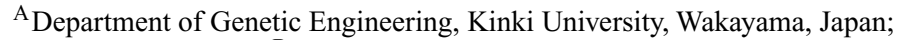 \\ ${ }^{B}$ Keari Co., Ltd., Osaka, Japan
}

\begin{abstract}
Interspecies nuclear transfer has been used as an invaluable tool for studying nucleus-cytoplasm interactions and it may also be used for rescuing endangered species whose oocytes are difficult to obtain. In this study, we investigated interaction of the cynomolgus monkey cell as a nuclear donor with the rabbit oocyte as a host cytoplasm. Whole cynomolgus fibroblast cells were injected into the rabbit enucleated oocytes (cynomolgus-rabbit cloned embryos) and cultured in TCM-199 and RPMI 1640 culture media. Rabbit-rabbit cloned embryos we used as control in this study. Karyotype analyses confirmed that genetic material of blastocysts was derived from the cynomolgus donor cells at blastocyst stage. Mitochondrial constitution analysis of the cynomolgus-rabbit cloned embryos indicated that mitochondria from both donor cells and enucleated oocytes coexisted. After culture for $168 \mathrm{~h}$ post-nuclear transfer, all cynomolgus-rabbit cloned embryos in TCM-199 were arrested at the 8-cell stage, but some of them developed to the blastocyst stage in RPMI $1640(11 / 59,18.6 \%)$. In this experiment, the nutrition requirement in vitro and the cleavage rate at each $24 \mathrm{~h}$ were examined. When TCM-199 was supplemented with lactate, some of these embryos developed to the blastocyst stage $(15.3 \%, 2 / 13)$. This means that cynomolgus-rabbit cloned embryos might be controlled by the donor nucleus even in these early developmental stages. However, the timing of cleavage of cynomolgus-rabbit cloned embryos is very similar to that of the rabbit-rabbit cloned embryos. Time of cleavage may depend on the protein accumulated in the cytoplasm. In the prolonged culture of reconstructed embryos on feeder cells, adhesion cells were observed. These cells are also very similar to the cells derived from cynomolgus embryos by the same method. Our results suggest that: (1) a cynomolgus nucleus can co-ordinate with rabbit oocyte cytoplasm in early embryo development, (2) the 8- to 16-cell stage block in the cynomolgus-rabbit cloned embryos may due to the same reason as that in the cynomolgus embryos, and (3) ooplasmic factors that control time of cleavage are highly conservative between the species.
\end{abstract}




\title{
40 EFFECT OF CHEMICAL ACTIVATION ON DEVELOPMENT AND APOPTOSIS OF PREIMPLANTATION PORCINE EMBRYOS DERIVED FROM NUCLEAR TRANSFER
}

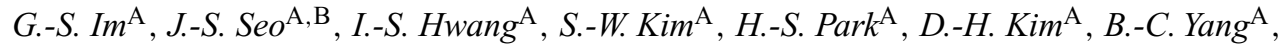 \\ I.-K. Kong ${ }^{\mathrm{B}}$, and B.-S. Yang ${ }^{\mathrm{A}}$

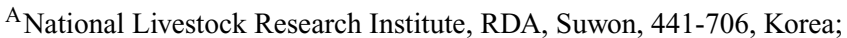 \\ ${ }^{B}$ Department of Animal Science and Technology, Sunchon National University, Sunchon, 540-742, Korea
}

\begin{abstract}
Activation is one of key factors for improving developmental ability of pre-implantation nuclear transfer (NT) embryos. This study investigated the effect of chemical activation following fusion/activation on the development and apoptosis of pre-implantation porcine embryos derived from NT. Oocytes were aspirated from ovaries collected from a local abattoir, and then matured in TCM-199 for 42 to 44 h. Donor cells were prepared from a 35-day-old porcine fetus. Matured oocytes were enucleated and donor cells were introduced into the perivitelline space. Fusion/activation was conducted with two electric pulse of $1.2 \mathrm{kV} / \mathrm{cm}$ for $30 \mu \mathrm{s}$. Fused embryos were divided into four groups. The first one was the control without chemical activation; the other three groups were treated with thimerosal $(0.2 \mathrm{mM}$ for $10 \mathrm{~min}$; $\mathrm{T})$ and then with dithiothreitol $(8 \mathrm{mM}$ for $30 \mathrm{~min}$; DTT), 6-dimethylaminopurine ( $2 \mathrm{mM}$ for $3 \mathrm{~h}$; 6-DMAP), or cycloheximide $(10 \mu \mathrm{g} / \mathrm{mL}$ for $6 \mathrm{~h} ; \mathrm{CH})$. Treated embryos were cultured in porcine zygote medium-3 (PZM-3) at $38.5^{\circ} \mathrm{C}$ under $5 \% \mathrm{CO}_{2}$ in air for 6 days. Cleavage and blastocyst rate were determined on Days 3 and 6 , respectively. Apoptosis was analyzed with a terminal deoxynucleotidyl transferase-mediated deoxyuridine 5-triphosphate nick-end labeling (TUNEL) assay from day 1 to 7. Embryos treated with chemicals following fusion/activation showed significantly higher blastocyst rates compared to control embryos fused/activated by electric pulse alone $(12.6 \%$ for control vs. $21.1 \%$ for DTT, $20.8 \%$ for 6 -DMAP, $20.6 \%$ for CH; $P<0.05)$. Although total cell number of blastocysts showed no significant difference, the ratio of inner cell mass to trophectoderm was significantly higher $(P<0.05)$ in embryos with chemical activation than in those without it $(11.9$ vs. $19.4,18.1$, and $24.1 \% ; P<0.05)$. Occurrence of apoptosis was first observed on Day 3 , but there was no significant difference among treatments until Day 6. It was significantly increased in embryos with chemical activation on Day 7 compared to control embryos (5.1 vs. 7.1, 7.8, and 7.8\%; $P<0.05$ ). These results indicate that chemical activation following fusion/activation could support significantly a higher blastocyst rate for pre-implantation porcine embryos derived from nuclear transfer; however, it can increase occurrence of apoptotic cells at blastocyst stage.
\end{abstract}

\section{STUDY ON THE ESTABLISHMENT OF SOMATIC CELL NUCLEAR TRANSFER USING IN VIVO-MATURED OOCYTES IN DOGS}

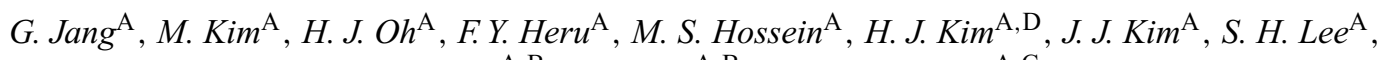 \\ S. K. Kang ${ }^{\mathrm{A}, \mathrm{B}}$, B. C. Lee $\mathrm{A}^{\mathrm{B}}$, and W.S. Hwang ${ }^{\mathrm{A}, \mathrm{C}}$ \\ ${ }^{\text {A }}$ Department of Theriogenology and Biotechnology, College of Veterinary Medicine, Seoul National University, \\ San56-1, Shillim-Dong, Kwanak-Gu, Seoul, 151-742 Korea; \\ ${ }^{B}$ Xenotransplantation Research Center, Seoul National University Hospital, \\ 28 Yongon-dong, Chongno-gu, Seoul, 110-799, Korea; \\ ${ }^{\mathrm{C}}$ School of Agricultural Biotechnology, San56-1, Shillim-Dong, Kwanak-Gu, Seoul, 151-742 Korea; \\ ${ }^{\mathrm{D}}$ Haemaru Institute of Small Animal Practice, Seohyun-dong, Bundang-gu, SungNam City, Kyunggi-do, Korea
}

The present study was performed to collect in vivo matured canine oocytes for somatic cell nuclear transfer (SCNT) and to investigate the developmental competence of canine parthenogenetic and SCNT embryos as the preliminary research for producing cloned dog. The day of ovulation as described by Hase et al. (2000 J. Vet. Med. Sci. 62, 243-248) was determined by serum progesterone levels and at that time vaginal cytology was performed to assess the cornified index. In vivo-matured oocytes were recovered by retrograde flushing of the oviducts at around $48 \mathrm{~h}(n=20)$ or $72 \mathrm{~h}(n=25)$ after the estimated time of ovulation. Overall size of each oocyte, as well as ooplasmic diameter, zona pellucida thickness, and perivitelline space width, was determined after removing the cumulus cells by pipetting (Exp. 1). To determine activation protocols, two treatments, (1) chemical activation $(10 \mu \mathrm{M} \mathrm{Ca}$ ionophore for $4 \mathrm{~min}$, followed by incubation for $4 \mathrm{~h}$ with $1.9 \mathrm{mM}$ 6-dimethylaminopurine) and (2) electrical stimulation $(3.1 \sim 3.4 \mathrm{kV} / \mathrm{cm}$ in $0.25 \mathrm{M}$ mannitol solution), were evaluated to induce parthenogenetic activation of oocytes (Exp. 2). Donor cells were obtained from the primary cell culture of a canine ear skin biopsy, and SCNT was performed according to our laboratory procedures (Jang et al. 2004 Theriogenology $62,512-521)$. Three voltages $(1.7 \sim 2.0 \mathrm{kV} / \mathrm{cm}, 2.1 \sim 2.4 \mathrm{kV} / \mathrm{cm}$, and $3.1 \sim 3.4 \mathrm{kV} / \mathrm{cm})$ were tested for fusion. The fused couplets were subjected to chemical or electrical stimulation as in parthenogenetic activation and in vitro developmental competence was monitored (Exp. 3). As a result, more in vivo-matured canine oocytes were obtained at $72 \mathrm{~h}(92 \%)$ than at $48 \mathrm{~h}(15 \%)$ after ovulation; the 72 -h occytes had progesterone concentrations of $4-8 \mathrm{ng} / \mathrm{mL}$ and a cornified index (vaginal cytology) of 83.34 . The average number of oocytes recovered was 12 and sizes of ooplasmic diameter, cytoplasm, zona pellucida, and perivitelline space in in vivo canine-matured oocytes $(n=120)$ were $178.8 \pm 9.3 \mu \mathrm{m}$, $125.0 \pm 8.2 \mu \mathrm{m}, 21.7 \pm 3.7 \mu \mathrm{m}$, and $12.7 \pm 3.5 \mu \mathrm{m}$, respectively. Parthenogenetically activated oocytes developed to the 16-cell and morula stages, but failed to develop to the blastocyst stage. Among the three voltages, in the highest voltage $(75.2 \%)$ the number of fused couplets was increased compared to either of the other voltages $(33.3 \%$ and $44.0 \%)$. Cleavage rates $(60.9 \%$ vs. $58.0 \%)$ of cloned embryos were not significantly affected by method of activation. In terms of in vitro developmental competence, cloned embryos developed to the 16-cell or morula stage in vitro after electrical or chemical activation, respectively. In conclusion, in the present study we demonstrated that measurement of progesterone levels, in combination with evaluation of vaginal cytology, can be used to determine the estimated time of ovulation in bitches. In addition, we determined fusion/activation protocols that resulted in in vitro development of a portion of parthenogenetically activated and cloned embryos to the 16-cell and morula stages.

This study was supported by grants from the Biogreen 21-1000520030100000. 


\title{
42 DEVELOPMENTAL POTENTIAL AND REPROGRAMMING EFFICIENCY OF BOVINE EMBRYOS CLONED WITH ADULT FIBROBLASTS TREATED BY A DEMETHYLATING AGENT, S-ADENOSYL-HOMOCYSTEINE
}

\author{
B.-G. Jeon ${ }^{\mathrm{A}}$, S. D. Perrault ${ }^{\mathrm{A}}$, G.-J. Rho ${ }^{\mathrm{B}}$, D. H. Betts ${ }^{\mathrm{A}}$, and W. A. King ${ }^{\mathrm{A}}$ \\ ${ }^{A}$ Department of Biomedical Sciences, Ontario Veterinary College, \\ University of Guelph, Guelph, Ontario, Canada; \\ ${ }^{\mathrm{B}}$ Department of Obstetrics and Theriogenology, College of Veterinary Medicine, \\ Gyeongsang National University, 900 Gazwa, Chinju 660-701, South Korea
}

\begin{abstract}
Animal cloning by somatic cell nuclear transfer (SCNT) has been successfully applied to several species although with low efficiency and often associated with severe abnormalities. These poor outcomes are thought to be a consequence of aberrant DNA methylation patterns that result from incomplete epigenetic reprogramming of the transplanted nucleus into recipient oocytes. Telomerase, an enzyme not expressed in most somatic cells, should be expressed in cloned embryos. Therefore its activity has been used as an index of reprogramming in SCNT embryos. The objective of this study was to investigate the DNA methylation status of donor fibroblasts treated with a non-cytotoxic transmethylation inhibitor, S-adenosyl homocysteine (SAH), and to assess the relative telomerase activity (RTA) and developmental potential of SCNT embryos derived from such cells. Adult ear skin fibroblasts were cultured in DMEM supplemented with $0,0.5,1.0$, or $2.0 \mathrm{mM} \mathrm{SAH}$ for $144 \mathrm{~h}$ by daily media change prior to nuclear transfer. The SAH-treated fibroblasts were immunostained with a fluorescein isothiocyanate (FITC) conjugated 5-methylcytosine antibody and the relative fluorescence intensity (RFI) was analyzed using a fluorescence microscope equipped with an Openlab ${ }^{\mathrm{TM}}$ program (Improvision, Coventry, UK). RTA was measured in Day 8 SCNT blastocysts using the real-time quantitative telomeric repeat amplification protocol (RQ-TRAP). Fibroblasts treated with $0.5,1.0$, and $2.0 \mathrm{mM} \mathrm{SAH}$ showed lower levels of DNA methylation compared to nontreated controls, and the values did not differ among the treatment groups. Cleavage rates did not differ between the SCNT embryos derived from $0.5 \mathrm{mM} \mathrm{SAH-treated} \mathrm{cells} \mathrm{and} \mathrm{nontreated} \mathrm{control}$ cells $(92.3 \%$ vs. $91.3 \%$, respectively). However, the rates of blastocyst development and hatching were significantly $(P<0.05)$ higher in SCNT embryos derived from $0.5 \mathrm{mM} \mathrm{SAH}$ treated donor cells compared to controls (60.0 and $40.0 \%$ vs. 34.3 and $26.4 \%$, respectively). Moreover, RTA of the $0.5 \mathrm{mM}$ SAH SCNT embryos was significantly $(P<0.05)$ increased $(1.5$-fold $)$ in relation to controls. S-adenosyl homocysteine treatment induces global DNA demethylation in donor fibroblasts and enhances the blastocyst frequencies for bovine SCNT embryos that also exhibit greater telomerase activity levels. These results suggest that use of hypomethylated donor somatic cells increases the developmental potential for SCNT embryos by enhancing the nuclear reprogramming efficiency.
\end{abstract}

This work was funded by NSERC, OMAF, OCAG, and CRC.

\section{PRODUCTION OF PORCINE EMBRYOS BY NUCLEAR TRANSFER OF BONE MARROW MESENCHYMAL STEM CELLS}

\author{
H.-F. Jin, B. Mohana Kumar, J.-G. Kim, H.-J. Song, S. Balasubramanian, S.-Y. Choe, and G.-J. Rho \\ College of Veterinary Medicine, Gyeongsang National University, Chinju, GN, Republic of Korea 660-701
}

Recent experimental evidence indicates that adult stem cells are more desirable than somatic cells for nuclear transfer (NT) because of their easy reprogrammability to resemble the genome of the zygote (Zhu et al. 2004 Biol. Reprod. 71, 1890-1897). Mesenchymal stem cells (MSCs) are a heterogeneous population of uncommitted and lineage-committed cells and may have a more flexible potential as donor cells for NT. The aim of this study was to characterize an isolated population of porcine MSCs from bone marrow and to compare the developmental potential of cloned (IVF) embryos with MSCs and porcine fetal fibroblasts ( $\mathrm{pFFs}$ ) by assessing the cleavage and blastocyst rate, total cell numbers, inner cell mass (ICM) ratio and apoptosis. MSCs were obtained from the aspirated bone marrow of 6-8-month-old pigs. Cells were centrifuged, resuspended, and plated in advanced-DMEM (ADMEM) supplemented with $5 \%$ fetal bovine serum (FBS). The differentiation potential was demonstrated by culture of MSCs (passage 3) under conditions that were favorable for adipogenic, osteogenic, and chondrogenic development (Pittenger et al. 1999 Science 284, 143-147). Oil red O staining revealed that MSCs produced lipid droplets after incubation in adipogenic media. Following osteoinduction, MSCs exhibited robust alkaline phosphatase activity and cells later transformed into mineralized nodules as demonstrated by von Kossa staining. Histological staining of proteoglycan indicated chondrogenic differentiation. Cumulus-oocyte complexes were matured, fertilized, and cultured by the following method (Abeydeera et al. 2000 Theriogenology 54, 787-797). NT embryos were produced as described by Kim et al. (2005 Mol. Rep. Dev. 70, 308-313). Cleavage rate was significantly $(P<0.05)$ higher in IVF embryos than in NT embryos derived from MSCs and $\mathrm{pFFs}(84.5 \% \pm 4.6$ vs. $52.2 \% \pm 5.4$ and $50.8 \% \pm 5.2$, respectively). However, blastocyst rates in IVF embryos and NT embryos derived from MSCs $(20.6 \% \pm 2.5$ and $18.5 \% \pm 3.0)$ did not differ but these rates were significantly $(P<0.05)$ higher than that for NT embryos derived from pFFs $(9.5 \% \pm 2.1)$. Total cell numbers and the ratio of ICM to total cells among embryos developed in NT from MSCs (29.4 \pm 5.2 and $0.38 \pm 0.08$, respectively) were significantly $(P<0.05)$ higher than for those from pFFs $(22.6 \pm 5.5$ and $0.18 \pm 0.12$, respectively). Proportions of TUNEL-positive cells in NT embryos from pFFs $(12.8 \pm 2.5)$ were significantly $(P<0.05)$ higher than in those from MSCs $(8.6 \pm 1.8)$ and in IVF embryos $(4.6 \pm 1.5)$. The results clearly demonstrate that multipotent bone marrow MSCs can make a suitable alternative to fibroblasts as donor cells and have a greater potential for producing viable cloned porcine embryos.

This work was supported by Grant No. R05-2004-000-10702-0 from KOSEF, Republic of Korea. 


\title{
44 IN VITRO AND IN VIVO SURVIVABILITY ON VITRIFIED EMBRYOS OBTAINED BY BOVINE SOMATIC CELL NUCLEAR TRANSFER
}

\author{
K. Kaneyama ${ }^{\mathrm{A}}, S$. Kobayashi $^{\mathrm{C}}, S$. Matoba $^{\mathrm{A}}$, Y. Hashiyada $^{\mathrm{B}}$, K. Imai $^{\mathrm{A}}$, M. Yonai $^{\mathrm{A}}$, and N. Saito $^{\mathrm{A}}$ \\ A National Livestock Breeding Center, Nishigo, Fukushima, Japan; \\ ${ }^{B}$ National Livestock Breeding Center Oou Station, Shichinohe, Aomori, Japan; \\ ${ }^{\mathrm{C}}$ National Agricultural Research Center for Kyushu Okinawa Region, Nishigoshi, Kumamoto, Japan
}

\begin{abstract}
Although many studies have been conducted on somatic cell nuclear transfer, there are only a few reports on cryopreservation of reconstructed embryos after nuclear transplantation. The objective of this study was to examine in vitro or in vivo development of vitrified blastocysts obtained by nuclear transfer. Nuclear transfer was carried out according to the procedure of Goto et al. (1999 Anim. Sci. J. 70, 243-245), and conducted using abattoir-derived oocytes and cumulus cells derived by ovum pickup from Holstein and Japanese Black cows. Embryos were vitrified as described by Saito et al. (1998 Cryobiol. Cryotech. 43, 34-39). The vitrification solution (GESX solution) was based on Dulbecco's PBS containing 20\% glycerol (GL), 20\% ethylene glycol (EG), $0.3 \mathrm{M}$ sucrose (Suc), $0.3 \mathrm{M}$ xylose (Xyl), and 3\% polyethylene glycol (PEG). The blastocysts were equilibrated in three steps, with $10 \%$ GL, $0.1 \mathrm{M}$ Suc, $0.1 \mathrm{M} \mathrm{Xyl}$, and 1\% PEG for $5 \mathrm{~min}$ (1); with 10\% GL, 10\% EG, $0.2 \mathrm{M} \mathrm{Suc,} 0.2 \mathrm{M}$ Xyl, and $2 \%$ PEG for 5 min (2) and GESX solution (3). After transfer to GESX, equilibrated embryos were loaded to $0.25-\mathrm{mL}$ straws and plunged into liquid nitrogen for 1 min. The vitrified blastocysts were warmed in water $\left(20^{\circ} \mathrm{C}\right)$ and diluted in $0.5 \mathrm{M}$ and $0.25 \mathrm{M}$ sucrose for 5 min each. Equilibration and dilution procedures were conducted at room temperature $\left(25-26^{\circ} \mathrm{C}\right)$. After dilution, the vitrified blastocysts were cultured in TCM- 199 supplemented with $20 \%$ fetal calf serum and $0.1 \mathrm{mM} \beta$-mercaptoethanol at $38.5^{\circ} \mathrm{C}$ under gas phase of $5 \% \mathrm{CO}_{2}$ in air. In Experiment 1, survival rates after vitrification were compared between the nuclear transfer and the IVF blastocysts. Survival rates of vitrified nuclear transfer blastocysts $(n=60$, Day 8$)$ at 24 and $48 \mathrm{~h}$ were $70.0 \%$ and $56.7 \%$, respectively, and those of vitrified IVF blastocysts $(n=41)$ were $82.9 \%$ and $82.9 \%$, respectively. There were no significant differences in survival rates at 24 and $48 \mathrm{~h}$ between the two groups. In Experiment 2, one (VIT-single) or two (VIT-double) vitrified and one (nonVIT-single) or two (nonVIT-double) nonvitrified reconstructed blastocysts per animal were transferred into Holstein dry cows. The result of Experiment 2 is shown in Table 1. This experiment demonstrated that the vitrification method in this study can be used for cloned embryo cryopreservation but the production rate should be improved.
\end{abstract}

Table 1. Comparison of survival rates of vitrified or nonvitrified cloned embryos after transfer

\begin{tabular}{lcccc}
\hline Group & Recipient cows & Pregnancy rate on day 40 & Cows that completed gestation & \% of viable calves \\
\hline VIT-single & 10 & $20.0 \%(2 / 10)$ & $10.0 \%(1 / 10)$ & $0.0 \%(0 / 10)$ \\
VIT-double & 21 & $28.6 \%(6 / 21)$ & $19.0 \%(4 / 21)$ & $9.5 \%(2 / 21)$ \\
nonVIT-single & 19 & $31.6 \%(6 / 19)$ & $21.1 \%(4 / 19)$ & $15.8 \%(3 / 19)$ \\
nonVIT-double & 6 & $66.7 \%(4 / 6)$ & $16.7 \%(1 / 6)$ & $16.7 \%(1 / 6)$ \\
\hline
\end{tabular}

\section{NUCLEAR LAMIN A/C EXPRESSION IN BOVINE PARTHENOTES AND NUCLEAR TRANSFER EMBRYOS}

\author{
R. D. W. Kelly, R. Alberio, and K. H. S. Campbell \\ University of Nottingham, Sutton Bonington Campus, Loughborough, Leicestershire, UK
}

Despite the apparent successes of nuclear transfer (NT) technology, numerous recent reports have indicated de-regulation of key gene expression patterns in NT embryos as compared to their in vivo and IVF counterparts. Aberrant expression of lamin A/C has been reported in mouse (Moreira et al. 2003 J. Cell Sci. 116, 3713-3720) and bovine (Sullivan et al. 2004 Biol. Rep. 70, 146-153) NT embryos, leading to the hypothesis that the presence of lamin $\mathrm{A} / \mathrm{C}$ might affect subsequent development. Lamin $\mathrm{A} / \mathrm{C}$ expression is a potential marker for reprogramming due to the induced expression and remodeling during differentiation. Previously using immunofluorescence in bovine IVF embryos, we have demonstrated the persistence of lamin A/C until the 2-cell stage (Kelly et al. 2005 Reprod. Fertil. Dev. 17, 205-206). The present study was initiated to further characterize lamin A/C expression in bovine parthenogenetic and NT embryos using a monoclonal antibody specific to lamin $\mathrm{A} / \mathrm{C}$. Bovine oocytes were matured in vitro as previously described (Fouladi-Nashta et al. 1998 Biol. Rep. 59, 255-262). NT embryos were constructed using lamin A/C-positive primary bovine fetal fibroblasts and in vitro-matured, enucleated MII bovine oocytes. Oocyte cell couplets were fused at $24 \mathrm{~h}$ post onset of maturation $1 \mathrm{~h}$ prior to activation. Oocyte activation was achieved with $7 \%$ ethanol for $7 \mathrm{~min}$ followed by a $6 \mathrm{~h}$ incubation in $\mathrm{mSOF}$ containing $10 \mu \mathrm{g} / \mathrm{mL}$ cycloheximide and $7.5 \mu \mathrm{g} / \mathrm{mL}$ cytochalasin B for the production of both NT and parthenogenetic embryos. Embryos were cultured in mSOF supplemented with $10 \%$ FCS and collected at various stages for immunofluorescence staining. Prior to fixation, embryos were incubated in $2 \mathrm{mg} / \mathrm{mL}$ protease to remove the zona pellucida. Samples were fixed in $100 \%$ methanol at $-20^{\circ} \mathrm{C}$ for $20 \mathrm{~min}$ and then blocked for $1 \mathrm{~h}(4 \%$ goat serum in PBS) at RT. Embryos were then incubated overnight at $4{ }^{\circ} \mathrm{C}$ with mouse anti-lamin A/C antibody (IgM; Santa Cruz Biotechnology, Santa Cruz, CA, USA) or with blocking solution as a control. Following the primary incubation, embryos were washed extensively in $1 \%$ BSA in PBS and then incubated with Cy3 goat anti-mouse IgM (1:400) (Chemicon International, Inc., Temecula, CA, USA) for $1 \mathrm{~h}$ at RT. Unbound secondary antibody was removed by washing with $1 \%$ BSA in PBS, and embryos were mounted in VectaShield mounting medium containing 4',6-diamidino-1-phenylindole (DAPI) (Vector Laboratories, Burlingame, CA, USA). Images were viewed using epifluorescence (Leica DMR; Leica Microsystems, Wetzlar, Germany) and confocal microscopy (Leica TCS). Inhibiting protein synthesis during the activation period with cycloheximide had no effect on lamin A/C assembly in $6 \mathrm{~h}$ post activation 
(hpa) parthenogenetic (35/35) and NT (7/7) embryos. The pronuclei of parthenogenetic (30/30) and NT (15/15) zygotes at 22 hpa were also positively labeled for lamin A/C. Nuclear labeling was observed in both parthenogenetic (25/25) and NT (12/12) 2-cell embryos. All parthenogenetic and NT embryos examined from the 4-cell stage through to blastocysts were stained negatively for lamin $\mathrm{A} / \mathrm{C}$. These results suggest that lamin $\mathrm{A} / \mathrm{C}$ present in bovine NT zygotes is not due to aberrant reprogramming and that remodeling of the nuclear lamina occurs correctly in bovine NT embryos.

\title{
46 ANTI-APOPTOTIC EFFECT OF INSULIN-LIKE GROWTH FACTOR-1 AND ITS RECEPTOR ON DEVELOPMENT OF PORCINE PRE-IMPLANTATION EMBRYOS PRODUCED BY IN VITRO FERTILIZATION AND SOMATIC CELL NUCLEAR TRANSFER
}

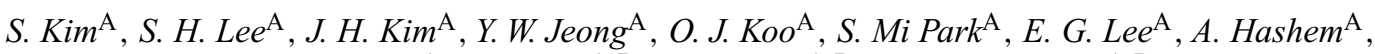

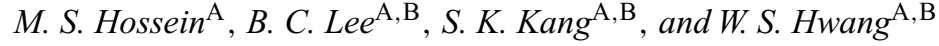 \\ ${ }^{A}$ Department of Theriogenology and Biotechnology, College of Veterinary Medicine, Seoul National University, Seoul, Korea; \\ ${ }^{\mathrm{B}}$ The Xenotransplantation Research Center, Seoul National University Hospital, Seoul, Korea; \\ ${ }^{\mathrm{C}}$ School of Agricultural Biotechnology, Seoul National University, Seoul National University, Seoul, Korea
}

\begin{abstract}
Insulin-like growth factor (IGF)-I is a receptor-mediated autocrine and/or paracrine growth and/or survival factor for mammalian embryo development. It is known to promote the growth and development of mouse pre-implantation embryos. The present study was designed to investigate the effects of IGF-I $(50 \mathrm{ng} / \mathrm{mL})$, anti-IGF-I receptor (IGR-IR) antibody $(0.05 \mu \mathrm{g} / \mathrm{mL})$, and their combination on porcine pre-implantation embryo development. Furthermore, the mechanism underlying the embryotropic effects of IGF-I was evaluated by monitoring the incidence of apoptosis and expression of apoptosis-related genes. In both IVF and SCNT embryos, culturing with IGF-I increased the rate of blastocyst formation and this embryotropic effect was neutralized by culturing with IGF-I and anti-IGF-I receptor antibody. Significant effects on the development of blastocysts $(P<0.05)$ were found in IVF $(16.9,22.6,9.3$, and $13.5 \%$ for control, IGF-I, anti-IGF-IR antibody, and their combination, respectively) and SCNT $(13.2,21.0,5.4$, and 15.7\%) embryos. Culturing IVF and SCNT embryos with IGF-I significantly increased the total number of cells in IVF blastocysts $(58.3,72.4,41.1$, and 55.2; $P<0.05)$, and SCNT blastocysts $(49.2,60.1,35.2$, and $43.1 ; P<0.05)$, and it decreased the number of apoptotic nuclei in IVF blastocysts $(3.9,2.8,5.5$, and $3.9 ; P<0.05)$ and SCNT blastocysts $(4.6,3.0,6.1$, and $4.9 ; P<0.05)$. These effects of IGF-I were also neutralized by culturing with IGF-I along with anti-IGF-IR antibody. Expression of the anti-apoptotic Bcl-2 gene was increased, whereas expression of the pro-apoptotic Bax gene was decreased in both IVF and SCNT embryos cultured with IGF-I. In both IVF and SCNT embryos, anti-IGF-IR antibody along with IGF-I neutralized the effect of IGF-I on expression of Bcl-2 and Bax genes. In conclusion, the present study demonstrated that IGF-I through its specific receptors improved the developmental competence of IVF and SCNT embryos by decreasing the incidence of apoptosis and regulating apoptosis-related genes in porcine pre-implantation embryos.
\end{abstract}

This study was supported by grants from the Korean MOST (Top Scientist Fellowship) and MAF (Biogreen 21 \#20050301-034-443-026-01-00).

\section{BLASTOCYST DEVELOPMENT RATE OF CLONED BOVINE EMBRYOS USING SERIAL NUCLEAR TRANSFER OF CELLS CONTAINING AN X-AUTOSOME-TRANSLOCATED CHROMOSOME $\mathbf{t}(\mathbf{X p + ; 2 3 q - )}$}

\author{
W. A. King, B.-G. Jeon, and D. H. Betts
}

Department of Biomedical Sciences, Ontario Veterinary College, University of Guelph, Guelph, Ontario, Canada

\begin{abstract}
Somatic cell nuclear transfer (SCNT) has been utilized to study various genetic and epigenetic contributions of specific biomedical diseases and developmental events by using various donor cell types such as mature lymphocytes, brain tumor cells, and other unique genotypes. Previously, we produced cloned fetuses and offspring derived from SCNT of adult ear skin fibroblasts obtained from a sub-fertile cow harboring an X-autosome translocation as a model to study $\mathrm{X}$-inactivation and chromosome dynamics during female meiosis. The aim of this study was to assess the cloning efficiency of the fibroblasts derived from a cloned calf with the $\mathrm{X}$-autosome translocation $\mathrm{t}(\mathrm{Xp}+; 23 \mathrm{q}-)$ compared to the original adult fibroblast donor containing the same chromosome translocation. Primary cultures of cells were established in DMEM $+15 \%$ fetal calf serum (FCS). To serve as nuclear donors, cells at 5-7 passages were cultured for 5 days until confluent. Oocytes matured for $18 \mathrm{~h}$ in TCM-199 with hormones were removed of their chromatin, and reconstructed by transfer of donor cells and fusion with two DC pulses $(1.2 \mathrm{kV} / \mathrm{cm}, 15 \mu \mathrm{s})$, delivered by a BTX 2000 Electro Cell Minupulator (BTX, Inc., San Diego, CA, USA), in $0.28 \mathrm{M}$ mannitol containing $0.01 \mathrm{mM} \mathrm{MgCl}_{2}$. After $1 \mathrm{~h}$ of fusion, the eggs were activated with $5.5 \mu \mathrm{M}$ ionomycin for $5 \mathrm{~min}$, followed by $11 \mu \mathrm{g} / \mathrm{mL}$ cyclohexamide for $5 \mathrm{~h}$. The eggs were cultured for 9 days in L-SOF at $39^{\circ} \mathrm{C}$ in a humidified atmosphere of $5 \% \mathrm{CO}_{2}, 5 \% \mathrm{O}_{2}, 90 \% \mathrm{~N}_{2}$. Chi-square analysis revealed no significant $(P>0.05)$ differences in the rates of cleavage, blastocyst frequencies, and cell numbers between the 1 st and 2 nd generation cloned embryos. Cleavage rates were $87.4 \%$ and $85.4 \%$ for 1 st and 2 nd generation cloned embryos, respectively. The frequencies of blastocyst development and hatched blastocyst formation on Day 9 were $41.4 \%(91 / 220)$ and $38.7 \%$ (92/238), and $26.4 \%(58 / 220)$ and $22.7 \%$ (54/238) for the 1st and 2nd generation cloned embryos, respectively. The numbers of total cells and inner cell mass (ICM) cells of Day 9 blastocysts were 183 and 52, respectively, in the 1st generation embryos and 167 and 51 cells in the 2nd-generation cloned embryos. In summary, 2nd generation cloned embryos derived from fibroblasts of a cloned calf with an X-autosome translocated chromosome showed embryo development and cell numbers similar to those of the 1st generation clones. These results demonstrate that serial nuclear transfer does not improve the blastocyst development rate of cloned embryos containing the $\mathrm{X}$-autosome translocation $\mathrm{t}(\mathrm{Xp}+; 23 \mathrm{q}-)$.
\end{abstract}

This work was funded by OCAG, OMAF, and CRC. 


\title{
48 MORPHOLOGICAL ANOMALIES OF FETAL MEMBRANES DURING GESTATION IN BOVINE CLONES DERIVED BY SOMATIC NUCLEAR TRANSFER
}

\author{
H. R. Kohan-ghadr ${ }^{\mathrm{A}}$, R. Lefebvre ${ }^{\mathrm{A}}$, G. Fecteau ${ }^{\mathrm{A}}$, L. C. Smith ${ }^{\mathrm{B}}$, and J. Durocher ${ }^{\mathrm{C}}$ \\ ${ }^{A}$ Department of Clinical Sciences, Faculty of Veterinary Medicine, University of Montreal, \\ Saint-Hyacinthe, Quebec, Canada; \\ ${ }^{\mathrm{B}}$ Department of Biomedicine, Faculty of Veterinary Medicine, University of Montreal, \\ Saint-Hyacinthe, Quebec, Canada; \\ ${ }^{\mathrm{C}}$ Alliance Boviteq, Inc., Saint-Hyacinthe, Quebec, Canada
}

High pregnancy loss and prenatal morbidity and mortality encountered in cloned animals might be explained by placental nutritional or steroidogenic insufficiency that would indirectly compromise fetal survival (Hashizume et al. 2002 Cloning and Stem Cells 4(3), 197-209). Our hypothesis is that ultrasonographic characterization of placenta in bovine clones could be used to predict anomalies in fetal development and well-being during pregnancy. We examined surrogate heifers bearing cloned fetuses $(n=37)$ and fetuses obtained by traditional embryo transfer $(n=5)$ in three stages of gestation (Day 70, 100 and 200). Morphological parameters based on direct ultrasonographic observations were collected: thickness and shape of amniotic membrane, utero-placental thickness, placentomes shape and length in fetal area, and umbilical cord shape and appearance. Analysis was done using repeated measures linear model, with day as a repeated factor. Of all clone pregnancies, 30 females were still pregnant at Day 70 and only 16 reached Day 100, from which $63 \%$ had different degrees of amniotic and/or umbilical cord anomalies. No placental anomalies or abortions were observed in the control group. The amniotic membrane abnormalities observed at Day 100 were irregularities and presence of nodules (rosary-like) in the cross-sectional view of the membrane. Hyperechogenic spikes or irregularities around the umbilical cord were observed at Day 100 in $38 \%$ of the clones $(n=6)$ and all of them also had amniotic anomalies. From the clone pregnancies with amniotic and/or umbilical cord problems, $30 \%$ aborted between Days 100 and 200, and for those that went to term (Days 264-278; $n=7$ ), all calves but one died at birth or during the first month of life. Statistical analysis results showed that placentomes in cloned embryos were larger than those in control embryos $(P=0.003$; least-squares mean $(\mathrm{LSM})$ for clone $=4.34 \mathrm{~cm}$, control $=2.97 \mathrm{~cm}$ ) in all three stages of gestation. During the same period, amniotic membranes of clones became thicker compared to those of the control group $(P=0.0001$; LSM for clone $=0.25 \mathrm{~cm}$, control $=0.11 \mathrm{~cm})$. Variation in utero-placental thickness was also observed. At Day 70, the utero-placental layer was thicker in clones in comparison to controls (LSM for clone $=0.32 \mathrm{~cm}, \mathrm{control}=0.30 \mathrm{~cm}$ ) before it became thinner at Day $200($ LSM for clone $=0.38 \mathrm{~cm}$, control $=0.40 \mathrm{~cm}$ ). The present results showed that morphological anomalies of placentomes, amniotic membrane and umbilical cord appear during gestation of bovine cloned fetuses that could potentially compromise normal fetal development. Also, ultrasonographic monitoring of pregnancies of cloned animals is a useful tool to characterize the fetal membrane changes.

This work was funded by a Strategic Project Grant from the Natural Sciences and Engineering Research Council (NSERC) Canada.

\section{INTRA-GENUS CLONED EMBRYOS PRODUCTION AND PREGNANCIES DERIVED FROM LEOPARD CAT NUCLEI AND DOMESTIC CAT ENUCLEATED EGGS}

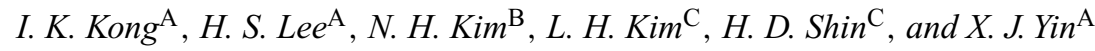 \\ ${ }^{\text {A }}$ Sunchon National University, Sunchon, JeonNam Province, South Korea; \\ ${ }^{\mathrm{B}}$ Chungbuk National University, Cheongju, Chungbuk Province, South Korea; \\ ${ }^{\mathrm{C}} \mathrm{SNP}$ Genetics, Inc., Seoul, South Korea
}

The leopard cat (Prionailurus bengalensis), a member of the felidae family, is currently listed as threatened by the Ministry of Environment in South Korea. In exotic or endangered species, the lack of oocytes and recipients precludes the use of traditional somatic cell nuclear transfer (NT), and an approach such as intragenus NT may be the only alternative for producing embryos and offspring. In the present study, we used the leopard cat (LC) as a somatic cell donor to evaluate the in vivo developmental competence, after transfer into domestic cat recipients, of cloned embryos produced by the fusion of LC fibroblast cell nuclei with domestic cat cytoplasts. A total of 412 enucleated domestic cat oocytes were reconstructed with either male (Treatment A) or female (Treatment B) adult LC fibroblasts. There was no significant difference in fusion rate (60.4 vs. $56.9 \%)$ between Treatment A and B. Of the fused couplets, the cleavage and blastocyst developmental rate in Treatment A were greater than those in Treatment B (69.5 vs. $60.9 \%$; 8.3 vs. $7.8 \% ; P<0.05)$. In treatment $\mathrm{A}$, in vivo developmental studies at $30-45$ days postimplantation demonstrated $4.8 \%(21 / 435)$ of reconstructed embryos $(n=435)$ had entered into the uterine lining of recipients, but only 1.4\% (6/435) formed fetuses. However, all of the reconstructed embryos failed to develop to term ( 65 days). Microsatellite analyses confirmed that the nuclear genome of the cloned fetuses were LC in origin.

\section{PRODUCTION OF THIRD-GENERATION CLONES OF A PIG BY SOMATIC CELL} NUCLEAR TRANSFER

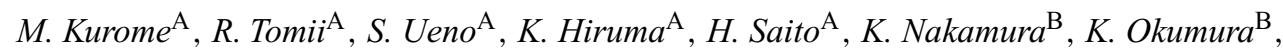 \\ S. Matsumoto ${ }^{\mathrm{B}}$, M. Matsumoto ${ }^{\mathrm{C}}$, Y. Kaji ${ }^{\mathrm{C}}, F$. Endo $^{\mathrm{B}}$, and H. Nagashima ${ }^{\mathrm{A}}$ \\ ${ }^{A}$ Laboratory of Developmental Engineering, Department of Life Science, Meiji University, Kawasaki 214-8571, Japan; \\ ${ }^{\mathrm{B}}$ Graduate School of Medical Science, Kumamoto University, Kumamoto 860-8556, Japan; \\ ${ }^{\mathrm{C}}$ National Agricultural Research Center for Kyushu Okinawa Region, Kumamoto 861-1192, Japan
}

There has been a dominant view that serial cloning, i.e., cloning of a cloned animal, is only possible for a few generations. In this study, we examined the reproduction efficiency and normality of porcine offspring generated by serial somatic cell cloning. Salivary gland progenitor (SGP) cells were 
collected from a 4-month-old female cloned Landrace large white Duroc (LWD) pig (first generation, G1), which had been cloned from a fibroblast, and used as nuclear donors for second-generation clones (G2). The third generation of clones (G3) was produced by nuclear transfer using SGP cells from the G2 clones. Nuclear transfer was carried out by electric cell fusion using in vitro matured oocytes as recipients. Reconstructed embryos were electroactivated 1 to $1.5 \mathrm{hr}$ after nuclear transfer, cultured for 1 to 2 days, and transplanted to the oviducts of estrus-synchronized surrogate gilts. A total of 391 embryos cloned from G1 animals were transplanted to three surrogates. All of the surrogates became pregnant and gave birth to a total of 13 (3.3\%) of G2 clones (including two stillbirths). The average birth weight and size of eleven live piglets were $1203.6 \pm 113.5 \mathrm{~g}$ and $27.1 \pm 1.2 \mathrm{~cm}$, both within the standard ranges of the original donor strain (LWD). Their growths until 8 months old were comparable to those of normal piglets of the same strain. For the generation of G3 clones, transplantation of 242 G2-derived embryos to two surrogate gilts resulted in one pregnant surrogate and three G3 clones (1.2\%; average weight $1196.7 \pm 267.1 \mathrm{~g}$ and average size $35.7 \pm 2.3 \mathrm{~cm})$, including a stillbirth. These results indicate that porcine serial cloning can efficiently generate up to three generations of apparently healthy clones, when SGP cells are used as nuclear donors

This study was supported by PROBRAIN.

\title{
51 AGGREGATION REDUCES THE VARIATION OF GENE EXPRESSION LEVELS IN BOVINE CLONES
}

\author{
S. Kurosaka, N. A. Leu, and K. J. McLaughlin \\ Center for Animal Transgenesis and Germ Cell Research, University of Pennsylvania, Kennett Square, PA 19348, USA
}

\begin{abstract}
Mammalian somatic cell clones frequently exhibit abnormal gene expression that presumably results from errors in reprogramming of the transplanted genome. In the mouse, aggregation of 4-cell stage clones with each other improves reprogramming with respect to Oct-4 expression in blastocysts and an increase in term development (Boiani et al. 2003 EMBO J. 22, 5304-5312). To determine if clone-clone aggregation has a similar beneficial effect in the bovine, we aggregated 8-16 cell bovine clones with each other and profiled gene expression levels in bovine clones and clone-clone aggregates at the blastocyst stage. Clone embryos were produced from fibroblasts and cultured in vitro in SOF supplemented with fetal bovine serum at $39^{\circ} \mathrm{C}$ in an atmosphere of $5 \% \mathrm{CO}_{2}, 5 \% \mathrm{O}_{2}$, and $90 \% \mathrm{~N}_{2}$. For aggregation of embryos, we first removed the zonae pepellucidae by treatment with $0.5 \%$ pronase at the $8-16$ cell stage and then placed two zona-free embryos per well into deep microwells produced on the bottom of a culture dish by pressing a heated darning needle onto the surface. Seven to 10 microwells in close proximity were covered by a culture $50-\mu \mathrm{L}$ drop of culture medium, and embryos were cultured until Day 7. Real-time RT-PCR analysis for Oct-4, DNA methyltransferase 1 (Dnmt1), Dnmt3, glucose transporter 1 (Glut1), Glut3, and Poly(A) polymerase (PolyA) was performed on reusable Dynabead Oligo (dT) 25 -cDNA libraries synthesized from individual blastocysts at Day 7. In vitro-fertilized embryos were used as controls. To compare the variation of gene expression in each embryo within the group, the coefficient of variation (COV; standard deviation/mean) was calculated. Although spatial distribution of Oct-4 transcript is normal in bovine blastocyst stage clones (Kurosaka et al. 2004 Reprod. Fertil. Dev. 16, 147), we detected disturbances in the level of Oct-4 expression in clones: $44.4 \%$ ( 8 of 18) of clones expressed Oct-4 within a range of 0.5 - and 1.5 -fold of the average level of expression in IVF embryos, compared to $81.8 \%$ ( 9 of 11 ) of IVF embryos. Only $22.2 \%$ ( 4 of 18 ) of clones expressed all genes examined within a range of 0.5 - and 2.0 -fold of the average level of IVF embryos, versus $45.5 \%$ ( 5 of 11) of IVF embryos. Clone-clone aggregation did not increase the proportion of clones with normal expression levels but did reduce the coefficient of variation of gene expression levels between individual clones for the genes Oct-4, Dnmt1, Dnmt3a and PolyA, but not for Glut1 and Glut3. Interestingly, bovine clone-clone aggregates $(n=25)$ had less variation between individual embryos compared to IVF aggregates $(n=11)$ for all genes except Glut1 and Glut3, although variation of single clones was larger than that of single IVF embryos. Analysis of Oct- 4 and $\beta$-Actin transcripts in mouse clone blastocysts indicated a similar decrease in gene expression variation subsequent to aggregation of mouse clones. These results demonstrate that bovine pre-implantation stage clones exhibit a high degree of variation in gene expression levels and suggest that aggregation of clones is beneficial in reducing the variation in expression of some genes.
\end{abstract}

\section{PRELIMINARY DATA ON PIG-BOVINE INTERSPECIES NUCLEAR TRANSFER EMBRYO DEVELOPMENT}

\author{
I. Lagutina ${ }^{\mathrm{A}}$, D. Brunetti ${ }^{\mathrm{A}}$, G. Lazzari $^{\mathrm{A}}$, and C. Galli ${ }^{\mathrm{A}, \mathrm{B}}$ \\ ${ }^{A}$ Laboratorio di Tecnologie della Riproduzione, ISILS, CIZ, Cremona, Italy; \\ ${ }^{\mathrm{B}}$ Dipartimento Clinico Veterinario, Universita di Bologna, Bologna, Italy
}

Interspecies nuclear transfer (NT) is a very important tool for study of nuclear-cytoplasm interactions and somatic cell nucleus reprogramming. We constructed, by means of a zona-free method, NT embryos using bovine (Bo) or porcine (Po) oocytes matured in vitro and bovine fetal fibroblasts (BFF), pig adult fibroblasts (PAF), and pig fetal (PFF) green fluorescent protein (GFP)-positive fibroblasts. Constructs were fused by a double pulse of DC $1.2 \mathrm{kV} / \mathrm{cm}$ for $30 \mu \mathrm{s}$. At 3-4 h post-fusion, embryos with Bo were activated by $5 \mu \mathrm{M}$ ionomycin for 4 min and incubated in $2 \mathrm{mM} 6-\mathrm{DMAP}$ in SOFaa for $4 \mathrm{~h}$, whereas embryos with Po were activated by a double pulse of DC $1.2 \mathrm{kV} / \mathrm{cm}$ for $30 \mu \mathrm{s}$ in the fusion medium with $1 \mathrm{mM} \mathrm{Ca}{ }^{++}$and incubated in SOFaa containing $5 \mu \mathrm{g} / \mathrm{mL}$ cytochalasin B in for $4 \mathrm{~h}$. Embryos were cultured in SOFaa in $5 \% \mathrm{CO}_{2}, 5 \% \mathrm{O}_{2}$ at $38.5^{\circ} \mathrm{C}$. The NT embryo development and GFP expression (D7) were checked. Our results (Table 1) showed that the blastocyst rate of control bovine and pig embryos was $74 \%$ and from 20 to $44 \%$, respectively. 'Pig fibroblasts into Bo' embryos were arrested at the 8-21-cell stage while 'BFF into Po' embryos were arrested at the 4-cell stage. About $84 \%$ of 'PFF GFP+ into Bo' NT embryos started to express GFP, but only 3.2\% (3/95) of the embryos were able to progress through the 16-cell stage suggesting insufficient embryonic genome activation. Overall significantly more 'Pig fibroblast into Bo' embryos were able to progress through the 4 -cell stage pig developmental block than normal pig NT embryos $(57.8 \pm 3.5 \%$ vs. $47.1 \pm 1.3 \% ;$-test, $P=0.02$ ). This study shows that early embryo development is driven by recipient cytoplasm up to the stage when genome activation should occur. The arrest of interspecies NT embryos at the stage of embryonic genome activation suggests that this developmental step is impaired. 
Table 1. Interspecies NT embryo development

\begin{tabular}{llccccc}
\hline Donor cells & Oocytes & $n$ & Number cleaved (\%) & $\geq$ Number at 8 cells (\%) & Blastocysts at Day 6 (\%) & GFP, \% \\
\hline BFF & Bovine & 39 & $38(97.4)$ & $29(74.4)$ & $29(74.4)$ & NA \\
& Pig & 34 & $25(73.5)$ & $18(52.9)^{*}$ & 0 & NA \\
PAF 1 & Pig & 51 & $46(90.2)$ & $25(49)$ & $10(19.6)$ & NA \\
& Bovine & 41 & $40(97.6)$ & $25(61)$ & 0 & NA \\
PAF 2 & Pig & 79 & $69(87.3)$ & $33(47.8)$ & $30(43.5)$ & NA \\
\multirow{2}{*}{ PFF-GFP+ } & Bovine & 99 & $99(100)$ & $61(61.6$ & 0 & NA \\
& Pig & 83 & $70(84.3)$ & $37(44.6)$ & $23(27.7)$ & 100 (BLD6) \\
& Bovine & 234 & $212(90.6)$ & $119(50.9)$ & 0 & 84.4 (all) \\
\hline
\end{tabular}

*All embryos were arrested at the 4 cell stage.

This work was funded by grant ISS CS 11 and ESF.

\title{
53 EVALUATION OF NUCLEAR REPROGRAMMING IN CLONED MINIATURE PIG EMBRYOS USING GENES OF Oct-4 FAMILY
}

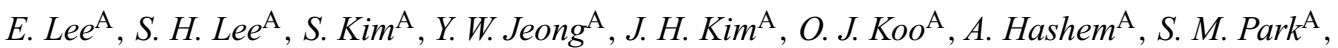 \\ M. S. H. Hossein ${ }^{\mathrm{A}}$, H. Y. S. Son ${ }^{\mathrm{B}}$, Ch.-K. Lee ${ }^{\mathrm{B}, \mathrm{C}}$, S. K. Kang ${ }^{\mathrm{A}, \mathrm{C}}$, B. C. Lee ${ }^{\mathrm{A}, \mathrm{C}}$, and W. S. Hwang ${ }^{\mathrm{A}, \mathrm{C}}$ \\ ${ }^{A}$ Department of Theriogenology and Biotechnololgy, College of Veterinary Medicine, \\ Seoul National University, Seoul 151-742, Korea; \\ ${ }^{B}$ School of Agricultural Biotechnology, Seoul National University, Seoul, 151-742, Korea; \\ ${ }^{\mathrm{C}}$ The Xenotransplantation Research Center, Seoul National University Hospital, Seoul, 110-144, Korea
}

\begin{abstract}
Xenotransplantation as a source of organs is a rapidly expanding field which can save thousands of human lives each year. Cloned miniature pigs have been considered as a model system for xenotransplantation. However, the efficiency of somatic cell nuclear transfer (SCNT) is extremely low, with most clones resulting in early lethality and several aberrancies. Possible explanation of the developmental failure of SCNT embryos is related to insufficient reprogramming of the somatic cell nucleus. In order to test this, we analyzed the reprogramming capacity of differentiated fibroblast cell nuclei and undifferentiated germ cell nuclei with Oct-4 and four Oct-4-related genes (Ndp5211, Dppa2, Dppa3, and Dppa5) as molecular markers using quantitative reverse transcription-polymerase chain reaction (RT-PCR). Oct-4 expression patterns were similar among IVF-derived embryos and cloned embryos derived from fibroblasts or germ cells during pre-implantation embryo development. However, the expression level was significantly lower $(P<0.05)$ in hatched blastocysts of fibroblast clones compared to other hatched blastocysts. Also, 9 of 13 cloned morulae and 12 of 40 cloned blastocysts failed to reactivate at least one of the five tested genes, whereas all of the germ cell clones and control embryos correctly expressed these genes. Analysis with miniature pig fetuses collected at Day 30 of gestation revealed that normal and cloned fetuses successfully expressed these genes. In conclusion, our results suggest that analysis of expression of Oct- 4 and related genes could be a reliable marker for evaluating the reprogramming status of transplanted donor nuclei in cloned embryos. The reprogramming of fibroblast cloned embryos is highly error-prone. This may contribute to their embryonic lethality because cloned embryos that fail to reactivate the marker genes may fail to be successfully implanted.
\end{abstract}

This study was supported by grants from the Ministry of Science and Technology (Top Scientist Fellowship), and the Biogreen 21-1000520030100000.

\section{ESTABLISHMENT OF PREGNANCY BY OVINE NUCLEAR TRANSFER EMBRYOS RECONSTRUCTED USING CAFFEINE-TREATED IN VITRO-MATURED OOCYTES AS CYTOPLAST RECIPIENTS}

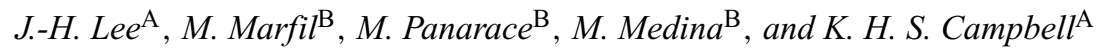 \\ ${ }^{A}$ School of Bioscience, University of Nottingham, Sutton Bonington Campus, \\ Loughborough, Lecis, LE12 5RD, UK; \\ ${ }^{B}$ Centro de Investigaciones Reproductivas Perez Companc, Goyaike SAACIyF, \\ Carmen de Areco, Buenos Aires, Argentina
}

In embryos reconstructed by somatic cell nuclear transfer (SCNT), components of the oocyte cytoplasm are capable of reprogramming the somatic genome to control subsequent development. Although the mechanisms that control nuclear reprogramming are unknown, we have previously hypothesized that the occurrence of nuclear envelope breakdown (NEBD) and premature chromosome condensation (PCC) in the donor nucleus are beneficial. In previous studies we have demonstrated that treatment of ovine oocytes with caffeine $(10 \mathrm{mM})$, a protein phosphatase inhibitor, increased the activities of both MPF and MAPK in enucleated oocytes (Lee and Campbell 2004 Reprod. Fertil. Dev. 16, 125) and additionally resulted in a significant increase in the occurrence of NEBD and PCC in donor nuclei. Furthermore, SCNT embryos reconstructed following caffeine treatment had significantly increased cell numbers at the blastocyst stage. More recently we have demonstrated that the use of caffeine 
treated ovine oocytes as cytoplast recipients can regulate the expression of several developmentally important genes in SCNT embryos, including Oct-4 and interferon-tau (Choi et al. 2006 Reprod. Fertil. Dev. 18, in press). This study was designed to establish the developmental potential of NT embryos reconstructed using caffeine treated oocytes as cytoplast recipients. Ear skin fibroblast cells established from a Merino ram were quiesced in DMEM containing $0.1 \%$ fetal bovine serum (FBS) for 3 days. Oocyte maturation and embryo reconstruction and culture were performed as previously described (Lee and Campbell 2004 Reprod. Fertil. Dev. 16, 125) with the exception that ovaries from Merino $\times$ Romney Marsh cross ewes were stimulated with FSH sponge (Folltropin ${ }^{\circledR}-\mathrm{V}$; Bioniche Animal Health, Beltsville, Ontario, Canada) and were collected at slaughter on Day 13 following sponging. Blastocyst stage embryos were surgically transferred to the uterine horn of synchronized Merino $\times$ Romney Marsh cross recipients (three blastocysts per recipient). Recipient ewes were scanned by ultrasonography at Days 30, 60, and 90 following embryo transfer. All data were analyzed by chi-square test. There were no differences in fusion $(145 / 167 ; 86.8 \%$ vs. $174 / 205 ; 84.9 \%)$, cleavage $(123 / 145 ; 84.8 \%$ vs. $135 / 174 ; 77.6 \%)$, or the development to blastocyst $(33 / 145 ; 22.8 \%$ vs. $34 / 174 ; 19.5 \%)$ between control SCNT embryos and caffeine treated SCNT embryos. However, although the frequency of pregnancy between control and caffeine-treated NT groups $(5 / 15 ; 33.3 \%$ vs. $7 / 14 ; 50.0 \%)$ at 30 days was not significantly different, control SCNT embryos showed significantly lower pregnancies $(1 / 15 ; 6.7 \%)$ than caffeine treated SCNT embryos $(4 / 14 ; 28.6 \%)$ at both 60 and 90 days. In conclusion, embryos reconstructed using caffeine-treated cytoplasts can induce pregnancy at the same frequency as untreated controls; furthermore, the results suggest that SCNT embryos produced in this way are more able to maintain pregnancy

\title{
55 INFLUENCE OF DONOR CELL TYPE ON THE DEVELOPMENT OF PORCINE NUCLEAR TRANSFER EMBRYOS
}

\author{
K. Lee ${ }^{\mathrm{A}}$, W. L. Fodor ${ }^{\mathrm{B}}$, and Z. Machaty ${ }^{\mathrm{A}}$ \\ ${ }^{A}$ Department of Animal Sciences, Purdue University, West Lafayette, IN 47907, USA; \\ ${ }^{B}$ Department of Molecular and Cellular Biology, University of Connecticut, Storrs, CT 06269, USA
}

Embryonic development after nuclear transfer is very low; the majority of cloned embryos do not survive the pre-implantation stage. Recent reports indicate that the characteristics of nuclear transfer embryos depend on the type of nuclear donor cells. It has been suggested that development after nuclear transfer improves if less differentiated cells are used as nuclear donors. The aim of the present study was to investigate the developmental potential of nuclear transfer embryos reconstructed using differentiated and non-differentiated cells. Two types of non-differentiated cells, skin stem cells and olfactory bulb progenitor cells, were used; fetal fibroblasts were used as differentiated control. Prior to nuclear transfer, the differentiated state of the cells was characterized by Oct-4 immunocytochemistry (Chemicon International, Inc., Temecula, CA, USA); Oct-4 is known to be expressed by pluripotent cells only. During nuclear transfer, the cells were transferred into the perivitelline space of in vitro-matured enucleated oocytes. After fusion, reconstructed oocytes were activated by an electrical pulse followed by incubation in $10 \mu \mathrm{g} / \mathrm{mL}$ cycloheximide and $5 \mu \mathrm{g} / \mathrm{mL}$ cytochalasin B for $5 \mathrm{~h}$. The embryos were subsequently cultured in NCSU-23 medium for 6 days; their developmental data were recorded and compared by ANOVA. Non-differentiated cell types showed strong Oct-4 expression, whereas the marker protein was completely absent in fetal fibroblast cells. A total of 161 embryos were reconstructed using skin stem cells, 171 embryos from olfactory bulb progenitor cells, and 189 embryos from fibroblasts. Of the skin stem cell-derived embryos, $32.9 \%$ cleaved, and during subsequent culture, $5.6 \%$ developed to the morula/blastocyst stage. In the olfactory bulb progenitor cell group, $19.8 \%$ cleaved, and the percentage of embryos that developed to the morula/blastocyst stage was $4.7 \%$. In the control group, $22.7 \%$ cleaved; the morula/blastocyst formation was $2.6 \%$. Embryos reconstructed from skin stem cells showed superior cleavage rate compared to embryos from the other cell types $(P<0.05)$. Also, morula/blastocyst formation from skin stem cells was significantly higher than that from fetal fibroblasts $(P<0.05)$, and morula/blastocyst formation from olfactory bulb progenitor cell-derived embryos also tended to be higher compared to control embryos $(P=0.08)$. Furthermore, the formation of morulae/blastocysts per cleaved embryos was the highest in embryos reconstructed with olfactory bulb progenitor cells (23.5\% vs. $17.0 \%$ using skin stem cells and $11.6 \%$ using fibroblasts) implying that embryos from olfactory bulb progenitor cells may have higher developmental potential in later stages of development. The results demonstrate that nuclei of different donor cells support development to various degrees and confirm previous reports that using non-differentiated cells as nuclear donors increases the efficiency of nuclear transfer in the pig.

\section{PRODUCTION OF TRANSGENIC RECLONED MINIATURE PIGS EXPRESSING HUMAN DECAY ACCELERATING FACTOR}

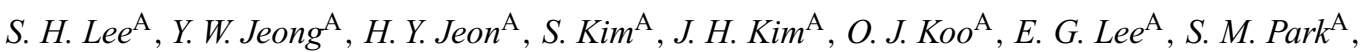

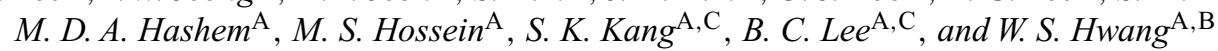 \\ ${ }^{\text {A }}$ Department of Theriogenology and Biotechnology, College of Veterinary Medicine, \\ Seoul National University, Seoul, South Korea; \\ ${ }^{B}$ School of Agricultural Biotechnology, Seoul National University, Seoul, South Korea; \\ ${ }^{\mathrm{C}}$ The Xenotransplantation Research Center, Seoul National University Hospital, Seoul, South Korea
}

This study was performed to produce transgenic recloned miniature pigs expressing human decay-accelerating factor (hDAF) to overcome the hyperacute rejection of pig-to-human xenotransplantation. The expression vector, named hDAF-Neo, was constructed by subcloning the amplified $2.2 \mathrm{~kb}$ hDAF cDNA fragment into the NheI and NotI site of the pEGFP-N1 (Clontech, Palo Alto, CA, USA) vector containing the chicken beta-actin promoter and rabbit globin poly A without a EGFP fragment. Day 30 fetal fibroblast cells (GN0) were used for producing the newborn cloned pigs (GN1). GN1 fibroblasts cultured from the newborn cloned pig derived from GN0 were used for transfection of the hDAF-Neo plasmid using FuGENE-6 ${ }^{\circledR}$ (Roche Diagnostics, Indianapolis, IN, USA). Transfected fibroblast cell colonies were selected with neomycin. All data were analyzed 
by one-way ANOVA and the protected least significant difference (LSD) test using general linear models in a statistical analysis system (SAS Institute, Inc., Cary, NC, USA) program to determine differences among experimental groups. Significant difference among the treatment groups was determined when the $P$ value was less than 0.05 . There was no significantly different development rate between GN0 and GN1 (12.8 vs. $13.0 \%)$ and between GN1-hDAF and GN2 (9.3 vs. 8.2\%). Transfected recloned embryos (GN1-hDAF) had no significantly different blastocyst formation compared to normal (GN1). After embryo transfer, we obtained two transgenic recloned pigs. hDAF gene integration in transgenic recloned piglets was confirmed by polymerase chain reaction. Cultured fibroblasts of transgenic recloned pigs showed 1.0-2.3 times higher levels of hDAF protein than did human umbilical vein endothelial cells. The levels of $\mathrm{C} 3$ deposition on cultured fibroblast cells after incubation with $10 \%$ human serum were decreased in transgenic recloned pigs compared to normal miniature pigs. In conclusion, the recloning procedure can be used to produce multiple genetically modified cloned pigs without any severe epigenetic abnormality.

This study was supported by grants from the Korean MOST (Top Scientist Fellowship) and MAF (Biogreen 21 \#20050301-034-443-026-01-00).

\title{
57 DEFECTIVE PLACENTAL VASCULARIZATION AND TROPHISMS IN FULL TERM SOMATIC CELL SHEEP CLONES
}

\author{
P. Loi, K. Matsukawa, C. Palmieri, L. Della Salda, and G. Ptak \\ Dipartimento di Scienze Biomediche Comparate, Teramo University, Teramo, Piazza Aldo Moro 45, 64100, Teramo, Italy
}

We report on cloning experiments designed to explore the causes of peri- and post-natal mortality of cloned lambs. A total of 93 blastocysts obtained by nuclear transfer of somatic cells (granulosa cells) were transferred into 41 recipient ewes, and pregnancies were monitored by ultrasound scanning. In vitro-derived, fertilized embryos (IVF; $n=123$ ) were also transferred in order to assess oocyte competence; naturally mated ewes $(n=120)$ were analyzed as well. Cloned embryos developed to the blastocyst stage and implanted at the same rate as IVF embryos. After Day 30 of gestation, however, dramatic losses occurred, and only 12 out of 93 (13\%) clones reached full-term development, compared to 51 out of 123 (41.6\%) lambs born from the IVF control embryos. Three full-term lamb clones were delivered stillborn as a result of placental degeneration. A further five clone recipients developed hydroallantois. Their lambs died within $24 \mathrm{~h}$ following delivery by Caesarean section; their carcasses displayed degenerative lesions in liver and kidney resulting from the severe hydroallantois. One set of twins was delivered by assisted parturition at Day 150 , but died $24 \mathrm{~h}$ later due to respiratory distress syndrome. The remaining two clone recipients underwent Caesarean section; the corresponding two lambs displayed signs of respiratory dysfunction and died at approximately one month of age due to a bacterial complication. Blood samples collected from the cloned lambs after birth revealed a wide range of abnormalities indicative of kidney and liver dysfunction. Macroscopical and histopathological examination of the placentae revealed a marked reduction in vascularization, particularly at the apex of the villous processes, as well as a loss of differentiation of the trophoblastic epithelium. Our results strongly suggest that post-mortality in cloned lambs is mainly caused by placental abnormalities.

\section{EFFICIENCY OF OVUM PICKUP AND EMBRYO PRODUCTION IN VITRO IN CLONED CATTLE}

\author{
A. Lucas-Hahn, E. Lemme, K.-G. Hadeler, H.-G. Sander, and H. Niemann \\ Institute for Animal Breeding (FAL), Mariensee, 31535 Neustadt, Germany
}

The reproductive performance of cloned cattle was investigated by assessing the efficiency of transvaginal ultrasound-guided ovum pickup (OPU) and embryo production in vitro. Fetal fibroblasts from the endangered species, German Blackpied Cattle, had been used for nuclear transfer to produce three live cloned offspring (Lucas-Hahn et al. 2002 Theriogenology 57, 433). In the three cloned animals at 12-20 months of age, OPU was performed once per week and the total number of collected oocytes was recorded. In the case of Blondie, the procedure was terminated due to too small ovaries associated with insufficient function. Oocytes suitable for IVF were matured in vitro for $24 \mathrm{~h}$ and fertilized in vitro with the semen of a fertile bull. Oocytes derived from abbatoir ovaries were processed in parallel as controls. Embryos were in vitro-cultured in SOFaaBSA medium. Cleavage and developmental rates up to the morula/blastocyst stage were recorded in all groups. Statistical significance was tested using ANOVA and the Student-Newman-Keuls test. The results are presented in Table 1. Embryos from clones had lower cleavage and blastocyst rates compared to those derived from abattoir oocytes. However, results may have been confounded by potential OPU effects. Some of the blastocysts produced from Blacky $(n=5)$ and Paula $(n=2)$ were transferred to recipients. Two pregnancies resulted from the Paula transfers. The two male calves were delivered normally. After the completion of this experiment, all three cloned animals were artificially inseminated, became pregnant, delivered healthy calves, and are pregnant again at present. Further studies are needed to explore the fertility of cattle derived from somatic cloning.

Table 1. OPU and in vitro embryo production in cloned cattle

\begin{tabular}{lcccccc}
\hline $\begin{array}{l}\text { Cloned } \\
\text { animals }\end{array}$ & $\begin{array}{c}\text { OPU sessions } \\
(n)\end{array}$ & $\begin{array}{c}\text { Total no. } \\
\text { oocytes }(n)\end{array}$ & $\begin{array}{c}\text { Oocytes/ } \\
\text { session }(n)\end{array}$ & $\begin{array}{c}\text { IVF suitable, } \\
(n)(\%)\end{array}$ & $\begin{array}{c}\text { Cleaved } \\
\text { embryos }(n)(\%)\end{array}$ & $\begin{array}{c}\text { Morulae and } \\
\text { Blastocysts }(n)(\%)\end{array}$ \\
\hline Blondie\#8352 & 4 & 25 & 6.2 & $12(48.0)$ & $4(33.3)^{\mathrm{b}}$ & $0^{\mathrm{d}}$ \\
Blacky\#7989 & 13 & 117 & 9.0 & $64(54.7)$ & $23(35.9)^{\mathrm{b}}$ & $6(9.4)^{\mathrm{d}}$ \\
Paula\#7990 & 15 & 99 & 6.6 & $54(54.5)$ & $25(46.3)^{\mathrm{b}}$ & $6(11.1)^{\mathrm{d}}$ \\
ControlAbattoir ovaries & - & - & - & 152 & $114(75.0)^{\mathrm{a}}$ & $63(41.4)^{\mathrm{c}}$ \\
\hline
\end{tabular}

${ }^{\mathrm{a}-\mathrm{d}}$ Values with different superscripts within a column differ significantly $\left({ }^{\mathrm{a}, \mathrm{b}} P<0.05\right.$; $\left.{ }^{\mathrm{a}, \mathrm{d}} P<0.001\right)$. 


\title{
59 BOVINE OOCYTE RECONSTRUCTION FOLLOWING CRYOPRESERVATION AND GERMINAL VESICLE TRANSPLANTATION
}

\author{
A. M. Luciano, F. Franciosi, F. PerazzouI, C. Galbusera, M. S. Beretta, V. Lodde, and S. Modina \\ Institute of Anatomy of Domestic Animals, Histology and Embryology, University of Milan, Milan, Italy
}

\begin{abstract}
The aim of the present study was to assess the maturational competence and the cytoskeletal pattern of bovine oocytes reconstructed by a germinal vesicle transplantation (GVT) technique using nuclear and/or cytoplasmic components from cryopreserved GV stage oocytes. Cumulus-oocyte complexes were isolated from slaughtered ovaries and denuded oocytes were cryopreserved by vitrification in open pulled straws (Vajta et al. 1998 Mol. Reprod. Dev. 51(1), 53-58) after cumulus cell removal (Modina et al. 2004 Eur. J. Histochem. 48(4), 337-346). Denuded oocytes were maintained in meiotic arrest with $0.5 \mathrm{mM}$ of 3-isobutyl-1-methylxanthine, centrifuged for 20 min in for visualization of the germinal vesicle and then treated with $7.5 \mu \mathrm{g} / \mathrm{mL}$ of cytochalasin B for $20 \mathrm{~min}$ before enucleation. By means of micromanipulation and electrofusion procedures, female gametes were reconstructed from karyoplasts and cytoplasts obtained from either cryopreserved or freshly isolated oocytes, and then in vitro-matured for $24 \mathrm{~h}$ (Luciano et al. $2005 \mathrm{Mol}$. Reprod. Dev. 71(3), 389-397). After in vitro maturation (IVM), the nuclear stage was assessed by Hoechst 33342 staining and oocytes were fixed for analysis of the organization of microtubules and microfilaments, which provide the framework for chromosomal alignment and meiotic division. A total of 179 oocytes were reconstructed by the GVT procedure. All data were analyzed by chi-square test. No differences were observed in the reconstruction efficiency after fusion between fresh and cryopreserved oocytes (77.6\% and $81.2 \%$, respectively). The maturational competence of artificial gametes originated from cryopreserved oocytes was not different from that of gametes reconstructed with fresh oocytes $(21.3 \%$ and $26.7 \%$, respectively) and cytoskeleton organization analysis of artificial oocytes demonstrated that the correct distribution of microtubules and microfilaments was associated with a proper progression through metaphase II (MII) of meiotic division. However, the maturation rate of reconstructed oocytes was significantly lower than that of the control group, represented by untreated oocytes $(86.7 \%$; $P<0.05)$, and preenucleation procedures significantly affected the capability of oocytes to reach the MII stage of meiotic division (73.3\%; $P<0.05)$. We conclude that both nuclear and cytoplasmic components derived from cryopreserved immature bovine oocytes are suitable for the GVT procedure, and that they generate oocytes able to progress to MII in vitro. The possibility of using cryopreserved immature oocytes as a source of nuclei and cytoplasm could help in applying the GV transfer procedure, both in research and clinical settings and in fertility preservation programs.
\end{abstract}

This work was supported by a PRIN 2003 MIUR Grant.

\section{EFFECTS OF CAFFEINE ON THE IN VITRO DEVELOPMENT OF BOVINE NUCLEAR TRANSFER EMBRYOS}

\author{
W. E. Maalouf, J. H. Lee, and K. H. S. Campbell
}

Animal Development and Biotechnology Group, School of Biosciences, The University of Nottingham, Loughborough, LE12 5RD, UK

Previous studies have demonstrated that treating ovine oocytes with caffeine increases the activities of both maturation-promoting factor (MPF) and mitogen-activated protein kinase (MAPK). When such oocytes are used as cytoplast recipients for nuclear transfer (NT), there is an increase in cell numbers at the blastocyst stage (Lee and Campbell 2004 Rep. Fert. Dev. 16, 125). The objective of this study was to determine the effects of caffeine on MPF and MAPK activities and the development of bovine NT embryos. Oocytes were matured in maturation medium (MM) composed of TCM199, $10 \%$ fetal bovine serum (FBS), $5 \mu \mathrm{g} \mathrm{mL}^{-1}$ follicle-stimulating hormone FSH, $5 \mu \mathrm{g} \mathrm{mL}^{-1}$ lutcinizing hormone (LH) and $1 \mu \mathrm{g} \mathrm{mL}-1$ estradiol for $24 \mathrm{~h}$. Subsequently, oocytes were cultured in MM supplemented with $0,5,10$, and $15 \mathrm{mM}$ caffeine for $6 \mathrm{~h}$. Groups of 10 oocytes were sampled and analyzed for MPF and MAPK activities as previously described (Ye et al. 2003 Reproduction 125, 645-656). Treatment with 15 mM caffeine significantly increased the levels of MPF and MAPK activities in MII oocytes. To study development potential, oocytes at $16 \mathrm{~h}$ post-onset of maturation (hpm) were stripped of cumulus cells and enucleated in HSOF containing $5 \mu \mathrm{g} \mathrm{mL}^{-1}$ Hoechst 33342 and $7.5 \mu \mathrm{g} \mathrm{mL} \mathrm{m}^{-1}$ cytochalasin B; enucleation was achieved using a blunt $(25-\mu \mathrm{m}$ i.d.) pipette after cutting a hole in the zona pellucida with a XYClone laser (Hamilton Thorne Research, Beverly, MA, USA). Enucleated oocytes were then cultured in MM $\pm 15 \mathrm{mM}$ caffeine for a further $6 \mathrm{~h}$. For NT, quiesced primary bovine foetal fibroblasts were used. Cell fusion was induced with two DC pulses of $35 \mathrm{~V}$ for $65 \mu \mathrm{s}$ at $24 \mathrm{hpm}$. At $2 \mathrm{~h}$ post-fusion, all reconstructed embryos were briefly exposed to ultraviolet light under a fluorescence microscope (Leica Microsystems AG, Wetzler, Germany) in order to assess nuclear morphology, and then activated in HSOF containing $5 \mu \mathrm{g} \mathrm{mL}^{-1}$ calcium ionophore (A23187), cultured in SOF with $10 \mu \mathrm{g} \mathrm{mL}^{-1} \mathrm{cycloheximide}$ and $7.5 \mu \mathrm{g} \mathrm{mL}^{-1}$ cytochalasin B for $5 \mathrm{~h}$, and transferred to mSOFaaBSA medium. On Day 2, cleavage was assessed and $10 \%$ FBS added to the medium. Development to blastocyst was assessed on Day 7. All data were analyzed using the chi-square test. There was a significant increase in the number of reconstructed embryos that underwent nuclear envelope breakdown (NEBD) and premature chromosome condensation (PCC) when caffeine-treated cytoplast recipients were used $(28.6 \pm 9.9 \%$ and $60.0 \pm 11.0 \%$ for control and caffeine groups respectively, $P<0.05)$. Cleavage rates $(47.6 \pm 10.9 \%$ and $50.0 \pm 11.1 \%)$, development to blastocyst $(20.0 \pm 4.0 \%$ and $30.0 \pm 4.6 \%)$, and mean cell number $(85.0 \pm 7.1$ and $122.5 \pm 3.5)$ were not statistically different between control and caffeine treated groups, respectively. In summary, treatment of bovine oocytes with $15 \mathrm{mM}$ caffeine increased the activities of two key cell-cycle regulators MPF and MAPK, and statistically increased the occurrence of NEBD and PCC in the donor nuclei. We previously hypothesized that the occurrence and extent of NEBD and PCC may increase nuclear reprogramming in NT embryos (Lee and Campbell 2004 Rep. Fert. Dev. 16, 125; Campbell et al. 2005 Rep. Dom. Anim. 40, 256-268); however, further studies are required to determine the developmental competence of these embryos. 


\title{
61 GENERATING A HERD OF TRANSGENIC DAIRY GOATS BY SOMATIC CELL NUCLEAR TRANSFER
}

\author{
D. Melican, N. Hawkins, and W. Gavin \\ GTC Biotherapeutics, Inc., Framingham, MA 01701, USA
}

Transgenic dairy goats expressing recombinant therapeutic molecules in the milk may offer an alternative manufacturing method compared to traditional cell culture. Typically, a herd of transgenic progeny can be efficiently generated by naturally mating a transgenic buck with multiple does. However, the ability to rapidly generate a herd of transgenic progeny from a female transgenic founder by natural mating represents a challenge. While a herd of transgenic progeny can be generated from a female transgenic founder by superovulation and either in vitro fertilization or embryo flushing, the time period required is dependent on the number and gender of progeny needed. In this work we determined the ability of using skin cells from two transgenic founder female dairy goats as karyoplasts for nuclear transfer to produce a herd of transgenic female dairy goats. Two transgenic founder female dairy goats expressing a recombinant therapeutic human protein in the milk were generated by nuclear transfer using a transfected fetal cell line. Primary cultures of transgenic skin cells were established from these two founder lines using tissue samples obtained by biopsy. The cells were used as nuclear donors following three or four days of low-serum culture [0.5\% fetal bovine serum (FBS)]. Donor cells were simultaneously fused and activated with in vivo produced oocytes from superovulated does. Nuclear transfer embryos were transferred to synchronized surrogate recipients at 24 to $48 \mathrm{~h}$ post-fusion and activation. Pregnancies were determined by ultrasonography starting at approximately Day 28 post-fusion and activation and then monitored weekly. The results of work conducted during two defined breeding periods are summarized in Table 1. While there were no significant differences in the number of Day 50 pregnancies (17 vs. 27), there were significantly more term pregnancies (14 vs. 12$)$ and offspring produced (20 vs. 15) during the traditional (September through December) compared with the nontraditional (January through May) breeding seasons, respectively $(P<0.05)$. In this work, 35 female NT offspring were produced, requiring 13 months from the start until the last offspring was born. These results demonstrate that somatic cell nuclear transfer using transgenic skin cells as karyoplast donors is an alternative method to produce a herd of transgenic female dairy goats.

Table 1. Caprine transgenic herd development by nuclear transfer

\begin{tabular}{lccccc}
\hline $\begin{array}{l}\text { Breeding } \\
\text { period }\end{array}$ & $\begin{array}{c}\text { No. } \\
\text { embryos }\end{array}$ & $\begin{array}{c}\text { No. } \\
\text { recipients }\end{array}$ & $\begin{array}{c}\text { No. day 50 } \\
\text { pregnancies (\%) }\end{array}$ & $\begin{array}{c}\text { No. term } \\
\text { pregnancies (\%) }\end{array}$ & $\begin{array}{c}\text { No. offspring } \\
(\% \text { embryos) }\end{array}$ \\
\hline $\begin{array}{l}\text { September-December } \\
\text { January-May }\end{array}$ & 757 & 114 & $17^{\mathrm{a}}(15)$ & $14^{\mathrm{a}}(82)$ & $20^{\mathrm{a}}(2.6)$ \\
Totals & 1101 & 175 & $27^{\mathrm{a}}(15)$ & $12^{\mathrm{b}}(44)$ & $15^{\mathrm{b}}(1.4)$ \\
\hline
\end{tabular}

${ }^{\mathrm{a}, \mathrm{b}}$ Differ significantly by chi-square test, $P<0.05$.

\section{REPROGRAMMING EVENTS AND DEVELOPMENTAL COMPETENCE OF RHESUS MONKEY EMBRYOS PRODUCED BY SOMATIC CELL NUCLEAR TRANSFER}

\author{
S. Mitalipov ${ }^{\mathrm{A}}, Q . Z_{\text {Zhou }}^{\mathrm{B}}, J$. Byrne $^{\mathrm{A}}, W \cdot-Z . J i^{\mathrm{C}}$, and D. Wolf ${ }^{\mathrm{A}}$ \\ ${ }^{\mathrm{A}}$ Oregon National Primate Research Center, Beaverton, OR 97006, USA; \\ ${ }^{B}$ Institute of Zoology, CAS, Beijing, People's Republic of China; \\ ${ }^{\mathrm{C}}$ Kunming Institute of Zoology, CAS, Kunming, People's Republic of China
}

Successful reprogramming of somatic cell nuclei after nuclear transfer requires active remodeling by factors present in the nonactivated cytoplast. High levels of maturation promoting factor (MPF) activity are associated with this remodeling process which includes nuclear envelope breakdown (NEBD), premature chromosome condensation (PCC), and spindle formation. In this study, we examined the extent of nuclear remodeling in monkey somatic cell nuclear transfer (SCNT) embryos by monitoring the dynamics of lamin A/C appearance, as detected immunocytochemically, following fusion of donor cells with recipient cytoplasts. In the control, intracytoplasmic sperm injection (ICSI) fertilized embryos, lamin A/C was readily detected at the pronuclear stage but disappeared in early cleaving embryos only to reappear by the morula stage in association with the activation of the embryonic genome. We initially documented lack or incomplete NEBD and PCC in SCNT embryos in the form of retention of lamin $\mathrm{A} / \mathrm{C}$ signal emanating from the donor nucleus. This observation was consistent with premature cytoplast activation due to the manipulation procedures. SCNT embryos produced by this approach typically arrested at the morula stage. Significant modifications in nuclear transfer protocols were then employed. Optimization of procedures resulted in robust NEBD and PCC, as indicated by loss of lamin A/C signal from the donor cell. Also, significant improvement of SCNT embryo development in vitro was observed, with a markedly improved blastocyst formation rate $(21 \%)$. Several different fetal and adult somatic cell types screened as nuclear donors supported blastocyst development. SCNT blastocysts displayed a pattern of Oct-4 expression similar to that of sperm fertilized counterparts, indicative of efficient nuclear reprogramming. However, no pregnancies were established following a preliminary trial of 8 embryo transfers with 48 cloned embryos. Nevertheless, our results represent a breakthrough in efforts to produce cloned monkeys and should provide the resources required for the derivation of embryonic stem cells from SCNT blastocysts. 
63 IMPROVEMENT OF SEMEN-DERIVED EPITHELIAL CELL PROLIFERATION BY FIBROBLAST CO-CULTURE

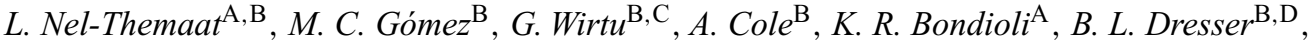 \\ C. E. Pope ${ }^{\mathrm{B}}$, and R. A. Godke $\mathrm{A}, \mathrm{C}$ \\ ${ }^{A}$ Department of Animal Sciences, LSU Agricultural Center, Baton Rouge, LA 70803, USA; \\ ${ }^{B}$ Audubon Center for Research of Endangered Species, New Orleans, LA 70131, USA; \\ ${ }^{\mathrm{C}}$ Department of Veterinary Clinical Sciences, LSU School of Veterinary Medicine, Baton Rouge, LA 70803, USA; \\ Department of Biological Sciences, University of New Orleans, New Orleans, LA 70148, USA
}

We previously isolated epithelial-like cells (ELC) from sheep (Ovis aries) and eland (Taurotragus oryx) semen (Nel-Themaat et al. 2004 Reprod. Fertil. Dev. 16, 152) and subsequently developed a system to separate ELC before plating (Nel-Themaat et al. 2005 Reprod. Fertil. Dev. 17, 314). Cells attached and proliferated in only $50 \%$ and $31 \%$ of the attempts, respectively. Therefore, the purposes of the present study were to improve ELC proliferation by co-culture with inactivated $3 \mathrm{~T} 3$ mouse fibroblasts and to characterize the obtained cells. Semen fractions from two mature Gulf Coast Native rams $(n=20$ ejaculates) and one common eland bull ( $n=2$ ejaculates) were plated on feeder layers (A), on collagen with feeder cell inserts (B), or on collagen alone (C). For B and C, cell attachment and division were assessed; proliferation and passage 1 (P1) confluence were evaluated for A, B, and C. No difference in attachment rates between B $(80 \%)$ and $\mathrm{C}(70 \%)$ were found for ovine cells, but $(P<0.05)$ cells divided more times in $\mathrm{B}(80 \%)$ than in $\mathrm{C}(35 \%)$. All colonies in $\mathrm{A}(60 \%)$ and $\mathrm{B}(70 \%)$ reached $\mathrm{P} 1$ confluence and no difference was detected between $\mathrm{A}$ and $\mathrm{B}$, but less proliferation $(10 \%)$ and $\mathrm{P} 1$ confluence $(5 \%)$ were observed in $\mathrm{C}$. Therefore, contact between epithelial cells and feeders was not necessary for growth stimulation by the feeder cells. No difference among A, B, and C was detected for eland ELC proliferation (100\%), but no P1 confluence was observed in C. Ram cells were subsequently characterized by immunohistochemical detection of keratin and vimentin, as well as morphology. The 3 T3 cells cross-reacted with keratin, and characterization was thus performed mainly on morphology and vimentin expression. Distribution of vimentin microfilaments differed between different epithelial morphologies and fibroblasts. Expression in epithelial cells was faint and patchy in confluent colonies and located around cytoplasmic extremities in semi-confluent colonies. In 3T3 cells, expression was very prominent throughout the cytoplasm and around the nucleus. For treatments A and B, 63 and 57\%, respectively, were characterized as only epithelial cells; 25 and $36 \%$, respectively, appeared to contain a mixture of epithelial and fibroblast cells; and 13 and $7 \%$, respectively, contained only fibroblast cells. Only one sample was evaluated from treatment $\mathrm{C}$ and only keratin was detected in the epithelial-like colony. We conclude that culture of semen-derived ELC is markedly improved by $3 \mathrm{~T} 3$ fibroblast co-culture. Further research on conditioned media may simplify the system and reduce chances of $3 \mathrm{~T} 3$ cell contamination.

\title{
64 X-LINKED GENE EXPRESSION IN BOVINE (BOS TAURUS) MALE AND FEMALE IN VITRO- FERTILIZED AND SOMATIC CELL NUCLEAR TRANSFER-DERIVED BLASTOCYSTS
}

\author{
M. Nino-Soto ${ }^{\mathrm{A}}$, G. Mastromonaco ${ }^{\mathrm{A}}$, P. Blondin $^{\mathrm{B}}$, and W. A. King ${ }^{\mathrm{B}}$ \\ ${ }^{A}$ Department of Biomedical Sciences, University of Guelph, Guelph, Ontario, Canada; \\ ${ }^{B}$ L'Alliance Boviteq, Inc., Saint-Hyacinthe, Qubec, Canada
}

\begin{abstract}
Expression of some X-chromosome linked genes has recently been shown to be altered in bovine somatic cell nuclear transfer (SCNT) derived embryos (Wrenzycki et al. 2002 Biol. Reprod. 66, 127), implying that the regulatory mechanisms of X-linked transcription are affected by embryo in vitro production (IVP) methods. We analyzed the transcriptional pattern of X-linked genes (BIRC4, GAB3, HPRT1, MECP2, RPS4X, SLC25A6, and $X I S T$ ) in bovine in vitro fertilized (IVF) and SCNT male and female blastocysts to determine X-inactivation status and changes resulting from IVP. We collected pools of male ( $n=5$ pools) and female $(n=3$ pools) IVF-derived blastocysts (Bousquet et al. 1999 Theriogenology 51,59$)$ and male ( $n=5$ pools) and female ( $n=3$ pools) SCNT-derived blastocysts (Mastromonaco et al. 2004 Reprod. Domest. Anim. 39, 462). Each pool consisted of five blastocysts. Embryos were washed in phosphate buffered saline (PBS) $+0.1 \%$ polyvinyl alcohol (PVA), collected, and stored at $-80^{\circ} \mathrm{C}$. Total RNA was extracted with an Absolutely RNA Microprep kit (Stratagene, La Jolla, CA, USA), DNase I treated, and precipitated with isopropanol and linear acrylamide (Ambion, Inc., Austin, TX, USA) as a carrier. Reverse transcription was performed with Oligo-dT (Invitrogen, Burlington, Ontario, Canada) and Superscript II RT (Invitrogen). Transcript quantification was performed by quantitative real-time PCR using SYBR Green I (LightCycler system, Roche, Diagnostics, Laval, Quebec, Canada). Data analysis was performed with SAS (SAS Institute, Inc., Cary, SC, USA) using a mixed-model factorial ANOVA and with results presented as estimates of the median, ratios of estimates, and $95 \%$ confidence intervals with $\alpha=0.05$. IVF-derived male and female blastocysts possessed similar levels of the transcripts analyzed, suggesting successful dosage compensation at this developmental stage for embryos fertilized in vitro. XIST was not detected in male IVF embryos. GAB3 was not detected in any of the female groups and, in addition, HPRT1 transcripts were not detected in SCNT derived female embryos. Male and female SCNT-derived blastocysts possessed marked differences in their transcript levels, with males showing statistically significantly higher levels of $B I R C 4$ and $R P S 4 X$ and females possessing higher levels of MECP2 and SLC25A6 transcripts although differences between the latter two were not statistically significant. XIST was detected in both male and female SCNT blastocysts. We conclude that dosage compensation between male and female IVF blastocysts is achieved at this developmental stage for the transcripts examined. However, this pattern was markedly changed in the SCNT group, affecting especially female SCNT blastocysts, suggesting that the regulatory mechanisms of X-inactivation and X-linked gene expression are substantially altered in SCNT embryos probably due to aberrant epigenetic patterns and faulty genome reprogramming. We are currently analyzing X-linked transcription in male and female in vivo-derived blastocysts in order to compare this group with IVP-derived embryos.
\end{abstract}

This work was funded by NSERC, CIHR, and CRC. 


\title{
65 ASSESSMENT OF LIVE OFFSPRING SIRED BY A SOMATIC CELL NUCLEAR TRANSFER BULL, STARBUCK II
}

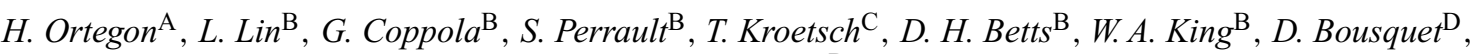 \\ and P. Blondin ${ }^{\mathrm{D}}$ \\ ${ }^{A}$ Department of Population Medicine, Ontario Veterinary College, University of Guelph, Guelph, Ontario, Canada N1G 2W1; \\ ${ }^{\mathrm{B}}$ Department of Biomedical Sciences, Ontario Veterinary College, University of Guelph, Guelph, Ontario, Canada N1G 2W1; \\ ${ }^{\mathrm{C}}$ Semex Alliance, Guelph, Ontario, Canada N1G 3Z2; \\ DL'Alliance Boviteq Inc., Saint-Hyacinthe, Quebec, Canada J2T 5H1
}

\begin{abstract}
It appears that overt phenotypic abnormalities observed in some domestic animal clones are not transmitted to their progeny, suggesting that epigenetic and genetic alterations are reset in the germline of clones. Still, little is known about the normalcy and performance of the offspring from cloned animals. The current study monitored Holstein heifers sired by a cloned bull, Starbuck II, from weaning to puberty. Genomic stability was assessed by telomere length status and chromosomal assessments. Growth parameters, blood profiles and physical exams were performed and the results were compared to those for a group of control heifers with matched breed and ages born from normal cows located in the Ponsonby Research Station (Guelph, Ontario, Canada). Once circulating progesterone concentrations were determined to be $>1 \mathrm{ng} / \mathrm{mL}$, the heifers were monitored by ultrasound to investigate follicular dynamics. Statistical analysis was performed using descriptive statistics, paired $t$-test, and a Tukey-Kramer post-hoc test for multiple comparisons. Compared to controls during the observation period, progeny sired by the cloned bull did not show any significant $(P>0.05)$ difference as in weight (mean \pm SEM) $(231.5 \pm 29.4$ vs. $237.6 \pm 31.0 \mathrm{~kg})$, length $(160.6 \pm 13.0 \mathrm{vs} .172 .4 \pm 2.7 \mathrm{~cm})$, height $(105.3 \pm 3.72$ vs. $95.5 \pm 11.0 \mathrm{~cm})$ and temperature $\left(39.06 \pm 0.01 \mathrm{vs} .39 .16 \pm 0.0^{\circ} \mathrm{C}\right)$. However, the cases displayed a relaxed behavior and were easier to handle than controls. This was reflected by significant differences in heart rates $(83.3 \pm 3.3 \mathrm{vs} .101 .3 \pm 2.6 \mathrm{bpm})(P=0.005)$ and respiratory rates $(35.4 \pm 2.7$ vs. $46.7 \pm 3.3 \mathrm{rpm})(P=0.04)$ for progeny and controls, respectively. Blood profiles were within normal ranges for both groups. External and internal genitalia were normal at palpation and ultrasound exam. Both groups began puberty at the expected age (10-12 months) and weight ( 318 to $365 \mathrm{~kg}$ ). Follicular dynamics and progesterone concentrations corresponded to normal adult cows and were similar between groups after puberty. Telomere lengths of spermatozoa and blood samples from Starbuck II ( $22.23 \mathrm{~kb}$ and $14.19 \mathrm{~kb}$, respectively) were not statistically different from normal telomere length profiles for non-cloned cattle. Telomere lengths of Starbuck II's progeny $(22.52 \pm 0.38 \mathrm{~kb}, n=32)$ were no different compared to age-matched controls $(22.20 \pm 0.56 \mathrm{~kb}, n=20)$. In addition, the progeny exhibited a normal karyotype in their peripheral blood leukocytes $(83.14 \pm 5.22 \%$ normal diploid cells). In summary, heifers sired by a cloned bull have normal chromosomal stability, growth, physical, hematological and reproductive parameters compared to normal heifers. Also, these heifers had a better response with lower stress to regular handling and restraining procedures.
\end{abstract}

This work was funded by OCAG, OMAF, and CRC.

\section{HISTIOTROPHIC NUTRITION AND IRON TRANSFER ACROSS THE MATERNO-FETAL INTERFACE} IN NUCLEAR TRANSFER-DERIVED SOMATIC CATTLE CLONES

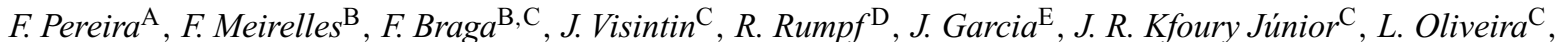

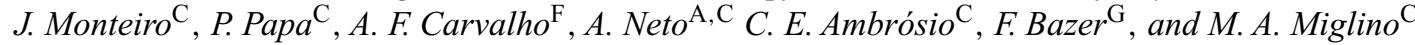

A UNESP-Dracena, Dracena, Sao Paulo, Brazil; ${ }^{\mathrm{B}}$ FZEA-USP, Pirassununga, Sao Paulo, Brazil; ${ }^{\mathrm{C}}$ FMVZ-USP, Sao Paulo, Sao Paulo, Brazil; DEmbrapa-Cenargen, Brasilia, Distrito Federal, Brazil; E UNESP-Jaboticabal, Jaboticabal, Sao Paulo, Brazil; F UNIFEOB, Sao Joao da Boa Vista, Sao Paulo, Brazil; ${ }^{\mathrm{G}}$ Texas A\&M University, College Station, TX 77843, USA

The histiotrophic nutrition by the endometrial glands and the materno-fetal interface in the cloned cattle placenta were analyzed in order to investigate the iron transfer. Placentomes and intercaruncular region samples were recovered at term Caesarean delivery from 14 cloned cattle and 10 controls, fixed in $4 \%$ paraformaldehyde and $10 \%$ formaldehyde in PBS, processed and stained for light microscopy (hematoxylin-eosin, picrosirius, and Masson's trichrome), histochemistry [Perls, acid phosphatase and periodic acid Schiff (PAS) reactions], and immunohistochemistry (with rabbit anti-pig uteroferrin antibody because the uteroferrin is an iron transporter protein). In the controls we verified blood extravasations in the maternofetal interface between the uterine and the trophoblast epithelium characterized by hemophagous areas with consequent erythrophagocytosis by the adjacent trophoblast. This content presented extravasated erythrocytes, plasm, cell debris, and cells in a probable apoptotic process. The Perls histochemical reactions that exposed the ferric iron in the placentomes were positive, as was the uteroferrin immunohistochemistry in the trophoblast cytoplasm and in other deep points in the placentomes. The histochemical reactions, demonstrating the acid phosphatase enzyme that detects the phagocytic activity, were positive in the mesenchyme and trophoblast, with a weak stain in an endometrial stroma. In the top of fetal villi, mainly in the binucleate cells, we visualized accumulations of PAS-positive secretions, indicating the presence of mucoid material. The uterine gland epithelium was columnar-type and in the gland lumina there were cell debris and PAS-positive mucoid secretions. We confirmed the reactivity of the uterine glands to the acid phosphatase enzyme and to the Perls reaction in the epithelium and in the gland lumina. The uteroferrin immunohistochemistry showed a strong stain in the cytoplasm of the endometrial glands cells and in the lumina. In the NT bovine placentae, the blood extravasations between uterine and trophoblast epithelium were aberrant. There was also the remodeling of the maternal connective tissue (endometrial stroma) in this area. We also demonstrated phagocytic uptake of uteroferrin by the trophoblast, although the histochemical and immunohistochemical reactions were weak in the trophoblast of the placentomes and in the endometrial glands of the intercaruncular region, when compared with the controls. The results obtained by the histochemistry and immunohistochemistry indicated that these sites of transfer substances from mother to fetus are very important in providing adequate nutrition to the fetus, key to a successful pregnancy in NT bovines.

This work was funded by FAPESP, Brazil. 


\title{
67 GENERATION OF PIGS TRANSGENIC FOR hCD59/DAF AND HUMAN THROMBOMODULIN BY SOMATIC NUCLEAR TRANSFER
}

\author{
B. Petersen, W. Kues, A. Lucas-Hahn, A.-L. Queisser, E. Lemme, M. Hoelker, J. W. Carnwath, and H. Niemann \\ Institute for Animal Science (FAL), Dept. of Biotechnology, Neustadt a.Rbge., Lower Saxony, Germany
}

\begin{abstract}
After a porcine-to-primate xenotransplantation, hyperacute rejection (HAR) destroys the transplanted organ within minutes. The HAR can be overcome either by a knockout of the gene for $\alpha$-1,3-galactosyltransferase or by overexpression of human complement regulatory proteins such as hCD59 and DAF. When HAR can be controlled, the next hurdle is acute vascular rejection (AVR) which is primarily due to an incompatibility of human protein $\mathrm{C}$ and porcine thrombomodulin, both of which are important factors in the coagulation cascade. This incompatibility leads to thrombosis and finally to a disseminated intravascular coagulation (DIC), causing rejection of the xenotransplant. Human thrombomodulin is a good candidate gene for improving survival time of porcine organs after xenotransplantation and for overcoming AVR. Here, we transfected adult fibroblasts obtained from a double transgenic boar (hCD59/DAF) with a construct for human thrombomodulin (hTM). After selection with $800 \mu \mathrm{g} / \mathrm{mL}$ G418 for 14 days, cells were analyzed for integration of the construct by PCR and were visually selected for expression of the hTM-GFP fusion protein under ultraviolet light with a fluorescein isothiocyanate (FITC) filterset. A total of 39 positve clones were obtained, of which two were used in somatic nuclear transfer. Ovaries were collected from a local slaughterhouse, and follicles of $2-5 \mathrm{~mm}$ in diameter were aspirated. After $38-42 \mathrm{~h}$ of in vitro maturation oocytes were denuded and enucleated. For cell cycle synchronization, the donor cells were serum-starved for $48 \mathrm{~h}$, subsequently trypsinized and placed into the perivitelline space of the enucleated oocytes. The complexes were fused and activated electrically followed by an incubation in DMAP for $3 \mathrm{~h}$. Puberal gilts were synchronized by treatment with $5 \mathrm{~mL}$ Regumate ${ }^{\circledR}$ (Intervet UK, Ltd., Milton Keynes, Buckinghamshire, UK) for 13 days. At the end of treatment, the animals received $1000 \mathrm{IU}$ pregnant mare serum gonadotropin (PMSG) intramuscularly followed by an injection of $500 \mathrm{IU}$ hCG $72 \mathrm{~h}$ later. Cloned embryos were transferred surgically $20 \mathrm{~h}$ after hCG injection. Maintenance of pregnancy was supported by injections of $1000 \mathrm{IU}$ PMSG on Day 11 and 500 IU hCG on Day 14 of the pregnancy. Out of 1409 reconstructed complexes, 1161 were fused $(82.4 \%)$ successfully. In total, 1040 embryos were transferred to 8 recipients $(\sim 130$ embryos/gilt, range $70-162)$. Five of the eight recipients $(62.5 \%)$ became pregnant as determined by ultrasound on Days 25,36, and 51 and will farrow within the next weeks. These results show that cloning with triple transgenic adult donor cells is compatible with high pregnancy rates. Porcine multitransgenic organs will be used in perfusion experiments to test the effectiveness of this novel approach to overcoming the incompatibilities between the porcine and the human coagulation systems.
\end{abstract}

This project is funded by the Deutsche Forschungsgemeinschaft (FOR 535). The hTM-construct was a gift of Dr. Wu which is gratefully acknowledged.

\section{GENE EXPRESSION COMPARISONS BETWEEN BOVINE IN VIVO AND CLONED EMBRYOS PRODUCED BY THREE DIFFERENT METHODS}

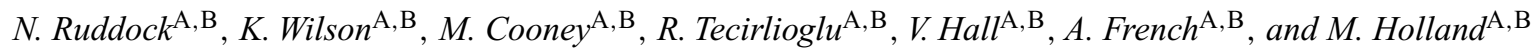 \\ ${ }^{\mathrm{A}}$ Monash Institute of Medical Research; Monash University, Clayton, Victoria, Australia; \\ ${ }^{\mathrm{B}} \mathrm{Co}$-operative Research Centre for Innovative Dairy Products, Melbourne, Victoria, Australia
}

Developmental pathways in the mammalian embryo are profoundly influenced by the epigenetic interaction of the environment and the genome. Loss of epigenetic control has been implicated in aberrant gene expression and altered imprinting patterns with consequence to the physiology and viability of the conceptus. Bovine somatic cell nuclear transfer (SCNT) is contingent on in vitro culture, and both SCNT and culture conditions are known to induce changes in embryonic gene expression patterns. Using these experimental models, this study compared gene expression of Day 7 cloned blastocysts created from three different SCNT protocols using the same cell line, with Day 7 in vivo blastocysts to elucidate mechanisms responsible for variations in phenotypic outcomes. SCNT methods included: (1) traditional SCNT by subzonal injection (SI); (2) handmade cloning (HMC); and (3) modified serial nuclear transfer (SNT), developed within the group. Four imprinted genes (Grb10, Ndn, Nnat, and Ube3a), four chromatin remodeling genes (Cbx1, Cbx3, Smarca4, and Smarcb1) and two genes implicated in polycystic liver disease (Prkcsh and Sec63) were analyzed in single blastocysts from each treatment $(n=5)$. All blastocysts expressed Actin, Oct-4 and Ifn-tau. All genes were sequence verified. Several genes were expressed ubiquitously across all groups, including Ndn, Ube3a, Cbx1, Cbx3, and Smarcb1. Interestingly, Grb10 was not expressed in two HMCs and one SNT blastocyst. Nnat was weakly expressed in one in vivo blastocyst and in the majority of cloned blastocysts in all groups. Prkcsh and Sec63 were expressed in all but one HMC blastocyst. While gene expression patterns were mostly maintained following SCNT, the imprinted genes Nnat and Grb10 showed instances of differential or abnormal expression in SCNT embryos. The chromatin remodeling genes were maintained in all SCNT treatments. Prkcsh and Sec63 were both absent in one HMC blastocyst, with implications for liver dysfunction, a condition previously reported in abnormal cloned offspring. The variable mRNA expression following SCNT provides an insight into genetic and environmental factors controlling implantation, placentation, organ formation, and fetal growth.

\section{RELATION OF SPATIAL GENE EXPRESSION PATTERNS IN BOVINE EMBRYOS RECONSTRUCTED WITH SOMATIC CELLS TO BLASTOCYST DEVELOPMENT}

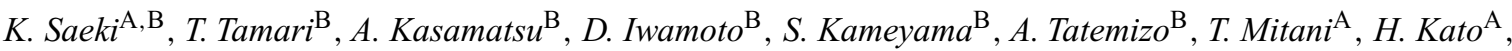

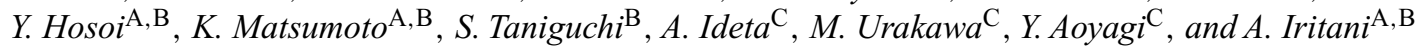 \\ A Institute of Advanced Technology, Kinki University, Wakayama, 642-0017, Japan; \\ ${ }^{B}$ Department of Genetic Engineering, Kinki University, Wakayama, 649-6493, Japan; \\ ${ }^{\mathrm{C}}$ ZEN-NOH Embryo Transfer Center, Hokkaido, 080-1407, Japan
}

Recently, enhanced development to full term was obtained with embryos reconstructed with bovine early $\mathrm{G}_{1}$ cells rather than with $\mathrm{G}_{0}$ cells (Kasinathan et al. 2001 Nat. Biotechnol. 19, 1176-1178; Urakawa et al. 2004 Theriogenology 62, 714-728). However, the reason why donor somatic 
cells at the early $\mathrm{G}_{1}$ phase are better for embryo reconstruction is unclear. In this study, we investigated the relation of spatial gene expression patterns at the 4- to 8-cell stage to blastocyst development of embryos reconstructed with early $\mathrm{G}_{1}$ cells. Bovine fibroblasts stably transfected with $\beta$-act/luc ${ }^{+} / I R E S / E G F P$ were used for embryo reconstruction. M phase cells were prepared as described by Urakawa et al. (2004). Early $\mathrm{G}_{1}$ cells were obtained from cultured $\mathrm{M}$ phase cells soon after the $\mathrm{M}$ phase cells divided. Quiescent cells (cultured in $0.4 \%$ serum for 7 days) were used as $\mathrm{G}_{0}$ cells for a control. The cells were electrofused with enucleated bovine oocytes matured in vitro, and activated with a calcium ionophore and cycloheximide. The reconstructed embryos were cultured until 60 hours post fusion (hpf), and zonae pellucidae of 4- to 8-cell embryos were removed by pronase. To determine gene expression, the $\mathrm{LUC}^{+}$activity (luminescence) in the embryo blastomeres was detected with an imaging photon counter (Hamamatsu Photonics, Hamamatsu City, Shikuoka Prefecture, Japan) for $10 \mathrm{~min}$. The embryos were categorized as being positive, mosaic, or negative depending on whether all, some or no blastomeres were luminescent, respectively. The embryos were cultured in mSOF medium individually until $168 \mathrm{hpf}$ to assess development to the blastocyst stage. Blastocyst development of reconstructed embryos without detection of luminescence was also examined. Experiments were repeated three times, and the data were analyzed with Fisher's PLSD test following ANOVA. At 60 hpf, 75\% (74/99) of embryos reconstructed with early $\mathrm{G}_{1}$ cells and $55 \%(46 / 83)$ of embryos with $\mathrm{G}_{0}$ cells developed to 4 - to 8 -cell stage embryos. The difference is significant $(P<0.05)$. The percentages of positive, mosaic, and negative embryos with $\mathrm{G}_{1}$ cells were 49,35 and $16 \%$, and blastocyst rates were 30,11 , and $0 \%$, respectively. With $\mathrm{G}_{0}$ cells, the percentages were 32,56 , and $12 \%$, and the blastocyst rates were 15,4 , and $0 \%$, respectively. More positive embryos were obtained with early $\mathrm{G}_{1}$ cells than with $\mathrm{G}_{0}$ cells $(P<0.05)$. Blastocyst rates of the positive embryos with early $\mathrm{G}_{1}$ cells were the same as with $\mathrm{G}_{0}$ cells $(P>0.05)$. Blastocyst development of positive embryos was higher than that of mosaic and negative embryos in early $\mathrm{G}_{1}$ and $\mathrm{G}_{0}$ groups $(P<0.05)$. Without detection of luminescence, the blastocyst rates from the reconstructed embryos were $43 \%(35 / 81)$ and $16 \%(20 / 125)$ with early $\mathrm{G}_{1}$ and $\mathrm{G}_{0}$ cells, respectively $(P<0.05)$. These results suggest that the higher developmental capacity of embryos reconstructed with early $\mathrm{G}_{1}$ cells might be related to the appropriate spatial gene expression at the 4 - to 8 -cell stage.

A part of this study was supported by a grant from the Wakayama Prefecture Collaboration of Regional Entities for the Advancement of Technological Excellence of the JST.

\title{
70 NON-PLATED GRANULOSA AND CUMULUS CELLS AND FIRST PASSAGE FIBROBLASTS AS NUCLEUS DONOR FOR GOAT CLONING
}

\author{
D. Salamone ${ }^{\mathrm{A}}$, M. Catala $^{\mathrm{A}}$, A. Gibbons $^{\mathrm{B}}$, F. Pereyra Bonnet $^{\mathrm{B}}$, and M. Cueto ${ }^{\mathrm{B}}$ \\ A Laboratorio de Biotecnología Animal, Facultad de Agronomía, Universidad de Buenos Aires, Argentina; \\ ${ }^{B}$ Instituto Nacional de Tecnología Agropecuaria, Estación Experimental Bariloche, CC 277 (8400) Bariloche, Argentina
}

Different types of somatic cells have been used as nucleus donors for cloning. Most of them were previously cultured in vitro as a monolayer through several plate passages. The experiment reported here was conducted to study the potential usages of granulosa and cumulus cells for cloning without previous culture as a monolayer. A first-plate-passage fibroblast was also used. Oocytes were aspirated by laparoscopy from Criolla goats and matured in TCM-199+5\% FCS at $39^{\circ} \mathrm{C}$ for $24 \mathrm{~h}$. Matured oocytes were denuded by vortexing for $3 \mathrm{~min}$ in TL HEPES with $1 \mathrm{mg} / \mathrm{mL}$ bovine testis hyaluronidase. Metaphases were assessed and oocytes were enucleated by visualization with Hoechst $33342(5 \mu \mathrm{g} / \mathrm{mL}) \mathrm{under} \mathrm{UV} \mathrm{light}(<6 \mathrm{~s})$. Granulosa and cumulus cells were also recovered by laparoscopy and maintained in maturation medium in cryotube for $20 \mathrm{~h}$ at room temperature or $39^{\circ} \mathrm{C}$, respectively. Goat adult ear fibroblasts were cultured for 1 or 2 weeks and used 2 days after confluence. All types of donor cells were transferred to the perivitlline space of enucleated oocytes and fused by an electrical pulse. After $2 \mathrm{~h}$, activation was induced by incubation in TLHEPES with $5 \mu \mathrm{M}$ ionomycin for $4 \mathrm{~min}$ and $2 \mathrm{mM}$ 6-DMAP for $3 \mathrm{~h}$. The oocytes were then washed with TL-HEPES and cultured in SOF medium and atmosphere of $5 \% \mathrm{CO}_{2}+5 \% \mathrm{O}_{2}+90 \% \mathrm{~N}_{2}$. Cleavage (Day 2) and development to blastocysts (Day 6) were recorded and analyzed by chi-square test. The cleavage rate for non-plated granulosa cells was higher than for the other treatment goups; cumulus cells had a lower rate of development to blastocysts (Table 1). These results suggest that granulosa cells collected and maintained for $24 \mathrm{~h}$ at room temperature could be used to produce cloned blastocysts.

Table 1. Effect of non-plated granulosa and cumulus cells and first passage fibroblasts as donor nucleus oocytes in goat cloning

\begin{tabular}{lcccc}
\hline Treatment & $n$ & Cleavage (\%) & Morula (\%) & Blastocyst (\%) \\
\hline Granulosa cells & 51 & $38(74.5)^{\mathrm{a}}$ & $8(15.7)$ & $5(9.8)^{\mathrm{a}}$ \\
Cumulus cells & 83 & $36(43.3)^{\mathrm{b}}$ & $9(10.8)$ & $0(0)^{\mathrm{b}}$ \\
Fibroblasts & 49 & $18(36.7)^{\mathrm{b}}$ & $11(24.4)$ & $1(2)^{\mathrm{a}, \mathrm{b}}$ \\
Total & 183 & 92 & 28 & 6 \\
\hline
\end{tabular}

${ }^{\mathrm{a}, \mathrm{b}}$ Percentages within columns with different superscripts are different $(P<0.05)$. 


\title{
71 WHAT IS THE UTERINE RESPONSE IN A CLONED BOVINE PREGNANCY?
}

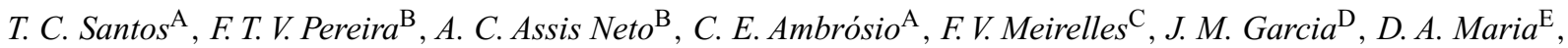 \\ A. F. Carvalho ${ }^{\mathrm{F}}$, and M. A. Miglino ${ }^{\mathrm{A}}$ \\ ${ }^{\mathrm{A}}$ Universidade de São Paulo - FMVZ/USP, São Paulo, São Paulo, Brazil; \\ ${ }^{B}$ Faculdade de Zootecnia /UNESP, Dracena, São Paulo, Brazil; \\ ${ }^{C}$ FZEA - São Paulo Univeristy, Pirassununga, São Paulo, Brazil; \\ ${ }^{D}$ FCAV/UNESP - Reproduction Department, Jaboticabal, São Paulo, Brazil; \\ ${ }^{\mathrm{E}}$ Intituto Butantan, São Paulo, São Paulo, Brazil; \\ FUNIFEOB, São João da Boa Vista, São Paulo, Brazil
}

Bovine has a synepitheliochorial placenta and characteristically there is no invasion of the trophoblast, but there is migration of the binucleate trophoblast giant cells into the maternal endometrium. The feto-maternal interface occurs in placentome where a tridimensional organization permits interactions between maternal epithelium and trophoblast, and in the intercaruncular area it is possible to observe a few mini-placentomes and the uterine glands opening. The objective of the present investigation was to study the morphological aspects of the uterus in bovine that had a cloned cattle gestations to understand the differences with natural gestation. The uterus and fetal membranes from natural and cloned cattle gestations were collected, fixed in 10\% formaldehyde, processed, and stained for light microscopy and immunohistochemistry. The morphological differences observed in the surrogate uterus were: extensive areas without placentome, hemorrhagic uterine areas, caruncular fusion giving a reduced number of caruncules, increase in size and weight (megacaruncules), and a significant number of mini-caruncules giving miniplacentomes (diameter $<1 \mathrm{~cm}$ ). In particular the mini-placentome showed functional trophoblastic cells with PAS+ granules in the binucleate trophoblast giant cells and an intense subepithelial capillary organization in maternal and fetal sides. The normal and clone placentomal cell populations were analyzed throughout pregnancy. The population of tetraploid and diploid trophoblastic cells was stained; detached cell cycle and DNA content was measured in FL2 using a FACscalibur flow cytometric system. We determined the percentage of cells in apoptosis (sub-G1), quiescent cells (G0/G1), synthesis (S), and proliferative cells (G2/M) with the aid of ModFit software. In addition, a cell cycle differential analysis was performed, and the tetraploid population presented statistical differences in cell cycle phases and populations relative to the apoptosis rate for the first $(7.5 \pm 3.1 \%)$, second (15.2 $\pm 5.0 \%)$ and third $(17.3 \pm 4.3 \%)$ trimesters. The number of apoptotic cells increased significantly during pregnancy stages. The results showed that first trimester presented the majority of its cells in the G0-G1 phase, starting the cell cycle. On the other hand, the second and third trimesters presented the majority of their cells in the G2-M phase, ending the cell cycle. The relationship between cell cycle phases/rate of apoptosis in mononucleate cells, days of normal and cloned pregnancy, the number of binucleate cells, and their metabolic activity as well as their developmental kinetics could be important data in several studies that involve placental development in natural pregnancy or that derived from laboratory-manipulated embryos.

This work was supported by FAPESP and CNPq.

\section{HISTONE METHYLATION AND GENE EXPRESSION PATTERNS IN PRE-IMPLANTATION EMBRYOS DERIVED FROM INTRA-AND INTER-SPECIES NUCLEAR TRANSFER}

\author{
W. Shi ${ }^{\mathrm{A}, \mathrm{B}}$, F. Yang ${ }^{\mathrm{A}}$, E. Wolf ${ }^{\mathrm{A}}$, and V. Zakharchenko ${ }^{\mathrm{A}}$ \\ ${ }^{A}$ Department of Molecular Animal Breeding and Biotechnology, University of Munich, Munich, Germany; \\ ${ }^{\mathrm{B}}$ Department of Development and Genetics, Uppsala University, Uppsala, Sweden
}

The differential epigenetic changes in embryos from different species provide a model to study how the nucleus from one species interacts with cytoplasm from another species. In this study we examined histone methylation at lysine 9 of histone 3 (K9H3) and lysine 20 of histone H4 (K20H4) and the expression levels of three early development-related genes (Oct-4, Hsp 70.1 and Hprt) in individual intra- and inter-species cloned and control embryos at the 1-, 2-, 4- and 8-cell stages. Mouse fetal fibroblast (MFF) nuclei were transferred into mouse, bovine, or rabbit oocytes. As control, we used in vivo derived (mouse and rabbit) or in vitro-produced (bovine) embryos. Histone methylation was detected by anti-MeK9H3 and anti-MeK20H4 antibodies. Gene expression analysis was performed using a quantitative RT-PCR technique (Daniels et al. 2000 Biol. Reprod. 63, 1034-1040). Data were analyzed by Student's $t$-test. No embryos from inter-species cloning (MFF-bovine and MFF-rabbit) survived beyond the 8-12 cell stage. MFF-mouse and MFF-bovine embryos exhibited demethylation of K9H3 and $\mathrm{K} 20 \mathrm{H} 4$ at the 2-cell stage and the methylation level was increased at the 4-cell stage, but no demethylation was observed at the 2-cell stage of MFF-rabbit embryos and the methylation level in these embryos was significantly higher than that of in vivo rabbit embryos. The level of Oct-4 mRNA was low at the 1- and 2-cell stages of in vivo mouse embryos and increased at the 8-cell stage. No significant increase in Oct-4 transcript was detected at the 8-cell stage of inter-species cloned embryos. The expression of Hsp 70.1 in in vivo mouse embryos was increased at the 2-cell stage and decreased to a level similar to that in the zygote at the 8-cell stage. In cloned embryos, Hsp 70.1 transcripts were also increased at the 2-cell stage, but there was no significant decrease of Hsp70.1 mRNA abundance at the 8-cell stage of inter-species embryos as compared to the corresponding 2-cell stage. For MFF-mouse embryos, Hsp 70.1 expression was increased at the 2-cell stage, but at the 8-cell stage the transcript level was at the level similar to that in inter-species clones. Hprt expression was increased at the 8-cell stage of in vivo mouse embryos. The dynamic change of Hprt transcript in MFF-mouse embryos was not significantly different from that of in vivo mouse embryos, but no significant change of Hprt expression occurred in the development of MFF-bovine and MFF-rabbit embryos. Differential epigenetic characteristics of mouse somatic nucleus after transfer into oocytes from different species suggest the existence of incompatibilities of nuclear-cytoplasm interaction between distantly related species. This abnormal interaction at the time of genome activation may affect normal development.

This work was supported by the Bayerische Forschungsstiftung and by Therapeutic Human Polyclonals, Inc. 


\title{
73 PROTEOMIC ANALYSIS OF THE MAJOR CELLULAR PROTEINS OF BOVINE TROPHECTODERM CELL LINES DERIVED FROM IVP, PARTHENOGENETIC, AND NUCLEAR TRANSFER EMBRYOS: REDUCED EXPRESSION OF ANNEXIN I IN NUCLEAR TRANSFER-DERIVED CELL LINES
}

\author{
N. Talbot ${ }^{\mathrm{A}}$, A. Powell $^{\mathrm{A}}$, T. Caperna $^{\mathrm{B}}$, and W. Garrett ${ }^{\mathrm{A}}$ \\ ${ }^{\mathrm{A}}$ Biotechnology and Germplasm Laboratory, Animal and Natural Resources Institute, USDA, Beltsville, Maryland, USA; \\ ${ }^{\mathrm{B}}$ Growth Biology Laboratory, Animal and Natural Resources Institute, USDA, Beltsville, Maryland, USA
}

Trophectoderm cell lines were established from 8-day in vitro cultured bovine embryos that were derived from the fertilization (IVF), somatic cell nuclear transfer (NT), or parthenogenetic activation (P) of in vitro-matured oocytes and from five 8-day-old in vivo (V) embryos. The most abundant cellular proteins of $2 \mathrm{~V}-, 16 \mathrm{NT}-, 12 \mathrm{P}-$, and $13 \mathrm{IVF}-$ derived cell lines were compared by 2-D gel electrophoresis and mass spectrometry; that is, the unaltered thiourea/urea extract of each cell culture was analyzed. One-hundred and eighteen in common protein spots were examined, and $95 \%$ were identified with significant scores from protein and gene database searches. Of the proteins detected and identified, actin and cytokeratin- 8 were found to be the most abundant. Other prominently expressed cellular proteins were metabolic enzymes such as aldose reductase, phosphoglycerate mutase, enolase, triosephosphate isomerase, cytoskeletal interacting proteins transgelin and stratifin, antioxidant proteins peroxiredoxin 1 and anti-oxidant protein 2 , and the calcium-dependent lipid-binding proteins annexin I and II. In comparative analysis of the 2-D gels, the NT-derived trophectoderm showed diminished annexin I expression in comparison to the IVF-derived trophectoderm. Since annexin I is abundantly expressed in the placenta and has functions important to the maintenance of placentation, the down-regulation of annexin I in the cultured NT trophectoderm may be related to the frequent failures of NT pregnancies.

\section{EXAMINATION OF ABNORMAL CELL DIVISION AND CHROMOSOME ABERRATION IN PIG PARTHENOTES AND CLONED EMBRYOS}

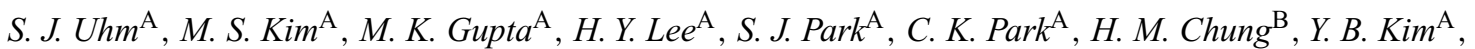 \\ K.S. Chung ${ }^{\mathrm{A}}$, and H.T. Lee $\mathrm{A}$ \\ ${ }^{\mathrm{A}}$ Department of Animal Science, Konkuk University, Seoul, Korea; ${ }^{\mathrm{B}}$ College of Medicine, Pochon CHA University, Seoul, Korea
}

Blastomere fragmentation is commonly observed in pig embryos and is associated with reduced blastocyst and pregnancy rates. This study examined the effect of the frequency of abnormal cell division and chromosome aberration on the embryonic developmental ability of pig parthenotes and nuclear transferred (NT) embryos. Pig immature oocytes cultured in TCM-199 supplemented with 10\% pig follicular fluid, $0.2 \mathrm{mM}$ pyruvate, $10 \mathrm{ng} / \mathrm{mL}$ epidermal growth factor (EGF), $5 \mu \mathrm{g} / \mathrm{mL}$ Folltropin $\mathrm{V}, 1 \mu \mathrm{g} / \mathrm{mL}$ estradiol-17 $\beta$, and $25 \mu \mathrm{g} / \mathrm{mL}$ gentamycin for $44 \mathrm{~h}$. Cumulus cells from matured oocytes were removed by vortexing for $1 \mathrm{~min}$ in TL-HEPES medium containing $0.1 \%$ hyarunonidase. Denuded oocytes were enucleated using 20 um micropipette in TCM-HEPES medium containing $7.5 \mu \mathrm{g} / \mathrm{mL}$ cytochalasin B (CB) and $10 \%$ fetal bovine serum, and were reconstructed with fetal fibroblasts by electrofusion (two DC pulses of $2.0 \mathrm{kV} / \mathrm{cm}$ for $30 \mu \mathrm{s}$ ). For production of parthenotes and reconstructed embryos, denuded oocytes were activated by a DC pulse of $1.0 \mathrm{kV} / \mathrm{cm}$ for $30 \mu \mathrm{s}$ and then cultured for $4 \mathrm{~h}$ in NCSU23 with $10 \mu \mathrm{g} / \mathrm{mL} \mathrm{CB}$ and $0.4 \%$ bovine serum albumin for inhibition of polar body extrusion. Subsequently, these oocytes were cultured in $50 \mu \mathrm{L}$ of NCSU23 containing $0.4 \%$ BSA for 7 days at $39^{\circ} \mathrm{C}$ in a humidified atmosphere of $5 \% \mathrm{CO}_{2}$ in air. The frequency of chromosome aberrations was evaluated using fluorescent in situ hybridization technique with a porcine chromosome-1 submetacentric specific probe. Data were analyzed by Student's $t$-test and ANOVA using SAS software as appropriate (SAS Institute, Inc., Cary, NC, USA). Parthenotes and NT embryos showed similiar cleavage rates (61.4 and 62.9\%), but the blastocyst rate of parthenotes $(18.4 \%)$ was significantly higher $(P<0.05)$ than that of NT embryos $(10.4 \%)$. The frequency of chromosome aberration in NT embryos (39.8\%) at the 4-cell stage on Day 3 of culture was significantly higher $(P<0.05)$ than that of parthenotes $(21.9 \%)$. The percentage of fragmentation was significantly higher $(P<0.05)$ in NT embryos $(51.7 \%)$ than in parthenotes $(27.1 \%)$. Furthermore, the developmental rates of non-fragmented parthenotes $(40.0 \%)$ and NT $(22.9 \%)$ embryos to the blastocyst stage were significantly higher $(P<0.05)$ than those of fragmented parthenote and NT embryos (17.3 and 5.9\% respectively). The total cell number of non-fragmented parthenote and NT embryos (34.4 \pm 10.0 and $29.7 \pm 7.5)$ were significantly higher $(P<0.05)$ than those of fragmented parthnote and NT embryos $(22.3 \pm 9.6$ and $18.4 \pm 6.2$ respectively). Therefore, these results indicate that chromosomal abnormality and embryonic fragmentation could be associated with reduced developmental ability in pig NT embryos.

This work was supported by the Research Project on the Production of Bio-organs, Ministry of Agriculture and Forestry, Republic of Korea.

\section{DEVELOPMENT OF BOVINE SCNT EMBRYOS RECONSTRUCTED USING RECIPIENT OOCYTES AND EGFP-TRANSFECTED CELLS THAT WERE CULTURED WITH VITAMIN C OR E}

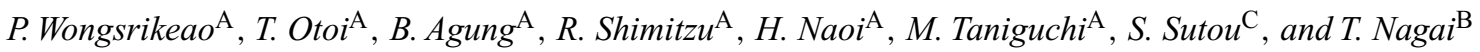 \\ ${ }^{A}$ Laboratory of Animal Reproduction, Department of Veterinary Sciences, Yamaguchi University, Yamaguchi, 753-8515, Japan; \\ ${ }^{B}$ Department of Research Planning and Coordination, National Institute of Livestock and Grassland Science, Tsukuba, Ibaraki, 305-0901, Japan; \\ ${ }^{\mathrm{C}}$ School of Pharmacy, Shujitsu University, 1-6-1 Nishigawara, Okayama, 703-8516, Japan
}

The objective of this study was to investigate the development of bovine embryos produced by somatic cell nuclear transfer (SCNT) after treatment of recipient oocytes and/or gene-transfected donor cells with vitamin $\mathrm{E}$ and/or vitamin $\mathrm{C}$. In the first experiment, oocytes derived from a 
local slaughterhouse were cultured in vitro for $22 \mathrm{~h}$ in TCM-199 medium supplemented with $100 \mu \mathrm{g} \mathrm{mL}^{-1}$ vitamin E, $100 \mu \mathrm{g} \mathrm{mL}-1$ vitamin C, a combination of vitamins $\mathrm{E}\left(100 \mu \mathrm{g} \mathrm{mL}^{-1}\right)$ and $\mathrm{C}\left(100 \mu \mathrm{g} \mathrm{mL}^{-1}\right)$, or no addition. After culture, a total of 1139 oocytes were randomly examined for their meiotic stage and glutathione (GSH) concentration, and 1171 oocytes were enucleated and reconstructed with fetal lung cells that had been transfected with a plasmid containing the enhanced green fluorescence protein (EGFP) and neomycin-resistant genes. As a control, the same cell line without gene transfection and recipient oocytes matured without vitamin treatment were used for SCNT. The SCNT was performed according to the established procedure in our laboratory (Murakami et al. 2005 Cloning and Stem Cells 7,77-81). The total cells and DNA fragmented cells of SCNT blastocysts were examined on Day 7 after SCNT (Day 0). Data were subjected to arcsin transformation before analysis, and tested by a post-hoc, Fisher's protected least significant difference PLSD test using the Statview programme (SAS Institute, Inc., Cary, SC, USA). Differences at a probability $P<0.05$ were considered significant. The levels of GSH synthesis in the matured ocytes were higher $(P<0.05)$ in vitamin supplementation groups (6.8-9.0 pmol/oocyte) than in the non-supplementation group (6.7 pmol/oocyte). As shown in Table 1, the vitamin E supplementation improved the blastocyst formation, total cell number, and DNA fragmentation of EGFP-embryos compared with those parameters for EGFP-embryos obtained from recipient oocytes without vitamin treatment. However, the numbers of total cells and DNA-fragmented cells of EGFP-embryos were significantly lower $(P<0.05)$ than those of control embryos obtained by the SCNT using somatic cells without transfection, irrespective of vitamin treatment. In the second experiment, SCNT embryos were produced using 312 recipient oocytes treated with $100 \mu \mathrm{g} \mathrm{mL}^{-1}$ vitamin $\mathrm{E}$ and EGFP-transfected donor cells that had been cultured in medium supplemented with various concentrations $\left(0,50, \mathrm{or}^{100 \mu \mathrm{g} \mathrm{mL}}{ }^{-1}\right)$ of vitamin E. Although the supplementation of $100 \mu \mathrm{g} \mathrm{mL}^{-1}$ vitamin E decreased the proportion (30.8\%) of blastocyst formation as compared with non-vitamin (42.8\%) and $50 \mu \mathrm{g} \mathrm{mL}^{-1}$ vitamin E (46.0\%), the proportion (2.0\%) of DNA fragmented cells in the SCNT blastocysts was significantly lower $(P<0.05)$ than that of other groups $(3.2-3.6 \%)$. In conclusion, supplementation of vitamin $\mathrm{E}$ during oocyte maturation and donor cell culture improved the development and quality of gene-transfected SCNT bovine embryos.

Table 1. Development of SCNT embryos* using recipient oocytes treated with vitamin $\mathrm{C}$ and $\mathrm{E}$

\begin{tabular}{lccccc}
\hline Treatment & MII (\%) & Fused (\%) & Blastocyst/fused (\%) & Total cell no. & DNA fragmentation (\%) \\
\hline Control & - & $74.5^{\mathrm{a}}$ & $47.6^{\mathrm{a}}$ & $155.0^{\mathrm{a}}$ & $1.9^{\mathrm{a}}$ \\
Non-vitamin & $77.4^{\mathrm{a}, \mathrm{b}}$ & $66.4^{\mathrm{b}}$ & $33.6^{\mathrm{b}}$ & $86.5^{\mathrm{c}}$ & $10.4^{\mathrm{b}}$ \\
Vitamin C & $80.2^{\mathrm{a}}$ & $78.1^{\mathrm{a}}$ & $41.9^{\mathrm{a} . \mathrm{b}}$ & $122.1^{\mathrm{b}}$ & $8.5^{\mathrm{b}}$ \\
Vitamin E & $82.0^{\mathrm{a}}$ & $76.8^{\mathrm{a}}$ & $47.1^{\mathrm{a}}$ & $118.9^{\mathrm{b}}$ & $4.7^{\mathrm{c}}$ \\
Vitamin C and E & $67.9^{\mathrm{b}}$ & $71.3^{\mathrm{a} . \mathrm{b}}$ & $34.0^{\mathrm{b}}$ & $113.9^{\mathrm{b}}$ & $5.0^{\mathrm{c}}$ \\
\hline
\end{tabular}

${ }^{\mathrm{a}-\mathrm{c}}$ Values with different superscripts within the same column were significantly different.

\title{
76 SCREENING Oct-4 PROMOTER ACTIVITY IN BOVINE FIRST AND SECOND ROUND SCNT EMBRYOS USING AN EGFP REPORTER CONSTRUCT
}

\author{
A. Wuensch ${ }^{\mathrm{A}}$, F. A. Habermann ${ }^{\mathrm{B}}$, R. Klose ${ }^{\mathrm{A}}$, V. Zakhartchenko $^{\mathrm{A}}$, F. Yang $^{\mathrm{A}}$ H. Wenigerkind $^{\mathrm{A}}$, \\ F. Sinowatz ${ }^{\mathrm{B}}$, and E. Wolf ${ }^{\mathrm{A}}$

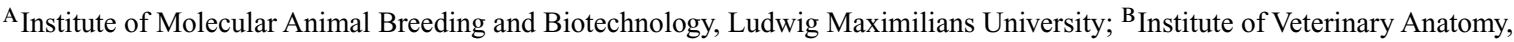 \\ Histology and Embryology, Ludwig Maximilians University, Munich, Germany
}

Oct-4, also known as Oct-3 or POU5F1, belongs to the POU (Pit-Oct-Unc) transcription factor family. So far it has been identified in mice, cattle, pigs, and humans. In mice, pluripotent cells of the early pre-implantation embryo express Oct-4. Therefore, Oct- 4 expression is considered to be a marker for pluripotency in mice. In bovine blastocysts, Oct-4 protein is not restricted to the pluripotent cells of the inner cell mass (ICM) but is also present in the trophectoderm (TE). It has been reported, however, that at this stage the transcript level of Oct-4 was already downregulated in the TE suggesting a similar regulation of Oct-4 transcription in bovine and mice. To obtain insight into the regulation of the Oct-4 promoter after somatic cell nuclear transfer (SCNT), we transfected bovine fetal fibroblasts (BFF) with GOF18- $\triangle$ PE-EGFP, a reporter gene construct for the Oct-4 promoter (kindly provided by Dr. Hans R. Schoeler, Director, Max Planck Institute for Molecular Biomedicine, Münster, Germany). Six stably transfected colonies (BFFGOF), none of which exhibited green fluorescence, were used for SCNT, with subsequent examination of the resulting embryos on Days 5-7 by fluorescence microscopy. SCNT embryos originating from BFF colonies 3, 12, and 82 showed fluorescence both in ICM and TE. SCNT with BFF colony 8 resulted in embryos with no detectable fluorescence. This might have been due to a positional effect of the reporter gene construct or an incomplete reprogramming of the used fibroblast colonies during SCNT. To study the consequences of another round of SCNT on Oct-4 promoter activity, cloned embryos from BFF colonies 12 (positive) and 8 (negative) were transferred to recipients. At Day 33, three BFFGOF12 and four BFFGOF8 fetuses were recovered and BFF cultures were established from all cloned fetuses. The presence of GOF18- $\triangle$ PE-EGFP was verified by PCR and Southern blot analysis. BFF cultures from two cloned BFFGOF12 and BFFGOF8 fetuses each were used for SCNT, and fluorescence was examined in Day 6 embryos. SCNT embryos derived from BFFGOF12 fetuses exhibited weaker fluorescence than embryos directly derived from the original transfected colony. SCNT embryos derived from BFFGOF8 fetuses showed no fluorescence. GFPpositive embryos will be examined by immunocytochemistry using an antibody against Oct-4 to evaluate the correlation between endogenous Oct-4 and Oct-4 reporter gene expression. Further fluorescence in situ hybridization (FISH) analyses are underway to localize the reporter gene integration sites in BFFGOF8 and BFFGOF12. In summary, stable GOF18- $\triangle$ PE-EGFP transfected cells are an interesting tool for monitoring Oct-4 promoter activation after using different protocols of SCNT or in consecutive rounds of SCNT. Furthermore, it will be possible to correlate Oct-4 promoter activity with epigenetic mechanisms, such as DNA methylation and histone modifications, in SCNT embryos. 


\title{
77 PRODUCTION OF BAN MINIPIG EMBRYO BY SOMATIC CELL NUCLEAR TRANSFER
}

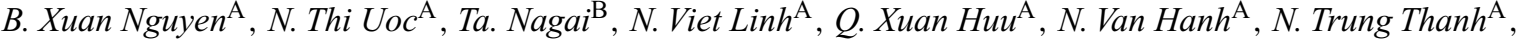 \\ N. Huи Duc ${ }^{\mathrm{A}}$, and B. Linh $C h i^{\mathrm{A}}$
}

${ }^{A}$ Laboratory of Biology of Reproduction and Development, Institute of Biotechnology, Vietnamese Academy of Science and Technology, Nghia Do, Hanoi, Vietnam; ${ }^{\text {B }}$ Department of Animal Reproduction, National Institute of Animal Industry, Tsukuba, Ibaraki 305-0901, Japan

\begin{abstract}
Ban pig is a one of the wild miniature pig breeds of the Vietnamese highland characterized by small body size and adult weight from 40 to $50 \mathrm{~kg}$. SCNT has been applied to this breed as a tool for its conservation to maintain biodiversity as well as to multiply the minipigs as models for research and application in human xenotransplantation. In this paper we present the results of experiments on the production of Ban minipig embryos by transfer of their somatic cell nuclei into enucleated oocytes prepared from domestic pig (Landrace). Skin explants were collected from a young Ban male. After disinfection, the samples were cut into small pieces and cultured for $7-10$ days in DMEM medium supplemented with $10 \%$ heat treated fetal bovine serum at $39^{\circ} \mathrm{C}$ under $5 \% \mathrm{CO}_{2}$. The cultures resulting in cell confluence were used for further passage and cryopreservation by rapid freezing in medium containing $10 \%$ DMSO and $0.1 \mathrm{M}$ sucrose. The ovaries collected from prepubertal Landrace gilts from a slaughterhouse were transported to the laboratory in PBS at $30^{\circ} \mathrm{C}$. Cumulus-oocyte complexes were aspirated from follicles of 3-6 mm in diameter using an 18-gauge needle. They were washed in Hepes-buffered TCM-199, in vitro matured (IVM) in a modified North Carolina State University-37 medium (NCSU-37) containing $10 \%(\mathrm{v} / \mathrm{v})$ porcine follicular fluid, $0.6 \mathrm{mM}$ cysteine, $1 \mathrm{mM}$ dibutytyl cyclic AMP (dbcAMP), $10 \mathrm{IU} / \mathrm{mL}$ eCG, and $10 \mathrm{IU} / \mathrm{mL}$ $\mathrm{hCG}$ for $22 \mathrm{~h}$, and then held for an additional $14 \mathrm{~h}$ in the modified NCSU-37 without dbcAMP, eCG, and hCG. The mature oocytes were separated from cumulus cells by vortexing in $0.1 \%$ hyaluronidase in TCM-199. Metaphase II oocytes showing a clear first polar body (PB) were selected for enucleation in the presence of cytochalasin B. For nuclear transfer, fibroblasts were induced to quiescent state by culture in a medium with a low fetal bovine serum (FBS) concentration, and a single cell was transferred into each enucleated oocyte. Fusion was induced with needle electrodes and two direct current (DC) pulses of $30 \mathrm{~V}$ for $25 \mu \mathrm{s}$ in Zimmerman medium with $0.3 \mathrm{M}$ mannitol. The fused oocytes were activated with $7 \%$ ethanol in HEPES-TCM-199 for $5 \mathrm{~min}$ and cultured in the medium containing cyclohexamide for $6 \mathrm{~h}$ before further culture in vitro. The percentage of reconstructed oocytes fused, cleaved, and developed to the morula and blastocyst stages was assessed under a stereomicroscope at Days 2, 3, and Day 6-7, after nuclear transfer. The results showed that the rate of oocytes with a clear PB, obtained after IVM for $36 \mathrm{~h}$, was $52.8 \%(n=500)$. From 294 reconstructed oocytes, 192 (72.7\%) fused; a total of $156(81.2 \%)$ cleaved to the 4-8-cell stage, 96 (50\%) developed to the morula stage, and $24(12.5 \%)$ developed to the blastocyst stage at Days 2-3, 5-6, and 7 after nuclear transfer, respectively. A karyotype study showed a normal distribution of chromosome number $(2 n=38)$ for the cell population after passage and for reconstructed embryos. These results suggest that IVM oocytes prepared from domestic pig can be successfully used for the production of the SCNT BAN minipig embryos.
\end{abstract}

This work was supported by grant from the JSPS-VAST Joint Research Project for FY 2004 and by Project 82.

\section{EFFECTS OF DIFFERENT ACTIVATION PROTOCOLS ON ACTIN FILAMENT DISTRIBUTION AND IN VITRO DEVELOPMENT OF MINIATURE PIG NT EMBRYOS}

\author{
K. Yamanaka, S. Sugimura, T. Wakai, T. Shoji, H. Sasada, and E. Sato \\ Laboratory of Animal Reproduction, Graduate School of Agricultural Science, Tohoku University, Sendai, Japan
}

In the process of producing reconstructed oocytes nuclear transfer (NT) embryos by somatic cell nuclear transfer, in vitro-matured oocytes can be used as recipient ones. It, however, has been well documented that after IVF porcine embryos derived from in vitro-matured oocytes have a small number of cells and low viability compared from those in vivo. As one possible reason, abnormal actin filament distribution has been detected in abnormal embryo cleavage and small cell numbers (Wang et al. 1999 Biol. Reprod. 60, 1020-1028). Artificial activation, which is necessary for development of NT embryos, can affect actin filament distribution of porcine oocytes matured in vitro, resulting in fragmentation (Kawahara et al. 2002 Theriogenology 58, 1081-1095). In the present study, we investigated effects of different activation protocols on actin filament distribution and in vitro development of miniature pig NT embryos. Porcine oocytes collected from ovaries were matured in vitro for 40 to $44 \mathrm{~h}$ in NCSU-23. First, we compared different activation protocols in development rates to blastocysts of oocytes activated. We used three activation methods $(15 \mu \mathrm{M}$ ionomycin treatment for $20 \mathrm{~min}$ (I), double DC pulses of $1.2 \mathrm{kV} / \mathrm{cm}$ for $60 \mathrm{~ms}$ in intervals of $5 \mathrm{~s}(\mathrm{E})$, and $5 \mathrm{mg} / \mathrm{mL}$ cycloheximide treatment for $5 \mathrm{~h}$ (C)) to prepare seven activation protocols $(\mathrm{I}, \mathrm{E}, \mathrm{C}, \mathrm{I}+\mathrm{C}, \mathrm{I}+\mathrm{E}, \mathrm{E}+\mathrm{C}$, and I $+\mathrm{E}+\mathrm{C})$. Second, we examined effects of different activation protocols on actin filament distribution and subsequent development of NT embryos activated by the different activation protocols. Matured oocytes were enucleated, and fused with miniature pig fetal fibroblasts in calcium-free medium; approximately $3 \mathrm{~h}$ later, the resultant NT embryos were activated with three activation protocols $(\mathrm{E}, \mathrm{I}+\mathrm{C}$, or $\mathrm{I}+\mathrm{E}+\mathrm{C})$. All data were analyzed by chi-square test. The developmental rates to blastocysts in the I, E, $\mathrm{C}, \mathrm{I}+\mathrm{C}, \mathrm{I}+\mathrm{E}, \mathrm{E}+\mathrm{C}$, and I $+\mathrm{E}+\mathrm{C}$ groups were $5.6,11.1,0.0,36.1,20.7,14.6$, and $24.7 \%$, respectively, showing that the rate in oocytes activated with $\mathrm{I}+\mathrm{C}$ was significantly higher $(P<0.05)$ than the rates in oocytes activated by other treatments. In NT embryos, the developmental rates to blastocysts in the $\mathrm{E}, \mathrm{I}+\mathrm{C}$, or I $+\mathrm{E}+\mathrm{C}$ groups were $4.1,14.3$, and $4.6 \%$, respectively, showing that the rate in NT embryos activated with $\mathrm{I}+\mathrm{C}$ was significantly higher $(P<0.05)$ than the rate in NT embryos activated with other treatments. The abnormal rate of actin filament distribution in NT embryos activated with $\mathrm{E}$ or $\mathrm{I}+\mathrm{E}+\mathrm{C}$ was significantly higher $(P<0.05)$ than that in $\mathrm{NT}$ embryos activated with $\mathrm{I}+\mathrm{C}(26.7 \%$ or $33.3 \%$ vs. $6.7 \%)$. The present results suggest that in miniature pig NT embryos an activation protocol by ionomycin combined with cycloheximide treatments may avoid physical damage to actin filaments with the resultant improvement of subsequent development. 


\title{
79 THE AMOUNT OF TELOMERIC DNA IN CLONED CATTLE AND THEIR CALVES IS LESS THAN THAT OF AGE MATCHED CATTLE
}

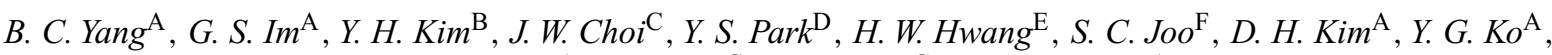 \\ H. H. Seong ${ }^{\mathrm{A}}$, E. J. Cho ${ }^{\mathrm{G}}$, S. H. Sohn ${ }^{\mathrm{G}}$, and B. S. Yang ${ }^{\mathrm{A}}$
}

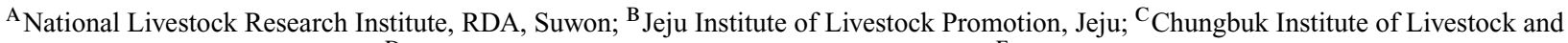
Veterinary Research, Chungbuk; ${ }^{\mathrm{D}}$ Gyongbuk Livestock Research Institute, Gyongbuk; ${ }^{\mathrm{E}}$ Gangwon Livestock Research Center, Gangwon;

F Gyeonggido Livestock Veterinary Service, Gyeonggido; G Jinju National University, Jinju, South Korea

A telomere is a structureconsisting of tandem repeats sequences of (TTAGGG) $n$ at the end of the eukaryotic chromosome. Telomere lengths in animals vary by species, age, and tissues, as well as environment. This experiment concentrated on the amount of telomeric DNA in cloned cattle, their calves, and age-matched normal cattle. Using somatic cell nuclear transfer (SCNT), we had obtained 16 cloned Korean Native cows derived from ear skin fibroblasts and two cloned bulls from fetal fibroblasts. In addition, four female calves were produced from each cloned cow by artificial insemination. Control cattle selected to have matched age and the same raising place served as counter-parts of cloned the cattle in this study. The lymphocytes of all cloned cattle, their calves, and the age-matched controls were examined for telomere quantity. The amount of telomeric DNA was analyzed by quantitative fluorescence after in situ hybridization (Q-FISH) with a human telomeric DNA repeat probe. A minimum of 100 interphase nuclei from each set of harvests was studied to determine the mean and medium percentages of telomeric DNA using the MetaMorph Imaging System (Universal Imaging Co., West Chester, PA, USA). The amount of telomeric DNA obtained was found to decrease in cloned and control animals during growth. The amounts of telomeric DNA in cloned cattle from both ear skin fibroblasts $(n=16)$ and fetal fibroblasts $(n=2)$ was less than that of age-matched controls $(P<0.01)$. Surprisingly, the amount of telomeric DNA of calves from cloned cattle was also lower than that of age matched controls $(n=4, P<0.01)$. The results showed a remarkable difference in the amount of telomeric DNA between SCNT cloned cattle and normal cattle. In conclusion, the telomeres of cloned animal and their calves are significantly shorter than those of normal cattle. Moreover, the short telomeres in calves could be inherited from their cloned mothers.

\section{EFFECT OF DNA METHYLATION ON SOMATIC CELL NUCLEAR TRANSFER EMBRYO DEVELOPMENT IN RHESUS MONKEY}

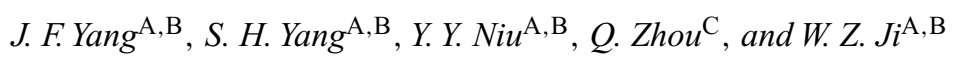

${ }^{A}$ Kunming Institute of Zoology, Chinese Academy of Sciences, Kunming, Yunnan, China; ${ }^{B}$ Kunming Primate Research Center, Chinese Academy of Sciences, Kunming, Yunnan, China; ${ }^{\mathrm{C}}$ Institute of Zoology, Chinese Academy of Sciences, Beijing, China

Up to now, no primate animals have been successfully cloned with somatic cell nuclear transfer (SCNT) and little is known about molecular events occurring in SCNT embryos. DNA methylation reprogramming is likely to have a crucial role in establishing nuclear totipotency in normal development and in cloned animals. Epigenetic characteristics of donor cell nuclei and their epigenetic reprogramming in oocyte cytoplasm have been supposed as major factors influencing the development of SCNT embryos. In Experiment 1, on donor cells used in a previous SCNT at our laboratory, global DNA methylation and histone 3 lysine 9 acetylation (H3K9ac) of three cell lines (S11, S1-04, and S1-03) derived from ear skin were examined after serum starvation by immunofluorescence with monoclonal antibody to 5-methyl cytosine (Oncogene, Science, Inc., Cambridge, MA, USA) and anti-acetyl-Histone H3 (Lys 9) (Upstate Jingmei Biotech, Ltd., Shenzhen, China). In the results, two cells lines, S11 and S1-04, supporting higher blastocyst development (about 20\%) than that (7.8\%) of S1-03, showed a higher level of H3K9ac than the S1-03 cell line. Global DNA methylation levels in the three cell lines were decreased after serum starvation, but no obvious correlation between the level and SCNT embryo developmental potential was found among the three cell lines. In Experiment 2, on SCNT and IVF embryos, global DNA methylation reprogramming during pre-implantation development was investigated with immunofluorescence and laser scanning microscopy techniques. In IVF embryos, active demethylation of paternal genome occurred soon after fertilization; subsequently, passive demethylation resulted in remarkably reduced global methylation level at the 8-cell stage and the morula stage. Thereafter, genomewide remethylation started at the late morula stage and an asymmetric methylation pattern was formed in blastocysts, with higher methylated trophectoderm than inner cell mass (ICM). Compared with IVF embryos, most SCNT 2-cell embryos and ICM in blastocysts showed higher methylation levels, and the asymmetric methylation pattern was not as evident as that in IVF blastocysts. Some SCNT 8-cell embryos showed higher methylation, but others were slightly stained, even lower than IVF embryos. In conclusion, the higher global H3K9 acetylation level of donor cells may benefit chromatin remolding and development of SCNT embryos. Abnormal methylation reprogramming in most SCNT embryos, especially in ICM of blastocysts, may be one main obstacle for primate cloning, although relatively high blastocyst development rate was obtained. DNA methylation reprogramming in rhesus monkey pre-implantation embryos, on the whole, was as conservative as that reported in other mammals.

\section{COMPARISON OF DEVELOPMENTAL ABILITY AMONG CLONED EMBRYOS WITH VARIOUS INDIVIDUAL RECIPIENT CYTOPLASMS IN BOVINE}

\author{
J. G. Zhao, X. Y. Yang, H. F. Liu, H. Li, S. Z. Huang, and Y. T. Zeng \\ Institute of Medical Genetics, Shanghai Jiao Tong University, Shanghai, China
}

Faithful reprogramming ensures the proper activation of genes during embryonic development of the somatic cell nuclear transfer (NT) in bovine. It is unambiguous that all these remodeling factors are presented in the oocyte cytoplasm (Du et al. 2002 Mol. Reprod. Dev. 63, 183-191). It will 
be interesting to determine if the recipient cytoplasms derived from individuals have different development ability and reprogramming competence during NT. Oocytes recovered by Ovum pickup from five Holstein heifers at 14 months of age were used as recipient cytoplasms. Cultured granulosa cells of the same origin were used as donor cells. Oocytes were enucleated at $20 \mathrm{~h}$ post-maturation and a single donor cell was transferred into the perivitelline space of a recipient oocyte. After fusion and activation, the reconstructed embryos were cultured in B2 medium (Laboratoire CCD, Paris, France) on a monolayer of Vero cells for 7 days. The oocyte number, development ability, and NT efficiency of recipient cytoplasm derived from each individual were compared (Table 1). Differences among individuals were verified using a chi-square test, SAS 6.12 version (SAS Institute, Cary, NC, USA). There were significant differences of survival after fusion and the rate of development to the blastocyst stage for embryos reconstructed with recipient cytoplasm from five different individual heifers $(P<0.05)$. However, maturation rate, fusion rate and cleavage rate of embryos reconstructed with recipient cytoplasm from five different individual heifers presented no significant differences $(P>0.05)$. Reconstructed embryos with recipient cytoplasm from one heifer ( 03025$)$ showed a lower survival after fusion $(61 \%$ vs. $80 \%, 86 \%, 77 \%, 91 \%)$ but a higher ability to develop to blastocyst stage $(61 \%$ vs. $24 \%, 31 \%, 52 \%, 31 \%)$ than the embryos from the other four heifers. The current study showed that recipient cytoplasm from various individuals may present great differences in developmental ability in nuclear transfer. This may result from different compatibility between nucleus and mitochondria or the content of maternal RNA as well as proteins in the oocyte. Further studies are needed to elucidate the genetic factors that affect the reprogramming in nuclear transfer.

Table 1. Nuclear transfer efficiency with various individual recipient cytoplasms

\begin{tabular}{lccccccc}
\hline Individual & $\begin{array}{c}\text { Oocytes, } \\
n(\text { batch) }\end{array}$ & $\begin{array}{c}\text { Maturation, } \\
n(\%)\end{array}$ & $\begin{array}{c}\text { Karyoplast-cytoplast } \\
\text { complexes, } n\end{array}$ & $\begin{array}{c}\text { Fused, } \\
n(\%)\end{array}$ & $\begin{array}{c}\text { Viable post- } \\
\text { fusion, } n(\%)\end{array}$ & $\begin{array}{c}\text { Cleaved Day 2, } \\
n(\%)\end{array}$ & $\begin{array}{c}\text { Blastocyst } \\
\text { Day 7, } n(\%)\end{array}$ \\
\hline 02099 & $60(14)$ & $55(92)^{\mathrm{a}}$ & 51 & $30^{\mathrm{a}}$ & $24(80)^{\mathrm{ab}}$ & $17(71)^{\mathrm{a}}$ & $4(24)^{\mathrm{a}}$ \\
03025 & $133(16)$ & $113(85)^{\mathrm{a}}$ & 95 & $49^{\mathrm{a}}$ & $30(61)^{\mathrm{b}}$ & $18(60)^{\mathrm{b}}$ & $11(61)^{\mathrm{b}}$ \\
03052 & $203(19)$ & $162(80)^{\mathrm{a}}$ & 148 & $94^{\mathrm{a}}$ & $81(86)^{\mathrm{a}}$ & $51(63)^{\mathrm{a}}$ & $16(31)^{\mathrm{ac}}$ \\
\hline
\end{tabular}

${ }^{\mathrm{a}-\mathrm{c}}$ Values with the same superscript within a column are not significantly different $(P>0.05)$.

\title{
Cryopreservation/Cryobiology
}

\section{IN VITRO SURVIVAL OF PORCINE BLASTOCYSTS VITRIFIED USING THE CRYOLOGIC VITRIFICATION METHOD}

\author{
L. Beebe, S. McIlfatrick, R. Ashman, and M. Nottle
}

Department of Obstetrics and Gynaecology, Research Centre for Reproductive Health, The University of Adelaide, Adelaide, SA, Australia

Porcine embryo cryopreservation is an important technology for the storage and transport of valuable genetic material. With many of the current vitrification and storage systems, such as the open pulled straws and microdrops, there is direct contact between the medium containing the embryos and the liquid nitrogen. This represents a possible contamination risk. One system with which there is no direct contact between the embryos and liquid nitrogen during the vitrification process is the Cryologic Vitrification System (CVM; Lindemans et al. Reprod. Fertil. Dev. 16, 174) which uses solid surface vitrification. Microdrops of vitrification medium containing the embryos are placed in contact with a metal block that has been precooled by partial submersion in liquid nitrogen, resulting in very rapid cooling rates. Blastocysts were collected surgically on day 5 of pregnancy from mature sows, and the embryos were randomly divided into two groups; each group was then vitrified and warmed with either of two previously published protocols except that the CVM replaced the open pulled straws plunged into liquid nitrogen in both protocols. The first method (OPS/CVM) was based on the open pulled straw method (Cuello et al. Theriogenology 61, 843-850), and used DMSO and ethylene glycol as cryoprotectants and TCM-199 as the basic medium. The second method (EG/CVM) used HEPES-buffered NCSU23 as the basic medium; the blastocysts were centrifuged prior to vitrification in ethylene glycol and polyvinylpyrrolidone (PVP) and the zona pellucida was removed immediately after warming (Cameron et al. Theriogenology 61, 1533-1543). Embryos were then cultured in NCSU23 $+10 \%$ fetal bovine serum for $48 \mathrm{~h}$ at $38.5^{\circ} \mathrm{C}$ in an humidified atmosphere of $5 \% \mathrm{CO}_{2}, 5 \% \mathrm{O}_{2}$, and $90 \% \mathrm{~N}_{2}$. Embryos that had reformed the blastocoel and continued to expand were considered to have survived. These were stained with Hoechst 33342 and the nuclei counted using fluorescence microscopy. There was no difference between the OPS/CVM or EG/CVM methods in either the survival rates $(27 / 29 ; 93 \%$, and 24/27; 89\%, respectively) or the number of cells (mean \pm SEM; $109 \pm 6$ and $112 \pm 6$, respectively). The survival rates are comparable to previously published rates using these two methods and open pulled straws. These data suggest that the CVM can successfully replace the open pulled straws in these two protocols. However, transfer of vitrified and warmed embryos into recipients would be needed to confirm the viability of the surviving embryos.

\section{VITRIFICATION OF IMMATURE AND IN VITRO-MATURED HORSE OOCYTES}

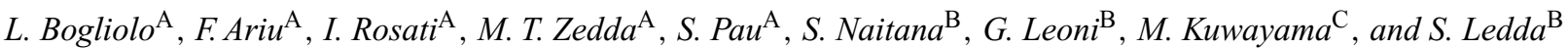

${ }^{A}$ Obstetrics Section of the Institute of General Pathology, Pathological Anatomy and Veterinary Obstetrics-Surgery Clinic, University of Sassari, Sassari, Italy; ${ }^{\mathrm{B}}$ Department of Animal Biology, University of Sassari, Sassari, Italy; ${ }^{\mathrm{C}}$ Kato Ladies' Clinic, Tokyo, Japan

Few attempts have been carried out to cryopreserve equine oocytes, and an effective cryopreservation protocol is not defined yet. Studies were conducted to compare the viability of immature and in vitro-matured horse oocytes vitrified by the minimal volume cooling (MVC) cryotop 
vitrification method (Kuwayama et al. 2005 Reprod. BioMed. Online 11, 300-308). Oocytes were recovered from slaughterhouse ovaries and divided, on the basis of the morphology of cumulus cells, into cumulus-expanded (CE) and cumulus-compacted (CC) oocytes. Groups of CC and CE oocytes were vitrified immediately after recovery [germinal vesicle (GV) stage] or matured in vitro (IVM) and cryopreserved at the MII stage as follows: oocytes were incubated $30 \mathrm{~min}$ in TCM-199 + 20\% FCS $+10 \%$ ethylene glycol (EG) $+10 \%$ DMSO, followed by $20 \mathrm{~min}$ in TCM-199 + $20 \%$ $\mathrm{FCS}+20 \% \mathrm{EG}+20 \% \mathrm{DMSO}+0.25 \mathrm{M}$ sucrose, loaded in cryotops $(2 \mu \mathrm{L})$, and plunged into liquid nitrogen. Warming was performed at $38.5^{\circ} \mathrm{C}$ by washing the oocytes in TCM-199+20\% FCS with decreasing sucrose concentrations $(1.25 \mathrm{M}, 0.62 \mathrm{M}, 0.31 \mathrm{M})$. After warming oocytes cryopreserved at the GV stage were matured in vitro for $24 \mathrm{~h}(\mathrm{CE})$ or $36 \mathrm{~h}(\mathrm{CC})$ in TCM-199 $+10 \% \mathrm{FCS}+\mathrm{FSH}$, LH each at $(0.1 \mathrm{UI} / \mathrm{mL})+$ cysteamine, fixed, and stained with glycerol-Hoechst 33342 to assess nuclear maturation. Oocytes vitrified at the MII stage were in vitro cultured for $2 \mathrm{~h}$ to evaluate their morphological survival on the basis of the presence of an intact zona pellucida and membrane. Nonvitrified oocytes undergoing the same maturation protocol were used as controls. Results (Table 1) indicated that the survival rate of oocytes vitrified at the GV stage, after IVM, was similar between CE and CC oocytes $(43.6 \%$ vs $42.6 \%)$. Significantly $(P<0.01)$ higher numbers of vitrified CE MII oocytes $(52.9 \%)$ survived, compared to CC (34.8\%), after 2 -h culture. The percentages of viable MII oocytes from CE and CC GV vitrified oocytes were $43.6 \%$ and $40.9 \%$ respectively and were comparable to those from vitrified MII oocytes (CE, 52.9\%; CC, 34.8\%) and control oocytes (CE, 56.4\%; CC, 53.3\%). In conclusion, the results of this study showed that vitrification by the MCV Cryotop method of horse oocytes at either the GV or the MII stage allows a similar number of viable mature oocytes to be recovered.

Table 1. Maturation and survival rates of immature and mature equine oocytes vitrified by the MCV Cryotop method

\begin{tabular}{lccc}
\hline GROUP/n & \% MII $(n)$ & $\begin{array}{c}\text { \% Viable post-thawing } \\
\text { and post-culture }(n)\end{array}$ & \% Viable to MII $(n)$ \\
\hline Immature & & & \\
CE/110 & $43.6(48)$ & $43.6(48)$ & $43.6(48)^{\mathrm{ab}}$ \\
CC/122 & $40.9(50)$ & $42.6(52)$ & $40.9(50)^{\mathrm{ab}}$ \\
Mature & & & \\
CE/117 & $55.5(65)$ & $52.9(62)$ & $52.9(62)^{\mathrm{a}}$ \\
CC/135 & $51.8(70)$ & $34.8(47)$ & $34.8(47)^{\mathrm{b}}$ \\
Control & & & $56.4(44)^{\mathrm{a}}$ \\
CE/78 & $56.4(44)$ & & $53.3(48)^{\mathrm{a}}$ \\
CC/90 & $53.3(48)$ & & \\
\hline
\end{tabular}

${ }^{a b}$ Values with different superscripts within a column differ significantly $(P<0.01 ;$ chi-square test $)$.

\title{
84 EVALUATION OF BULL SEMEN AT 1 H VS. 48 H OF POST-FROZEN STABILIZATION TIME
}

\author{
G. M. Brogliatti, G. Larraburu, R. Cavia, and M. E. Carini
}

Centro Genetico Bovino, Eolia SA, Libertad 6910, Marcos Paz, Buenos Aires, Argentina

The process of cryopreservation of bull semen in liquid nitrogen at $-196^{\circ} \mathrm{C}$ is usually carried out after 3 to $6 \mathrm{~h}$ of refrigeration at $4^{\circ} \mathrm{C}$ post-collection. To guarantee the quality of the final product, the frozen straws are evaluated after cryopreservation. The seminal samples are usually stabilized during $48 \mathrm{~h}$ before being analyzed (Hafez, Reproduction and Artificial Insemination in Animals, 1989); this would retard the possible commercialization. The objective of the present study was to determine motility parameters and viability of semen doses stabilized by $1 \mathrm{~h}$ or more than $48 \mathrm{~h}$ in liquid nitrogen at $-196^{\circ} \mathrm{C}$. A total of 122 ejaculated from 23 different adult bulls (Angus, Brangus, Braford, and Hereford) were evaluated in an artificial insemination center between January and April 2005. The semen was diluted in a semi-defined semen extender (Andromed, Minitub, Germany) and frozen in an automatic freezer (Digicool, IMV, France). Parameters of velocity average path (VAP, $\mu \mathrm{m} / \mathrm{s})$, velocity straight line (VSL, $\mu \mathrm{m} / \mathrm{s}$ ), amplitude lateral head (ALH, $\mu \mathrm{m}$ ), linearity (LIN, \%), percentage of rapid cells (RAPID, \%), and viability (VIA, \%) were determined by Computer Assisted Semen Analysis (CASA, HTM-ceros 12.1, Berkeley, CA, USA). The obtained results were analyzed statistically with T Student and are summarized in Table 1 . The results indicate that there is no difference in the velocity of the spermatozoa evaluated $1 \mathrm{~h}$ or $48 \mathrm{~h}$ post-frozen. There is no difference in VAP, VSL, movement of amplitude lateral head (ALH), or linearity (LIN). The percentage of viable spermatozoa was not affected in either group. Statistical analysis indicates that there is no difference $(P>0.05)$ in any of the evaluated parameters. The results demonstrate that spermatic motility and viability of frozen bull semen could be evaluated before $48 \mathrm{~h}$ post-frozen. This allows reduction of the time between freezing and evaluation and immediate availability of the bull straws.

Table 1. Parameters of motility and viability at $1 \mathrm{~h}$ vs. $48 \mathrm{~h}$ of post-frozen stabilization time

\begin{tabular}{lcccccc}
\hline Evaluation (time) & VAP $(\mu \mathrm{m} / \mathrm{s})$ & VSL $(\mu \mathrm{m} / \mathrm{s})$ & ALH $(\mu \mathrm{m})$ & LIN (\%) & Rapid (\%) & VIA (\%) \\
\hline $1 \mathrm{~h}$ & $100.9 \pm 4.6$ & $77.7 \pm 4.3$ & $7.5 \pm 0.3$ & $45.3 \pm 1.8$ & $44.3 \pm 1.1$ & $96.3 \pm 0.3$ \\
$48 \mathrm{~h}$ & $99.8 \pm 4.6$ & $76.9 \pm 4.3$ & $7.4 \pm 0.3$ & $45.5 \pm 1.8$ & $42.8 \pm 1.3$ & $96.1 \pm 0.3$ \\
\hline
\end{tabular}




\title{
85 VITRIFICATION IN VIVO OF LARGE EQUINE EMBRYOS AFTER VITRIFICATION OR CULTURE
}

\author{
L. F. Campos-Chillon, T. J. Cox, G. E. Seidel Jr, and E. M. Carnevale \\ Colorado State University, Fort Collins, CO 80521, USA
}

Cryopreservation of large $(>300 \mu \mathrm{m})$ equine embryos has been unsatisfactory using slow-cooling or vitrification techniques. The objective of the present experiments was to compare three methods for vitrification of large embryos using modified vitrification protocols for equine (EldridgePanuska et al. 2005 Theriogenology 63, 1308-1319) and bovine (2003 J. Anim. Sci. 81 Suppl. 1, 143) embryos. For Method 1, embryos ( $n=14)$ 350-550 or 550-750 $\mu \mathrm{m}$ were exposed, respectively to, VS1 [1.4 M glycerol (G) in mPBS] for $5 \mathrm{~min}$ or 7 min, moved to VS2 [1.4 M G, 3.6 M ethylene glycol (EG) in mPBS] for $5 \mathrm{~min}$ or $7 \mathrm{~min}$, and then transferred to VS3 (3.4 M G, 4.6 M EG in mPBS) for 40 or $60 \mathrm{~s}$. In Method 2, embryos ( $n=13)$ $350-550$ or $550-750 \mu \mathrm{m}$ were exposed, respectively, to VS1 for 10 or $14 \mathrm{~min}$, VS2 for 10 or $14 \mathrm{~min}$ and VS3 for 60 or $90 \mathrm{~s}$. Straws $(0.25 \mathrm{~mL})$ for Methods 1 and 2 were loaded with two columns of DS ( $0.5 \mathrm{M}$ galactose in $\mathrm{mPBS})$ and a small column of VS 3 containing the embryo. Straws were heat-sealed and deposited in a goblet suspended in liquid N2 and containing vapor for 1 min and then plunged. Straws were warmed in air $\left(24^{\circ} \mathrm{C}\right)$ for $10 \mathrm{sec}$ and then in water at $20^{\circ} \mathrm{C}$ for $10 \mathrm{sec}$. Straws were shaken to mix the columns; after $3 \mathrm{~min}$, embryos were expelled and re-hydrated in two additional solutions containing 0.3 and $0.15 \mathrm{M}$ galactose for $3 \mathrm{~min}$ each. For Method 3, embryos $(n=17) 300-750 \mu \mathrm{m}$ were placed in $1.5 \mathrm{M}$ EG in $\mathrm{mPBS}$ for $5 \mathrm{~min}$; $3 \mathrm{M} \mathrm{EG}$ in mPBS for $10 \mathrm{~min} ; 5 \mathrm{M} \mathrm{EG}$ in $\mathrm{mPBS}$ for $5 \mathrm{~min}$ and $7 \mathrm{M} \mathrm{EG}, 0.5 \mathrm{M}$ galactose, $18 \% \mathrm{w} / \mathrm{v}$ Ficoll 70 in $\mathrm{mPBS}$ for less than a minute. The droplet containing an embryo was loaded into a $0.25 \mathrm{~mL}$ straw that was preloaded with two columns of DS and followed by a small column of DS. Straws were heat-sealed and plunged vertically, sealed end fist, into liquid nitrogen covering the embryo, then the remainder of the straw was slowly immersed. Straws were warmed in air $\left(24^{\circ} \mathrm{C}\right)$ for $10 \mathrm{~s}$ and then in water at $37^{\circ} \mathrm{C}$ for $10 \mathrm{~s}$. Straws were shaken to mix the columns; after $3 \mathrm{~min}$ at $37^{\circ} \mathrm{C}$, embryos were rehydrated as in Methods 1 and 2. Embryos were transferred nonsurgically to recipients $5 \mathrm{~d}$ after ovulation, and pregnancy diagnoses were performed 5 to $9 \mathrm{~d}$ after transfers. No embryonic vesicles were observed for embryos vitrified with Methods 1 and 2 . The pregnancy rate for embryos vitrified with Method 3 was 35\% (6/17) overall, and 55\% (6/11) for embryos between 300-400 $\mu \mathrm{m}$. No pregnancies resulted from embryos $>400 \mu \mathrm{m}$. More studies are needed to optimize methodologies for dehydration, equilibration, and warming of large equine embryos.

\section{MOTILITY AND VIABILITY OF BULL SEMEN DURING 24 HOURS OF STORAGE AT $4^{\circ} \mathrm{C}$}

\author{
M. E. Carini, R. Cavia, G. Larraburu, and G. M. Brogliatti
}

Centro Genetico Bovino, Eolia SA, Libertad 6910, Marcos Paz, Buenos Aires, Argentina

Currently, cryopreservation process of fresh bull semen is carried out between 3 and 6 hours of refrigeration at $4{ }^{\circ} \mathrm{C}$ post-collection (Hafez, 1989). However, it is sometimes difficult when the cryopreservation process is not available at the site of collection. The objective of this study was to determine seminal motility and viability in samples maintained at $4^{\circ} \mathrm{C}$ during 24 hours. A total of 98 ejaculates from 23 adult bulls (Angus, Brangus, Braford and Hereford) were collected and diluted in a semi-defined semen extender (Andromed, Minitüb, Tiefenbach, Germany) and stored at $4^{\circ} \mathrm{C}$. Parameters of velocity average path (VAP, $\mu \mathrm{m} / \mathrm{s}$ ), velocity straight line (VSL, $\left.\mu \mathrm{m} / \mathrm{s}\right)$, amplitude lateral head (ALH, $\left.\mu \mathrm{m}\right)$, linearity (LIN, \%), percentage of rapid cells (RAPID, \%), percentage of slow and static cells (SL/ST, \%), and viability (VIA, \%) were determined by Computer Assisted Semen Analysis (CASA, HTM-ceros 12.1, Berkeley, CA, USA). Measurements were done at 6, 9, 12, and $24 \mathrm{~h}$. The obtained results were analyzed statistically with one-way ANOVA and Dunnet Multiple Comparison Test and are summarized in Table 1. There were no significant differences $(P>0.05)$ in the VAP, RAPID, or SL/ST during $24 \mathrm{~h}$ of storage at $4^{\circ} \mathrm{C}$. Measurements were significantly different $(P<0.01)$ for VSL and VIA at $24 \mathrm{~h}$. Measurements of ALH were increased from $12 \mathrm{~h}(P<0.01)$ and consequently, LIN decreased at the same time $(P<0.01)$. These results suggest that there are no differences in velocity, except in VSL at the end of the storage time. The type of movement of the spermatozoa change, because ALH increases and the trajectory loses linearity. A decrease in viability suggests that from $24 \mathrm{~h}$ of storage, the membrane of the spermatozoa becomes more susceptible. More research needs to be done to evaluate the competence of this time-storage semen in the artificial insemination trial.

Table 1. Parameters of motility and viability of semen maintained at $4^{\circ} \mathrm{C}$ during $24 \mathrm{~h}$

\begin{tabular}{lccccccc}
\hline Hours & $\operatorname{VAP}(\mu \mathrm{m} / \mathrm{s})$ & $\operatorname{VSL}(\mu \mathrm{m} / \mathrm{s})$ & $\operatorname{ALH}(\mu \mathrm{m})$ & $\operatorname{LIN}(\%)$ & $\operatorname{Rapid}(\%)$ & SL/ST $(\%)$ & VIA (\%) \\
\hline 6 & $123.5 \pm 15.3^{\mathrm{a}}$ & $90.3 \pm 10.7^{\mathrm{a}}$ & $8.5 \pm 0.9^{\mathrm{a}}$ & $42.6 \pm 3.7^{\mathrm{a}}$ & $66.8 \pm 15.2^{\mathrm{a}}$ & $26.8 \pm 14.3^{\mathrm{a}}$ & $96.1 \pm 2.2^{\mathrm{a}}$ \\
9 & $123.8 \pm 15.6^{\mathrm{a}}$ & $90.4 \pm 10.9^{\mathrm{a}}$ & $8.6 \pm 0.9^{\mathrm{a}}$ & $42.4 \pm 3.6^{\mathrm{a}}$ & $66.8 \pm 14.8^{\mathrm{a}}$ & $27.1 \pm 13.9^{\mathrm{a}}$ & $96.2 \pm 2.1^{\mathrm{a}}$ \\
12 & $123.7 \pm 14.8^{\mathrm{a}}$ & $89.6 \pm 9.7^{\mathrm{a}}$ & $8.8 \pm 1^{\mathrm{b}}$ & $41.6 \pm 3.8^{\mathrm{b}}$ & $67.1 \pm 14.8^{\mathrm{a}}$ & $26.6 \pm 13.7^{\mathrm{a}}$ & $96.0 \pm 2.2^{\mathrm{a}}$ \\
24 & $122.6 \pm 15.9^{\mathrm{a}}$ & $86.2 \pm 9.1 \mathrm{~b}$ & $9.1 \pm 1^{\mathrm{b}}$ & $39.5 \pm 3.6^{\mathrm{b}}$ & $67.2 \pm 15.0^{\mathrm{a}}$ & $26.6 \pm 14.0^{\mathrm{a}}$ & $95.1 \pm 3.1^{\mathrm{b}}$ \\
\hline
\end{tabular}

${ }^{\mathrm{ab}}$ Values with different superscripts differ significantly $(P<0.01)$. 


\title{
87 THE EFFECT OF LOW TEMPERATURE ON THE DETECTABILITY/VISUALIZATION OF THE MEIOTIC SPINDLE IN MII MOUSE OOCYTES
}

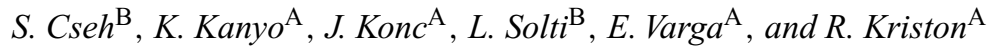 \\ ${ }^{\mathrm{A}}$ Infertility and IVF Center of Buda, Saint Janos Hospital, Budapest, Hungary; ${ }^{\mathrm{B}}$ Department of Animal Reproduction, Faculty of Veterinary \\ Science, Budapest, Hungary
}

The polscope uses novel electrooptical hardware and digital processing to image macromolecular structures in cells on the basis of their birefringence (Sato et al. $1975 \mathrm{~J}$. Cell Biol. 76, 501-517). Imaging of the spindle in living oocytes with the polscope is based on birefringence of an inherent physical property of the microtubules. De-polymerization of microtubules arrests meiosis and induces abnormal meiosis generating chromosomally abnormal oocytes which are not capable of being fertilized and developing. In this study we examined whether spindles in living mouse oocytes can be safely imaged/examined by the polscope and investigated the influence of sub-optimal temperature on spindle detection/visualization. For oocyte collection, 6- to 8-week-old CB6F1 female mice were superovulated with PMSG (10 IU, i.p.; Sigma, USA) and 46 to $48 \mathrm{~h}$ later they were injected with hCG (10 IU, i.p.; Sigma). Oocytes were collected 20 to $23 \mathrm{~h}$ after hCG treatment in MOPS buffered medium (G-MOPS ${ }^{\mathrm{TM}}$; Vitrolife, Sweden AB, Kungsbacka, Sweden) supplemented with human serum albumin (HAS ${ }^{\mathrm{TM}}$; Vitrolife). Cumulus cells were removed from the zonae pellucidae by exposure to a solution of $40 \mathrm{IU} / \mathrm{mL}$ hyaluronidase $\left(\mathrm{HYASE}^{\mathrm{TM}}\right.$; Vitrolife). For spindle examination, each oocyte was placed in a $5-\mu \mathrm{L}$ drop of G-MOPS medium, covered with oil (Ovoil; Vitrolife), and examined in a Delta T.C.O. dish with a specially coated glass bottom (WillcoDish, Willco Wells, Amsterdam, The Netherlands). Oocytes were imaged by a Nikon Diaphot microscope with a video camera, objective lens, and controller, combined with a computerized imaging analysis system (CRI, Great Britain). For evaluation of the effect of sub-optimal temperature on the visualization/detection of the spindle, mouse oocytes were cultured for a short period of time at sub optimal temperatures $\left(18\right.$ to $-20^{\circ} \mathrm{C} / 25 \mathrm{~min}$ and -10 to $-15^{\circ} \mathrm{C} / 2 \mathrm{~min}$ ). After that, the oocytes were immediately checked for presence and condition of the meiotic spindle and re-checked after 30-min culture at $37^{\circ} \mathrm{C}$ with $6 \% \mathrm{CO}_{2}, 5 \% \mathrm{O}_{2}, 89 \% \mathrm{~N}_{2}$ and maximal humidity in air. There was no difference in the detect ability of the spindle in mouse oocytes with or without polar body (PB+: 21/24, 87\%; PB-: 29/32, 90\%). We found that the treatment with sub-optimal temperature has an effect to the spindle visualization. Immediately after treatment, the spindle could be detected in $80 \%$ of the oocytes $(16 / 20)$. However, after 30 -min culture, the meiotic spindle was detectable in only $25 \%$ of the treated oocytes $(5 / 20 ; P<0.05)$. Sub optimal temperature at first increased the birefringence of the meiotic spindle for a short period of time; then the spindle became completely undetectable. The increased sensitivity of the meiotic spindle to low temperature is one of the biggest obstacles to the oocyte cryopreservation. Our results provide further evidence that the spindle in mammalian oocytes is very sensitive to temperature fluctuations. The increase in the birefringence was the first sign that the structure of microtubules and other cytoskeletal factors forming the spindle had changed. Investigations are underway to learn more about the capability of the meiotic spindle to become reorganized.

\section{IS STEREOMICROSCOPY AN EFFICIENT METHOD FOR EVALUATING THE VITRIFIED PORCINE EMBRYO QUALITY?}

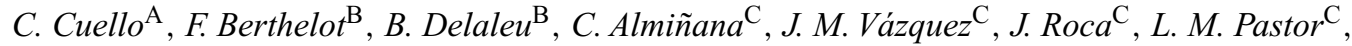 \\ E. A. Martínez ${ }^{\mathrm{C}}$, and F. Martinat Botté ${ }^{\mathrm{B}}$ \\ ${ }^{\text {A }}$ Swedish University of Agricultural Sciences (SLU), Uppsala, Sweden; ${ }^{\mathrm{B}}$ INRA, Nouzilly, France; \\ ${ }^{\mathrm{C}}$ Department of Medicine and Animal Surgery, University of Murcia, Murcia, Spain
}

The development of the open pulled straw vitrification has provided excellent results of in vitro porcine embryo development. Embryo quality evaluation after vitrification has been traditionally focused on morphological assessment performed by stereomicroscopy. The objective of this experiment was to evaluate the efficiency of the stereomicroscopic evaluation of vitrified-warmed (V) porcine blastocysts. Unhatched blastocysts were obtained after slaughter from Large-White gilts $(n=9)$. Blastocysts $(n=75)$ were vitrified and warmed using the protocol described by Cuello et al. (2004 Theriogenology 61,353-361). After warming, vitrified blastocysts were cultured for $24 \mathrm{~h}$. Then blastocysts were morphologically assessed for their progression and morphology by stereomicroscopy. Blastocysts that reformed their blastocoelic cavities showing an excellent appearance were considered viable. Some of the viable blastocysts kept their zonae pellucidae (V viable expanded blastocysts) and others hatched during the in vitro culture (V viable hatched blastocysts). The remaining blastocysts were classified as degenerated embryos. A group of fresh blastocysts was not vitrified and cultured in vitro for $24 \mathrm{~h}$ (control group). All of the control blastocysts were considered viable by stereomicroscopy. Some fresh, $\mathrm{V}$ viable expanded, $\mathrm{V}$ viable hatched, and $\mathrm{V}$ degenerated blastocysts $(n=13, n=19, n=9$, and $n=9$, respectively) were processed for ultrastructural study by light and transmission electron microscopy or stained with Hoechst-33342 and TUNEL for cell death evaluation $(n=16, n=21, n=11$, and $n=6$, respectively). All $\mathrm{V}$ hatched blastocysts showed ultrastructure similar to that of control hatched blastocysts. However, $26.3 \%$ of the $\mathrm{V}$ viable expanded blastocysts revealed important ultrastructural alterations in comparison with control expanded blastocysts. These observations suggest that stereomicroscopic evaluation was not efficient enough for $\mathrm{V}$ expanded blastocysts. As expected, degenerated blastocysts showed ultrastructural disintegration and disorganization. Hatched $\mathrm{V}$ blastocysts did not differ $(P<0.05)$ from control hatched blastocysts with regard to the total cell number and ratio of death cells ( $173 \pm 4.8$ vs. $202.1 \pm 10.9$ and $2.8 \pm 0.5 \%$ vs. $1.9 \pm 0.3 \%$, respectively). However, $\mathrm{V}$ expanded blastocysts a had higher $(P<0.01)$ cell death level $(4.3 \pm 3.4 \%)$ than that observed in the control expanded blastocysts $(1.1 \pm 0.3 \%)$. Degenerated embryos showed the lowest $(P<0.01)$ total cell number $(45.7 \pm 4.0)$. The $66.7 \%$ of the degenerated blastocysts exhibited wide TUNEL-labeled areas, and the remaining $33.3 \%$ showed TUNEL label over $19.4 \pm 6.3 \%$ of the cells. In conclusion, the hatching rate assessed by stereomicroscopy is a more efficient parameter than assessing the in vitro viability (ratio of blastocysts that reformed their blastocoelic cavities after warming) for estimating the quality of $\mathrm{V}$ blastocysts. 


\title{
89 OPEN PULLED STRAW VITRIFICATION FOR IN VITRO-PRODUCED BUFFALO (BUBALUS BUBALIS) EMBRYOS
}

\author{
A. De Rosa, R. Di Palo, L. Attanasio, E. Monaco, G. Campanile, and B. Gasparrini \\ Discizia, Federico II University, Naples, Italy
}

\begin{abstract}
The aim of this study was to compare the efficiency of different combinations of cryoprotectants for vitrification of IVP buffalo (Bubalus bubalis) embryos at different developmental stages by the open pulled straw (OPS) method. In method A, we evaluated the vitrification and warming solutions previously used to vitrify buffalo embryos in French straws (Gasparrini et al. 2001 Theriogenology 55, 307). Embryos were equilibrated in 1.4 M glycerol for $5 \mathrm{~min}$ before being placed into $1.4 \mathrm{M}$ glycerol $+3.6 \mathrm{M}$ ethylene glycol (EG) for $5 \mathrm{~min}$. Then, embryos were transferred into $3.4 \mathrm{M}$ glycerol + 4.6 M EG for $25 \mathrm{~s}$ and loaded into the OPSs. For warming, OPSs were briefly immersed in a $0.5 \mathrm{M}$ sucrose solution; the embryos were exposed to $0.25 \mathrm{M}$ sucrose for $5 \mathrm{~min}$ before transfer to SOF medium for culture. In Method B, we examined the vitrification and warming solutions previously used for OPS vitrification of cattle embryos (Vajta et al. $1998 \mathrm{Mol}$. Reprod. Dev. 51, 53-58). Buffalo embryos were equilibrated in 7.5\% $\mathrm{EG}+7.5 \%$ dimethyl sulfoxide (DMSO) for 3 min before transfer into $16.5 \% \mathrm{EG}+16.5 \%$ DMSO and $0.5 \mathrm{M}$ sucrose. After $25 \mathrm{~s}$, they were loaded into the OPSs. For warming, embryos were recovered in a $0.25 \mathrm{M}$ sucrose solution and transferred into a $0.15 \mathrm{M}$ sucrose solution for $5 \mathrm{~min}$ before being placed in SOF medium. A total of 293 IVP buffalo embryos (eight replicates) were vitrified at Day 7 of culture (Day $0=$ in vitro fertilization) Embryos were vitrified at the following developmental stages: early blastocyst (eBL, $n=26$ and 34 with methods A and B, respectively), blastocyst (B1, $n=31$ and 35 for Methods A and B, respectively), expanded blastocyst (XB1, $n=29$ and 38 for Methods A and $\mathrm{B}$, respectively), and hatched blastocyst ( $\mathrm{HBl}, n=46$ and 54 for Methods A and B, respectively). Embryo survival rate was determined as the percentage of vitrified-warmed embryos undergoing further development during a 24-h in vitro culture period. Differences between methods were analyzed by ANOVA following arcsine transformation of data. The overall embryo survival rate recorded at $24 \mathrm{~h}$ was not significantly different between Methods A and B (70\% vs. $62 \%$, respectively). Specifically, no differences were observed in embryos vitrified at the eBL ( $70 \%$ vs. $73 \%$, A and B, respectively), Bl (69\% vs. $70 \%$, A and B, respectively), and $\mathrm{HBl}$ ( $46 \%$ vs. $36 \%$, A and B, respectively) stages. In contrast, a significantly higher survival rate was recorded for XBl-stage embryos vitrified-warmed by Method A as compared to Method B ( $90 \%$ vs. $53 \%$, respectively; $P<0.01)$. In Method A, survival rate of $\mathrm{XBl}$ was significantly higher than that of $\mathrm{HBl}(P<0.05)$, but it was not different from that of $\mathrm{eBl}$ and $\mathrm{Bl}$. Within Method $\mathrm{B}$, the survival efficiency was similar for eBL, $\mathrm{BL}$, and $\mathrm{XB1}$, whereas survival rate of $\mathrm{HB} 1$ was significantly lower $(P<0.05)$. In conclusion, although overall embryo survival in vitro was similar between methods, the combination of cryoprotectants used in Method A seemed more suitable for vitrification of IVP buffalo embryos at the XB1 stage.
\end{abstract}

\section{SUCCESSFUL VITRIFICATION OF PARTHENOGENETIC PORCINE BLASTOCYSTS PRODUCED FROM DELIPATED IN VITRO-MATURED OOCYTES}

\author{
Y. Du ${ }^{\mathrm{A}, \mathrm{B}}, P . M$. Kragh $^{\mathrm{A}, \mathrm{B}}, X . Z_{\text {Zhang }}^{\mathrm{C}}, H$. Yang $^{\mathrm{C}}$, G. Vajta $^{\mathrm{A}}$, and L. Bolund $^{\mathrm{A}}$ \\ ${ }^{\mathrm{A}}$ Danish Institute of Agricultural Sciences, Tjele, Denmark; ${ }^{\mathrm{B}}$ Institute of Human Genetics, Aarhus University, Aarhus, Denmark; \\ ${ }^{\mathrm{C}}$ Beijing Genomics Institute, Beijing, China
}

Cryopreservation of cloned porcine embryos may improve the output of somatic cell cloning considerably by alleviating logistic problems. However, the high lipid content of porcine oocytes and embryos compromises their cryotolerance. Recently a noninvasive procedure was published for delipation of porcine embryos with centrifugation but without subsequent micromanipulation (Esaki et al. 2004 Biol. Reprod. 71, 432-436). This method was applied to our present work with few modifications to compare the cryosurvival of porcine blastocysts produced from delipated vs. intact oocytes with parthenogenetic activation. In four replicates, a total of 192 oocytes were used for the experiments. After in vitro maturation for $44 \mathrm{~h}$, cumulus cells were removed and oocytes were randomly distributed into two groups. For delipation, oocytes were digested with $1 \mathrm{mg} / \mathrm{mL}$ pronase in the presence of 50\% cattle serum (CS) for 3 min, and washed in HEPES-buffered TCM-199 medium supplemented with 20\% CS. Subsequently, 40-50 oocytes were centrifuged $(12000 \mathrm{~g}, 20 \mathrm{~min})$ in HEPES-buffered TCM-199 medium supplemented with $2 \% \mathrm{CS}, 3 \mathrm{mg} / \mathrm{mL}$ polyvinyl alcohol and $7.5 \mu \mathrm{g} / \mathrm{mL}$ cytochalasin B (CB). Zonae pellucidae of both centrifuged and intact oocytes were removed completely by further digestion in $2 \mathrm{mg} / \mathrm{mL}$ pronase solution. For activation, a single direct current of $85 \mathrm{kV} / \mathrm{cm}$ for $80 \mu \mathrm{s}$ was applied to both groups, followed by 4-h treatment with $5 \mu \mathrm{g} / \mathrm{mL} \mathrm{CB}$ and $10 \mu \mathrm{g} / \mathrm{mL}$ cycloheximide. All embryos were then cultured in modified NCSU37 medium. Day 7 blastocysts were vitrified and warmed by using the Cryotop technique (Kuwayama et al. 2005 RBM Online $11,300-308$ ) at $38.5^{\circ} \mathrm{C}$. Survival of vitrified blastocysts was determined according to re-expansion rates after $24 \mathrm{~h}$ recovery in culture medium supplemented with $10 \% \mathrm{CS}$. Cell numbers of reexpanded blastocysts from both groups were determined after Hoechst staining. Results were compared by ANOVA. Partial zona digestion and centrifugation resulted in successful delipation in $173 / 192(90 \%)$ of oocytes. The development to blastocysts was not different between delipated and intact oocytes ( $28 \pm 7 \%$ vs. $28 \pm 5 \%$, respectively; $P>0.05$ ). However, survival rates of blastocysts derived from delipated oocytes were significantly higher than those developed from intact oocytes ( $85 \pm 6 \%$ vs. $32 \pm 7 \%$, respectively; $P<0.01)$. There was no difference in average cell number of re-expanded blastocysts derived from either delipated or intact oocytes ( $36 \pm 7$ vs. $38 \pm 9$, respectively; $P>0.05$ ). Our results prove that the simple delipation technique does not hamper the in vitro developmental competence of activated porcine oocytes, and improves the cryosurvival of the derived blastocysts without significant loss in cell number. Future investigations are required to prove the value of the method in an analogue system with blastocysts produced by somatic cell nuclear transfer. 


\title{
91 PRELIMINARY RESULTS OF SURGICAL EQUINE EMBRYO TRANSFER AFTER OPEN PULLED STRAW VITRIFICATION
}

\author{
G. Duchamp, F. Guignot, J. Grizelj, M. Vidament, and P. Mermillod \\ INRA Unité de Physiologie de la Reproduction et des Comportements, 37380 Nouzillly, France
}

\begin{abstract}
In equine species, embryo cryopreservation is not as widely developed as in some other species. Slow freezing has been applied to equine embryos but with relatively low success rates. This higher sensitivity to conventional freezing procedures may be explained by the presence of a capsule surrounding the equine embryo that may impair cryoprotectant penetration. Recently, good in vitro embryo survival rate was obtained after open pulled straw (OPS) vitrification (Moussa et al. 2005 Theriogenology 64, 1619-1632). The aim of the present study was to evaluate in vivo survival of vitrified embryos five days after surgical transfer into Welsh pony mares. Morulae (M), early blastocysts (EB), and blastocysts (B) ranging from 140 to $320 \mu \mathrm{m}$ in diameter were collected $(n=20)$ in a Ringer lactate solution on Day 6.75 after ovulation. Before vitrification, embryos were assessed morphologically and their size was measured (McKinnon and Squires 1988 J. Am. Vet. Med. Assoc. 192, 401-406). Then, embryos were vitrified using the OPS method described by Berthelot et al. (2001 Reprod. Nutr. Dev. 41, 267-272). Briefly, embryos were washed twice in HEMES-TCM$199+20 \%$ newborn calf serum (NBCS) for 1 min, equilibrated in HEPES-TCM-199+20\% NBCS with 7.5\% dimethyl sulfoxide (DMSO) $+7.5 \%$ ethylene glycol (EG) for $3 \mathrm{~min}$, and then with $18 \% \mathrm{DMSO}+18 \% \mathrm{EG}+0.4 \mathrm{M}$ sucrose for $45 \mathrm{~s}$. One embryo was then loaded per straw. For transfer, four straws were quickly thawed ( $5 \mathrm{~s}$ in air) and the narrow end of the straw containing the embryo was immersed in HEPES-TCM-199 $+20 \%$ $\mathrm{NBCS}+\mathrm{PBS}+0.2 \mathrm{M}$ sucrose. Five to 8 min after thawing, four embryos were surgically transferred into the cranial portion of the uterine horn in each of five pony mare recipients. Five days after transfer, embryos recovered by transcervical flushing of the uterus were classified as viable if morphology was normal, no dark inner cells were present, the capsule was intact, and the diameter was at least $1000 \mu \mathrm{m}$. The results are shown in the table. One recipient of vitrified embryos had an endometritis and no embryo was recovered. From the four other recipients, nine embryos were recovered out of $16(56 \%)$ transferred, seven of which were viable (44\%). The results of the present preliminary study demonstrating survival of equine embryos transferred after OPS vitrification is very encouraging. However, the results should be confirmed by birth of foals after transfer of OPS-vitrified embryos to recipients.
\end{abstract}

Table

\begin{tabular}{lllll}
\hline $\begin{array}{l}\text { No./stage of embryos } \\
\text { transferred }\end{array}$ & Size $(\mu)$ & $\begin{array}{c}\text { No. embryos } \\
\text { received }\end{array}$ & Size $(\mu)$ & $\begin{array}{c}\text { No. viable } \\
\text { embryos }\end{array}$ \\
\hline $1 \mathrm{M}, 3 \mathrm{~EB}$ & $140-190$ & 3 & $2200,1000,320$ & 2 \\
$3 \mathrm{~EB}, 1 \mathrm{~B}$ & $150-260$ & 0 & - & 0 \\
$4 \mathrm{~B}$ & $200-320$ & 3 & $6200,5700,333$ & 2 \\
$3 \mathrm{~EB}, 1 \mathrm{~B}$ & $145-225$ & 3 & $7600,5000,1100$ & 3 \\
$1 \mathrm{M}, 1 \mathrm{~EB}, 2 \mathrm{~B}$ & $140-270$ & 0 & Endometritis & 0 \\
Total =20 & $140-320$ & $9(56 \%)$ & - & $7(44 \%)$ \\
\end{tabular}

\section{EVALUATION OF BOAR SPERM FUNCTIONALITY AFTER A CUSHIONED CENTRIFUGATION TECHNIQUE}

\author{
J. Gadea ${ }^{\mathrm{A}}$, S. Martínez-Miró ${ }^{\mathrm{A}}$, G. Decuadro-Hansen ${ }^{\mathrm{C}}$, and C. Matás ${ }^{\mathrm{A}}$
}

${ }^{A}$ Department of Physiology, Faculty of Veterinary Science, University of Murcia, Murcia, Spain; ${ }^{\mathrm{B}}$ Department of Animal Production, Faculty of Veterinary Science, University of Murcia, Murcia, Spain; ${ }^{\mathrm{C}}$ IMV Technologies, L'Aigle, France

Separation of sperm from seminal plasma is required in most semen freezing procedures. Semen is typically subjected to centrifugation to concentrate sperm into a pellet and allow removal of the seminal plasma prior to dilution in freezing extender. Centrifugation is a relatively effective method to recover sperm, however, the process also causes considerable sperm damage. The use of a dense, inert, and isotonic solution as a cushion in the bottom of the centrifuge tube allows a greater centrifugation speed to be applied and results in greater sperm recovery. The aim of the present work was to evaluate the effects of this cushioned centrifugation technique on in vitro sperm viability and functionality. Sperm-rich fractions from 16 fertile boars were diluted and cooled to $15^{\circ} \mathrm{C}$; then subsamples were centrifuged by one of two different techniques. A standard method (SM), $800 \mathrm{~g}$ for $10 \mathrm{~min}$ in 50-mL tubes (Westendorf et al. 1975 Dtsch. Tierärztl. Wschr. 82, 261-267) and a cushioned method (CM), $1000 \mathrm{~g}$ for $20 \mathrm{~min}$ using $45 \mathrm{~mL}$ of diluted semen on $5 \mathrm{~mL}$ of an isotonic iodixanol solution $(60 \% \mathrm{w} / \mathrm{v}$ gradient) were performed. Sperm samples were stained with merocyanine 540 (M540) and Yo-Pro 1 (Harrison et al. 1996 Mol. Rep. Dev. 45, 378-391) to detect changes in lipid packing disorder of the plasma membrane. Another set of sperm samples was incubated in the presence of $(0.7 \mu \mathrm{M}) 2^{\prime}, 7^{\prime}$-dichlorodihydrofluorescein diacetate (Gadea et al. 2005 J. Androl. 26, 396-404) to estimate production of reactive oxygen species (ROS). A final set of sperm samples was stained with peanut aggultinin-fluorscein isothiocyanate (PNA-FITC) and propidium iodide to evaluate the acrosome reaction. All of these parameters were evaluated by flow cytometry before and after centrifugation. ANOVA analysis revealed that centrifugation altered lipid packing disorder and viability. Raw semen (RS) had a larger number of viable low lipid disorder sperm than centrifuged semen ( $\mathrm{RS}=86.9 \mathrm{a}$ vs. $\mathrm{SM}=81.64 \mathrm{~b}$ vs. $\mathrm{CM}=80.6 \mathrm{~b}, P<0.01)$ and a decreased number of dead sperm cells $(\mathrm{RS}=9.5 \mathrm{a}$ vs. $\mathrm{SM}=15.0 \mathrm{~b}$ vs. $\mathrm{CM}=16.3 \mathrm{~b}, P<0.01)$. However, the cushioned and standard centrifugation methods yielded similar results for all the parameters measured. No significant differences were found for generation of ROS or in the number of sperm exhibiting the acrosome reaction. In conclusion, compared to the standard centrifugation method, this simple cushioned modification is a more efficient means of processing boar semen for freezing because significantly less sample losses are detected; also, it provides similar levels of sperm viability and functionality, and consequently a higher number of doses per ejaculation can be produced. 


\title{
93 CALCIUM REMOVAL INCREASES THE PROTECTIVE EFFECTS OF $\beta$-CYCLODEXTRIN PLUS CHOLESTEROL ON PORCINE SPERM DURING COLD SHOCK
}

\author{
H. Galantino-Homer, W. Zeng, S. Megee, M. Modelski, and I. Dobrinski \\ University of Pennsylvania School of Veterinary Medicine, Kennett Square, PA, USA
}

Porcine sperm are extremely sensitive to the damaging effects of cold shock and cryopreservation. Cholesterol-binding molecules, such as 2hydroxypropyl- $\beta$-cyclodextrin (HBCD), improve post-thaw and post-cooling porcine sperm viability when added to an egg yolk-based extender, but also enhance sperm capacitation in other species. Depending upon the environmental cholesterol content, HBCD can act either as a cholesterol shuttle or sink to increase or decrease, respectively, sperm plasma membrane cholesterol content. Increasing the sperm cholesterol to phospholipid ratio reduces cold shock sensitivity whereas decreasing the ratio initiates the process of sperm capacitation. An increase in protein tyrosine phosphorylation correlates with sperm capacitation and has been shown to be dependent upon the presence of extracellular calcium. Sperm intracellular calcium also increases during cold shock. The objective of this study was to determine the combined effects of extracellular calcium and membrane cholesterol manipulation on porcine sperm viability and protein tyrosine phosphorylation following cold shock $\left(10^{\circ} \mathrm{C}\right.$ for $\left.10 \mathrm{~min}\right)$. Viability was assessed using CFDA/propidium iodide staining. Protein tyrosine phosphorylation, previously shown to correlate with porcine sperm capacitation, was evaluated via antiphosphotyrosine (clone 4G10) immunoblots. We report here that following cold shock, porcine sperm incubated in defined medium containing both $0.8 \mathrm{mM}$ HBCD and $0.5 \mathrm{mM}$ cholesterol 3-sulfate $(\mathrm{ChS})$ incubated in the absence of added extracellular calcium and the presence of $6 \mathrm{mM}$ EGTA have significantly improved viability $(90.5 \pm 6.3 \%, n=3)$ when compared with cold-shocked sperm incubated in either the same medium with calcium ( $46.1 \pm 3.8 \%$ ), without $\mathrm{HBCD}$ or $\mathrm{ChS}(26.5 \pm 7.4 \%$ with calcium; $46.5 \pm 13.1 \%$ without calcium), or with $\mathrm{HBCD}$ alone $(17.0 \pm 7.4 \%$ with calcium, $36.8 \pm 7.5 \%$ without calcium). As we have found previously, treatment with $0.8 \mathrm{mM} \mathrm{HBCD}$ plus $0.5 \mathrm{mM}$ ChS completely inhibited the increase in protein tyrosine phosphorylation induced by the cold shock treatment. Although protein tyrosine phosphorylation correlates with porcine sperm capacitation, the ability of cold shock treatment to induce the same phosphorylation pattern indicates that other processes or pathways may contribute to its appearance. Removing extracellular calcium consistently decreased, but did not completely eliminate, the protein tyrosine phosphorylation induced by cold shock. These results indicate that cold shock-induced protein tyrosine phosphorylation is not dependent upon, but can be modulated by, extracellular calcium. The combined effects of calcium, HBCD and ChS on viability suggest that porcine sperm viability following cold shock is best maintained by removing extracellular calcium and increasing membrane cholesterol content via the cholesterol shuttle activity of HBCD.

This work was supported by grants from PA Dept. Ag. (ME 443291) and the NIH (5-K08-HD041430).

\section{ADDITION OF REDUCED GLUTATHIONE TO THAWING MEDIUM IMPROVED THE SPERM MOTILITY AND REDUCED ROS GENERATION IN FROZEN OVINE AND CAPRINE SPERMATOZOA}

\author{
J. C. Gardón $^{\mathrm{A}, \mathrm{B}}$, J. A. Rodriquez ${ }^{\mathrm{A}}$, and J. Gadea ${ }^{\mathrm{A}}$

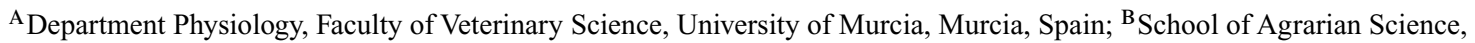 \\ National University of Lomas de Zamora, Lomas de Zamora, Argentina
}

The processes of cooling and freezing/thawing produce physical and chemical stress on the sperm membrane, and this stress is associated with oxidative stress and reactive oxygen species (ROS) generation that further reduce sperm viability and fertilizing ability. It is known that the process of freezing is associated with a significant reduction of the intracellular reduced glutathione (GSH) content. The aim of these experiments was to investigate the effects of addition of GSH to thawing extenders on motility parameters and ROS generation in frozen-thawed ovine and caprine spermatozoa. Frozen spermatozoa from eight rams (Ovis aries) and eight bucks (Capra hircus) (generously provided by Ovigen, Zamora, Spain) were thawed in a water bath at $37^{\circ} \mathrm{C}$ for $30 \mathrm{~s}$ and resuspended in sperm-TALP medium (Parrish et al. 1986 Theriogenology 25, 591-600) without (control) and with addition of $1 \mathrm{mM}$ or $5 \mathrm{mM} \mathrm{GSH}$. After $30 \mathrm{~min}$ of incubation at $37^{\circ} \mathrm{C}$, sperm motility was evaluated using a computer-assisted sperm analysis (CASA) system (SCA, Microptic, Barcelona, Spain). The recorded parameters of motility were: $\%$ total, $\%$ progressive, curvilinear velocity, straight-line velocity, average path velocity, linearity of the curvilinear trajectory, straightness, amplitude of lateral head displacement, wobble of the curvilinear trajectory and beat cross frequency. Another set of sperm samples was incubated in the presence of $(0.7 \mu \mathrm{M}) 2^{\prime}, 7^{\prime}$-dichlorodihydrofluorescein diacetate (Gadea et al. $2005 \mathrm{~J}$. Androl. 26, 396-404) to estimate production of ROS by flow cytometry. Data were analyzed by two-way ANOVA, considering the specific sperm treatment (GSH addition) and the males as the main variables. In ram frozen spermatozoa, all of the motility parameters were significantly improved when the medium was supplemented with GSH $(P<0.01)$ with even better results when $5 \mathrm{mM}$ GSH was used. As an example, progresive motility increased from $31.16 \%$ (control) to 39.17 and $43.97 \%$, respectively, for 1 and $5 \mathrm{mM} \mathrm{GSH}$. Despite of the male effect detected $(P<0.01)$, all eight rams studied presented a similar pattern (interaction $P>0.05$ ). The generation of ROS was significantly reduced when GSH was added (6.23a for control vs. $5.32 \mathrm{~b}$ and $3.85 \mathrm{c}$ for 1 and $5 \mathrm{mM}$, respectively; $P<0.01$ ). In buck frozen spermatozoa, \% motility and progressive motility were significantly higher in GSH groups than in the control $(P<0.01)$, with no differences between 1 and $5 \mathrm{mM}$ GSH. However, for the other motility parameters, the differences were not significant, which probably could be related to differences in the pattern shown by different animals (interaction of buck by treatment $P<0.05$ ). ROS generation was significantly reduced when GSH was added (7.50a for control vs. $4.32 \mathrm{~b}$ and $2.70 \mathrm{~b}$ for 1 and $5 \mathrm{mM}$, respectively; $P<0.01$ ). The addition of GSH to the thawing medium had a positive influence on the parameters studied in both species, increasing the motility patterns and reducing the ROS generation. In conclusion, we can assume that the addition of reduced glutathione to the thawing medium exerts a protective effect on spermatozoa functionality. 


\title{
95 EFFECT OF CRYOPROTECTANT ADDITION AND REMOVAL AT $4^{\circ} \mathrm{C}$ ON MOTILITY AND PLASMA MEMBRANE INTEGRITY OF BOVINE CAUDAL EPDIDYMAL SPERMATOZOA
}

\author{
C. Guerrero ${ }^{\mathrm{A}}$, S. Leibo ${ }^{\mathrm{C}}$, D. Paccamonti ${ }^{\mathrm{B}}$, B. Eilts $^{\mathrm{B}}$, K. Bondioli $^{\mathrm{A}}$, and R. Godke $\mathrm{A}$
}

${ }^{A}$ Department of Animal Sciences, Louisiana State University Agricultural Center, Baton Rouge, LA 70803, USA; ${ }^{B}$ Department of Veterinary Clinical Sciences, School of Veterinary Medicine, Louisiana State University, Baton Rouge, LA 70803, USA;

${ }^{\mathrm{C}}$ Department of Biological Sciences, University of New Orleans, New Orleans, LA 70803, USA

Cryopreservation of spermatozoa harvested from the epididymides would be a means of salvaging germplasm from genetically valuable males that die unexpectedly from injury, disease, or poaching. It is well known that the addition of cryoprotective agents (CPAs) is essential for sperm survival following the freezing process. However, CPAs can cause loss in sperm viability due to osmotic damage or chemical toxicity. The objective of this study was to determine the effects of single-step addition and/or removal of glycerol (GLY) or ethylene glycol (EG) on motility and plasma membrane integrity of bovine epididymal spermatozoa. Paired testes were obtained from mature bulls $(n=10)$ at a local abattoir and transported to the laboratory at $25-28^{\circ} \mathrm{C}$ within $4-6 \mathrm{~h}$ post-mortem. Epididymal spermatozoa were harvested by multiple incisions from the caudae epididymides of each bull, pooled, and washed in Brackett-Oliphant medium by centrifugation for $5 \mathrm{~min}$ at $500 \mathrm{~g}$. Pellets were resuspended in egg yolk-Tris-glucose-citric acid monohydrate medium (EYT-GC) at a concentration of $120 \times 10^{6} \mathrm{cells} / \mathrm{mL}$ and cooled to $4^{\circ} \mathrm{C}$ at a rate of $0.1{ }^{\circ} \mathrm{C} / \mathrm{min}$. Specimens were allocated to each of five treatment groups: control (no CPA), 7\% GLY, and 14\% GLY, 7\% EG, 14\% EG. Then, replicate samples were diluted 1:1 in EYT-GC medium containing twice the final desired concentration of CPA. After being exposed for $10 \mathrm{~min}$, each sample was diluted directly into EYT-GC at $4^{\circ} \mathrm{C}$. Motility was assessed by means of a computer assisted semen analysis system and plasma membrane integrity was determined by SYBR 14 and propidium iodide staining followed by fluorescence microscopy. Differences among treatments were analyzed using one way ANOVA $(P<0.05)$. The results (Table 1) show that maximum survival, as assessed by measurements of motility and membrane integrity, was achieved with spermatozoa exposed to 7\% EG. Almost identical intermediate levels of survival were observed with spermatozoa exposed to $7 \%$ GLY or $14 \%$ EG. The lowest survival was observed for spermatozoa exposed to $14 \%$ GLY. The results indicate that the use of EG as a cryoprotectant may minimize toxicity and osmotic damage to fresh bovine epididymal spermatozoa. Its efficacy as a CPA is currently being determined.

Table 1. Sperm motility and membrane integrity (mean \pm SEM) after addition of CPA to epididymal sperm

\begin{tabular}{lccc}
\hline Treatment & Overall motility (\%) & Progressive motility (\%) & Plasma membrane integrity (\%) \\
\hline Control & $73 \pm 1.6^{\mathrm{a}}$ & $54 \pm 3.2^{\mathrm{a}}$ & $78 \pm 2.5^{\mathrm{a}}$ \\
GLY 7\% & $49 \pm 3.8^{\mathrm{b}}$ & $30 \pm 3.8^{\mathrm{b}}$ & $54 \pm 3.0^{\mathrm{b}}$ \\
GLY 14\% & $23 \pm 4.5^{\mathrm{c}}$ & $10 \pm 3.9^{\mathrm{c}}$ & $29 \pm 3.0^{\mathrm{c}}$ \\
EG 7\% & $64 \pm 2.8^{\mathrm{a}}$ & $46 \pm 3.9^{\mathrm{a}}$ & $70 \pm 2.5^{\mathrm{a}}$ \\
EG 14\% & $48 \pm 4.4^{\mathrm{b}}$ & $31 \pm 4.0^{\mathrm{b}}$ & $48 \pm 3.7^{\mathrm{b}}$ \\
\hline
\end{tabular}

${ }^{\mathrm{a}-\mathrm{c}}$ Different superscripts within columns indicate significant differences $(P<0.05)$.

\section{VITRIFICATION OF PORCINE OOCYTES - A NEW APPROACH TO GAMETE CRYOPRESERVATION}

\author{
M. K. Gupta, H. Y. Lee, S. J. Uhm, and H. T. Lee \\ Animal Resources Research Center, BORC/IBST, Konkuk University, 1 Hwayang-dong, Gwangjin-Gu, Seoul, South Korea
}

Cryopreservation of porcine oocytes has a special importance in reproductive biotechnologies owing to the high lipid content of the oocytes. The objective of this study was to investigate the efficiency of a new and simple method of oocyte vitrification for cryopreservation of porcine oocytes. Abattoir ovary-derived prepubertal porcine oocytes at germinal vesicle (GV) or metaphase II (MII) stage were exposed for 10-15 min to vitrification solution I that contained 4\% (v/v) ethylene glycol (EG) in either TCM-199 supplemented with 20\% FCS or phosphate-buffered NCSU23 medium supplemented with $0.4 \%$ BSA. Oocytes were then washed thrice in EG $(35 \%$; v/v)-based vitrification solution II containing sucrose $(13.7 \%$; w/v) and polyvinyl pyrrolidone $(5 \% ; \mathrm{w} / \mathrm{v})$ and placed in groups of $25-50$ oocytes per $1-2 \mu \mathrm{L}$ droplet onto aluminum foil kept directly over the liquid nitrogen $\left(\mathrm{LN}_{2}\right)$. After visually observed vitreous formation, they were transferred to cryovials and directly plunged into $\mathrm{LN}_{2}$ for storage. Thawing was performed in sucrose- or EG-based media. After warming, the viability was assessed by fluorescein diacetate (FDA) staining and in vitro maturation (IVM) of GV-stage oocytes was determined based on cumulus expansion and polar body extrusion. In addition, the developmental capacity of oocytes was assessed by in vitro fertilization (IVF). Effects of pretreatment with cytochalasin B (CB) on the post-thaw viability, nuclear maturation, and developmental capacity were also investigated. The results demonstrated that the rate of oocytes having normal morphology was more than $90 \%$ in all groups. Viability based on esterase enzyme activity and oolemma integrity, as determined by FDA staining, ranged from $80 \%$ (without $\mathrm{CB}$ ) to $86 \%$ (with $\mathrm{CB}$ ) for MII-stage oocytes and $79 \%$ (without CB) to $90 \%$ (with CB) for GV-stage oocytes. The IVM rate of pelleted GV-stage oocytes reached $70 \%$ based on cumulus expansion and $55 \%$ based on polar body extrusion. Sucrose-based thawing media was found to be superior to EG-based media. Although the vitrification method resulted in a high survival rate based on the morphological appearance, FDA staining, and IVM rate, developmental rates of pelleted oocytes were found to be compromised. However, we believe that there is a scope for improving the developmental rates with further modifications in the method. 


\title{
97 DIFFERENCES IN SPERM CHROMATIN STRUCTURE AMONG GOOD AND BAD BOAR SPERM FREEZERS
}

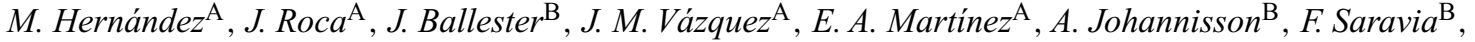 \\ and H. Rodríguez-Martínez ${ }^{\mathrm{B}}$

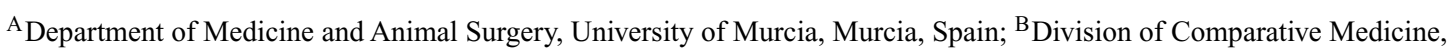 \\ Swedish University of Agricultural Sciences (SLU), Uppsala, Sweden
}

\begin{abstract}
Inter- and intra-boar differences in sperm freezability are observed independent of the sperm quality before freezing, the breed, or the genetic line. This study aimed to determine whether boars with different post-thaw sperm quality also show differences in sperm DNA integrity. Sperm-rich fractions ( 3 to 10 ejaculates per boar) from 19 fertile mature boars were extended in Beltsville thawing solution (BTS) and cooled to $17^{\circ} \mathrm{C}$ for $16 \mathrm{~h}$. Then, samples were centrifuged at $2400 \mathrm{~g}$ for $3 \mathrm{~min}$, extended in freezing extender (lactose/egg yolk/glycerol/Equex STM; Nova Chemical Sales, Inc., Scituate, MA, USA) to a final concentration of $1 \times 10^{9}$ spermatozoa/mL, dispensed into $0.5 \mathrm{~mL}$ straws, and frozen in a programmable cell freezer at a rate of $-20^{\circ} \mathrm{C} \mathrm{min}$. Thawing was carried out in a water bath at $37^{\circ} \mathrm{C}$ for $20 \mathrm{~s}$. Frozen-thawed spermatozoa were evaluated for progressive sperm motility (PSM) using a computer-assisted sperm analysis (CASA) system, and sperm viability (PMI) using flow cytometry. All data generated were used for a multivariate pattern analysis (PATN; CSIRO, Canberra, Australia) which objectively classified all boars into two groups, categorized as $\operatorname{good}(n=10 ;>60 \%$ PSM and PMI) or bad $(n=9 ;<40 \%$ PSM and PMI) based on their sperm freezability. Post-thaw sperm quality was consistent for different ejaculates within boars $(P<0.05)$. The DNA-integrity of frozen-thawed spermatozoa was evaluated according to the sperm chromatin structure assay (SCSA; Evenson et al. 1980 Science 210, 1131-1133). All SCSA variables (X mean, DNA fragmentation index (DFI), and the standard deviation of the DFI), were significantly higher for bad freezers $(P<0.001)$. The percentage of spermatozoa with abnormal chromatin structure ranged from 1.06 to $3.42 \%$ for good and 3.06 to $6.04 \%$ for bad freezers. Although these differences exist between good and bad sperm freezers, and can only to some extent be the product of cryopreservation, the levels of affected spermatozoa can not explain the differences on post-thaw sperm survival seen in the two categories of sires.
\end{abstract}

This work was supported by CICYT, AGL05-0471 (Spain), SLF and Formas (Sweden).

\section{SUCCESSFUL PREGNANCIES FOLLOWING TRANSFER OF VITRIFIED PORCINE EMBRYOS DERIVED FROM IN VITRO-MATURED OOCYTES}

\author{
K. Hiruma, H. Ueda, H. Saito, C. Tanaka, N. Maeda, M. Kurome, R. Tomii, S. Ueno, and H. Nagashima
}

Laboratory of Developmental Engineering, Department of Life Science, School of Agriculture, Meiji University, Kawasaki, Kanagawa, Japan

To date only in vivo-produced embryos have successfully produced live piglets after cryopreservation. In this study, we aimed to produce piglets from vitrified embryos derived from in vitro matured (IVM) oocytes. Cumulus-oocyte complexes collected from ovaries obtained at a local slaughterhouse were matured for 44 to $45 \mathrm{~h}$ in NCSU23 MEDIUM supplemented with $0.6 \mathrm{mM}$ cysteine, $10 \mathrm{ng} / \mathrm{mL}$ epidermal growth factor, $10 \%$ (v/v) porcine follicular fluid, $75 \mu \mathrm{g} / \mathrm{mL}$ potassium penicillin $\mathrm{G}, 50 \mu \mathrm{g} / \mathrm{mL}$ streptomycin sulfate, and $10 \mathrm{IU} / \mathrm{mL}$ eCG/ hCG. These IVM oocytes were either activated for parthenogenesis or in vitro-fertilized (IVF). For IVF, oocytes were incubated with $5 \times 10^{6} / \mathrm{mL}$ of cryopreserved epididymal sperm in PGM-tac medium (Yoshioka et al. 2003 Biol. Reprod. 69, 2092-2099) for $20 \mathrm{~h}$. Embryos were treated for removal of cytoplasmic lipid droplets (delipation; Nagashima et al. 1995 Nature 374, 416) at the 4- to 8-cell stages, around 50 to $54 \mathrm{~h}$ after activation or insemination. After culture in NCSU23 for $15 \mathrm{~h}$, they were vitrified by the minimum volume cooling (MVC) method. Embryos were equilibrated with equilibration solution containing $7.5 \%(\mathrm{v} / \mathrm{v})$ ethylene glycol (EG), 7.5\% (v/v) dimethylsulfoxide (DMSO), and 20\% (v/v) calf serum for 4 min, followed by exposure to vitrification solution containing $15 \%$ EG, $15 \%$ DMSO, $0.5 \mathrm{M}$ sucrose, and $20 \%$ calf serum. Embryos were then loaded onto a Cryotop (Kitazato Supply Co., Tokyo, Japan) and immediately plunged into liquid nitrogen. Vitrified embryos were examined for viability in vitro and in vivo after warming. Their in vitro developmental competence was compared to that of corresponding control (nonvitrified) embryos. Vitrified 4- to 8-cell stage embryos, both parthenogenetic and IVF, showed developmental competence into blastocysts comparable to that of control embryos (parthenogenetic: 46.8\%, 36/77 vs. $51.7 \%, 31 / 60$; IVF: $40.0 \%, 30 / 75$ vs. $44.3 \%, 35 / 79)$. Of four surrogate gilts that received a total of 251 vitrified parthenogenetic embryos, three became pregnant and had 20 fetuses $(8.0 \%, 22$ to 23 days old). Three surrogates gilts that received 267 vitrified IVF embryos all became pregnant. Of those, the one that received 47 embryos was confirmed to have eight fetuses $(17.0 \%, 22$ days old) by autopsy. The other two were examined by ultrasonography at 56 and 95 days of gestation and found to be pregnant. These results suggest that porcine embryos derived from IVM oocytes have a potential to develop into live offspring after delipation and MVC vitrification. 


\title{
99 EFFECT OF CYSTEAMINE ON SURVIVAL OF BOVINE AND OVINE OOCYTES VITRIFIED USING THE MINIMUM VOLUME COOLING (MVC) CRYOTOP METHOD
}

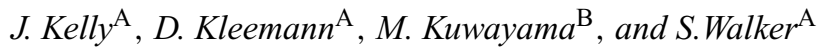 \\ ${ }^{A}$ South Australian Research and Development Institute, Rosedale, Australia; ${ }^{B}$ Kato Ladies' Clinic, Toyko, Japan
}

The addition of cysteamine to maturation (IVM) media increases glutathione (GSH) synthesis in bovine oocytes and improves embryo development and quality (de Matos et al. 1995 Mol. Reprod. Dev. 42, 432-436). This study assesses the effect of adding cysteamine to IVM media on the survival of bovine and ovine oocytes following vitrification using the MVC cryotop method (Kuwayama and Kato 2000 J. Assist. Reprod. Genet. 17, 477 abst.). Abattoir-sourced bovine and ovine cumulus-oocyte complexes (COC) were matured in IVM media with or without $100 \mu \mathrm{M}$ cysteamine for $24 \mathrm{~h}$. After maturation, the COC were partially denuded and a proportion vitrified. Oocytes were equilibrated with $10 \%$ ethylene glycol (EG) and $10 \%$ dimethyl sulfoxide (DMSO) for $30 \mathrm{~s}$ and then exposed to $20 \% \mathrm{EG}, 20 \%$ DMSO, $0.5 \mathrm{M}$ sucrose, and 20\% FCS for $15 \mathrm{~s}$. Oocytes were loaded onto a MVC plate (Cryotop, Kitazato Supply, Tokyo), and plunged into liquid nitrogen. After 5 days, oocytes were thawed directly into $1.25 \mathrm{M}$ sucrose solution at $38.5^{\circ} \mathrm{C}$, followed by stepwise dilution of the cryoprotectants. Ova were subsequently fertilized (Day 0) and cultured in modified SOF. Oocyte survival was assessed by cleavage and development to Day 8 compared with the development of fresh oocytes. Main effects, interactions and individual treatment differences were tested using procedure CATMOD in SAS. Cleavage rate was higher $(P<0.001)$ for fresh oocytes than for vitrified oocytes and it increased $(P<0.05)$ only for fresh ovine oocytes when cysteamine was added to maturation media. Blastocyst development was influenced by a significant $(P<0.001)$ interaction between species and whether or not oocytes were vitrified. This interaction occurred because cysteamine improved blastocyst rate in fresh ovine and vitrified bovine oocytes but not in other treatments. These results show that bovine oocytes (38.3\% blastocyst rate) can be vitrified successfully when maturation occurs in the presence of cysteamine; however, a comparable result did not occur in ovine oocytes $(10.6 \%$ blastocyst rate) despite $>70 \%$ cleavage.

\begin{tabular}{llcccc}
\hline & Fresh/vitrified & Cysteamine IVM & No. oocytes & No. (\%) cleaved & No. (\%) blastocysts \\
\hline Bovine & Fresh & - & 37 & $31(83.8)^{\mathrm{aef}}$ & $17(54.8)^{\mathrm{ae}}$ \\
& & + & 107 & $96(89.7)^{\mathrm{a}}$ & $64(66.7)^{\mathrm{a}}$ \\
& Vitrified & - & 38 & $20(52.6)^{\mathrm{b}}$ & $1(5.0)^{\mathrm{b}}$ \\
& & + & 69 & $47(68.1)^{\mathrm{beg}}$ & $18(38.3)^{\mathrm{ce}}$ \\
Ovine & Fresh & - & 90 & $79(87.8)^{\mathrm{a}}$ & $53(67.1)^{\mathrm{a}}$ \\
& & + & 226 & $216(95.6)^{\mathrm{c}}$ & $174(80.6)^{\mathrm{d}}$ \\
& Vitrified & - & 88 & $64(72.7)^{\mathrm{dfg}}$ & $6(9.4)^{\mathrm{b}}$ \\
& & + & 157 & $113(72.0)^{\mathrm{dfg}}$ & $12(10.6)^{\mathrm{b}}$ \\
\hline
\end{tabular}

${ }^{\mathrm{a}-\mathrm{g}}$ Percentages within columns with a similar superscript are not different.

\section{TIMING OF BLASTOCOELIC CAVITY RE-EXPANSION REPRESENTS A QUALITY INDEX OF IN VITRO PRODUCED OVINE BLASTOCYSTS}

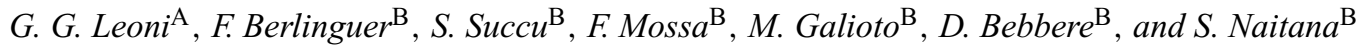 \\ ${ }^{A}$ Deptartment of Physiological, Biochemical and Cellular Sciences, University of Sassari, Sassari, Italy; ${ }^{\mathrm{B}}$ Department of Animal Biology, \\ University of Sassari, Sassari, Italy
}

There is general consensus that the quality of in vitro-produced embryos is inferior to that of their in vivo counterparts and that along with other factors this may be responsible for the poorer cryosurvival and the lower pregnancy rates reported for these embryos. The feasibility to accurately select viable embryos would be valuable for improving pregnancy rates and avoiding futile transfer attempts. The aim of our study was to assess whether in vitro-produced ovine embryo quality could be determined by the timing blastocoelic cavity re-expansion after vitrification, thawing, and in vitro culture. Embryos were produced in vitro from ovine ovaries collected at the local slaughterhouse following a standard protocol developed for ovine oocytes, as previously described (Berlinguer et al. 2005 Reprod. Fertil. Dev. 17, 201). Blastocysts were then vitrified/warmed according to a simple method (Leoni et al. 2002 Cryobiology 45, 204-212) and cultured in vitro in TCM-199 supplemented with 10\% FCS for $72 \mathrm{~h}$ in $5 \%$ $\mathrm{CO}_{2}$ in air at $39^{\circ} \mathrm{C}$. Vitrified embryos were divided into two groups: (A) expanded within $8 \mathrm{~h}$ of in vitro culture after warming; (B) expanded during 8 to $16 \mathrm{~h}$ of in vitro culture after warming. Of the 338 vitrified/warmed embryos, $173(51.1 \%)$ showed a re-expanded blastocoelic cavity after $8 \mathrm{~h}$ of in vitro culture, whereas $58(17.1 \%)$ re-expanded during 8 to $16 \mathrm{~h}$ of in vitro culture. We also analyzed by semiquantitative RT-PCR a panel of genes expressed during pre-implantation embryo development (glucose transporter, HPS-10, e-caderin, poly(A)polimerase, and ubiquitine); our results showed that these genes, apart from ubiquitine which showed no difference in the two groups, were more expressed in Group A blastocysts compared to Group B. Group A blastocysts showed higher hatching rates $(67.6 \%)$ than Group B blastocysts $(43.1 \% ; P<0.01)$. The total cell number calculated for the hatched blastocysts after staining with Hoechst 33342 was significantly higher in Group A $(140.7 \pm 8.3, n=42)$ than Group B $(102.2 \pm 8.4, n=27 ; P<0.01)$. Pregnancy rate (detected by ultrasonography 30 days after embryo transfer) after laparoscopic embryo transfer to 12 synchronized recipient Sarda sheep was 40\% (6/15) in Group A and 13.4\% (2/15) in Group B. The results indicated that timing of blastocoelic cavity re-expansion after vitrification/warming and in vitro culture can be considered as a reliable index of in vitro-produced ovine embryo quality, and in addition, a better prediction for improved survival rate after transfer to synchronized recipients. 


\title{
101 CRYOPRESERVATION OF PORCINE EMBRYOS DERIVED FROM SOMATIC CELL NUCLEAR TRANSFER
}

\author{
R. Li, L. Lai, D. Wax, Y. Hao, Z. Zhong, C. N. Murphy, A. Rieke, and R. S. Prather \\ Department of Animal Sciences, University of Missouri, Columbia, MO 65201, USA
}

In the production of cloned pigs, a large number of nuclear transfer (NT) embryos generally need to be transferred into a single surrogate. Thus, attempts to conduct embryo transfer can be frustrating when either a synchronized surrogate is not available, or enough NT embryos are not produced. This problem would be solved if one could cryopreserve the porcine nuclear transfer embryos. Cryopreservation of porcine embryos has been successful only for in vivo-derived embryos. In vitro-derived porcine embryos are sensitive to chilling, and this sensitivity has been attributed to the lipid droplets in the cytoplasm. In previous reports, the viability of cyropreserved embryos was improved by removal of lipid drops from the cytoplasm. Therefore we designed a procedure to cryopreserve cloned blastocysts by a combination of the open pulled straw (OPS) vitrification method with removal of lipid drops from the oocyte. In vitro-matured MII oocytes were enucleated, and centrifuged ( 10000 rpm, 5 min) to polarize the lipid droplets. This was followed by removal of the polarized lipid droplets and transfer of a donor fetal-derived fibroblast cell into the perivitelline space by micromanipulation. After electrical activation and fusion, the NT embryos were cultured in PZM-3 medium with 4 mg/mL BSA. Day 5 and Day 6 blastocysts (manipulation day was Day 0) were vitrified by equilibration with 25 mM HEPES-buffered TCM-199 containing $10 \%$ ethylene glycol, $10 \%$ DMSO, and 20\% fetal calf serum for $2 \mathrm{~min}$, followed by exposure to $20 \%$ ethylene glycol and $20 \%$ DMSO. Embryos were loaded into an OPS straw and immediately plunged into liquid nitrogen. The process from exposure of embryos to vitrification solution to plunging was $25-30 \mathrm{~s}$ Embryos were thawed by immersing the end of the OPS straw in $0.3 \mathrm{M}$ sucrose in which embryos were kept for 5 min, and then in $0.2 \mathrm{M}$ sucrose for $5 \mathrm{~min}$. Some embryos were cultured in PZM-3 for $12 \mathrm{~h}$ to determine the percentage and cell number of re-expanded blastocysts. The others were transferred to the uterus of a surrogate gilt within $3 \mathrm{~h}$ of thawing. Lipid removal appeared to have no harmful effect on embryo development and cell number of the blastocysts. Interestingly, a higher blastocyst percentage $(28.8 \%, 178 / 619)$ was obtained with NT embryos from which the lipid had been removed as compared to normal NT $(19.6 \%, 44 / 225 ; P<0.01)$. The cell number $(31.2 \pm 7.7)$ of re-expanded blastocysts in the delipation group was comparable with normal NT blastocysts $(33.6 \pm 14.1, P=0.33)$. The survival rate of blastocysts after freezing and thawing was enhanced after delipation (delipation group: $66.7 \%, 14 / 21$; normal NT group: $21.9 \%, 9 / 42 ; P<0.01$ ). Two hundred and fourteen delipatized NT blastocysts were transferred to four surrogates after freezing and thawing. Three of the surrogates showed a delayed estrus cycle and one is still pregnant as confirmed by ultrasound scanning. We show that the combination of the OPS vitrification method with removal of lipid drops of oocyte cytoplasm might be an efficient way to cryopreserve porcine NT blastocysts.

Funding for this project was from the NIH HL51670 and RR018877 and Food for the 21st Century.

\section{DEVELOPMENTAL COMPETENCE OF BUFFALO (BUBALUS BUBALIS) OOCYTES VITRIFIED AT DIFFERENT STAGES OF MATURATION IN VITRO}

\author{
K. Loganathasamy, R. Rajhans, G. SaiKumar, and G. T. Sharma \\ Sharma Reproductive Physiology and ETT Laboratory, P \& C Division, India Veterinary Research Institute, Izatnagar-243122, UP, India
}

Cryopreservation of unfertilized oocytes at very low temperature $\left(-196^{\circ} \mathrm{C}\right)$ is carried out to ensure their continuous availability during different assisted reproductive techniques. However, various problems associated with the freezing of oocytes influence their developmental competence, resulting in suboptimal embryo production. The present study was planned to assess the developmental competence of buffalo oocytes vitrified at different meiotic stages of maturation. Expression profile of developmentally important genes, viz, heat shock protein 70 (HSP 70) and glucose transporter 1 (Glut1), was verified in these vitrified warmed oocytes. Buffalo cumulus-oocyte complexes were collected from slaughterhouse ovaries and divided into six groups: control (no vitrification); $0 \mathrm{~h}$ group (vitrified before maturation), and 6-, 12-, 18-, and 24-h groups [vitrified respectively at $6,12,18$, and $24 \mathrm{~h}$ post-in vitro maturation (IVM)]. Vitrification solution consisted of propylene glycol $(40 \% \mathrm{w} / \mathrm{v})$, and trehalose $(0.25 \mathrm{~mol} / \mathrm{L})$ in PBS + BSA $(4 \% \mathrm{w} / \mathrm{v})$ and vitrification was carried out by directly plunging $0.25-\mathrm{mL}$ French mini-straws into liquid nitrogen. After a minimum storage period of 7 days, the straws were thawed at $37^{\circ} \mathrm{C}$ for $30 \mathrm{sec}$. In all groups, the oocytes completed $24-\mathrm{h}$ of maturation. After $24 \mathrm{~h}$ maturation, a few oocytes from each of the six groups were stained with ethidium bromide to reveal their nuclear status. The remaining oocytes were co-incubated with frozen thawed buffalo semen in fertilization TALP with $6 \mathrm{mg} / \mathrm{mL}$ fatty acid free BSA and $10 \mu \mathrm{g} / \mathrm{mL}$ heparin sodium salt for $18 \mathrm{~h}$. Presumptive zygotes were cultured in mSOF for 8 days. Vitrified warmed oocytes were subjected to total RNA isolation and RT-PCR for detection of mRNA transcripts of HSP 70 and Glut1 genes. Data were analyzed by one-way ANOVA and F-test analysis. Differences of $P<0.05$ were considered significant. The percentage of oocytes recovered from all five vitrification groups varied from 89 to 92 out of which $84-91 \%$ of oocytes were morphologically normal. A higher proportion of nonvitrified control oocytes $(72.8 \% ; 40 / 55)$ reached the metaphase II stage than for the oocytes vitrified at $24(60 \% ; 36 / 60), 18(54.4 \% ; 31 / 57), 12(42.3 \% ; 22 / 52), 6(33.3 \% ; 20 / 60)$, and $0(31.7 \% ; 19 / 60)$ h of IVM. The cleavage rate of nonvitrified control oocytes was higher (36.8\%) than that of oocytes vitrified at $0(1.6 \%), 6(2.0 \%), 12(3.2 \%), 18(5.3 \%)$, and $24(5.2 \%) \mathrm{h}$ of IVM. With regard to subsequent development, 0 - and 6-h oocytes were blocked at 8 cells, whereas in other groups development reached the late morula (4.8\%) and blastocyst (3.5\%) stages, confirming that the stage of maturation at which oocytes are vitrified influenced the nuclear maturation and developmental competence. Total RNA content was $2.24 \pm 0.40 \mathrm{ng} /$ oocyte in the control group and $2.11 \pm 0.22 \mathrm{ng} / \mathrm{oocyte}$ in the group vitrified after $24 \mathrm{~h}$ of IVM. The expression pattern of HSP 70 and Glut 1 was identical in control and vitrified groups, indicating that the vitrification protocol did not alter the expression pattern of these genes. 


\title{
103 GENE EXPRESSION PROFILES OF VITRIFIED MOUSE EMBRYOS DETECTED BY MICROARRAYS
}

\author{
S. Mamo, Sz. Bodo, Z. Polgar, and A. Dinnyes \\ Agricultural Biotechnology Center, Department of Animal Biology, Hungarian Academy of Sciences and Szent Istvan University, \\ Gödöllö, Hungary
}

\begin{abstract}
Very little is known about the effect of vitrification on gene functions after warming. The goals of our study were to examine the transcript variations and identify genes most affected by the treatment. For this, 8-cell-stage embryos were collected from female ICR mice mated with ICR males. The embryos were washed with CZB-HEPES base medium and suspended briefly in equilibrium medium consisting of $4 \%$ ethylene glycol (EG) in base medium at room temperature. Following equilibration, the embryos were vitrified in a 35\% EG, $0.4 \mathrm{M}$ trehalose, $5 \%$ polyvinylpyrrolidone (PVP) solution by means of a solid-surface vitrification (SSV) technique as described earlier (Dinnyes 2000 Biol. Reprod. 63, 513-518). Then 40 embryos each from the control and the vitrified/warmed groups were cultured in CZB medium for $3 \mathrm{~h}$. Total RNAs were extracted from cultured embryos in each group using TRIzol (Invitrogen, Bio-Science, Ltd., Budapest, Hungary), following the manufacturer's instructions. Two rounds of amplification were employed to produce labeled RNA, using low input RNA amplification kit (Agilent Technologies, Kromat, Ltd., Budapest, Hungary) procedures with modifications. Three micrograms of contrasting RNA samples were hybridized on the Agilent Mouse 22K oligonucleotide slides with subsequent analysis of the results. Moreover, as an independent analysis tool, real time PCR was used with eight designed primers. All of the vitrified embryos were recovered after warming with no morphological signs of cryodamage and used for analysis. The two rounds of amplification yielded $15-16 \mu \mathrm{g}$ of cRNA. The analysis of repeated hybridizations by Rosetta luminator software (Agilent) showed 20183 genes and expressed sequence tags (ESTs) that passed the selection criteria and were identified as common signatures in all of the slides. Unsupervised analysis of the gene expression data identified a total of 631 differentially expressed $(P<0.01)$ genes. However, to support the reliability of the results, only those variations above 1.5 fold differences were considered as significant in the final analysis. Therefore, with this stringent criterion 183 genes were differentially expressed $(P<0.01)$, of which 109 were up-regulated and the remainder down-regulated. Although genes have multiple and overlapping functions, most of the differentially expressed genes were functionally classified into various physiological categories. These include stress response (8), apoptosis related (6), metabolism (51), temperature response (4), and transcription regulation (15). Moreover, the independent analysis with real time PCR and unamplified samples verified the results of microarray. Thus, based on confirmation of the results by an independent analysis and support by the previous studies for some of the genes, it is possible to conclude that the expression patterns reflect the true biological image of embryos after vitrification, with most effects on stress- and cell metabolism-related genes.
\end{abstract}

This work was supported by EU FP6 (MEXT-CT-2003-59582), Wellcome Trust Foundation (Grant No. 070246), and National Office of Research and Technology (NKTH) (\#BIO-00017/2002, \#BIO-00086/2002).

\section{COMPARING DIFFERENT LEVELS OF OSMOLALITY OF SUCROSE EXTENDER ON THE VIABILITY OF SPERMATOZOA IN BACTRIAN CAMEL (CAMELUS BACTRIANUS)}

\author{
S. Mosaferi ${ }^{\mathrm{A}, \mathrm{E}}$, A. Niasari-Naslaji ${ }^{\mathrm{A}, \mathrm{B}}$, N. Bahmani $^{\mathrm{A}}$, A. A. Gharahdaghi $^{\mathrm{B}}$, A. Abarghani $^{\mathrm{C}}$, \\ A. Ghanbari ${ }^{\mathrm{C}}$, and A. Gerami ${ }^{\mathrm{D}}$ \\ ${ }^{A}$ Department of Clinical Sciences, Faculty of Veterinary Medicine, University of Tehran, Tehran, Iran; \\ ${ }^{B}$ Animal Science Research Institute, karaj, Tehran, Iran; \\ ${ }^{\mathrm{C}}$ Research Center for Agriculture and Natural Resources, Ministry of Jihad-e-Agriculture, Ardabil, Iran; \\ DStatistical Reserach Center, Tehran, Iran; \\ ${ }^{E}$ Islamic Azad University of Tabriz, Tabriz, East Azarbayejan, Iran
}

\begin{abstract}
Disaccharides have been used as an extender for dromedary camel semen (Bravo et al. 2000 Anim. Reprod. Sci. 62, 173-193). More recently we have investigated the effect of different concentrations of lactose extender on the viability of Bactrian camel spermatozoa (Mosafer et al. 2005 Reprod. Fertil. Dev. 17, 197). Considering the osmolality $(316.1 \pm 1.48 \mathrm{mOsm} / \mathrm{kg}$ ) and $\mathrm{pH}(7.4 \pm 0.03)$ of Bactrian camel semen (Mosaferi et al. 2005 Theriogenology 63, 92-101), the objective of this study was to investigate the effect of osmolality of sucrose extender on the viability of Bactrian camel spermatozoa. Sucrose at the concentrations of $9,10,11,12$, and $13 \%$ with osmolalities of $292,331,356,386$, and $410 \mathrm{mOsm} / \mathrm{kg}$, respectively, were prepared. All extenders contained $20 \%$ egg yolk and antibiotics, with pH adjusted to 6.9. Semen was collected from camels with a sound history of semen quality and fertility $(n=3)$ using a modified artificial vagina and divided into different treatments after mechanical reduction of semen viscosity (3). Progressive forward motility of spermatozoa was examined at the time of semen collection and at 4,12 , and $24 \mathrm{~h}$ after incubation at $4{ }^{\circ} \mathrm{C}$. Data were analyzed using the GLM procedure in SAS/STAT after arcsin transformation. At the time of semen dilution, the progressive forward motility of spermatozoa was greater at osmolality of $331(23 \%)$ compared with $292(1 \%), 386(6 \%)$, and $410(3.5 \%) \mathrm{mOsm} / \mathrm{kg}$ $(P<0.05)$. No progressive forward motility of spermatozoa was noticed after $4 \mathrm{~h}$ incubation at $4^{\circ} \mathrm{C}$ at osmolalities of 292,386 , and $410 \mathrm{mOsm} / \mathrm{kg}$. At this time, a significant decrease $(P<0.05)$ of progressive forward motility occurred at osmolalities of $331(4 \%)$ and $356(0.5 \%)$ compared with that of the time of dilution. After 12 and $24 \mathrm{~h}$ incubation at $4{ }^{\circ} \mathrm{C}$, no progressive forward motility of spermatozoa was detected in any of these extenders. In conclusion, $10 \%$ sucrose $(331 \mathrm{mOsm} / \mathrm{kg})$ at the adjusted $\mathrm{pH}$ of 6.9 was the most suitable concentration of this disaccharide for preserving Bactrian camel semen for less than $4 \mathrm{~h}$ under chilled conditions.
\end{abstract}




\title{
105 COMPARISON BETWEEN TWO EXTENDERS FOR CRYOPRESERVATION OF BACTRIAN CAMEL SEMEN
}

\author{
A. Niasari-Naslaji ${ }^{\mathrm{A}, \mathrm{B}}$, S. Mosaferi ${ }^{\mathrm{A}} \mathrm{\textrm {E }}$, A. A. Gharahdaghi ${ }^{\mathrm{B}}$, A. Abarghani $^{\mathrm{C}}$, A. Ghanbari $^{\mathrm{C}}$, \\ A. Gerami ${ }^{\mathrm{D}}$, and N. Bahmani ${ }^{\mathrm{A}}$ \\ ${ }^{A}$ Department of Clinical Sciences, Faculty of Veterinary Medicine, University of Tehran, Tehran, Iran; \\ ${ }^{\mathrm{B}}$ Animal Science Research Institute, karaj, Tehran, Iran; \\ ${ }^{\mathrm{C}}$ Research Center for Agriculture and Natural Resources, Ministry of Jihad-e-Agriculture, Ardabil, Iran; \\ D Statistical Reserach Center, Tehran, Iran; \\ EIslamic Azad University of Tabriz, Tabriz, East Azarbayejan, Iran. Email: niasari@ut.ac.ir
}

A Tris-based extender (SHOTOR ${ }^{\dagger}$ diluent) has been developed for preserving Bactrian camel semen at $4^{\circ} \mathrm{C}$ (Niasari-Naslaji et al. 2005 Reprod. Fertil. Dev. 17, 198 (abstr.)). The present study investigated the possibility of utilizing the SHOTOR diluent for the cryopreservation of Bactrian camel semen. A modified bovine artificial vagina (Masaferi et al. 2005 Theriogeology 63, 92-101) was used to collect semen from three fertile bulls. The viscosity of the semen was reduced mechanically (Mosateri et al. 2005) and the homogenized semen was divided equally into two parts. Each part was sequentially diluted with either IMV buffers (Green buffer: first extender; White buffer: second extender; IMV, France) or SHOTOR diluents (without glycerol: first extender; with $12 \%$ glycerol: second extender). SHOTOR diluent consists of $2.6 \mathrm{~g}$ TIS, $1.35 \mathrm{~g}$ citric acid, $1.2 \mathrm{~g}$ glucose, and $0.9 \mathrm{~g}$ fructose in $100 \mathrm{~mL}$ of deionized water, with an osmolality of $330 \mathrm{mOsm} / \mathrm{kg}$ and $\mathrm{pH}$ of 6.9 . All extenders had $20 \%$ egg yolk and antibiotics. The semen was diluted at the ratio of $1: 1$ with the first extender. The diluted semen was then cooled within $2 \mathrm{~h}$ to $4^{\circ} \mathrm{C}$. At this temperature, the second extender was added at the same volume as the diluted semen in three steps with an equal volume, 10 min apart. After a 30 -min equilibration time, beginning after addition of the last fraction of the second extender, the diluted semen was loaded into 0.5 -mL straws at a concentration of $50 \times 10^{6}$ sperm per straw. The straws were maintained for $20 \mathrm{~min}$ at $4 \mathrm{~cm}$ above the liquid nitrogen surface, after which they were plunged into liquid nitrogen. The semen was thawed at $40^{\circ} \mathrm{C}$ water bath for $20 \mathrm{~s}$. Progressive forward motility of spermatozoa was assessed at the time of dilution and immediately after thawing of the semen. The experiment was replicated four times. Data were analyzed using GLM procedure in SAS/STAT after arcsine transformation. At the time of dilution, there was no significant difference in progressive forward motility of spermatozoa between IMV buffers $(51.8 \%)$ and SHOTOR diluent $(61 \% ; P>0.05)$. However, after thawing, there was a significant decrease in progressive forward motility of spermatozoa in IMV buffers $(4.2 \%)$ compared to SHOTOR diluent $(29.9 \%, P<0.05)$. In conclusion, in this experiment, SHOTOR diluent was more efficient for cryopreserving Bactrian camel semen than IMV extender.

$\dagger$ Shotor means camel in the Persian language.

\section{EFFECT OF GLYCEROL AND ETHYLENE GLYCOL ON THE DEVELOPMENT OF IN VITRO BOVINE EMBRYOS}

\author{
A. C. Nicacio, R. Simões, M. A. Peres, J. S. A. Gonçalves, M. E. O. D’Ávila Assumpção, and J. A. Visintin \\ University of São Paulo - FMVZ, São Paulo, Brazil
}

\begin{abstract}
The aim of this study was to evaluate the viability of in vitro-produced bovine embryos after exposure to different cryoprotectant solutions and cryopreservation. Bovine ovaries were collected at slaughterhouse and oocytes were matured, fertilized, and cultured in vitro. The embryos were co-cultured on a granulosa cell monolayer in SOF $+5 \%$ FCS and nonessential amino acids. In Experiment 1, expanded blastocysts were exposed to $10 \%$ ethylene glycol (EG) solution for $10 \mathrm{~min}$ (Group EG) or to $10 \%$ EG solution for $10 \mathrm{~min}$ and to $20 \% \mathrm{EG}+20 \%$ glycerol (Gly) solution for $30 \mathrm{~s}$ (Group EG/Gly). Cryoprotectants were diluted with PBS $+0.2 \%$ BSA $+0.3 \mathrm{M}$ sucrose and PBS $+0.2 \%$ BSA solutions, both for 3 min, and the hatching rate was evaluated after culture. In Experiment 2, after exposure, EG Group was cryopreserved by slow freezing procedure $\left(1.2^{\circ} \mathrm{C} / \mathrm{min}\right)$ and EG/Gly Group was vitrified on nitrogen vapor for $2 \mathrm{~min}$. After thawing, cryoprotectants were diluted using PBS $+0.2 \% \mathrm{BSA}+0.3 \mathrm{M}$ sucrose and PBS $+0.2 \%$ BSA solutions, both for $3 \mathrm{~min}$; hatching rate was evaluated after culture. As a control group for both experiments, non exposed embryos were cultured and evaluated for hatching rate. In Experiment 1, the hatching rates were 59.72\% (43/72) for control, 62.38\% (63/101) for EG, and 69.00\% (69/100) for EG/Gly groups. In Experiment 2, hatching rates were 59.72\% (43/72) for control, 15.22\% (7/46) for EG, and 0.00\% $(0 / 46)$ for EG/Gly groups. Results were analyzed by chi-square test. In Experiment 1, no differences were observed among groups $(P>0.05)$ and in Experiment 2, differences were observed among control, EG, and EG/Gly groups $(P<0.05)$. In conclusion, the cryoprotectants were not deleterious to the development of in vitro bovine embryos until hatching, but the cryopreservation procedures decreased embryo viability.
\end{abstract}

This work was supported by FAPESP 04/05335-1.

\section{COMPARISON OF PRIMATE SPERM CRYOPRESERVATION PROTOCOLS: POST-THAW SPERM RECOVERY AND HYPERACTIVATION IN CULTURE}

\author{
S. Nichols and B. Bavister \\ Department of Biological Sciences, University of New Orleans, New Orleans, LA 90148, USA
}

Cryopreservation of spermatozoa provides material for gene banking of genetically valuable males and offers convenience for in vitro fertilization (IVF). In addition, cryobanking of spermatozoa allows less frequent ejaculation collections from males. The present study compared the effectiveness of several published techniques in non-human primates to find the most efficient one for rhesus macaque (Macaca mulatta) semen cryopreservation. The effectiveness of each method was assessed by longevity (post-thaw motility $\%$ and duration) and ability to hyperactivate in culture in response 
to chemical activators (caffeine, dbcAMP) needed for rhesus sperm capacitation (Boatman and Bavister 1984 J. Reprod. Fertil. 71, 357-366). The ability to hyperactivate provides a reasonable assessment of the fertilizing capability of spermatozoa prior to performing IVF; the latter was impractical for this study, given the large number of treatments and endpoints. Spermatozoa were collected via electroejaculation from one male on three occasions to avoid confounding treatments with male effects. Each ejaculate was divided into one of four treatment groups for cryopreservation: Method A (Seier et al. 1993 J. Med. Primatol. 22, 355-359); Method B (Wei et al. 2000); Method C (Sanchez-Partida et al. 2000, Biol. Reprod. 63, 1092-1097); and Method D (Isachenko et al. 2005 Reprod. Biomed. Online 10, 350-354). Protocols were followed according to each published technique. Upon thawing, each sample was split into different incubation conditions: $37^{\circ} \mathrm{C}, 5 \% \mathrm{CO}_{2}$ in air or room temperature for $0-24 \mathrm{~h}$. One dose of activators was used according to standard protocol. Statistical analyses of motility rates were performed using $2 \times 2 \mathrm{G}$ tests (Sokal and Rohlf 1981 Biometry. New York: W. H. Freeman Co.) to determine significance. Samples cryopreserved using method D did not survive the method $($ motility $=0)$ and were not included in the statistical analysis. Methods A-C all demonstrated reasonable post-thaw motility recovery rates $(68 \%$, $73 \%$, and $62 \%$, respectively) and underwent capacitation within $30 \mathrm{~min}$ of exposure to activators. Sperm motility decreased over time in culture within each treatment at $37^{\circ} \mathrm{C}$. However, spermatozoa in Method A were significantly less motile at 4 and $24 \mathrm{~h}$ than those in Methods B and C, and Method B spermatozoa were significantly less motile at $24 \mathrm{~h}$ than those in Method C. Sperm motility also decreased over time in samples incubated at room temperature, with motility of sperm in Method A motility being significantly less at $24 \mathrm{~h}$ than that of sperm in Methods B and C. Method C best preserved motility over time regardless of temperature of incubation upon thawing. Overall, incubation at room temperature preserved motility better than incubation at $37^{\circ} \mathrm{C}$. Methods A-C yielded satisfactory post-thaw recovery of progressively motile spermatozoa despite the various differences among their protocols. For long-term use of each sample, however, it would be beneficial to incubate spermatozoa at room temperature after using Method C. This technique appears to be more appropriate for gene banking rhesus semen, and applying this protocol would allow more efficient usage of each semen sample, potentially providing for multiple IVF cases over a 24 -h period.

This work was supported by NIH Grant RR15395.

\title{
108 NEGLIGIBLE LEVEL OF DNA DAMAGE IN BOVINE OOCYTES VITRIFIED USING MINIMUM VOLUME METHODS
}

\author{
K. Papis ${ }^{\mathrm{A}}$, E. Stachowiak ${ }^{\mathrm{A}}$, M. Kruszewski ${ }^{\mathrm{B}}$, T. Iwanenko $^{\mathrm{B}}$, and T. Bartlomiejczyk ${ }^{\mathrm{B}}$ \\ Anstitute of Genetics \& Animal Breeding, Jastrzebiec, Poland; ${ }^{\mathrm{B}}$ Institute of Nuclear Chemistry and Technology, Warsaw, Poland
}

A relatively high number of bovine cryopreserved oocytes analyzed by the comet assay (Men et al. 2003 Mol. Reprod. Dev. 64, 245) showed compromised DNA integrity. The DNA fragmentation (comet tails) was found in $29 \%$ of slow cooled oocytes, in $20 \%$ of oocytes vitrified in straws and in $24 \%$ of oocytes vitrified in open pulled straws (OPS). Present study used the comet assay to compare the DNA status of 151 in vitro matured bovine oocytes vitrified in straws, in OPS or in droplets. It was assumed that the droplet method (Papis et al. 2000 Theriogenology 54, 651), which has gentle pre-equilibration prior to vitrification, would offer better protection of DNA. OPS vitrification was performed using a solution consisting of $20 \%$ DMSO, $20 \%$ ethylene glycol (EG), and $0.5 \mathrm{M}$ sucrose. For in-straw and in-droplet vitrification, VS14 (5.5 M EG and $1.0 \mathrm{M}$ sucrose) solution was used. In these two methods pre-equilibration in $3 \%$ EG solution for $15 \mathrm{~min}$ was applied. Fresh oocytes exposed to $0.5 \mathrm{mM}$ of hydrogen peroxide for $5 \mathrm{~min}$ served as the positive control. Fresh M II oocytes served as the negative control. The comet assay was performed according to the procedure of Men et al. (2003) with some modifications aimed at enhancing the sensitivity of the method. The zona pellucida was removed using $0.5 \%$ pronase solution, followed by placing of the oocytes in droplets of low-melting agarose on slides. These were subjected to overnight treatment in lysis buffer, followed by $40 \mathrm{~min}$ of DNA unfolding and $30 \mathrm{~min}$ electrophoresis. Following air drying, the slides were stained with DAPI fluorochrome and photographed. The pictures were saved as anonymous consecutive files to enable objective assessment. Of 119 vitrified oocytes, 112 (94\%) were evaluated. The remainder were lost or displayed atypical pictures. The comets could not be analyzed with the Comet v.3.0 software, possibly due to the large size of each oocyte. Six main classes of comet tails were distinguished ranging from no tail (class 0 ) to heavy and long tail (class 5). Positive control oocytes displayed class $4(36 \%)$ or $5(64 \%)$ tails. Negative control oocytes formed class $0(18 \%)$ to class $3(47 \%)$ comet tails. The oocytes vitrified using minimum volume methods fell within the same range, with $80 \%$ and $76 \%$ of oocytes vitrified in droplets and OPS, respectively, forming class 1 or 2 tails. One OPS vitrified oocyte (2.2\%) expressed a class 5 tail. A small but significant proportion of oocytes vitrified in straws $(15.4 \%, P \leq 0.05$, ANOVA) formed class 4 tails typical of positive control oocytes. In conclusion, in spite of pre-equilibration, a significant proportion of oocytes vitrified in straws contained detectable levels of DNA fragmentation, due probably to the lower cooling rate. The minimum volume protocols (the droplet and OPS methods) caused virtually no damage as assessed by the DNA comet assay. Results presented here differ from those reported previously. Reasons for differences remain to be established.

\section{HYDROSTATIC PRESSURE INDUCED INCREASE IN POST-THAW MOTILITY OF FROZEN BOAR SPERMATOZOA}

\author{
C. Pribenszky ${ }^{\mathrm{A}}$, M. Molnar ${ }^{\mathrm{A}}, A$. Horvath $^{\mathrm{A}}$, A. Harnos $^{\mathrm{B}}$, and O.Szenci ${ }^{\mathrm{A}}$ \\ ${ }^{A}$ Clinic for Large Animals, Faculty of Veterinary Science, Szent Istvan University, Dora major, Ullo, Hungary; \\ ${ }^{B}$ Department of Biomathematics and Informatics, Faculty of Veterinary Science, Szent Istvan University, Budapest, Hungary
}

Formerly we reported that a sublethal shock, high hydrostatic pressure (HHP), significantly improves the post-thaw survival of frozen mouse blastocysts and post-thaw motility of frozen bull sperm, presumably from the HHP induced changes in the protein profile [e.g. heat shock proteins (HSPs)] (Pribenszky et al. 2005 Rep. Fert. Dev. 17, 199-200; Pribenszky et al. 2005 Anim. Rep. Sci. 87, 143-150). We now report the effect of HHP on the motility of fresh boar semen, and we compare post-thaw motility of HHP-treated frozen boar semen with non-pressurized frozen-thawed controls. Pressurization was done both at RT and at body temperature (BT). Exp. 1: Semen was extended with Beltsville thawing solution (BTS), centrifuged, diluted with lactose-egg yolk diluent with a final concentration of $6 \%$ glycerol and $0.5 \%$ Equex (Minitüb, Tiefenbach, Germany) to 
a sperm concentration of $3 \times 10^{9} / \mathrm{mL}$. Diluted sperm were loaded into 0.25 -mL straws (IMV, Paillette Crista, France) at RT. Straws were heat sealed then assorted to one of the treatment groups $(100,200,400$, or 800 bar for 40, 80, or $120 \mathrm{~min})$. Non-pressurized samples were left at RT for the corresponding time. For freezing, straws were put at $15^{\circ} \mathrm{C}$ for $3 \mathrm{~h}$, and then $5^{\circ} \mathrm{C}$ for $2 \mathrm{~h}$, followed by $3 \mathrm{~cm}$ above $\mathrm{LN}_{2}$ for $10 \mathrm{~min}$ before plunging into $\mathrm{LN}_{2}$. Exp. 1 was repeated on two boars. Exp. 2: Sperm was treated with 400 bar for $80 \mathrm{~min}$ at RT or BT, and was then prepared and frozen as described above, together with non-pressurized controls. Exp. 2 was repeated in five boars. For evaluation, straws were thawed in a $37^{\circ} \mathrm{C}$ water bath for $2 \mathrm{~min}$. Motility was analyzed with CASA SpermVision 3.0 (Minitüb). For hypothesis testing, a linear mixed model was fit to the motility data. The analysis was carried out in R statistical software. In Exp. 1, the 100-, 200-, and 400-bar treatments did not affect motility; 800 bar treatments resulted in reduced motility compared to control. After $5 \mathrm{~h}$ of cold acclimatization, only the groups with 800 bar treatments and the non-pressurized controls had significantly reduced motility. After freezing-thawing motility $(\% \pm \mathrm{SE})$ in groups pre-treated for 80 min with 200 or 400 bar was $43.2 \pm 5.24$ and $42 \pm 3.24$, respectively; control: $23.2 \pm 1.83$ motility in groups pre-treated for 120 min with 200 or 400 bar was $51 \pm 2.33 ; 55.5 \pm 3.63$, respectively; control: $41.88 \pm 2.97$. The pre-treated groups displayed significantly enhanced motility compared to the nontreated controls. In Exp. 2, the HHP treatment performed at BT yielded the highest post-thaw motility compared to the HHP treatment at RT, or the non-pressurized controls $(59.75 \pm 2.59 ; 46.43 \pm 2.05 ; 37.37 \pm 2.19$, respectively $)$. All of these results differed significantly from each other. In conclusion, HHP treatment, simply inserted before the freezing step, can significantly increase post-thaw motility and yield consistent acceptable results. The effect of the treatment is even stronger at BT, but great care has to be taken to maintain BT from the time of sperm collection till the end of the treatment. Further experiments are being conducted concerning the pressure-induced alterations in the protein profile of boar spermatozoa (fresh and frozen-thawed)

This work was supported by OTKA061975 and TST050157.

\title{
110 VITRIFICATION DEVICES AFFECT DEVELOPMENTAL COMPETENCE AND BIOCHEMICAL PROPERTIES OF IVM OVINE OOCYTES
}

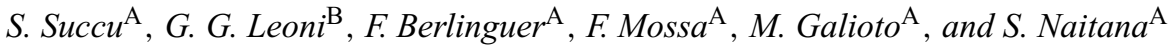 \\ ${ }^{A}$ Dept. of Animal Biology, Sassari, Italy; ${ }^{B}$ Dept. of Physiological, Biochemical and Cellular Sciences, Sassari, Italy
}

This study was designed to evaluate the effects of 3 different vitrification devices on the developmental ability and on the maturation-promoting factor (MPF) and mitogen-activated protein kinase (MAPK) activity of ovine oocytes in vitro-matured. Cumulus-oocytes complex (COCs) were in vitro-matured in TCM-199 supplemented with $10 \% \mathrm{FCS}, 10 \mu \mathrm{L} / \mathrm{mL}$ of $\mathrm{FSH} / \mathrm{LH}$, and $1 \mu \mathrm{g} / \mathrm{mL}$ estradiol at $39^{\circ} \mathrm{C}$ and $5 \% \mathrm{CO}_{2}$ atmosphere. For vitrification, oocytes were incubated in HEPES-buffered TCM-199 containing 20\% FCS supplemented with DMSO (7.5\%) and ethylene glycol (7.5\%). After $3 \mathrm{~min}$, oocytes were loaded into the same medium containing $0.5 \mathrm{~m}$ sucrose, $16.5 \%$ DMSO, and $16.5 \%$ EG, and then immersed into $\mathrm{N}_{2}$ using open pull straw (OPS; Vajta et al. 1998 Mol. Reprod. Dev.), cryoloop (CL; Lane et al. 1999 Nat. Biotechnol.), or cryotop (CT; Kuwayama and Kato 2000 J. Assist. Reprod. Genet.). After warming a part of oocytes were fertilized and cultured in vitro up to blastocyst stage in standard conditions (Leoni et al. 2005 Anim. Reprod. Sci., in press). The fertilization $(23.8 \%, 31.6 \%$, and $36.8 \%$ vs. $61.5 \%)$ and blastocyst rates $(0,12.5$, and $0 \%$ vs. $50.0 \%$ ) were lower for oocytes vitrified in OPS, CL, and CT, compared with control group. Control and vitrified IVM oocytes were valued for MPF and MAPK activity at 0 and $2 \mathrm{~h}$ after warming. In both post-warming experimental groups, the MAPK activity did not differ from control group. Immediately post-warming, MPF activity was lower in the vitrified groups compared with control oocytes $(P<0.01)$. If $100 \%$ is assigned to MPF activity in the control oocytes, those in the OPS, CL, and CT groups were, respectively, $63.3 \%, 65.5 \%$, and $26.2 \%$. After warming and culture for $2 \mathrm{~h}$ in standard condition, the activity of MPF was restored to values similar to control oocytes ( 87.0 and $76.8 \%$, respectively) in the OPS and CL groups, whereas it was at the similar value in the CT group. To evaluate if the lowered MPF activity could cause parthenogenetic activation, the vitrified-warmed oocytes were cultured in $\mathrm{SOF}+2 \%$ oestrus sheep serum in $5 \% \mathrm{O}_{2}, 5 \% \mathrm{CO}_{2} ;$ after $27 \mathrm{~h}$ of culture, the oocytes were fixed and stained with propidium iodide to evaluate chromatin configuration. Results showed significantly higher parthenogenetic activation rates in the CT group compared with OPS and CL groups (54.5\% vs. 22.6 and $27.4 \%$, respectively). Our results indicate that the success of cryopreservation of the ovine oocyte is still very limited. The use of different vitrification devices not only modifies the ability to survive cryopreservation and developmental competence of oocytes but is also associated with important molecular alterations in the warmed oocyte cytoplasm.

This work was supported by Cofin 2003.

\section{ESTIMATION OF SPERM QUALITY IN FRESH AND FROZEN-THAWED SEMEN FROM ASTURIANA DE LOS VALLES BULLS}

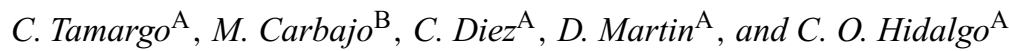 \\ ${ }^{\mathrm{A}}$ Serida, Gijon, Spain; ${ }^{\mathrm{B}}$ Veterinary Faculty, Leon, Spain
}

Artificial insemination and semen cryopreservation have significantly improved the breeding potential of male animals. However, current freezing techniques commonly result in reduced semen quality (Januskauskas et al. 1999 Theriogenology 52, 641-658), and surviving cells are affected postthaw either structurally or functionally (Nagy et al. 2004 Anim. Reprod. Sci. 80, 225-235). In this work we analyze the impact of cryopreservation on Asturiana de los Valles bull sperm. Ejaculates $(n=373)$ from seven adult bulls were weekly collected by means of artificial vagina. Immediately after collection, routine parameters including volume (V), mass motility (MM), and concentration (C) of sperm cells were evaluated. Then the semen was extended with a commercial extender, loaded into $0.25-\mathrm{mL}$ plastic straws at a concentration of $23 \times 10^{6}$ per straw, frozen and stored for further analysis. Four straws per ejaculate were thawed, pooled and analyzed for motion characteristics by means of a CASA system (Sperm Class 
Analyzer, SCA $2002^{\circledR}$ Microptic S. L., Barcelona, Spain) added to an optical phase-contrast microscope with heatable $\left(37^{\circ} \mathrm{C}\right)$ stage. Immediately after thawing, we analyzed the \% of motile spermatozoa (MS) and the \% of progressively motile spermatozoa (PMS); then samples were incubated for $3 \mathrm{~h}$ at $37^{\circ} \mathrm{C}$ and MS and PMS were measured again (MS3 and PMS3, respectively). Functional integrity of the plasmallema was evaluated by the hypoosmotic swelling test (HOST) together with the \% of typical tail coiling/swelling (percentage of HOST-positive spermatozoa, HOST-PS). The $\%$ of viable spermatozoa (VS) [membrane integrity was evaluated by fluorescence microscopy with a dual staining system (propidium iodide (PI) and 6-carboxyfluorescein diacetate (CFDA)]. Sperm showing partial or complete red fluorescence (PI staining) were considered nonviable, whereas sperm showing complete green fluorescence were considered viable. Altered acrosomes (AA) and morphological abnormalities were also determined. The $\%$ of morphological abnormalities was classified according to their location in head (HA), midpiece (MA), and tail (TA). Proximal and distal cytoplasmic droplets were counted as separate abnormalities (CD). Data were analyzed by the MEANS procedure of SAS (SAS Institute, Inc., Cary, NC, USA). A significant $(P<0.05)$ decrease in the sperm motility was observed after freezing/thawing (MS: $80.20 \pm 0.75 \mathrm{vs.} 47.36 \pm 1.04$, and PMS: $68.73 \pm 0.73$ vs. $42.14 \pm 0.96$ for fresh and frozen-thawed semen, respectively). Also, the frozen-thawed sperm showed increased $\%$ of HA, MA, AA, HOST-PS, and VS $(P<0.05)$. These morphological abnormalities could contribute to decreasing sperm motility. The new computer and video technologies provide useful information about sperm quality and can be used in the daily routine of processing semen.

This work was performed in collaboration with ASEAVA.

\title{
112 LLAMA LAMA GLAMA PREGNANCIES FROM VITRIFIED/WARMED BLASTOCYSTS USING A NOVEL COAXIAL CRYOPROTECTANT MICROINJECTION SYSTEM
}

\author{
P. Taylor ${ }^{\mathrm{A}}$, S. Taylor ${ }^{\mathrm{A}}$, M. Sansinena ${ }^{\mathrm{B}}$, and R. Godke $\mathrm{B}$ \\ ${ }^{\mathrm{A}}$ GeneSearch, Inc., Bozeman, MT 59715, USA; ${ }^{\mathrm{B}}$ Department of Animal Sciences, \\ Louisiana State University Agricultural Center, Baton Rouge, LA 70803, USA
}

There has been only limited progress in efforts to cryopreserve llama embryos, and no live offspring have been reported to date. The main obstacle is the large diameter of in vivo-recovered llama embryos that results in high embryo volume to surface ratio. First, a novel coaxial microinjection device was designed and constructed to allow the injection of a cryoprotectant (CPA) solution directly into the cavity of hatched llama blastocysts in an effort to reduce the exposure time to toxic CPAs. The coaxial device consisted of a holding borosilicate pipette (120- $\mu \mathrm{m}$ OD; 60- $\mu \mathrm{m}$ ID) and an injection pipette (20- $\mu \mathrm{m}$ OD; $17-\mu \mathrm{m}$ ID) placed inside the holding pipette for injection of CPAs and for aspiration of aqueous fluids from the blastocele cavity. In this preliminary study, two methods of cryopreservation were evaluated. Treatment (Trt) A consisted of injection of a CPA solution using the coaxial microinjection system followed by a vitrification (VITC) protocol. Day 7 in vivo-derived, hatched llama blastocysts ( $>500-\mu \mathrm{m}$ diameter) were held in PBS during injection with an equilibration solution (EQUL) consisting of $15 \%$ glycerol, $10 \%$ butanediol, $1 \%$ polyethylene glycol, 20\% fetal bovine serum (FBS) and $0.5 \mathrm{~m}$ sucrose in ViGro Holding Plus medium (Bioniche Animal Health, Melleville, Ontario, Canada). Embryos were immobilized with the holding pipette and EQUL solution was injected until the embryos were hyperinflated. The contents of the blastocele cavity were then aspirated and the embryos were released and held in EQUL solution for 8 min. After equilibration, embryos were transferred to a 3 step VITC solution ( $20 \%$ glycerol, $20 \%$ ethylene glycol, $0.3 \mathrm{~m}$ sucrose, $0.375 \mathrm{~m}$ glucose, $3 \%$ polyethylene glycol in three steps; Aller et al. 2002 Anim. Reprod. Sci. 73, 121-127), loaded into 0.25-mL plastic straws, and plunged into $\mathrm{LN}_{2}$. Trt B consisted of VITC only, without microinjection of CPAs. After 60 days, the straws containing the vitrified embryos in Trt A were removed from the $\mathrm{LN}_{2}$ and placed in a water bath at $32^{\circ} \mathrm{C}$ for $10 \mathrm{~s}$. Embryos were injected with diluent medium $(0.3 \mathrm{M}$ sucrose in ViGro Holding Plus) into the blastocele cavity using the coaxial system until re-inflated to their original spherical shape. The warming of embryos in Trt B was performed without injection of diluent medium. Embryos from both treatments were held in diluent medium for $7 \mathrm{~min}$ and then placed in ViGro culture medium. Randomly chosen, VITC-thawed hatched blastocysts were nonsurgically transferred ( 1 or $2 /$ female) to GnRH-treated llama recipients. Two pregnancies resulted from the transfer of three embryos (67\%) from Trt A, with normal heart beats confirmed by ultrasonography 24 days post-transfer (Table 1). No pregnancies resulted from the transfer of embryos from Trt B. The pregnancies remain ongoing (60 and 210 days) at the time of this submission. These preliminary results demonstrate the successful use of a novel microinjection system in the cryopreservation of llama embryos. Research is now underway to determine the optimal VITC solutions and embryo exposure time.

Table 2. Comparison of two vitrification methods for hatched llama blastocysts

\begin{tabular}{lcccccc}
\hline Treatment & $\begin{array}{c}\text { No. embryos } \\
\text { vitrified }\end{array}$ & $\begin{array}{c}\text { No. (\%) } \\
\text { warmed }\end{array}$ & $\begin{array}{c}\text { No. (\%) embryos } \\
\text { re-expanded }\end{array}$ & $\begin{array}{c}\text { No. embryos } \\
\text { transferred }\end{array}$ & $\begin{array}{c}\text { No. } \\
\text { recipients }\end{array}$ & $\begin{array}{c}\text { No. (\%) } \\
\text { pregnant }\end{array}$ \\
\hline A (coaxial + VITC) & 20 & $20(100)$ & $20(100)$ & 3 & 2 & $2(67)$ \\
B (VITC only) & 20 & $20(100)$ & $1(5)$ & 1 & 1 & 0 \\
\hline
\end{tabular}

\section{EFFECT OF SPERM COATING ON THE QUALITY OF BOVINE FROZEN-THAWED SPERMATOZOA}

\author{
M. Thys, A. Van Soom, J. Dewulf, T. Rijsselaere, and A. de Kruif
}

Department of Reproduction, Obstetrics and Herd Health, Faculty of Veterinary Medicine, Ghent University, Merelbeke, Belgium

The substantial decrease of sperm quality after cryopreservation remains an important issue in the artificial insemination industry. Sperm coating with Triladyl ${ }^{\circledR}$ (Minitübe, Tiefenbach, Germany) during ejaculation can preserve sperm characteristics and oocyte penetrating capacity of fresh bovine 
spermatozoa stored in egg yolk diluent for up to 6 days (De Pauw et al. 2003 Theriogenology 59, 1109-1122). Since collecting semen in a tube containing egg yolk-Tris extender (sperm coating) limits the period of contact between spermatozoa and seminal plasma, the present experiment was conducted to assess if this slightly adjusted method of sperm collection could also have a significant effect on bovine sperm quality after cryopreservation. Semen of five young Holstein Friesian bulls was collected by means of an artificial vagina connected to an empty tube (Group 1; five ejaculates per bull) or a tube containing $4 \mathrm{~mL}$ of an egg yolk-Tris extender (Groups 2 and 3 ; each five ejaculates per bull). The semen samples of Group 1 were conventionally diluted in straws $\left(60 \times 10^{6} \mathrm{sperm} / \mathrm{mL}\right)$, frozen, and stored in liquid nitrogen. The samples of Group 3 were centrifuged, and after removing diluent and seminal plasma, the sperm pellet was conventionally diluted and processed. The samples of Group 2 were processed without removal of the supernatant. After thawing each ejaculate was analyzed for average path velocity (VAP), beat cross frequency (BCF), and progressive motility (PROG) using CASA (Minitübe, Tiefenbach, Germany). Furthermore, the membrane integrity of each sample was evaluated using fluorescent SYBR ${ }^{\circledR}-14 /$ PI staining (BD Biosciences, Erembodegem, Belgium). All parameters were compared among the three groups of sperm using univariate analysis of variance (SPSS 12.0; SPSS, Inc., Chicago, IL, USA). No significant differences could be observed among the three groups for all of the evaluated sperm characteristics (Table 1). A significant effect of the bull could be determined for all analyzed parameters $(P \leq 0.02)$, except for the percentage of moribund cells. Nevertheless, the group-bull interaction was never statistically significant. Coating bovine sperm with an egg yolk-Tris extender during ejaculation cannot prevent the substantial deterioration of the spermatozoa that occurs during freezing and thawing since this method of sperm collection does not significantly influence the motility parameters or the membrane integrity after thawing.

Table 1. VAP, BCF, PROG, and percentage of membrane-intact, dead, and moribund spermatozoa for the three groups of sperm

\begin{tabular}{|c|c|c|c|c|}
\hline Group & Parameter & Mean \pm SD & Parameter & Mean \pm SD \\
\hline 1 & $\operatorname{VAP}(\mu \mathrm{m} / \mathrm{s})$ & $95.54 \pm 6.82$ & Membrane intact (\%) & $42.16 \pm 13.33$ \\
\hline 2 & & $94.79 \pm 6.82$ & & $40.27 \pm 10.17$ \\
\hline 3 & & $96.94 \pm 6.63$ & & $39.12 \pm 11.01$ \\
\hline 1 & $\mathrm{BCF}(\mathrm{Hz})$ & $30.13 \pm 4.15$ & Dead $(\%)$ & $49.64 \pm 14.90$ \\
\hline 2 & & $31.63 \pm 3.02$ & & $52.92 \pm 11.38$ \\
\hline 3 & & $29.83 \pm 4.24$ & & $52.44 \pm 13.10$ \\
\hline 1 & PROG (\%) & $32.34 \pm 9.87$ & Moribund (\%) & $8.20 \pm 4.26$ \\
\hline 2 & & $31.84 \pm 6.31$ & & $6.81 \pm 5.01$ \\
\hline 3 & & $31.53 \pm 7.64$ & & $8.44 \pm 5.96$ \\
\hline
\end{tabular}

This research was supported by IWT (no. IWT/020727).

\title{
114 VITRIFICATION OF BOVINE EMBRYOS IN MEDIUM WITH POLYVINYL ALCOHOL REPLACING BSA
}

\author{
D. J. Walker and G. E. Seidel Jr \\ Colorado State University, Fort Collins, CO 80523-1683, USA
}

Embryos vitrified in medium supplemented with $4.25 \mu \mathrm{g} / \mathrm{mL}$ sodium hyaluronate (SH) and $0.1 \%$ polyvinyl alcohol (PVA) survived vitrification better than embryos vitrified in medium supplemented with $0.25 \%$ FAF-BSA (Walker and Seidel 2005 Reprod. Fert. Dev. 17, 153). The purpose of the present study was to determine if the small amount of SH was beneficial to in vitro survival and to examine the effects of different concentrations of PVA in vitrification solutions. Day 7 blastocysts $(n=360)$ were produced in vitro with semen from three bulls, two replicates each. Cryoprotectant solutions were prepared in a $2 \times 3$ factorial combination with two SH concentrations $(0$ or $4.25 \mu \mathrm{g} / \mathrm{mL})$ and three PVA concentrations $(0.05,0.1 \%$, or $0.2 \%)$. For vitrification, embryos were placed into chemically defined HEPES-buffered medium (HCDM-2) at room temperature $\left(22-24^{\circ} \mathrm{C}\right)$ and then transferred to V1 (5 M ethylene glycol in HCDM-2) for $3 \mathrm{~min}$. Next, embryos were placed in a $6 \mu \mathrm{L}$ drop of V2 ( $7 \mathrm{M}$ ethylene glycol, $0.5 \mathrm{M}$ galactose, and $18 \% \mathrm{w} / \mathrm{v}$ Ficoll 70 in HCDM-2) for $45 \mathrm{~s}$. During these $45 \mathrm{~s}$, dilution medium ( $0.5 \mathrm{~m}$ galactose in HCDM-2) was aspirated into $0.25-\mathrm{mL}$ straws, followed by the $6 \mu \mathrm{L}$ drop of $\mathrm{V} 2$ plus embryos and a final short column of dilution medium. When $45 \mathrm{~s}$ had elapsed, the heat-sealed end of straw was dipped into liquid nitrogen to cover the embryo, and then the remainder of the straw was immersed slowly. Straws were thawed in air for $10 \mathrm{~s}$ and then in $37^{\circ} \mathrm{C}$ water for $20 \mathrm{~s}$. Next, straws were shaken like a clinical thermometer four times to mix columns, and held in $37^{\circ} \mathrm{C}$ water for $10 \mathrm{~min}$ before embryos were expelled, rinsed and cultured in CDM- $2+5 \% \mathrm{FCS}$. At $48 \mathrm{~h}$, embryo survival (as determined by expansion of blastocysts), embryo quality $(1=$ excellent, $2=$ fair, $3=$ poor $)$, inner cell mass (ICM) quality $(1=$ large and compact, $2=$ clearly visible, $3=$ not discernable) and blastocyst stage $(5=$ early, $6=$ full, $7=$ expanded, $8=$ hatching, $9=$ hatched $)$ were evaluated and replicate averages were analyzed by ANOVA. Neither bull nor SH concentration nor PVA concentration significantly affected any response $(P>0.10)$. Averaged over PVA concentrations, vitrification of embryos in $0 \mu \mathrm{g} / \mathrm{mL}$ or $4.25 \mu \mathrm{g} / \mathrm{mL} \mathrm{SH}$ resulted in similar survival rates $(67 \%$ vs. $62 \%$, respectively). When averaged over SH concentrations, $0.2 \%$ PVA had a numerically higher survival rate of blastocysts as compared to $0.1 \%$ or $0.05 \%$ ( $71 \%$ vs. $63 \%$ and $60 \%$, respectively). The main effects of $0 \mu \mathrm{g} / \mathrm{mL} \mathrm{SH}$ and $0.2 \%$ PVA also resulted in numerically higher, but nonsignificant improvements in quality score, ICM score and blastocyst stage as compared to the other doses of SH and PVA. Vitrification of Day 7 in vitro-produced bovine blastocysts in medium containing $0.2 \%$ PVA in the absence of SH resulted in a subclass mean of $80 \%$ embryo survival. Results of this experiment show no benefit of $4.25 \mu \mathrm{g} / \mathrm{mL} \mathrm{SH}$ and that $0.2 \%$ PVA may be slightly better than $0.05 \%$ or $0.1 \%$ in terms of embryo survival. Therefore, our results indicate that $0.2 \%$ PVA can be used alone as an effective alternative to animal products in this vitrification procedure for in vitro-derived bovine blastocysts. 


\title{
115 SPERM CRYOPRESERVATION IN TRAGELAPHINE ANTELOPES
}

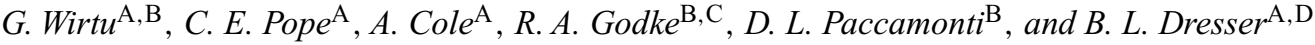 \\ AAudubon Center for Research of Endangered Species, New Orleans, LA 70131, USA; \\ ${ }^{B}$ Veterinary Clinical Sciences, LSU School of Veterinary Medicine, Baton Rouge, LA 70803, USA; \\ ${ }^{\mathrm{C}}$ Embryo Biotechnology Laboratory, Department of Animal Sciences, Louisiana State University Agricultural Center, \\ Baton Rouge, LA 70803, USA; D Department of Biological Sciences, \\ University of New Orleans, New Orleans, LA 70148, USA
}

\begin{abstract}
As an integral aspect of our program to develop assisted reproductive technologies in tragelaphine antelopes, we have been evaluating the effects of various extenders on cryosurvival of spermatozoa. Semen was collected by electroejaculation (EEJ) from an eland (Taurotragus oryx, $n=$ 14 EEJs) and a bongo (Tragelaphus euryceros, $n=7$ EEJs) male during sedation and standing restraint in a hydraulic chute. Epididymal spermatozoa were collected from the proximal vas deferens, and distal epididymides recovered from four common eland bulls after elective castration at the Hattiesburg Zoo, Mississippi (age = 46 months) and the San Diego Wild Animal Park, California (ages=18, 20, and 23 months). Testes from a bongo $(\mathrm{age}=6.5 \mathrm{yr}$ ) and a greater kudu bull (Tragelaphus strepsiceros, age $=7.5 \mathrm{yr}$ ) at the Audubon Nature Institute were recovered and processed immediately postmortem. Kudu, bongo, and the Hattiesburg eland testes were transported at ambient temperature whereas the testes from San Diego elands were shipped overnight in a cold-pack container. Sperm processing was done at room temperature $\left(\mathrm{RT}=23^{\circ} \mathrm{C}\right)$ in $\mathrm{HEPES}$-buffered Tyrode's solution. Four extenders containing 7\% glycerol were evaluated: Biladyl ${ }^{\circledR}$, TEST Yolk buffer (TYB), Beltsville extender (BF5F), and skim milk. For freezing, sperm samples were initially extended and gradually cooled to $4^{\circ} \mathrm{C}$ before sequential addition of extender containing glycerol at $4^{\circ} \mathrm{C}$; the procedure was then modified by addition of the glycerol fraction at RT before the sample was cooled to $4^{\circ} \mathrm{C}$. Spermatozoa were vaporfrozen in $0.5 \mathrm{~mL}$ straws placed $5 \mathrm{~cm}$ above liquid nitrogen before storage at $-196^{\circ} \mathrm{C}$. Straws were thawed in a water bath $\left(38^{\circ} \mathrm{C}, 30 \mathrm{~s}\right)$ to evaluate cryosurvival. All eland and bongo electroejaculation procedures produced spermatozoa, although sperm quality varied. Ejaculate volume averaged $5.4 \pm 1.2 \mathrm{~mL}$ and $3.7 \pm 1.1 \mathrm{~mL}$ in the bongo and the eland, respectively. Sperm motility at collection, after cooling, and after freezing in the different extenders is presented in Table 1. No immediate decline in sperm motility was observed after adding glycerol to samples, whether at $23^{\circ} \mathrm{C}$ or $4^{\circ} \mathrm{C}$. Bongo spermatozoa lost more motility during freezing than during cooling $(15.0 \pm 4.4$ vs. $5.8 \pm 2.0 \% ; P=0.006)$; whereas, in eland, motility loss during cooling and freezing was similar $(25.3 \pm 16.2 \mathrm{vs.} 21.1 \pm 7.7 ; P=0.44)$. The present results indicate that cooling and freezing tolerance of spermatozoa in tragelaphine antelopes is influenced by species, extender type, and temperature at which a cryoprotectant is added.
\end{abstract}

Table 1. Cryosurvival of ejaculated or epididymal spermatozoa of three antelope species in different extenders

\begin{tabular}{|c|c|c|c|c|c|}
\hline Species & Source & Method & Initial motility* & Cooled motility* & Post-thaw motility* \\
\hline \multirow[t]{8}{*}{ Eland } & \multirow[t]{4}{*}{ Ejaculate } & Biladyl & 70 & 30 & 10 \\
\hline & & Milk & $75 \pm 10$ & $41 \pm 10$ & $21 \pm 12$ \\
\hline & & Milk-RT & $73 \pm 12$ & $58 \pm 11$ & $33 \pm 9$ \\
\hline & & BF5F-RT & 60 & 50 & 45 \\
\hline & \multirow[t]{4}{*}{ Epididymal } & Biladyl & $67 \pm 15$ & $47 \pm 15$ & $23 \pm 15$ \\
\hline & & TYB & 80 & 70 & 30 \\
\hline & & BF5F & 40 & 30 & 5 \\
\hline & & BF5F-RT & 40 & 30 & 5 \\
\hline \multirow[t]{4}{*}{ Bongo } & \multirow[t]{3}{*}{ Ejaculate } & Milk & $70 \pm 0$ & $63 \pm 4$ & $45 \pm 0$ \\
\hline & & Milk-RT & $72 \pm 3$ & $67 \pm 3$ & $52 \pm 3$ \\
\hline & & BF5F-RT & 75 & 70 & 60 \\
\hline & Epididymal & TYB or Milk & 85 & 75 & 40 \\
\hline \multirow[t]{2}{*}{ Kudu } & \multirow[t]{2}{*}{ Epididymal } & Milk & 90 & 70 & 30 \\
\hline & & TYB & 90 & 75 & 50 \\
\hline
\end{tabular}

${ }^{*} \%$ of single observation or mean $\% \pm \mathrm{SD}$.

\section{EFFECTS OF L-GLUTAMINE ON CRYOPRESERVATION OF IMMATURE BOVINE OOCYTES}

\author{
C. Yamada, M. D. Goissis, H. V. A. Caetano, A. R. S. Coutinho, M. E. O. A. Assumpção, and J. A. Visintin \\ Department of Animal Reproduction, FMVZ, University of São Paulo, São Paulo, SP, Brazil
}

The cryopreservation of bovine oocytes remains a challenge despite significant reported progress. Immature bovine oocytes have a complex structure and the conventional cryoprotectants (penetrating cryoprotectants, sugars, and macromolecules) appear to be not sufficient to preserve them efficiently during freezing. Studies on semen and fibroblast cryopreservation indicate that amino acids, particularly L-glutamine, protect enzymes during freezing and increase the post-thaw viability. Therefore, the amino acids may optimize oocyte cryopreservation when associated with conventional cryoprotectants. This work evaluated the effect of L-glutamine on cryopreservation of immature bovine oocytes after in vitro maturation. Oocytes with homogeneous cytoplasm and several cumulus cell layers from slaughterhouse ovaries were distributed randomly in three groups: non-vitrified control, vitrified control, and vitrified with L-glutamine. Oocytes from vitrified groups were exposed for 10 min to PBS $+10 \%$ FCS $+10 \%$ ethylene glycol $(\mathrm{EG})+0.25 \mathrm{~m}$ trehalose $(\mathrm{T})$, and for $30 \mathrm{~s}$ to $\mathrm{PBS}+10 \% \mathrm{FCS}+25 \% \mathrm{EG}+25 \%$ dimethylsulfoxide $+0.5 \mathrm{~m} \mathrm{~T}$ at room temperature, adding $80 \mathrm{~mm}$ 
L-glutamine for the third group. Oocytes were loaded into OPS and plunged in liquid nitrogen. For thawing, OPS were immersed in PBS $+10 \%$ $\mathrm{FCS}+10 \% \mathrm{EG}+1 \mathrm{~m} \mathrm{~T}$ for three min. Oocytes werethen placed in PBS $+10 \% \mathrm{FCS}+0.5 \mathrm{~m} \mathrm{~T}$ and in PBS $+10 \% \mathrm{FCS}$, remaining three min in each solution. For in vitro maturation, oocytes were washed three times on holding medium (TCM-HEPES + FCS + pyruvate + gentamycin), washed three times in maturation medium (TCM-bicarbonate $+\mathrm{FCS}+$ pyruvate + gentamycin $+\mathrm{hCG}+\mathrm{FSH}+$ estradiol), and cultured in microdrops $(90 \mu \mathrm{L})$ of maturation medium covered with mineral oil at $38.5^{\circ} \mathrm{C}$ under $5 \% \mathrm{CO}_{2}$ in air and high humidity for $24 \mathrm{~h}$. Oocytes were denuded, fixed in paraformaldehyde and triton, stained with Hoechst 33342, and evaluated under epifluorescence microscopy. Oocytes at metaphase II were considered matured. The group vitrified with L-glutamine had a significantly higher maturation rate than the group vitrified without L-glutamine; however, both had significantly lower maturation rates than the non-vitrified control group. In conclusion, L-glutamine improved the viability of vitrified oocytes.

Table 1. Oocyte maturation rates of non-vitrified control, vitrified control, and vitrified with glutamine groups

\begin{tabular}{lccc}
\hline & Non-vitrified control & Vitrified control & Vitrified with glutamine \\
\hline Oocytes $(n)$ & 149 & 145 & 144 \\
Maturation $(\%)$ & $71.8^{\mathrm{a}}$ & $17.4^{\mathrm{c}}$ & $32.8^{\mathrm{b}}$ \\
\hline
\end{tabular}

${ }^{\mathrm{a}-\mathrm{c}}$ Different superscripts indicate significantly different values (Tukey, $P<0.05$ ).

This work was supported by FAPESP 03/08543-1.

\title{
117 VITRIFICATION OF CHAROLAIS AND HOLSTEIN BLASTOCYSTS USING LN SLUSH
}

\author{
S. Yavin ${ }^{\mathrm{A}, \mathrm{B}}$, D. Ditesheim ${ }^{\mathrm{C}}$, Y. Zeron $^{\mathrm{C}}$, and A. Arav ${ }^{\mathrm{A}}$ \\ Anstitute of Animal Science, Volcani Centre, Bet Dagan 50250, Israel; ${ }^{B}$ Department of Animal Science, Faculty of Agricultural, Food and \\ Environmental Quality Sciences, The Hebrew University of Jerusalem, Rehovot 76100, Israel; ${ }^{\mathrm{C}}$ Sion, AI Center, Shikmim 79800 , Israel
}

Cryopreservation of embryos for transfer in outdoor conditions is a challenge; therefore a rapid and simple technique should be applied. Two different experiments were performed to develop a functional and efficient vitrification technique. Charolais (for Exp. 1) and Holstein (for Exp. 2) female cows were superovulated and artificially inseminated (AI). Seven days after AI, embryos were flushed from the uterus and vitrified. For vitrification, blastocyst were exposed to $10 \%$ vitrification solution (VS) for $3 \mathrm{~min}$, transferred into $50 \% \mathrm{VS}$, and immediately thereafter into $87.5 \% \mathrm{VS}$ (100\% VS containing $38 \%(\mathrm{v} / \mathrm{v})$ ethylene glycol (EG), $0.5 \mathrm{M}$ trehalose, and $6 \%$ BSA in PBS). The embryos were then loaded into super open pulled straws (SOPS) (Minitub, Tiefenbach, Germany) in a minimum-volume droplet $(0.5 \mu \mathrm{L})$. The SOPSs containing the embryos were sealed by applying a soldering device to the narrow end and by inserting an identification rod into the wide end. Samples were vitrified at a rapid cooling rate in LN Slush (VitMaster apparatus, IMT Ltd, Ness-Ziona, Israel). On the day of transfer, blastocysts were warmed by plunging the SOPS into the warming chamber of the device, which contained $70 \%$ ethanol at $37^{\circ} \mathrm{C}$, for $5 \mathrm{~s}$. The straws were then withdrawn from the warming chamber and the sealed end was cut off carefully. The embryos were immersed in a $200-\mu \mathrm{L}$ drop of $0.6 \mathrm{M}$ trehalose in PBS solution for $4 \mathrm{~min}$ and then transferred through a series of solutions containing decreasing concentrations $(0.5,0.4,0.3,0.2$, and $0.1 \mathrm{M})$ of trehalose for $2 \mathrm{~min}$ each. Viability was evaluated according to the ability of the embryos to re-expand. Blastocysts with the highest morphology rank were selected for transfer and loaded into the transfer gun (Minitub). Holstein recipients were in estrus 6-8 days prior to transfer. In Exp. 1, all blastocysts $(n=15)$ re-expanded after the vitrification and warming processes and were transferred into the uteruses of 15 Holstein recipient cows. One recipient cow became pregnant and gave birth to a healthy calf. In Exp. 2, all blastocysts $(n=6)$ re-expanded after warming and were transferred, one each into the uterus of a recipient Holstein heifer. Three pregnancies are still ongoing. In summary, the results obtained demonstrate that plunging embryos in SOPSs into LN Slush in outdoor conditions offers a potential technique for embryo cryopreservation and transfers. Further field trails are required to examine the effect of recipient age (cow vs. heifer) and embryo breed (Charolais vs. Holstein) on the suggested procedure.

\section{Developmental Biology}

\section{EXPRESSION PROFILES OF CIRCADIAN CLOCK GENES IN MOUSE OOCYTES AND PRE-IMPLANTATION EMBRYOS}

\author{
T. Amano, A. Matsushita, R. Kakegawa, K. Matsumoto, K. Saeki, Y. Hosoi, and A. Iritani \\ Kinki University, Wakayama, Japan
}

\begin{abstract}
Matsuo et al. reported that circadian clock genes regulate the timing of cell division in mouse regenerating liver cells (2003). Their results suggested the importance of circadian clock genes for organs or tissues for which functions are characterized by cell division, such as pre-implantation embryos. To obtain basic information on the molecular functions of circadian clock genes in pre-implantation embryos, we investigated the expression profiles of transcripts and proteins of some circadian clock genes, clock, bmall, cryl, and per2, in mouse germinal vesicle oocytes (GV), MII oocytes (MII), and pre-implantation embryos using real-time PCR and immunocytochemistry (ICC). Germinal vesicle oocytes were collected from ICR females at $48 \mathrm{~h}$ after PMSG priming. The mouse at $48 \mathrm{~h}$ after PMSG priming was primed with hCG, and MII were collected at $15 \mathrm{~h}$ after hCG priming. The pre-implantation embryos were collected at $6,12,24,36,48,60,72,84$, and $96 \mathrm{~h}$ after insemination, and they corresponded to early 1-cell, late
\end{abstract}


1-cell, early 2-cell, late 2-cell, 4-cell, 8-cell, early morula, late morula, and blastocyst stages, respectively. cDNA was produced by mRNA isolated from 20 oocytes or embryos using oligo dT and was subjected to real-time PCR using a TaqMan Probe system (ABI). Three sets of 20 oocytes or embryos at each developmental stage were applied to mRNA extraction and real-time PCR analysis to ensure equal mRNA extraction efficiency between samples. The level of mRNA of each clock gene contained in 3 samples from each developmental stage was almost the same. Statistical analysis of the transcripts of each gene were done by ANOVA. Germinal vesicles, MII and embryos collected at each time point were subjected to ICC using antibodies of CLOCK, BMAL1, CRY1, and PER2. The oocytes or embryos treated with only secondary antibody did not produce any signal. All of the examined genes except per 2 were expressed in oocytes and pre-implantation embryos. The transcript level of clock, bmall, and cry 1 in MII were significantly lower than those in GV $(P<0.05)$. After fertilization, transcript levels of clock, bmall, and cryl significantly decreased from early 1 -cell stage to late 2 -cell stage $(P<0.05)$. These decreased transcript levels were maintained until the blastocyst stage after the late 2-cell stage. Immunocytochemistry analysis showed the nuclear localization of CLOCK and BMAL1 in early and late 2-cell embryos and of CRY1 in early 2-cell embryos but no signals of PER2 in oocytes or pre-implantation embryos. Because mouse oocytes and 1- to 2-cell embryos are transcriptionally inert, the abundant transcripts of clock, bmall, and cryl in these stages seemed to indicate that they were synthesized and stored during the oocyte growth phase. Moreover, the nuclear localization of CLOCK, BMAL1, and CRY1 in the oocytes and 1- to 2-cell stage embryos suggested that some clock genes were translated and worked for oocyte maturation and early embryogenesis

This study was supported by a Grant-in-Aid for the 21st Century COE Program of the Japan Mext and by a grant for the Wakayama Prefecture Collaboration of Regional Entities for the Advancement of Technology Excellence of the JST.

\title{
119 EARLY BOVINE GESTATIONAL DEVELOPMENT: MANIPULATED (IVF) AND NOT MANIPULATED IN LABORATORY
}

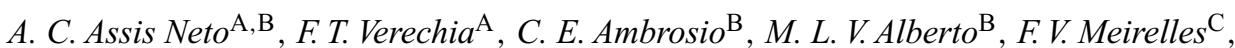

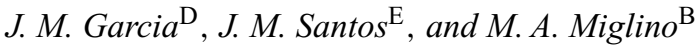 \\ A Faculdade de Zootecnia/UNESP, Dracena, São Paulo, Brazil; ${ }^{\mathrm{B}}$ Universidade de Sao Paulo/USP, São Paulo, Brazil; ${ }^{\mathrm{C}}$ Faculdade de Zootecnia e \\ Engenharia de Alimentos/FZEA USP, Pirassununga, São Paulo, Brazil; ${ }^{D}$ Faculdade de Ciencias Agrarias e Veterinaria/UNESP, Jaboticabal, São \\ Paulo, Brazil; ${ }^{\mathrm{E}}$ Universidade Anhembi Morumbi, São Paulo, Brazil
}

High embryonic and fetal death rates in manipulated embryos in laboratory suggest that the process of early placentation can be inefficient. This investigation aimed to evaluate the development of placenta, and organogenesis of Nelore bovine embryos, and fetuses by natural stud and in vitro fertilization (IVF) over the period from 15 to 70 days of pregnancy. Fifty-nine embryos (15 to 50 days of gestation), 9 fetuses in initial period ( 60 to 70 days of gestation), and 10 embryos originated by IVF technique ( 35 to 46 days of gestation) were used. The same semen was used for all IVF, except the embryos originated by natural stud. The embryos were prepared by serial sections and the fetal membranes were fixed in $4 \%$ paraformaldehyde for light and scanning electron microscopy (SEM) and 2.5\% glutaraldehyde for transmission electron microscopy (TEM). All material was routinely processed and stained. The embryos and fetuses originating from natural stud showed an increase in growth based on the weight of the gestational sac, and related with cranio-caudal and dorso-ventral length of the chorioallantois and amniotic membrane in periods from 20 to 30 and from 50 to 60 days of the pregnancy. The gross appearance of the first cotyledons in development $(9.39 \pm 0.73 \mathrm{~cm})$ was quantified at 30 to 40 days of pregnancy. The IVF embryos on days 35 to 46 of gestation showed discreet cotyledons presenting a functional decrease of development related to embryo derived naturally. Ultrastructurally, the trophoblast showed binucleate trophoblast giant cells with a cytoplasm rich in electrodense vesicles and few mitochondria located in the apical poles suggesting lower cell activity. The yolk sacs of IVF embryos were shorter $(1.07 \pm 0.55 \mathrm{~cm})$ when compared with the natural group $(5.53 \pm 3.14 \mathrm{~cm})$ over 30 to 40 days of pregnancy. In both groups, the epithelium of the yolk sac presented cells with round nuclei, hemangioblast cells, and blood islands with a great number of primitive mononuclear cells. Embryos by natural stud ( $10 \%$ of analyzed ones) showed malformations in cephalic and frontal curvature, encephalocele, gastroschisis, and hepatomegaly. In conclusion, these results indicate occurrence of alteration in the organogenesis in bovine embryos originated by natural stud and retardation of fetal membrane development in bovine embryos by IVF.

This work was supported by FAPESP and CAPES.

\section{EMBRYO SURVIVAL FOLLOWING LIPID-BASED TRANSFECTION OF 1-CELL STAGE BOVINE EMBRYOS WITH SMALL INTERFERING RNA (siRNA) FRAGMENTS AND/OR DNA}

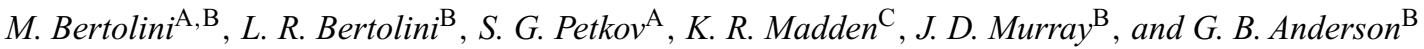 \\ ${ }^{A}$ Embryo Transfer Laboratory, Department of Animal Science, University of California, Davis, CA, USA; ${ }^{\mathrm{B}}$ Transgenics Laboratory, \\ Department of Animal Science, University of California, Davis, CA, USA; ${ }^{\mathrm{C}}$ Invitrogen Co., Carlsbad, CA, USA
}

The RNA interference (RNAi) technology is a powerful tool for studies in functional genomics. The aim of this study was to evaluate the effects of a cationic lipid-based small interfering RNA (siRNA) and/or DNA delivery to 1-cell-stage bovine embryos on survival to the blastocyst stage. In vitro-produced (IVP) embryos were generated according to Bertolini et al. 2002 (Theriogenology 58, 973), and cloned embryos were produced by the handmade cloning technique (Vajta et al. 2003 Biol. Reprod. 68, 571) using green fluorescent protein (GFP)-expressing fibroblast cells as nuclear donors. Lipofections were performed on zona-free 1-cell-stage IVP embryos at $24-28 \mathrm{~h}$ post-fertilization by exposure to $1 \%$ (v/v) Lipofectamine 2000 (Invitrogen Co., CA, USA), 0.002\% (w/v) GFP plasmid (pEFGP-N1, Clontech Laboratories, CA, USA) and/or various doses of siRNA GFP-specific siRNA oligonucleotide (Invitrogen) or DNA methyltransferase 1 (Dnmt1)-specific siRNA fragments for 60 min at $39^{\circ} \mathrm{C}$, according to 
5 treatment groups: (1) zona-intact IVP embryos (controls), (2) zona-free control embryos (controls for embryo development after zona removal), (3) embryos treated with GFP + GFP-siRNA at $0,50,100,200,400$, or $800 \mathrm{~nm}$, (4) embryos treated with Dnmt1-siRNA at 0, 50, 100, 250, or $500 \mathrm{nM}$, and (5) cloned embryos (positive controls for GFP expression). After treatment, embryos were in vitro-cultured in a WOW culture system (Vajta et al. $2000 \mathrm{Mol}$. Reprod. Dev. 55, 256) for 7 days. Cleavage and developmental rates to at least 8-cell and to blastocyst stages were assessed at 48,96 , and $168 \mathrm{~h}$ post-fertilization (hpf), respectively. Data were analyzed by the chi-square test. Cleavage rates in embryos treated with higher doses of siRNA were lower than in all other groups (Table 1). Embryo survival to at least 8-cell stage at $48 \mathrm{~h}$, based on cleavage, was similar among all treatments (data not shown), but survival to blastocyst stage was affected by higher doses of GFP- or Dnmt1-siRNA (Table 1). After a qualitative assessment by fluorescence microscopy at $168 \mathrm{hpf}, 40$ to $63 \%$ of GFP-transfected blastocysts showed various levels of fluorescence, irrespective of the siRNA treatments. Fragments of siRNA are known to be short-lived in cultured cells, although we are still uncertain of their behavior and effects in early bovine embryos. We are currently analyzing the effectiveness of the siRNA transfection in the early IVP and clone embryo. In conclusion, liposome transfection of 1-cell-stage embryos did not affect survival and development to the blastocyst stage. However, survival followed an siRNA dose-response effect, with doses higher than $400 \mathrm{~nm}$ appearing to be detrimental to embryo development, with a developmental arrest at or close to the embryonic genome activation period.

Table 1. Developmental rate of bovine embryos following lipid-based transfection at the 1-cell-stage

\begin{tabular}{lccccr}
\hline Embryo treatment & siRNA, nM & Cleavage rate & $\%$ & Blastocyst rate & $\%$ \\
\hline Zona-intact & $\mathrm{nt}$ & $91 / 109^{\mathrm{a}}$ & 83 & $22 / 91^{\mathrm{a}}$ & 24 \\
Zona-free & $\mathrm{nt}$ & $51 / 63^{\mathrm{a}}$ & 81 & $16 / 51^{\mathrm{a}}$ & 31 \\
Clone & $\mathrm{nt}$ & $37 / 54^{\mathrm{ab}}$ & 69 & $9 / 37^{\mathrm{a}}$ & 24 \\
Dnmt1 & 0 & $23 / 27^{\mathrm{a}}$ & 85 & $9 / 23^{\mathrm{a}}$ & 39 \\
& 50 & $35 / 43^{\mathrm{a}}$ & 81 & $13 / 35^{\mathrm{a}}$ & 37 \\
& 100 & $41 / 46^{\mathrm{a}}$ & 89 & $14 / 41^{\mathrm{a}}$ & 34 \\
& 250 & $31 / 45^{\mathrm{ab}}$ & 69 & $5 / 31^{\mathrm{abc}}$ & 16 \\
GFP+ siRNA & 500 & $7 / 14^{\mathrm{b}}$ & 50 & $0 / 7^{\mathrm{c}}$ & 0 \\
& $05 / 79^{\mathrm{a}}$ & 82 & $17 / 65^{\mathrm{ab}}$ & 26 \\
& 50 & $39 / 45^{\mathrm{a}}$ & 87 & $9 / 39^{\mathrm{ab}}$ & 23 \\
& 100 & $37 / 42^{\mathrm{a}}$ & 88 & $4 / 37^{\mathrm{b}}$ & 11 \\
& 200 & $37 / 47^{\mathrm{a}}$ & 79 & $8 / 37^{\mathrm{ab}}$ & 22 \\
& 400 & $25 / 43^{\mathrm{b}}$ & 58 & $4 / 25^{\mathrm{bc}}$ & 16 \\
& 800 & $16 / 21^{\mathrm{ab}}$ & 76 & $0 / 16^{\mathrm{c}}$ & 0 \\
\hline
\end{tabular}

$\mathrm{nt}=$ not treated; ${ }^{\mathrm{a}-\mathrm{c}} P<0.05$ (columns).

\title{
121 DEMETHYLATION OF MAMMALIAN SOMATIC DNA BY XENOPUS EGG AND
} OOCYTE EXTRACTS

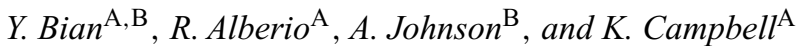 \\ ${ }^{A}$ School of Biosciences, University of Nottingham, Loughborough, Leics, LE12 5RD, UK; \\ ${ }^{B}$ Institute of Genetics, University of Nottingham, Queens Medical Centre, Nottingham, NG7 2UH, UK
}

In mammals, the successful development of live offspring by somatic cell nuclear transfer (SCNT) has demonstrated the ability of oocyte or egg cytoplasm to reprogram the differentiated status of somatic DNA. However, the efficiency of development is low, and this has been attributed to incomplete or inappropriate reprogramming of epigenetic status. One such epigenetic marker is methylation of genomic DNA at CpG islands. In SCNT, derived embryo abnormal DNA methylation patterns have been reported by a number of groups; in particular, it has been observed that the methylation pattern of embryonic cells resembles that of the donor cell (Santos et al. 2003 Curr. Biol. 13, 1116-1121). One strategy to improve reprogramming and, hence, development is to erase or reprogram the epigenetic status of the donor cell prior to nuclear transfer. We have previously reported that Xenopus egg and oocyte extracts show a differential effect on transcription. In oocyte extracts Pol I and II transcripts are maintained in the somatic cells; in egg extracts, these are abolished (Alberio et al. 2005 Exp. Cell. Res. 307, 131-141). To extend these studies, we have investigated the ability of oocyte and egg extracts to demethylate bovine somatic DNA. Preparation of Xenopus oocyte and egg extracts, culture, permeabilization of donor cells, and incubation conditions were all as previously described (Alberio et al. 2005 Exp. Cell. Res. 307, 131-141). Cells were incubated in extracts for 1 and $3 \mathrm{~h}$ at $21^{\circ} \mathrm{C}$, centrifuged onto glass slides fixed in $4 \%$ Para formaldehyde for $15 \mathrm{~min}$, followed by $4 \mathrm{M}$ HCL for $1 \mathrm{~h}$ at $39^{\circ} \mathrm{C}$, and blocked for $1 \mathrm{~h}$. Cells were stained with mouse monoclonal anti-1MeC $(1: 50)$ overnight at $4^{\circ} \mathrm{C}$ followed by FITC-conjugated goat anti-mouse antibody $(1: 20)$ for $1 \mathrm{~h}$ at room temperature and mounted in Vectashied containing $10 \mu \mathrm{g}$ of propidium iodide/mL. Nuclei were scored as positive or negative for $5 \mathrm{MeC}$ staining. In control cells, $90 \%$ of nuclei stained positively for $5 \mathrm{MeC}$. In both oocyte and egg extracts the number of positive nuclei decreased with time showing demethylation of the somatic DNA 68 and 58\% and 38 and 42\% positive, respectively, after 1 and $3 \mathrm{~h}$ of incubation. Addition of apyrase (2\%) to hydrolyze ATP inhibited demethylation in both extracts ( $90 \%$ nuclei positive). High rates of DNA replication were observed in somatic cells in egg extracts in contrast to no replication in oocyte extracts. Aphidicolin $(1 \mu \mathrm{g} / 20 \mu \mathrm{L})$ was added to egg extracts to inhibit DNA replication, and under these conditions, DNA demethylation was abolished, suggesting a passive DNA demethylation mechanism as a result of DNA replication. In conclusion, Xenopus laevis oocyte and egg extracts can demethylate mammalian somatic DNA in an energy-dependent manner. In oocyte extracts, demethylation is independent of DNA replication, suggesting an active mechanism. In egg extracts, 
DNA replication is required, suggesting a passive mechanism. These studies further demonstrate the differences in reprogramming activities between oocyte and egg cytoplasm and suggest that interspecies extracts may provide a tool for nuclear reprogramming.

\title{
122 UNIQUE EXPRESSION PATTERNS OF DIFFERENTIATION, GROWTH, AND CELL STRUCTURE FACTORS IN THE ELONGATING PORCINE CONCEPTUS
}

\author{
L. A. Blomberg, J. R. Miles, and K. A. Zuelke \\ USDA-ARS, Biotechnology and Germplasm Laboratory, Beltsville, MD 20705, USA
}

\begin{abstract}
Elongation of the trophectoderm and gastrulation of the embryonic disc, observed during gestational Days 11 (D11) through 12 (D12), denote a critical period of porcine conceptus development. Serial analysis of gene expression identified genes involved in cellular differentiation/structure (cytokeratin-8 and -18) and growth/cell migration/mesoderm-epithelial interaction (stratifin and midkine), which could potentially be regulated by steroids such as estrogen. Characterization of these factors is lacking in porcine conceptuses, therefore, the current study investigated mRNA expression of these factors in elongating conceptuses and primordial tissues as well as protein expression and cellular localization to better define their biological significance. Conceptuses examined were of ovoid (D11;6-10 mm), tubular (D11; 11-50 mm), or filamentous (D12; >100 mm) morphology. Cells of the conceptus were highly proliferative at all stages and the embryonic disc of the ovoid conceptus was already polarized as indicated by the protein expression of Ki67 and brachyury. Real-time PCR was utilized to determine the transcript expression profiles. Differential expression of cytokeratin-18 and midkine were not apparent; however, cytokeratin-8 was clearly down-regulated in filamentous compared to ovoid conceptuses. In contrast, stratifin mRNA levels were greatest in tubular conceptuses of $42-50 \mathrm{~mm}$ size. Transcripts for cytokeratin- 8 and -18 , stratifin, and midkine were detected in both cell types (endoderm and trophoblast) of the trophoectoderm. Western blotting and/or immunohistochemistry were utilized to examine protein expression and cellular localization. The embryonic disc of ovoid conceptuses was almost devoid of cytokeratin-18 protein, however, its distribution was uniform throughout the trophectoderm at all stages of elongation. Stratifin and midkine proteins demonstrated more unique expression patterns within the conceptus. Distinct cell populations of the embryonic disc and the trophoectoderm contained stratifin; cellular localization was predominantly cytoplasmic but occasional nuclear translocation was evident. Furthermore, total protein levels of stratifin were not different among ovoid, tubular, and filamentous conceptuses, but proteolysis of the protein was apparent at the filamentous stage. Midkine protein expression was prominent in the embryonic disc of ovoid conceptuses. In tubular conceptuses, midkine was associated with cells that appeared to be migrating away from embryonic disc as well as some concentrating in the tips of the trophectoderm. Our findings suggested that cytokeratin- 8 and -18 are associated primarily with the trophectoderm, as seen in other species. Furthermore, the distribution and localization of stratifin and midkine proteins could reflect attributed functions of these factors, minimal anti-proliferative activity in the rapidly growing conceptuses and cell migration important for gastrulation/trophectoderm elongation, respectively.
\end{abstract}

\section{HISTONE H3 MODIFICATIONS IN PIG OOCYTES DURING GROWTH, MATURATION, AND ACTIVATION}

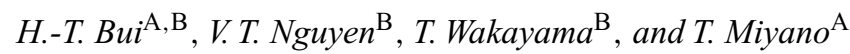 \\ ${ }^{A}$ Department of Life Science, Graduate School of Science and Technology, Kobe University, Kobe, Japan; \\ ${ }^{B}$ Laboratory for Genomic Reprogramming, RIKEN-Center for Developmental Biology, Kobe, Japan
}

Oocyte growth, maturation, and activation are complex processes that include transcription, heterochromatin formation, chromosome condensation and decondensation, two consecutive chromosome separations, and genomic imprinting for producing the mature egg. The first sign of oocyte maturation is phosphorylation of histone H3, which leads to the chromosome condensation (Bui et al. 2004 Biol. Reprod. 70, 1843-1851). The objective of this study was to investigate the change in chromosome morphology in relation to histone modifications in pig oocytes during growth, maturation, and activation. Growing oocytes were collected from follicles at various diameters (from 0.1 to $6 \mathrm{~mm}$ ) in pig ovaries. For maturation, oocyte-cumulus-granulosa cell complexes (OCGC) were collected from follicles that were 3 to $6 \mathrm{~mm}$ in diameter and cultured in modified TCM 199 for different periods of time to obtain meiotic stages of oocytes. For activation, oocytes were cultured for maturation in $42 \mathrm{~h}$ and were activated using a protocol that was described previously (Nguyen et al. 2003 Theriogenology 59, 719-734). Then, oocytes were examined by immunostaining with antibodies: anti-phospho-histone $\mathrm{H} 3$ at serine 10 or serine 28 (S10 or S28), anti-trimethyl-histone $\mathrm{H} 3$ at lysine 9 (K9), and anti-acetyl-histone H3 at lysine 9, 14, or 28 (K9, K14, or K28). Some oocytes were examined for double assay of Cdc2 and H3 kinase, which were measured by phosphorylation of histone $\mathrm{H} 1$ and myelin basic protein as their substrates. To examine the effects of histone deacetylase (HDAC) inhibition, OCGC were cultured in maturation medium supplemented with or without $100 \mathrm{nM}$ trichostatin $\mathrm{A}$ for $42 \mathrm{~h}$. The results show that, during the growth phase, histone $\mathrm{H} 3 \mathrm{became}$ methylated at $\mathrm{K} 9$ and is acetylated at $\mathrm{K} 9, \mathrm{~K} 14$, and $\mathrm{K} 18$. When the fully grown oocytes start maturation, histone $\mathrm{H} 3$ becomes phosphorylated at $\mathrm{S} 28$ and then $\mathrm{S} 10$ and is deacetylated at $\mathrm{K} 9, \mathrm{~K} 14$, and $\mathrm{K} 18$. After oocyte activation, reacetylation and dephosphorylation of histone $\mathrm{H} 3$ correlates to the decondensation of chromosomes. We also found that the activity of histone $\mathrm{H} 3$ kinase occurred at a similar time course to that of phosphorylation of histone H3-S28. This suggests that phosphorylation of H3-S28 might be one of the key events initiating meiotic chromosome condensation. The inhibition of HDAC induces maintenance of acetylation of H3-K14 and dephosphorylation of histone $\mathrm{H} 3$ at S10 and S28. Therefore, the chromosome could not condense and affect meiotic progression. It is possible that deacetylation is required for the phosphorylation of histone $\mathrm{H} 3$. The results suggest that chromatin morphology of pig oocytes is regulated by acetylation/deacetylation and phosphorylation/dephosphorylation of histone $\mathrm{H} 3$ and that histone deacetylase activity is essential for the process of chromatin remodeling in pre-ovulatory oocytes. Although histone acetylation and phosphorylation were reversible, histone methylation has energetic stability and is established during the oocyte growth phase. It is also suggested that the ordered phosphorylation of histone $\mathrm{H} 3$ at S10 and S28 is influenced by acetylation of neighboring lysines in the histone $\mathrm{H} 3$ molecule. 


\title{
124 KNOCKING-IN OF A FOREIGN GENE ON A BOVINE BETA-CASEIN GENE INTO BOVINE PRIMARY FIBROBLASTS USING HOMOLOGOUS RECOMBINATION EVENTS
}

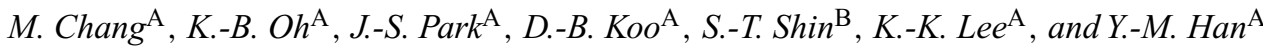 \\ ${ }^{A}$ Korea Research Institute of Bioscience and Biotechnology, Daejeon, 305-106, South Korea; \\ ${ }^{B}$ College of Veterinary Medicine, Chungnam National University, Daejeon, 305-164, South Korea
}

Knocking-in of a foreign gene on a tissue-specific genomic region has not been reported in livestock. In this study, we constructed two knock-in vector cassettes specific for the bovine beta-casein gene which is expressed only in the mammary gland during lactation. The targeting vector cassettes, pBCKI1 and pBCKI2, have homology regions for the bovine beta-casein gene and contain $13.1 \mathrm{~kb}$ and $9.1 \mathrm{~kb}$ targeting arms with different long arm lengths, respectively. The targeting vector cassettes have unique restriction enzyme sites for insertion of foreign therapeutic genes in front of the neo gene which was inserted into the vector as a selection marker. The human thrombopoietin (hTPO) gene was inserted into the restriction enzyme sites of both targeting vectors, which were named $\mathrm{pBCTPOKI} 1$ and $\mathrm{pBCTPOKI}$. When the two targeting vectors were transfected into bovine ear skin fibroblasts using Lipofectamine (Invitrogen, Seoul, South Korea), 6.3\% of neo resistant clones (2/32) were homologously targeted with the pBCTPOKI2 vector. Cells from the targeted colonies were nuclear-transferred into enucleated bovine oocytes and cultured to the blastocyst stage. A total of 66 blastocysts were generated of which 46 were transferred into recipients. No offspring have been produced at this time. Here we first describe knock-in vectors specific for a bovine beta-casein gene, which will be employed to generate animal bioreactors that produce therapeutic proteins secreted into the milk.

\section{ISOLATION OF MULTILINEAGE STEM CELLS FROM THE PORCINE MAMMARY FAT PAD}

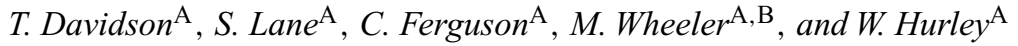 \\ ${ }^{A}$ Department of Animal Sciences, University of Illinois, Urbana, IL, USA; \\ ${ }^{B}$ Department of Bioengineering, University of Illinois, Urbana, IL, USA
}

Porcine mammary tissue is a dynamic system that undergoes multiple cycles of growth, differentiation, and regression during the life cycle of a female; however, the mechanisms of mammary morphogenesis are not fully understood. The current hypothesis suggests that epithelial stem cells in the terminal end buds interact with surrounding epithelial and stromal cells during mammary tissue development and regeneration. Because the mammary gland of the virgin animal consists largely of a pad of adipose tissue, we propose that adipose-derived stem cells from the mammary fat pad (MFP) may also play a role in the regeneration process. Therefore, the objective of this experiment was to determine whether multilineage stem cells are present in the MFP of gilts. Adipose tissue from the MFP and back fat (BF) from the loin region were harvested from six nulliparous, cycling Yorkshire gilts, approximately 16 mo of age. Methods for isolation of adipose-derived stem cells were modified from those of Malusky and Wheeler (2004; International Society for Stem Cell Research, Proceedings 2nd Annual Meeting, abstr. 248:124). Presumptive stem cells isolated from each tissue type were maintained in vitro in DMEM supplemented with either $10 \%$ newborn calf serum (NCS; Rep 1 and 2) or $10 \%$ fetal calf serum (Rep 3). BF and MFP cell types formed colonies of fibroblast-like cells within 3 to $5 \mathrm{~d}$. Growth characteristics are shown in Table 1 . When the cells were $80 \%$ confluent, they were trypsinized and reseeded into $75-\mathrm{cm}^{2}$ flasks. When cultured in the presence of NCS, both cell types had a progressive decrease in viability, and these cultures could not be maintained past Passage 4. Differentiation of both cell types (Passage 1) into adipogenic, myogenic, and osteogenic lineages are currently underway. Based on morphological evaluations, both cell types are able to differentiate into the aforementioned lineages; however, differentiation of MFP-derived cells occurred at a slower rate and was less pronounced than that of BF-derived cells. These preliminary findings suggest that the MFP likely contains a population of multipotent stem cells; however, at this time, it is not possible to make meaningful statistical comparisons. Further experiments are needed to fully characterize these cells and determine their role in mammary gland morphogenesis.

Table 1. Growth of porcine adipose-derived stem cells obtained from the BF or from the MFP

\begin{tabular}{|c|c|c|c|c|c|c|}
\hline & \multicolumn{2}{|c|}{ Rep 1} & \multicolumn{2}{|c|}{ Rep 2} & \multicolumn{2}{|c|}{ Rep 3} \\
\hline & $\mathrm{BF}$ & MFP & $\mathrm{BF}$ & MFP & $\mathrm{BF}$ & MFP \\
\hline \multicolumn{7}{|c|}{ Pop Dblgs ${ }^{\mathrm{a}}$} \\
\hline Pas 1 & 4.0 & 3.3 & 2.1 & 2.1 & 2.2 & 1.8 \\
\hline Pas 2 & 4.0 & 4.0 & 2.4 & 2.2 & 1.8 & 2.2 \\
\hline Pas 3 & 3.5 & 1.7 & - & - & - & - \\
\hline \multicolumn{7}{|c|}{ Hours/Dblgb } \\
\hline Pas 1 & 36.4 & 43.2 & 104.5 & 103.3 & 55.8 & 65.6 \\
\hline Pas 2 & 48.2 & 48.2 & 69.7 & 76.4 & 93.9 & 76.0 \\
\hline Pas 3 & 48.0 & 99.4 & - & - & - & - \\
\hline
\end{tabular}

a Pop Dblgs = population doublings; number of times the original cell population doubled before passage

${ }^{\mathrm{b}}$ Hours/Dblg = hours per doubling; number of hours for one population doubling to occur. 


\title{
NUCLEAR TRANSLOCATION OF NUCLEAR FACTOR KAPPA B AND ITS ROLE IN MOUSE PRE-IMPLANTATION DEVELOPMENT
}

\author{
Y. Fumiiwa, H. Imai, and M. Yamada \\ Laboratory of Reproductive Biology, Graduate School of Agriculture, Kyoto University, Kyoto, Japan
}

\begin{abstract}
In mouse pre-implantation development, it has been reported that RelA, one of the subunits of nuclear factor kappa B (NF-kB), is expressed in eggs and embryos from the Metaphase II oocyte to the blastocyst stage. However, the role of NF- $\mathrm{KB}$ in the pre-implantation development has not yet been elucidated in detail. In this study, we examined (1) the activation of NF- $\kappa \mathrm{B}$ during mouse pre-implantation development and (2) the effect

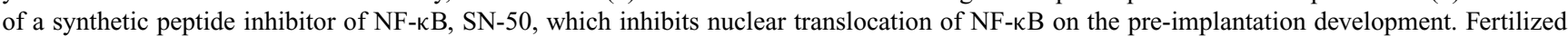
one-cell embryos were collected $17 \mathrm{~h}$ post-hCG from the ampullae of oviducts of superovulated ICR mouse females that had been mated with the same strain of males and then were cultured in $\mathrm{KSOM}$ medium at $37^{\circ} \mathrm{C}$ under $5 \% \mathrm{CO}_{2}$ in air for $4 \mathrm{~d}$. To elucidate the timing of $\mathrm{NF}-\mathrm{kB}$ activation, we examined the localization of NF- $\mathrm{kB}$ in the nucleus by an immunofluorescence approach using RelA antibody with a laser confocal microscope. RelA was distributed mainly in the cytoplasm of embryos from the one-cell stage through the blastocyst stages. The presence of RelA in the nucleus, evidence for NF- $\mathrm{kB}$ activation, was observed in embryos from the one-cell to the compacted 8-cell stages. Moreover, we observed RelA punctate localization in nucleoplasm of embryos from the one-cell to the 4-cell stages, and nuclear dots were enriched conspicuously in the one-cell embryos and the late 2-cell embryos. These results suggest that NF-кB is activated in embryos from the one-cell to the compacted 8-cell stages and that its activation seems to be particularly strong at the developmental stage when RelA appeared to be concentrated in nuclear dots, as it has been reported that NF- $\mathrm{KB}$ and other transcription factors and co-activators form punctate structures called 'enhanceosom' in association with particular promoters in the nucleus. Next, we examined the effect of SN-50 on the pre-implantation development of mouse embryos. When embryos were treated with SN-50 at $20 \mu \mathrm{g} / \mathrm{mL}$ from the 2-cell stage, $63 \%$ (33 of 52) of the embryos developed to blastocysts, but 55\% (18 of 33) of the blastocysts showed abnormal morphology, such as poor cavitation, and many degenerating cells extruded into the perivitelline space. The percentages of 2 -cell embryos that formed morphologically normal blastocysts were significantly lower in the $\mathrm{SN}-50$ treatment group $(29 \% ; 15$ of 52$)$ than in the untreated control group $(76 \% ; 35$ of 46$)$ and in the SN-50M (inactive analogue of SN-50, $20 \mu \mathrm{g} / \mathrm{mL})$ treatment group $(72 \% ; 38$ of 53$)$. These experiments were done in 4 replicates, and the statistical analyses of the data were done by ANOVA and Fisher's PLSD test. Nuclear location of RelA was not observed in the embryos at the 4-cell stage when treated with SN-50 from the 2-cell stage, although observed in control and SN-50M-treated embryos. Furthermore, it was found that most of embryos (23 of 37) treated with SN-50 from the compacted 8-cell or morula stages developed normally to the blastocyst stage as control embryos ( 25 of 36 ). These results suggest that morphological aberration at the blastocyst stage is elicited by inhibiting NF-kB activation.
\end{abstract}

\section{BINDING RETINOID RECEPTORS BY SPECIFIC AGONISTS AFFECTS THE BOVINE} BLASTOCYST DEVELOPMENT IN VITRO

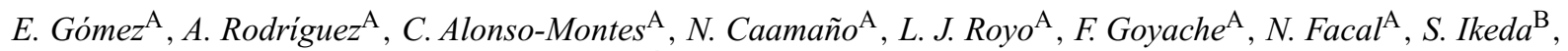 \\ I. Álvarez ${ }^{\mathrm{A}}$, and C. Díez \\ ${ }^{\mathrm{A}}$ Genética y Reproducción, SERIDA, Gijón, Asturias, Spain; \\ ${ }^{B}$ Livestock Farm of Graduate School of Agriculture, Kyoto University, Tamba, Kyoto Prefecture, Japan
}

\begin{abstract}
Production of embryos in vitro with improved inner cell mass (ICM) and high ICM per total cell rate is a major objective in reproductive biotechnology. Exogenous all-trans retinoic acid (ATRA), a vitamin A metabolite, and endogenous retinoid regulate development and differentiation during bovine morula to blastocyst transition in vitro. ATRA binds to retinoic acid-receptor (RAR), and the ATRA isomere 9-cis-retinoic acid (9-cis-RA) binds to both RAR and the retinoid X receptor (RXR). The unspecific binding of 9-cis-RA to receptors makes it difficult to study RXR transactivation. Therefore, in this work we studied blastocyst development and cell counts by using a specific synthetic RXR agonist [LG100268 LG; a gift of Ligand Laboratories] as opossed to the effect exerted by ATRA upon RAR binding. Cumulus-oocyte complexes from slaughterhouse ovaries were matured and fertilized in vitro. Presumptive zygotes were cultured in B2 medium with Vero cells until $139 \mathrm{~h}$ post-insemination (Day 6), the time at which embryos [morulae $(\geq 90 \%)+$ early blastocysts] underwent treatments for $48 \mathrm{~h}$ in $400 \mu \mathrm{L}$ of SOFaaci $+5 \%$ FCS. Data $(5$ replicates per experiment) were analyzed by CATMOD for effects, processed by GLM and Duncan's test, and expressed as LSM \pm SE (a,b,c $P \leq 0.05)$. After a LG dose-response experiment ( $n=480$ morulae), blastocysts rates from LG $1 \mu \mathrm{M}$ on Day 7 were higher than LG $10 \mu \mathrm{M}, \mathrm{LG} 0.1 \mu \mathrm{M}$, and LG $0 \mu \mathrm{M}$ (Day 7: $42.8 \pm 4.1$ vs. $34.4 \pm 3.7,36.8 \pm 3.7$, and 32.4 \pm 3.7 , respectively). On Day 8, LG $1 \mu \mathrm{M}$ also yielded more blastocysts than LG $0.1 \mu \mathrm{M}$ $(50 \pm 4.2$ vs. $44.4 \pm 3.7$, respectively). By differential cell counting ( $n=113$ blastocysts), hatched blastocysts with LG $10 \mu \mathrm{M}$ showed proliferation in the ICM, while trophectoderm (TE) cells decreased conversely to LG concentration. These effects were not obvious in expanded blastocysts. In a subsequent experiment $(n=340$ morulae), ATRA led to blastocysts rates on Day 8 that were higher than negative, untreated controls, but not different from LG $1 \mu \mathrm{M}(42.4 \pm 2.4$ vs. $33.1 \pm 2.0$ and $36.0 \pm 2.4$, respectively). ATRA and LG 1 increased TE in expanded blastocysts $(n=42)(102 \pm 13.2$ and $96.23 \pm 13.2$, respectively vs. $72.8 \pm 10.9$ in the untreated group) but not in their hatched counterparts $(n=44)$. There were no differences in the ICM; but percentages of ICM per total cells were higher in hatched blastocysts cultured with ATRA than in expanded LG $1 \mu$ M blastocysts and expanded controls ( $39.5 \pm 5.5$ vs. $24.2 \pm 5.7$, and $20.9 \pm 4.7$, respectively). Manipulation of retinoid receptor-specific pathways make it possible to control blastocyst development and differentiation, leading to embryos of improved quality and viability. Work is in progress to analyze gene expression in these blastocysts.
\end{abstract}




\title{
128 MITOCHONDRIAL DNA DELETIONS IN RHESUS MACAQUE OOCYTES, EMBRYOS, AND ADULT AND EMBRYONIC STEM CELLS
}

\author{
T. Gibson, T. Quebedeaux, S. Rajasekaran, and C. Brenner \\ University of New Orleans, New Orleans, LA, USA
}

\begin{abstract}
Mitochondria are the most abundant organelles in mammalian oocytes and early embryos. Previous data have shown that mitochondrial DNA (mtDNA) deletions are present both in human oocytes and in embryos from in vitro fertilization (IVF) patients and suggest that accumulation of these deletions may contribute to mitochondrial dysfunction and impaired ATP production. In addition, high levels of mitochondrial mutations are present in skeletal muscle fibers from aged rhesus macaques. The specific aims of this study were to determine whether the mitochondrial common deletion is present in non-human primate oocytes and embryos generated by IVF and to determine whether mtDNA mutations are already present in immature oocytes from rhesus ovaries. Using a nested primer polymerase chain reaction (PCR) strategy, we determined the frequency of the rhesus common deletion in immature oocytes compared with stimulated oocytes and embryos. There was a low incidence $(21 \%)$ of the rhesus common deletion present in immature, unstimulated oocytes derived from necropsied ovaries of 2 to 10 -yr-old rhesus macaques. However, there was $>3$-fold increase (71.4\%) in the frequency of deleted mtDNA in stimulated oocytes and IVF embryos from age-matched fertile monkeys. We postulated that, in addition to skeletal muscle, a similar time-dependent accumulation of mtDNA deletions occurs in fertile rhesus macaque oocytes and embryos. We are now investigating the effects of culture and passage number on mtDNA deletions in primate adult and embryonic stem cells. We propose the rhesus monkey to be an excellent model to assess the quality of gametes and embryos, as well as stem cells, and their developmental competence in human and non-human primates.
\end{abstract}

This study was supported by National Institutes of Health grants RR15395 and HD045966.

\section{INSULIN-LIKE GROWTH FACTOR-I PROMOTES BLASTOCYST DEVELOPMENT OF HEAT-SHOCKED BOVINE EMBRYOS INDEPENDENT OF ITS ANTI-APOPTOTIC EFFECTS REQUIRING P13K SIGNALING}

\author{
F. D. Jousan and P. J. Hansen \\ University of Florida, Gainesville, FL, USA
}

Insulin-like growth factor-I (IGF-I) reduces effects of heat shock on blastocyst development and induction of apoptosis. The present objective was to test whether IGF-I would allow for blastocyst development following heat shock because of its anti-apoptotic effects. Because anti-apoptotic actions of IGF-I require signaling through the phosphatidylinositol 3-kinase (PI3K) pathway, an inhibitor of PI3K (LY 294002) was used in Exp. 1 to determine whether it would prevent the thermoprotective effects of IGF-I on development. Embryos were produced in vitro in KSOM-BE2 medium $\pm 100 \mathrm{ng} \mathrm{mL} \mathrm{m}^{-1}$ of IGF-I. Embryos $\geq 16$ cells at $5 \mathrm{~d}$ post-insemination (dpi) were placed in fresh drops containing the same IGF-I treatment as well as LY $294002(100 \mu \mathrm{M})$ or vehicle $(0.1 \% \mathrm{DMSO})$ and cultured at either $38.5^{\circ} \mathrm{C}$ or $41^{\circ} \mathrm{C}$ for $15 \mathrm{~h}$. All groups were then cultured at $38.5^{\circ} \mathrm{C}$ until $8 \mathrm{dpi}$ when blastocyst development was assessed (10 replicates; 112 to 142 embryos per treatment). For embryos in DMSO, IGF-I did not increase the percentage that became blastocysts when cultured at $38.5^{\circ} \mathrm{C}(59.1$ and $57.1 \%$ for control and IGF-I embryos, respectively; SEM $=3.6 \%)$. Heat shock reduced blastocyst development $(P<0.01)$, and IGF-I blocked this decrease (percent blastocyst $=42.0$ and $54.7 \%$ for control and IGF-I embryos, respectively; SEM $=3.6 \%$ ). Similar results were obtained for embryos cultured with LY 294002. The percentage of embryos becoming blastocysts for control embryos was 53.5 and $35.5 \%$, respectively, at 38.5 and $41^{\circ} \mathrm{C}$, development for IGF-I-treated embryos was, respectively, 55.8 and $49.4 \%$ at 38.5 and $41^{\circ} \mathrm{C}(\mathrm{SEM}=4.8 \%)$. Analysis of the entire data set revealed an IGF-I $\times$ temperature interaction $(P<0.05)$ but no interactions with inhibitor treatment. Thus, IGF-I protected embryos from heat shock in the presence and absence of LY 294002. In Exp. 2, procedures were similar, except that embryos were cultured with a caspase-3 inhibitor (z-DEVD-fmk; $100 \mu \mathrm{M}$ ) instead of LY 294002 (12 replicates; 114 to 137 embryos per treatment). For embryos in DMSO, blastocyst development was reduced by heat shock $(P<0.06)$ and increased by IGF-I $(P<0.06)$. The percentage of embryos becoming blastocysts was 67.0 and $55.8 \%$ for control embryos at 38.5 and $41{ }^{\circ} \mathrm{C}$, respectively, vs. 74.7 and $70.8 \%$ for IGF-I embryos at 38.5 and $41^{\circ} \mathrm{C}$, respectively $(\mathrm{SEM}=5.2 \%$ ). For embryos cultured with $\mathrm{z}$-DEVD-fmk, heat shock reduced blastocyst development $(P<0.01)$, and IGF-I was no longer effective in blocking the reduction in blastocyst development caused by heat shock (IGF-I $\times$ inhibitor treatment; $P<0.01$ ). Blastocyst development was 66.4 and $58.7 \%$ for control embryos at 38.5 and $41^{\circ} \mathrm{C}$, respectively, vs. 61.8 and $48.6 \%$ for IGF-I embryos at 38.5 and $41^{\circ} \mathrm{C}$, respectively $(\mathrm{SEM}=4.6 \%)$. Note that $\mathrm{z}-\mathrm{DEVD}-\mathrm{fmk}$ did not protect embryos from the anti-developmental effects of heat shock but exacerbated heat shock effects for IGF-I-treated embryos. In conclusion, the ability of IGF-I to allow heat-shocked embryos to continue development to the blastocyst stage is independent of its anti-apoptotic effects involving the PI3K pathway but may depend on active caspase-3.

This work was supported by USDA NRICGP 2002-35203-12664, BARD US-3551-04, and USDA TSTAR 2004-34135-14715.

\section{ANTI-APOPTOTIC PTEGT SHOWS A DIFFERENTIAL mRNA TRANSCRIPT LEVEL DURING IN VITRO MATURATION AND EARLY DEVELOPMENT OF PORCINE IVP EMBRYO}

\author{
J.-Y. Kim, J.-H. Kim, H.-Y. Choi, B.-W. Kim, S. J. Uhm, H. T. Lee, and S.-G. Cho
}

Department of Animal Biotechnology, BMIC, and IBST, Konkuk University, Seoul 143-101, South Korea

It is well known that very early development of the mammalian pre-implantation embryo is regulated by gene transcripts and proteins stored in the oocyte and that the embryonic genome gains control of development following 1 to 3 cleavage divisions. An active transcription and translation 
is required for chromatin condensation and germinal vesicle breakdown in pig oocyte. The transition from maternal to embryonic control of development is a gradual event, and following this transition, the maternally derived transcripts and proteins are gradually degraded. Successful embryonic development is dependent on the temporal and stage-specific expression of proper genes, but information on specific gene expression during early stages before zygotic gene activation (ZGA) is limited. Before activation of the embryonic genome, mRNA and proteins synthesized during oocyte growth and maturation contribute to early development. In this study, we compared the mRNA transcripts level among porcine immature, in vitro-matured and cleaved 2- to 4-cell stage embryos after in vitro fertilization to identify genes that show differential mRNA transcript levels during maturation and very early embryonic development. For the first strand cDNA synthesis, oligo (dT) primers were added to the total RNA isolated from each sample. Using annealing control primer (ACP)-based GeneFishing PCR, we detected tens of different genes showing differential mRNA transcript level (DRTL) and nine DRTL genes were identified to be KCRF, CAMSAP1, SMP1, FLJ20647, LOC132321, NADH1, NADH6, HERC3, and TEGT. Of 9 DRTL genes, TEGT showed higher mRNA transcript level at the immaturation stage, and mRNA transcript levels of the other 8 genes were increased after in vitro maturation. Therefore, we focused on TEGT (testis enhanced gene transcript), which is highly expressed in testis and also in oocytes before in vitro maturation. Differential mRNA transcripts pattern of CAMSAP1 and TEGT were confirmed using RT-PCR and real-time RT-PCR. Porcine TEGT (pTEGT) was cloned and sequenced to have an ORF of 714 bp nucleotides and to encode an integral membrane protein. When overexpressed in HEK293 cells, pTEGT suppressed apoptosis induced by etoposide. We found that pTEGT, but not TEGT-C (C-terminal deletion mutant), inhibited etoposide- and staurosporine-induced cell death. Next, we found that introduction of TEGT siRNA suppressed the anti-apoptotic effect of TEGT. Interestingly, expression of TEGT suppressed etoposide-induced ERK activation, suggesting that ERK phosphorylation is involved in the anti-apoptotic function of the gene. Several reports showed that apoptosis and MAP kinase signaling pathways play important roles in oocyte maturation and early embryo development. Therefore, the anti-apoptotic effect of TEGT was suggested to play a key role in the normal ooctye maturation and early embryo development.

This work was supported by the Research Project on the Production of Bio-organs, Ministry of Agriculture and Forestry, Republic of Korea.

\title{
131 DYNAMICS OF HISTONE H3 METHYLATION AT POSITIONS K4 AND K9 IN MOUSE, RABBIT, AND BOVINE PRE-IMPLANTATION EMBRYOS
}

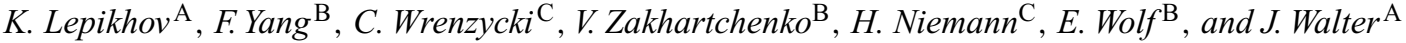 \\ ${ }^{A}$ University of Saarland, Natural Sciences-Technical Faculty III, FR 8.3, Biological Sciences, Genetics/Epigenetics, \\ Saarbrücken, Germany; ${ }^{B}$ Department of Molecular Animal Breeding and Biotechnology, Ludwig-Maximilian University, \\ Munich, Germany; ${ }^{\mathrm{C}}$ Institute for Animal Breeding (FAL), Department of Biotechnology, Neustadt/Mariensee, Germany
}

In mammals, upon the penetration of sperm into the oocyte, the paternal genome undergoes dramatic epigenetic changes. Protamin packaging of DNA is replaced by histones that acquire specific modifications. In mouse zygotes, paternal DNA gets rapidly demethylated by an active mechanism. In bovine zygotes the methylation from paternal DNA is erased only partially, and in rabbit zygotes it persists at the initial level. To understand whether these reprogramming differences are also reflected in histone modifications, we examined the dynamic changes of histone $\mathrm{H} 3$ methylation at positions $\mathrm{K} 4$ and $\mathrm{K} 9$ in mouse, bovine, and rabbit zygotes and in preimplantation embryos using an immunofluorescence staining procedure (Lepikhov and Walter 2004 BMC Dev. Biol. 4, 12). In zygotes, maternal chromatin contains both types of histone H3 methylation. After fertilization protamines in sperm are very quickly replaced by histones. After the formation of nucleosomes, histone $\mathrm{H} 3$ acquires methylation at position $\mathrm{K} 4$ in a stepwise manner: first as mono-methylated form and later as tri-methylated. In the late zygote, both paternal and maternal pronuclei show equal levels of histone H3 methylation at position K4. Regardless of the differences in DNA reprogramming in these 3 species, H3/K9 di-methylation is not detected on paternal genomes and is only associated with maternal genomes. During the subsequent cleavage stages, H3/K9 di-methylation decreases gradually and becomes hardly detectable in 4-cell bovine and rabbit embryos. In mouse embryos, it is detectable through all the stages. Bovine embryos reacquire this type of modification at the $8-16$ cell stage, and it remains at the very low levels in rabbit, embryos until the blastocyst stage. In conclusion, mouse, rabbit and bovine zygotes show similar patterns of H3/K4triMe and H3/K9diMe distribution despite the difference in paternal DNA demethylation. The dynamics of H3/K9diMe distribution patterns in cleavage stage embryos from all embryos do not correlate with embryonic genomic activation events.

\section{DIFFERENCES IN RESPIRATION RATES BETWEEN IN VIVO-AND IN VITRO-PRODUCED BOVINE EMBRYOS}

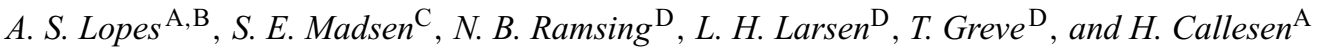 \\ ${ }^{A}$ Danish Institute of Agricultural Sciences, 8830, Tjele, Denmark; \\ ${ }^{B}$ Royal Veterinary \& Agricultural University, 1870, Frederiksberg C, Denmark; \\ ${ }^{\mathrm{C}}$ Trans - Embryo Genetics, 8740 Brædstrup, Denmark; \\ DFertiliTech ApS, 8000 Aarhus C, Denmark
}

In vitro-produced (IVP) bovine embryos differ (e.g. morphology and physiology) from their in vivo counterparts. Oxygen consumption is an indicator of the overall metabolic activity of a single embryo. Therefore, the aim of this study was to determine and compare respiration rates of in vivo- and in vitro-produced bovine day 7 embryos. Diameters of these two embryo types were also compared. In vivo embryos $(n=28)$ were recovered from 8 superovulated Holstein Frisian cows on day 7 following AI, while IVP embryos ( $n=160$; Holm et al. 1999 Theriogenology 52, 683-700) were used on day 7 after fertilization. Embryos were measured (outer diameter) and morphologically evaluated (Quality 1 to 4, IETS Manual, 1998). Only 
transferable in vivo embryos were used (i.e. excluding Quality 4). Respiration rates were measured on each embryo by Nanorespirometer technology (Lopes et al. 2005 Reprod. Fertil. Develop. 17, 151). Data were analyzed using Proc Mixed, and values are presented as mean \pm SEM. Values with different superscripts differ significantly $(P<0.05)$. The average respiration rates were $0.82 \pm 0.06^{\mathrm{a}} \mathrm{nL} / \mathrm{h}$ for in vivo vs. $1.37 \pm 0.06^{\mathrm{b}} \mathrm{nL} / \mathrm{h}$ for IVP embryos. The average respiration rates for the different morphological qualities were as follows ( $\mathrm{nL} / \mathrm{h}$, numbers in brackets): IVP: $2.1 \pm 0.08^{\mathrm{a}}$ (38), $1.37 \pm 0.07^{\mathrm{b}}(55), 1.08 \pm 0.07^{\mathrm{c}}(48)$ and $0.62 \pm 0.11^{\mathrm{d}}(19)$ for Quality $1,2,3$, and 4 , respectively. In vivo: $1.17 \pm 0.21^{\mathrm{b}, \mathrm{c}, \mathrm{e}}(6), 0.80 \pm 0.15^{\mathrm{c}, \mathrm{d}, \mathrm{e}}$ (12), and $0.64 \pm 0.16^{\mathrm{d}, \mathrm{f}}(10)$ for Quality 1,2 , and 3, respectively. The average diameter (mm) of in vivo and IVP embryos was $0.157 \pm 0.002^{\mathrm{a}}$ and $0.176 \pm 0.002^{\mathrm{b}}$, respectively. Respiration rates were directly related to embryo diameter; larger embryos were associated with higher respiration rates $(\mathrm{y}=17.55 \pm 1.32 \mathrm{~nL} / \mathrm{h} \times \mathrm{mm}, n=188)$. Respiration rates of in vivo embryos were significantly lower than those of IVP embryos, regardless of quality. This difference could reflect an effect of the culture conditions on IVP embryos because media components affect embryo metabolism. Moreover, the different ages (day 7 for IVP vs. approximately Day 6.5 for in vivo embryos, because in vivo embryos are less than 7 days after fertilization at recovery) and stages (IVP: up to expanded blastocyst stage; in vivo: morula or early blastocyst stage) could have influenced the results and also partly explain the smaller diameter of the in vivo embryos. Finally, respiration rates decreased proportionately to the morphological quality within embryo type, indicating that morphological differences are reflected at the physiological level. In conclusion, this study further outlines metabolic differences between in vivo and IVP bovine embryos. Whether such differences are a manifestation of metabolic stress associated to the separation from the natural environment or reflect suboptimal culture conditions is yet to be determined.

ASL is supported by FCT, Portugal.

\title{
133 ROLE OF CASPASE-9 AND STAGE OF DEVELOPMENT IN INDUCTION OF APOPTOSIS BY HEAT SHOCK AND TUMOR NECROSIS FACTOR- $\alpha$ IN BOVINE PRE-IMPLANTATION EMBRYOS
}

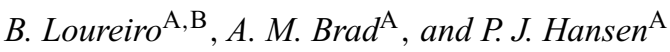 \\ ${ }^{A}$ Dept. of Animal Sciences, University of Florida, Gainesville, FL, USA; ${ }^{\mathrm{B}}$ Departamento de Medicina Veterinária, \\ Universidade Federal Rural de Pernambuco, Recife, PE, Brasil
}

\begin{abstract}
Heat shock and tumor necrosis factor- $\alpha(\mathrm{TNF}-\alpha)$ can increase apoptosis in bovine embryos in a developmental-dependent manner. It was hypothesized that addition of the caspase-9 inhibitor, z-LEHD-fmk, would block induction of apoptosis caused by heat shock of $41^{\circ} \mathrm{C}$ and TNF- $\alpha$. Furthermore, it was hypothesized that the magnitude of induced apoptosis would increase with stage of development. Embryos were collected on day 4,5 , and 6 after in vitro insemination and were cultured for $24 \mathrm{~h}$ in the presence of either $100 \mu \mathrm{M}$ z-LEHD-fmk reconstituted in $0.5 \%$ (v/v) dimethyl sulfoxide or vehicle dimethyl sulfoxide at either (1) $38.5^{\circ} \mathrm{C}$ for $24 \mathrm{~h}$ (control), (2) $41^{\circ} \mathrm{C}$ for $15 \mathrm{~h}$ followed by $38.5^{\circ} \mathrm{C}$ for $9 \mathrm{~h}$, or (3) $38.5^{\circ} \mathrm{C}$ for $24 \mathrm{~h}$ with $10 \mathrm{ng} / \mathrm{mL}$ murine TNF- $\alpha$. Embryos were then fixed, and the proportion of blastomeres undergoing apoptosis was determined using TUNEL labeling. Heat shock did not increase the percentage of blastomeres that were TUNEL-positive ( $\%$ apoptosis) at day 4 ( $n=100$ embryos total). In contrast, heat shock increased \% apoptosis at day 5 and day $6(P<0.04)$ and this effect was blocked by $z$-LEHD-fmk (temperature $\times$ inhibitor, $P<0.04)$. At day $5, \%$ apoptosis in the absence and presence of $z$-LEHD-fmk was $3.8 \pm 1.9 \%$ and $3.7 \pm 1.7 \%$ at $38.5^{\circ} \mathrm{C}$ vs. $8.9 \pm 1.5 \%$ and $4.1 \pm 1.7 \%$ at $41{ }^{\circ} \mathrm{C}\left(n=75\right.$ embryos total). At day $6, \%$ apoptosis in the absence and presence of z-LEHD-fmk was $3.6 \pm 1.1 \%$ and $3.7 \pm 1.2 \%$ at $38.5^{\circ} \mathrm{C}$ vs. $11.1 \pm 1.1 \%$ and $6.1 \pm 1.2 \%$ at $41^{\circ} \mathrm{C}(n=168$ embryos total $)$. Mean cell number at the end of culture ranged from 21 to 26 cells at day 4,43 to 73 cells at day 5, and 101 to 114 cells at day 6 . Treatment with TNF- $\alpha$ also increased apoptosis at all days $(P<0.01)$, and z-LEHD-fmk blocked this effect (TNF $\times$ inhibitor, $P=0.05 ; n=361$ embryos total). Across days, $\%$ apoptosis was $3.6 \pm 1.4 \%$ (control), $3.3 \pm 1.3 \%$ (inhibitor), $11.1 \pm 1.3 \%$ (TNF- $\alpha$ ), and $6.0 \pm 1.4 \%$ (TNF- $\alpha+$ inhibitor). Mean cell number at the end of culture ranged from 21 to 27 cells at day 4,59 to 74 cells at day 5 , and 105 to 115 cells at day 6 . In conclusion, activation of caspase-9 dependent pathways is involved in the induction of apoptosis by heat shock and TNF- $\alpha$. Moreover, the magnitude of induced apoptosis increases as embryos advance in development.
\end{abstract}

This work was supported by USDA Grant No. 2004-34135-14715 and BARD Grant No. US-3551-04.

\section{BRAHMA-RELATED-PROTEIN 1 OVEREXPRESSION IN PARTHENOGENETIC PORCINE EMBRYOS}

\section{Magnani and R. Cabot}

Animal Sciences Department, Purdue University, West Lafayette, IN 47907, USA

The chromatin remodeling protein Brahma-related-protein 1 (Brg1) has been implicated in several cellular processes that affect chromatin structure and transcription. Brg1 is a member of the SWI/SNF family, a large group of chromatin remodeling proteins, all of which have the characteristic of utilizing the energy from ATP hydrolysis to catalyze the repositioning of nucleosomes on DNA templates. Various members of this class of proteins are known to be differentially expressed throughout vertebrate development. We hypothesize that the balance of chromatin remodeling factors present in cleavage stage porcine embryos has an impact on embryonic developmental potential. As a first step to testing this hypothesis, we attempted to overexpress a wildtype version of Brg1 in cleavage stage porcine parthenogenic embryos. The Brg1 construct was a gift from Dr. Anthony Imbalzano (University of Massachusetts, Worster, MA). The Brg1 cassette ligated into the NheI site of pIRES2-eGFP, Clontech. This construct was sequenced to verify the orientation. Oocytes were matured in vitro in defined culture medium (TCM 199 supplemented with $0.1 \%$ polyvinyl chloride, $10 \mathrm{ng} / \mathrm{mL}$ epidermal growth factor, $0.5 \mathrm{IU} / \mathrm{mL} \mathrm{LH}, 0.5 \mathrm{IU} / \mathrm{mL} \mathrm{FSH}, 1 \mathrm{~mm}$ cysteine) for $42-44 \mathrm{~h}$ at $39^{\circ} \mathrm{C}$ in $5 \% \mathrm{CO}_{2}$. Following in vitro maturation, oocytes were denuded and activated by electroporation and cultured in NCSU23 supplemented with BSA $(4 \mu \mathrm{g} / \mathrm{mL})$ at $39^{\circ} \mathrm{C}$ 
in $5 \% \mathrm{CO}_{2}, 5 \% \mathrm{O}_{2}$. For microinjection, activated oocytes were divided into 3 treatment groups $3-5 \mathrm{~h}$ after activation. Oocytes in Treatment 1 were microinjected with pIRES2-eGFP-Brg1 vector; oocytes in Treatment 2 were microinjected with pIRES2-eGFP vector; and oocytes in Treatment 3 were not injected with any construct. Approximately $40 \mathrm{pL}$ were injected at a concentration of $1 \mathrm{mg} / \mathrm{mL}$. Following injection, lysed embryos were discarded, and the intact embryos were placed in embryo culture medium for 6 days. At this time, embryos were stained with Hoechst 33342 , and the number of nuclei for each embryo was determined. Expression of the injected construct was determined by the presence of GFP $24 \mathrm{~h}$ following microinjection. There was a tendency for embryos that developed from oocytes injected with the Brg1 construct to have increased cell numbers at day 6 of embryo culture, as compared with the other 2 groups $(21.4 \pm 15, n=12 ; 17.7 \pm 9.6, n=7 ; 16.8 \pm 5.82, n=24$ for treatments 1,2 , and 3, respectively; $P=0.3$ ). Data were analyzed using GLM procedures in SAS; embryos with cell number $\geq 8$ were analyzed. There was no difference in fragmented embryos among the 3 groups. The results come from 5 independent replicates. To the best of our knowledge, this is the first time that a chromatin remodeling protein has been overexpressed in cleavage stage porcine embryos. Further experiments using an ATPase defective subunit will be performed to further examine the role that ATP dependent chromatin remodeling processes play during porcine embryogenesis.

\title{
135 THE EFFECT OF DIFFERENT TREATMENTS OF PORCINE EJACULATED AND EPIDIDYMAL SPERMATOZOA ON ROS GENERATION
}

\author{
C. Matás, M. Sansegundo, S. Ruiz, and J. Gadea \\ Department of Physiology, School of Veterinary, University of Murcia, Murcia, Spain
}

\begin{abstract}
The production of pig embryos in vitro is still relatively inefficient compared with results obtained with oocytes matured and fertilized in vivo. The main reasons for this limited performance are polyspermy after IVF and the poor developmental ability of embryos produced by IVM-IVF (Kikuchi et al. 2002 Biol. Reprod. 1033-1041). Between factors affecting polyspermy are the sperm procedures before IVF. Usually, these procedures including centrifugations that increase reactive oxygen species (ROS) formation in spermatozoa. ROS play an important role in sperm physiology including capacitation. Physiological concentrations of ROS have been proposed to enhance sperm capacitation by increasing cAMP synthesis and by inhibiting protein tyrosine phosphatases whilst activating tyrosine kinases. In general, epididymal spermatozoa appear to be able to capacitate and fertilize eggs in vitro much more easily than ejaculated spermatozoa (Yanagimachi Mammalian Fertilization. In: The Physiology of Reproduction, Raven Press 1988; 135-182). In this study, we investigated how different sources (ejaculated spermatozoa vs. epididymal spermatozoa) and sperm capacitating methods, usually employed in porcine IVF, could be affect ROS generation. Sperm-rich fractions from five fertile boars and sperm from five different epididyimides were used. The semen samples were then: (i) washed in Dulbecco's phosphate-buffered saline (DPBS) supplemented with $0.1 \%$ BSA, (ii) left unwashed, or (iii) washed on a Percoll (Pharmacia, Uppsala, Sweden) gradient (Matás et al. 2003 Reproduction 125, $133-141$ ). Production of ROS was measured by incubating the spermatozoa in the in vitro fertilization medium (TALP) in the presence of $0.7 \mu \mathrm{M}$ $2^{\prime}, 7^{\prime}$-dichlorodihydrofluorescein diacetate at $37^{\circ} \mathrm{C}$ under $5 \% \mathrm{CO}_{2}$ in $100 \%$ humidified air. Every $15 \mathrm{~min}$ (from 15 to 135 ) the samples were analyzed and evaluated by flow cytometry. Measurements were expressed as the mean green intensity fluorescence units and it was used as index of ROS generation (Gadea et al. $2005 \mathrm{~J}$. Androl. 26, 396-404). ANOVA analysis revealed a significant effect of sperm treatment on the ROS generation $(P<0.001)$. The highest value was obtained in sperm washed on a Percoll gradient and the lowest in unwashed semen. When ejaculated vs. epididymal semen was analyzed, the same tendency was observed in both. However, the values were always lower in epididymal semen than in ejaculated semen $(P<0.001)$. As a conclusion, ROS generation is different between treatments and between semen procedures for the time interval studied, and this finding may help to explain the different outcome in IVF among laboratories.
\end{abstract}

This work was supported by Ministerio de Educación y Ciencia, AGL2003-03144.

\section{FUNCTIONAL ANALYSIS OF RHOPHILIN-2 GENE IN PRE-IMPLANTATION MOUSE EMBRYO}

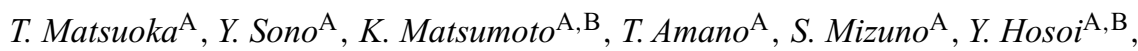 \\ K. Saeki ${ }^{\mathrm{A}, \mathrm{B}}$, and A. Iritani $^{\mathrm{A}, \mathrm{B}}$

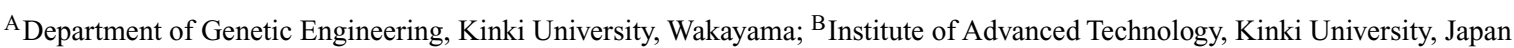

Zygotic gene activation (ZGA), which starts at the G2 phase at the 1-cell stage (Latham 1999), promotes the reprogramming of gene expression and is critical for the subsequent development of pre-implantation embryos. We have investigated the molecule function of many gene clusters, DD clones obtained by Differential-Display assays for ovulated eggs at the M II stage, and 1-cell embryos at the G2 phase. The differential expression of rhophilin-2 shown in DD assays was also confirmed by 3 independent real-time PCR analyses $(P<0.05)$. For these reasons, in this study, we focused on the rhophilin-2 gene, which regulates cytoskeletal organization (Peck et al. 2002). At first, we identified a protein that interacts with the Rhophilin-2 protein by a yeast 2-hybrid system. To confirm the interaction between Rhophilin-2 and the putative protein obtained by a yeast two-hybrid system, we used a co-immunoprecipitaion assay. We also investigated the expression profiles of rhophilin-2 and the transcripts of the identified protein in ovary and pre-implantation embryos using real-time PCR and immunofluorescence (IF) analysis. The ICR mice at $48 \mathrm{~h}$ after PMSG priming were primed with hCG, and ovaries were collected at $7 \mathrm{~h}$ after hCG priming. Pre-implantation embryos were collected at 1-cell, 2-cell, and 4-cell stages, and cDNA was produced by mRNA isolated from 10 oocytes or embryos in each group and was subjected to real-time PCR using a TaqMan Probe system (ABI). Sectioned ovaries and pre-implantation embryos were analyzed by IF analysis using antibody of Rhophilin-2 and the identified protein. This is the first report that GABA receptor-association protein (GABARAP) was identified as a protein that interacts with Rhophilin-2, as a result of using the yeast 2-hybrid system and subsequent co-immunoprecipitation assay. After fertilization, transcript levels of rhophilin-2 significantly decreased from the 1 -cell stage to the 2 -cell stage $(P<0.05)$, but transcript levels of GABARAP significantly increased 
from the 1-cell stage to the 2-cell stage $(P<0.05)$. The IF analysis revealed localization of Rhophilin-2 and GABARAP at the nucleolus of all follicle stage in the ovary. Moreover, Rhophiln-2 and GABARAP were found to be localized on the microtubules of 1-cell and 2-cell embryos, but no signal of Rhophilin-2 was detected in 4-cell embryos. These results suggest that Rhophilin-2 protein regulates the cytoskeletal organization in 1-cell to 2-cell embryos and is involved in the molecular mechanism of cell division by coupling with GABARAP.

This study was supported by a Grant-in-Aid for the 21st Century COE Program of the Japan Mext and by a grant for the Wakayama Prefecture Collaboration of Regional Entities for the Advancement of Technology Excellence of the JST.

\title{
137 IRON TRANSFER ACROSS THE LLAMA PLACENTA (LAMA GUANICOE GLAMA)
}

$$
\begin{gathered}
\text { M. A. } \text { Miglino }^{\mathrm{A}}, \text { D. Iturrizaga } \\
\text { J. M. Monteiro }{ }^{\mathrm{A}} \text {, and F.W. Bazer } \\
\text { C. }
\end{gathered}
$$

${ }^{A}$ Universidade de São Paulo/USP, São Paulo, Brazil; ${ }^{\mathrm{B}}$ Faculdade de Zootecnia de Dracena/UNESP, Dracena, São Paulo, Brazil;

${ }^{\mathrm{C}}$ Center for Animal Biotechnology and Genomics, Texas A\&M University, College Station, TX 77843, USA

The placenta of the llama has been described as epitheliochorial in type, but recent studies have not shown extensively the fetal nutrition aspects in this animal. In epitheliochorial placentation there is development of structures called areolae, as well as inter-microvillous attachment of the trophoblast, with irregular contact, to the uterine epithelium. This attachment is interrupted and the transfer of substances between the mother and the fetus takes place across the areolar cavity. These areolae appeared as small rounded or dome-shaped elevated areas of the chorioallantoic membrane over the narrow uterine gland openings. In order to detail their mechanisms of iron transfer in the llama placenta, we collected the samples of nine uteri between 28 to 36 weeks of pregnancy in association with fetal membranes. These samples were fixed in $4 \%$ paraformaldehyde in PBS, processed, and stained for light microscopy (HE, picrosirius, and Masson's trichrome), histochemistry (Perls, acid phosphatase, and PAS reactions) and immunohistochemistry with rabbit anti pig uteroferrin antibody to confirm the iron transfer, because the uteroferrin is an iron transporter and a progesterone-induced hematopoietic growth factor. The trophoblast formed a columnar-type single layer that was comprised of cells of various sizes and shapes with basal nuclei, including the giant binucleate cells. The trophoblast formed chorionic projections which presented ramifications in number from 4 to 5. A great quantity of blood vessels were found in the materno-fetal interface, between the cells of uterine epithelium and around of the chorionic projections. A PAS-positive reaction was observed with diffuse cytoplasmic PAS staining at the apical region of the trophoblast at the materno-fetal interface as well as in the endometrial glands. Collagen fibers were observed in the mesenchyme and inside the chorionic projections. In the areolae we confirmed the positive reaction of the acid phosphatase enzyme that detects phagocytic activity. In the basal region of the uterine gland epithelium, which is columnar type, and in the gland lumina, this reaction demonstrated a strong positive stain. The Perls histochemical reaction that reveals ferric iron was positive in the areola, as well as in the uterine glands. The uteroferrin immunohistochemistry showed a strong stained in the areolae and in the epithelium and lumina of the uterine glands. Our findings suggest that the areola region and the endometrial glands play an important role in histiotrophic nutrition in llamas, and in fetal red blood cell formation by iron transfer from mother to the fetus.

This work was supported by FAPESP, CNPq, CAPES, PRONEX, Brazil.

\section{IN VITRO CULTURE OF CD9-EXPRESSING CELLS ENRICHED BY MAGNETIC CELL SORTING FROM TESTES OF CRYPTORCHID ADULT AND PUP IN MICE}

\author{
T. Mitani ${ }^{\mathrm{A}, \mathrm{B}}, Y_{\text {. Tanaka }}^{\mathrm{C}}$, Y. Ozaki $^{\mathrm{C}}, K$. Saeki $^{\mathrm{A}, \mathrm{C}}, K_{\text {. }}$ Kato $^{\mathrm{A}, \mathrm{B}}$, K. Matsumoto $^{\mathrm{A}, \mathrm{C}}$, \\ Y. Hosoi ${ }^{\mathrm{A}, \mathrm{C}}$, and A. Iritani ${ }^{\mathrm{A}, \mathrm{C}}$ \\ ${ }^{\mathrm{A}}$ Institute of Advanced Technology, Kinki University, Kainan, Wakayama 642-1017, Japan; ${ }^{\mathrm{B}}$ Gene Control Corporation, Kainan, \\ Wakayama 642-1017, Japan; CDepartment of Genetic Engineering, Kinki University, Uchita, Wakayama 649-1493, Japan
}

Recently, studies on cell surface markers of spermatogonia in combination with germ cell transplantation techniques have made possible the functional analysis of germline stem cells (GS cells). The GS cells are downstream of the stem cells such as ES cells and embryonic germ cells (EG cells), which are derived from primordial germ cells (PGCs). Therefore, GS cells are expected to be useful in the production of genetically modified animals. In this study, we examined the enrichment and cultivation of mouse GS cells by magnetic cell sorting (MACS). Testicular cell suspensions were collected from C57BL/6J cryptorchid adult testes at 2 to 3 months after surgery and ICR pup (6 to $8 \mathrm{dpp}$ ) testes. They were digested by $0.1 \%$ collagenase followed by $0.25 \%$ trypsin with gentle shaking. Dissociated cell suspensions were filtrated through a glass-wool column followed by a Falcon cell strainer $(40-\mu \mathrm{m}$ mesh). They were then treated with biotin-conjugated anti-mouse CD9 antibody, whose antigen, CD9, is localized on the GS cell surface, followed by the streptavidin-microbeads treatment. The cell suspension was passed through a MACS-separation column. In Experiment I, MACS-treated fractions were analyzed by flow cytometry (FCM) on the rates of recovery and enrichment and their cellular characteristics. In Experiment II, CD9-positive $\left(\mathrm{CD}^{+}\right)$cells were cultured on gelatin-coated MultiDish $\left(176740\right.$, Nunc) with $4-5 \times 10^{5}$ cells/well in StemPro34-SFM supplemented with $1 \%$ fetal bovine serum, leukemia inhibitory factor, GDNF, bFGF, EGF, insulin, transferrin, putrescine, MEM vitamin solution, MEM-NEAA and some other reagents at $32^{\circ} \mathrm{C}$ or $37^{\circ} \mathrm{C}$ under $5 \% \mathrm{CO}_{2}$ in air. They were examined for their proliferation and cytological changes such as CD9, $\alpha 6$-integrin and Oct-1 expression by immunohistochemistry. In Experiment I, MACS selection effectively enriched CD $9^{+}$cells from mouse testes. However, FCM analysis revealed that the CD9-negative (CD9 $\left.{ }^{-}\right)$cells partially remained in MACS-selected fraction from cryptorchid adult testes. In contrast, the $\mathrm{CD} 9^{+}$subpopulation could be successfully separated from $\mathrm{CD}^{-}{ }^{-}$subpopulation from pup testes. Therefore $\mathrm{CD} 9^{+}$ 
subpopulation from pup testes was used for the following cultivation. In Experiment II, the cells proliferated in the first few days in suspension. Then they attached to the dish and formed colonies after 5 days or 3 days of culture at $32^{\circ} \mathrm{C}$ or $37^{\circ} \mathrm{C}$, respectively. Immunohistochemical analysis showed that the cells maintained the expression of CD9 for at least 14 days, but their expression of $\alpha 6$-integrin gradually diminished. It was demonstrated by immunohistochemistry and FCM analysis that the cells in colonies expressed Oct- 1 , and its expression level was stronger in culture at $37^{\circ} \mathrm{C}$ than at $32^{\circ} \mathrm{C}$. These findings indicate that the $\mathrm{CD} 9^{+}$cells collected from mouse pup testes have stem cell properties.

This work was supported by the Wakayama Prefecture Collaboration of Regional Entities for the Advanced Technological Excellence, JST; by a Grant-in-Aid for the 21st Century Center of Excellence Program of the MEXT, Japan; and by a Grant-in Aid for Scientific Research from the Japan Society for the Promotion of Science.

\title{
139 INJECTION OF SOMATIC CELL CYTOPLASM INTO OOCYTES BEFORE ICSI IMPAIRED FULL-TERM DEVELOPMENT AND INCREASED PLACENTA WEIGHT IN MICE
}

\author{
V.T. Nguyen ${ }^{\mathrm{A}}$, S. Wakayama ${ }^{\mathrm{B}}$, S. Kishigami ${ }^{\mathrm{A}}, H_{\text {. Ohta }}^{\mathrm{A}}$, T. Hikichi $^{\mathrm{A}}$, E. Mizutani $^{\mathrm{A}}$, \\ H.-T. Bui ${ }^{\mathrm{A}}$, and T. Wakayama ${ }^{\mathrm{A}}$ \\ ${ }^{\mathrm{A}}$ Center for Developmental Biology, RIKEN-Kobe, Kobe, Hyogo, Japan; ${ }^{\mathrm{B}}$ Graduate School of Science and Technology, Kobe University, \\ Kobe, Hyogo, Japan
}

During the process of somatic cell nuclear transfer, cytoplasm is introduced into the enucleated oocytes, in addition to the genomic material, regardless of the electrofusion methods (Wilmut et al. 1997) or direct injection of somatic nucleus by the Honolulu method (Wakayama et al. 1998). Only 1 to $2 \%$ of cloned embryos, however, develop to term with many incidences of developmental anomalies. These cloning failures may be explained by incomplete reprogramming of the donor cell genome, although it is not yet clear whether cytoplasmic materials of the somatic cell also have an affect on development of the cloned embryo. In an attempt to answer this question, this study investigates the effects of somatic cytoplasm of different mouse strains and cytoplasm of fertilized embryos at different stage by injecting them into intact mouse oocytes before intracytoplasmic sperm injection (ICSI). Mature oocytes collected from B6D2F1 female after 14 to $16 \mathrm{~h}$ of hCG injection were injected with (1) B6D2F1 cumulus cell cytoplasm with different volumes (collected by 2 to $3 \mu \mathrm{m}$ of injection pipette and piezo pulses), (2) cumulus cell cytoplasm from different mouse strains (B6D2F1, ICR, C57BL/6), (3) cytoplasm of 1- to 8-cell embryos. After subsequent culture for 1h, B6D2F1 sperm were injected into those oocytes and examined for preimplantation developmental competence. The total number of cells, inner cell mass (ICM), and expression of Oct4 in expanded blastocysts were also examined. In order to examine the effects of somatic cytoplasm on full-term development, we transferred 2-cell embryos at $24 \mathrm{~h}$ or morula and blastocysts at $72 \mathrm{~h}$ after ICSI to the oviduct or uterus of surrogate mothers (ICR) on Day 1 or 3 of pseudopregnancy. The control group received a sham injection with PVP before ICSI. The results showed that an increase the volume of cytoplasm from 1-fold to 4-fold (equivalent with the volume of 1 cumulus cell) resulted in impairing full-term development ( 28 and $7 \%$, respectively, vs. 56 to $63 \%$ in the control group, $P<0.01)$. There was no difference in the frequency of embryos developing to the blastocyts stage between B6D2F1 and ICR somatic cytoplasms at the same volume. However, C57BL/6 somatic cytoplasm induced the 2-cell block to B6D2F2 embryos. Fertilized embryo cytoplasm did not reduce the frequency of blastocyst stage and full-term development. Interestingly, we found that somatic cytoplasm increased the placenta weight of ICSI embryo $(0.2002 \pm 0.03, n=32$; vs. $0.1198 \pm 0.02$ in control group, $n=87 ; P<0.01)$. We also obtained placenta with no fetus when the volume of somatic cytoplasm was the same size as cumulus cell. We found that an increase in the volume of somatic cytoplasm led to low expression of Oct4 in expanded blastocysts. These findings indicated that injection of somatic cytoplasm into oocytes before ICSI decreased the preimplantation development, clearly impaired full-term development, and caused placental overgrowth in fertilized embryos. This study suggested that somatic cell cytoplasmic material is one cause of the low rate of full-term development of cloned animals.

\section{IMMUNOLOCALIZATION OF INDOLEAMINE 2,3-DIOXYGENASE IN PLACENTA FROM BOVINE CLONED CONCEPTUS PREGNANCY}

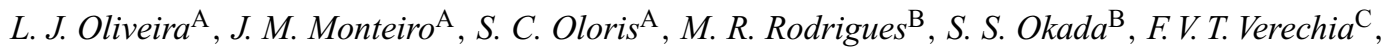 \\ F. V. Meirelles ${ }^{\mathrm{D}}$, J. A. Visintin ${ }^{\mathrm{A}}$, A. Campa ${ }^{\mathrm{B}}$, and J. R. Kfoury Jr ${ }^{\mathrm{A}}$ \\ ${ }^{\mathrm{A}}$ University of Sao Paulo, FMVZ-USP, Sao Paulo, SP, Brazil; ${ }^{\mathrm{B}}$ IQ-USP, Sao Paulo, SP, Brazil; ${ }^{\mathrm{C}}$ FZ-Unesp, Dracena, SP, Brazil; \\ DFZEA-USP, Pirassununga, SP, Brazil
}

The catabolism of tryptophan by indoleamine 2,3-dioxygenase (IDO) activity is strongly correlated to maternal-fetal tolerance in hemochorial placentation. Several studies have been conducted on the role of IDO on maternal-fetal tolerance in human and mice placental tissue. Changes in IDO expression and activity are related to fertility problems in humans and lower IDO activity was found in women who had recurrent abortions. In mice, IDO was localized in blastocyst, cytotrophoblast, placental macrophages, stromal cells and invasive trophoblast cells, but not in the syncytiotrophoblast. Also, IDO staining was detected on villous trophoblast cells; probably due to the fact that this is the site where fetal antigen is most exposed to the maternal immune system during pregnancy. Our previous studies in bovine showed that IDO activity is present throughout pregnancy, increasing as it develops. IDO staining can be observed at the maternal-fetal interface, in the trophoblast, especially in giant cells where it increases as gestation progresses. IDO staining in the uterine epithelium remained constant during gestation and absent in the fetal mesenchyma. This study aimed to qualitatively immunolocalize IDO staining in placenta from bovine cloned conceptuses at term. Fibroblasts originated from one bull were cultured and fused with enucleated oocytes to produced clone embryos. Placentomes were collected from four cloned conceptuses and 
10 normal conceptuses (control) at parturition. Samples were fixed in $10 \%$ buffered formalin and embedded in paraffin. Sections were made and processed for immunohistochemistry using a commercial monoclonal antibody against IDO. Placenta from two cloned conceptuses showed IDO staining on fetal mesenchyma, differing from normal pregnancy where the staining of this enzyme was absent in all control samples. IDO staining was absent at the fetomaternal interface in one sample from cloned placenta and present in all normal pregnancy animals. It was difficult to establish a staining profile for giant and binucleate cells in cloned placenta, since IDO staining varied greatly among individuals. IDO immunolocalization in placenta from cloned conceptus was altered in all animals studied. It may raise the question that cloning could interfere in maternal immune modulation during pregnancy and IDO misexpression in fetomaternal tolerance could be involved in a decreased maintenance of cloned pregnancies

\title{
141 EXPRESSION OF GENE PRODUCTS ENCODING CNN PROTEINS DURING THE FIRST WEEK OF DEVELOPMENT
}

\author{
A. Pin $^{\mathrm{A}, \mathrm{B}}$ and A. Watson ${ }^{\mathrm{A}, \mathrm{B}}$ \\ ${ }^{\mathrm{A}} \mathrm{OB} / \mathrm{GYN}$ and Physiology and Pharmacology, The University of Western Ontario, London, Ontario, Canada; \\ ${ }^{B}$ Children's Health Research Institute, Lawson Health Research Institute,, London, Ontario, Canada
}

CCNs (cysteine-rich 61 (Cyr61), connective tissue growth factor (CTGF), and nephroblastoma overexpressed (Nov)) are a newly characterized gene family encoding 30-40 kDa proteins that are cysteine-rich and associated with the extracellular matrix. CCNs bind integrins and activate intracellular signaling, resulting in kinase activation and gene transcription. They are involved in events essential to development, including implantation and placentation. For example, CTGF is increased during decidualization and both CTGF and Cyr61 are produced by trophoblast giant cells. Furthermore, Cyr61-null mice display early embryonic lethality due to placental defects. Although CTGF protein has been reported in mouse 4.5-day embryo, no studies to date have focused on defining the expression of CCN mRNAs during pre-implantation development. The objective of this study was to characterize the expression of CCN mRNAs during the first week of in vivo mouse and in vitro cow development. CD-1 mice were superovulated by i.p. injection of pregnant mare serum gonadotrophin $(10 \mathrm{U})$ and human chorionic gonadotrophin $(10 \mathrm{U}) 22$ hours later. Following mating of the mice with CD-1 males, embryos were flushed from oviducts and uteri at 2-cell, 4-cell, 8-cell, morula, and blastocyst stages. Cow oocytes were obtained from slaughterhouse ovaries. Cow embryos were produced by aspiration of early antral follicles followed by in vitro oocyte maturation, fertilization, and embryo culture methods, and collected at 2-5-cell, 6-8-cell, morula, and blastocyst stages. Groups of five embryos of each stage were pooled for mRNA extraction using a magnetic bead protocol. Reverse transcription-polymerase chain reaction was used to detect CCN mRNAs at each time point, and product identity was verified by sequencing. CCN mRNAs are detectable during the first week of development in both species. In the mouse, Cyr61 mRNAs were present in all embryo stages investigated. CTGF mRNAs were variable at the 2-cell stage, but were consistently present from the 4-cell stage to the blastocyst stage. Interestingly, Nov appeared transiently at the 4- to 8-cell stage only. In the cow, CTGF and Cyr61 mRNAs were also detectable. Nov was undetectable in the cow. This is the first report of CCN mRNA expression in mouse or cow pre-implantation development. The early appearance and continued presence of Cyr61 and CTGF indicate a specific role in early development. The transient nature of Nov expression may indicate a specific role during compaction in the mouse. The expression of CTGF and Cyr61 in the cow indicates that these proteins are conserved between species and supports the hypothesis that they are important in this early phase of development.

This research was funded by NSERC, Canada.

\section{INTRACELLULAR CALCIUM UPTAKE AS A RESPONSE TO DIFFERENT CAPACITATION TREATMENTS IN EJACULATE AND EPIDIDYMAL BOAR SPERMATOZOA}

\author{
M. Sansegundo ${ }^{\mathrm{A}}$, S. Ruiz ${ }^{\mathrm{A}}$, A. Gonzalez ${ }^{\mathrm{B}}$, N. T. Atucha ${ }^{\mathrm{A}}$, and C. Matás ${ }^{\mathrm{A}}$ \\ ${ }^{\mathrm{A}}$ Department of Physiology, University of Murcia, Murcia, Spain; ${ }^{\mathrm{B}}$ Department of Physiology, \\ School of Veterinary Sciences, University of Extremadura, Caceres, Spain
}

Both cauda epididymal and ejaculated spermatozoa are considered fully mature, although these two types of spermatozoa do not necessarily show the same behavior in vitro. It has been reported that both types of spermatozoa fertilize eggs in vitro at the same rate, but, in general, epididymal ones achieve this objective easier than the ejaculated ones. Intracellular $\mathrm{Ca}^{2+}$ influx is one of the crucial biochemical events occuring capacitation and $\mathrm{Ca}^{2+}$ requirements for capacitation varies among different species. In this study, we investigated how different source of spermatozoa (ejaculated vs. epididymal) and commonly employed sperm capacitating methods can affect calcium uptake. Sperm-rich fractions from seven fertile boars and sperm from seven different epididymides were used. Semen samples were kept unwashed (method A), washed in Dulbecco's phosphate-buffered saline (DPBS) supplemented with 0.1\% BSA (method B), or washed on a Percoll gradient (method C) (Matás et al. 2003 Reproduction 125, 133-141) To measure intracellular free $\mathrm{Ca}^{2+}$, spermatozoa, treated as described above, were incubated with $2.5 \mu \mathrm{M}$ fura-1/AM in a non capaciting medium (Tardif et al. 2003 Biol. Reprod. 68, 207-213) for $45 \mathrm{~min}$ at $37^{\circ} \mathrm{C}$. Then, spermatozoa were resuspended in TALP medium, incubated $\left(5 \% \mathrm{CO}_{2}\right.$, $38.5^{\circ} \mathrm{C}$ ) for a further $60 \mathrm{~min}$ and then analyzed in a fluorescence spectrofluorometer with excitation wavelength set at $340-880 \mathrm{~nm}$ and emission held at $510 \mathrm{~nm}$. The calculation of intracellular free $\mathrm{Ca}^{2+}$ was performed according to the equation of Grynkiewicz et al. (1985 J. Biol. Chem. 260 , $3440-3450)$. Results showed that calcium uptake is affected by treatment and semen source $(P<0.001)$. The intracellular free $\mathrm{Ca}^{2+}$ concentrations $(\mathrm{nM})$ in ejaculated semen and in epididymal spermatozoa were 269.52 vs. $208.52,1134.58$ vs. 137.37 and 1224.79 vs. 216.54 for A, B, and C methods, respectively. As a conclusion, it can be stated that sperm capacitation treatment affects calcium uptake. In addition, capacitation pathways may be modified or regulated in some way by seminal plasma, thus increasing intracellular calcium levels in ejaculated sperm (methods B and C) in comparison to those in epididymal spermatozoa.

This work was supported by Ministerio de Educación y Ciencia, AGL2003-03144. 


\title{
143 PRESENCE OF PROSTAGLANDIN $F_{2 \alpha}$ RECEPTOR IN IN VITRO-DERIVED MORULA AND BLASTOCYST STAGE BOVINE EMBRYOS
}

\author{
F. N. Scenna, J. L. Edwards, G. M. Pighetti, and F. N. Schrick \\ Department of Animal Science, University of Tennessee, Knoxville, TN, USA
}

Culture of in vitro and in vivo-derived embryos in medium containing prostaglandin $\mathrm{F}_{2 \alpha}$ (PGF) decreased embryonic development to blastocyst stage and reduced hatching rates (Scenna et al. Prostaglandins 73, 215-226). Moreover, administration of an inhibitor of PGF synthesis at the time of embryo transfer in bovine recipients improved pregnancy rates (Schrick et al. 2001 Theriogenology 59, 335 abstr.). These findings indicate a direct negative effect of PGF on embryonic development. However, to our knowledge, no evidence of PGF receptor expression in morula or blastocyst stage bovine embryos is available in the literature. Therefore, the objective of the current study was to determine the presence of PGF receptor mRNA using real-time RT-PCR and protein expression by Western blotting in morula or blastocyst stage in vitro bovine embryos. Briefly, isolated total RNA from compact morula or blastocyst stage embryos and from bovine tongue epithelium (positive control for PGF receptor mRNA) were reverse-transcribed into cDNA. A volume from the RT reaction equivalent to 10 embryos per tube was utilized to determine transcripts for PGF receptor and Histone H2A (standard PCR control). Polymerase chain reaction was performed, and identity of PCR fragments was confirmed by ethidium-bromide-stained 2\% agarose gel electrophoresis and by DNA sequencing. To determine protein expression, morula and blastocyst stage embryos were lysed in lysis buffer ( $10 \% \mathrm{SDS}, 1 \mathrm{~m} \mathrm{Tris} \mathrm{pH} \mathrm{7.5,} 1 \mathrm{~m} \mathrm{NaF}, 1 \mathrm{~m}$ DTT, $0.1 \mathrm{~m}$ EGTA with protease inhibitors) and stored at $-20^{\circ} \mathrm{C}$. Crude proteins isolated from bovine corpora lutea (positive control for PGF receptor protein), embryo samples, and prestained standards were separated by $12 \%$ SDS-PAGE under reducing conditions. Proteins were electrotransferred onto a PVDF membrane. Nonspecific binding sites in the PVDF membrane were blocked with $10 \%$ nonfat dry milk, and the blot was washed and incubated for $1 \mathrm{~h}$ at room temperature with a 1:1000 dilution of the primary antibody (rabbit polyclonal antibody against PGF receptor protein). Subsequently, the blot was washed and incubated for $1 \mathrm{~h}$ at room temperature with 1:1000 dilution of mouse anti-rabbit IgG conjugated with horseradish peroxidase. Finally, the blot was washed and revealed by chemiluminescence in a CCD camera. Results indicated that transcripts as well as the protein for PGF receptor were present in early stage bovine embryos. Identification of PGF receptor in morula and blastocyst stage bovine embryos may, in part, explain the increase in pregnancy rates after administration of a PGF synthesis inhibitor at the time of embryo transfer, which opens up the possibility to develop new strategies to prevent detrimental effects of PGF during early embryonic development.

\section{EPIGENETIC ASYMMETRY IN HISTONE H3 LYSINE 9 DIMETHYLATION STATUS IN PRONUCLEAR STAGE PORCINE EMBRYOS}

\author{
M. Sega and R. Cabot
}

Purdue University, Department of Animal Sciences, West Lafayette, IN, USA

Covalent modification of specific residues on the tail regions of core histone proteins has been shown to play a key role in regulation of the genome. Methylation of lysine 9 on histone H3 (H3K9) is associated with repression of transcription and formation of heterochromatin domains. The maternal- and paternal-derived pronuclei from pronuclear stage murine embryos have an asymmetric distribution of H3K9 dimethylation between the 2 pronuclei; maternal pronuclei have the H3K9 dimethylation, whereas the paternal pronuclei lack this modification. The aim of this study was to characterize the H3K9 dimethylation pattern in cleavage stage porcine embryos. Indirect immunocytochemical staining was performed using a commercially available antibody from Upstate (Charlottesville, VA) that recognizes the dimethylated form of lysine 9 on histone H3 and fluorescein isothiocyanate (FITC)-conjugated secondary antibody on germinal vesicle (GV) stage porcine oocytes and cleavage stage porcine embryos. Germinal vesicle stage oocytes were matured in vitro for $44 \mathrm{~h}$ in a chemically defined maturation medium (TCM 199 supplemented with $0.1 \%$ PVA, $0.069 \mathrm{mg} / \mathrm{mL}$ cysteine, $10 \mathrm{ng} / \mathrm{mL}$ EGF $0.5 \mathrm{IU} / \mathrm{mL} \mathrm{LH}$, and $0.5 \mathrm{IU} / \mathrm{mL} \mathrm{FSH}$ ) at $39^{\circ} \mathrm{C}$ in $5 \% \mathrm{CO}_{2}$. Following in vitro fertilization, presumptive zygotes were cultured in NCSU23 medium containing $4 \mathrm{mg} / \mathrm{mL}$ BSA at $39^{\circ} \mathrm{C}$ in $5 \% \mathrm{CO}_{2}, 5 \% \mathrm{O}_{2}$. Embryos were fixed in $3.7 \%$ paraformaldehide for $2 \mathrm{~h}$ and washed in PBS and $0.1 \%$ Tween20. H3K9 dimethylation is present in the nuclei of GV-stage oocytes $(n=24)$ and pronuclear $(n=13)$, 2-cell $(n=4)$, 4-cell $(n=9)$, blastocyst $(n=8)$ stage embryos. The analysis revealed 2 interesting findings. First, examination of thin optical sections through the nuclei of processed embryos on a confocal microscope revealed that in GV-stage oocytes and pronuclear, 2-cell and 4-cell stage embryos the dimethylated form of H3K9 was distributed throughout the nuclei at these developmental time points, whereas in blastocyst stage embryos, the dimethylated $\mathrm{H} 3 \mathrm{~K} 9$ was restricted to the nuclear periphery. Second, not all pronuclei within pronuclear stage embryos were positive for the dimethylated form of $\mathrm{H} 3 \mathrm{~K}$ 9. To determine if differential $\mathrm{H} 3 \mathrm{~K}$ 9 methylation pattern observed among pronuclei in 1-cell stage embryos was due to parental origin, we produced parthenogenic porcine embryos by electrically activating porcine oocytes in calcium containing activation medium and processed them as indicated above for fertilized oocytes $(n=13)$. All pronuclei found in pronuclear stage parthenogenic porcine embryos were positive for dimethylation of $\mathrm{H} 3 \mathrm{~K} 9$. Chi square analysis revealed this pattern to be different from that observed in pronuclear embryos produced by fertilization $(P<0.05)$. In summary we conclude that the pronuclei in 1-celled embryos produced by in vitro fertilization are differentially dimethylated at $\mathrm{H} 3 \mathrm{~K} 9$ and that the localization of dimethylated $\mathrm{H} 3 \mathrm{~K} 9$ changes during cleavage development.

\section{STAGE-SPECIFIC EFFECT OF OXIDATIVE STRESS ON DEVELOPMENTAL COMPETENCE, ROS GENERATION AND DNA DAMAGE OF PORCINE PARTHENOGENETIC EMBRYOS}

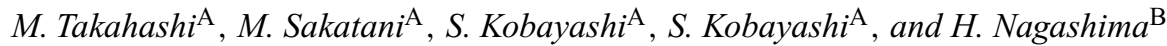 \\ ${ }^{A}$ National Agricultural Center for Kyushu Okinawa Region, Kumamoto, Japan; ${ }^{B}$ Meiji University, Kawasaki, Kanagawa, Japan
}

We investigated the effect of oxidative stress on stage specific developmental ability, reactive oxygen species (ROS) generation and DNA damage of parthenogenetically activated porcine embryos. Cumulus-oocyte complexes (COCs) were aspirated from follicles on the surface of ovaries. The 
COCs were matured in NCSU-23 containing $10 \%$ (vol/vol) porcine follicular fluid and $10 \mathrm{IU} / \mathrm{mL}$ hCG during the first $22 \mathrm{~h}$ followed by an extra $22 \mathrm{~h}$ of culture in the hormone free NCSU-23. After $44 \mathrm{~h}$ of maturation, oocytes were denuded of cumulus cells and used for activation. Oocytes were activated by a $100-\mu \mathrm{sec}$ pulse of $1.5 \mathrm{kV} / \mathrm{cm}$ DC with 1 -mm electrodes in $0.3 \mathrm{M}$ mannitol, $0.1 \mathrm{~mm} \mathrm{MgSO}_{4}$, and $0.05 \mathrm{~mm} \mathrm{CaCl} 2$. Activated oocytes were then cultured for $5 \mathrm{~h}$ in NCSU-23 containing $5 \mathrm{mg} / \mathrm{mL}$ BSA, $10 \mu \mathrm{g} / \mathrm{mL}$ EGF and $7.5 \mu \mathrm{g} / \mathrm{mL}$ cytochalasin B. Embryos were then cultured for 6 days in PZM-5. In Experiment 1, after parthenogenetic activation, embryos were cultured at $38.5^{\circ} \mathrm{C}$ under $5 \% \mathrm{O}_{2}, 5 \% \mathrm{CO}_{2}$ and $90 \% \mathrm{~N}_{2}$ (defined as $5 \%$ $\left.\mathrm{O}_{2}\right)$ or $5 \% \mathrm{CO}_{2}$ in air $\left(20 \% \mathrm{O}_{2}\right)$. The oxygen concentration for embryo culture was changed from $5 \%$ to $20 \%$ on day $1,2,3,4$, and 5 post-activation, respectively. Embryos were also cultured throughout 6 days in 5 and $20 \% \mathrm{O}_{2}$. About 100 embryos were used in each experiment. The number of embryos cleaved and developed to blastocyst stage was observed on day 2 and 6, respectively. In Experiment 2, 10 to 20 embryos cultured in 5 and $20 \% \mathrm{O}_{2}$ were collected on Days 2, 4, and 6 for the detection of ROS, intracellular glutathione (GSH) levels and DNA damage. Intracellular ROS and GSH levels, were measured with fluorescent dyes $\left(2^{\prime}, 7^{\prime}\right.$-dichlorodihydrofluorescein diacetate for ROS and Cell Tracker ${ }^{\mathrm{TM}}$ Blue for GSH). DNA damage of individual embryos was detected with a comet assay. DNA damage was quantified by measuring the length of the streak of DNA comet tail between the edge of the zona pellucida and the end of the visible comet tail by image analysis software. The rate of migrated DNA area per total DNA was also quantified. In Exp. 1, the rate of blastocyst formation was significantly decreased $(P<0.001)$ when embryos were cultured for 6 days under $20 \% \mathrm{O}_{2}(17.8 \pm 4 \%)$ than $5 \% \mathrm{O}_{2}(38.5 \pm 5 \%)$. The rates of blastocyst formation were significantly decreased $(P<0.05)$ when $\mathrm{O}_{2}$ concentration was changed from 5 to $20 \%$ before Day 3. After Day 4, high $\mathrm{O}_{2}$ concentration did not affect the development. In Exp. 2, relative ROS levels were significantly higher $(P<0.05)$ on Day $2(1.5 \pm 0.03)$ and Day $4(1.4 \pm 0.06)$ in embryos cultured under $20 \% \mathrm{O}_{2}$ than in those cultured under $5 \% \mathrm{O}_{2}(1.0)$. No difference was observed in GSH level. DNA damage was significantly increased $(P<0.05)$ in Day 2 embryos cultured under $20 \% \mathrm{O}_{2}(161 \pm 54 \mu \mathrm{m})$ than $5 \% \mathrm{O}_{2}(65 \pm 8.8 \mu \mathrm{m})$. These results indicate that the oxidative stress to embryo development by high $\mathrm{O}_{2}$ concentration is stage specific, that embryos are more sensitive in early stages, and that the oxidative stress has correlation with the increase of intracellular ROS and DNA damage.

\title{
146 EFFECT OF MATERNAL AGE AND PARITY ON THE PRESENCE AND DISTRIBUTION OF CADHERIN-E IN HAMSTER EMBRYOS DURING PRE-IMPLANTATION DEVELOPMENT AND TRANSPORT
}

\author{
A. Trejo, C. Navarro-Maldonado, F. Jimenez, and A. Rosado \\ Universidad Autonoma Metropolitana-Iztapalapa, Mexico City, Iztapalapa, Mexico
}

Our knowledge of the factors that contribute to in vivo embryo development and transport are still incomplete. Although there is solid evidence that the hormonal state of the female regulates ovum transport in mammals, recently, because of significant differences between the rate of oviduct transport of fertilized and unfertilized eggs in several mammalian species, there have been some reports showing that the conceptus itself may auto regulates its own transport. During the initial phases of embryonic development the distribution of E-cadherin, a calcium dependent cell adhesion molecule, changes from a general distribution over the whole surface of the blastomeres to become almost exclusively localized over the points of contact between blastomeres. This change on E-cadherin localization has been related to the beginning of embryo compactation and is considered as the initial stage of differentiation. We have recently shown that pre-implantation embryo development and transport is highly synchronous in nuliparous young females (NYF), but not in adults. Nuliparous adult (8 months) females (NAF) and multiparous adult females (MAF) show significant asynchrony and retard on early embryo development. In NYF, all 8 cells embryos reached the uterus by $62 \mathrm{~h}$ after coitus. In adult females, both nuliparous and multiparous, a considerable number of 8 cells embryos fail to migrate into the uterus even at $67 \mathrm{~h}$ after coitus. E-cadherin distribution was studied by indirect immunofluorescence and confocal microscopy during preimplantation embryo development in three groups, NYF, MAF, and NAF, of regularly cycling golden hamster (Mesocricetus auratus) females. Females were mated with males of proven fertility. Only 15 minutes were allowing for mating. Time of ejaculation was registered. Females were sacrificed from 60 to $69 \mathrm{~h}$ after coitus. Corpora lutea were counted in both ovarian surfaces. Oviducts and uterine horns were flushed separately and embryo number, stage of development, and distribution were recorded. Cell numbers, cadherin distribution and the number of cadherin-dependent cell to cell contacts were established in at least 20 embryos per group and at each of the different hours of the study. Presence and distribution of E-cadherin on 4-, 6-, and 8-cell blastomeres embryos differs accordingly with the parity and age of the females. Differences are particularly important at the eight-cell stage 80 to $93 \%$ of the embryos from NYF showed normal cadherin dependent cell contacts with all neighboring cells. In the case of multiparous adult females (MAF) only 52 to $68 \%$ of the embryos showed normal cell to cell contacts. These results will be discussed in relation to the respective role that the female hamster and the conceptus itself play in early embryo development and transport. Our results also indicate that in spite of the significantly higher ovulation rate of old females, their reproductive efficiency were significantly lower than that of NYF.

\section{IDENTIFICATION OF PRIMORDIAL GERM CELLS IN PORCINE EMBRYOS FROM THE PRIMITIVE STREAK STAGE}

\section{Vejlsted, H. Offenberg, and P. Maddox-Hyttel}

Department of Animal and Veterinary Science, Royal Veterinary and Agricultural University, 1870 Frederiksberg C, Denmark

In embryonic stem cell research, Oct-4 is one of the most widely used markers of pluripotency. Moreover, at least in the mouse, this marker is restricted to primordial germ cells (PGCs) after gastrulation. Vimentin is often used as a marker of mesoderm/mesenchyme in embryonic tissues and appears to localize to the same embryonic cells as Oct-4, at least in the bovine epiblast. The expression of neither of these markers has been completely addressed in the pig. Therefore, the purpose of the present study was to examine the expression of Oct- 4 and vimentin in the porcine epiblast during differentiation and establishment of the three germ layers, i.e. the process of gastrulation. A total of 410 porcine embryos were collected at 8 to 17 days post-insemination from 29 sows of the Danish Landrace breed. Embryos were categorized based on stereo-microscopic observations into the following stages: pre-streak stages 1 and 2, primitive streak stage, neural groove stage, and somite stage. Specimens were 
fixed at all stages, dehydrated and embedded in paraffin wax. Selected embryos at each stage $(n=28)$ were completely cut into serial sections for immunohistochemical evaluation of Oct- 4 and vimentin. Pre-streak stage 1 embryos were defined by lack of polarization of the embryonic disk, whereas in pre-streak stage 2 embryos a crescent shaped thickening was seen at the posterior pole of the disk. This thickening, marking the first morphological anterior-posterior polarization of the embryo proper, was shown to be a site of incipient ingression of cells from the epiblast. Immunohistochemical analyses localized Oct-4 to nuclei and vimentin to cytoplasm of both founding and ingressing epiblast cells. During formation of mesoderm and endoderm, at the primitive streak stage, solitary Oct-4 positive cells, i.e. potential PGCs, were seen scattered in the endoderm. Cells of the epiblast displayed positive labeling for Oct-4 until specification for the ectoderm cell lineage at the subsequent neural groove stage. In mesoderm, Oct-4 likewise disappeared by the time of formation of the first somites, defining the following somite stage. Thus, at this stage the only cells labeled for Oct-4, i.e. potential PGCs, were seen solitarily scattered in the endoderm. By the 15 -somite stage, such cells were no longer visible in the endoderm but were seen located in the mesoderm, spreading from the stalk of the yolk sac and allantois and extending through the mid- and hindgut areas into the incipient genital ridge. Vimentin localized to the mesenchyme and most other derivatives of neural crest and mesodermal origin. In conclusion, based on Oct-4 labeling and distribution pattern, we strongly believe that we have identified the porcine PGCs from the primitive streak stage.

\title{
148 INDUCTION OF HYPOMETHYLATION BY EPIGENETIC ALTERATION OF SOMATIC NUCLEI IN CLONED BOVINE BLASTOCYSTS
}

\author{
G. Wee, J.-J. Shim, B.-S. Song, J.-S. Kim, D.-B. Koo, K.-K. Lee, and Y.-M. Han \\ Laboratory of Development and Differentiation, Korea Research Institute of, Daejon, Korea
}

Epigenetic reprogramming such as DNA methylation is incomplete in cloned embryos during early development as compared with normal embryos. The increased methylation levels of cloned bovine blastocysts are showed in centromeric heterochromatin. The aim of the present study was to investigate the change of methylation state by treatment of trichostatin A (TSA), a specific inhibitor of histone deacetylase in somatic donor nuclei and cloned blastocyst reconstructed with TSA-treated cells or nontreated cells. Bovine ear skin fibroblast cells (bESF) were used as donor cell and treated with TSA for $60 \mathrm{~h}$ at a final concentration of $1 \mu \mathrm{M}$. To methylation analysis of satellite I as specific DNA sequence, genomic DNA from $7 \times 10^{4}$ cells and a blastocyst were isolated, and then the genomic DNA was analyzed by bisulfite sequencing. The reduction of HDAC1, 2 and Dnmt family such as Dnmt1, Dnmt3a, Dnmt3b, and Dnmt3L after TSA treatment were shown by Western blot in bESF, but histone acetyltransferases such as Tip60 and HAT1 were not changed. Satellite I DNA in nontreated cells was highly methylated in CpG sequences, whereas methylation level of TSA-treated cells was significantly decreased (64 vs. $48 \%, P<0.05)$. After nuclear transfer using normal or altered donor cells, methylation levels of satellite were measured at the blastocyst stage of NT and TSA-NT embryos as compared with IVF embryos. In nontreated NT blastocysts, methylation levels were significantly higher than IVF blastocysts ( 66 vs. $29 \%, P<0.05$ ) and were similar to that of nontreated bESF cells. The reduction of methylation levels in TSA-NT blastocysts were showed and were significantly lower than NT blastocyst derived with nontreated cells (37 vs. $66 \%, P<0.05$ ), but no significant differences were found between TSA-NT and IVF blastocysts. Also, the levels of methylation were similar to that of TSA-treated donor cells. In blatocyst formation, TSA-NT embryos were improved significantly compared with NT or IVF embryos ( 45.9 vs. 31.7 or $28 \%, P<0.05$ ). These results demonstrated that somatic methylation status after epigenetic alteration affect in early cloned embryo development, suggesting epigenetic control may help to solve of inherent problems in cloning.

\section{Early Pregnancy/Pregnancy Recognition}

\section{EXPRESSION OF ANGIOGENIC FACTORS AND THEIR MAJOR RECEPTORS IN THE SHEEP PLACENTA THROUGHOUT THE EARLY PREGNANCY}

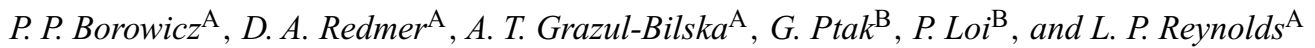 \\ ${ }^{A}$ Center for Nutrition and Pregnancy, North Dakota State University, Fargo, ND, 58105-5727, USA; \\ ${ }^{\mathrm{B}}$ Dipartimento di Scienze Biomediche Comparate, Universita di Teramo, 64100 Teramo, Italia
}

Embryonic losses are high in mammals, with more than $30 \%$ of fertilized eggs not resulting in an offspring. The development of the placenta is critical for normal fetal growth and development as it provides for exchange of respiratory gases, nutrients, and wastes between the fetal and maternal systems. Placental vascular development determines the rate of placental blood flow, which is a primary determinant of placental function. Recent studies suggest that vascular endothelial growth factor (VEGF), its receptors (VEGFR), along with angiopoietins (Ang-1 and Ang-2) and their common receptor Tie-2, are major placental angiogenic factors, along with fibroblast growth factor-2 (FGF-2) and its receptor (FGFR). To evaluate the patterns of placental expression of these factors during early placental development, gravid uteri were obtained from ewes ( $n=6$ per day) on Days $12,18,24,30$, and 40 of gestation (day of mating = Day 0 ). At slaughter the uterine and embryonic tissues were weighed and representative samples of utero-placenta (CAR - caruncle, maternal placenta; ICAR - intracarunclar, endometrium; FM, fetal membranes) were snap frozen on dry ice and analyzed for relative mRNA levels by real-time RT-PCR (ABI Prism 7000, Sequence Detection System, Applied Biosystems, Monza, Italy) of vascular endothelial growth factor (VEGF), vascular endothelial growth factor receptor-1 (VEGFR-1), vascular endothelial growth factor receptor-2 (VEGFR-2), angiopoietin-1 (Ang-1), angiopoietin-2 (Ang-2), receptor for both angiopoietins (Tie-2), fibroblast growth factor-2 (FGF-2), and fibroblast growth factor receptor (FGFR). The data were analyzed by nonlinear procedures using proc reg of SAS (SAS Institute, Inc., Cary, NC, USA). In CAR, the data showed the exponential increase from Days 12 to 40 in mRNA expression for VEGFR-1 $\left(P<0.0004 ; 0.04398 \mathrm{e}^{0.08794 \times \text { day }}\right)$, VEGFR-2 $(P<0.01$; $\left.0.119208 \mathrm{e}^{0.06537 \times \text { day }}\right)$, Ang-1 $\left(P<0.005 ; 0.00488 \mathrm{e}^{0.10881 \times \text { day }}\right)$, Ang-2 $\left(P<0.0001 ; 0.01591 \mathrm{e}^{0.07864 \times \text { day }}\right)$, Tie- $2\left(P<0.03 ; 0.00488 \mathrm{e}^{0.06852 \times \text { day }}\right)$, and FGFR $\left(P<0.08 ; 0.24577 \mathrm{e}^{0.04721 \times \text { day }}\right)$. In the CAR, we also observed an exponential decrease in mRNA concentration for VEGF $(P<0.05$; 
28.193 $\left.\mathrm{e}^{-1.0719 \times \text { day }}\right)$. In ICAR, we observed an exponential increase in mRNA concentration for VEGF $\left(P<0.05 ; 1.11685 \mathrm{e}^{0.06865 \times \text { day }}\right)$, VEGFR-1 $\left(P<0.07 ; 0.09853 \mathrm{e}^{0.0383 \times \text { day }}\right)$, Ang-1 $\left(P<0.09 ; 0.009318 \mathrm{e}^{0.05711 \times \text { day }}\right)$, and Ang-2 $\left(P<0.004 ; 0.012647 \mathrm{e}^{0.09973 \times \text { day }}\right)$. For FM, no changes in mRNA levels were observed from Days 12 to 40, but levels of all mRNAs were similar to those in CAR and ICAR. Based on the patterns of mRNA expression, these data indicate that these angiogenic factors may play an important role in early placental angiogenesis in sheep.

This work was supported by NIH grant HL64141 to LPR and DAR.

\title{
150 EFFECT OF TIMELY FLUNIXIN MEGLUMINE TREATMENT ON PREGNANCY RATES IN HOLSTEIN HEIFERS
}

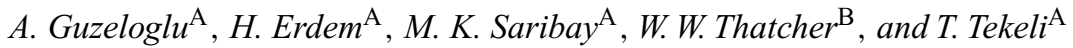 \\ ${ }^{A}$ Selcuk University, Faculty of Veterinary Medicine, Department of Obstetrics and Gyneacology, Konya, Turkey; \\ ${ }^{\mathrm{B}}$ Department of Animal Sciences, University of Florida, Gainesville, FL 32611, USA
}

The objective was to determine if administration of flunixin meglumine (FM) to heifers following insemination would increase pregnancy rate due to its inhibitory effect on prostaglandin F2 $\alpha$ (PGF2 $\alpha$ ) synthesis. Fifty-two 15-month-old Holstein heifers were synchronized with single or double injections of PGF2 $\alpha$ followed by an injection of gonadotropin-releasing hormone (GnRH) $48 \mathrm{~h}$ later and a timed artificial insemination at $12-14 \mathrm{~h}$ after injection of GnRH (Day 0). Heifers randomly assigned to the treatment group (FMG) were injected twice with FM (1.1 mg/kg BW; i.m.), given $12 \mathrm{~h}$ apart on the evening of Day 15 and the morning of Day 16 . The control group (CG) was not treated. Pregnancy rates were defined as the percentage of heifers diagnosed pregnant by ultrasound at Days 29 and 65 after AI. Effects of treatment on pregnancy rates and pregnancy losses were analyzed by chi-square test and logistic regression analysis. Pregnancy rates in the heifers treated with FM were higher at Day $29(76.9 \% ; 20 / 26$ [FMG] vs. 50\%; 13/26 [CG]; $P<0.04)$ and tended to be higher at Day $65(69.2 \% ; 18 / 26$ [FMG] vs. 46.2\%; $12 / 26$ [CG]; $P<0.09)$. Administration of FM two times at a critical stage leading up to pregnancy recognition, associated with corpus luteum maintenance, increased both early embryo survival and pregnancy rate via an additive antiluteolytic effect due to a delay in the luteolytic secretion of PGF2 $\alpha$; this provided conceptuses extra time to develop the capability to inhibit the luteolytic process.

\section{PROTEOMICS ANALYSIS OF PREGNANCY-SPECIFIC SERUM PROTEINS IN BOVINE}

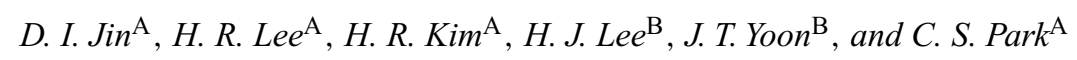

${ }^{A}$ Research Center for Transgenic Cloned Pig, Chungnam National University, Daejoen City, Korea;

${ }^{\mathrm{B}}$ Genetic Engineering Institute, Hankyong National University, Ansung City, Korea

To identify early pregnancy-specific serum proteins in bovine, we performed proteomics analysis using blood serum samples of pregnant and nonpregnant Holstein dairy cattle Days 21 and 35 after AI. A total of eight pregnant and eight non-pregnant cattle were used for collection of the blood samples. The global proteomics approach was exploited by the use of 2-D gel electrophoresis and mass spectrometry to sort out pregnancy-specific proteins. Serum proteins within isoelectric point ranges of 4.0 to $7.0,6.0$ to 9.0 , and 5.5 to 6.7 were analyzed separately by 2 -D electrophoresis with three replications of each sample. The stained gels were scanned and calibrated at an optical resolution of $63.5 \mu \mathrm{m} /$ pixel using a GS-710 imaging densitometer (Bio-Rad Laboratories, Philadelphia, PA, USA). A total of approximately 1200 spots were detected in 2-D gels stained with Coomassie-blue. In the comparison of serum samples from pregnant and non-pregnant cattle, nine pregnancy-specific spots were detected unanimously in Day 21 and Day 35 serum samples. Pregnancy-specific proteins were identified as transferrin, albumin, IgG2a heavy chain constant region, and immunoglobulin gamma heavy chain variable region by means of MALDI-TOF-MS (PerSeptive Biosystems, Framingham, MA, USA). Even though the identified spots were abundant serum proteins, their molecular weights and $\mathrm{pI}$ values were different from those of the main serum proteins. Most proteins identified in this analysis appeared to be related - with pregnancy-specific subunits or fragments of transferrin, albumin, and IgG. One of the pregnancy-specific proteins, transferrin, is known to be related to iron transport during pregnancy. Western blot analysis using polyclonal anti-transferrin antibody revealed specific transferrin expression in the serum samples from the pregnant cattle but no detectable expression in the serum samples from the non-pregnant cattle. Our results revealed composite profiles of key proteins involved in early pregnancy and suggest the potential use of identified proteins to detect early pregnancy in bovine.

\section{SURVIVAL OF BIOPSIED DAY 15 BOVINE CONCEPTI RE-TRANSFERRED TO SYNCHRONIZED RECIPIENT HEIFERS}

\author{
R. Lee, M. Berg, L. McGowan, J. Peterson, A. Ledgard, and N. Li
}

AgResearch, Hamilton, Waikato, New Zealand

In cattle, a significant proportion of in vitro-produced (IVP) blastocysts do not result in viable pregnancies after transfer to recipient surrogates. Betteridge et al. (1980 J. Reprod. Fert. 59, 205-216) showed that it was possible after superovulation to recover elongated bovine embryos up to Day 17, transfer them into synchronized recipient cows, and have them develop further. We investigated the feasibility of recovering cattle embryos at Day 15, taking a sample of the trophoblast and transferring the embryos into recipients afterward for further development. The biopsied material could be used later to evaluate gene expression and correlate the profile retrospectively with developmental potential. With this approach, a larger amount of material is available for study and only embryos surviving to the elongation stage would be examined. In our experience, $30-40 \%$ of transferred blastocysts do not develop to the elongation stage. In three separate experiments, IVP embryos were generated using abattoir derived oocytes and cultured in SOF-aa supplemented with BSA (Thompson et al. $2000 \mathrm{~J}$. Reprod. Fert. 118, 47-55). Six graded Day 7 (Day $0=$ day of IVF) blastocysts were transferred into synchronized recipient heifers $(n=10$ for each experiment). At Day 15 of gestation, concepti were flushed from the 
uteri after slaughter with EmCare Flush (ICPbio, Ltd., Suckland, New Zealand) containing $25 \mathrm{mg} / \mathrm{mL}$ kanamycin sulfate and then put into EmCare Hold. Conceptus lengths were measured and a proportion of those $>30 \mathrm{~mm}$ long were cut off $(5-15 \mathrm{~mm})$ at one end and the trophoblast kept for future analysis. Pairs of cut or uncut (control) concepti were loaded into $0.25-\mathrm{mL}$ embryo transfer straws. Each pair was transferred nonsurgically into recipients synchronized at Days 15 (Expt. 1,n=17) or 13 (Expts. 2 and 3, $n=16$ and 17, respectively) of the estrous cycle. The time between embryo flush and transfer to a recipient was noted. At Day 30, embryo survival was assessed at slaughter and compared using the Fisher's exact and chi-square. Day 15 conceptus lengths varied between 1 and $140 \mathrm{~mm}$. The time between flush and transfer varied between 13 and $126 \mathrm{~min}$ and did not affect the ability of the concepti to subsequently establish pregnancies. Transfer to an earlier uterine environment did not significantly improve embryo survival. The proportion of embryos recovered at Day 30 was not affected by the biopsy. Up to $10 \mathrm{~mm}$ can be removed from $40-10 \mathrm{~mm}$ concepti without effect on subsequent survival. However, the overall survival post-Day 15 transfer is still too low for practical application.

Table 1.

\begin{tabular}{lccc}
\hline Parameter & $\begin{array}{c}\text { Expt. 1 } \\
\text { (D15 into D15) }\end{array}$ & $\begin{array}{c}\text { Expt. 2 } \\
\text { (D15 into D13) }\end{array}$ & $\begin{array}{c}\text { Expt. 3 } \\
\text { (D15 into D13) }\end{array}$ \\
\hline D15 embryo recovery (\%) & 64 & 62 & 66 \\
D15 conceptus length $^{\mathrm{a}}$ & $38 \pm 4$ & $14 \pm 3$ & $37 \pm 7$ \\
D30 conceptus recovery (\%) $^{\mathrm{b}}$ & 20 & 28 & 26 \\
Biopsied embryos recovered $^{\mathrm{c}}$ & $3 / 14$ & $1 / 5$ & $4 / 14$ \\
Control embryos recovered $^{\mathrm{c}}$ & $4 / 20$ & $8 / 27$ & $5 / 21$ \\
\hline
\end{tabular}

${ }^{\mathrm{a}}$ Mean \pm SEM (mm); ${ }^{\mathrm{b}}$ Both biopsied and controls; ${ }^{\mathrm{c}}$ Number recovered from number transferred.

\title{
153 ALLEVIATIVE EFFECTS OF ANTIOXIDANT ADMINISTRATION ON MATERNAL HYPERTHERMIA-INDUCED EARLY EMBRYONIC DEATH IN MICE
}

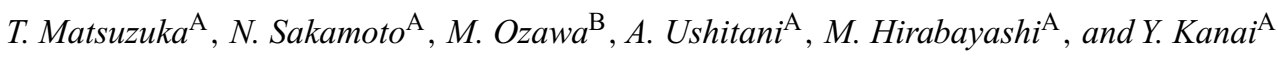 \\ ${ }^{\mathrm{A}}$ Graduate school of Life and Environmental Sciences, University of Tsukuba, Tsukuba, Iaraki, Japan; \\ ${ }^{\mathrm{B}}$ Genetic Diversity Department, National institute of Agrobiological Sciences, Tsukuba, Iaraki, Japan
}

Hyperthermia-induced early embryonic death is generally ascribed to the high susceptibility of early embryos to elevated maternal body temperature. However, recent studies have indicated that the disruption of embryonic development by maternal hyperthermia has relevance to not only high temperature exposure to the embryo, but also hyperthermia-associated changes in the maternal body. Hyperthermia enhances physiological production of reactive oxygen species (ROS) systemically. Early embryos are susceptible to oxidative stress and it becomes easy to arrest their development when the oxidative stress is exposed. These findings led us to speculate that maternal hyperthermia-induced early embryonic death is caused by an increase in oxidative stress to the embryo. Vitamin E and melatonin are both well known to function as antioxidants in vivo when they are administered exogenously. In this study, we administered vitamin $\mathrm{E}$ or melatonin to heat-stressed pregnant mice, aiming to alleviate the hyperthermia-induced embryonic death. Female mice were heat-stressed $\left(35^{\circ} \mathrm{C}\right.$ with $60 \%$ relative humidity) for $12 \mathrm{~h}$ on the day of mating. In Experiment 1 , vitamin $\mathrm{E}$ $(1000 \mathrm{mg} / \mathrm{kg}$ body weight) was injected intraperitoneally just before stress treatment, or melatonin $(3 \mathrm{mg} / \mathrm{kg}$ body weight $)$ was injected subcutaneously for every $2 \mathrm{~h}$ during heat exposure. Then, zygotes were collected and in vitro developmental ability was assessed. In Experiment 2, intracellular glutathione (GSH) content in the zygote, ROS level and free radical scavenging activity (FRSA) in the oviduct, and lipid peroxidation level in the liver were measured to evaluate the effects of melatonin administration on physiological redox status. All data were analyzed using one-way ANOVA followed by Fisher's protected least-significant test. The percentage of embryos that developed to the morula or blastocyst stage was significantly $(P<0.01)$ increased by administration of either vitamin E $(58.6 \%)$ or melatonin $(47.9 \%)$ compared with that in heat-stressed mice that were administered placebos (14.8\%). Intracellular GSH content in zygotes derived from melatonin-administered mice was not significantly different from that in unstressed mice (1.67 and $1.82 \mathrm{pmol} /$ zygote, respectively), whereas in heat-stressed mice that were administered placebo, intracellular GSH content was significantly decreased ( $1.48 \mathrm{pmol} /$ zygote, $P<0.05)$ compared to that in unstressed mice. There were no significant differences in lipid peroxidation levels in the liver and in ROS levels in the oviduct between melatonin-administered stressed mice and unstressed mice, although these parameters in heat-stressed mice with placebo were significantly higher than in unstressed mice $(P<0.05)$. Furthermore, FRSA in the oviduct was significantly $(P<0.05)$ higher in the melatonin-administered mice than in the heat-stressed mice. These findings suggest that antioxidant administration to heat-stressed mice alleviates the hyperthermia-induced early embryonic death, and this may be accomplished in part by maintaining a neutral redox status within the mother.

\section{EFFECTS OF THE BREED AND SEASON ON THE FERTILITY OF TROPICAL DUAL PURPOSE COWS}

\author{
H. Nava-Trujillo ${ }^{\mathrm{A}}$, A. Hernández ${ }^{\mathrm{A}}$, H. Hernández-Fonseca ${ }^{\mathrm{A}}$, E. Soto-Belloso $^{\mathrm{A}}$, and F. Perea-Ganchou ${ }^{\mathrm{B}}$
}

A Facultad de Ciencias Veterinarias, Universidad del Zulia, Maracaibo, Zulia, Venezuela;
B Nucleo Universitario Rafael Rangel, Universidad de los Andes, Valera, Trujillo, Venezuela

First service fertility is an important factor affecting the calving-conception interval and the number of services per conception. In the present study, first service fertility of 618 multiparous dual purpose cows was analyzed according to breed predominance (Bos indicus and Bos taurus) and service 
season: dry (December-March); sub-humid (April-August); and humid season (September-November). All cows were located in a sub-humid tropical forest area, characterized by $1950 \mathrm{~mm} /$ year of rainfall and a mean daily temperature of $28.3^{\circ} \mathrm{C}$. Cows were milked and suckled twice a day. Estrus was detected twice a day with a minimum observation period of $30 \mathrm{~min}$. All cows were serviced according to the international rule AM-PM with semen of one of two Brahman bulls (A and B). All cows received water and mineral salt ad libitum during the entire year. Additionally, during the dry season cows received an energy supplementation. Data were analyzed using the chi-square procedure of SAS (SAS/STAT User's Guide, 8.2 ed. Cary, NC: SAS Institute, Inc., 2001). No bull effects were observed on first service fertility (bull A: 37.70\%, 105/305; and bull B: 40.26\%, 126/313; $P>0.05)$. Breed predominance significantly affected the first service fertility, being higher in Bos indicus cows (47.48\%) than in Bos taurus cows $(32.78 \% ; P<0.05)$. The higher first service fertility of Bos indicus cows seen during the year was probably because Bos indicus cattle are more thermotolerant than Bos taurus cattle (Hansen 2004 Anim. Reprod. Sci. 82-83, 349-360). Moreover, season of service did not affect the first service fertility of Bos indicus cows (dry: 48.95\%, 70/143; sub-humid: 50\%, 33/66; and humid: 41.30\%, 19/46; $P>0.05$ ). While in Bos taurus cows, first service fertility was higher in cows serviced during the dry season $(43.24 \%)$ in comparison with those serviced during the sub-humid $(28.26 \% ; P<0.05)$ or humid season $(23.58 \% ; P<0.05)$. No differences were observed between Bos taurus cows serviced in the sub-humid and the humid season $(P>0.05)$. First service fertility did not differ between Bos indicus and Bos taurus cows serviced during the dry season $(P>0.05)$, which is likely due to the lower relative humidity and extra energy supplementation. This may improve oocyte quality. In conclusion, fertility of $B o s$ taurus cattle is more sensitive than that of Bos indicus cattle under tropical conditions; therefore, the implementation of a reproductive seasonality scheme is recommended to increase the reproductive efficiency of Bos taurus cattle.

\title{
Embryo Culture
}

\section{QUALITY OF PORCINE EMBRYO PRODUCED IN TWO EMBRYO CULTURE MEDIA AS ASSESSED BY TOTAL CELL NUMBER AND TIME COURSE OF BLASTOCYST HATCHING}

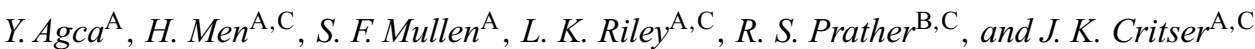 \\ ${ }^{A}$ Comparative Medicine Center, University of Missouri-Columbia; ${ }^{\mathrm{B}}$ Department of Animal Sciences, University of Missouri-Columbia; \\ ${ }^{\mathrm{C}}$ National Swine Resource and Research Center, University of Missouri-Columbia, Columbia, MO 65201, USA
}

The ability to produce porcine embryos of good quality will have a significant impact on a number of porcine assisted reproductive technologies, such as cloning, intracytoplasmic sperm injection, and embryo cryopreservation. However, porcine embryos resulting from current serum-free embryo culture systems differ significantly both structurally and functionally from those derived in vivo (Wang et al. 1999 Mol. Reprod. Dev. 53, 99-107). In this experiment, the quality of porcine embryos produced by North Carolina State University (NCSU)-23 medium (Petters and Wells 1993 J. Reprod. Fertil. Suppl. 1993, 48, 61-73) and porcine zygote medium (PZM)-1 (Yoshioka et al. 2002 Biol. Reprod. 66, 112-119) were compared by assessing the total cell number and the time course of in vitro blastocyst hatching. Porcine embryos were produced by in vitro maturation and fertilization using serum-free systems. After fertilization, presumptive zygotes were randomly allocated to either PZM-1 or NCSU-23 for subsequent development. On Day 4 of culture, the embryo culture media were supplemented with $10 \%$ fetal bovine serum (FBS). Day 6 blastocysts from each group were counted and the blastocysts were subsequently fixed in $4 \%$ formalin for counting the total cell number. The cell number in each embryo was determined by counting the nuclei after staining with bisbenzimide (Hoechst 33342). To assess the hatching ability of blastocysts, Day 6 blastocysts were cultured until Day 9 and hatched blastocysts were counted daily. Day 6 blastocyst rates (ratio of blastocysts to oocytes) and total cell number count were replicated three times. The time course of blastocyst hatching experiment was repeated four times. The data were analyzed using a chi-square test, Fisher's exact test, or Student's $t$-test. The blastocyst rate from culture in PZM-3 was $19.4 \pm 0.96 \%$ (mean \pm SEM), which was similar to that $(16.7 \pm 3.2 \%)$ resulting from culture in NCSU-23 $(P>0.05)$. However, the total cell number in Day 6 blastocysts cultured in PZM-3 was significantly higher than for blastocysts cultured in NCSU-23 ( $57 \pm 3.1 \mathrm{vs.} 46 \pm 1.7 ; P<0.01)$. The total hatching rates (ratio of hatched blastocysts to total blastocysts) by Day 9 were similar between the two culture systems $(50.1 \pm 9.1 \%$ vs. $50.7 \pm 4.1 \% ; P>0.05)$. However, on Day $6,2.1 \%$ of blastocysts from PZM-3 culture hatched whereas no blastocysts from NCSU-23 culture hatched. The cumulative hatching rates from PZM-3 culture on Day 7 were significantly higher than those from NCSU-23 culture $(15.1 \pm 3.8 \%$ vs. $2.6 \pm 1.1 \% ; P<0.01)$. In conclusion, these data suggest that blastocysts produced in PZM-3 medium have better quality than blastocysts produced in the NCSU-23 culture system as assessed by the total cell number and the time course of blastocyst hatching.

This project was supported by a grant from the National Institutes of Health (U42 RR 018877).

\section{PRETREATMENT WITH THE ANTIOXIDANT EPIGALLOCATECHIN GALLATE (EGCG) COUNTERACTS THE NEGATIVE EFFECTS OF MATERNAL HYPERTHERMIA ON EMBRYONIC DEVELOPMENT IN MICE}

\author{
A. Aroyo ${ }^{\mathrm{A}, \mathrm{B}}, S . \operatorname{Yavin}^{\mathrm{A}, \mathrm{B}}, Z$. $\operatorname{Roth}^{\mathrm{B}}$, and A. Arav ${ }^{\mathrm{A}}$ \\ ${ }^{A}$ Institute of Animal Science, Agricultural Research Organization, The Volcani Center, Bet Dagan 50250, Israel; \\ ${ }^{\mathrm{B}}$ Department of Animal Science, Faculty of Agricultural, Food and Environmental Quality Sciences, \\ The Hebrew University of Jerusalem, Rehovot 76100, Israel
}

Hyperthermia-induced oxidative stress is one of the suggested mechanisms underlying the loss of developmental competence in heat-stressed embryos. The objective of the present study was to determine whether pretreatment with the antioxidant epigallocatechin gallate (EGCG) would 
counteract the negative effects of maternal hyperthermia on oocyte competence and improve subsequent embryonic development. Exp. 1 examined the effect of pretreatment with EGCG on ex vivo embryonic development under normal culture conditions ( $\mathrm{KSOM}, 37^{\circ} \mathrm{C}, 5 \% \mathrm{CO}_{2}, 95 \% \mathrm{RH}$ in air). Female mice $\left(\mathrm{CB}_{6} \mathrm{~F}_{1}\right)$ were synchronized (PMSG $\left.+\mathrm{hCG}\right)$ and injected with $0.4 \mathrm{~mL}$ EGCG $(100 \mathrm{mg} / \mathrm{kg}$ body weight) or with saline. Both EGCGand saline-treated females were paired with stud males overnight. Mated mice were sacrificed and putative zygotes recovered and cultured in vitro. Cleavage and blastocyst formation rates were recorded on Days 1 and 5 post-fertilization, respectively. The percentage of putative zygotes that cleaved into the two-cell stage did not differ between the groups; however, blastocyst formation rate was higher $(P<0.05)$ in the EGCG group $(85 \pm 2 \%)$ than in the saline group $(75 \pm 2 \%)$. In Exp. 2 (a $2 \times 2$ factorial study), both EGCG- and saline-treated mice were exposed to normothermal conditions (NT; $22^{\circ} \mathrm{C}, 45 \% \mathrm{RH}$ ) or heat stress (HS; $40^{\circ} \mathrm{C}, 70 \% \mathrm{RH}$ ) for 1.5 to $2 \mathrm{~h}$, the latter to induce a rise of $2^{\circ} \mathrm{C}$ in body temperature. Synchronized mice were paired with stud males overnight and mated mice were then sacrificed. Putative zygotes were recovered and cultured in vitro as described above. The number of putative zygotes recovered, cleavage and blastocyst formation rates and percentage of hatched blastocysts are presented in Table 1. Blastocyst formation rate was higher $(P<0.05)$ in the HS-EGCG group than in the HS-saline group. In addition, HS-EGCG embryos exhibited developmental competence similar to that of embryos from both NT groups. In summary, pretreatment with EGCG improved developmental competence under the described culture conditions. Furthermore, pretreatment with the antioxidant EGCG counteracted the negative effects of maternal hyperthermia. Further studies are warranted to evaluate the effect of pretreatment with EGCG on embryo quality.

Table 1. Effect of pretreatment with EGCG on developmental competence of heat stressed oocytes

\begin{tabular}{lcccl}
\hline Group & Recovered putative zygotes/mouse & Cleavage rate (\%) & Blastocysts (\%) & Hatching (\%) \\
\hline NT-saline & 39 & $119 / 142(84)^{\mathrm{a}}$ & $107 / 119(90)^{\mathrm{a}}$ & $59 / 107(55)^{\mathrm{a}}$ \\
NT-EGCG & 56 & $141 / 170(83)^{\mathrm{a}}$ & $133 / 141(94)^{\mathrm{a}}$ & $62 / 133(47)^{\mathrm{ab}}$ \\
HS-saline & 47 & $183 / 212(86)^{\mathrm{a}}$ & $143 / 183(78)^{\mathrm{b}}$ & $54 / 143(38)^{\mathrm{b}}$ \\
HS-EGCG & 54 & $148 / 166(89)^{\mathrm{a}}$ & $132 / 148(89)^{\mathrm{a}}$ & $62 / 132(47)^{\mathrm{ab}}$ \\
\hline
\end{tabular}

${ }^{a b}$ Values within columns followed by different superscript letters are significantly different $(P<0.05)$.

\title{
157 EFFECTS OF FETAL CALF SERUM OR PHENAZINE ETHOSULFATE AND FRUCTOSE OR GLUCOSE ON EMBRYONIC DEVELOPMENT AND LIPID ACCUMULATION OF BOVINE EMBRYOS
}

\author{
M. Barcelo-Fimbres and G. Seidel Jr
}

Animal Reproduction and Biotechnology Laboratory, Colorado State University, Fort Collins, CO 80521, USA

Slaughterhouse oocytes $(n=6222)$ were maturated in a chemically defined medium (CDM) similar to SOF plus $0.5 \%$ fatty acid-free BSA (FAF$\mathrm{BSA}$ ) and hormones (M-CDM) for $23 \mathrm{~h}$ at $38.5^{\circ} \mathrm{C}$ in $5 \% \mathrm{CO}_{2}$ in air. Oocytes and frozen-thawed sperm, centrifuged through a Percoll gradient, were co-cultured for $18 \mathrm{~h}$ in F-CDM (CDM + heparin). Zygotes were cultured at $38.5^{\circ} \mathrm{C}$ in $5 \% \mathrm{CO}_{2} / 5 \% \mathrm{O}_{2} / 90 \% \mathrm{~N}_{2}$ in CDM-1 (CDM + nonessential amino acids, $10 \mu \mathrm{M}$ EDTA, 0.5\% FAF-BSA, and $0.5 \mathrm{mM}$ fructose or glucose in Expt 1 and glucose in Expt 2). In both experiments, after $48 \mathrm{~h}$, 8-cell embryos were cultured $135 \mathrm{~h}$ in CDM-1 (CDM-1 + essential amino acids, no EDTA, and $2 \mathrm{mM}$ fructose or glucose). A factorial design with two hexoses and three additives in CDM-2 (control; $10 \%$ fetal calf serum (FCS); and $0.3 \mu \mathrm{M}$ phenazine ethosulfate (PES), an electron acceptor that oxidizes NADPH) was used for both experiments, each replicated eight times. For Expt 1, Day 7.5 blastocysts were fixed and stained with Sudan Black B to quantify cytoplasmic lipid droplets. A digital photo at $600 \times$ of the equatorial part of the embryo was evaluated by classifying lipophilic droplet diameters as small $(\mathrm{S},<2 \mu \mathrm{m})$, medium $(\mathrm{M}, 2$ to $6 \mu \mathrm{m})$, or large $(\mathrm{L},>6 \mu \mathrm{m})$, reported as number of lipid droplets (LD) per $1000 \mu \mathrm{m}^{2}$. Data were analyzed by ANOVA. For Expt 1, 8-cell embryo production per oocyte matured was not affected by fructose or glucose $(P>0.1)(70 \mathrm{vs} .68 \%$, respectively); however, blastocyst rates per oocyte matured $(\mathrm{B} / \mathrm{O})$ and per 8 -cell embryo $(\mathrm{B} / \mathrm{E})$ were higher $(P<0.01)$ for fructose than glucose (Table 1). There were no differences between control, PES, and FCS $(P>0.1)$ for $\mathrm{B} / \mathrm{O}$, or B/E. For Expt $2, \mathrm{~B} / \mathrm{O}$ and $\mathrm{B} / \mathrm{E}$ were higher $(P<0.01)$ for fructose than for glucose. No differences were found for additives $(P>0.1)$ control, FCS, or PES for B/O or B/E. There was an interaction $(P<0.05)$ between additives and hexoses for blastocyst production, because the benefit of fructose compared to glucose was greater for controls than for FCS or PES (means not presented). Accumulations of each size of LD were less for PES $(P<0.05)$ than for control and FCS. Control and PES were lower than FCS $(P<0.05)$ for S, M, and L droplets. There was no effect of fructose or glucose $(P>0.1)$ on numbers of S, M, or L droplets (Table 1). In conclusion, fructose produced more blastocysts than glucose after 8-cell development, but there was no hexose effect either before this stage or in lipid accumulation. PES reduced and FCS increased lipid accumulation relative to controls.

Table 1. Main effects of additives and hexoses on development of bovine embryos $( \pm \mathrm{SE})$

\begin{tabular}{lccccccc}
\hline Main effect & $\mathrm{B} / \mathrm{O}(\%)$ & $\mathrm{B} / \mathrm{O}(\%)$ & $\mathrm{B} / \mathrm{E}(\%)$ & $\mathrm{B} / \mathrm{E}(\%)$ & $\mathrm{LD} / 10^{3} \mu \mathrm{m}^{2}$ & $\mathrm{LD} / 10^{3} \mu \mathrm{m}^{2}$ & $\mathrm{LD} / 10^{3} \mu \mathrm{m}^{2}$ \\
\hline & Expt 1 & Expt 2 & Expt 1 & Expt 2 & $\mathrm{S}$ & $\mathrm{M}$ & $\mathrm{L}$ \\
Fructose & $23 \pm 0.9^{\mathrm{a}}$ & $37 \pm 0.9^{\mathrm{a}}$ & $33 \pm 1.2^{\mathrm{a}}$ & $51 \pm 1.2^{\mathrm{a}}$ & $45 \pm 3$ & $31 \pm 2$ & $10 \pm 1$ \\
Glucose & $19 \pm 0.9^{\mathrm{b}}$ & $33 \pm 0.9^{\mathrm{b}}$ & $26 \pm 1.2^{\mathrm{b}}$ & $45 \pm 1.2^{\mathrm{b}}$ & $47 \pm 3$ & $32 \pm 2$ & $12 \pm 1$ \\
PES & $24 \pm 1.4$ & $35 \pm 1.5$ & $34 \pm 1.5$ & $49 \pm 1.5$ & $32 \pm 4^{\mathrm{e}}$ & $23 \pm 3^{\mathrm{e}}$ & $5 \pm 2^{\mathrm{e}}$ \\
\hline
\end{tabular}

${ }^{\mathrm{a}, \mathrm{b}} P<0.01 ;{ }^{\mathrm{c}, \mathrm{d}, \mathrm{e}} P<0.05$. 


\title{
EFFECTS OF APOTRANSFERRIN ON IN VITRO MATURATION OF CUMULUS-OOCYTES COMPLEXES (COCS) IN HANWOO, KOREAN NATIVE COWS
}

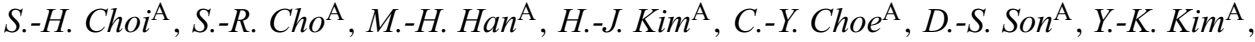 \\ Y.-G. Chung ${ }^{\mathrm{B}}$, and H. Hoshi ${ }^{\mathrm{A}}$ \\ A Animal Genetic Resources Station, NLRI, RDA, Namwon, Cheonbuk, Korea; \\ ${ }^{B}$ ET Biotech, Daegu, Kyungbuk, Korea; \\ ${ }^{\mathrm{C}}$ Institute of Functional Peptide, Yamagata, Japan
}

For in vitro production of embryos, animal sera have been used as energy sources, maturation promoters, vitamins, growth factors, and antioxidative compounds. However, the sera had risk of virus and mycoplasma infections which could result in too big offspring and cause dystocia in ovine and bovine. Apotransferrin (apo-Tf) is a component of mammalian sera and has played a role as an antioxidant in media. A study was conducted to investigate the effects of apo-Tf on in vitro maturation of cumulus-oocytes complexes (COCs) in Hanwoo, Korean native cows. Ovaries were collected from a slaughterhouse and COCs were taken from 2-6-mm antral follicles. The collected COCs were washed three times with $0.1 \mathrm{M}$ polyvinyl alcohol (PVA)-TCM199 and matured in 0, 1, 10, or $100 \mu \mathrm{g} / \mathrm{mL}$ apo-Tf with TCM- 199 at $39^{\circ} \mathrm{C}, 5 \% \mathrm{CO}_{2}, 95 \%$ air for 6,12 , or $24 \mathrm{~h}$. Mature COCs were fertilized with frozen-thawed Korean native cattle semen treated with BO medium (Brackett and Oliphants 1975 Biol. Reprod. 12, 260-274) containing $5 \mathrm{mM}$ caffeine and $1 \mu \mathrm{g} / \mathrm{mL}$ heparin for $8 \mathrm{~h}$ and developed to the blastocyst stage in 5\% FBS and 0.3\% BSA in TCM199-IVMD (IFP, Japan). To evaluate the morphology of nuclear types, the matured COCs were fixed in 1:3 acetic acid-ethanol for $30 \mathrm{~s}$ and stained with 3\% basic Fuchsin. IVM and IVF were replicated three times. All of the results were analyzed by ANOVA using the STATVIEW program. The maturation rates of control were $34.2 \%, 37.3 \%$, and $45.8 \%$ for 6,12 , and $24 \mathrm{~h}$, respectively. There were no differences among the concentrations of apo-Tf, and nuclear types at $78.3-87.0 \%$ GVBD for $6 \mathrm{~h}, 82.8-91.3 \%$ MI for $12 \mathrm{~h}$, and $88.9-100.0 \% \mathrm{MII}$ for $24 \mathrm{~h}$, with 1,10 , and $100 \mu \mathrm{g} / \mathrm{mL}$ apo-Tf, respectively. Conversely, there was significant difference between $1 \mu \mathrm{g} / \mathrm{mL}$ and $10 \mu \mathrm{g} / \mathrm{mL}$ in terms of cleavage rates, although the others did not vary significantly $(P<0.05)$. There were significant differences among the concentrations of apo-Tf for blastocyst formation $(P<0.05)$. Blastocysts matured with 1, 10, and $100 \mu \mathrm{g} / \mathrm{mL}$ apo-TF and developed in 5\% FBS and 0.3\% BSA in TCM199-IVMD showed rates of 8.8-21.6\%, 9.4-35.3\%, and $9.1-19.1 \%$, respectively. The control groups developed to the blastocyst stage showed rates of $8.6 \%, 10.8 \%$, and $10.5 \%$ in $5 \%$ FBS and $0.3 \%$ BSA in TCM199-IVMD, respectively. These results suggest that apo-Tf is an important factor for the in vitro maturation and in vitro development of bovine COCs.

\section{THE EFFECT OF DIFFERENT ZWITTERIONIC BUFFERS AND PBS ON IN VITRO DEVELOPMENT AND MORPHOLOGY OF BOVINE EMBRYOS}

\author{
J. De la Fuente, A. Gutiérrez-Adán, P. Beltrán Breña, S. S. Pérez-Garnelo, and A. T. Palasz \\ Ministerio de Educación y Ciencia, INIA Ctra de la Coruña Km 5.9, Madrid 28040, Spain
}

It is assumed that, contrary to phosphate buffers, zwitterionic buffers are neutral. However, zwitterionic buffers containing hydroxymethyl or hydroxyethyl residues may interact with OH-groups in the media and produce formaldehyde (Shiraishi et al. 1993 Free Radic. Res. Commun. 19, 315-321). Also, it was shown that three zwitterionic buffers tested in this study interact with DNA (Stellwagen et al. 2000 Anal. Biochem. 287, 167-175). Our objective was to evaluate the effect of the following buffers: TES (T), MOPS (M), HEPES (H) (pKa values at 20 $\left.{ }^{\circ} \mathrm{C}: 7.2-7.5\right)$, and PBS on in vitro development and morphology of bovine embryos. Zwitterionic buffers and PBS were prepared at a concentration of $10 \mathrm{mM}$ in TALP medium and the final $\mathrm{pH}$ was adjusted to 7.2. Bovine follicular fluid was aspirated from abattoir-derived ovaries and evenly divided into four tubes. Collected oocytes (five replicates) from each tube were processed separately through the entire IVM, IVF, and IVC procedures using washing medium buffered with: PBS $(n=490)$, Group 1; H $(n=438)$, Group 2; M $(n=440)$, Group 3; and T $(n=394)$, Group 4 . All buffers contained $4 \mathrm{mg} / \mathrm{mL}$ BSA. Oocytes were matured in TCM-199+10\% FCS and $10 \mathrm{ng} / \mathrm{mL}$ of epidermal growth factor and fertilized in Fert-TALP containing $25 \mathrm{mM}$ bicarbonate, $22 \mathrm{mM}$ sodium lactate, $1 \mathrm{mM}$ sodium pyruvate, $6 \mathrm{mg} / \mathrm{mL}$ BSA-FAF, and $10 \mu \mathrm{g} / \mathrm{mL}$ heparin with $1 \times 10^{6}$ spermatozoa/mL. After $24 \mathrm{~h}$, oocytes-sperm co-incubation presumptive zygotes were cultured in SOFaa medium with $8 \mathrm{mg} / \mathrm{mL}$ BSA at $39^{\circ} \mathrm{C}$ under paraffin oil and $5 \% \mathrm{CO}_{2}$ in humidified air. Cumulus-oocyte complexes and zygotes were held in designated buffers $\sim 16$ min before oocyte maturation, $\sim 7 \mathrm{~min}$ after IVM and before IVF, and $\sim 18 \mathrm{~min}$ after IVF and before culture. The total time of oocyte/embryo exposure to each buffer was $\sim 41 \mathrm{~min}$ Embryo development was recorded on Days 4, 7, 8, and 9. A total of ten, Day 8 blastocysts were taken randomly from each treatment and fixed in $4 \%$ paraformaldehyde for total and apoptotic cells counts, and five blastocysts from each replicate and treatment were frozen for later mRNA analysis. Apoptosis were determined by TUNEL, using commercial In situ Cell Death Detection Kit (Roche Diagnostic, SL, Barcelono, Spain). Embryo development among groups was compared by chi-square analysis. The cleavage rates were not different among the groups: PBS, $70.8 \%$; $\mathrm{H}, 76.5 \% ; \mathrm{M}, 77.5 \%$ and T, 73.6\%. The number of embryos that developed to $\leq 8$ cells at Day 4 was higher in $\mathrm{M}, 36.2 \%$, and PBS, $37.6 \%$, than in $\mathrm{H}, 30.6 \%$, and $\mathrm{T}, 29.7 \%$, but was not significantly different. However, more $(P<0.05)$ blastocysts developed at Days 7,8 , and 9 in $\mathrm{H}$ and $\mathrm{M}$ than in PBS and T groups $(21.9 \%$ and $22.9 \%$ vs. $16.9 \%$ and $14.9 \%$, respectively). No difference was found between groups in total cell number $(98.8 \pm 7, \mathrm{PBS} ; 111.8 \pm 11.9, \mathrm{M} ; 106.8 \pm 12.9, \mathrm{H} ;$ and $104.3 \pm 9.7, \mathrm{~T})$ and the number of apoptotic cells $(9.2 \pm 1.0, \mathrm{P} ; 9.2 \pm 0.8, \mathrm{M} ; 12.9 \pm 1.8, \mathrm{H} ;$ and $9.7 \pm 0.9, \mathrm{~T})$. Based on the results of this study, we conclude that within our protocol choice of buffer may affect embryo developmental rates but not morphology. 


\title{
160 EFFECT OF BSA SOURCES AND TYPES ON IN VITRO-PRODUCED LIVESTOCK EMBRYO DEVELOPMENT
}

\author{
J. R. Dobrinsky, A. M. Paprocki, V. L. Chrostowski, C. M. Penfield, K. R. Rozeboom, and M. E. Wilson \\ Livestock Embryology Laboratory, Minitube International Center for Biotechnology, \\ Minitube of America, Mt. Horeb, WI 53572, USA
}

\begin{abstract}
Bovine serum albumin (BSA) is a macromolecule supplement used in embryo and cell culture media. Other chemicals have been used as macromolecule substitutes in embryo culture with variable effectiveness. There are BSA products available that are defined in their disease status, collection method, and manufacturing process. They are compliant for use in raw form or in culture medium in the USA and EU. It was the purpose of this study to compare the effectiveness of highly defined and internationally compliant BSA with typically used BSA on in vitro-produced pig and cow embryo development. Pig oocyte-cumulus complexes were matured in two stages for $44 \mathrm{~h}$ in vitro. Semen from one boar of known high fertility was used across the study to fertilize mature oocytes. After 6-h co-incubation with sperm (50 motile sperm/oocyte), presumptive zygotes were cultured for $120 \mathrm{~h}$ in modified NCSU-23 containing $4 \mathrm{mg} / \mathrm{mL}$ of one of the following heat-shocked BSA fractions: Sigma A-7906 (control), Minitube Reproductive Biology Grade Fraction-V (RBG-V1, RBG-V2, RBG-V3), or Minitube Reproductive Biology Grade Fatty Acid-Free (RBG-FAF1). Across all treatments, Day 5 morulae were removed from BSA culture and placed into modified NCSU-23 with 10\% fetal bovine serum (FBS) (no BSA) and cultured for $48 \mathrm{~h}$. After 168-h total culture, the following blastocyst development was observed: Sigma A-7906, 12.3\% (40/324); RBG-V1, $21.1 \%$ (36/171); RBG-V2, 19.0\% (30/158); RBG-V3, 16.8\% (27/161); and RBG-FAF1, 13.4\% (21/157). These data show that culture medium supplemented with Minitube Reproductive Biology Grade BSA meets or exceeds $(P<0.05$; ANOVA-GLM of SAS; SAS Institute, Inc., Cary, NC, USA) blastocyst development potential when compared to culture medium supplemented with undefined standard BSA preparations, such as Sigma A-7906. In vitro-matured cow cumulus-oocyte complexes were fertilized and cultured in one of two CR-1aa-based media. Oocytes were fertilized in IVF medium containing $6 \mathrm{mg} / \mathrm{mL}$ Sigma A-8806 (FAF) or $6 \mathrm{mg} / \mathrm{mL}$ Minitube RBG-FAF1. After $24 \mathrm{~h}$ in IVF medium, presumptive zygotes from a specific BSA-supplemented medium were cultured for $144 \mathrm{~h}$ in CR-1aa supplemented with their respective BSA $(8 \mathrm{mg} / \mathrm{mL})$. Day $6 \mathrm{morulae}$ were removed from BSA culture and placed into CR-1aa with 10\% FBS (no BSA) and cultured for $48 \mathrm{~h}$. After 192-h total culture, the following blastocyst development from oocytes matured was observed: Sigma A-8806, 21.4\% (88/411); RBG-FAF1, 18.9\% (70/370). These data show that culture medium supplemented with Minitube Reproductive Biology Grade BSA meets blastocyst development potential $(P>0.05)$ when compared to culture medium supplemented with undefined standard BSA preparations, such as Sigma A-8806. The inclusion of internationally compliant BSA meets or exceeds blastocyst development rates in comparison to standard BSA preparations in common in vitro embryo production systems for swine and cattle. Although manufacturing differences remain the prominent variant in BSA sources and types, continued monitoring and documentation of BSA preparations tested in livestock in vitro embryo production systems will ensure a safe global supply of BSA products for future culture media production.
\end{abstract}

\section{LIPID CONTENT, RESISTANCE TO CRYOPRESERVATION, AND SEX RATIO OF IN VITRO BOVINE BLASTOCYSTS PRODUCED IN A SERUM-FREE SYSTEM}

\author{
F. George, C. Daniaux, G. Genicot, F. Focant, B. Verhaeghe, P. Lambert, and I. Donnay \\ Université Catholique de Louvain, Institut des Sciences de la Vie, Louvain-la-Neuve, Belgium
}

\begin{abstract}
In vitro-produced (IVP) bovine blastocysts are known to be more sensitive to cryopreservation than their in vivo counterparts. Removing serum from the culture medium decreases sanitary risk and could improve embryo resistance to cryopreservation by preventing the accumulation of intracellular lipids. Our objectives were to evaluate the lipid content, resistance to cryopreservation, and sex ratio of IVP embryos cultured in a serum-free system. Oocytes from slaughterhouse ovaries were matured in a serum-free enriched medium (Donnay et al. 2004 Reprod. Fertil. Dev. 16, 274) and cultured in 5\% $\mathrm{O}_{2}$ in modified SOF supplemented with 5\% FCS (FCS) or with insulin-transferrin-selenium (ITS) and $0.1 \mathrm{mg} / \mathrm{mL}$ polyvinylpyrrolidone (PVP) (ITS-PVP) or $4 \mathrm{mg} / \mathrm{mL}$ BSA (ITS-BSA) (Daniaux et al. 2005 Reprod. Fertil. Dev. 17, 217). Day 5 morulae were stained with the fluorescent dye Nile Red in order to evaluate their lipid content (Genicot et al. 2005 Theriogenology 63, 1181). Day 7 blastocysts (diameter $\geq 160 \mu \mathrm{m}$ ) were selected, classified according to their size, and frozen in HEPES-SOF containing $1.5 \mathrm{M}$ ethylene glycol, $0.1 \mathrm{M}$ sucrose, and $1.8 \mathrm{mg} / \mathrm{mL}$ wheat peptones (George et al. 2002 Reproduction 29, 51). The lipid content was significantly lower in morulae cultured in ITS-BSA compared with the two other media $(320 \pm 10$ arbitrary fluorescence units vs. $383 \pm 12$ in FCS and $406 \pm 10$ in ITS-PVP; $n=271$; ANOVA2: $P<0.01$ ). After cryopreservation, a higher total hatching rate was found $24 \mathrm{~h}$ post-thawing in blastocysts cultured in ITS-BSA and for both serum-free conditions at $48 \mathrm{~h}$ (Table 1). In particular, embryos $\leq 180 \mu \mathrm{m}$ cultured in FCS were less resistant to cryopreservation than embryos of the same size produced without serum. Expanded blastocysts cultured in ITS-BSA were sexed by PCR (Grisart et al. 1995 Theriogenology 43, 1097) and a higher proportion of male embryos was found $(62.7 \% ; n=51)$. In conclusion, a complete serum-free system was set up from oocyte maturation to embryo cryopreservation that gave high quality embryos resistant to cryopreservation. Embryos produced in ITS-BSA presented a lower lipid content, but a shift of the expanded blastocyst sex ratio toward males was observed.
\end{abstract}


Table 1. Hatching rates post-thawing as a function of the blastocyst size and the culture medium

\begin{tabular}{lcccc}
\hline Diameter $(\mu \mathrm{m})$ & Culture medium & $n$ & $\begin{array}{c}\text { Hatched embryos }(\%) \\
\text { After } 24 \mathrm{~h}\end{array}$ & After $48 \mathrm{~h}$ \\
\hline $160-180$ & ITS-PVP & 22 & $31.8^{\mathrm{a}, \mathrm{b}, \mathrm{e}}$ & $81.8^{\mathrm{a}, \mathrm{e}}$ \\
& ITS-BSA & 35 & $51.4^{\mathrm{a}, \mathrm{e}}$ & $68.6^{\mathrm{a}, \mathrm{e}}$ \\
$>180$ & FCS & 40 & $22.5^{\mathrm{b}, \mathrm{e}}$ & $37.5^{\mathrm{b}, \mathrm{e}}$ \\
& ITS-PVP & 30 & $50.0^{\mathrm{b}, \mathrm{e}}$ & $90.0^{\mathrm{a}, \mathrm{e}}$ \\
& ITS-BSA & 45 & $73.3^{\mathrm{a}, \mathrm{f}}$ & $93.3^{\mathrm{a}, \mathrm{f}}$ \\
Total & FCS & 42 & $61.9^{\mathrm{a}, \mathrm{b}, \mathrm{f}}$ & $85.7^{\mathrm{a}, \mathrm{f}}$ \\
& ITS-PVP & 52 & $42.3^{\mathrm{b}}$ & $86.6^{\mathrm{a}}$ \\
& ITS-BSA & 80 & $63.7^{\mathrm{a}}$ & $82.5^{\mathrm{a}}$ \\
& FCS & 82 & $42.7^{\mathrm{b}}$ & $62.2^{\mathrm{b}}$ \\
\hline
\end{tabular}

Different superscripts within a column are significantly different within a size category $\left({ }^{\mathrm{a}, \mathrm{b}}\right)$ or within a medium $\left({ }^{\mathrm{e}, \mathrm{f}}\right)(P<0.05)$.

\title{
162 IMPROVED EMBRYO SURVIVAL AND QUALITY WITH EMCARE II
}

\author{
A. Harvey, M. Lane, and J. Thompson
}

Research Centre for Reproductive Health, Department of Obstetrics and Gynaecology, The University of Adelaide, Adelaide, SA, Australia

Collection of embryos exposes them to a number of stresses, including light, air, and changes in temperature. Improvement of holding media to reduce the impact of handling stresses on the embryo during in vivo collection and transfer is therefore beneficial to ensure maintenance of viability following transfer. The aim of this study was to compare the effect of holding IVP-derived blastocysts at $25^{\circ} \mathrm{C}$ in Emcare I (ECMI, Emcare, Dallas, TX, USA) with those held in Emcare II (ECMII), a proprietry formulation designed to reduce in vitro-induced stress. In vitro-produced bovine embryos were generated using standard protocols. Blastocysts were randomly allocated to either ECMI or ECMII (ICPBio, Aukland, New Zealand) on Day 7 and were held at $25^{\circ} \mathrm{C}$ for a period of $24 \mathrm{~h}$, after which they were cultured in Cook Bovine Blast (Cook Australia, Brisbane, Australia) supplemented with $10 \%$ fetal calf serum for $48 \mathrm{~h}$. At 24 and $48 \mathrm{~h}$, embryos were scored for hatching, and a cohort removed for TUNEL staining at each time point. Differences were analyzed by Student's $t$-test. At both 24- and 48-h culture, hatching rates tended to be higher for embryos held in ECMII than in ECMI (Table 1). The level of apoptosis at $48 \mathrm{~h}$ was reduced in blastocysts held in ECMII $(P=0.06)$. Moreover, the total cell number of hatched blastocysts at $48 \mathrm{~h}$ was significantly increased $(1.5$-fold $)$ in those held in ECMII $(P=0.01)$. Results suggest that the formulation of ECMII improves the ability of IVP bovine blastocysts to re-expand and hatch following an imposed stress $\left(25^{\circ} \mathrm{C}\right.$ for $\left.24 \mathrm{~h}\right)$. Furthermore, ECMII improves overall embryo quality through a reduction in the percentage of cells undergoing apoptosis as well as through increased cell numbers, evident $48 \mathrm{~h}$ following cessation of the stress. We suggest that Emcare II reduces the impact of (or increases the embryo's tolerance to and recovery from) an imposed stress, which, although severe in the present study, may provide improved outcomes following embryo transfer in field situations.

This work was supported with funding by ICPBio (NZ).

Table 1. Hatching and apoptosis of blastocysts held at $25^{\circ} \mathrm{C}$ for $24 \mathrm{~h}$ in Emcare I or Emcare II

\begin{tabular}{lcccccc}
\hline Formulation & $\begin{array}{c}\text { Hatching at } \\
24 \mathrm{~h}(\%)\end{array}$ & $\begin{array}{c}\text { Hatching at } \\
48 \mathrm{~h}(\%)\end{array}$ & $\begin{array}{c}\text { Total cell no. } \\
\text { at } 24 \mathrm{~h}\end{array}$ & $\begin{array}{c}\text { Apoptosis at } \\
24 \mathrm{~h}(\%)\end{array}$ & $\begin{array}{c}\text { Total cell no. } \\
\text { at } 48 \mathrm{~h}\end{array}$ & $\begin{array}{c}\text { Apoptosis at } \\
48 \mathrm{~h}(\%)\end{array}$ \\
\hline Emcare I & $26.5 \pm 9.0$ & $48.9 \pm 12.7$ & $120.6 \pm 5.3$ & $6.8 \pm 1.5$ & $213.2 \pm 10.6^{\mathrm{a}}$ & $7.4 \pm 0.6$ \\
Emcare II & $37.1 \pm 8.3$ & $61.2 \pm 8.8$ & $132.0 \pm 4.7$ & $8.5 \pm 1.2$ & $296.9 \pm 29.4^{\mathrm{b}}$ & $5.7 \pm 0.5$ \\
\hline
\end{tabular}

${ }^{\mathrm{ab}}$ Different superscripts represent statistically significant differences $(P<0.05)$.

\section{SUCROSE ADDITION AT EARLY CULTURE STAGE IMPROVES DEVELOPMENT OF PRE-IMPLANTATION PORCINE NT AND IVF EMBRYOS}

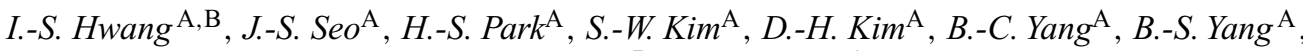 \\ H.-T. Cheong ${ }^{\mathrm{B}}$, and G.-S. Im ${ }^{\mathrm{A}}$

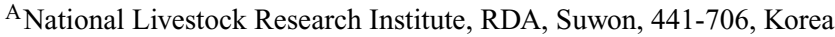 \\ ${ }^{B}$ Kangwon National University, Chunchon, 200-702, Korea
}

Apoptosis is a form of cell death leading to fragmentation of the DNA, shrinkage of the cytoplasm, membrane changes, and cell death without lysis or damage to neighboring cells. It might contribute to the low developmental rate of in vitro-produced (IVP) embryos, but apoptosis in porcine embryos is still unclear. This study investigated the effect of sucrose in the culture medium on the development of porcine NT and IVP embryos. Oocytes were aspirated from the follicles in ovaries collected from a local abattoir, and then matured in TCM-199 for 40-44 h. Fresh semen was diluted and 
equilibrated at $16^{\circ} \mathrm{C}$. A final concentration of motile spermatozoa was adjusted to $5 \times 10^{5}$ cells $/ \mathrm{mL}$ in fertilization medium. Fetal fibroblast cells were prepared from a 35-day-old porcine fetus and used as donor cells. Embryos were cultured in PZM-3 supplemented with $0.05 \mathrm{M}$ sucrose for 2 days, and then cultured in the PZM-3 without sucrose for 4 days at $38.5^{\circ} \mathrm{C}$ under $5 \% \mathrm{CO}_{2}$ in air. Apoptotic cell death was analyzed by using a terminal deoxynucleotidyl transferase-mediated deoxyuridine 5-triphosphate nick-end labeling (TUNEL) assay. All data were subjected to a generalized linear model procedure (PROC-GLM) of the statistical analysis system (SAS; SAS Institute, Inc., Cary, NC, USA). NT and IVF embryos cultured in the medium supplemented with sucrose showed a significantly higher blastocyst formation rate than those cultured with no addition ( 28.8 vs. $20 \%$ and 32.3 vs. $17.9 \% ; P<0.05$, respectively). For apoptosis, both NT and IVF embryos cultured in the medium with sucrose showed significantly lower frequency of apoptosis compared to embryos cultured in the medium without sucrose ( 3.4 vs. $6.3 \%$ and 0.6 vs. $1.8 \% ; P<0.05$, respectively). Finally, the number of nuclei in NT blastocysts cultured in the medium with sucrose was higher than that of NT blastocysts cultured without sucrose (30.8 vs. 25.5; $P<0.05$, respectively). However, the number of nuclei in the IVF blastocysts was not significantly different between groups. These results indicate that sucrose addition may increase the development of porcine NT and IVF embryos to the blastocyst stage and decrease the rate of apoptotic cells.

\title{
164 HEAT STRESS ENHANCES THE DEVELOPMENT OF PORCINE PARTHENOGENETIC EMBRYOS, BUT NOT IN VITRO-FERTILIZED EMBRYOS
}

\author{
S. C. Isom, R. S. Prather, and E. B. Rucker III \\ Department of Animal Sciences, University of Missouri-Columbia, Columbia, MO 65201, USA
}

Recent research in our laboratory has demonstrated that a borderline supra-physiological heat stress $\left(42^{\circ} \mathrm{C}\right)$ of moderate duration $(9 \mathrm{~h})$ can have beneficial effects on the development of porcine parthenogenetic embryos - depending on the timing of heat stress application relative to oocyte activation. A 9-h heat stress (HS) applied to presumptive parthenotes immediately after oocyte activation by electrical stimulus $(0-9 \mathrm{~h}$ post-activation [hpa]; 09HS) significantly accelerated the timing of the first cleavage event: only $26 \%$ of the non-heat stressed (NHS) controls had cleaved by $24 \mathrm{hpa}$, but more than $60 \%$ of the 09HS embryos had cleaved after $24 \mathrm{~h}(P<0.0001$; ANOVA as determined using the PROC GLM commands in SAS; SAS Institute, Inc., Cary, NC, USA). This discrepancy had all but disappeared by $48 \mathrm{hpa}(P>0.1)$. After 7 days in culture, however, $38.0 \%$ of the $09 \mathrm{HS}$ embryos developed to blastocyst whereas $18.5 \%$ of the NHS embryos developed to that stage $(P<0.0001)$. Pharmaceutical and biomolecule inhibitors were used to block several candidate biochemical pathways and processes that were thought to be involved in bringing about the phenotype observed in the 09HS embryos: alpha amanitin (an RNA polymerase inhibitor), roscovitine (a cdc2/MPF inhibitor), cordycepin (an inhibitor of polyadenylation), SP600125 (a stress-activated protein kinase inhibitor), LY294002 (a phosphoinositide 3-kinase inhibitor), and MG132 (a 26S proteasome inhibitor). NHS and 09HS embryos were incubated with and without inhibitors. At least three replicates of each experiment were performed. Although some interesting data have been generated, none of these pathways individually appears to be responsible for the observed beneficial effect of the heat stress on early embryo development in parthenotes $(P>0.01)$. Efforts to replicate in in vitro-fertilized (IVF) embryos the beneficial effects of the heat stress observed in parthenogenetic embryos have been largely unsuccessful, even if the timing of stressor application was altered. IVF embryos were heat stressed at $42^{\circ} \mathrm{C}$ for $9 \mathrm{~h}$ at different time points after insemination: $4-13 \mathrm{~h}$ post-insemination (hpi; $413 \mathrm{HS}$ ), 13-22 hpi (1322HS), or 22-31 hpi (2231HS). The 24- and 48-h cleavage rates, and Day 7 blastocyst rates were assessed. These observations were repeated at least five times. Although the heat stress was able to alter the 24 -h cleavage rates $(P<0.0001), 48$-hpi cleavage rates and Day 7 blastocyst rates were not significantly different between groups $(P>0.1)$. We have observed similar results in nuclear transfer (NT) embryos (Lai et al. 2005), leading us to postulate that the nuclear remodeling that is necessary in IVF and NT embryos prevents these embryos from responding positively to the HS in a manner similar to that in the parthenogenetic embryos. Experiments to test this hypothesis as well as further genomic and proteomic analyses of this phenomenon are ongoing in our laboratory.

This work was funded in part by Food for the 21st Century.

\section{EFFECTS OF GLUCOSE CONCENTRATIONS ON IN VITRO DEVELOPMENT AND THE INTRACELLULAR OXIDATIVE STATE OF IN VITRO-PRODUCED PORCINE EMBRYOS}

\author{
N.W.K.Karja ${ }^{\mathrm{A}, \mathrm{B}}, K_{\text {Kikuchi }}^{\mathrm{B}}$, M. Fahrudin $^{\mathrm{B}}$, O. Manabu $^{\mathrm{B}}$, T. Somfai $^{\mathrm{B}}$, J. Noguchi $^{\mathrm{B}}$, \\ H. Kaneko ${ }^{\mathrm{B}}$, and T. Nagai ${ }^{\mathrm{A}}$ \\ ${ }^{A}$ Department of Research Planning and Coordination, National Institute of Livestock and Grassland Science, \\ Tsukuba, Ibaraki 305-0901, Japan; \\ ${ }^{\mathrm{B}}$ Genetic Diversity Department, National Institute of Agrobiological Sciences, Tsukuba, Ibaraki 305-8602, Japan
}

The present study was conducted to elucidate the effects of glucose addition during Days 0 to 2 (the day of in vitro fertilization was defined as Day 0 ) on the developmental competence, intracellular reactive oxygen species (ROS) level, and glutathione (GSH) concentration of in vitro-produced pig embryos. Zygotes were obtained as reported previously (Kikuchi et al. 2002 Biol. Reprod. 66, 1033-1041), and cultured in NCSU-37 supplemented with $1.5,3.5,5.5,10$, and $20 \mathrm{mM}$ glucose (glucose groups) or in NCSU-37 supplemented with $0.17 \mathrm{mM}$ pyruvate and $2.73 \mathrm{mM}$ lactate (Pyr/Lac group) from Days 0 to 2. Subsequently, embryos in all groups were then cultured in NCSU-37 added with 5.5 mM glucose until Day 6. Data were analyzed by ANOVA and are presented as percentage for blastocyst rate and as mean \pm SEM for blastocyst cell number. The blastocyst rates and blastocyst cell numbers in glucose groups were significantly lower compared to those in the Pyr/Lac group (blastocyst rate: 5.2, 13.8, 12.6, 16.3, and $13.5 \%$, respectively, vs. $26.3 \%$ ); blastocyst cell number: $41.8 \pm 3.2,41.6 \pm 2.3,42.2 \pm 3.2,43.0 \pm 3.3$, and $39.2 \pm 2.8$, respectively, vs. $52.7 \pm 4.1$ ). The ROS levels of Day 1 embryos were significantly higher when they were exposed to glucose, whereas the ROS levels of Day 2 embryos were higher only in the $1.5 \mathrm{mM}$ and $3.5 \mathrm{mM}$ glucose groups, compared to levels in embryos in Pyr/Lac group. There were no differences in the GSH 
concentrations of Day 1 embryos among the glucose groups and the Pyr/Lac group. Of Day 2 embryos, the GSH concentration was significantly lower only in $1.5 \mathrm{mM}$ glucose group, compared to that in embryos in the Pyr/Lac group. These results indicate that (1) the presence of glucose during the first 2 days of culture causes a decrease in the development of in vitro-produced embryos to the blastocyst stage, which might be related to the rise in ROS generation in Day 1 embryos; and (2) except for the $1.5 \mathrm{mM}$ glucose group, the ability of Day 2 embryos in glucose groups to maintain GSH concentration at levels needed for their development might provide them with preferable intracellular conditions for the development to the blastocyst stage.

\title{
166 EFFECTS OF LEPTIN AND IGF-1 ON PRE-IMPLANTATION DEVELOPMENT, DNA FRAGMENTATION, AND GENE EXPRESSION OF BOVINE EMBRYOS CULTURED IN VITRO
}

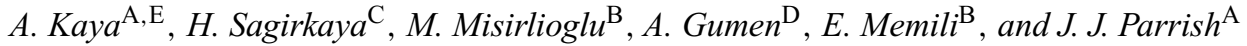 \\ ${ }^{A}$ Department of Animal Sciences, University of Wisconsin, Madison, WI 53706, USA; \\ ${ }^{B}$ Department of Animal \& Dairy Sciences, Mississippi State University, Mississippi State, MS 39762, USA; \\ ${ }^{\mathrm{C}}$ Deptartment of Reproduction \& Artificial Insemination, University of Uludag, Veterinary Faculty, Bursa, Turkey; \\ DDepartment of Obstetrics \& Gynecology, University of Uludag, Bursa, Turkey; \\ ${ }^{\mathrm{E}}$ Altagenetics USA, Watertown, WI 53094, USA
}

Adequate regulatory proteins, growth factors, and hormones in in vitro embryo culture systems are important for improving the quality of embryos to a level similar to that in vivo conditions. The objective of this study was to define the effects of leptin, insulin-like growth factor-1 (IGF-1), and their combination on embryonic development, apoptosis, and expression profiles of a panel of developmentally important genes. Presumptive zygotes (16-18 h post-insemination) were randomly assigned and cultured in control (no supplementation), $5 \mathrm{ng} / \mathrm{mL}$ leptin (Group I), $100 \mathrm{ng} / \mathrm{mL}$ IGF-1 (Group II), and $5 \mathrm{ng} / \mathrm{mL}$ leptin and $100 \mathrm{ng} / \mathrm{mL}$ IGF-1 (Group III), all supplemented with 10\% FCS on Day 4. On Day 8, the embryos reaching blastocyst stage were randomly either fixed for determination of DNA-fragmented nuclei by using terminal deoxynucleotidyl transferase biotin-dUTP nick end labeling (TUNEL) or frozen for real-time relative quantitative RT-PCR analysis. The RT-PCR was performed to assess gene transcripts of glucose transporter-1 (Glut-1), heat shock protein 70.1 (Hsp70.1), interferon tau (IF-tau), insulin-like growth factor II receptor (IGF-IIr), desmosomal glycoprotein desmocollin III (DcIII), and DNA methyltransferase 3a (Dnmt3a). A total of 349, 322, 347, and 360 zygotes were used for the control group and Groups I, II, and III, respectively. Data were analyzed with a randomized complete block design and arcsine square root transformation of the dependent variables consisting of four treatments and six replicates. Cleavage rates were 79.5, 84.2, 87.3, and 82.4\% for the control group and Groups I, II, and III, respectively, and only Group II was different from the control $(P<0.05)$. The percentages of embryos developed beyond the 8-16 cell stage were 44.2, 48.2, 49.0, and 50.7 for the control group and Groups I, II, and III, respectively, and Group III was different from the control $(P<0.05)$. Percentages of blastocyst development were 26.7, 29.6, 31.5, and 29.8, and the mean blastocyst cell numbers were $96.6,98.6$, 104.4, and 104.1 for the control group and Groups I, II, and III, respectively. The percentage of nuclei with fragmented DNA were 4.2, 3.3, 2.5, and 1.9 for the control group and Groups I, II, and III, respectively. Addition of IGF-1 and/or combination with leptin (Groups II and III) decreased the number of nuclei with fragmented DNA $(P<0.01)$ as compared to the control group. Although the expression of Glut1, DcIII, and Igf2r did not change among the groups, IF-tau and Dnmt3a were down-regulated in Group II. Hsp70 and IF-tau were up regulated in Group III. Results indicate that addition of IGF-I in culture media improved the cleavage rate; combination with leptin also improved the development rates to 8-16-cell-stage embryos, decreased the TUNEL-positive nuclei, and altered expression of some of the developmentally important genes.

\section{THE SUPPLEMENTATION OF GRANULOCYTE-MACROPHAGE COLONY STIMULATING FACTOR (GM-CSF) PROMOTES THE DEVELOPMENT OF NUCLEAR TRANSFERRED BOVINE EMBRYOS}

\author{
D. H. Kim, S. W. Kim, B. C. Yang, G. S. Im, H. S. Park, I. S. Hwang, J. S. Seo, H. H. Seong, and B. S. Yang \\ Animal Biotechnology Division, National Livestock Research Institute, Suwon, South Korea
}

Granulocyte-macrophage colony stimulating factor (GM-CSF) is secreted by epithelial cells lining the female reproductive tract in mice and several other species. GM-CSF receptors are present in the fertilized oocyte and in all subsequent stages of development, and in blastocysts it is expressed in both inner cell mass and trophectoderm cells. Recent studies suggest that GM-CSF can act as a survival factor for the developing embryo. The purpose of this study was to examine the effect of GM-CSF, as a medium supplement, on the development of nuclear-transferred bovine embryos. Oocytes were enucleated after in vitro maturation in TCM-199 supplemented with $10 \%$ fetal bovine serum (FBS), $1 \mathrm{mg} / \mathrm{mL} \mathrm{FSH,} \mathrm{and} 1 \mathrm{mg} / \mathrm{mL}$ estradiol-17 $\beta$ for $20 \mathrm{~h}$. Enucleated oocytes were fused with bovine ear skin fibroblast cells by a DC pulse of $25 \mathrm{~V} / 150 \mathrm{~mm}$ for $20 \mathrm{~ms}$ in Zimmerman cell fusion medium. For activation, reconstructed embryos were exposed to $10 \mathrm{mM} \mathrm{Ca}$-ionophore for $5 \mathrm{~min}$, followed by $2 \mathrm{mM} 6$-dimethylaminopurine for $3 \mathrm{~h}$. NT embryos were subsequently cultured in CR2 medium without or with $10 \mathrm{ng} / \mathrm{mL}$ recombinant porcine $\mathrm{GM}-\mathrm{CSF}$ at $39.0^{\circ} \mathrm{C}$ in $5 \% \mathrm{O}_{2}, 5 \% \mathrm{CO}_{2}$ and $90 \% \mathrm{~N}_{2}$. After 7 days of culture, blastocyst formation was observed. The number of inner cell mass (ICM) and trophectoderm (TE) cells was examined by differential staining. Apoptotic cells in blastocysts were detected by a terminal deoxynucleotidyl transferase-mediated d-UTP nickend labeling (TUNEL) assay. Data were analyzed by chi-square and Student's $t$-test. Addition of GM-CSF to the medium significantly $(P<0.05)$ increased the proportion of embryos developing to the blastocyst stage (37.6 \pm 12.0 and $54.7 \pm 13.9 \%$ for control and GM-CSF groups respectively). No differences in the total cell number and the ratio of ICM to total cells were detected between the control group (125.4 \pm 35.7 and $38.5 \pm 9.7 \%)$ and the GM-CSF group $(123.8 \pm 35.1$ and $34.2 \pm 13.1 \%)$. The mean proportion of apoptotic cells in blastocysts was not different between the control $(5.4 \pm 5.4 \%)$ and the GM-CSF $(5.3 \pm 3.9 \%)$ group. Our results showed the beneficial effect of GM-CSF on the development of NT bovine embryos. These results suggest that GM-CSF might be a useful molecule for increasing development of NT bovine embryos. Further studies are necessary to verify the mechanism of GM-CSF on the development of bovine NT embryos. 


\title{
168 IMPROVED MITOCHONDRIAL MEMBRANE POTENTIAL AND PRE-IMPLANTATION DEVELOPMENT OF PORCINE EMBRYOS BY DIBUTYRYL CAMP TREATMENT
}

\author{
J.-S. Kim, D.-B. Koo, B.-S. Song, G. B. Wee, K.-K. Lee, and Y.-M. Han \\ Laboratory of Development and Differentiation, Korea Research Institute \\ of Bioscience and Biotechnology, Daejeon, Korea
}

\begin{abstract}
Mitochondria play a pivotal role in energy metabolism and apoptosis during embryo development. In general, cAMP that exists at high level in GV oocytes inhibits germinal vesicle breakdown (GVBD), and the amount of cAMP in oocyte cytoplasm is gradually decreased for meiotic resumption. We first examined the effects of dibutyryl cAMP (dbcAMP) on nuclear maturation, fertilization, and early embryonic development. To determine whether mitocondrial activity is related to embryonic development, mitochondrial membrane potential $(\Delta \Psi \mathrm{m})$ was measured in porcine embryos. Porcine oocytes were cultured in NCSU-23 medium supplemented with $1 \mathrm{mM}$ dbcAMP for $22 \mathrm{~h}$ and further cultured without dbcAMP for $22 \mathrm{~h}$. After in vitro fertilization, porcine eggs were cultured in NCSU-23 medium with $4 \% \mathrm{BSA}$ at $39^{\circ} \mathrm{C}, 5 \% \mathrm{CO}_{2}$ in air for $6 \mathrm{~d}$. Porcine embryos were obtained at various developmental stages and stained with the mitochondrial membrane potential-sensitive dye JC-1 (5, $5^{\prime}, 6,6^{\prime}$-tetrachloro-1, $1^{\prime}, 3,3^{\prime}$ tetraethylbenzimidazolyl-carbocyanine iodide). The mitochondrial membrane potential of porcine embryos was quantitatively evaluated by the ratio of green (presumptively alive mitochondria) to red fluorescent signal (presumptively dead mitochondria) using a fluorescence microscope. Acquired images were analyzed using DeltaVision Software (Applied Precision, Inc., Hsin-Chu City, Taiwan, ROC). After completion of IVM, dbcAMP-treated oocytes $(91.3 \pm 0.9 \%)$ showed a higher proportion of the metaphase II stage than nontreated ones $(72.8 \pm 2.6 \%)(P<0.05)$. In the dbcAMP-treated group, sperm penetration rate was increased and polyspermic rate was reduced as compared to the nontreated group. Furthermore, the rate $(37.3 \%, 47 / 126)$ of blastocyst formation in dbcAMP-treated group was higher than that $(19.2 \%, 28 / 146)$ of the nontreated group $(P<0.05)$. After JC-1 staining, the number of blastomeres having live mitochondria per embryo increased in the dbcAMP-treated group at various developmental stages, whereas the number of blastomeres having dead mitochondria per embryo was enhanced in the nontreated group (43.3\% vs. 30.2\%). Our results indicate that in vitro maturation of porcine oocytes may affect mitochondrial membrane potential, apoptosis, and embryonic quality during pre-implantation development.
\end{abstract}

\section{FORSKOLIN INDUCED INCREASE IN LIPOLYTIC ACTIVITY I PORCINE EMBRYOS PRODUCED IN VITRO}

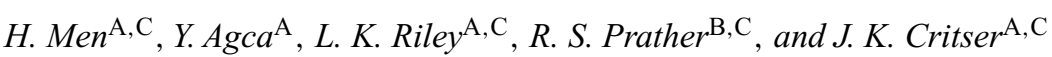 \\ ${ }^{A}$ Comparative Medicine Center, University of Missouri-Columbia; \\ ${ }^{\mathrm{B}}$ Department of Animal Sciences, University of Missouri-Columbia; \\ ${ }^{\mathrm{C}}$ National Swine Resource and Research Center, University of Missouri-Columbia, \\ Columbia, MO 65201, USA
}

\begin{abstract}
The sensitivity of porcine embryos to cryopreservation is largely due to their high level of intracellular lipid content (Polge and Willadsen 1978 Cryobiology 15,370-373). Delipation through centrifugation and micromanipulation resulted in a significant proportion of porcine embryos produced in vivo being able to survive cryopreservation and produce live births (Nagashima et al. 1995 Nature 374, 416). However, due to the intense resources needed for delipation via micromanipulation, this approach has limited practical value. In this experiment, we tested the hypothesis that delipation can be achieved through chemical stimulation of intracellular lipolysis in porcine embryos produced in vitro. Day 6 porcine blastocysts cultured in the presence of $10 \mu \mathrm{M}$ forskolin, a lipolytic agent (Ho and Shi 1982 Biochem. Biophys. Res. Commun. 107, 157-164), in a group of 101-202 blastocysts per $50 \mu$ L of NCSU-23 $+4 \mathrm{mg} / \mathrm{mL}$ BSA (Sigma-Aldrich, St. Louis, MO, USA) yielded approximately $125000-250000$ cells/mL (Zalatan et al. 2001 Endocrinology 142, 3783-3790). Blastocysts cultured in $50 \mu \mathrm{L}$ NCSU-23 $+4 \mathrm{mg} / \mathrm{mL}$ BSA without the supplementation of forskolin served as control. Samples $(12 \mu \mathrm{L})$ of the media were taken from culture at 0,3 , and $6 \mathrm{~h}$ and frozen at $-20^{\circ} \mathrm{C}$ for lipolytic assay. Because the major content of intracellular lipids in porcine embryos is triacylglycerol and the hydrolysis of triacylglycerol results in the production of fatty acid and glycerol, therefore, the lipolytic activity in porcine blastocysts was measured by detection of glycerol concentration in the culture media. A commercially available Free Glycerol Regent (Sigma) was used with modifications. This kit only measures free glycerol released into the media as a result of endogenous lipase activity because the kit itself doesn't contain lipase. Ten $\mu \mathrm{L}$ of sample medium was mixed with $80 \mu \mathrm{L}$ of Free Glycerol Regent and incubated in a $37^{\circ} \mathrm{C}$ water bath for $5 \mathrm{~min}$. The resulting samples were read using a Cary 50 UV Spectrophotometer (Varian, Inc., Palo Alto, CA, USA). The actual concentration of glycerol in the culture medium was calculated using the glycerol standard curve. The glycerol concentration at $0 \mathrm{~h}$ was regarded as 0 for both the treatment group and the control group. The glycerol concentrations at other time points were calculated accordingly. The measurement was conducted four times for the treatment group and three times for the control group. The data were analyzed using a Student's $t$-test. Glycerol concentrations in the treatment group at $3 \mathrm{~h}$ and $6 \mathrm{~h}$ were $5.99 \pm 1.74$ (mean $\pm \mathrm{SEM}$ ) $\mu \mathrm{M}$, and $10.49 \pm 0.81 \mu \mathrm{M}$, respectively, and both values were significantly different from their counterparts in the control group, which were $0.95 \pm 0.62 \mu \mathrm{M}$ and $4.43 \pm 1.31 \mu \mathrm{M}$, respectively. These results indicate that the hydrolysis of intracellular lipids in porcine embryos can be stimulated by lipolytic agents and result in the partial reduction of the intracellular lipid content. This approach may be used for designing a better protocol for the cryopreservation of porcine embryos produced in vitro.
\end{abstract}




\title{
POST-THAW VIABILITY OF IN VITRO-PRODUCED BOVINE EMBRYOS CULTURED EITHER IN HOST CAPRINE REPRODUCTIVE TRACTS OR IN VITRO
}

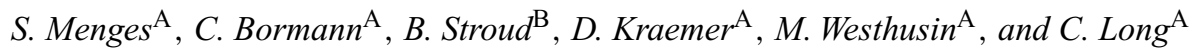 \\ ${ }^{A}$ Department of Physiology and Pharmacology, Texas A\&M University, College Station, TX 77843, USA; \\ ${ }^{\mathrm{B}}$ Stroud Veterinary Clinic, Weatherford, TX 76086, USA
}

In vitro culture of bovine embryos is usually associated with poor pregnancy rate following cryopreservation. The objective of this study was to compare the post-thaw viability of in vitro-produced bovine zygotes, cultured in vitro or in the reproductive tract of a host goat. Cumulus-oocyte complexes were matured in vitro, and in vitro fertilization was carried out with frozen-thawed semen as per standard laboratory procedures. At $18-20 \mathrm{~h}$ post-fertilization, zygotes were stripped of remaining cumulus cells and randomly separated into culture treatments. In three replicates, a total of 606 embryos were surgically transferred 12 to $24 \mathrm{~h}$ post-ovulation to the oviducts of an estrous-synchronized goat (VIVO) and 550 embryos were cultured in G1.3 for $72 \mathrm{~h}$ and then moved to G2.3 medium for $96 \mathrm{~h}$ and in a humidified atmosphere of $5 \% \mathrm{CO}_{2}, 5 \% \mathrm{O}_{2}$, and $90 \% \mathrm{~N}_{2}$ (IVC). On Day 7, embryos were flushed from the excised tract with a $69.5 \%$ recovery rate or removed from culture. Embryos were classified according to IETS criteria with grades and stages recorded. All data were analyzed using the one-way analysis of variance and means were compared using Student's $t$-test. No differences were seen in the percentage of freezable quality embryos per total recovered between the two groups ( $34.3 \% \mathrm{vs.} 32.3 \%$ for IVC and VIVO, respectively). However, there was a significant difference in the pre-freezing stage between the two culture groups (Stage $5.5 \pm 0.22 \mathrm{vs}$ Stage $4.8 \pm 0.26$ for IVC and VIVO, respectively; $P<0.05$ ), but no difference in the quality grade. All embryos greater than Stage 4 , Grade 2 were frozen in groups of 5-10 in ethylene glycol with sucrose (Vigro Ethylene Glycol Freeze Plus; Bioniche Animal Health, Belleville, Ontario, Canada) in 0.25 -mL straws. After thawing, embryo groups were washed, rehydrated, and incubated in G2.3 as above. Morphology was assessed by assigning grade and stage objectively at $24 \mathrm{~h}$ and $48 \mathrm{~h}$ post-thaw. Post-thaw viability in vitro was not different between groups $(73.4 \%$ vs. $72.7 \%$ for IVC and VIVO, respectively). The average changes in morphology post-thaw from pre-freezing to $24 \mathrm{~h}$ and from $24 \mathrm{~h}$ to $48 \mathrm{~h}$ within each freezing group were determined. There was no significant difference in the mean change in stage $(0.67 \pm 0.15 \mathrm{vs} .0 .82 \pm 0.17$ at $24 \mathrm{~h}$ and $0.31 \pm 0.09 \mathrm{vs}$. $0.37 \pm 0.10$ at $48 \mathrm{~h}$ for IVC and VIVO, respectively) or grade $(0.60 \pm 0.15 \mathrm{vs.} 0.41 \pm 0.17$ at $24 \mathrm{~h}$ and $0.03 \pm 0.06 \mathrm{vs} .0 .14 \pm 0.07$ at $48 \mathrm{~h}$ for IVC and VIVO, respectively) at either observation point. These results suggest that culture of in vitro-fertilized bovine embryos in the caprine reproductive tract did not alter post-thaw development or improve post thaw viability compared to in vitro cultured controls. However, morphological evaluation is too subjective to successfully predict pregnancy rate after transfer; therefore, further study is needed to determine if there are differences in pregnancy rates between these culture methods.

\section{EFFECT OF LONG-TERM TRANSPORTATION OF OVARIES ON THE DEVELOPMENT OF IN VITRO-PRODUCED BOVINE EMBRYOS}

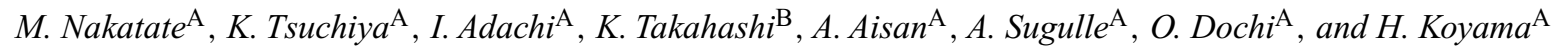 \\ ${ }^{\mathrm{A}}$ Rakuno Gakuen University, Ebetsu, Hokkaido, Japan; ${ }^{\mathrm{B}}$ Genetics Hokkaido, Shimizu, Hokkaido, Japan
}

The transportation of bovine ovaries would allow the shipment of oocytes for research purposes after the slaughter of valuable cows. The objective of this study was to investigate the effect of long-term transportation of ovaries on the development of in vitro-produced bovine embryos. After collection of the ovaries from a slaughterhouse, they were placed inside a thermos flask and transported to the laboratory. The thermos flask was covered with a freezer pack in a foam polystyrene box. The transportation time was $17-18 \mathrm{~h}$, and the temperature of the thermos flask changed from $20^{\circ} \mathrm{C}$ to $28^{\circ} \mathrm{C}$ (average $23.8^{\circ} \mathrm{C}$ ) during the transportation. Cumulus-oocyte complexes (COCs) were collected by the aspiration of follicles with a diameter of $2-6 \mathrm{~mm}$. The COCs were matured for $20 \mathrm{~h}$ in IVMD101 (RIFP: Research Institute for the Functional Peptides, Yamagata, Japan) containing DM199 supplemented with $5.56 \mathrm{mM}$ glucose, $0.91 \mathrm{mM}$ pyruvate, $5 \mathrm{mM}$ taurine, $5 \mathrm{mM}$ selenium, $5 \mathrm{mM}$ HEPES, and $10 \mu \mathrm{g} / \mathrm{mL}$ gentamicin at $38.5^{\circ} \mathrm{C}$ under an atmosphere of $5 \% \mathrm{CO}_{2}$ in air (Hoshi 2003 Theriogenology 59, 675-685). The matured COCs were inseminated with $5 \times 10^{6}$ sperm $/ \mathrm{mL}$ in IVF100 (RIFP) medium comprising a modified BO medium supplemented with $1.25 \mathrm{mM}$ sodium pyruvate, $0.5 \mathrm{mM}$ cysteine, $5 \mathrm{mg} / \mathrm{mL} \mathrm{BSA}, 7.5 \mu \mathrm{g} / \mathrm{mL}$ sodium heparin, $5 \mathrm{mM}$ caffeine, and $10 \mu \mathrm{g} / \mathrm{mL}$ gentamicin. After $6 \mathrm{~h}$ of gamete co-culture, the presumed zygotes were cultured in IVD101 (RIFP) medium comprising DM199, $2.48 \mathrm{mM}$ lactate, $0.27 \mathrm{mM}$ pyruvate, and $2.22 \mathrm{mM}$ of glucose for 9 days at $38.5^{\circ} \mathrm{C}$ under an atmosphere of $5 \% \mathrm{CO}_{2}$, $5 \% \mathrm{O}_{2}$, and $90 \% \mathrm{~N}_{2}$ in air. As controls, bovine ovaries were transported to the laboratory within 1-1.5 h. Embryo development was evaluated based on the cleavage rate, blastocyst rate, and total number of cells on Days 7-9 after in vitro fertilization. The experiment was replicated five times, and data were analyzed by chi-square test and ANOVA. Results are presented in Table 1 . There were no differences in the cleavage rate between the treatments. The blastocyst rate and the number of cells in the blastocyst after long-term transportation of ovaries were significantly lower than those in the controls. These results suggest that the long-term transportation of bovine ovaries does not affect on the cleavage; however, the blastocyst rate and the quality of blastocysts may be affected. Therefore, additional experiments are required to determine suitable conditions for long-term transportation of bovine ovaries.

Table 1. Effect of long-term transportation of ovaries on the development of bovine IVM/IVF embryos

\begin{tabular}{lcccc}
\hline Treatment & Oocytes, $n$ & Cleaved, $n(\%)$ & Blastocysts at Days 7-9, $n(\%)$ & Total number of cells in blastocyst, mean \pm SE \\
\hline Transportation & 516 & $337(65.3)$ & $111(21.5)^{\mathrm{a}}$ & $118.2 \pm 2.1^{\mathrm{c}}$ \\
Control & 700 & $477(68.1)$ & $200(28.6)^{\mathrm{b}}$ & $139.5 \pm 2.8^{\mathrm{d}}$ \\
\hline
\end{tabular}

Values with different superscripts within a column differ significantly ( ${ }^{\mathrm{ab}} P<0.05,{ }^{\mathrm{cd}} P<0.01$ ). 


\title{
172 ADMINISTRATION OF GLUTATHIONE OR THIOREDOXIN TO MEDIUM REDUCES INTRACELLULAR REDOX STATUS AND IMPROVES EMBRYONIC DEVELOPMENT TO THE BLASTOCYST STAGE OF PORCINE IVM/IVF OOCYTES
}

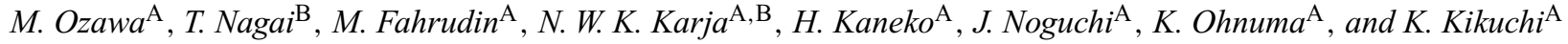

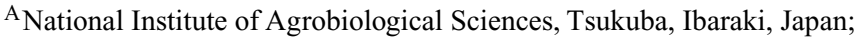 \\ ${ }^{B}$ National Institute of Livestock and Grassland Science, Tsukuba, Ibaraki, Japan
}

\begin{abstract}
Successful in vitro production of blastocysts from immature oocytes can be carried out using in vitro oocyte maturation (IVM), fertilization (IVF), and embryo culture (IVC) at a high level of repeatability in the porcine. However, the rates of in vitro development of IVM/IVF oocytes to the blastocyst stage remained around $20 \%$. The environment in vitro is so simple and materially limited that there exist several stressors in vitro that disturb normal embryo development. Oxidative stress, which is caused by excess production of reactive oxygen species, is a major disturbing factor for the development of pre-implantation embryos in vitro. The series of present experiments were conducted using culture conditions with enhanced reducing capacity by the addition of glutathione $(\mathrm{GSH})$ or thioredoxin to the culture medium to monitor developmental competence of porcine embryos and to verify their intracellular redox status. Cumulus-oocyte complexes were obtained from ovaries recovered from prepubertal gilts. Putative zygotes were produced by IVM of oocytes, followed by IVF (designated as Day 0). They were then cultured in modified NCSU-37 media containing GSH or thioredoxin as an antioxidant, or without any antioxidant (control), and blastocyst development rates on Day 6 were monitored. In addition, intracellular GSH content as a reducing parameter and intracellular $\mathrm{H}_{2} \mathrm{O}_{2}$ level as an oxidative parameter were measured; the intracellular redox status in the embryo was verified by the ratio of the GSH to the $\mathrm{H}_{2} \mathrm{O}_{2}$. Measurements in each group were replicated six times. Percentages of the embryos that developed to the blastocyst stage were significantly increased when 0.5 or $1.0 \mu \mathrm{M} \mathrm{GSH}(29.6 \pm 2.7 \%$ or $30.4 \pm 3.5 \%$, and $P<0.05$ or 0.01 , respectively) or $1.0 \mathrm{mg} / \mathrm{mL}$ thioredoxin $(30.6 \pm 2.4 \%, P<0.01)$ was added to the medium compared to the percentage in the control group $(20.1 \pm 2.2 \%)$. Intracellular redox status in embryos at the 8- to 12-cell stage or blastocysts was drastically reduced in GSH- or thioredoxin-added groups compared to that in the control group $(P<0.05$ to 0.001$)$. Furthermore, GSH or thioredoxin addition to the medium increased total cell numbers $(48.3 \pm 2.1$ to $49.2 \pm 2.1)$ and lowered ratios of apoptotic cells $(6.2 \pm 0.6 \%$ to $7.0 \pm 0.7 \%)$ in blastocyst compared to those values in the control group $(P<0.05$; cell number $=39.3 \pm 2.0$, apoptosis rate $=11.1 \pm 1.1 \%)(37$ to 53 embryos in each group were used for the TUNEL assay). These results suggest that the administration of GSH or thioredoxin to the culture medium improves in vitro embryonic development after IVM/IVF of oocytes, and that these beneficial effects are associated with maintenance of the intracellular redox status in a reduced state in porcine embryos.
\end{abstract}

\section{THE EFFECT OF AMINO ACIDS AND HYALURONAN ON BOVINE EMBRYO IN VITRO DEVELOPMENT AND GENE EXPRESSION PATTERN}

\author{
A. T. Palasz ${ }^{\mathrm{A}}$, J. Beltrán Breña ${ }^{\mathrm{A}}$, P. De la Fuente ${ }^{\mathrm{A}}$, M. F. Martinez ${ }^{\mathrm{B}}$, and A. Gutiérrez-Adán ${ }^{\mathrm{A}}$ \\ A Ministerio de Educación y Ciencia, INIA, Ctra de la Coruña Km 5.9, Madrid 28040, Spain; \\ B2WCVM, University of Saskatchewan, Saskatoon, SK S7N 5B4, Canada. Email: palasz@inia.es
}

\begin{abstract}
We have previously shown that bovine embryos cultured in SOFaa (BME + MEM amino acids) culture medium with hyaluronan (HA) + BSA are of better quality (Gutiérrez-Adán et al. 2005 Reprod. Fertil. Dev. 17, 219). Our objective was to examine the effect of essential (BME) or non-essential (MEM) amino acids with or without HA (MAP-5; Bioniche, Inc., Belleville, Ontario, Canada) on bovine embryo in vitro development and mRNA transcription of five developmentally important genes; apoptosis (Bax), growth factor (IGF-II), glucose (Glut-1) and fructose (Glut-5) transport and metabolism, and cell to cell adhesion $(\mathrm{Cx}-43)$. A total of 1474 presumptive zygotes ( 5 replicates) were initially cultured in $40 \mu \mathrm{L}$ drops in the following groups: Group 1, control, SOFaa; Group 2, SOF-1 (MEM only); and Group 3, SOF-2 (BME only). On Day 4 ( $\sim 96 \mathrm{~h}$ post-insemination (pi) the number of zygotes that had developed to $\leq 8$ cells was recorded and $10 \mu \mathrm{L}$ of SOF-1 and SOF-2, each with $2.5 \mathrm{mg} / \mathrm{mL} \mathrm{HA}$, was added to half of the embryos from Groups 2 and 3, respectively; the other half of Groups 2 and 3 and control group received $10 \mu \mathrm{L}$ of corresponding medium without HA. Embryos were cultured under paraffin oil at $39^{\circ} \mathrm{C}$ and $5 \% \mathrm{CO}_{2}$ in humidified air. Cleavage rates were recorded on Day 2 and the number of blastocysts on Days 7, 8, and 9. Five blastocysts from each replicate from each treatment were frozen for determination of gene expression patterns later. Cleavage rates and embryo development $96 \mathrm{~h}$ pi were compared among groups by chi-square analysis. The effects of HA and medium on blastocyst rates were analyzed by logistic regression and the data on mRNA expression by one-way repeated-measures ANOVA. Cleavage rates were $81.1 \%$ in SOFaa and $79.3 \%$ in SOF-1 $(P=0.48)$ and different from those in the SOF-2 group $(72.4 \% ; P<0.02)$. The proportion of embryos that developed to $\leq 8$ cells at Day 4 was higher in the control (46.7\%) and SOF-1 (46.8\%) groups than in the SOF-2 group (32.6\%). The number of blastocysts that developed in SOFaa $(37.0 \%)$, SOF-1 $(37.7 \%)$, and SOF-1 + HA $(37.8 \%)$ were higher $(P<0.001)$ than those in SOF-2 $(19.6 \%)$ and SOF-2 + HA (21.8\%). The level of expression of Glut-5 was not different among the groups. However, SOF-2 was the only group that had significantly lower expression of Glut-5, Igf II, and $\mathrm{Cx} 43$, and higher expression of BAX $(P<0.05)$ as compared to the control group and the SOF-1 groups with or without HA. Addition of HA to SOF-2 medium increased expression of Glut-1 and Igf II and decreased expression of BAX as compared to the SOF-1 only and control groups and the SOF-2 groups with or without HA $(P<0.05)$. The level of expression of Cx43 was higher in the control than in four remaining groups, and lower in the SOF-2 than in the SOF-1 group $(P<0.05)$. Addition of HA increased expression of Cx43 in both SOF-1 and SOF-2 groups but this level of expression was lower than in the control group; the level in the SOF-2 + HA group was lower $(P<0.05)$ than in the SOF-1 + HA group. We conclude that, within our protocol, MEM amino acids only stimulate embryo development to the blastocyst stage and the addition of HA to the SOF-MEM and SOF-BME media on Day 4 of culture improved embryo quality.
\end{abstract}




\title{
174 DEVELOPMENT OF IN VITRO-FERTILIZED BOVINE EMBRYOS AFTER EXPOSURE TO TRYPLETMEXPRESS (RECOMBINANT TRYPSIN)
}

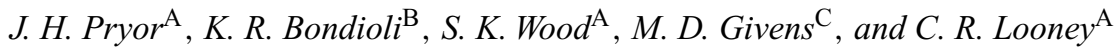 \\ A Ovagenix, Bryan, TX, USA; \\ ${ }^{B}$ Department of Animal Sciences, Louisiana State Uniersity Agricultural Center, Baton Rouge, LA 70803, USA; \\ ${ }^{\mathrm{C}}$ College of Veterinary Medicine, Auburn University, Auburn, AL 36849, USA. Email: charles@ovagenix.com
}

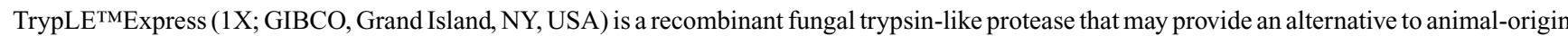
trypsin for inactivation of embryo-associated virus. This experiment was designed as an embryo safety study to determine if different exposure times

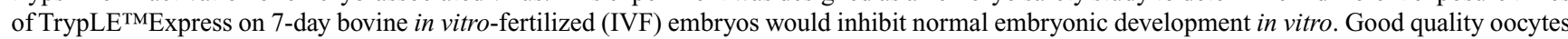
were harvested from ovaries of slaughtered animals and matured in vitro (Looney et al. 1994 Theriogenology 41, 67). Oocytes were fertilized (Day 0) with frozen-thawed, Percoll-separated Brangus spermatozoa in TALP-fertilization medium for $18 \mathrm{~h}$. Presumptive zygotes were cultured in $0.5 \mathrm{~mL}$ of G1.3/G2.3 medium (Lane et al. 2003 Theriogenology 60, 407) supplemented with $8 \mathrm{mg} / \mathrm{mL}$ of Pentex BSA (Serologicals Corporation, Norcorss, GA, USA) under oil for 3 days in a $5 \% \mathrm{CO}_{2}, 5 \% \mathrm{O}_{2}, 90 \% \mathrm{~N}_{2}$ humidified modular incubator (Billups-Rothenberg, Inc., Del Mar, CA, USA) at $38.5^{\circ} \mathrm{C}$. On Day 4, embryos were washed and moved to new culture wells containing G2.3 medium. Cleavage rates of $74 \%$ (750/1014) from three replicates produced 322 viable embryos on Day 7 which were then evenly distributed by grade and stage into three treatment groups (1-, 5-, and 10-minute exposure times to TrypLETMExpress). Embryos were washed in accordance with International Embryo Transfer Society standards (Stringfellow, IETS Manual, 1998, 79-84) substituting TrypLETMExpress with phenol red for the porcine-origin trypsin. Washed embryos were cultured in G2.3 medium with hatched rates assessed at 24, 48, and $72 \mathrm{~h}$. Hatched embryos were fixed and stained using a modified Hoechst method (Damiani et al. 1996 Mol. Reprod. Dev. 45, 521-534) to count cells. The results are presented in Table 1 . There were no significant differences $(P<0.05$, chi-square analysis) between treatment groups for hatched rates or average cell counts. The results indicate that exposure to TrypLETMExpress did not inhibit normal embryonic development. The efficacy of TrypLETMExpress to remove or inactivate certain embryo-associated viruses is being investigated

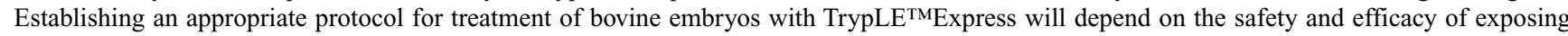
embryos to this recombinant protease.

Table 1. Comparison of hatched IVF bovine embryo rates and average cell counts after exposure to TrypLETMExpress

\begin{tabular}{lccccc}
\hline \multicolumn{5}{c}{ Treatment groups } \\
& Control (\%) & 1 min (\%) & $5 \min (\%)$ & $10 \min (\%)$ & Total (\%) \\
\hline Total in culture & 80 & 82 & 78 & 82 & 322 \\
24-h hatched & $19(24)$ & $24(29)$ & $17(22)$ & $18(22)$ & $78(24)$ \\
48-h hatched & $45(56)$ & $41(50)$ & $47(60)$ & $42(51)$ & $175(54)$ \\
72-h hatched & $54(68)$ & $44(54)$ & $53(68)$ & $51(62)$ & $202(63)$ \\
Avg. cell count & 209 & 218 & 196 & 196 & \\
Cell range & $80-330$ & $110-330$ & $100-360$ & $80-380$ & \\
\hline
\end{tabular}

This work was funded by Invitrogen Corporation.

\section{BOVINE EMBRYO DEVELOPMENT AND MRNA EXPRESSION IN BLASTOCYSTS CULTURED IN ISOLATED MOUSE OVIDUCTS MAINTAINED IN SOF OR KSOM}

\author{
D. Rizos ${ }^{\mathrm{A}}$, B. Pintado $\mathrm{A}, J$. de la Fuente $\mathrm{A}$, P. Lonergan ${ }^{\mathrm{B}}$, and A. Gutierrez-Adan ${ }^{\mathrm{A}}$ \\ ${ }^{A}$ Departamento de Reproduccion Animal, INIA, Ctra de la Coruna km 5.9, Madrid 28040, Spain; \\ ${ }^{B}$ Department of Animal Science, University College Dublin, Lyons Research Farm, Newcastle, County Dublin, Ireland
}

It is well known that modification of the post-fertilization culture environment of mammalian pre-attachment embryos can affect blastocyst quality, manifested in terms of morphology, cryotolerance, and relative abundance of certain gene transcripts. Culture of in vitro-produced bovine zygotes in the ewe oviduct leads to the development of blastocysts of a quality similar to those derived totally in vitro (Rizos et al. 2002 Biol. Reprod. 66 , 589-595). However, such a system has disadvantages from a practical and animal welfare point of view. The isolated mouse oviduct (IMO) culture system is a potential alternative and has been successfully used in the in vitro culture of mouse, rat, hamster, and pig embryos from the one-cell stage to the morula/blastocyst stage. The aim of this study was to examine (1) the development of bovine zygotes in the IMO maintained in two different media (SOF and KSOM) in organ culture, and (2) the quality of the resultant blastocysts assessed in terms of the relative abundance of transcripts for several genes that have been previously implicated in embryo quality. Mouse oviducts were isolated from adult Swiss females (CD1, Harlan) the day after mating with an intact male. Approximately 10-15 presumptive bovine zygotes, produced by in vitro oocyte maturation and fertilization, were transferred to the ampullae of the isolated oviducts and were cultured in Transwell plates (Costar, Corning, NY, USA) over $1.1 \mathrm{~mL}$ of culture medium (SOF, $n=241$ or KSOM, $n=320$ ) at $39^{\circ} \mathrm{C}$ in an atmosphere of $5 \% \mathrm{CO}_{2}$ in air at maximum humidity. A control group of embryos was cultured in droplets $(25 \mu \mathrm{L})$ of the same culture medium and conditions in parallel (SOF, $n=278$, KSOM, $n=225)$. Five replicates (=days of bovine ovary collection) were carried out. Following 6 days of culture, embryos were recovered from the oviducts/culture drops and blastocysts were snap-frozen in liquid nitrogen. Quantification of all gene transcripts was carried out by real time quantitative RT-PCR. Data on embryo development 
were analyzed by chi-square analysis and differences in transcript abundance by ANOVA. Culture in the IMO did not affect the proportion of zygotes developing to the blastocyst stage compared to the respective control droplets (SOF: 21.0 vs. $21.9 \%$; KSOM: 22.0 vs. $22.2 \%$ ). Culture in the IMO in SOF resulted in an increase $(P \leq 0.05)$ in the abundance of transcripts for Oct- 4 and SOX and reduced abundance of Glut- $1, \mathrm{Na} / \mathrm{K}$ transporter, $\mathrm{Cx} 43$, and survivin, compared to control embryos. In contrast, culture in the IMO in KSOM resulted in increased abundance of transcripts for Glut-1, Cx43, Oct-4, and survivin and a reduced expression of $\mathrm{Na} / \mathrm{K}$ transporter and SOX. Transcripts for G6PDH, IFN, and E-Cad were unaffected by culture environment. In conclusion, culture in the IMO leads to alterations in the relative abundance of transcripts that have been previously associated with embryo quality following culture in the ewe oviduct. However, the effect is dependent on the basal medium used.

\title{
176 EFFECT OF HYPERTHERMIA ON DEVELOPMENTAL COMPETENCE OF GERMINAL-STAGE OOCYTES: IN VIVO AND EX VIVO STUDIES IN MICE
}

\author{
Z. Roth $^{\mathrm{A}}, A$. Aroyo $^{\mathrm{A}, \mathrm{B}}, S$. Yavin $^{\mathrm{A}, \mathrm{B}}$, and A. Arav ${ }^{\mathrm{B}}$ \\ ${ }^{A}$ Department of Animal Science, Faculty of Agricultural, Food and Environmental Quality Sciences, The Hebrew University of Jerusalem, \\ Rehovot, 76100 Israel; \\ ${ }^{B}$ Institute of Animal Science, Agricultural Research Organization, The Volcani Center, Bet Dagan, 50250 Israel
}

Mammalian oocytes are susceptible to thermal stress at various stages of follicular development. The present study was performed to determine the effects of maternal hyperthermia on germinal vesicle $(\mathrm{GV})$-stage oocytes in mice. Evaluation parameters included (a) the oocyte's ability to become fertilized, (b) ex vivo embryonic development, (c) pregnancy outcome, and (d) quality of offspring. Female mice $\left(\mathrm{CB}_{6} \mathrm{~F}_{1}\right)$ were synchronized with pregnant mare serum gonadotropin (PMSG) followed by hCG $48 \mathrm{~h}$ later. One hour after hCG administration, control mice were kept under normo-thermal conditions $\left(22^{\circ} \mathrm{C}, 45 \% \mathrm{RH}\right)$, whereas the treated mice were exposed to thermal stress $\left(40^{\circ} \mathrm{C}, 70 \% \mathrm{RH}\right)$ for 1.5 to $2 \mathrm{~h}$ to induce a rise of $1.8^{\circ} \mathrm{C}$ in rectal temperature. After a 1-h recovery period, both treated and control mice were paired with stud males overnight. In Exp. 1 , mated mice were sacrificed $20 \mathrm{~h}$ after hCG administration and embryos were recovered and cultured in vitro. The number of putative zygotes recovered was counted, and cleavage and blastocyst-formation rates were recorded on Days 1 and 5 post-fertilization, respectively. Examination of oocyte developmental stage during hyperthermia induction revealed that oocytes were at the GV stage. The percentage of putative zygotes that cleaved into the two-cell stage was higher $(P<0.05)$ in the control $(91.2 \pm 1.3)$ than in the treated group $(84.5 \pm 1.7)$. In addition, blastocyst-formation rate was higher in the control $(P<0.05 ; 84.3 \pm 1.8 \%)$ than in the treated group $(57 \pm 2.6 \%)$. In Exp. 2, females were left with the stud males until litter delivery. The date of delivery and the number of pups were documented. Eight-week-old offspring were submitted to behavioral (locomotor activity and episodic memory) tests. The one-way ANOVA procedure of JAMPIN (SAS Institute, Inc., Cary, NC, USA, 2004) was used for statistical analysis. The percentage of females that gave birth during the first and second estrous cycles after hyperthermia induction was $59 \%$ and $71 \%$ for treated and control groups, respectively. The average number of pups in the first estrous cycle was lower $(P<0.05)$ for the treated group, and only became equal to that in the control group in the third estrous cycle. Behavioral tests did not reveal any differences between pups from the two experimental groups. In summary, GV-stage oocytes from stimulated ovaries are highly sensitive to thermal stress, since induction of maternal hyperthermia during this stage disrupts oocyte developmental competence both in vivo and ex vivo. However, oocytes that survive thermal stress exhibit normal developmental potential, since heat-stressed mice produced normal pups, at least as reflected by offspring behavioral tests. Thus, strategies designed to protect the ovarian pool of oocytes from thermal stress are likely to improve fertility in heat-stressed females.

\section{ANTHOCYANIN ISOLATED FROM PURPLE SWEET POTATO IMPROVES DEVELOPMENT AND REDUCES OXIDATIVE STRESS OF BOVINE PRE-IMPLANTATION EMBRYOS EXPOSED TO HEAT SHOCK}

\author{
M. Sakatani, I. Suda, T. Oki, S.-I. Kobayashi, S. Kobayashi, and M. Takahashi \\ National Agricultural Research Center for Kyushu Okinawa Region, Kumamoto, Japan
}

Development of cleavage-stage pre-implantation embryos is disrupted by exposure to heat shock. Heat shock also increases intracellular reactive oxygen species (ROS) in pre-implantation embryos. Therefore, reduction of intracellular ROS levels might improve the development of heat-shocked embryos. Recently the antioxidative activities of polyphenols have been widely reported to reduce the oxidative stress. In this study, we investigated the effect of purple sweet potato anthocyanin, a kind of polyphenol that is a strong ROS scavenger, on development and intracellular redox status of bovine pre-implantation embryos exposed to heat shock. Experiment 1: In vitro-produced 8-16-cell-stage embryos on Day 2 after fertilization were exposed to $41.5^{\circ} \mathrm{C}$ for $6 \mathrm{~h}$ in CR1aa containing $0,0.1,1$, and $10 \mu \mathrm{g} / \mathrm{mL}$ anthocyanin at $5 \% \mathrm{CO}_{2}, 5 \% \mathrm{O}_{2}$, and $90 \% \mathrm{~N}_{2}$. After heat shock, embryos were cultured at $38.5^{\circ} \mathrm{C}$ at $5 \% \mathrm{CO}_{2}, 5 \% \mathrm{O}_{2}$ until Day 8. On Day 8, the proportion of embryos developing to the blastocyst stage was evaluated. Blastocyst total cell number and the ratio between inner cell mass and tropheoderm were evaluated by differential staining. The experiment was replicated five times with more than 70 embryos used in each treatment. Experiment 2: Heat shock treatment of in vitro-produced 8-16-cell-stage embryos was carried out as described in experiment 1. After heat shock, intracellular ROS and glutathione (GSH) levels were measured in individual $8-16$ cell stage embryos with fluorescent probes $\left(2^{\prime}, 7^{\prime}\right.$-dichlorodihydrofluorescein diacetate for ROS and CellTracker ${ }^{\mathrm{TM}}$ Blue (Invitrogen Japan K. K., Tokyo, Japan) for GSH). The fluorescence emissions of each treatment were normalized to those of $8-16$ cell stage embryos cultured at $38.5^{\circ} \mathrm{C}$ without anthocyanin to obtain the relative fluorescence emission. This experiment was replicated four times. Embryos treated with heat stress without anthocyanin $(0 \mu \mathrm{g} / \mathrm{mL})$ showed low development $(14.6 \pm 3.6 \%)$ and blastocyst total cell number $(88.2 \pm 9.4)$. However, embryos treated with $0.1 \mu \mathrm{g} / \mathrm{mL}$ anthocyanin improved development $(31.7 \pm 4.5 \%, P<0.05)$ and increased the total cell number $(96.5 \pm 11.3)$. The higher concentrations of anthocyanin $(1$ and $10 \mu \mathrm{g} / \mathrm{mL})$ did not affect development and cell number. The intracellular ROS levels in heat-shocked embryos were significantly reduced by all concentrations of anthocyanin $(P<0.05)$. In addition, anthocyanin increased GSH levels at all doses tested $(P<0.05)$. These results indicate that an appropriate concentration of anthocyanin improves development by regulating intracellular redox balance in bovine embryos exposed to heat shock. 
178 IMPROVED DEVELOPMENTAL COMPETENCE OF BOVINE EMBRYOS BY PGI2 ANALOG TREATMENT

\author{
B. S. Song, J. S. Kim, D. B. Koo, J. S. Park, K. K. Lee, and Y. M. Han \\ Laboratory of Development and Differentiation, Korea Research Institute of Bioscience and Biotechnology (KRIBB), Daejeon 305-600, Korea
}

The microenvironment of the follopian tube, in which the oviductal fluid contains a variety of cytokines and growth factors, affects pre-implantation development of fertilized embryos in mammals. Prostaglandin I2 (PGI2, prostacyclin) exists in oviductal fluid and is synthesized from arachidonic acid by prostacyclin synthetase. PGI2 also enhances the implantation rate of mouse embryos. In this study, the effect of PGI2 analog on the development of bovine embryos was examined. Bovine cumulus oocytes complexes (COCs) were matured in TCM-199 medium supplemented with $10 \mathrm{IU} / \mathrm{mL}$ pregnant mare serum gonadotropin (PMSG), $10 \mathrm{IU} / \mathrm{mL}$ hCG, and $10 \mathrm{ng} / \mathrm{mL}$ epidermal growth factor $(\mathrm{EGF})$ at $39^{\circ} \mathrm{C}, 5 \% \mathrm{CO} 2 \mathrm{in}$ air for 20 22 h. Following in vitro maturation, COCs were fertilized in Fert-TALP medium containing $0.6 \%$ BSA using frozen semen. Also, oocytes matured in vitro were enucleated, individually reconstructed with bESF cells, fused, and then activated by treatment with $5 \mu \mathrm{M}$ ionomycin for $5 \mathrm{~min}$ and $2 \mathrm{mM} \mathrm{6-DMAP} \mathrm{for} 4 \mathrm{~h}$. In vitro-fertilized (IVF) and nuclear-transferred (NT) eggs were cultured in $50 \mu \mathrm{L}$ drops of CR1-aa medium supplemented with $0.3 \%$ BSA in the absence or presence of $1 \mu \mathrm{M}$ PGI2 analog at $39^{\circ} \mathrm{C}, 5 \% \mathrm{CO}_{2}$ in air, respectively. At 3 days of culture, cleaved embryos were further cultured in the same culture media supplemented with 10\% FBS for 4 days. Allocations of blastocysts to inner cell mass (ICM) and trophoblast (TE) cells were investigated to assess embryo quality. All experiments were repeated more than three times. All data were analyzed by using the Duncan test of ANOVA by the Statistical Analysis System (SAS Institute, Inc., Cary, NC, USA) and numbers of nuclei in blastocysts were expressed as mean \pm SE. No difference was detected in the cleaved rate of the eggs between the treated-and nontreated groups. IVF zygotes treated with PGI2 analog represented a higher developmental rate $(33 \%, 122 / 418)$ to the blastocyst stage than nontreated controls $(24 \%, 107 / 456)$ $(P<0.05)$. Among IVF-derived blastocysts, interestingly, the proportion $(46 \%, 84 / 181)$ of expanded blastocysts was significantly higher in the PGI2 analog-treated group compared with that in the nontreated group $(28 \%, 46 / 164)$. The number of nuclei in $(165 \pm 6.1, n=15)$ in blastocysts in the PGI2 analog-treated group was higher than that $(146.12 \pm 5.7, n=18)$ in the nontreated group $(P<0.05)$. No difference was detected in the ratio of ICM to total cells between PGI2 analog-treated $(42.0 \pm 3.0 \%)$ and nontreated groups $(41.9 \pm 2.9 \%)$. Like the IVF embryos, NT embryos in the PGI2 analog-treated group showed a higher in vitro developmental rate $(33.6 \%, 43 / 128)$ than the nontreated embryos $(24.2 \%, 32 / 132)(P<0.05)$. Our results indicate that PGI2 analog improves the kinetics of embryo development in cattle.

\title{
179 EFFECTS OF AN ENDOCANNABINOID, ANANDAMIDE, ON BOVINE BLASTOCYST DEVELOPMENT IN VITRO
}

\author{
M. Y. Turco, K. Matsukawa, P. Loi, and G. Ptak
}

Department of Comparative Biomedical Sciences, University of Teramo, Teramo, TE, Italy

Cannabinoids cause many adverse effects on reproductive functions including fetal loss and pregnancy failure (Paria et al. 1995 PNAS 92, 94609464). N-arachidonylethanolamine (anandamide), an endogenous cannabinomimetic lipid derivative, binds with high affinity to brain type and spleen type cannabinoid receptors (CB1-R and CB2-R) and mimics most of the effects of $\Delta$ 9-tetrahydrocannabinol $[(-)$ THC], a psychoactive derivative of marijuana. In this study, we investigated the effects of anandamide on ovine blastocyst development in vitro. In vitro-matured oocytes were chemically activated and cultured to the blastocyst stage in our standard media (Ptak et al. 1999 Biol. Reprod. 61, 1568-1574). The development rate of blastocysts was $41 \%$. Day 7 blastocysts were exposed to $28 \mathrm{nM}$ anandamide with or without $20 \mathrm{nM} \mathrm{SR} 141716 \mathrm{~A}$ (an antagonist of CB1-R) for $48 \mathrm{~h}$. In Experiment 1, we examined the CB1-R protein localization on blastocysts by anti CB1-R antibody (Santa Cruz Biotechnology, Inc., Santa Cruz, CA, USA). In Experiment 2, to investigate the possible effect of anandamide on blastocyst development, we used the cell viability assay by propidium iodide, a cell proliferation assay (5-bromo-1'-deoxyuridine incorporation), and the terminal deoxynucleotidyl transferase-mediated dUTP nick-end labeling (TUNEL) assay (Qbiogene, Inc., Carlsbad, CA, USA). Data were analyzed by one-way ANOVA. CB1-R signals were detected on ovine blastocysts, and these signals were effectively improved in the anandamide co-cultured group. The results of Experiment 2 are summarized in Table 1. Our results demonstrate that CB1-R was expressed in the ovine blastocyst, and anandamide exerts negative effects on in vitro blastocyst development by inhibiting cell proliferation and increasing apoptotic rate, but not cell viability. Furthermore, SR141716A can effectively block the negative effects of anandamide.

Table 1. Effect of anandamide (AEA) on sheep blastocyst development assessed by viability, cell proliferation, and TUNEL staining assays

\begin{tabular}{llc}
\hline Method of assay & Treatment & \% Positive cells/total cells (mean \pm SEM) \\
\hline Viability & AEA & $4.1 \pm 1.1$ \\
& AEA + SR141716A & $3.0 \pm 0.6$ \\
Centrol proliferation & AEA & $3.7 \pm 0.5$ \\
& AEA + SR141716A & $19.3 \pm 1.3^{\mathrm{a}}$ \\
& Control & $26.9 \pm 2.3^{\mathrm{b}}$ \\
& Control & $27.1 \pm 2.7^{\mathrm{b}}$ \\
& Control & $3.1 \pm 0.3^{\mathrm{b}}$ \\
& & $3.1 \pm 0.3^{\mathrm{b}}$ \\
\hline
\end{tabular}

${ }^{\mathrm{ab}}$ Values with different superscripts within the same column differed significantly $(P<0.05)$. 


\title{
180 DETECTION OF CASPASE-3 AND -7 IN NORMAL AND SLOW DEVELOPING BOVINE IN VITRO EMBRYOS
}

\author{
L. Vandaele, B. Mateusen, D. Maes, and A. Van Soom
}

Department of Reproduction, Obstetrics and Herd Health, Faculty of Veterinary Medicine, Ghent University, 9820 Merelbeke, Belgium

The incidence of apoptosis is an important indicator of embryo quality that can be measured by detection of active caspase- 3 and -7 at various stages of in vitro development. The aim of the present study was to investigate the expression of these apoptotic markers in bovine in vitro embryos at different developmental stages using a fluorescent staining method. Additionally, expression of both caspases was compared between normal and slow-developing embryos. A total of 2640 immature bovine oocytes (four replicates) were matured and fertilized in vitro. Presumed zygotes $(n=2400)$ were denuded $24 \mathrm{~h}$ post-insemination (hpi) after fertilization and cultured in $50-\mu \mathrm{L}$ droplets of modified SOF medium with $10 \%$ fetal calf serum at $39.0^{\circ} \mathrm{C}$ in $5 \% \mathrm{CO}_{2}, 5 \% \mathrm{O}_{2}$ and $90 \% \mathrm{~N}_{2}$. Cleavage rate was determined at 45 hpi for all embryos, except 400 that were used as positive controls for the caspase staining. At different time points, embryos were allocated to the normal developing group if they reached the 5-8-cell stage at $45 \mathrm{hpi}$; the 9-16-cell stage at $80 \mathrm{hpi}$, the morula stage at $117 \mathrm{hpi}$ and the expanded blastocyst stage or beyond at $168 \mathrm{hpi}$. Embryos that lagged one cell cycle behind at each time point were designated as slow-developing embryos. All selected embryos were stained using CaspaTag Pan-Caspase In Situ Assay kit, Fluorescein ${ }^{\circledR}$ (Chemicon International, Temecula, CA, USA). Positive controls were incubated in $0.5 \mu \mathrm{M}$ staurosporine (Sigma, Belgium) for $24 \mathrm{~h}$. The apoptotic cell ratio (ACR) was determined for each embryo by means of confocal laser scanning microscopy (ACR $=$ number caspase-positive cells/total cell number $\times 100)$. Control embryos $(n=400)$ were cultured undisturbed until 168 hpi and evaluated for blastocyst yield. Mixed model analyses of variance with group as fixed factor and replicate as random factor were used to evaluate the ACR at $45,80,117$, and $168 \mathrm{hpi}$, respectively. The mean cleavage rates at $45 \mathrm{hpi}$ and the blastocyst yield at $168 \mathrm{hpi}$ were $60.5 \%$ and $26.9 \%$, respectively. The ACR over all time points and at each individual time point was significantly lower in normal-developing embryos compared with slow-developing embryos, as determined by caspase staining $(P<0.01)$ (Table 1$)$. In conclusion, this study demonstrated that slow-developing embryos express a higher level of apoptosis. Further research is necessary to investigate the cause of the higher ACR in slow developing embryos, which may be the result of altered gene expression or environmental influences.

Table 1. Apoptotic cell ratio (ACR; mean $\% \pm$ SD) at four time points in normal and slow-developing embryos

\begin{tabular}{lrccccccr}
\hline Group & \multicolumn{2}{c}{$45 \mathrm{hpi}$} & \multicolumn{2}{c}{$80 \mathrm{hpi}$} & \multicolumn{2}{c}{$117 \mathrm{hpi}$} & \multicolumn{2}{c}{$168 \mathrm{hpi}$} \\
& \multicolumn{1}{c}{$n$} & ACR & $n$ & ACR & $n$ & ACR & $n$ & ACR \\
\hline Normal & 59 & $16.3 \pm 18.8^{\mathrm{a}}$ & 61 & $22.8 \pm 21.7^{\mathrm{a}}$ & 72 & $14.8 \pm 10.8^{\mathrm{a}}$ & 53 & $9.0 \pm 9.8^{\mathrm{a}}$ \\
Slow & 186 & $38.0 \pm 36.9^{\mathrm{b}}$ & 68 & $36.3 \pm 24.1^{\mathrm{b}}$ & 58 & $27.1 \pm 18.2^{\mathrm{b}}$ & 58 & $19.0 \pm 17.4^{\mathrm{b}}$ \\
\hline
\end{tabular}

${ }^{a b}$ Within a column, values with a different superscript differ significantly $(P<0.01)$.

This study was supported by a grant of Research Foundation - Flanders (aspirant 1.1.084.04N00).

Embryo Manipulation

\section{MOUSE TRIPLETS DEVELOPED FROM SINGLE BLASTOMERES OF AN 8-CELL-STAGE EMBRYO SUPPORTED WITH TETRAPLOID EMBRYOS}

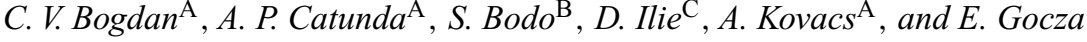 \\ ${ }^{A}$ Department of Animal Biology, Agricultural Biotechnology Center, Godollo, Hungary; \\ ${ }^{B}$ Faculty of Animal Science and Biotechnology, Timisoara, Romania; \\ ${ }^{\mathrm{C}}$ Research Institute for Animal Breeding and Nutrition, Herceghalom, Hungary
}

Chimera production using altered ES cells became a key tool for generating transgenic mice. However, chimeras are more than just a tool for making mouse mutants; they play a crucial role in analyzing the biological effects of genetic changes. Chimeras can be made by combining two whole 8 -cell embryos or by combining subsets of blastomeres of two cleavage stage embryos. Because, at these stages, the early embryonic cells are not yet restricted in their lineage potency, they are equally capable of contributing to the inner cell mass or the trophectoderm. Pluripotency of single blastomeres of the 4-cell and 8-cell mouse embryo has been proved indirectly by aggregating them with carrier blastomers of a different genotype, giving rise to chimeric blastocyst (Tarkowski et al. 1967 J. Embryol. Exp. Morphol. 18, 155-180). In our study we wanted to demonstrate that a single blastomere of an 8-cell stage embryo, supported with tetraploid embryos at the 4-cell stage, is capable of developing into a healthy animal. When tetraploid embryos are used to make chimeras together with diploid cells, tetraploid cells rarely contribute to the embryo itself, but contribute mainly to the primitive endoderm and the trophectoderm, so in this case the newborns will be derived only from the diploid blastomers used (Nagy et al. 1990 Development $110,815-821)$. We produced chimeras by aggregating a single blastomere [(2n)(1-cell)] derived by combining sexed, GFP-expressing, diploid 8-cell stage embryo (Hadjantonakis et al. 1998 Nat. Genet. 19, 220-222) with either a sexed diploid 7-cell embryo [(2n)(7-cells)] (one of the 8 -cells was taken for sexing) or a non-sexed tetraploid embryo [(4n)(4-cells)]. The aggregates were cultured in vitro and transferred as blastocysts to the uteruses of pseudo-pregnant females. Fetuses were removed by Caesarian section and raised by lactating foster mothers. From the transferred 84 $[\mathrm{XY}(2 \mathrm{n})(1-\mathrm{cell})] /[\mathrm{XX}(2 \mathrm{n})(7$-cells) $]$ aggregates we obtained $12(14.3 \%)$ newborns, $11(91.7 \%)$ males and one $(8.3 \%)$ female. From the transferred $27[\mathrm{XY}(2 \mathrm{n})(1-\mathrm{cell})] /[(4 \mathrm{n})(4$-cells)] aggregates, where one XY blastomere was combined with a tetraploid embryo, we obtained $7(25.9 \%)$ male newborns: 1 triplet and 2 pairs of twins. We also transferred four $[\mathrm{XX}(2 \mathrm{n})(1-\mathrm{cell})] /[(4 \mathrm{n})(4-\mathrm{cells})]$ chimera embryos, where an XX-blastomere was 
aggregated with a tetraploid embryo; we obtained a set of living female triplets (75\%) from this aggregate. We demonstrated that a single blastomere of the 8-cell-stage embryo is capable of developing into a living newborn. We obtained identical pups from three blastomeres isolated from the same embryo (triplets). This way we produced single blastomere clones and could control the sex of the new generation.

This research was supported by grants from OTKA T037582 and RO-1/2002 Intergovernmental S\&T cooperation program.

\title{
182 FAST STAINING METHODS FOR DIFFERENTIAL STAINING OF INNER CELL MASS AND TROPHECTODERM CELLS OF MAMMALIAN BLASTOCYSTS
}

\author{
S. W. Kim, J.-K. Park, J.-H. Han, C. G. Park, and W.-K. Chang
}

National Livestock Research Institute, Suwon, Gyeonggi, Republic of Korea

The present study was undertaken to develop a simple differential staining method for inner cell mass (ICM) and trophectoderm (TE) cells of mammalian blastocysts using the permeabilizing agent, saponin, without species-specific antibodies and complements. The nuclei of whole embryos were pre-stained to green by $5 \mu \mathrm{M}$ SYTO 13 for $10 \mathrm{~min}$. After washing, the green color of TE was turned to red by exposure to $100 \mu \mathrm{g} / \mathrm{mL}$ propidium iodide and 50 to $100 \mu \mathrm{g} / \mathrm{mL}$ saponin solution for 5 to $10 \mathrm{~min}$. To confirm the exactness of staining patterns, the fluorescent nuclei of ICM and TE from mouse, pig, and bovine blastocysts were compared with 3D location by confocal microscopy. By the saponin mixture treatment method, in vitro-cultured mouse, pig, and bovine blastocysts were shown to have an ICM:TE ratio of 1:2.5, 1:4.5, and 1:3.6, with an average total cell number of $78 \pm 14(n=45), 65 \pm 18(n=49)$, and $150 \pm 20(n=45)$, respectively. Although a few TE cells were stained to a yellowish-green color, the successful protection of the green color of ICM depended on the exposure time of blastocysts to the saponin mixture. The total time lapse of the procedure did not exceed $1 \mathrm{~h}$. These results indicate that saponin could be used as a practical substitute for special antibodies and complements. So this differential staining for examining the ICM:TE ratio and the total cell count of mammalian blastocysts would be a fast and reliable method.

\section{DEVELOPMENT OF PORCINE EMBRYOS MICROINJECTED WITH PORCINE EMBRYONIC} GERM CELLS

\author{
C.-H.Park ${ }^{\mathrm{A}, \mathrm{B}}$, S.-G. Lee $e^{\mathrm{A}, \mathrm{B}}$, D.-H. Choi ${ }^{\mathrm{A}, \mathrm{B}}, M .-G . K_{i m}^{\mathrm{A}, \mathrm{B}}$, and C. K. Lee $\mathrm{A}^{\mathrm{A}, \mathrm{B}}$

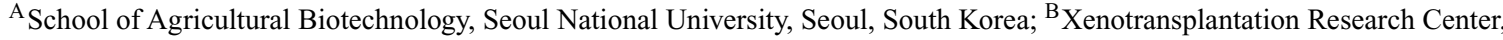 \\ Seoul National University Hospital, Seoul, South Korea
}

Embryonic germ (EG) cells, derived from primordial germ cells in the developing fetus, are similar to embryonic stem (ES) cells in terms of expression pattern of undifferentiated markers and their ability to colonize both the somatic and the germ cell lines following injection into a host blastocyst, which has been proven in mouse. Several studies using porcine EG cells have shown that it is possible to produce somatic chimeras after blastocyst injection. However, not only was the degree of reported chimerism low, but also there has been no report about the fate of injected EG cells in porcine blastocysts. This study was designed to observe the distribution pattern of porcine EG cells in chimeric blastocyst after injection into cleavage-stage porcine embryos. To ascertain development of microinjected porcine embryos with EG cells, 10 to 15 EG cells were injected into cleavage stage of in vitro fertilized embryos and cultured up to blastocyst. Also, porcine EG cells were labeled with DiO (Invitrogen, Carlsbad, CA) on the cell membrane or transfected with green fluorescent protein gene to observe whether the EG cells injected in the host embryo would incorporate into the inner cell mass (ICM) or trophectoderm (TE). Chimeric embryos were produced and allowed to develop into blastocysts to investigate the injected EG cells would come to lie in ICM and/or TE of the blastocyst, by scoring their position. In result, developmental rate was similar in all treatments. In all treatments, EG cells were mainly allocated in both ICM and TE of the chimeric blastocysts. These results suggest that examining the allocation pattern of injected EG cells, maintained pluripotency in vitro, could provide clues of differentiation process in vivo. Furthermore, to enhance the allocation of EG cells into the embryonic lineage, it would be required to optimize the culture condition for EG cells as well as embryos. Further experiment are needed to determine whether the injected EG cells could maintain their properties throughout the environment in the embryonic development in vitro.

Table 1. Distribution of the porcine EG cells microinjected into cleavage-stage embryos

\begin{tabular}{lcclc}
\hline $\begin{array}{l}\text { Stage of } \\
\text { embryo }\end{array}$ & $\begin{array}{c}\text { No. embryos } \\
\text { examined }\end{array}$ & $\begin{array}{c}\text { No. (\%) embryos } \\
\text { developed to blastocyst }\end{array}$ & \multicolumn{2}{c}{ No. (\%) embryos detected in } \\
ICM only & ICM and TE \\
\hline 4-cell & 45 & $26(57.7 \pm 3.99)$ & $2(7.6)$ & $24(92.4)$ \\
8-cell & 78 & $47(60.2 \pm 4.76)$ & $5(10.6)$ & $44(89.4)$ \\
16-cell & 45 & $28(62.2 \pm 2.19)$ & $2(7.1)$ & $25(92.9)$ \\
morula & 21 & $21(100)$ & $0(0.0)$ & $21(100)$ \\
\hline
\end{tabular}


184 EFFECT OF LIVE CONFOCAL FLUORESCENCE IMAGING ON EMBRYONIC DEVELOPMENT

\author{
P. Ross and J. Cibelli \\ Cellular Reprogramming Laboratory, Michigan State University, East Lansing, MI 48824, USA
}

\begin{abstract}
The aim of this study was to examine the effects of fluorescent confocal imaging on embryo development. DNA damage, caspase activation, and reactive oxygen species (ROS) production were also measured. In vitro-generated bovine 8-cell embryos and in vivo-produced mouse 2-cell embryos were stained with Mitotracker Red and individually imaged using a spinning-disc confocal module (CARV) attached to a Nikon TE2000-U microscope. The sample was excited with a $120 \mathrm{~W}$ metal halide lamp (X-Cite 120) using a fluorescent filter set (Excitation: $575 \mathrm{~nm}$; Emission: $640 \mathrm{~nm}$ ). A variety of number and durations of exposures were tested. Continuous variables were analyzed by $t$-test, whereas categorical data were analyzed using the homogeneity chi square test. Bovine embryos showed no differences in blastocyst development (Day 7.5 ; Day $0=\mathrm{IVF})$ or hatched blastocysts (Day 11) between groups of imaged and non-imaged embryos (Table 1). Despite numerous attempts, we were unable to find a level of exposure that disturbed embryo development, indicating that bovine embryos have a high degree of tolerance to the imaging system. After exposure, mouse embryos were cultured in vitro to the blastocyst stage and transferred into surrogate synchronized females. Embryos subjected to five exposures of $50 \mathrm{~ms}$ developed normally both in vitro and in vivo (Table 1). However, exposing the embryos 30 times significantly affected in vitro and in vivo development. Subsequently, we examined the effect of confocal imaging on DNA damage (Comet assay), ROS production (dichlorofluoresceindiacetate, DCF), and caspase 3 activation (PhiPhiLux ${ }^{\circledR}$-G1D2). Bovine embryos were exposed to $60 \mathrm{z}-50 \mathrm{~ms}$ or $60 \mathrm{z}-500 \mathrm{~ms}$, and mouse embryos to $5 \mathrm{z}-50 \mathrm{~ms}$ or $30 \mathrm{z}-50 \mathrm{~ms}$ after Mitotracker Red staining. We observed that caspase 3 was not activated when measured $1.5 \mathrm{~h}$ after imaging. Similarly, DNA damage was not different among embryos exposed at different levels of imaging. However, ROS production significantly increased in both bovine and mouse embryos after exposure to confocal imaging, and this increase correlated with fluorescence exposure level. We conclude that imaging bovine pre-implantation embryos with this system increased ROS production but did not disturb in vitro development (even at unnecessary high levels of exposure). In mouse embryos, in vitro development after imaging directly correlated with in vivo development. It remains to be determined whether the increase in ROS production and decreased developmental potential is due to the fluorescence exposure alone or the combined effect of fluorescence exposure and Mitotracker labeling.
\end{abstract}

Table 1. Embryonic development after confocal fluorescence imaging

\begin{tabular}{llccccl}
\hline Experiment & Treatment & z planes $(n)$ & Exposure time $(\mathrm{ms})$ & $n$ & Blastocyst rate & Hatched blastocyst rate \\
\hline Bovine 1 & Stained & - & - & 69 & $31 \%$ & $16 \%$ \\
& $30 \mathrm{z}-100 \mathrm{~ms}$ & 30 & 100 & 67 & $33 \%$ & $18 \%$ \\
& $60 \mathrm{z}-100 \mathrm{~ms}$ & 60 & 100 & 68 & $32 \%$ & $21 \%$ \\
\multirow{5}{*}{ Bovine 2 } & $90 \mathrm{z}-100 \mathrm{~ms}$ & 90 & 100 & 68 & $37 \%$ & $19 \%$ \\
& Stained & - & - & 55 & $25 \%$ & $22 \%$ \\
& $60 \mathrm{z}-100 \mathrm{~ms}$ & 60 & 100 & 56 & $23 \%$ & $23 \%$ \\
& $60 \mathrm{z}-250 \mathrm{~ms}$ & 60 & 250 & 56 & $29 \%$ & $25 \%$ \\
& $60 \mathrm{z}-500 \mathrm{~ms}$ & 60 & 500 & 56 & $25 \%$ & $20 \%$ \\
\multirow{5}{*}{ Mouse } & & - & & & Pups born \\
& Control & - & - & 87 & $79 \%^{\mathrm{a}}$ & $17 / 28(57 \%)$ \\
& Stained & - & 50 & $91 \%{ }^{\mathrm{a}}$ & $22 / 36(61 \%)$ \\
& $5 \mathrm{z}-50 \mathrm{~ms}$ & 5 & 50 & 77 & $95 \%^{\mathrm{a}}$ & $32 / 56(55 \%)$ \\
& $30 \mathrm{z}-50 \mathrm{~ms}$ & 30 & 50 & 82 & $33 \%^{\mathrm{b}}$ & $0 / 27$ \\
\hline
\end{tabular}

${ }^{\mathrm{a}, \mathrm{b}}$ Values with the same superscript are not significantly different $(P<0.01$, chi-square).

\title{
185 EMBRYONIC LOSS IN PIGS ASSOCIATED WITH OVIDUCT TRANSPLANTATION OF EARLY-STAGE EMBRYOS WITH DAMAGES IN THE ZONA PELLUCIDA
}

\author{
S. Ueno, M. Kurome, R. Tomii, K. Hiruma, N. Maeda, H. Saito, and H. Nagashima \\ Laboratory of Reproduction Engineering, Department of Life Science, School of Agriculture, Meiji University, Kawasaki, Kanagawa, Japan
}

It is assumed that if porcine early-stage embryos with damages in their zonae pellucidae are transplanted to the recipient's oviduct, they may suffer from mechanical and immunological stresses by oviduct contraction and the recipient's immune response. This study aimed to examine the impact of zona pellucida damages, which might arise during nuclear transfer and intra cytoplasmic sperm injection (ICSI), on the development and survival of transplanted embryos. Cumulus-oocyte complexes were collected from ovaries obtained at a local slaughterhouse and matured in vitro in NCSU23 to prepare MII-stage oocytes. The zonae pellucidae of these oocytes were either penetrated with 8 - to 10 - $\mu \mathrm{m}$ square-ended microinjection pipettes or incised with 35- to $40-\mu \mathrm{m}$ beveled enucleation pipettes. Intact oocytes were used as controls. The oocytes were electroactivated to induce parthenogenesis and transplanted to the oviducts of estrus-synchronized recipient gilts (estrus-synchronized with $1000 \mathrm{IU}$ eCG and $1500 \mathrm{IU}$ hCG). After 5 to 7 days, the recipient uteri were flushed with PBS supplemented with $1 \%$ fetal bovine serum (FBS) to collect embryos, and their development (morula-blastocyst stage embryos/collected embryos) and survival (viable embryos/collected embryos) were determined. In total, 221 zona-penetrated, 129 zona-incised, and 57 intact embryos were transplanted to four, two and two gilts, respectively. The efficiency of embryo recovery 
was similar in all groups (59.0 to $81.8 \%$ ). However, the zona-penetrated and zona-incised embryos showed inconsistent development and survival compared with controls; the development and survival rate were $92.6 \%(25 / 27)$ to $96.7 \%(29 / 30)$ and $77.8 \%(21 / 27)$ to $96.7 \%$ (29/30) in control embryos, respectively, whereas those of zona-penetrated embryos were $57.1 \%(28 / 49)$ to $95.7 \%(22 / 23)$ and $8.2 \%(4 / 49)$ to $78.3 \%(18 / 30)$, and those of zona-incised embryos were $47.6 \%$ (30/63) to $92.3 \%$ (36/39) and $23.8 \%$ (15/63) to $92.3 \%$ (22/23), respectively. Large foci of cells that appeared to be macrophage giant cells were observed at the surface or inside of the degenerated zona-damaged embryos. These results indicate that the recipient's immune response may impair development after transplantation of the embryo to the oviduct, when there is damage in the zona pellucida. This may be one of the factors attributable to the reduced efficiency of live progeny production by ICSI and nuclear transfer.

This work was supported by PROBRAIN.

Embryo Transfer

\title{
186 COMPARISON OF IVF AND ICSI TREATMENTS ON THE FERTILIZATION, EMBRYO CLEAVAGE, EMBRYO TRANSFER, PREGNANCY, AND EMBRYO IMPLANTATION RATES IN IMMUNOLOGICALLY INFERTILE PATIENTS
}

\author{
S. Al-Anssari ${ }^{\mathrm{A}}$, M. Ridha-Barzanchi ${ }^{\mathrm{A}, \mathrm{B}}, N . \operatorname{Hawa}^{\mathrm{A}}, S \cdot$ Mahmood $^{\mathrm{A}}$, and S. Khunda ${ }^{\mathrm{A}}$ \\ A IVF Center, Department of Obstetric and Gynecology and Physiology, Baghdad University Medical School, Baghdad Teaching \\ Hospital, Baghdad, Republic of Iraq; \\ ${ }^{B}$ Reproductive Sciences Laboratory, Department of Veterinary Physiology and Pharmacology, College of Veterinary Medicine \\ and Biomedical Sciences, College Station, TX 77843-4466, USA
}

The objective of the present work was to study the response to in vitro fertilization (IVF) and intracytoplasmic sperm injection (ICSI) techniques in regard to fertilization, embryo cleavage, embryo transfer (ET), pregnancy, and embryo implantation rates using semen from immunologically infertile men. Ninety-four men with antisperm antibodies (ASA) detected in their semen and serum by slide agglutination and tray microagglutination tests were involved in IVF (IVF group) or ICSI (ICSI group) treatment. All male patients underwent sperm penetration assay (SPA) using zona-free hamster oocytes. Men with positive SPA were admitted to the IVF protocol $(n=46)$ and men with negative SPA were involved in the ICSI protocol $(n=48)$. The female patients had normal ovulatory cycles and normal reproductive hormone concentrations (FSH, LH, prolactin, estradiol and progesterone). They received human menopausal gonadotropin (hMG) and human chorionic gonadotropin (hCG) for the induction of ovulation. Follicular growth was monitored by serial vaginal sonography and measurement of estradiol concentrations. Sperm viability was assayed by a hypo-osmotic swelling test (HOST) prior to oocyte insemination. Student t-test and Chi-Square test were used for statistical analysis of the data. The clinical data of the female patients (including age, body mass index $\left(\mathrm{kg} / \mathrm{m}^{2}\right)$, infertility duration, total number of hMG ampoules, estradiol concentration on cycle Day 3 and at day of hCG injection, and endometerial thickness) in the IVF and ICSI groups were not significantly different between the groups $(P>0.05)$. The oocyte recovery rate per patient was $6.34(292 / 46)$ in the IVF group and $6.87(330 / 48)$ in the ICSI group. The fertilization and embryo cleavage rates were similar $(P>0.05)$ in the IVF and ICSI groups $(61.86 \%(133 / 215)$ vs. $56.50 \%(139 / 246)$ and $78.95 \%$ $(105 / 133)$ vs. $72.66 \%(101 / 139)$, respectively). The percentage of embryos of transferable quality (ET rate) and the number of transferred embryos per patient were significantly higher $(P<0.05)$ in the IVF group compared to the ICSI group $(73.33 \%(77 / 105)$ vs. $58.42 \%(59 / 101)$ and 1.67 vs. 1.22 , respectively). The pregnancy rate per patient and embryo implantation rate were similar $(P>0.05)$ in both IVF and ICSI groups $(28.26 \%$ vs. $25 \%$ and $21.74 \%$ vs. $18.75 \%$, respectively). It was concluded from the results of the present study that the higher ET rate and the number of the transferred embryos per patient in the IVF group may be due to the superior quality of IVF sperm compared to ICSI sperm. Both IVF and ICSI embryos showed no significant differences in their pregnancy and embryo implantation rates; this may indicate that both types of embryos are viable and have similar pregnancy and embryo implantation potentials following ET, provided that the female patients have normal ovulatory cycles and normal concentrations of reproductive hormones. SPA and HOST were found to be useful tests for the selection of spermatozoa in ASA positive male patients for involvement in IVF or ICSI procedures.

\section{RE-ESTABLISHMENT OF AN EXTINCT STRAIN OF SHEEP FROM A LIMITED SUPPLY OF FROZEN SEMEN}

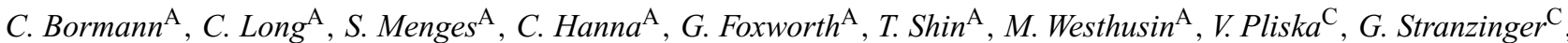

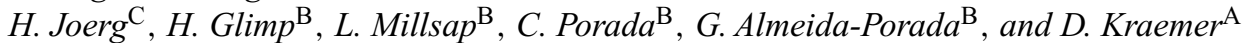 \\ ${ }^{A}$ Department of Veterinary Physiology and Pharmacology, Texas A\&M University, College Station, TX 77843, USA; \\ ${ }^{B}$ Agriculture, Biotechnology, and Natural Resources, University of Nevada, Reno, NV 89507, USA; \\ ${ }^{\mathrm{C}}$ ETH Zurich, Zurich, Switzerland
}

The objective of this study was to restore a line of sheep that exhibits spontaneous X-linked factor VIII deficiency closely mimicking human hemophilia A. Six straws of frozen semen from an affected Alpine White male were obtained from Switzerland. In the first experiment the straw of semen thawed was of poor quality. Two ewes were synchronized for use as embryo donors (MOET) by means of CIDRs for 14 days and superovulated with declining doses of FSH (184 mg) twice daily for 3 days. PMSG (200 IU) was given with the final dose of FSH and $1000 \mathrm{IU}$ of hCG $12 \mathrm{~h}$ postCIDR removal. The ewes were surgically inseminated $24 \mathrm{~h}$ later. Oviducts were flushed $48 \mathrm{~h}$ post-insemination producing 13 unfertilized ova (UFO). Spermatozoa were used for intracytoplasmic sperm injection (ICSI) utilizing oocytes collected from superstimulated ewes by laporatomy. These 
ewes were synchronized with CIDRs ( 15 days) and superovulated with a declining dose of FSH ( $204 \mathrm{mg}$ ) twice daily for 3.5 days. Utilizing 236 oocytes, ICSI produced 189 embryos, an $80 \%$ embryo/oocyte rate. Embryos were transferred surgically to the oviducts of 17 synchronized recipients. Recipients were synchronized using sponges (Ovakron; Heriot Agvet, Rowville, Victoria, Australia) containing $30 \mathrm{mg}$ of flugestone acetate (14 days) and given PMSG (400 IU) at sponge removal, followed by $1000 \mathrm{IU}$ of hCG $12 \mathrm{~h}$ post-sponge removal. Eleven recipients produced 17 lambs for a lamb/embryo rate of $8.9 \%$. The straw of semen utilized for the second experiment was of higher quality. Three ewes were superstimulated for use as MOET donors, as above, with increased doses of FSH $(228 \mathrm{mg})$ and PMSG (500 IU). Donors were surgically inseminated and oviductal flushes were performed $40 \mathrm{~h}$ post-insemination, yielding $19 \mathrm{UFO}$ and 12 embryos for an embryo/oocyte rate of $38.7 \%$. Embryos were transferred to four recipients, synchronized as above with an increased dose of PMSG (600 IU). These MOET recipients produced nine lambs for a lamb/embryo rate of $75 \%$. Semen was used to produce embryos via in vitro fertilization (IVF) using oocytes collected from superstimulated ewes (as above with an increase of FSH to $252 \mathrm{mg}$ ). IVF produced 91 embryos from 247 oocytes for an embryo/oocyte rate of $36.8 \%$. Embryos were transferred to 20 recipients 24 to $48 \mathrm{~h}$ post-fertilization. Seven recipients maintained pregnancy and produced $10 \mathrm{lambs}$ with a lamb/embryo rate of $11 \%$. ICSI was also utilized, producing 54 embryos from 98 oocytes, an embryo/oocyte rate of $55.1 \%$. Embryos were transferred to eight recipients; none maintained pregnancy. Through the use of multiple reproductive technologies, 36 lambs (22 carriers) were produced from two straws of semen. Carriers will be bred back to their sire in a similar program to produce affected lambs.

The authors would like to acknowledge J. Liu and M. Ridha for their contributions. This work was supported by NIH Grant HL073737-12.

\title{
188 EFFECT OF TRANSFER OF FROZEN-THAWED IVF EMBRYOS ON PREGNANCY IN REPEAT-BREEDER INSEMINATED OR NON-INSEMINATED HOLSTEIN CATTLE
}

\author{
O. Dochi ${ }^{\mathrm{A}}$, M. Tanisawa ${ }^{\mathrm{B}}, S$. Goda $^{\mathrm{A}}$, and H. Koyama ${ }^{\mathrm{A}}$ \\ ${ }^{\mathrm{A}}$ Department of Dairy Science, Rakuno Gakuen University, Ebetsu, Hokkaido, Japan; ${ }^{\mathrm{B}}$ Severity Confidence Breeding Service, \\ Betsukai, Hokkaido, Japan
}

Repeat-breeding is one of the important factors that affect dairy management. The objective of this study was to investigate the effect of transfer of frozen-thawed IVF embryos on pregnancy in repeat-breeder Holstein cattle. Cumulus-oocyte complexes (COCs) were collected by aspiration of $2-1-\mathrm{mm}$ follicles from ovaries obtained at a local abattoir. COCs were matured for $20 \mathrm{~h}$ in TCM-199 supplemented with $5 \%$ calf serum (CS) and $0.02 \mathrm{mg} / \mathrm{mL}$ of FSH at $38.5^{\circ} \mathrm{C}$ under a $5 \% \mathrm{CO}_{2}$ atmosphere in air. Matured oocytes were inseminated with spermatozoa of $5 \times 10^{6} / \mathrm{mL}$ in BO solution (Brackett and Oliphant 1975 Biol. Reprod. 12, 260-274) containing $10 \mathrm{mM}$ hypotaurine and 4 units/mL heparin. After $18 \mathrm{~h}$ of gamete co-culture, presumptive zygotes were cultured in CR1aa (Rosenkrans et al. 1991 Theriogenology 35,266 ) supplemented with $5 \% \mathrm{CS}$ for 8 days at $38.5^{\circ} \mathrm{C}$ under $5 \% \mathrm{CO}_{2}, 5 \% \mathrm{O}_{2}, 90 \% \mathrm{~N}_{2}$ atmosphere in air. After in vitro fertilization, Day 7 and Day 8 blastocysts were frozen in $1.5 \mathrm{M}$ ethylene glycol (EG) in Dulbecco's PBS (DPBS) supplemented with $0.1 \mathrm{M}$ sucrose and $20 \%$ CS. Embryos were transferred into a freezing medium, loaded into 0.25 -mL straws, and allowed to stand for $15-20 \mathrm{~min}$ for equilibration. The straws were then plunged into a $-7^{\circ} \mathrm{C}$ methanol bath of a programmable freezer for $1 \mathrm{~min}$, seeded at $-7^{\circ} \mathrm{C}$, maintained at $-7^{\circ} \mathrm{C}$ for $15 \mathrm{~min}$, cooled to $-30^{\circ} \mathrm{C}$ at the rate of $-0.3^{\circ} \mathrm{C} / \mathrm{min}$, and then plunged into liquid nitrogen. Recipient animals (43 heifers, 131 cows) included those that did not conceive after being artificially inseminated (AI) 3 to 15 times. The frozen-thawed IVF embryos were directly transferred to the recipient animals 7 days after estrus or AI. Pregnancy rates were analyzed by chi-square test. The results are presented in Table 1. There were no significant differences in the pregnancy rates between treatments. However, a slightly higher pregnancy rate was achieved by embryo transfer after AI. These results suggest that embryo transfer may increase the pregnancy rate in repeat-breeder Holstein cattle.

Table 1. Pregnancy rates after transfer of IVF frozen-thawed embryos in repeat-breeder Holstein cattle

\begin{tabular}{llcc}
\hline Parity & Treatment & No. recipients & No. pregnancies (\%) \\
\hline Heifers & Without AI & 33 & $13(39.4)$ \\
& With AI & 10 & $6(60.0)$ \\
Cows & Without AI & 62 & $14(22.6)$ \\
& With AI & 69 & $27(39.1)$ \\
\hline
\end{tabular}

\section{PREGNANCY RATES IN HEAT-STRESSED DAIRY CATTLE RECEIVING ONE OR TWO IN VITRO-PRODUCED EMBRYOS IN A TIMED EMBRYO TRANSFER PROGRAM}

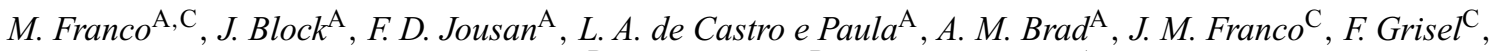 \\ R. L. Monson ${ }^{\mathrm{B}}$, J. J. Rutledge ${ }^{\mathrm{B}}$, and P. J. Hansen ${ }^{\mathrm{A}}$ \\ ${ }^{\mathrm{A}}$ Department of Animal Sciences, University of Florida, Gainesville, FL 32611, USA; ${ }^{\mathrm{B}}$ Department of Animal Sciences, \\ University of Wisconsin, Madison, WI 53706, USA; ${ }^{\mathrm{C}}$ Embogen, Santa Cruz, Bolivia
}

The objective was to determine whether transfer of two embryos would increase pregnancy rates in heat-stressed dairy recipients receiving an in vitro-produced embryo transferred into the uterine horn ipsilateral to the corpus luteum $(\mathrm{CL})$. Such a treatment would increase the likelihood that the cow receives at least one embryo competent for sustained development. In addition, transfer of two embryos into the ipsilateral uterine horn is likely to increase the amounts of interferon-tau and other embryonic-signaling molecules in the uterus. A total of 32 virgin crossbreed heifers and 26 
lactating crossbreed cows were used as timed embryo transfer recipients after being subjected to an ovulation synchronization protocol as follows: GnRH $(100 \mu \mathrm{g})$ and insertion of previously used progesterone-containing CIDR on Day -10 , prostaglandin $\mathrm{F}_{2 \alpha}$ and CIDR removal on Day -3 , and GnRH $(100 \mu \mathrm{g})$ on Day 0 (day of anticipated ovulation). All recipients had a palpable CL on Day 6 and were randomly selected to receive one ( $n=31$ recipients) or two $(n=27)$ embryos on Day 7. At Day 64, the pregnancy rate tended to be higher $(P=0.07)$ for cows than for heifers. While not significant, heifers that received two embryos tended to have lower pregnancy rates than those that received a single embryo ( $20 \%$ vs. $41 \%$ ); there was no difference in pregnancy rate in cows (50\% for two embryos vs. 57\% for one embryo). Pregnancy losses between Day 64 and Day 127 occurred in one group only - cows receiving two embryos. In that group, pregnancy rate was $50 \%$ at Day 64 but $17 \%$ at Day 127 . Overall, there was no difference in pregnancy rates at day 127 between cows and heifers, but recipients that received two embryos (17\% for cows and $20 \%$ for heifers) had lower pregnancy rates $(P<0.03)$ than recipients that received one embryo $(57 \%$ for cows and $41 \%$ for heifers $)$. Only one animal, a cow, had twin fetuses at day 127. In conclusion, unilateral transfer of two embryos failed to improve pregnancy rates of dairy cattle exposed to heat stress. The fact that fetal loss occurred sooner for heifers than for cows points out the importance of uterine capacity as a limiting factor for maintenance of fetal development of two conceptuses. The suitability of timed embryo transfer was evident from the high pregnancy rates achieved with crossbreed females that received a single embryo.

This work was supported by BARD Grant No. US-1551-14, USDA TSTAR Grant No. 2004-14135-14715, Grant No. 2001-12101-11318 from the USDA-IFAFS Program, and CAPES Grant No. 134202-1).

\title{
190 EFFECT OF THE PROPERTIES OF CERVICAL MUCUS ON PREGNANCY RATES IN HOLSTEIN HEIFERS AND COWS AFTER EMBRYO TRANSFER
}

\author{
T. Maekawa, S. Morita, O. Douchi, and H. Koyama
}

Department of Dairy Science, Rakuno Gakuenn University, Ebetsu, Hokkaido, Japan

Selection of animals as recipients of embryo transfer is an important procedure of embryo transfer on farms. Most animals are evaluated for their acceptability as recipients based on the quality of their corpus leteum (CL). However, since rectal palpation is a subjective evaluation method, a more objective method of assessing the suitability of the recipient is required. Cervical mucus may be able to be used to evaluate the condition of the uterus indirectly. The purpose of this study was to investigate the relationship between the properties of cervical mucus and pregnancy rates after embryo transfer in Holstein heifers and cows. Cervical mucus was collected using a swab off the ostium uteri externum and was stained with $5 \%$ Giemsa's solution for 20 min one day before embryo transfer. The stained cervical mucus were classified based on the type of staining pattern (Kitamura et al. 2003 Theriogenology 59,307) into five groups: filiaceous (Type 1), taenia (Type 2), claustral (Type 3), nubecula (Type 4), or aqueous (Type 5). Proportions of the types of cervical mucus and pregnancy rates were analyzed by chi-square test. In Experiment 1, 113 heifers and 266 cows were examined for cervical mucus type. No significant difference was observed in the proportions of the types of cervical mucus between heifers and cows (heifers: $35.4 \%, 18.6 \%, 16.8 \%, 25.7 \%$, and $3.5 \%$; cows: $24.4 \%, 14.3 \%, 20.3 \%, 30.8 \%$, and $10.2 \%$ for Types $1 \sim 5$, respectively) In Experiment 2, either a fresh or frozen-thawed embryo was implanted in vivo in 84 heifers and 163 cows 7 days after estrus. The heifers and cows were judged to have normal sized CLs (normal, $17 \mathrm{~mm}$ or more) and have no vaginal abnormalities such as cervical mucus contaminated with pus and urovagina as per vaginal examination. The proportions of acceptable Type 5 recipients was lower than that of Type $1(P<0.05)$. The pregnancy rates were $47.6 \%$ for heifers and $45.4 \%$ for cows (Table 1$)$. The pregnancy rates of Types $1-3(53.5 \%)$ were significantly higher than for Types 4 and $5(29.9 \%)$ in the cows $(P<0.05)$. Although there was no statistically significant difference, the same tendency was observed in the heifers. Pregnancy was unsuccessful in Type 5 recipients, both heifers and cows. The total pregnancy rates of Types 1-3 were significantly higher than for Types 4 and $5(53.5 \%$ vs. $29.9 \%, P<0.001)$. These results suggest that cervical mucus type can serve as an objective selection criterion for embryo recipients. Further, embryo transfer should be avoided in Type 5 recipients.

Table 1. Cervical mucus type and pregnancy rates $(\%)$ in dairy cattle

\begin{tabular}{llllllc}
\hline Parity & \multicolumn{1}{c}{ Filiaceous } & \multicolumn{1}{c}{ Taenia } & \multicolumn{1}{c}{ Claustral } & \multicolumn{1}{c}{ Nubecula } & \multicolumn{1}{c}{ Aqueous } & \multicolumn{1}{c}{ Total } \\
\hline Heifers & $48.3(14 / 29)$ & $58.8(10 / 17)$ & $57.1(8 / 14)$ & $36.4(8 / 22)$ & $0.0(0 / 2)$ & $47.6(40 / 84)$ \\
Cows & $47.7^{\mathrm{a}}(21 / 44)$ & $59.3^{\mathrm{a}}(16 / 27)$ & $56.4^{\mathrm{a}}(22 / 39)$ & $35.7^{\mathrm{bc}}(15 / 42)$ & $0.0^{\mathrm{c}}(0 / 11)$ & $45.4(74 / 163)$ \\
Total & $47.9(35 / 73)$ & $59.1(26 / 44)$ & $56.6(30 / 53)$ & $35.9(23 / 64)$ & $0.0(0 / 13)$ & $46.2(114 / 247)$
\end{tabular}

${ }^{\mathrm{a}-\mathrm{c}}$ Means with different superscripts are significantly different $(P<0.05)$.

\section{INFLUENCE OF THE DIFFERENT TIME COMPONENTS BETWEEN FLUSHING AND TRANSFER ON PREGNANCY RATES OF FROZEN CATTLE EMBRYOS}

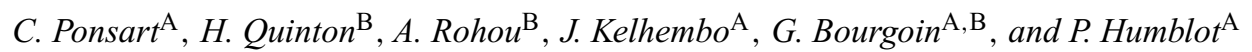 \\ ${ }^{A}$ UNCEIA, R\&D, Maisons-Alfort, 94704, France; ${ }^{\mathrm{B}}$ Embryotop, URCEO, BP 1978, 35019 Rennes cedex, France
}

Previous studies have shown that the time between flushing and freezing of bovine embryos can influence pregnancy rates (PRs) following embryo transfer (ET). The aim of this study was to determine which time components can influence ET results. Time components between flushing of a superovulated donor and freezing of the collected embryos were investigated under field conditions. Embryos were frozen in $1.5 \mathrm{M}$ ethylene glycol 
(EG) for direct transfer. During January 2003, ET technicians (EmbryoTop, Rennes cedex, France) recorded systematically times corresponding to each step comprising the time spent in vitro (TIV) from 153 recovery sessions (RS) with freezing: end of flushing, beginning and end of search of embryos, start of equilibration in EG, beginning and end of straw loading, introduction to $-7^{\circ} \mathrm{C}$ in the freezer, and seeding. Numbers of donor cows and ET technicians doing the freezing $(n=5)$ were noted for each RS. Embryo (stage, quality) and recipient (breed, parity) characteristics were also noted. A total of 548 frozen embryos were transferred and PRs were assessed. Variability of time components was investigated (Bourgoin et al. 2004 Reprod. Fertil. Dev. 16, 207). The influence of time components and other variation factors was tested on PRs $(t$-tests and chi-square analysis). The TIV averaged $210 \pm 80 \mathrm{~min}$ and did not influence PR $(\leq 4 \mathrm{~h}=51.9 \%(n=393)$ vs. $>4 \mathrm{~h}=55.5 \%(n=155) ; P>0.05)$, as well as duration of flushing (32 $\pm 8 \mathrm{~min})$, interval between end of flushing and search $(31 \pm 27 \mathrm{~min})$, duration of search $(45 \pm 25 \mathrm{~min})$ and interval between end of search and beginning of freezing $(101 \pm 63 \mathrm{~min})$. Only significant factors were kept for further analysis. The effects of recipient parity, number of donor cows per RS, and interval between introduction of straw to $-7^{\circ} \mathrm{C}$, and seeding were tested in a multivariate logistic model. PR varied strongly with parity of recipient $(+25 \%$ in heifers vs. cows; $P=0.001)$. PRs were higher when the interval between straw introduction in the freezer and seeding lasted at least $5 \min (2-4 \mathrm{~min}=48.0 \%(n=254)$ vs. $5-8 \mathrm{~min}=57.1 \%(n=294) ; P=0.009)$. Time and operator effects were confounded. Overall PR results for the two technicians who used mostly $2-4 \mathrm{~min}$ intervals averaged $47 \%$ (operator values $=35.6,48.9$, and 54.5 ) whereas PRs were 54.9 and $60.5 \%$ for those waiting $5 \mathrm{~min}$ or more before inducing seeding $(n=2)$. PRs were higher when at least two donor cows were collected per RS ( 1 donor cow $=49 \%(n=259)$ vs. $\geq 2$ donor cows $=56.4 \%(n=289) ; P=0.003)$. This was not in agreement with previous observations in fresh embryos (Bourgoin et al. 2004). However, the number of donor cows strongly influenced the number of viable embryos per RS (1 donor cow $=11 \pm 5$ vs. $\geq 2$ donor cows $=18 \pm 8.5 ; P<0.05$ ) and could permit the choice of more embryos to be frozen. These results show that good PR may be achieved with a delay of several hours between flushing and freezing, when heifers are used as recipients. Moreover, confirmed from higher numbers of operators, these data show that it is better to wait at least $5 \mathrm{~min}$ to achieve equilibration of the embryo before seeding.

\title{
192 THE USE OF FIXED-TIME TECHNIQUES AND eCG TO SYNCHRONIZE RECIPIENTS FOR FROZEN-THAWED BOVINE IVF EMBRYOS
}

\author{
R. Remillard $^{\mathrm{A}}$, M. Martínez ${ }^{\mathrm{B}}$, G. Bó ${ }^{\mathrm{C}}$, and R. Mapletoft ${ }^{\mathrm{B}}$ \\ ${ }^{A}$ IND Lifetech, Inc., Delta, British Columbia, V3M 6S7 Canada; \\ ${ }^{B}$ Department of Large Animal Clinical Sciences, University of Saskatchewan, Saskatoon, Saskatchewan, S7N 5B4 Canada; \\ ${ }^{\mathrm{C}}$ Instituto de Reproducción Animal Córdoba (IRAC), Córdoba, 5000 Argentina
}

Two experiments were designed to investigate the use of eCG in a fixed-time embryo transfer (FTET) protocol for Chinese Yellow crossbred recipients receiving in vitro-produced (IVP) bovine embryos. In Experiment 1, cows were observed for spontaneous estrus (Group 1) or following $500 \mu \mathrm{g}$ cloprostenol (PGF; Schering-Plough Animal Health, Montreal, Quebec, Canada; Group 2) with nonsurgical embryo transfer 7.5 or 8.0 days later. Cows in Groups 3, 4, and 5 were treated with a CIDR insert (Bioniche Animal Health, Beijing, China), 2 mg estradiol benzoate (EB), and $50 \mathrm{mg}$ progesterone (Sigma-Aldrich, Canada) i.m. on Day 0, PGF on Day 5, CIDR removal on Day 7, and $1 \mathrm{mg}$ EB i.m. $24 \mathrm{~h}$ later with nonsurgical transfer of Holstein IVP embryos 8.5 days later, without estrus detection. In addition, cows in Group 3 received no further treatment, whereas cows in Group 4 received 400 IU eCG i.m. (Pregnecol; Bioniche) on Day 5, and cows in Group 5 received eCG on Day 7. Holstein embryos were produced utilizing slaughterhouse ovaries and standard IVF procedures. Expanded blastocysts of quality grade 1 (IETS) were cryopreserved in $10 \%$ ethylene glycol and $20 \%$ fetal bovine serum using standard procedures. Straws were thawed in a $30^{\circ} \mathrm{C}$ water bath, and embryos were expelled directly into holding medium and evaluated prior to nonsurgical transfer (NT) ipsilateral to the corpus luteum (CL). Pregnancy was diagnosed ultrasonically $30 \mathrm{~d}$ later. Although pregnancy rates, based on numbers of recipients synchronized, numerically favored the administration of eCG on Day 5 , there were no significant differences $(P=0.40)$ among groups (Group 1: $15.4 \%, n=52$; Group 2: $20.0 \%, n=50$; Group 3: 19.2\%, $n=99$; Group 4: $28.1 \%, n=96$; Group 5: 21.3\%, $n=75$ ). In Experiment 2, Chinese Yellow crossbred cattle were synchronized with a CIDR insert plus estradiol and progesterone on Day 0, PGF on Day 5, CIDR removal on Day 7 and estradiol on Day 8, and were randomly assigned to received no further treatment (Group $1 ; n=400$ ) or an injection of $400 \mathrm{IU}$ eCG on Day 5 (Group $2 ; n=391$ ). Recipients with a detectable CL received a frozen-thawed IVP Holstein embryo by NT 8.5 days after the second injection of estradiol without estrus detection, as in the first experiment. Pregnancy diagnosis was done ultrasonically 30 days later. Although CL size, cow age, and embryo quality, prior to transfer, were recorded, no effects on pregnancy rates were demonstrated $(P=0.30)$. Pregnancy rates, based on recipients receiving embryos, did not differ $(P=0.5)$ between groups (Group 1 : $21.4 \%, n=154$; Group 2: $24.5 \%, n=290)$. Overall pregnancy rates (based on the total number of recipients synchronized) were significantly higher $(P<0.001)$ in Group $2(\mathrm{eCG} ; 18.2 \%)$ than in Group 1 (no eCG; 8.3\%), because of the significantly higher $(P<0.03)$ percentage of recipients used following treatment with eCG $(74.2 \%$ vs. $38.5 \%)$. Results indicate that the administration of eCG on Day 5 of an 8-d synchronization protocol for FTET of frozen-thawed bovine IVP embryos will improve pregnancy rates in beef recipients, especially those of marginal quality.

\section{EVALUATION OF A NOVEL ELECTRONIC ESTRUS DETECTION DEVICE IN RECIPIENTS SYNCHRONIZED FOR EMBRYO TRANSFER}

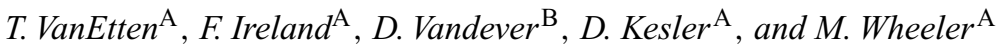 \\ ${ }^{A}$ Department of Animal Sciences, University of Illinois at Urbana-Champaign, Urbana, IL 61801, USA; \\ ${ }^{\mathrm{B}}$ Cedarburg Veterinary Clinic, Cedarburg, WI 53012, USA
}

The ability to detect estrus in cattle is an important aspect for both successful artificial insemination (AI) and successful embryo transfer (ET). The use of estrus detection devices has allowed producers to more precisely determine when to breed their cattle. There have been many types of devices including chin-ball marking harnesses, dye patches, and even electronic devices that monitor the animal's activity. With the growing popularity in 
timed AI and ET, these devices have again become of great interest. The latest tool for estrus detection is the multi-use TattleTale ${ }^{\mathrm{TM}}$ Heat Detector (Microdyne Co., LLC., St. Joseph, MO, USA). The TattleTale ${ }^{\mathrm{TM}}$ is an electronic device that is placed in a patch and affixed to the tail head. It is activated by a 3-s mount with subsequent mounts recorded per $\mathrm{h}$ through $12 \mathrm{~h}$. Twelve hours after activation the device flashes indicating that the animal is ready for breeding. Such devices may allow breeders to determine exactly when estrus began as well as identify the peak estrus period, resulting in more accurate determination of optimal time for AI or ET. The objective of this study was to compare the accuracy of estrus detection devices when analyzing detection records and pregnancy rates. Cows from the Dixon Springs Agricultural Center (Simpson, IL, USA) were randomly separated into two groups based on estrus detection devices, one group receiving Kamar ${ }^{\circledR}$ patches (Kamor, Inc., Steamboat Springs, CO, USA) and the other receiving TattleTales. Both groups were synchronized using the OvSynch protocol (Syntex, Buenos Aires, Argentina). Cows were checked twice each day for four consecutive days, with a fifth day occurring a week later when the embryos were transferred. Estrus results were analyzed using a PROC UNIVARITE and GLM analyses with differences between treatment means determined by the LSD method of SAS (SAS Institute, Inc., Cary, NC, USA). The TattleTale detected a higher percentage $(P<0.05)$ of the cows in estrus $(92$ vs. $70 \%)$ than the Kamar ${ }^{\circledR}$ patch. Further, the TattleTale detected more asynchronous recipients (in estrus $>48 \mathrm{~h}$ before expected) and synchronous recipients (in estrus $<24 \mathrm{~h}$ than expected), although not significant with this sample size. In the present study, the TattleTale was more accurate in detecting estrus (i.e. fewer pregnancies were observed without estrus detected). Using the TattleTale information, a breeder would be able detect more animals in estrus and determine the most accurate time to breed or transfer embryos. Practically, the use of estrus detection devices in combination with timed AI and ET could allow for the exclusion of animals not in estrus in the optimal time windows for AI or ET, thus saving valuable semen and embryos that would be otherwise wasted on asynchronous females. The advantages of this device to producers include improved pregnancy rates based on timed breeding, lower herd management costs due to reduced quantities of wasted semen or embryos and fewer cows being resynchronized. This device also records any mounting activity missed by visual observation alone.

This work was supported by USDA RRF W-1171.

\title{
Embryonic Stem Cells
}

\section{ESTABLISHMENT OF GOAT EMBRYONIC STEM CELL-LIKE LINES DERIVED FROM IN VIVO-PRODUCED BLASTOCYST-STAGE EMBRYOS}

\author{
E. Behboodi, I. Begin, K. Rao, N. Neveu, J. Pierson, H. Baldassarre, and C. Karatzas \\ PharmAthene, Vaudreuil-Dorion, Quebec, Canada
}

Pluripotent embryonic stem cells (ESC) derived from the inner cell mass (ICM) of mammalian blastocysts provide AN unlimited number of cells that can be used in gene targeting and be of great value to agriculture and medicine. Embryonic stem cells with capacity for germ line transmission have been verified only in mouse despite many efforts to derive ESC from other mammalian species. The methods for the derivation, propagation and differentiation of ESC from domestic animals have not been fully established. The objective of this study was the generation and initial characterization of goat embryonic stem cells (GESC) derived from in vivo-produced blastocyst-stage embryos. Goat compact morulae and blastocysts were collected from superovulated adult Saanen crossbreed donors 7 days after insemination with a fertile male. Embryos were collected by uterine flushing using a $12 \mathrm{Fr}$. Foley catheter by means of a laparoscopically assisted mid-ventral laparotomy under general anesthesia. Twenty eight in vivo-derived blastosyst-stage embryos were cultured on a goat fetal fibroblast feeder layer (inactivated by Mitomycin C) in a medium of DMEM containing $0.1 \mathrm{~mm} \beta$-mercaptoethanol, $0.1 \mathrm{~mm}$ MEM nonessential amino acids, $200 \mathrm{~mm}$ L-glutamine, and $10 \%$ FCS. Following three days in culture 25 of 28 embryos hatched. Ten embryos attached to the feeder layer, nine degenerated, and nine embryos were floating in the medium and expanding in size. After 5-7 days in culture four of the tehn attached embryos appeared with a prominent an ICM outgrowth and 3 of the nine floating embryos formed structures resembling ICM disc surrounded by trophectoderm cells. The ICM and the embryonic discs were isolated mechanically and cultured on goat feeder cells in DMEM medium containing $10 \mathrm{ng} / \mathrm{mL}$ horse leukemia inhibitory factor (hLIF) and $10 \%$ FCS. The ICM and embryonic disc outgrew into colonies on Day 4 post-culture. Compact colonies of cells from these outgrowths were isolated mechanically and passed onto fresh goat feeder cells every 4-5 days with the addition of $10 \mathrm{ng} / \mathrm{mL}$ hLIF to the culture media. Established colonies at passage 6 were tested for immunoreactivity against alkaline phosphatase (AP) and Oct-1 using standard protocols. The result was the estabilshment of embryonic stem cell-like colonies (57\%) from both ICM and embryonic discs cultured on goat fetal fibroblast feeder cells. Colonies forming from these outgrowths (50\%) of either ICM or embryonic disc stained positive for both AP and Oct-4. Embryoid bodies formed from colonies of either ICM or embryonic disc in suspension (DMEM containing 10\% FCS with no hLIF). Two cell lines (one from ICM and one from embryonic disc) have been maintained so far through passage 8 and have been cryopreserved. These GESC-like lines will be used in further characterization and ultimately in transgenic animal production studies.

\section{DEVELOPMENT OF INNER CELL MASS AND FORMATION OF EMBRYOID BODIES ON A GELATIN-COATED DISH AND ON THE FEEDER LAYER IN BUFFALO (BUBALUS BUBALIS)}

\author{
M. S. Chauhan, V. Verma, R. S. Manik, P. Palta, S. K. Singla, and S. L. Goswami \\ Animal Biotechnology Center, National Dairy Research Institute, Karnal, Haryana, India
}

Isolation and culture of embryo-derived cell lines have been reported in many mammals, however, there is not even a single report toward initiation of such work in buffalo (Bubalus bubalis). Therefore the present study was carried out to isolate the inner cell mass from in vitro-produced buffalo 
blastocysts and to grow this inner cell mass for formation of embryoid bodies in a gelatin-coated dish and on an homologous fetal fibroblast feeder layer. Immature buffalo oocytes were isolated from the slaughterhouse ovaries. In vitro production of blastocysts was carried as reported by Chauhan et al. (1999 J. Dairy Science 82, 918-926). A total of 26 buffalo blastocysts were produced in vitro. These blastocysts were transferred into a 100-mL drop of Dulbecco's modified Eagle's medium (DMEM) supplemented with 20\% fetal bovine serum (FBS) for further culturing. Thirteen blastocysts hatched on the next day of culture. The hatched mass was separated, suspended in Dulbecco's phosphate buffer saline (DPBS, without Ca ${ }^{++}$and $\mathrm{Mg}^{++}$) containing 5\% FBS $+0.25 \%$ trypsin and examined under the zoom stereomicroscope until disappearance of the trophectoderm cells. The remaining cells of seven blastocysts were cultured in DMEM supplemented with $20 \%$ FBS on $0.2 \%$ gelatin coated culture dish (group 1), and cells of six blastocysts in DMEM medium supplemented with $20 \%$ FBS were cultured on a mitomycin-c-treated $(10 \mu \mathrm{L} / \mathrm{mL})$ feeder layer (group 2), for 14 days. The isolated cells attached to the bottom of the dish in both the groups, spreading was noticed on Day 5 of the culture in group 1 and on Day 3 of culture in group 2. The attached cells were trypsinized using DPBS with $0.25 \%$ trypsin, isolated, and subcultured further. Attachment and spreading was noticed only in group 2 subcultured cells. The cellular integrity was homogeneous and the plasma membrane was clearly visible in group 2, but not in group 1. Less than $10 \%$ of the attached cells formed embryoid bodies in group 1, where as more than $30 \%$ attached cells in group 2 formed embryoid bodies; the latter expressed alkaline phosphatase activity and were blue after staining. These results indicate that the culturing of the inner cell mass on an homologous fetal fibroblast feeder layer is a better choice for production of embryonic stem cells in buffaloes.

\title{
196 EFFECT OF MITROMYCIN-C ON VIABILITY AND CELL PROLIFERATION OF DOMESTIC CAT AND MOUSE EMBRYONIC FIBROBLASTS
}

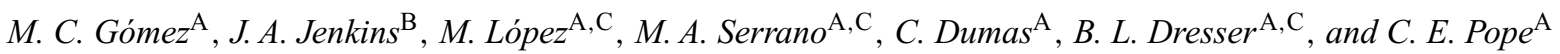 \\ ${ }^{A}$ Audubon Center for Research of Endangered Species, New Orleans, LA 70178, USA; ${ }^{B}$ National Wetland Research Center, U.S. Geological \\ Survey, Lafayette, LA 70506, USA; ${ }^{C}$ Department of Biological Sciences, University of New Orleans, New Orleans, LA 70148, USA
}

Nondomestic cat cloned embryos created by intergeneric nuclear transfer (ig-NT) have lower rate of blastocyst development than that observed in cloned embryos produced by inter-species NT. One promising technique for increasing the efficiency of ig-NT is to use embryonic stem cells (ESC) derived from the pluripotent inner cell mass (ICM) of pre-implantation blastocysts. Mouse embryonic fibroblasts (MEF) are commonly used as feeder cells to support the growth of human and mouse ESC, but they do not always maintain human ESC in an undifferentiated state and carry the risk of transmitting mouse retroviral virus and other pathogens. Thus, it is important to determine whether domestic cat embryonic fibroblasts (DCEF) are able to support cat ESC growth. Proliferation of MEF cells is inhibited before their use as feeder layers by exposure to gamma irradiation or Mitomycin-C. Therefore, in the present study, we determined what concentration of Mitomycin-C was required to inhibit proliferation of DCEF and MEF cells without affecting viability. Embryonic fibroblasts were generated from fetuses collected from a single pregnant domestic cat and mouse at 30 and 14 days of gestation, respectively. Tissue was minced and cultured in DMEM for 7 to 10 days before freezing and storage at $-196^{\circ} \mathrm{C}$. After thawed DCEF and MEF cells reached 80 to $100 \%$ confluence, proliferation was inhibited by exposure to 10, 30, or $40 \mu \mathrm{g}$ of Mitomycin-C for 2.5 or $5 \mathrm{~h}$. Cells were then washed and labeled with BrdU to measure DNA synthesis as a specific marker for replication. For cytotoxicity, cells were labeled with a dual fluorescent stain to evaluate membrane integrity and esterase activity. The amounts of DNA-labeled BrdU as well as calcein and ethidium homodimer-1 incorporated into the cells were quantified by flow cytometry (see Table 1). Proliferation of DCEF and MEF cells was affected similarly by both concentration and exposure time to Mitomycin-C, with the highest inhibition of DCEF cells occurring after treatment with $40 \mu \mathrm{g}$ for $5 \mathrm{~h}(92 \%$ not replicating). Cell viability was influenced by both concentration and exposure time to Mitomycin-C, with the highest survival of DCEF cells occurring after exposure to $30 \mu \mathrm{g}$ for $5 \mathrm{~h}(90 \%)$ and in MEF cells after exposure to $40 \mu \mathrm{g}$ for $2.5 \mathrm{~h}(88 \%$ viable). In summary, these results indicate that cell proliferation of DCEF can be inhibited by Mitomycin-C, but a higher dosage and longer exposure time should be considered for use compared to the dosage commonly employed for inhibiting cell proliferation of MEF. High concentrations of Mitomycin-C did not significantly increase cytotoxicity of cells in either species.

Table 1.

\begin{tabular}{lcccc}
\hline $\begin{array}{l}\text { Type of feeder } \\
\text { layer }\end{array}$ & $\begin{array}{c}\text { Exposure time } \\
(\mathrm{h})\end{array}$ & $\begin{array}{c}\text { Mitomycin-C } \\
(\mu \mathrm{g} / \mathrm{mL})\end{array}$ & $\begin{array}{c}\text { Cells expressing } \\
\text { BRDU }(\%)\end{array}$ & $\begin{array}{c}\text { Dead cells } \\
(\%)\end{array}$ \\
\hline DCEF & 2.5 & 10 & 25 & 19 \\
DCEF & 2.5 & 30 & 16 & 17 \\
DCEF & 2.5 & 40 & 19 & 13 \\
DCEF & 5 & 10 & 11 & 34 \\
DCEF & 5 & 30 & 22 & 10 \\
DCEF & 5 & 40 & 8 & 18 \\
MEF & 2.5 & 10 & 6 & 15 \\
MEF & 2.5 & 30 & 8 & 16 \\
MEF & 2.5 & 40 & 6 & 12 \\
MEF & 5 & 10 & 6 & 20 \\
MEF & 5 & 30 & 10 & 25 \\
MEF & 5 & 40 & 9 & 15 \\
\hline
\end{tabular}


197 PRODUCTION OF IN VITRO-AND IN VIVO-DERIVED CAT BLASTOCYSTS FOR THE ESTABLISHMENT OF FELINE EMBRYONIC STEM CELLS

\author{
J. Kehler ${ }^{\mathrm{A}, \mathrm{B}}$, M. Roelke-Parker ${ }^{\mathrm{B}}$, B. Pukazhenthi ${ }^{\mathrm{C}}$, W. Swanson ${ }^{\mathrm{D}}$, C. $_{\text {. }}$ Ware $^{\mathrm{E}}$, \\ I. Dobrinski ${ }^{\mathrm{A}}$, and $S . \mathrm{O}^{\prime}$ Brien $^{\mathrm{B}}$ \\ ${ }^{\mathrm{A}}$ Center For Animal Transgenesis and Germ Cell Research, School of Veterinary Medicine, University of Pennsylvania, \\ Kennett Square, PA 19348, USA; \\ ${ }^{B}$ National Cancer Institute Laboratory of Genomic Diversity, Frederick, MD 21702, USA; \\ ${ }^{\mathrm{C}}$ Department of Reproductive Sciences, Smithsonian Institution National Zoological Park, Washington, DC 20008, USA; \\ ${ }^{\mathrm{D}}$ Cincinnati Zoo and Botanical Garden, Cincinnati, OH 45220, USA; \\ ${ }^{\mathrm{E}}$ School of Medicine, University of Washington, Seattle, WA 98105, USA
}

Identification and characterization of spontaneously occurring genetic diseases in cats has permitted the development of valuable models for testing potential treatments of similar human diseases. With the near completion of the feline genome project, establishment of pluripotential feline embryonic stem (ES) cells would facilitate the targeting of specific genetic loci to produce new feline medical models. Two approaches were used to produce feline blastocysts in an attempt to establish feline ES cells in culture. Naive queens were superovulated with an intramuscular (i.m.) injection of $150 \mathrm{IU}$ of equine chorionic gonadotropin (eCG) followed by an i.m. injection of $100 \mathrm{IU}$ of human chorionic gonadotropin (hCG) $80 \mathrm{~h}$ later; follicles were aspirated laparoscopically 24-26 h later for subsequent in vitro fertilization (IVF). On average, 29 mature cumulus oocyte cell complexes (COCs) were recovered from each queen. IVF was performed in 50 microliter drops of complete Hams F-10 medium containing 30000 fresh, motile sperm. COCs were cultured overnight in $5 \%$ carbon dioxide at $38^{\circ} \mathrm{C}$, and residual adherent cumulus cells were removed 12 to $16 \mathrm{~h}$ later by trituration in $0.1 \%$ hyaluronidase. Embryos were cultured in fresh drops of Hams F-10, and on average $25 \%$ developed to the early blastocyst stage after 7 days. Alternatively, estrus was induced in queens with a single i.m. injection of $100 \mathrm{IU}$ of eCG, and then $72 \mathrm{~h}$ later queens were permitted six supervised matings with a fertile tom over the next two days. Queens underwent ovariohysterectomy 7 days after their first copulation, and compacted morulae and early blastocysts were flushed from the oviducts and uterine horns. On average, eight embryos were recovered from the reproductive tract of each queen. Both in vivo- and in vitro-matured blastocysts were subsequently cultured in standard mouse ES cell medium on inactivated mouse embryonic fibroblasts. When they failed to hatch in culture after 3 days, a $0.5 \%$ pronase solution was used to dissolve the zonae pellucidae under microscopic visualization. Denuded expanded blastocysts adhered to the heterotypic feeder layer and primary inner cell mass (ICM) outgrowths formed within 4 days. Outgrowths were mechanically disaggregated into small clusters of 15 to 20 cells and re-plated on fresh feeders. These colonies grew slowly and were transferred after one week onto new feeder layers. The addition of murine or human recombinant leukemia inhibitory factor had no effect on the survival and proliferation of primary outgrowths or subsequent colonies. After 3 weeks, all colonies derived from both in vivoand in vitro-matured blastocysts had either differentiated or died. Additional experiments are ongoing to test the effects of homotypic feeder layers and alternative growth factors on promoting the establishment and survival of feline ES cell lines. Ultimately, germline transmission of any putative feline ES cell lines will need to be demonstrated in vivo for their utility in gene targeting experiments to be realized.

\title{
198 REPROGRAMMING FIBROBLAST CELL CULTURES WITH EMBRYONIC STEM-CELL EXTRACTS
}

\author{
J. Kobolak ${ }^{\mathrm{A}}$, H. Rozsar ${ }^{\mathrm{A}}$, E. Fellinger ${ }^{\mathrm{B}}$, and A. Dinnyes ${ }^{\mathrm{A}, \mathrm{C}}$ \\ ${ }^{\mathrm{A}}$ Micromanipulation and Genetic Reprogramming Group, Agricultural Biotechnology Center, Godollo, Hungary; \\ ${ }^{B}$ Department of Anatomy, Cell- and Developmental Biology, Eotvos Lorand University, Budapest, Hungary; \\ ${ }^{\mathrm{C}}$ Research Group for Applied Animal Genetics and Biotechnology, HAS and Szent Istvan University, Godollo, Hungary
}

Reprogramming differentiated cells could be a effective method to dedifferentiate and transdifferentiate cells and thereby to replace or treat tissues. The goal of our study was to examine the effectiveness of embryonic stem (ES) cell extract on reprogramming fibroblast cell cultures and to use these cells as nucleus donors in subsequent cloning experiments. Based on the method of Collas and Hakelien (2003 Trends Biotechnol. 21, 354-361), cell extracts were produced from ES cells after homogenization and sucrose gradient centrifugation. The ES cells were suspended in one volume of $0.3 \mathrm{M}$ sucrose then one volume of $2 \mathrm{M}$ sucrose was added, and cells were homogenized. Finally 5.6 volumes of deionized $\mathrm{H}_{2} \mathrm{O}$ were added to the suspension, and the nucleus compartments were separated with centrifugation ( $2000 \mathrm{rpm}, 10 \mathrm{~min}$; Janetzki K24 angle rotor). The supernatant was separated (cytosol fragment), the precipitate was rehomogenized in the same way (nuclei fraction), and both fractions were centrifuged ( $8000 \mathrm{rpm}$, $1 \mathrm{~h}$, Janetzki K24 angle-rotor). The supernatants were ultracentrifuged ( $40000 \mathrm{rpm}, 30 \mathrm{~min}$; Beckman 40 angle rotor) and used immediately or stored at $-80^{\circ} \mathrm{C}$. Fibroblast cells were cultured and treated in 24-well plates. Cell extracts were administered to culture media [DMEM $+10 \%$ fetal bovine serum (FBS)] directly. Cell numbers were calculated after MTT-assay (Roche, Budapors, Hungary). Cell divisions were monitored by BrDU-tests (Roche) and RT-PCRs (Cdk2 and p21; Invitrogen, Budapest, Hungary). Results were statistically analyzed with Student's $t$-test. In our experiments the crude cell fractions killed $98 \%(P<0.005)$ of cells after 1 -h treatment, thus the administration of protease inhibitors was necessary. The supplemented extracts were tested as to dosage and treatment time: 4-h treatment $(P<0.05)$ and $10^{5}$ or $10^{6}$ cells $/ \mathrm{mL}(P<0.05 ;$ cell number in $1 \mathrm{~mL}$ of starting material) were the most appropriate combination. When $5 \mathrm{mM} \mathrm{CaCl}_{2}$ was administered, the cell cycle-arresting effect was increased using either the nuclear $(+24 \%, P<0.05)$ or the cytosol extract $(+30 \%, P<0.05)$, but the effect of the nuclear extract was more intensive. When steptolysin $\mathrm{O}(0.1 \mu \mathrm{g}, 5 \mathrm{~min})$ treatment of fibroblast was used prior to addition of ES cell extracts, 1-h treatment and $10^{4}$ cells $/ \mathrm{mL}$ were the most effective combination, as higher concentration or longer treatment was toxic. Further experiments are needed to test the effect of treatment on nuclear transfer procedures. 


\title{
199 DIFFERENTIATION OF HIGHLY ENRICHED OLIGODENDROCYTE PRECURSORS AND MATURE OLIGODENDROCYTES FROM RHESUS MONKEY EMBRYONIC STEM CELLS
}

\author{
T. $L i^{\mathrm{A}, \mathrm{B}}, Y . X i e^{\mathrm{A}, \mathrm{B}}$, and $W . J i^{\mathrm{A}, \mathrm{B}}$ \\ ${ }^{A}$ Department of Reproduction and Development, Kunming Institute of Zoology, Chinese Academy of Sciences, \\ Kunming, Yunnan, 650223, China; \\ ${ }^{\mathrm{B}}$ Kunming Primate Research Center, Chinese Academy of Sciences, Kunming, Yunnan, 650223, China
}

Generating homologous oligodendrocytes are required for studying the molecular mechanisms of oligodendrogliogenesis and for providing donor cells for transplantation therapies. Previous studies have shown that embryonic stem (ES) cells can be induced to generate neural stem cells with many kinds of culture systems; however, few or no oligodendrocytes were obtained from these culture systems. Here we present a simple method containing five steps for obtaining highly enriched oligodendrocyte precursors $(75 \pm 6.8 \%)$ and mature oligodendrocytes $(81 \pm 8.6 \%)$ from rhesus monkey embryonic stem (rES) cells. We expanded rES cells on a feeder layer of irradiated MESF (ear skin fibroblasts from a one-week-old rhesus monkey), formed embryoid bodies (EBs), promoted Day 9 (3 days in hanging drop and 6 days in suspension) differentiation into highly enriched $(90.2 \pm 6.1 \%)$ neural progenitors (NPs) with hepatocyte growth factor (HGF) and G5 supplement [containing $5 \mathrm{ng} / \mathrm{mL}(\mathrm{bFGF}) \mathrm{and} 10 \mathrm{ng} / \mathrm{mL}$ epidermal growth factor (EGF)], purified NPs with $0.0625 \%$ trypsin in $0.04 \%$ EDTA ( $98 \%$ of cells were nestin-positive), amplified those progenitors in HGF and G5 media for two months, and then induced oligodendrocyte precursors differentiation in the absence of G5, but in the presence of $20 \mathrm{ng} / \mathrm{mL}$ HGF for 2 days. To obtain terminal oligodendrocytes, neurospheres cultured for 2 months were plated on laminin-coated plates for 3 weeks in the presence of HGF. The results showed that differentiated cells expressed myelin basic protein (MBP) and had typical mature oligodendrocyte morphology. Our studies also revealed that HGF significantly increased the NP proliferation speed $(P<0.05)$ by both decreasing cell apoptosis rate $(P<0.05)$ and shortening cell cycle time $(P<0.05)$ in the presence of G5. Additionally, HGF promoted oligodendrocyte maturation by increasing the length and number of branches and the expression of MBP. To test whether the original HGF had similar functions for oligodendrocyte specification, a series of experiments were evaluated by adding HGF or G5 to differentiation or expansion media at different differentiation stages. The results demonstrated that the ability of HGF responsiveness to initiate oligodendrocyte differentiation was regulated by G5 and by HGF alone without G5-induced rES cell differentiation into neurons. Further studies showed that the crucial time point of G5 action was from EBs to NPs; the early addition of HGF to EBs in the presence of G5 increased oligodendrocyte differentiation rate, but was not necessary, and the treatment during the first 2 days was enough to produce a similar effect; and HGF was required for terminal oligodendrocyte differentiation from NPs. Taken together, these results showed that HGF and G5 cooperatively promote rES cell differentiation into highly enriched oligodendrocyte precursors and mature oligodendrocytes. These observations set the method for obtaining highly enriched oligodendrocytes from ES cells in the nonhuman primate for clinical application and provide a platform to probe the molecular mechanisms that control oligodendrocyte differentiation.

\section{TRANSPLANTATION AND IN VIVO DIFFERENTIATION OF ADIPOSE-DERIVED STEM CELLS IN SWINE}

\author{
A. S. Lima ${ }^{\mathrm{A}}$, S. A. Malusky ${ }^{\mathrm{A}}$, M. R. B. Mello $\mathrm{A}$, S. J. Lane ${ }^{\mathrm{A}}$, J. R. Rivera ${ }^{\mathrm{A}}$, and M. B. Wheeler ${ }^{\mathrm{A}, \mathrm{B}}$ \\ ${ }^{A}$ Department of Animal Sciences, University of Illinois; \\ ${ }^{\mathrm{B}}$ Department of Bioengineering, University of Illinois, Urbana, IL 61801, USA
}

\begin{abstract}
A primary concern in stem cell biology is that observations made in vitro may be an artifact of the in vitro culture environment. In vitro derived stem cells can be implanted into the environment from which they are derived so that their response to physiological conditions may be observed. Several important cellular characteristics need to be examined following the cell's reintroduction to the in vivo environment, including the potential for differentiation, proliferative ability, and life span. Studying implanted stem cells will assist in determining the potential for stem cell use in clinical therapies and provide further understanding of the role adult stem cells have in the adult body. Currently, the scientific literature is lacking a detailed description of the cellular response of adipose-derived stem cells (ADSCs) reintroduced to their exact tissue of origin. Thus, the aim of this study was to evaluate porcine ADSC growth in vivo and to analyze cell differentiation in vivo following injection of undifferentiated ADSCs into subcutaneous fat. Subcutaneous adipose tissue was isolated from the back fat of male pigs ( 11 months of age) and digested with $0.075 \%$ collagenase at $37^{\circ} \mathrm{C}$ for $90 \mathrm{~min}$. The digested tissue was centrifuged at $200 \mathrm{~g}$ for $10 \mathrm{~min}$ to obtain a cell pellet. The pellet was re-suspended with DMEM and the ADSCs were plated onto $75 \mathrm{~cm}^{2}$ flasks (5000-10 000 cells per $\mathrm{cm}^{2}$ ) and cultured in DMEM supplemented with 10\% fetal bovine serum (FBS) and $1 \%$ gentamicin. Passage 3 ADSCs were labeled with fluorescent dye (PKH26; Sigma, St. Louis, MO, USA) and sorted by flow cytometry. After sorting, positive cells were washed and re-suspended in culture medium. For transplantation, $100 \mu \mathrm{L}$ of cell suspension in DMEM containing one of four cell concentrations ( 0 (control); $30000 ; 300000$; and 900000 cells) were placed in a 1-mL syringe and injected into the subcutaneous back fat of recipient pigs $(n=2)$. Each pig had previously been tattooed with $121^{\prime \prime} \times 1^{\prime \prime}$ squares to mark injection sites. The treatments were replicated three times within each animal. Two and three weeks after transplantation, animals were euthanized, the back fat containing the transplantation site was harvested, and the cells were disaggregated as described above. The buoyant adipocytes and pelleted ADSCs cells were then analyzed by flow cytometry. The results indicated that there were dose- and time-dependent increases in labeled ADSCs and labeled adipocytes in the fat samples with increasing cell number (from 0 to 300000 cells). There was, however, a decrease in labeled ADSCs at the 900000 -cell dose, which is likely due to excess cells being transplanted or an immune reaction. Both of these aspects are currently being evaluated. In conclusion, undifferentiated ADSCs from swine can be isolated from and returned to the subcutaneous adipose layer and differentiate into mature adipocytes.
\end{abstract}

This work was supported by the Council for Food and Agricultural Research (C-FAR) Sentinel Program, University of Illinois. 


\title{
201 CHARACTERIZATION OF CANINE EMBRYONIC GERM CELLS: AN EXPERIMENTAL MODEL FOR CELL THERAPY
}

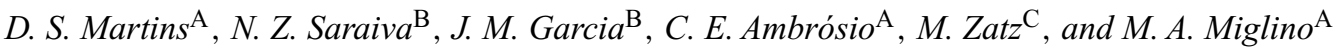 \\ ${ }^{A}$ Surgery Department, FMVZ, University of São Paulo, São Paulo, Brazil; \\ ${ }^{B}$ Reproduction Department, UNESP, Paulista State University, Jaboticabal, São Paulo, Brazil; \\ ${ }^{\mathrm{C}}$ Human Genome Research Center, IB, University of São Paulo, São Paulo, Brazil
}

\begin{abstract}
Dogs represent excellent models to test different approaches before use for human therapy. Studies with animal models have suggested that the transplant of stem cells may have success in the treatment of degenerative diseases such as Parkinson's disease, diabetes, Duchenne muscular dystrophy (DMD), and acquired lesions. Embryonic stem cells are pluripotent and therefore have the potential to form all tissues. Our research aims to contribute to the treatment of DMD through the isolation and identification of embryonic germ cells and the development of the methodology of cellular differentiation for future transplantation into dystrophic dogs. Mongrel female dogs were ovariehysterectomized between 25 and 30 days of pregnancy. For recovery of embryos, the excised uterine horns were flushed with heparinized PBS. Samples collected from somites near the mesonephros area of four embryos recovered at 22 to 24 days of pregnancy and designated as A1 through A4 were dissociated and placed in culture. Isolated embryonic cells were allowed to plate onto monolayers of canine fibroblast cells. Flow cytometry was used to identify CD34+ markers. Isolated compact colonies of embryonic germ cells were seen growing around tissue fragments at 7 days of culture and remained in the undifferentiated stage until approximately 21 days in culture. At 14 days of explant, cell colonies were analyzed by flow cytometry. Cells from the A2 embryos contained the highest number of CD34+ cells, whereas no cells from A4 embryos showed specificity for the marker. A small proportion $(2.12 \%)$ of cells from embryos A1 and A3 showed specificity for the CD34 marker. A quantity of A2 embryo cells that had maintained stem cell characteristics were frozen for future studies. Our results suggest that although spontaneous differentiation occurred, a small population of cells maintain the characteristics of stem cells. We are currently trying to improve the methodology to maintain cells undifferentiated for longer periods and to better control the specific differentiation process.
\end{abstract}

\section{IN VITRO DIFFERENTIATION OF ADULT PORCINE ADIPOSE-DERIVED STEM CELLS AFTER LABELING WITH PKH26 AND FLOW CYTOMETRY}

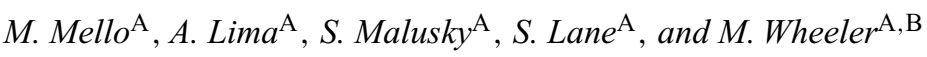 \\ ${ }^{A}$ Department of Animal Sciences, University of Illinois, Urbana, Illinois, USA; \\ ${ }^{B}$ Department of Bioengineering, University of Illinois, Urbana, Illinois, USA
}

\begin{abstract}
The purpose of this study was to investigate the possible effects of the fluorescent dye PKH26 and flow cytometry on adult porcine adipose-derived stem cells (ADSCs) after exposing them to adipogenic and osteogenic differentiation conditions. Adipose tissue was isolated from swine (11 months of age) and digested with $0.075 \%$ collagenase at $37^{\circ} \mathrm{C}$ for $90 \mathrm{~min}$. The digested adipose tissue was centrifuged at $200 \mathrm{~g}$ for $10 \mathrm{~min}$ to obtain a cell pellet. The pellet was re-suspended with DMEM, and the ADSCs were plated onto $75 \mathrm{~cm}^{2}$ flasks $\left(5000-10000\right.$ cells per $\left.\mathrm{cm}^{2}\right)$ and cultured in DMEM supplemented with $10 \%$ fetal bovine serum (FBS) and 1\% gentamicin. Passage 3 cells were labeled with fluorescent dye (PKH26 red fluorescent cell linker kit; Sigma Chemical, St. Louis, MO, USA) and sorted by flow cytometry. After labeling and sorting, the sorted and unsorted (control group) cells were replated and exposed to adipogenic $\left(1 \mu \mathrm{M}\right.$ dexamethasone, $0.5 \mathrm{mM}$ isobutylmethylxantine, $10 \mu \mathrm{M}$ insulin and $200^{\circ} \mathrm{M}$ indomethacin) and osteogenic $\left(0.1 \mu \mathrm{M}\right.$ dexamethasone, $10 \mathrm{mM} \alpha$-glycerophosphate, and $50^{\circ} \mathrm{M}$ ascorbic acid) differentiation conditions when the cells were $90 \%$ confluent. Cells were evaluated based on morphology and specific staining properties. Adipogenic differentiation was confirmed by oil red O-positive staining of large lipid vacuoles, and osteogenic differentiation by Von Kossa staining 2 weeks after initiation of differentiation. The frequency of oil red O-positive colonies in both sorted and unsorted group was similar ( $15.0 \%$ vs. $13.2 \%$, respectively). The number of osteogenic nodules, confirmed by the presence of calcium by Von Kossa staining, in the sorted and unsorted group was 17 and 184 per flask, respectively. In conclusion, this study demonstrates that adult porcine adipose-derived stem cells maintain their differentiation potential after labeling with fluorescent dye and sorting by flow cytometry. This should allow for more rapid evaluation of the differentiation potential of ADSCs in vitro.
\end{abstract}

This work was partially supported by the Council for Food and Agricultural Research (C-FAR) Sentinel Program, University of Illinois and CNPq, Brazil (M. Mello).

\section{EFFECT OF OXYGEN TENSION AND SUBSTRATE ON GROWTH AND DIFFERENTIATION OF MOUSE EMBRYONIC STEM CELLS}

\author{
M. A. Ramírez, E. Pericuesta, M. Pérez-Crespo, R. Fernández-González, P. N. Moreira, D. Rizos, \\ B. Pintado, and A. Gutiérrez-Adán \\ Department of Animal Reproduction, INIA, Madrid, Spain
}

Normally the majority of mammalian cells, including murine embryonic stem (mES) cells, are immersed in a low oxygen environment (hypoxia); however, $\mathrm{mES}$ are generally cultured in an atmosphere containing $21 \% \mathrm{O}_{2}$ (normoxia). Such conditions may not be the most appropriate for mES propagation. We have tested the effects of hypoxia and culture on either feeder fibroblasts or gelatin substrate on mES cell growth and spontaneous differentiation. Two ES cell lines (R1 129/Sv from the laboratory of A. Nagy and MAR B6D2 F1 generated in our laboratory) were cultured 
under two different oxygen tensions ( 5 and $21 \%$ ), and on two different substrates, $0.1 \%$ gelatin or murine embryonic fibroblasts (mEF). Cell cycle, cell proliferation, mRNA expression of pluripotency and differentiation markers, as well as spontaneous differentiation to cardiomyocytes, were monitored. For cell proliferation measurements, $\mathrm{mES}$ after 7 days of culture at the different conditions were labeled with 5-(and-6)-carboxyfluorescein diacetate succinimidyl ester, and cultured for up to three more days. Cells were then harvested for flow cytometry analysis every $24 \mathrm{~h}$ after labeling (Cell TraceTM CFSE Cell Proliferation Kit; Molecular Probes, Inc., Eugene, OR, USA). For cell cycle analysis, cells grown on mEF under the two different oxygen tensions were fixed after 10 days of culture, and then stained with propidium iodide/Triton-X-100 for flow cytometry analysis (Current Protocols in Cytometry, Chap. 7, 2001). The spontaneous differentiation of embryoid bodies [formed by ES cells in the absence of leukemia inhibitory factor (LIF)] to cardiomyocytes was also monitored. For mRNA expression of pluripotency (Nanog, Oct-3/4, Rex1, GENESIS, FGFR-4, TERF1, Cx43, and GLUT1) and differentiation markers (GATA4, GATA2, AFP, Msx-1, Brachyury, and Myf5), RT-PCR analysis was performed on $\mathrm{mES}$ cells from Day 0 to Day 10. Under hypoxia conditions, the proliferation of both types of mES cells was greater than under normoxia, independent of substrate used, and a higher number of apoptotic cells was detected. Moreover, only under normoxia conditions did mES cells lose the expression of pluripotency markers GENESIS and GLUT1. In addition, under lower oxygen tension, the rate of differentiation to beating cardiomyocytes was significantly lower on the feeder layer than that under normoxia $(11.9 \%$ vs. $28.1 \% ; P=0.012)$. The feeder layer supported significantly higher cardiomyocyte formation when compared to $0.1 \%$ gelatin at $21 \% \mathrm{O}_{2}(28.1 \% \mathrm{vs} .8 .3 \% ; P<0.001)$. Our results show that normoxia may not be the most appropriate condition for $\mathrm{mES}$ cell propagation and that hypoxic culture may be necessary to maintain full pluripotency of $\mathrm{mES}$ cells.

\title{
204 SPONTANEOUS DIFFERENTIATION OF PORCINE INNER CELL MASS-DERIVED CELLS INTO CELLS DISPLAYING NEURAL AND GLIAL MARKERS
}

\author{
K. Schauser ${ }^{\mathrm{A}}$, S. Friis ${ }^{\mathrm{A}}$, M. Schmidt ${ }^{\mathrm{B}}$, and P. Maddox-Hyttel ${ }^{\mathrm{A}}$ \\ ${ }^{A}$ Department of Basic Animal and Veterinary Sciences, Royal Veterinary and Agricultural University; \\ ${ }^{B}$ Department of Large Animals, Royal Veterinary and Agricultural University, 1870 Frederiksberg C, Denmark
}

The pig is needed as a model for human embryonic stem (ES) cell therapy. Our aims were to isolate, culture, and characterize porcine inner cell mass (ICM) derived cells. Porcine blastocysts $(n=22)$ were flushed from sows on Day 8 after insemination and two blastocysts were immediately fixed. A total of $18(90 \%)$ ICMs were microsurgically isolated from the remaining blastocysts and cultured on mitomycin-inactivated mouse embryonic fibroblasts (SLN cells) in a human ES cell medium without $(n=9)$ or with $10^{6} \mathrm{U} / \mathrm{mL}$ leukemia inhibitory factor (LIF, $\left.n=9\right)$. The colonies were inspected by stereomicroscopy every second day. After approximately one week, ES-like portions of the primary outgrowth colonies were passaged physically. Subsequently, passages were performed approximately once a week. Colonies from different passages were fixed in $4 \%$ paraformaldehyde and processed for immunocytochemistry together with the blastocysts. LIF had no apparent effect on rates of attachment and growth during initial outgrowth and after passages, or on differentiation patterns. A total of $15(83 \%)$ ICMs attached during the outgrowth culture and $12(80 \%)$ of these developed ES-like portions, i.e. compact masses of small tightly packed cells. In blastocysts, nuclei of the ICM stained exclusively for Oct-4. After 6 days of culture, however, Oct-4 staining was lacking in outgrowth colonies. After 14 days of culture, 6 out of 13 passage $(\mathrm{P}) 1$ colonies had developed morphological characteristics compatible with neural differentiation, i.e. large bipolar perikarya and long axon-like structures; after one month, 15 out of 17 P2-4 colonies displayed neural differentiation. Cells in such colonies displayed cytoplasmic staining for $\beta$-III-tubulin in the perikarya and axon-like extensions. After 2-3 months of culture, cell populations in colonies with neural differentation displayed cytoplasmic staining for the intermediate filaments nestin (marker for neural progenitor cells) and vimentin (in the nervous system a marker for glial cells), and cytoplasmic staining for $\beta$-III-tubulin and TUC-4 (markers of post-mitotic neurons). Double-immunostaining revealed a co-localization pattern suggesting the existence of a heterogeneous neural cell population that included neural progenitor cells (staining for nestin only), maturing neural progenitor cells (staining for nestin throughout the cytoplasm combined with $\beta$-III tubulin in the axon-like extensions only), early neurons (staining for $\beta$-III-tubulin, TUC-4, and nestin in the complete cytoplasm), and glial cells (staining for vimentin alone or in combination with nestin). At no time point studied so far were the neural transcription factors Pax6 and Sox 2 detected. In conclusion, ICMs were efficiently isolated from Day 8 porcine blastocysts and attached to feeder cells. However, during initial outgrowth culture they lost their Oct-4 expression, and over the subsequent passages they developed into a heterogeneous population of cells at different stages of neural differentiation.

\section{DERIVATION OF CAT EMBRYONIC STEM-LIKE CELLS FROM IN VITRO-PRODUCED BLASTOCYSTS AND THEIR SUPPORT BY INTRASPECIFIC VS. INTERSPECIFIC FEEDER CELLS}

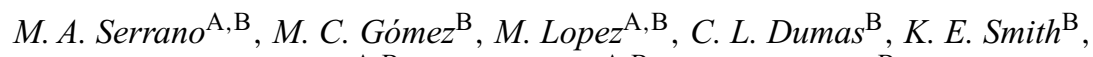 \\ S. P. Leibo ${ }^{\mathrm{A}, \mathrm{B}}$, B. L. Dresser ${ }^{\mathrm{A}} \mathrm{B}$, and C. E. Pope ${ }^{\mathrm{B}}$ \\ ${ }^{A}$ University of New Orleans, Department of Biological Sciences, New Orleans, LA 70148, USA; \\ ${ }^{B}$ Audubon Center for Research of Endangered Species, New Orleans, LA 70131, USA
}

Interspecific nuclear transfer has been successfully demonstrated in nondomestic cats (Gomez et al. 2004 Cloning Stem Cells 6, 247); however, the efficiency remains low and may be attributable to nuclear reprogramming errors. Embryonic stem cells (ESC) may complete nuclear reprogramming more efficiently than somatic cells and, therefore, are potentially useful for increasing cloning success (Jaenisch et al. 2002 Cloning Stem Cells 4, 389). The objectives of this study were to: (1) compare efficiency of immunosurgery vs. mechanical separation for isolating the inner cell mass (ICM) of in vitro-derived cat blastocysts; and (2) determine the influence of mouse (MEF: CF-1) and cat (CEF) embryonic fibroblast feeder layers on ICM attachment and growth of ES-like cells. After ICMs were isolated from in vitro-derived blastocysts $(n=142)$ by immunosurgery or mechanically, 
they were plated either on mitotically inactivated CEF $(40 \mu \mathrm{L} / \mathrm{mL}$ Mitomycin-C; $5 \mathrm{~h})$ or MEF $(30 \mu \mathrm{L} / \mathrm{mL}$ Mitomycin-C; $2.5 \mathrm{~h})$. Cells were cultured in DMEM-F12, $1 \mathrm{mM}$ L-Glutamine, $0.1 \mathrm{mM}$ 2-mercaptoethanol, 1.25\% nonessential amino acids, $15 \%$ knock-out replacement serum, $5 \%$ fetal bovine serum, $40 \mathrm{ng} / \mathrm{mL}$ leukemia inhibitory factor, $5 \mathrm{ng} / \mathrm{mL}$ basic fibroblast growth factor, $100 \mathrm{IU}$ penicillin, $100 \mu \mathrm{g} / \mathrm{mL}$ streptomycin, and $25 \mu \mathrm{L} / \mathrm{mL}$ amphotericin-B in a humidified atmosphere of $5 \% \mathrm{CO}_{2}$ in air at $38^{\circ} \mathrm{C}$. Our results indicated that ICM isolation and attachment were not affected by either the method of isolation (immunosurgery: $75.8 \pm 6.9 \%$ vs. mechanical: $89.5 \pm 6.4 \%$ ) or the feeders (MEF: $74.6 \pm 6.7 \%$ vs. CEF: $90.7 \pm 6.6 \%$ ). However, the incidence of ES-like cell colony formation was significantly affected by the feeder layer (CEF: $55.4 \pm 7.2 \%$ vs. MEF: $12.7 \pm 7.2 \%$; $P<0.001)$. A total of 32 ES-like cell lines were derived on CEF $(n=26)$ and $\operatorname{MEF}(n=6)$, of which $50 \%$ were alkaline phosphatase (AP)-positive. One ES-like cell line derived on MEF spontaneously differentiated into myocardiocytes after 14 days in culture. Three ES-like cell lines derived on CEF were immunostained for ESC-markers Oct-4, SSEA-1, and SSEA-4, and for AP. Positive results for all markers were observed in a few colonies of each line, with colonies from one cell line appearing on Day 23 and remaining in culture for 102 days (12 passages). Colonies from the other two cell lines appeared on Day 17 and remained in culture for 78 days ( 9 passages). Colonies derived on MEF appeared on average at 17.9 days and remained in culture an additional 15 to 61 days without further characterization. The present results describe the first isolation of cat ES-like cells. We have demonstrated an important species-specific relationship between feeder layers and the derivation of cat ESCs. Further studies are in progress to improve culture conditions for the derivation and expansion of stable cat ESC lines.

\title{
206 COMPARATIVE EVALUATION OF VARIOUS PROTOCOLS FOR NEURAL DIFFERENTIATION OF MOUSE EMBRYONIC STEM CELLS
}

\author{
A. $\operatorname{Tas}^{\mathrm{A}, \mathrm{B}}, S$. Arat $^{\mathrm{B}}$, and H. Dalcik ${ }^{\mathrm{A}}$ \\ A Department of Histology and Embryology, Kocaeli University, Kocaeli, Turkey; \\ ${ }^{\mathrm{B}}$ TUBITAK, Research Institute for Genetic Engineering and Biotechnology, Gebze, Kocaeli, Turkey
}

\begin{abstract}
Mouse embryonic stem (ES) cells derived from the inner cell mass of blastocysts can differentiate into neuronal cells by treatment with retinoic acid (RA). ES cells cultured as aggregates and as single cell suspensions were then exposed to RA which induced multiple phenotypes of neuronal cells. Differentiation was dependent on the concentration of RA and the time of exposure. In this study, we cultured ES cells as a suspension in which they formed embryoid bodies (EBs). The EBs were treated with varying concentrations of RA for differing times. We used increasing concentrations of RA $(50 \mathrm{nM}, 100 \mathrm{nM}, 1 \mu \mathrm{M}$, and $3 \mu \mathrm{M})$ prepared from a stock of $10 \mathrm{mM} \mathrm{RA}$ in DMSO. Immunocytochemistry staining was carried out on 2, 5, 7, and 9 days of culture. We formed EBs for 4 days with standard ES cell medium (without LIF) plus an additional 4 days of treatment with $1 \mu$ M RA. ES cells were treated with $1 \mu \mathrm{M}$ RA for 2 days in suspension culture. Two-day-old EBs plated on culture dishes were treated with $1 \mu \mathrm{M}$ RA for 3 days. To test for the effect of RA concentration on embryonic differentiation, 2-day-old EBs were treated with $50 \mathrm{nM}, 100 \mathrm{nM}, 1 \mu \mathrm{M}$, and $3 \mu \mathrm{M}$ RA for 3 days. The time-dependent effects of RA on the 4-/4+ RA group were investigated. Results showed that neuronal precursor cells appeared on the second day of culture; they were stained with nestin antibody. On the 5th day of culture, neurons were detected with NCAM antibody. On the 7th day of culture, glial cells were observed with GFAP, and on the 9th day of culture GFAP, expression increased. In EBs that were plated and then treated with RA, the same results were obtained. RA induced neuronal differentiation in a concentration-dependent manner. Low concentrations $(50 \mathrm{nM}$ and $100 \mathrm{nM})$ of RA induced neuronal differentiation besides mesenchymal differentiation; however, higher concentrations $(1 \mu \mathrm{M}$ and $3 \mu \mathrm{M})$ of RA did not induce mesenchymal differentiation. The most efficient neuronal differentiation was obtained at $3 \mathrm{mM}$ RA concentration.
\end{abstract}

This study was performed in TUBITAK Research Institute for Genetic Engineering and biotechnology.

\section{ESTABILSHMENT OF CYNOMOLGUS MONKEY EMBRYONIC STEM CELL LINES AND CONFIRMATION OF THE POSSIBILITY FOR GERMINAL COMPETENCY}

\author{
T. Teramura ${ }^{\mathrm{A}}$, N. Kawata ${ }^{\mathrm{B}}$, N. Fujinami ${ }^{\mathrm{B}}$, M. Takenoshita $^{\mathrm{C}}$, N. Sagawa $^{\mathrm{A}}$, K. Matsumoto $^{\mathrm{B}}$, \\ K. Saeki ${ }^{\mathrm{B}}$, . Hosoi $^{\mathrm{B}}$, and A. Iritani ${ }^{\mathrm{B}}$

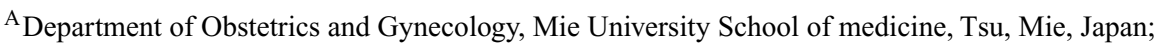 \\ ${ }^{B}$ Department of Genetic Engineering, Kinki University, Naga, Wakayama, Japan; \\ ${ }^{\mathrm{C}}$ Keari Co., Ltd., Osaka, Japan
}

Embryonic stem cells (ESCs) of nonhuman primate are important tools for human gametogenesis research. Generally, ESCs, embryos, and fetuses of nonhuman primates are similar to these of human. Recently, germ cell formation of mouse ESCs in vitro has been reported. In this study, we established new cynomolgus monkey ES (cyES) lines and determined germinal competency by assessing expression of mRNA markers. CyES lines were established using blastocysts produced by intracytoplasmic sperm injection (ICSI). For inducing super-ovulation, females were treated with $25 \mathrm{IU} / \mathrm{kg}$ pregnant mare serum gonadotropin (PMSG) once a day for 9 days, followed by $400 \mathrm{IU} / \mathrm{kg} \mathrm{hCG}$. Oocytes were collected $40 \mathrm{~h}$ after injection of hCG. After sperm injection, embryos were cultured in mCMRL medium to the blastocyst stage. For ES line establishment, inner cell masses (ICMs) were isolated by immunosurgery. ESC colonies emerged at about 10 days after ICM plating; three cyES cell lines were successfully obtained $(3 / 11 ; 27.3 \%)$. We characterized these lines by immunocytochemistry for Oct-3/4, SSEA-3, and SSEA-4, which are diagnostic markers for primate ESCs, and by assay for alkaline phosphatase (ALP) activity. All cell lines expressed Oct-3/4, SSEA-4 and ALP activity. The previously reported SSEA-3 weak expression in cyES cells was not observed. These lines differentiated spontaneously when they were replaced in non-adherent culture (embryoid body: EB) or injected into SCID mice subcutaneously. To assess germ cell competency in vitro, we analyzed for the presence of vasa mRNA which shows a restricted expression pattern to germ cell formation, and DMC1 and SYCP1 which show specific existence on synaptonema 
complex in meiosis. Detection of these germ cell markers was performed by RT-PCR with total cDNA from ESCs and EBs. Nanog mRNA was detected only in ESCs. Oct-4 was detected in gonadal tissue of both sexes, ESCs, and EBs. Vasa was expressed in testis, but not in ESCs or somatic cells. Interestingly, we recognized weak expression of Vasa in Day 12-16 EBs. DMC1 and SYCP1 as meiosis markers were not detected. Because Oct-4 and Vasa mRNA are transcribed simultaneously, similar to that in the early part of gametogenesis such as the latter period of primordial germ cell (PGC) migration, PGC formation in cynomolgus EBs could occurr as in some cases of mouse or human EBs previously reported. Although detailed properties such as the functions of these Vasa-positive cells have not been confirmed, these results demonstrate that cyES cells obtained in the current study might contribute to putative germ cells in vitro by differentiating to EBs.

This study was supported by a Grant-in-Aid for the 21st Century COE Program of the Japan Mext and by a grant for the Wakayama Prefecture Collaboration of Regional Entities for the Advancement of Technology Excellence of the JST.

\title{
208 CYTOLYTIC ANALYSIS AND NUCLEAR TRANSFER OF hCD46-TRANSGENIC PORCINE EMBRYONIC GERM CELLS TO DEVELOP AND IN VITRO MODEL OF XENOTRANSPLANTATION
}

\author{
J. Y. Won, K. S. Ahn, S. Y. Heo, J. H. Kang, and H. Shim \\ Department of Physiology, Dankook University School of Medicine, Cheonan, Korea
}

Pigs are considered the most likely source of organs for xenotransplantation due to their anatomical and physiological similarities to humans. Production of transgenic pigs including addition of human complement-regulatory protein genes and deletion of alpha-1,3-galactosyl transferase gene may overcome hyperacute rejection (HAR), the first and currently the most critical immunological hurdle in the development of xenogeneic organs for human transplantation. However, even after resolving HAR in pig-to-human xenotransplantation, a series of other transgenic pigs may be required to alleviate subsequent acute and chronic rejection and incompatibility of porcine proteins to human counterparts. The production of transgenic pigs is not only labor-intensive, time-consuming, and costly, but also the usefulness of such pigs in transplantation to humans is unpredictable. For these reasons, development of a reliable in vitro procedure to pre-evaluate effectiveness of the transgenic approach would be beneficial. This study was preformed to establish an in vitro model of xenotransplantation using porcine embryonic germ (EG) cells, undifferentiated stem cells derived from culture of primordial germ cells. Porcine EG cells were maintained in feeder-free state in DMEM containing $15 \%$ (v/v) fetal bovine serum and 1000 units $/ \mathrm{mL}$ leukemia inhibitory factor. Human complement down-regulator hCD46 (also known as MCP, membrane cofactor protein) gene under the regulation of cytomegalovirus promoter was introduced into porcine EG cells. Transfected cells were selected by antibiotic treatment and confirmed by PCR. To test the resistance of hCD46-transgenic EG cells to human xenoreactive natural antibody and complement, EG cells were cultured for 1.5 days in DMEM containing $15 \%(\mathrm{v} / \mathrm{v})$ normal human serum. The treatment with human serum did not affect the survival of hCD46-transgenic EG cells, whereas with the same treatment approximately one half of non-transfected EG cells failed to survive $(P<0.01)$. Transgenic EG cells presumably capable of overcoming HAR were used as nuclear donors for subsequent transfer of nuclei into enucleated oocytes. Among 110 reconstituted oocytes, 19 (17.3\%) developed to the blastocyst stage. Analysis of individual nuclear transfer embryos by PCR indicated that $89.5 \%$ (17/19) of embryos contained transgene hCD46. The PCR-negative embryos might be due to an incomplete antibiotic selection of cells after transfection. Overall, the results of the present study demonstrate that the cell culture-based model of xenotransplantation may validate the usefulness of particular transgenic pigs prior to actual production. Further experiments on differentiation of transgenic EG cells into various cell types, cytolytic analysis of such cells to assess efficiency of xenotransplantation, and subsequent production and transfer of transgenic clone embryos to recipients may provide a useful new procedure to accelerate xenotransplantation research.

Epidemiology/Diseases

\section{RISKS OF TRANSMITTING MYCOBACTERIUM AVIUM SSP. PARATUBERCULOSIS BY TRANSFER OF IN VIVO-DERIVED AND IN VITRO-FERTILIZED BOVINE EMBRYOS}

\author{
A. Bielanski, J. Algire, G. Randall, and O. Surujballi \\ Animal Diseases Research Institute, CFIA-OLAF, Germplasm Centre of Expertise, Ottawa, Ontario, Canada
}

Paratuberculosis (Johne's disease) is a chronic infectious disease of cattle and other domestic and wild ruminants caused by Mycobacterium avium ssp. paratuberculosis (Map) which is widespread throughout the world. The disease is characterized by persistent diarrhea, weight loss, and eventually death. In addition to interest in the economic losses, there has been an increasing interest in eradicating the disease due to the potential involvement of the causative microorganism in Crohn's disease, a debilitating chronic enteritis in man. Experiments were conducted to determine the possibility of transmission of Map by embryo transfer (ET) and the association of Map with in vivo-derived and in vitro-fertilized (IVF) embryos. These experiments involved (1) collection of embryos from naturally infected donors $(n=5)$ and transfer of those embryos to uninfected recipients $(n=12)$ and testing of others for Map by culture on Herrold's medium with mycobactin or by PCR; and (2) in vitro exposure of in vivo-derived and IVF embryos to Map and their subsequent transfer to uninfected recipients. Experiment 1 revealed the presence of Map in the uterine horns of all five subclinically infected donors, but it was not detected in association with sequentially washed in vivo-derived embryos by culture or by PCR. In Experiment 2 , a high proportion of both in vivo-derived and IVF embryos exposed to Map in vitro tested positive for Map even after sequential washings as recommended by IETS. Transfer of in vivo-derived and IVF embryos that had been exposed to Map, and then washed, into 18 recipients resulted in 13 pregnancies and 8 calves born without evidence of disease transmission to either the recipients or the offspring over the following 5 year period. In conclusion, it is unlikely that Map will be transmitted by ET when the embryos have been processed according to the washing protocols recommended by the IETS. 


\title{
210 EFFECTS OF INTERFERON- $\tau$ ON REPLICATION OF BOVINE VIRAL DIARRHEA VIRUS AND BOVINE HERPESVIRUS-1
}

\author{
P. Galik, J. Waldrop, and S. Marley \\ College of Veterinary Medicine, Auburn University, Auburn, AL 36849, USA
}

\begin{abstract}
Bovine viral diarrhea virus (BVDV) and bovine herpesvirus-1 (BHV-1) are the most likely viruses to be associated with abattoir-origin materials used in in vitro embryo production. Further, both viruses are known to associate with zona pellucida-intact embryos after exposure and washing, and limited evidence indicates that developing, transferable embryos are able to inhibit viral replication in adjacent cells. Interferon- $\tau$ is known to have anti-BVDV and anti-BHV-1 activities, but it is not known whether interferon- $\tau$ which is secreted by developing embryos has the same effects. The objectives of this study were to evaluate the cytotoxicity and anti-viral effect of interferon- $\tau$ against a non-cytotoxic high affinity strain of BVDV (SD-1) and against a strain of BHV-1 (Colorado) in cell culture. Madin Darby bovine kidney (MDBK) cells were seeded in 96-well plates and then inoculated with serial dilutions (1:10) beginning with an initial concentration of $0.2 \mu \mathrm{g}$ of interferon- $\tau$. Cells and interferon were incubated at $37.5^{\circ} \mathrm{C}$ in $5 \% \mathrm{CO}_{2}$ and air for $24 \mathrm{~h}$ prior to addition of virus. Five concentrations of BVDV were added to the wells to give 500, 50, 25, 10 or 5 cell culture infective doses (50\% endpoint) per well. Three concentrations of BHV-1-50,10, and 5 plaque-forming units - were evaluated in separate cell cultures. Virus isolation (for BVDV) or plaque assays (for BHV-1) were utilized to determine if the addition of interferon- $\tau$ decreased the amount of infective virus. The interferon- $\tau$ produced no observable cytotoxicity in MDBK cells in any of the assays. At its three highest concentrations, the interferon- $\tau$ significantly decreased the amount of BVDV but it had no significant effect on the amount of BHV-1 in cell cultures. Thus, it is possible that interferon- $\tau$ produced by developing embryos might limit or prevent transmission of BVDV to recipients if this virus were to be inadvertently associated with the embryos that are transferred. However, a similar effect is not expected for BHV-1.
\end{abstract}

\section{LACTOFERRIN INHIBITS BOVINE HERPESVIRUS-1 IN CELL CULTURE AND ALLOWS NORMAL DEVELOPMENT OF IN VITRO-PRODUCED EMBRYOS}

\author{
M. Givens, M. Marley, P. Galik, K. Riddell, and D. Stringfellow \\ College of Veterinary Medicine, Auburn University, Auburn, AL 36849, USA
}

Lactoferrin is an iron-binding glycoprotein found in milk, saliva, tears, and other exocrine secretions. It is known to have in vitro antiviral effects against human, feline, and canine herpesviruses. In addition, lactoferrin is known to be safe in cell culture. Bovine herpesvirus-1 (BHV-1) is a likely contaminant of in vitro embryo production. Further, trypsin treatment is not completely effective in removing the virus from these embryos. We hypothesized that a nontoxic concentration of lactoferrin might prevent replication of BHV-1 within in vitro embryo production systems. Thus, the specific objectives of this research were to determine if lactoferrin from bovine milk would inhibit BHV-1 in cell culture and to determine if in vitro-produced embryos could develop normally when cultured in lactoferrin. Two-fold dilutions of lactoferrin (from 10 to $0.625 \mathrm{mg} / \mathrm{mL}$ ) were added to Madin Darby bovine kidney cells, followed in $15 \mathrm{~min}$ by the addition $10^{4} \mathrm{PFU} / \mathrm{mL}$ of BHV-1 (Colorado strain). Samples of cell lysate were taken at Day 2 and virus was quantified by plaque assay. The percent of virus inhibited by the antiviral agent at each concentration was determined by comparison to equivalent samples from temporal control cultures in which no compound was added before or after inoculation (Percentage of virus inhibited $=$ [Quantity of virus in the control sample - Quantity of virus in the compound sample]/Quantity of virus in the control sample $\times 100$ ). Next, the effect of lactoferrin was determined on in vitro-produced embryos. Cumulus oocyte complexes were received from an abattoir, matured in transit, placed in fertilization drops for $6 \mathrm{~h}$, and then placed in culture drops containing lactoferrin $(10,5$, and $2.5 \mathrm{mg} / \mathrm{mL})$. At Day 3.5 , embryos $>4$ cell stage were placed into fresh culture drops containing lactoferrin. On Day 7.5, blastocyst development was noted and the developed embryos were stained to count viable cells. Blastocyst development rate and nucleated cell count of the treated embryos were compared to those of the controls using Chi square test, and ANOVA and Tukey-Kramer HSD, respectively. Lactoferrin $(10 \mathrm{mg} / \mathrm{mL})$ inhibited 2 to 5 logs of virus. At concentrations of 5 and $2.5 \mathrm{mg} / \mathrm{mL}, 1$ to $3 \operatorname{logs}$ of virus were inhibited, and concentrations of 1.25 and $0.625 \mathrm{mg} / \mathrm{mL}$ inhibited 0 to 2 logs of virus. Lactoferrin did not affect the nucleated cell count of the treated embryos. In addition, unlike 10 and $5 \mathrm{mg} / \mathrm{mL}, 2.5 \mathrm{mg} / \mathrm{mL}$ of lactoferrin did not affect blastocyst development. These preliminary results indicate that lactoferrin from bovine milk can significantly inhibit BHV-1 in cell culture. Furthermore, supplementation of in vitro culture with $2.5 \mathrm{mg} / \mathrm{mL}$ of lactoferrin does not affect blastocyst development or cell count of in vitro-produced embryos.

\section{TRYPLE SELECT (10X) EFFECTIVELY REMOVES BOVINE HERPESVIRUS-1 ASSOCIATED WITH IN VIVO-DERIVED EMBRYOS}

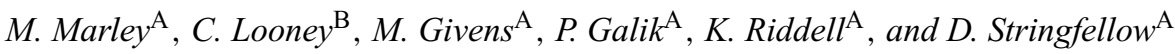 \\ ${ }^{\mathrm{A}}$ College of Veterinary Medicine, Auburn University, Auburn, AL 36849, USA; ${ }^{\mathrm{B}}$ OvaGenix, Navasota, TX 77868, USA
}

Porcine-origin trypsin will effectively remove bovine herpesvirus-1 (BHV-1) from in vivo-derived embryos. It is not known if TrypLE (Invitrogen, Carlsbad, CA, USA) could be used to remove BHV-1, but this recombinant porcine sequence trypsin-like protease would be an attractive alternative because it is highly stable at room temperature and does not pose the same threat for contamination as animal-origin trypsin. Thus, the objective of this study was to determine if TrypLE Express (1X) for $1.5 \mathrm{~min}$ of exposure or TrypLE Select (10X) for 10 min of exposure would be effective at removing BHV-1 from Day 7 zona pellucida-intact, in vivo-derived embryos after they had been exposed to the virus. Day 7 bovine in vivo-derived morulae and blastocysts and non-fertile degenerate (NFD) embryos were collected and shipped overnight to our facility. Upon arrival, the zona pellucida intact embryos were washed according to the International Embryo Transfer Society protocol. Developed embryos were washed separately 
from NFD embryos. One group of 5 or 10 NFD or developed embryos was not exposed to virus and served as the negative control. The remaining embryos and $10 \mathrm{NFD}$ were exposed to $10^{6} \mathrm{PFU} / \mathrm{mL}$ BHV-1 (Colorado strain) for $1 \mathrm{~h}$. Following exposure, one group of 5 or $10 \mathrm{NFD}$ or developed embryos was washed and served as the positive control. One group of 10 developed embryos was washed and treated with porcine origin trypsin. The remaining developed embryos were divided into groups of 5 or 10 and washed and treated with TrypLE Express for 1.5 min or TrypLE Select (10X) for $10 \mathrm{~min}$. Following treatment, the embryos were sonicated in groups of 5 or 10 and assayed by virus isolation. The negative control embryos, porcine origin trypsin treated embryos, and TrypLE Select treated embryos were negative for virus. The positive control embryos and the TrypLE Express treated embryos were positive on virus isolation (Table 1). When it was determined that TrypLE Express was not effective at $1.5 \mathrm{~min}$, TrypLE Select (10X) was used for $10 \mathrm{~min}$. These preliminary results indicate that use of TrypLE Select (10X) for $10 \mathrm{~min}$ is effective for removal of BHV-1 associated with Day 7, zona pellucida-intact, in vivo-derived embryos. In addition, TrypLE Select has the advantage of being an animal-origin-free product. However, use of TrypLE Express (1X) for $1.5 \mathrm{~min}$ was not effective. Because it is not practical to expose embryos to trypsin for $10 \mathrm{~min}$, further research is needed to determine the ideal treatment concentration and time that will effectively remove BHV-1 without harming in vivo-derived bovine embryos.

Table 1. Virus isolation results

\begin{tabular}{lc}
\hline Embryo group & Positive/total \\
\hline Porcine-origin trypsin-treated & $0 / 6$ \\
TrypLE Select-treated & $0 / 2$ \\
TrypLE Express-treated & $11 / 11$ \\
\hline
\end{tabular}

\title{
213 ASSESSMENT OF THE SUSCEPTIBILITY OF PORCINE PRE-IMPLANTATION EMBRYOS TO PSEUDORABIES VIRUS (PRV) AND PORCINE REPRODUCTIVE AND RESPIRATORY SYNDROME VIRUS (PRRSV) USING SUBZONAL MICROINJECTION
}

\author{
B. Mateusen, A. Van Soom, D. Maes, and H. Nauwynck
}

Department of Reproduction, Obstetrics and Herd Health, Faculty of Veterinary Medicine, Ghent University, 9820 Ghent, Belgium

It is known that porcine pre-implantation embryos before the morula stage are refractory to infection with pseudorabies virus (PRV) and porcine reproductive and respiratory syndrome virus (PRRSV) (Bolin et al. 1981 Am. J. Vet. Res. 42, 1711-1712; Prieto et al. 1996 Theriogenology 46, 687-693, respectively). The effects of PRV and PRRSV on embryonic cells of morulae and blastocysts are unknown. Therefore, the objectives of the present study were to (1) assess the effects of PRV and PRRSV exposure on further embryo development, and (2) determine whether PRV and PRRSV are able to replicate in embryonic cells. Zona pellucida (ZP)-intact morulae (6 days post-insemination, 6 dpi) and early blastocysts (7 dpi) were microinjected subzonally with approximately $3 \mathrm{~nL}$ of $10^{9} \mathrm{TCID}_{50} / \mathrm{mL}$ PRV (strain $89 \mathrm{v} 87$, second passage in swine testicle cells) or $10^{8.6} \mathrm{TCID}_{50} / \mathrm{mL}$ PRRSV (Lelystad virus strain, 13th passage in swine alveolar macrophages). Control embryos were microinjected under the same circumstances with phosphate-buffered saline (PBS). Hatched blastocysts $(8 \mathrm{dpi})$ were exposed for $1 \mathrm{~h}$ at $39^{\circ} \mathrm{C}$ to $10^{5} \mathrm{TCID} 0_{5} / \mathrm{mL}$ of PRV or PRRSV of the same strains used for injecting earlier embryonic stages. Control hatched blastocysts were incubated with PBS. Each group of morulae and blastocysts consisted of approximately 20 embryos. Embryonic development was assessed every $12 \mathrm{~h}$. At $48 \mathrm{~h}$ post injection, the percentage of infected embryos and the percentage of viral antigen positive cells per embryo were determined by immunofluorescence. Subzonal microinjection of ZP-intact morulae and blastocysts with PRV inhibited in vitro development in comparison to the controls. Moreover, under direct immunofluorescence, PRV antigen-positive cells were detected in association with the embryos. Exposure of hatched blastocysts to PRV inhibited further embryo development; the majority (16/20) of the embryos degenerated $24 \mathrm{~h}$ after incubation. Perivitelline microinjection of ZP-intact morulae and blastocysts with PRRSV and incubation of hatched blastocysts with PRRSV did not inhibit in vitro development in comparison to the controls. No PRRSV antigen positive cells were detected in association with the embryos. Based on these results, it can be deduced that embryonic cells of morulae and blastocysts are susceptible to PRV infection but refractory to PRRSV infection. Another argument substantiating insusceptibility of embryos to certain viral pathogens is the demonstration of the lack of virus receptors at a given embryonic cell stage. Therefore, the expression of sialoadhesin, the receptor that mediates the internalization of PRRSV in cells, was investigated in hatched blastocysts $(n=10)$. By indirect immunofluorescence using monoclonal antibody 41D3 directed against porcine sialoadhesin, no positive signals were detected. The result of this experiment strengthens the statement that embryonic stages up to the hatched blastocyst stage are refractory to PRRSV infection.

\section{USE OF VIRUS ISOLATION AND QUANTITATIVE POLYMERASE CHAIN-REACTION TECHNIQUES TO DETECT BOVINE VIRAL DIARRHEA VIRUS (BVDV) IN SINGLE OR SMALL GROUPS OF PRE-IMPLANTATION BOVINE EMBRYOS}

\author{
J. Waldrop, M. Givens, K. Riddell, P. Galik, and D. Stringfellow \\ College of Veterinary Medicine, Auburn University, Auburn, AL 36849, USA
}

Because of its broad distribution among populations of cattle and its association with materials of animal origin used in embryo production, bovine viral diarrhea virus (BVDV) is a potential problem in applications of embryo technologies. While some isolates of BVDV are known to associate with both in vivo-derived and in vitro-produced bovine embryos, it has yet to be determined if the quantity of virus associated with exposed 
zona pellucida-intact embryos is sufficient to infect susceptible recipient cows via the intrauterine route. Techniques to detect and quantify BVDV associated with single transferable embryos are important to determine the risk of transmitting BVDV via embryo transfer. The objectives of this study were to define reproducible techniques to detect and quantify BVDV associated with single or small groups of bovine embryos contained in small aliquots of medium using virus isolation (VI) or real time quantitative polymerase chain reaction (Q-PCR) assays. In vivo-derived and in vitro-produced embryos were exposed for $2 \mathrm{~h}$ to approximately $10^{6}$-cell culture infective doses (50\% endpoint) per mililiter of a high affinity strain of BVDV, SD-1, and then washed according to IETS guidelines. Embryos were assayed in groups of five or two embryos, or single. There were 5 replicates of the group of five embryos, 4 of the group of two embryos, and 3 of the single embryos for the in vivo-derived embryos undergoing VI; 5,4 , and 2 replicates, respectively, undergoing Q-PCR, and 2, 5, and 2 replicates, respectively, for the in vitro-produced embryo groups undergoing VI and Q-PCR. Those to be assayed by VI were sonicated and the sonicate fluids were layered onto Madin Darby Bovine Kidney (MDBK) cells and passaged to allow for viral replication; an immunoperoxidase monolayer assay was then used for viral detection. A Roche ${ }^{\circledR}$ RNA/DNA extraction kit (Roche Diagnostic Systems, Inc., Somerville, NJ, USA) was used to extract RNA from virally exposed embryos, and extracted samples were assayed in duplicate Q-PCR reactions consisting of $100 \mu \mathrm{L}$. The primers used were L1 and U3 which are specific for conserved areas of the 5 prime nontranslated regions of the viral genome of BVDV. The PCR product was detected using hybridization probes s1 and s2 as in Struder et al. 2002 Biologicals 40, 289-296. In vivo-derived groups of five or two embryos, or single embryos, were positive for BVDV 100, 50, and 30\% of the time, respectively, when VI was used and 100, 75 and 100\%, respectively, when Q-PCR was used. The virus was detected in all of the in vitro-produced embryo groups of five, or two embryos, or single embryos, $100 \%$ of the time using VI, and in 100, 80, and $100 \%$ respectively, using Q-PCR. The virus isolation technique is highly sensitive but the need to destroy embryos by sonication to identify any embryo-associated virus precludes its use for embryos intended for transfer. Techniques for Q-PCR were sufficiently sensitive to detect and quantify 10 copies of RNA in a sample and to detect BVDV associated with single embryos.

\title{
Exotic Species
}

\section{EPIDIDYMAL SPERM CRYOPRESERVATION OF ONE SOMALIA WILD ASS (EQUUS AFRICANUS SOMALIENSIS) USING SIX DIFFERENT EXTENDERS}

\author{
M. Álvarez ${ }^{\mathrm{A}}$, F. Martínez-Pastor ${ }^{\mathrm{A}, \mathrm{B}}$, V. García-Macias $^{\mathrm{A}}$, S. Borragán ${ }^{\mathrm{C}}$, M. Celada $^{\mathrm{C}}$, J. Bernardo $^{\mathrm{A}}$, \\ N. Gonzalez ${ }^{\mathrm{A}}$, S. Alves ${ }^{\mathrm{A}}$, and L. Anel ${ }^{\mathrm{A}}$ \\ ${ }^{A}$ Animal Reproduction, University of León, León, Spain; ${ }^{B}$ Cell Biology, University of León, León, Spain; \\ ${ }^{\mathrm{C}}$ Cabarceno Park, Santander, Cantabria, Spain
}

The Somalia wild ass (Equus africanus somaliensis) is a critically endangered taxon (IUCN 2004 red list) which could benefit from biological resource banking. In this work, we studied the effect of different extenders applied to the cryopreservation of epididymal sperm obtained from one male of this subspecies. This animal (13 years old; housed in Cabarceno Park, Cantabria, Spain) was castrated because of very aggressive behavior with other mature males. Genitalia were dissected and weighed (testicles: right, $166 \mathrm{~g}$, and left, $179 \mathrm{~g}$; cauda epididymis: right, $9.3 \mathrm{~g}$, and left, $11.8 \mathrm{~g}$ ). Sperm were flushed from the cauda epididymis, yielding $15 \mathrm{~mL}$ of sample. Sperm concentration was $15 \times 10^{9}$ spermatozoa $/ \mathrm{mL}$, totaling $225 \times 10^{9}$ (allowing 4500 doses at $50 \times 10^{6}$ sperm/dose). Sperm motility $(\mathrm{TM}=\%$ total motile; $\mathrm{PM}=\%$ progressive; $\mathrm{VAP}=$ average path velocity) was assessed by CASA (Microptic, Barcelona, Spain). Viability (VIAB $=\%$ viable sperm) and acrosomal status (ACR $=\%$ viable spermatozoa with intact acrosomes) were assessed using propidium iodide $(37 \mu \mathrm{mol} / \mathrm{L})$ and PNA-FITC $(1 \mathrm{ng} / \mathrm{L})$ and flow cytometry. Chemicals were purchased from Sigma (Madrid, Spain). Part of the sample was divided into six aliquots and diluted 1:1 with different extenders: UL4: Tes-Tris-Fructose (TTF), $10 \%$ egg yolk (EG), and 4\% glycerol (G); UL8: TTF, 20\% EG, and 8\% G; AND4: Andromed ${ }^{\circledR}$ (Minitüb, Tiefenbach, Germany) and 4\% G; AND7: Andromed $^{\circledR}$ and 7\% G; GENT: Gent 1045; and INRA: INRA96 and 4\% G. Andromed, Gent, and INRA are commercial extenders. Samples were cooled to $5^{\circ} \mathrm{C}\left(-0.2^{\circ} \mathrm{C} / \mathrm{min}\right)$ and then diluted to $200 \times 10^{6} \mathrm{sperm} / \mathrm{mL}$. Samples were packed $(0.5-\mathrm{mL}$ straws) and frozen using a biofreezer (from $5^{\circ} \mathrm{C}$ to $-15^{\circ} \mathrm{C}$ at $-15^{\circ} \mathrm{C} / \mathrm{min}$, and from $-15^{\circ} \mathrm{C}$ to $-100^{\circ} \mathrm{C}$ at $-25^{\circ} \mathrm{C} / \mathrm{min}$ ). Samples were thawed at $65^{\circ} \mathrm{C}$ for $6 \mathrm{~s}$, and assessed as for pre-freezing (Table 1). Post-thawing motility recovery using AND7 was excellent. The highest viability recovery was achieved by UL4, although that in AND7 was similar. The poor results of equine commercial extender Gent 1045 in this species are remarkable. Our results highlight the importance of species differences in the field of sperm cryopreservation. It is necessary to carry out continuous research for optimizing cryopreservation protocols in order to create germplasm banks for wild species.

Table 1. Quality assessment results

\begin{tabular}{lllrccc}
\hline & Extender & TM & PM & VAP & VIAB & ACR \\
\hline Fresh sample & & 67.1 & 19.5 & 65.5 & 84.4 & 99.9 \\
Post-thawing & UL4 & 37.7 & 7.6 & 17.3 & 62.7 & 99.8 \\
& UL8 & 36.3 & 6.5 & 20.4 & 30.4 & 99.6 \\
& AND4 & 21.3 & 5.9 & 35.0 & 28.0 & 99.6 \\
& AND7 & 67.6 & 25.0 & 36.9 & 57.3 & 99.9 \\
& GENT & 19.0 & 2.7 & 18.9 & 32.4 & 99.5 \\
& INRA & 23.0 & 9.1 & 30.0 & 26.1 & 99.6
\end{tabular}




\title{
216 IN VITRO OOCYTE MATURATION, FERTILIZATION, AND CULTURE AFTER LAPAROSCOPIC OVUM PICK-UP IN AN ENDANGERED GAZELLE (GAZELLA DAMA MHORR)
}

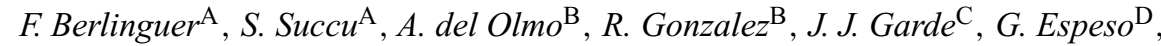 \\ M. Gomendio ${ }^{\mathrm{B}}$, S. Ledda $\mathrm{A}$, and E. R. S. Roldan ${ }^{\mathrm{B}}$ \\ ${ }^{\mathrm{A}}$ Università degli Studi di Sassari, 07100-Sassari, Italy; ${ }^{\mathrm{B}}$ Museo Nacional de Ciencias Naturales (CSIC), \\ 28006-Madrid, Spain; ${ }^{\mathrm{C}}$ IREC, Universidad de Castilla-La Mancha, 02071-Albacete, Spain; \\ ${ }^{D}$ Estacion Experimental de Zonas Aridas (CSIC), 04001-Almeria, Spain
}

\begin{abstract}
The recovery of immature oocytes followed by in vitro maturation, fertilization and culture (IVMFC) allows the rescue of biological material of great genetic value for the establishment of genetic resource banks. Studies have been carried out on endangered Mohor gazelle sperm cryopreservation (Garde et al. 2003 Biol. Reprod. 69, 602-611), but there are no studies on oocytes in this species. The purpose of this work was to develop a protocol for ovarian stimulation for the recovery of oocytes and subsequent IVMFC. The study was conducted using six reproductively mature female Mohor gazelles from the breeding herd at the Estacion Experimental de Zonas Aridas. Animals were synchronized by insertion of controlled progesterone internal drug release (CIDR) devices for 14 days and removal of the devices on the day of ovum pickup (OPU). Follicular growth was stimulated by a total of $5.28 \mathrm{mg}$ of oFSH (Ovagen, ICP, Auckland, New Zealand) given in four equal doses every $12 \mathrm{~h}$. OPUs were performed (Berlinguer et al. 2004 Theriogenology 61, 1477-1486) on Day 15 from the beginning of treatment, and follicles were aspirated with a syringe and a $25 \mathrm{G}$ needle using TCM199-HEPES with $50 \mu \mathrm{g} / \mathrm{mL}$ streptomycin, $50 \mathrm{IU} / \mathrm{mL}$ penicillin, $0.1 \%$ polyvinyl alcohol, and $15 \mathrm{IU} / \mathrm{mL}$ heparin. Degenerated oocytes and those with expanded cumulus were removed. Oocytes were cultured in TCM-199 plus $10 \% \mathrm{FCS}, 10 \mu \mathrm{g} / \mathrm{mL}$ ovine FSH/LH, $1 \mu \mathrm{g} / \mathrm{mL}$ estradiol, and $0.1 \mathrm{mg} / \mathrm{mL}$ glutamine at $38.5^{\circ} \mathrm{C}$ under $5 \% \mathrm{CO}_{2} /$ air and maximum humidity. Spermatozoa were cryopreserved in Tes-Tris with $5 \%$ egg yolk and $6 \%$ glycerol, and selected by swim-up in SOF medium. After $24 \mathrm{~h}$ sperm-oocyte coincubation (sperm concentration: $1 \times 10^{6} / \mathrm{mL}$ ) in SOF with $2 \%$ estrus sheep serum under $5 \% \mathrm{CO}_{2} 5 \% \mathrm{O}_{2} 90 \% \mathrm{~N}_{2}$, presumptive zygotes were transferred to SOF with $0.4 \% \mathrm{BSA}$ and amino acids under $5 \% \mathrm{CO}_{2}, 5 \% \mathrm{O}_{2}$ $90 \% \mathrm{~N}_{2}$ and cultured for 4 days. Oocytes and embryos were stained with Hoechst 33342 and propidium iodide $(1 \mu \mathrm{g} / \mathrm{mL}$ each) and visualized under a fluorescence microscope. A total of 35 oocytes were recovered from 56 punctured follicles $(62.5 \%)$. This recovery rate was similar to those in wildlife in earlier reports, but more studies are needed to improve hormonal stimulation and oocyte harvesting. Out of 29 cumulus-oocyte complexes matured in vitro, $3.5 \%$ were found at GV and $6.9 \%$ at MI; $20.7 \%$ were degenerated and $68.9 \%$ had advanced to MII. Fertilization and cleavage rates were $40 \%$ and $30 \%$, respectively, of matured oocytes. Out of eight zygotes, six showed cleavage (ranging from 2 to 8 cells). None of the developing embryos progressed to the blastocyst stage, suggesting the existence of a developmental block and the need to improve culture conditions. Although more trials will help to improve IVMFC, this study demonstrates for the first time the feasibility of in vitro fertilization with frozen-thawed semen of in vitro matured oocytes collected by OPU from FSH-stimulated endangered gazelles.
\end{abstract}

This work was supported by the Spanish Ministry of Education and Science (REN 2003-11587) and Acciones Integradas (HI20030336).

\section{QUALITY CHANGES IN POST-THAW SPRINGBOK (ANTIDORCAS MARSUPIALIS) EPIDIDYMAL SPERM MAINTAINED IN FERTILIZATION MEDIUM}

\author{
F. P. Chatiza ${ }^{\mathrm{A}, \mathrm{B}}$, G. M. Pieterse ${ }^{\mathrm{B}}$, and P. Bartels ${ }^{\mathrm{A}}$ \\ ${ }^{A}$ University of Johannesburg, Johannesburg, South Africa; \\ ${ }^{B}$ Wildlife Biological Resource Center of National Zoological Gardens of South Africa, Pretoria, South Africa
}

The availability of gametes from the cropping of excess wildlife species provides the opportunity for the advancement of knowledge into assisted reproductive technology for possible future conservation measures. Little is known about the longevity of springbok (Antidorcas marsupialis) spermatozoa maintained in fertilization medium. The aim of this project was to determine the quality changes of post-thawed springbok spermatozoa incubated in fertilization medium by measuring plasma membrane integrity over time. Testes $(n=12)$ were obtained from two geographically distinct free-ranging springbok populations in South Africa. Spermatozoa were flushed from the cauda epididymides within three hours of the animals' death. Samples from an individual male were pooled, diluted to $400 \times 10^{6} \mathrm{sperm} / \mathrm{mL}$ with Biladyl (Minitüb, Tiefenbach, Germany) fraction A (no glycerol) and equilibrated in a water bath for $6 \mathrm{~h}$ at $4{ }^{\circ} \mathrm{C}$. An equal volume of Biladyl fraction B (containing $12 \%$ glycerol) was added to the sample to make a final concentration of $200 \times 10^{6} \mathrm{sperm} / \mathrm{mL}$. Samples were loaded into $0.25-\mathrm{mL}$ straws and frozen in liquid nitrogen vapor $(5 \mathrm{~cm}$ above the liquid nitrogen level) for $20 \mathrm{~min}$ after which they were plunged into liquid nitrogen. Straws from each sample were thawed for $20 \mathrm{~s}$ at $36^{\circ} \mathrm{C}$ in a water bath. Thawed spermatozoa $(100 \mu \mathrm{L}$ ) was added to $1 \mathrm{~mL}$ IVF-TALP medium containing heparin and PHE (Vajta et al. 1996 Theriogenology 45, 683-689) in 2-mL Nunc tubes (AEC, Amersham, South Africa) and incubated at $38.7^{\circ} \mathrm{C}$, in humidified $5 \% \mathrm{CO}_{2}$ balance air for $30 \mathrm{~h}$. Aliquots were extracted from the incubating spermatozoa to determine plasma membrane integrity at 6-h intervals. Propidium iodide (Sigma, South Africa) at $50 \mathrm{ng} / \mathrm{mL}(10 \mathrm{~min}$ at RT) was used to evaluate membrane integrity under fluorescence microscopy at $\times 400$, with a 450 -nm excitation filter, a 510-nm dicroic beam splitter, and a 520-nm barrier filter. Cells with damaged plasma membrane have nuclei that fluorescence red. Eosin/nigrosin was also used to evaluate membrane integrity under $\times 400$ bright-field microscopy. Cells with damaged plasma membrane stain purple-red, whereas the balance of cells remain translucent. The average post-thaw motility of spermatozoa in populations A and B was $69 \%(n=6)$ and $68 \%(n=6)$, respectively. Plasma membrane integrity of post-thawed springbok spermatozoa deceased steadily in IVF-TALP medium over the 30-h period (Table 1). Cryopreserved epididymal sperm derived from free-ranging springbok populations survive in IVF-TALP media and may be useful in future conservation activities where an isolated gene pool requires genetic supplementation through one or more assisted reproduction techniques such as IVF or AI. Further research is required to confirm and extend these findings. 
Table 1. Percentage plasma membrane integrity of post-thawed springbok sperm over time

\begin{tabular}{llccllll}
\hline Characteristic measured & Population & & & & & & \\
\hline Time (hours) & & 0 & 6 & 12 & 18 & 24 & 30 \\
Propidium iodide & A & 50.2 & 42.7 & 36.8 & 28.0 & 12.1 & 3.6 \\
& B & 67.2 & 54.5 & 44.2 & 32.4 & 11.8 & 2.6 \\
Eosin/nigrosin & A & 77.5 & 58.1 & 33.1 & 26.1 & 26.1 & 13.2 \\
& B & 76.6 & 50.6 & 34.8 & 25.1 & 20.8 & 12.3 \\
\hline
\end{tabular}

\title{
218 EJACULATE TRAITS OF THE NAMIBIAN CHEETAH (ACINONYX JUBATUS)—INFLUENCE OF ANIMAL AGE, SEASON, AND CAPTIVITY
}

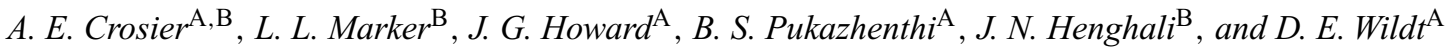 \\ A Smithsonian's National Zoological Park Conservation and Research Center, Washington, DC 20008, and Front Royal, VA 22630, USA; \\ ${ }^{\mathrm{B}}$ The Cheetah Conservation Fund, Otjiwarongo, Namibia
}

Wild cheetahs are threatened with extinction, and ex situ populations are not self-sustaining due to poor reproductive efficiency. Sperm cryopreservation is a valuable tool for genetic management; however, increased knowledge of ejaculate traits is essential to improve cryopreservation protocols. The objective of this study was to characterize ejaculate traits of wild-born cheetahs in Namibia, Africa. Specifically, the influences of animal age, season and captive status on electroejaculate volume, sperm concentration, motility, forward progressive status (FPS scale $0-5,5=$ best), morphology, and acrosomal integrity were evaluated. Animal age was divided into categories: juvenile (14-24 mo; $n=16$ males, 23 ejaculates), adult ( $25-120 \mathrm{mo} ; n=76$ males, 175 ejaculates), and aged (over $120 \mathrm{mo} ; n=5$ males, 5 ejaculates). Namibian seasons were divided into hotwet (Jan-Apr), cold-dry (May-Aug) and hot-dry (Sep-Dec). Cheetahs were considered wild-caught ( $n=29$ males; 44 ejaculates) if trapped on farmland $\leq 30$ days before semen collection. Raw ejaculates contained $69.0 \pm 1.1 \%$ motile sperm (mean \pm SEM) and $73.6 \pm 1.5 \%$ sperm with intact acrosomes. Overall, $18.4 \pm 0.9 \%$ of sperm were morphologically normal, with midpiece abnormalities being the most prevalent defects $(\sim 39 \%)$. To determine treatment differences, data were analyzed by General Linear Model procedures and means were separated with Duncan's multiple-range test. Juvenile cheetahs produced ejaculates with reduced $(P<0.05)$ sperm motility $(56.7 \pm 3.3 \%)$ and FPS $(2.9 \pm 0.1)$ compared to adult $(69.8 \pm 1.4 \%$ and $3.4 \pm 0.1$, respectively) and aged $(78.9 \pm 6.7 \%$ and $3.7 \pm 0.3$, respectively) animals. Ejaculates from juvenile animals also had reduced $(P<0.05)$ volume $(0.69 \pm 0.3 \mathrm{~mL})$ and fewer $(P<0.05)$ total motile sperm $\left(7.1 \pm 9.3 \times 10^{6}\right)$ compared to adult $(2.2 \pm 0.1 \mathrm{~mL}$ and $\left.42.3 \pm 4.1 \times 10^{6}\right)$ and aged $\left(2.3 \pm 0.6 \mathrm{~mL}\right.$ and $23.5 \pm 20.0 \times 10^{6}$, respectively) males. For all ejaculates combined, seminal quality was poorest during the hot-dry season with lower $(P<0.05)$ sperm motility and intact acrosomes as well as an increased $(P<0.05)$ percent of sperm with head abnormalities. Ejaculates from captive cheetahs $(n=68$ males, 159 ejaculates $)$ had increased $(P<0.05)$ volume $(2.0 \pm 0.2 \mathrm{~mL})$ and intact acrosomes $(80.1 \pm 3.6 \%)$, but lower $(P<0.05)$ sperm density $\left(14.3 \pm 3.9 \times 10^{6} / \mathrm{mL}\right)$ than wild-caught animals $(1.5 \pm 0.3 \mathrm{~mL}, 71.9 \pm 4.6 \%$, and $24.1 \pm 5.1 \times 10^{6} / \mathrm{mL}$, respectively). These are the first large-scale data acquired to examine the reproductive biology of male cheetahs in Namibia, including those recently captured from the wild. Results reveal that this species demonstrates seasonal and age-based variations in ejaculate quality, and that all individuals (including those recently derived from the wild) produce unusually high proportions of pleiomorphic spermatozoa. These data are being used to select the ideal donor age and season during which spermatozoa should be collected for addition to a genome resource bank, thereby enhancing effective genetic management for cheetahs propagated ex situ.

\section{MORPHOMETRIC CHARACTERIZATION OF EPIDIDYMAL AND EJACULATED SPERMATOZOA FROM BROWN BEAR (URSUS ARCTOS)}

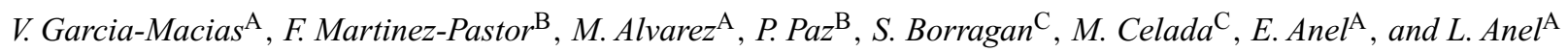 \\ ${ }^{\mathrm{A}}$ Reproduction and Obstetrics, University of León, León, Spain; ${ }^{\mathrm{B}}$ Cell Biology, University of León, León, Spain; \\ ${ }^{\mathrm{C}}$ Cabarceno Park, Cantabria, Spain
}

Sperm morphology is an useful characteristic for estimating potential fertility. Currently, we are obtaining baseline information on various aspects of reproduction in the brown bear (Ursus arctos) with the intention of using the knowledge to establish a germplasm bank for the species. In the present report, we describe the results obtained using assisted sperm morphology analysis (ASMA, Sperm Class Analyzer ${ }^{\circledR}$; Microptic S.L, Barcelona, Spain) to analyze the morphological differences in epidydimal (caput, corpus, and cauda) and ejaculated brown bear spermatozoa. A post-mortem epididymal sperm sample was obtained from an adult brown bear after accidental death. The epididymides were excised, washed, and dissected into the three major segments; caput, corpus and cauda. Then multiple incisions were made in the tissue to allow migration of spermatozoa into the surrounding medium. Semen was collected by electroejaculation from five adult brown bears living in a semi-free ranging environment in the Cabarceno Park (Cantabria, Spain). Anesthesia was induced using tiletamine + zolazepan (Zoletil 100 ${ }^{\circledR}$; Virbac, Carras, France; $7 \mathrm{mg} / \mathrm{kg}$ ), and ketamine (Imalgene $1000^{\circledR}$; Rhone Merieux, Lyon, France; $2 \mathrm{mg} / \mathrm{kg}$ ). The electroejaculation unit (PT Electronics ${ }^{\circledR}$; Boring, Oregon) was connected to a 3-lateral electrode transrectal probe ( $26 \mathrm{~mm}$ in diameter, $320 \mathrm{~mm}$ in length). Ejaculation occurred at 6-10 V/250-300 mA. For head morphometry assessment, sperm samples were fixed in glutaraldehyde and slides were smeared and air-dried for $2 \mathrm{~h}$. The samples were then stained with Diff-Quik ${ }^{\circledR}$ 
staining $\left(37^{\circ} \mathrm{C} ; 10 \mathrm{~min}\right.$ in the red component and $15 \mathrm{~min}$ in the blue component). The area, perimeter, length and width, and ellipticity (length/width) of heads were measured from at least 100 spermatozoa/slide. As shown in Table 1, values obtained for each measure were similar in both epididymal and ejaculated spermatozoa. These results provide normal morphometry values for brown bear spermatozoa, a potentially useful characteristic for predicting fertility.

Table 1. Head morphometry for epididymis and ejaculated bear sperm (mean \pm SD)

\begin{tabular}{lccccc}
\hline & Length $(\mu)$ & Width $(\mu)$ & Area $(\mu)$ & Perimeter $(\mu)$ & $\begin{array}{c}\text { Ellipticity } \\
\text { (length/width) }\end{array}$ \\
\hline Caput & $5.77 \pm 0.45$ & $4.44 \pm 0.33$ & $20.94 \pm 2.69$ & $17.49 \pm 1.09$ & $1.30 \pm 0.11$ \\
Corpus & $5.39 \pm 0.36$ & $4.33 \pm 0.16$ & $19.54 \pm 1.28$ & $17.11 \pm 0.59$ & $1.25 \pm 0.08$ \\
Cauda & $5.44 \pm 0.17$ & $4.35 \pm 1.41$ & $19.90 \pm 0.62$ & $16.96 \pm 0.07$ & $1.25 \pm 0.02$ \\
Ejaculated & $5.87 \pm 0.39$ & $4.32 \pm 0.21$ & $21.42 \pm 1.69$ & $17.73 \pm 0.81$ & $1.36 \pm 0.09$ \\
\hline
\end{tabular}

This work was supported in part by CANTUR S.A. and CICYT (CGL 2004-0278/BOS).

\title{
220 LIVE BIRTH OF A MOHOR GAZELLE (GAZELLA DAMA MHORR) CALF FOLLOWING INTRAUTERINE INSEMINATION OF MOTHER WITH FROZEN-THAWED SEMEN
}

\author{
J. J. Garde ${ }^{\mathrm{A}}$, M. Gomendio ${ }^{\mathrm{B}}$, G. Espeso ${ }^{\mathrm{C}}$, and E. R. S. Roldan ${ }^{\mathrm{B}}$ \\ A IREC, Universidad de Castilla-La Mancha, 02071-Albacete, Spain; ${ }^{\mathrm{B}}$ Museo Nacional de Ciencias Naturales (CSIC), 28006-Madrid, Spain; \\ ${ }^{\mathrm{C}}$ Estacion Experimental de Zonas Aridas (CSIC), 04001-Almeria, Spain
}

Gazella dama mhorr is an endangered species, and no animals have been observed in the wild since 1968. Assisted reproductive techniques have been used to propagate endangered species. However, no live offspring has been produced after cryopreservation of semen from gazelle species. Therefore, the objective of this study was to evaluate whether cryopreserved Mohor gazelle spermatozoa can fertilize in vivo after artificial insemination. Semen was collected by electroejaculation from four males and centrifuged at $700 \mathrm{~g}$ for $5 \mathrm{~min}$ at room temperature. The supernatant was discarded, and the semen pellet was resuspended with a TEST- $1 \%$ egg yolk diluent containing $6 \%$ glycerol to provide $400 \times 10^{6}$ spermatozoa $/ \mathrm{mL}$. The extended semen was loaded into 0.25 -mL plastic straws, cooled slowly to $5^{\circ} \mathrm{C}$, and equilibrated at $5^{\circ} \mathrm{C}$ for $2 \mathrm{~h}$. Straws were frozen in nitrogen vapors for $10 \mathrm{~min}$ and then plunged into liquid nitrogen. After thawing $\left(37^{\circ} \mathrm{C}, 30 \mathrm{~s}\right)$, the effects of cryopreservation on sperm motility and acrosomal integrity were examined. Percentage of motile sperm in fresh samples was $88.7 \pm 3.8 \%$ (mean \pm SEM), decreased $(P<0.0001)$ to $58.7 \pm 3.8 \%$ after freezing and thawing, and then to $40.0 \pm 3.8 \%$ after $120 \mathrm{~min}$ incubation at $37^{\circ} \mathrm{C}$. Spermatozoa with normal acrosomes decreased $(P<0.0001)$ after freezing and thawing (from $94.5 \pm 4.2 \%$ to $56.0 \pm 4.2 \%$ ), but did not significantly decrease after sperm incubation. Frozen spermatozoa from two males were used in an intrauterine insemination trial. Estrus of females $(n=15)$ was synchronized with controlled internal drug release (CIDR, InterAg, Hamilton, New Zealand). Single CIDRs (type G, $330 \mathrm{mg}$ progesterone/device) were inserted intravaginally for a total of 13 days. On Day 10 , devices were replaced in each animal and all females received an injection of prostaglandin $\mathrm{F}_{2 \alpha}$ ( $\left.\mathrm{PGF}_{2 \alpha} ; 125 \mathrm{mg} / \mathrm{female}\right)$. At CIDR withdrawal, females received 250-300 IU equine chorionic gonadotropin (eCG: Folligon; Intervet, Salamanca, Spain). After anesthesia with an intravenous injection of xylazine hydrochloride (Rompun; Bayer, Madrid, Spain; $0.8 \mathrm{mg} / \mathrm{kg}$ live weight) and ketamine hydrochloride (Imalgene; Leti \& Merieux, Madrid, Spain; $2.0 \mathrm{mg} / \mathrm{kg}$ live weight), eight females were inseminated with $100 \times 10^{6}$ frozen-thawed spermatozoa $56-57 \mathrm{~h}$ after removal of the CIDRs, and seven were inseminated after 64-65 h. Females were inseminated directly into the uterus using laparoscopy. Anesthesia was reversed with yohimbine hydrochloride $(0.3 \mathrm{mg} / \mathrm{kg}$ live weight). One female in the second group became pregnant. After a 202-day gestation, the female gave birth to one healthy Mohor gazelle male calf. These results demonstrate for the first time the successful use of frozen-thawed semen by means of artificial insemination for the rescue of endangered gazelle species. However, our results reveal that a number of unresolved technical problems remain to be addressed.

This work was supported by the Spanish Ministry of Education and Science (REN2003-1587).

\section{BASAL SEMINAL TRAITS AND IN VITRO FERTILIZATION IN THE SAND CAT (FELIS MARGARITA)}

\author{
J. Herrick ${ }^{\mathrm{A}}$, K. Leiske $^{\mathrm{B}}$, G. Magarey ${ }^{\mathrm{A}}$, and W. Swanson ${ }^{\mathrm{A}}$ \\ ${ }^{A}$ Center for Conservation and Research of Endangered Wildlife, Cincinnati Zoo and Botanical Garden, Cincinnati, \\ OH 45220, USA; ${ }^{B}$ The Living Desert Zoo and Gardens, Palm Desert, CA 92260, USA
}

The sand cat (Felis margarita) is one of five small-sized cat species given priority for conservation in North American zoos. An improved understanding of sand cat reproductive biology would benefit captive breeding and facilitate use of assisted reproduction for genetic management. In this study, our objectives were to: (1) characterize basal seminal traits, (2) assess ovarian responses to exogenous gonadotropins, and (3) compare Ham's (HF10) F-10 with 5\% fetal bovine serum (FBS) and feline optimized culture medium (FOCM) with $0.4 \%$ BSA for supporting gamete function and embryonic development in vitro. Semen was collected by electroejaculation from seven males $\left(n=10\right.$ ejaculates), washed, and resuspended $\left(10 \times 10^{6}\right.$ motile sperm $/ \mathrm{mL})$ in $\mathrm{HF} 10$ or FOCM for culture $\left(6 \% \mathrm{CO}_{2}\right.$ in air at $\left.38.7^{\circ} \mathrm{C}\right)$. Sperm motility ( $\%$ motile and rate of forward progress, $0-5$ scale) was evaluated at $0,1,3$, and $6 \mathrm{~h}$ of culture and used to calculate a sperm motility index (SMI; $[\%+(5 *$ rate) $] / 2)$. Acrosomal integrity was evaluated by staining (fast 
green FCF-rose bengal) at 0 and $6 \mathrm{~h}$. For IVF, ovarian follicles were aspirated laparoscopically from female sand cats $(n=4)$ treated at random times of the estrous cycle with $150 \mathrm{IU}$ eCG and $100 \mathrm{IU}$ hCG $(84 \mathrm{~h}$ post-eCG) prior to oocyte recovery $(25 \mathrm{~h}$ post-hCG). Grade 1 oocytes were co-incubated with $2 \times 10^{5}$ motile sperm $/ \mathrm{mL}$ in HF10 $(n=32)$ or FOCM $(n=33)$ for $20 \mathrm{~h}$ before transfer to fresh medium. Resulting embryos were either cryopreserved $(n=42)$ at $30 \mathrm{~h}$ post-insemination (hpi) or cultured until Day 7 pi after being moved to fresh medium (FOCM with $5 \%$ FBS $(n=10)$ or HF10 $(n=6)$ ) on Day 3 pi. Ejaculates contained (mean \pm SEM) $43.5 \pm 11.0 \times 10^{6}$ total spermatozoa, with $77.0 \pm 2.3 \%$ motility, $43.8 \pm 3.9 \%$ normal morphology, and $93.1 \pm 1.3 \%$ intact acrosomes. During $6 \mathrm{~h}$ of culture, SMI and \% intact acrosomes declined $(P<0.05)$ slightly (SMI, $73.8-74.8$ at $0 \mathrm{~h}$ and $68.5-68.8$ at $6 \mathrm{~h} ; \%$ intact acrosomes, $87.1-87.6 \%$ at $0 \mathrm{~h}$ and $69.0-74.2 \%$ at $6 \mathrm{~h})$, but similarly $(P>0.05)$ in both media. Females produced $24.3 \pm 5.6$ follicles, with $19.3 \pm 5.1$ total oocytes and $16.5 \pm 4.6$ Grade 1 oocytes recovered per female. The proportions of oocytes cleaving at 20 and $30 \mathrm{hpi}$ and the quality of the resulting embryos at $30 \mathrm{hpi}$ were higher $(P<0.05)$ in FOCM $(20 \mathrm{hpi}, 76.5 \pm 8.7 \% ; 30 \mathrm{hpi}, 92.9 \pm 7.1 \%$; $89.2 \pm 7.9 \%$ Grade 1$)$ than in HF10 (20 hpi, $29.8 \pm 11.7 \% ; 30$ hpi, $55.9 \pm 20.6 \% ; 62.9 \pm 7.2 \%$ Grade 1$)$. Two blastocysts developed in FOCM (69.0 \pm 19.0 cells), but the final cell numbers of all cultured embryos were not different $(P>0.05)$ between FOCM $(26.9 \pm 8.0$ cells $)$ and HF10 (19.3 \pm 6.4 cells). Compared to other small felid species, sand cats exhibited excellent seminal traits, gonadotropin-induced ovarian responses, and fertilization success in vitro. Although sperm motility and acrosomal integrity were similar in FOCM and HF10, the medium developed specifically for domestic cat embryos (FOCM) better supported IVF and early embryonic development. These results indicate that IVF with fresh spermatozoa could be a valuable tool for genetic management of captive sand cat populations.

This work was supported by MAF \#D04ZO-72.

\title{
222 IN VITRO FERTILIZATION OF OOCYTES OBTAINED THROUGH TRANSVAGINAL OOCYTE RETRIEVAL FROM CYCLIC MURRAH BUFFALOES (BUBALUS BUBALIS)
}

\author{
R. S. Manik, M. S. Chauhan, V. Gupta, S. K. Singla, and P. Palta \\ Animal Biotechnology Center, National Dairy Research Institute, Karnal, Haryana, India
}

Very limited information is available in the literature on in vitro maturation (IVM), in vitro fertilization (IVF) and in vitro culture (IVC) of oocytes collected through the transvaginal oocyte retrieval (TVOR) technique in buffaloes. Therefore, the present study was undertaken to examine the post IVF cleavage rates and embryonic development up to the blastocyst stage in the Murrah breed of buffalo subjected to TVOR. The five cyclic Murrah buffaloes were synchronized for estrus by a single prostaglandin injection. The animals were subjected to TVOR once weekly for seven weeks. TVOR was performed using an ultrasound machine with a transvaginal convex transducer (5 MHz), a needle guide, a single-lumen 19-gauge 60 -cm-long needle, and a vacuum pressure of $50 \mathrm{mmHg}$. The number and size of follicles in each ovary was determined before puncture. The follicles were characterized on the basis of their diameter as small $(3-5 \mathrm{~mm})$, medium $(6-9 \mathrm{~mm})$, and large $(\geq 10 \mathrm{~mm})$. The oocytes recovered were classified as grade A, cumulus-oocytes complexes with $\geq 5$ layers of cumulus cells; grade B, those with two to four layers; grade C, partially denuded oocytes; and grade D, completely denuded oocytes. IVM, IVF and IVC were carried as reported by Chauhan et al. (1999 J. Dairy Science 82, 918-926). Briefly, the oocytes were cultured for $24 \mathrm{~h}$ in a $\mathrm{CO}_{2}$ incubator $\left(5 \% \mathrm{CO}_{2}\right.$ in air) at $38.5^{\circ} \mathrm{C}$ for in vitro maturation. Frozen-thawed semen was used in Bracket and Olyphant medium for capacitation and fertilization. The in vitro-fertilized and cleaved embryos were cultured further for 9 days in modified synthetic oviductal fluid. The small follicles constituted a major proportion (60\%) of the total observed follicles, although a substantial proportion of medium (19\%) and large (21\%) follicles were also present. A total of 76 oocytes were recovered by aspiration of 110 follicles, with an overall recovery rate of $70 \%$ (range $67-74 \%$ ). Of these, $45(59 \%)$ were of grades A and B, and $31(41 \%)$ were of grades $\mathrm{C}$ and D. The mean number of total follicles and the oocytes recovered per session did not differ significantly among individual donors. Out of the 37 oocytes subjected to IVM and IVF, 19 (51\%) cleaved at Day 2 post-insemination. A total of four embryos (11\%) developed into morulae/blastocysts. This study demonstrates the use of TVOR as a mean of obtaining oocytes, their fertilization, and further embryo development in the Murrah breed of buffalo.

\section{DESCRIPTION OF GENITALIA AND SPERM RECOVERED POSTMORTEM FROM A PYGMY SPERM WHALE, KOGIA BREVICEPS}

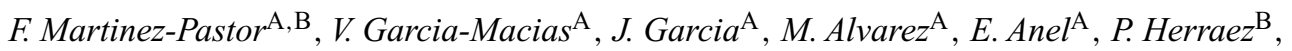 \\ P. de $P a z^{\mathrm{B}}$, and L. Anel ${ }^{\mathrm{A}}$ \\ A Animal Reproduction, University of León, León, Spain; ${ }^{\mathrm{B}}$ Cell Biology, University of León, León, Spain
}

A pygmy sperm whale (Kogia breviceps; adult male; $350 \mathrm{~kg}$ ) was stranded and died on a beach near Cabo Bustos (Asturias, North of Spain) on March 12th, 2005. Finding specimens of this species is a rare event on Spanish shores, although this whale is not considered endangered. Postmortem examination was performed $24 \mathrm{~h}$ later. Genitalia (testicles and epididymides) were extracted. The postmortem report indicated that vas deferens and seminal glands seemed to contain an important amount of semen, which was not recovered. Refrigerated genitalia were send to our laboratory, arriving around $40 \mathrm{~h}$ postmortem. The refrigerated testicles were in poor physical condition upon arrival, indicating advanced tissue detoriation The epididymides (very long) were not closely attached to the testicles, but were connected by a loose conjunctive membrane. We divided the epididymides into four regions that approximated the (1) caput, (2) mid-region, (3) corpus, and (4) cauda. Physical characteristics of the genitalia are described in Table 1 . The left testicle was larger, and possibly more active, than the right one. A sperm sample was obtained from the cauda region after incising the tissue. Osmolality and $\mathrm{pH}$ of the sample were $428 \mathrm{mOsm} / \mathrm{kg}$ and 6.62 , respectively (maybe due to tissue breakdown) and the sperm concentration was $1194 \times 10^{6} / \mathrm{mL}$. Spermatozoa were immotile, even after diluting in buffered medium; it is possible that postmortem damage occurred quickly. However, using flow cytometry we determined that $57 \%$ of cauda spermatozoa had intact plasma membranes and acrosomes (determined by staining with $37 \mathrm{mmol} / \mathrm{mL}$ propidium iodide and $1 \mu \mathrm{g} / \mathrm{mL}$ PNA-FITC; Sigma, Madrid, Spain). Examination by phase contrast 
microscopy $(\times 600)$ showed many spermatozoa with abnormal heads and bent midpieces and flagella, even in the cauda $(13 \%$ and $21 \%$, respectively). Sperm head morphometry was studied using DiffQuick staining and an automated analysis system (SCA2000; Microptic, Barcelona, Spain). Mean sperm head size was $3.71 \pm 0.19 \times 2.61 \pm 0.12 \mu \mathrm{m}$ in width and length, respectively. Computer analysis (AnalySiS-GmbH, Cologne, Germany) of phase contrast images revealed that the mean size of the sperm midpiece and flagellum were $3.44 \pm 0.19$ and $40.95 \pm 2.02 \mu \mathrm{m}$, respectively. The information obtained after postmortem recovery of the testes and epididymis should be useful to future conservation efforts of the pygmy sperm whale and similar species. The rapid deterioration of the testicular tissue by $40 \mathrm{~h}$ postmortem was not expected since good quality sperm samples have been obtained at similar postmortem intervals in other species. Therefore, we recommend that postmortem sperm recovery should be accomplished as rapidly as possible in this species.

Table 1. Genitalia measurements

\begin{tabular}{llll}
\hline Region & Parameter & Left & Right \\
\hline Testicles & Weight $(\mathrm{g})$ & 229.92 & 167.46 \\
& Length $\times$ width $\times$ breadth $(\mathrm{cm})$ & $22.8 \times 5.8 \times 4.2$ & $21.5 \times 4.7 \times 3.7$ \\
\multirow{5}{*}{ Epididymides } & Volume $\left(\mathrm{cm}^{3}\right)$ & 230 & 220 \\
& Weight $(\mathrm{g})$ & 31.42 & 23.76 \\
& Length $(\mathrm{cm})$ & 36 & 27 \\
& Region $1\left(\mathrm{~cm}^{*}\right)$ & $11.9 \times 1.0$ & $18.0 \times 1.0$ \\
& Region 2 $\left(\mathrm{cm}^{*}\right)$ & $8.9 \times 1.7$ & $8.3 \times 1.4$ \\
& Region 3 $\left(\mathrm{cm}^{*}\right)$ & $3.8 \times 1.6$ & Lost \\
& Region 4 $\left(\mathrm{cm}^{*}\right)$ & $11.0 \times 1.5$ & Lost \\
\hline
\end{tabular}

*Length $\times$ width.

\title{
224 OVARIAN FOLLICLE DEVELOPMENT DURING SEASONAL TRANSITION IN WAPITI
}

\author{
R. McCorkell, M. Woodbury, and G. Adams
}

Western College of Veterinary Medicine, University of Saskatchewan, Saskatoon, Saskatchewan, Canada

Wapiti are seasonally polyestrous. The transition into and out of the breeding season is marked by resumption of ovulation in autumn and cessation of ovulation in winter. Onset of ovulatory cyclicity is distinct and associated with aggressive breeding behavior of stags in rut. Cessation of ovulation at the end of the breeding season is not distinguished by behavioral patterns. The objective of the present study was to characterize follicular and luteal dynamics in wapiti during the transitional periods into and out of the breeding seasons. Transition from anestrus to estrus was monitored in 15 hinds, aged 2 to 14 years, over two successive seasons ( 11 in year 1 , with 5 hinds from year 1 used again in year 2 along with 4 new hinds; $n=20$ observations). Transition from estrus to anestrus was monitored in 11 hinds over 1 season ( $n=11$ observations). Hinds were maintained on a farm near Saskatoon, Saskatchewan $\left(52^{\circ} 07^{\prime} \mathrm{N}, 106^{\circ} 38^{\prime} \mathrm{W}\right)$. The ovaries were examined daily during September through October by transrectral ultrasonography using a B-mode ultrasound machine and a $7.5 \mathrm{MHz}$ linear array transducer for transition to estrus, and December through April for transition to anestrus. The first ovulation was recorded on September 15 and all hinds had ovulated for the first time by October 7 . In 17 of 20 observations, the duration of the first interovulatory interval (IOI) was $9.3 \pm 0.4$ days (mean \pm SEM). With one exception, these IOIs were characterized by one wave of follicular development. The remaining three IOIs ranged from 16 to 23 days and consisted of two or three waves of follicle development. The second ovulation occurred by October 15 in hinds with a short IOI and by October 17 in all remaining hinds. The mean dates of first and last ovulation were September 25 and February 7, respectively, an interval of 135 days. The median date of the last ovulation was February 15 and the range was from December 3 to March 22. Duration of the last IOI of the season (21.2 \pm 0.6 days) was similar to the notional 21-day cycle for wapiti, but longer $(P<0.05)$ than the duration of the first IOI $(10.9 \pm 1.0$ days $)$. Maximum diameters of the first 2 ovulatory follicles were similar $(11.3 \pm 0.4 \mathrm{vs}$. $11.3 \pm 0.2 \mathrm{~mm})$, but were larger $(P<0.05)$ than the last 2 ovulatory follicles of the breeding season $(10.3 \pm 0.3 \mathrm{vs} .10 .1 \pm 0.4 \mathrm{~mm})$. Maximum diameter of the corpus luteum $(\mathrm{CL})$ tended $(P=0.06)$ to be smaller for the short IOI than for longer IOI of the first and last cycles. Diameter of the last CL of the season was not different from that of the previous CL (12.8 $\pm 0.6 \mathrm{vs} .12 .5 \pm 0.6 \mathrm{~mm})$; however, it was detected for a longer period $(22.3 \pm 1.2$ vs. $19.3 \pm 0.7$ days; $P<0.05)$. Estrous cycles during transition into the breeding season have been described as being irregular and those out of the breeding season as increasingly long. In the present study, the transition periods were characterized by regular events. Transition to regular estrous cycles was preceded by one short ( 9 days) IOI. The last IOI of the breeding season was the same as that reported during the rut. Transition to anestrus occurred most commonly in February and was marked by a failure of the dominant follicle to ovulate after luteal regression.

\section{IN VITRO MATURATION OF LION OOCYTES}

\author{
B. Merlo, E. Iacono, G. Mari, and D. Zambelli
}

Veterinary Clinical department, University of Bologna, Ozzano Emilia, Bologna, Italy

The potential for rescuing immature oocytes from the ovaries of females of rare species of felids that die or undergo ovariohysterectomy has been reported in only two studies (Johnston et al. 1991 Biol. Reprod. 45, 898-906; Jewgenow et al. 1997 J. Reprod. Fertil. Suppl. 51, 33-39), in which oocytes were maturated in complex media (Eagle's MEM, M199, respectively). The domestic cat is used as a research model for endangered species 
but there may be some differences that perturb the adaptation of in vitro maturation (IVM), fertilization, or culture methods of lion oocytes compared to cat oocytes. Therefore, in the present study we evaluated the in vitro response of lion oocytes to our system for in vitro maturation of domestic cat oocytes in a simple medium (Merlo et al. 2005 Theriogenology 63, 2032-2039). A 14-year-old lioness, referred to our clinic because of pyometra, underwent ovariohysterectomy. After premedication with acepromazine $0.1 \mathrm{mg} / \mathrm{kg}$ i.m. (Prequillan; ATI, Bologne, Italy) and ketamine $5 \mathrm{mg} / \mathrm{kg}$ i.m. (Ketavet 100; Intervet, Boxmeer, The Netherlands), anesthesia was induced with ketamine $0.05 \mathrm{mg} / \mathrm{kg}$ i.v. and diazepam $0.02 \mathrm{mg} / \mathrm{kg}$ i.v. (Diazepam; Intervet) and maintained with isoflurane (Forane; Abbott, Rome, Italy) after intubation. Ovaries were removed and stored in saline solution at room temperature until collection of oocytes (within $1 \mathrm{~h}$ ). A total of 53 small follicles $(2-4 \mathrm{~mm}$ ), five corpora lutea and a 15 -mm follicle were present on the ovaries. Oocytes were collected by aspirating visible follicles of each ovary with a 21-ga needle connected to a vacuum pump (K-MAR-5100; Cook Australia, Brisbane, Australia) at $-75 \mathrm{mmHg}$; then CL were removed and the ovaries were minced with a scalpel blade in a 60 -mm petri dish containing HEPES-SOF (H-SOF) for recovery of additional oocytes. A total of 45 oocytes were recovered, of which 19 were degenerate (42.2\%); of the remaining 26, 12 were fully surrounded by cumulus cells, 9 had only corona radiata, and 5 were denuded. Degenerate oocytes were discarded and all other oocytes were washed twice in H-SOF and matured in a $35 \mathrm{~mm}$ petri dish containing SOFaaBSA $5 \mathrm{mg} / \mathrm{mL}$ plus $0.1 \mathrm{IU}$ of porcine FSH-LH (Pluset; Laboratorios Calier, Barcelona, Spain), $25 \mu \mathrm{L} / \mathrm{mL}$ insulin-transferrin-selenium (ITS) (Sigma, Madrid, Spain), $1.2 \mathrm{~mm}$ L-cysteine (Sigma), and $25 \mathrm{ng} / \mathrm{mL}$ epidermal growth factor (EGF) (Sigma) for $24 \mathrm{~h}$ in a humidified atmosphere of $5 \% \mathrm{CO}_{2}$ in air at $38.5^{\circ} \mathrm{C}$. After maturation, cumulus cells were removed by pipetting oocytes into a $0.25 \%$ trypsin solution for $2 \mathrm{~min}$. Then, denuded oocytes were washed once in $\mathrm{H}-\mathrm{SOF}$ plus $10 \%$ FCS to inactive trypsin and twice in H-SOF before being stained with Hoechst 33342 (10 $\mu \mathrm{g}$ in $10 \mathrm{~mL}$ PBS) for 30 min at room temperature After washing in PBS, oocytes were observed using fluorescence microscopy to determine maturation rate. Oocytes in telophase I or metaphase II were considered mature. Of 26 oocytes, $19(73.1 \%)$ were mature and $7(26.9 \%)$ were at the GV stage. These results demonstrated that lion oocytes can undergo successful IVM at a frequency that is similar to that of cat oocytes cultured in the same system $(76.9 \%, P>0.05)$. Furthermore, the maturation rate obtained in a simple medium was higher or similar to those previously reported (mean: $22.9 \%$ and $69.7 \%$ respectively, by Johnston et al. 1991 and Jewgenow et al. 1997).

\title{
226 BASIC CHARACTERISTICS OF CAPTIVE BROWN BEAR (URSUS ARCTOS) SPERMATOZOA COLLECTED BY ELECTROEJACULATION AND FROM THE POSTMORTEM EPIDIDYMIS
}

\author{
S. S. Pérez-Garnelo ${ }^{\mathrm{A}}$, C. Borque ${ }^{\mathrm{A}}$, M. Delclaux ${ }^{\mathrm{B}}$, C. Talavera $^{\mathrm{B}}$, E. Martínez $^{\mathrm{B}}$, \\ A. T. Palasz ${ }^{\mathrm{A}}$, and J. De la Fuente ${ }^{\mathrm{A}}$ \\ ${ }^{A}$ Departmento de Reproduccion Animal y Conservacion de Recursos Zoogeneticos, INIA, Madrid, Spain; \\ ${ }^{B}$ Zoo-Aquarium Madrid, Madrid, Spain
}

The objective of the present study was to describe the basic characteristics of brown bear spermatozoa collected by electroejaculation and from the cauda epididymis after necropsy. Semen was collected during the mating season of 2004 and 2005 from five mature males, aged from 3.5 to 22 years. Animals were anesthetized with 7 to $10 \mathrm{mg} / \mathrm{kg}$ body weight of Zoletil ${ }^{\circledR}$ (tiletamine + zolazepam; Virbac, Carros, France). Bears were placed on a special stretcher in ventral recumbency. A rectal probe with three longitudinal electrodes and a diameter of $2 \mathrm{~cm}$ (Electrojac IV; Minitübe, Tiefenbach, Germany) was placed into the rectum. Two series of stimuli were administered with a 2-3 min rest between series. Each stimulus lasted $2 \mathrm{~s}$ and was administered at $2 \mathrm{~s}$ intervals increasing intensity of stimulation from 0 to $15 \mathrm{~V}$. Of 23 electroejaculation attempts, 17 ejaculates were collected and two were eliminated because of poor semen quality. Epididymal spermatozoa were collected from three animals after euthanasia by flushing cauda epididymis with the freezing medium. Two animals showed unilateral testicular hypoplasia and only one testicle was processed from each of them. Volumes were recorded and concentration was evaluated in the laboratory. Semen quality of epididymal and ejaculated samples was assessed by: progressive sperm motility (\%IM); quality of movement on a scale of 0 to $5(\mathrm{Q})$; normal acrosome status (\%NAS) and normal sperm morphology (\%NOR), assessed by phase contrast microscopy of glutaraldehyde fixed samples; membrane response to hypo-osmotic test (\%HOST); and sperm viability estimated using eosin-nigrosin vital staining $(\% \mathrm{~V})$. Results were evaluated by Student $t$-test. Mean volume of ejaculates was $0.79 \pm 0.73 \mathrm{~mL}$. The average concentration of samples collected by electroejaculation was $519 \pm 278 \times 10^{6} \mathrm{sperm} / \mathrm{mL}$. The average number of spermatozoa collected per ejaculate was lower than the average number of spermatozoa collected from the epididymis, $476 \pm 352 \times 10^{6}$ vs. $640 \pm 164 \times 10^{6}$, respectively $(P>0.05)$. Sperm quality was not different between epididymal and electroejaculated samples (Table 1$)$, except that the $\%$ NOR was higher in electroejaculated samples than in epididymal samples $(62 \pm 16 \mathrm{vs} .3 \pm 15 ; P<0.01)$. A high percentage of spermatozoa with abaxial midpiece attachment (78.6 $\pm 10.4 \%)$ was observed in all samples. All electroejaculates contained leukocytes and $53 \%$ of the ejaculates had agglutinated spermatozoa. All 15 ejaculate and all epididymal samples were frozen in TES-Tris extender containing $4 \%$ glycerol using a standard protocol for bull semen. Our results indicate that electroejaculated and epididymal brown bear spermatozoa may be of sufficient quality for use in AI programs to increase gene flow between isolated captive populations. Whether cryopreserved samples can be used for artificial breeding remains to be determined.

Table 1. Quality parameters of spermatozoa from ejaculated and epididymal samples (mean \pm SD)

\begin{tabular}{lcccccc}
\hline & \%IM & Q & \%NAS & \%NOR & \%HOST & $\% \mathrm{~V}$ \\
\hline Ejaculated $(n=15)$ & $76.3 \pm 10.9$ & $4.0 \pm 0.3$ & $82.0 \pm 8.5$ & $62.53 \pm 16.7^{\mathrm{a}}$ & $79.9 \pm 6.9$ & $92.9 \pm 3.5$ \\
Epididymal $(n=3)$ & $71.6 \pm 7.6$ & $4.0 \pm 0.0$ & $81.0 \pm 7.5$ & $23.3 \pm 15.2^{\mathrm{b}}$ & $72.0 \pm 21.6$ & $96.0 \pm 4.3$ \\
\hline
\end{tabular}

${ }^{\mathrm{a}, \mathrm{b}}$ Values with different subscripts within a column differ significantly $(P<0.01)$. 


\title{
227 REPRODUCTIVE ANATOMY OF THE FEMALE GREY SHORT-TAILED OPOSSUM (MONODELPHIS DOMESTICA): CONSIDERATIONS FOR DEVELOPING ARTIFICIAL INSEMINATION BY CATHETER
}

\author{
D. B. B. P. Paris \\ Institute of Biomedical \& Life Sciences, University of Glasgow, Glasgow, Scotland, UK
}

\begin{abstract}
Due to the variability in vaginal anatomy among marsupials, the female reproductive tract must be examined for each species prior to developing artificial insemination using catheters. The grey short-tailed opossum (Monodelphis domestica) is a nonseasonal, polyovular didelphid and, as in all marsupials studied, has two completely separate uteri each opening into an anterior vaginal expansion through two distinct cervices. In the opossum, however, a septum divides the anterior vaginal expansion into two vaginal culs-de-sac, two lateral vaginae connect the anterior vaginal culs-de-sac to the urogenital sinus and opening, and the median vagina is a transient pseudo-vaginal birth canal. In this study we examined the site for insertion of artificial insemination catheters and the distribution of fluids inseminated within the vaginal complex of the opossum. Reproductive tracts were examined from four similarly sized unpaired adult one- to two-year-old females. A $2.3 \mathrm{~mm}$ diameter silicon balloon HSG catheter or a $0.8 \mathrm{~mm}$ diameter malleable obturator (Cook Australia, Brisbane, Australia) was introduced at the urogenital opening and navigated through the urogenital sinus, lateral vaginae, and cervices and the depth of insertion noted. Second, $0.15 \mathrm{~mL}$ of $1 \%$ Methylene blue dye (Sigma, Poole, UK) was injected by HSG catheter into the anterior urogenital sinus and the extent of dye distribution in the tract noted. In all tracts, the HSG catheter easily reached the anterior urogenital sinus $(1.5 \pm 0.3 \mathrm{~cm}$; mean $\pm \mathrm{SEM})$, but would not pass into the lateral vaginae or bladder. The malleable obturator easily passed into the bladder or through each lateral vagina into the anterior vaginal cul-de-sac $(2.9 \pm 0.2 \mathrm{~cm})$. The obturator also passed through the each cervix into the opening of the uterus $(3.1 \pm 0.3 \mathrm{~cm})$, but the tract required manipulation to navigate it through the convoluted lateral vaginae. Dye injected into the anterior urogenital sinus was distributed throughout the lateral vaginae and anterior vaginal culs-de-sac. No dye was detected in the bladder or had passed through the cervices into the uteri. In two of three tracts studied, dye was observed to migrate between each anterior vaginal cul-de-sac across the posterior end of the median septum that divides them. In conclusion, the use of larger diameter catheters $(\sim 2.3 \mathrm{~mm})$ is recommended for unguided insemination of the anterior urogenital sinus in this species. Catheters less than $1 \mathrm{~mm}$ diameter require ultrasonic or endoscopic guidance to reach the uteri in order to avoid insemination into the bladder. Dye studies indicate that the median septum may not completely separate the cervices in the opossum, suggesting that inseminates delivered to one side of the tract may be able to fertilize oocytes on the contralateral side. In addition, the cervices appear to restrict inseminates from entering the uteri. Spermatozoa may thus be required to pass through the cervices under their own motility.
\end{abstract}

This work was funded by the European Commission under a Marie Curie Incoming International Fellowship. Catheters were provided by Cook Australia.

\section{COLLECTION AND EVALUATION OF SEMEN OF SLOTH (BRADYPUS TRIDACTYLUS)}

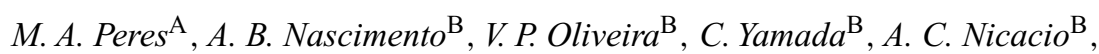 \\ J. A. Visintin ${ }^{\mathrm{B}}$, and M. E. O. A. Assumpção ${ }^{\mathrm{B}}$ \\ ${ }^{\mathrm{A}}$ Department of Anatomy, University of Sao Paulo - SP Brazil; ${ }^{\mathrm{B}}$ Animal Reproduction, University of Sao Paulo - SP Brazil
}

Sloths are animals that suffer with the destruction and fragmentation of forests. They experience a low population growth rate and need to be studied further for the preservation of the species. The objective of this study was to contribute data relevant to the reproductive physiology of this species, selecting a semen collection method and evaluating seminal characteristics that have never before been described in the literature. Fifteen Bradypus tridactylus males were captured in Manaus, Brazil. Nine of them were captured during the first half of 2004 (Group 1) and the others during the second half (Group 2). The animals were anesthetized with an i.m. injection of a combination of ketamine $(10 \mathrm{mg} / \mathrm{kg})$ and xylasine $(1 \mathrm{mg} / \mathrm{kg})$. Semen was collected by electroejaculaton using a rectal probe designed for domestic cats. Electrostimulations were given with a $0-100 \mathrm{~mA} / 0-12 \mathrm{~V}$ variable electrostimulator in sequences of three progressive intensities, with ten repetitions at each intensity and variation of $10 \mathrm{~mA}$ between them. They started with $20 \mathrm{~mA}$ and peaked at $60 \mathrm{~mA}$. Each stimulus lasted about $3 \mathrm{~s}$. It was not possible to define the best intensity of stimulus to use and ejaculation could take place at any time of the stimulation (Fisher's exact test). Sperm motility and vigor were immediately analyzed. Sperm count was determined in a Neubauer chamber at a 1:50 (v:v) dilution in formol-saline. Morphology was examined at the same dilution. Fresh semen smears were made and stained using Spermac Stain ${ }^{\circledR}$ (Minitüb, Tiefenbach, Germany) protocol for a better evaluation of the spermatozoa acrosome and midpiece. In both methods 200 cells were counted for morphological evaluation. All animals ejaculated approximately $30 \mu \mathrm{L}$ to $90 \mu \mathrm{L}$ of semen. In some ejaculates the semen was too thin and flowed down the penis, so that the volume effectively collected was not sufficient for a complete spermiogram. Spermatozoa presented a wide variety of defects, and some physical characteristics differed (not significantly) between samples collected during the first and second halves of the year. Motility and vigor were very low, the sperm did not show forward progression, only oscillatory movement. However, a high percentage $(80 \%)$ of spermatozoa were moving. The concentration in Group 1 ranged from 5000 spermatozoa $/ \mathrm{mm}^{3}$ to 685500 spermatozoa/mm 3 (mean $\pm 218571.4 \pm 242499.4$ ). Sperm concentation was not assessed in Group 2. The morphology of the head could be elongated or squared, or the head could have a base narrower than the apex. The tail showed a unique feature: the midpiece narrowed abruptly, forming a nip in its transition to the tail. This was similar in appearance to the segmental aplasia of the mitochondrial sheath, but it was considered normal because it was observed in all spermatozoa. Although further studies are necessary to standardize the semen evaluation of sloths and to define the best protocol for electroejaculation, this pioneering study has shown the characteristics of sloth spermatozoa and the possibility of collecting semen throughout the electroejaculation process in this species.

This work was supported by Fapesp 03/07457-4. 


\title{
229 OOCYTE RECOVERY, IN VITRO FERTILIZATION AND EMBRYO TRANSFER IN THE SERVAL (LEPTAILURUS SERVAL)
}

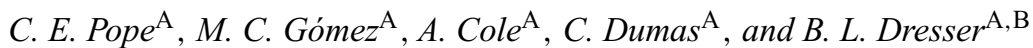 \\ A Audubon Center for Research of Endangered Species, New Orleans, LA 70178, USA; \\ ${ }^{B}$ Department of Biological Sciences, University of New Orleans, New Orleans, LA 70148, USA
}

\begin{abstract}
Servals are medium size ( 9 to $18 \mathrm{~kg}$ ) spotted cats found in sub-Saharan Africa that are protected by CITES under Appendix II regulations. There are at least six sub-species, one of which is listed as Endangered by the U.S. Endangered Species Act. In vitro-derived embryos have been produced in at least one-half of the 36 species of nondomestic cats, and kittens have been born after embryo transfer in six species. In the present study we evaluated (1) ovarian response of servals to repeated exogenous gonadotropin stimulation, and (2) in vitro and in vivo developmental ability of in vitro-derived embryos. One two-year-old and one five-year-old female were treated six and three times, respectively, over a 3.5-year period, with a total of 20 or 25 IU of porcine FSH (i.m.; Sioux Biochem, Sioux City, IA, USA) administered daily over four days during interestrus. On Day 5 , $15 \mathrm{IU}$ of porcine LH (i.m.; Sioux Biochem) was given, and laparoscopic oocyte retrieval was performed $24 \mathrm{~h}$ later. A total of 234 preovulatory oocytes were recovered: $182($ mean $=30.3)$ from the two-year-old and $52($ mean $=17.3)$ from the five-year-old female. A total of 91 and 91 oocytes were recovered at retrievals 1 through 3 and 4 through 6, respectively, from the two-year-old donor. Eighty oocytes from the two-year-old donor were inseminated with cooled $\left(24 \mathrm{~h}, 4^{\circ} \mathrm{C}\right)$ semen. Frozen semen from the same male was used to inseminate 102 oocytes from the two-year-old female and 52 oocytes from the five-year-old female. Overall, 136 embryos ( $58 \%$ cleavage frequency) were produced: 119 ( $65 \%$ cleavage frequency) from the two-year-old and 17 (33\% cleavage frequency) from the five-year-old female. Cleavage frequency of oocytes from the two-year-old female inseminated with cooled or frozen semen was similar, 68\% (54/80) and 64\% (65/102), respectively. Embryos were cultured for 5 or 6 days before controlled rate cryopreservation or uterine transfer (Gómez et al. 2003 Theriogenology 60, 239-251). On Day 5, 66 early to mid-stage morulae were cryopreserved at a slow controlled rate. Sixty Day 5 and 18 Day 6 embryos were auto-transferred to a recipient ( 8 to 26/transfer) in a total of six surgical procedures, of which five were with fresh embryos $(n=70)$ and one was with cryopreserved embryos $(n=8)$. The sixth embryo transfer procedure (26 fresh embryos) resulted in the unassisted birth of a live male kitten on Day 77 of gestation. We have shown that in vitro-derived embryos can be generated in the serval and that oocyte retrieval rates and cleavage frequencies are comparable to those reported for other species of mid-sized nondomestic cats. The nominal incidence of pregnancy and frequency of embryo survival may be improved by transferring early cleavage staged embryos into the oviduct, as demonstrated in the African wildcat (Felis silvestris lybica; Gómez et al. 2004 Cloning and Stem Cells 6, 247-258).
\end{abstract}

This work was partially funded by the Dan Heard Conservation Challenge Grant.

\section{IN VITRO MATURATION OF OOCYTES FROM ENDANGERED DORCAS GAZELLE (GAZELLA DORCAS NEGLECTA)}

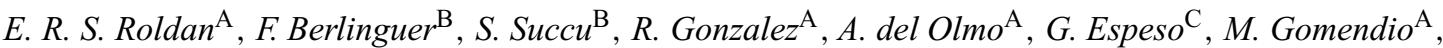 \\ and S. Ledda ${ }^{\mathrm{B}}$ \\ ${ }^{\mathrm{A}}$ Museo Nacional de Ciencias Naturales (CSIC), 28006-Madrid, Spain; ${ }^{\mathrm{B}}$ Università degli Studi di Sassari, 07100-Sassari, Italy; \\ ${ }^{\mathrm{C}}$ Estacion Experimental de Zonas Aridas (CSIC), 04001-Almeria, Spain
}

In vitro maturation of oocytes recovered from dead animals provides an opportunity for rescuing genetic material for biodiversity conservation. The dorcas gazelle (Gazella dorcas) is regarded by the World Conservation Union (IUCN) as 'vulnerable' but the subspecies G. dorcas neglecta is thought to be endangered due to excessive hunting. A captive breeding program for dorcas gazelles has been developed at the Estacion Experimental de Zonas Aridas (CSIC) in the South of Spain where efforts have so far concentrated on natural breeding and on the development of sperm cryopreservation protocols. The aim of the present study was to explore the possibility of recovering and maturing in vitro healthy oocytes from animals that die suddenly for the establishment of a program to rescue female gametes. Ovaries of a dorcas female that died unexpectedly were collected about $7 \mathrm{~h}$ after death of the animal. Cumulus-oocyte complexes (COCs) were recovered by slicing the ovaries. Collection and washing of COCs were performed in warmed TCM-199-HEPES with antibiotics and polyvinyl alcohol. Degenerated oocytes or those with expanded cumulus cells were removed. A total of 15 COCs were cultured in TCM-199 with $10 \%$ heat-treated fetal calf serum, $10 \mu \mathrm{g} / \mathrm{mL}$ ovine FSH/LH, $1 \mu \mathrm{g} / \mathrm{mL}$ estradiol, and $0.1 \mathrm{mg} / \mathrm{mL}$ glutamine at $38.5^{\circ} \mathrm{C}$ under $5 \% \mathrm{CO}_{2} /$ air with high humidity. After $24 \mathrm{~h}$ of culture, matured oocytes, as revealed by the presence of a polar body, were activated with 7\% ethanol for $10 \mathrm{~min}$ and further incubation for $3 \mathrm{~h}$. Meiotic progression and activation were evaluated by staining with Hoechst 33342 and propidium iodide $(1 \mu \mathrm{g} / \mathrm{mL}$ each) and visualization under a fluorescence microscope. Results at the end of incubations showed that $4 / 15$ oocytes were degenerated, $4 / 15$ were arrested at the MI stage, and 7/15 (46.7\%) progressed to the MII stage. One oocyte was found to be at the 2-cell stage but it could not be established whether this was the result of the activation method used. These results demonstrate that it is possible to recover viable oocytes several hours after death and rescue them for subsequent in vitro maturation and fertilization. More studies are needed to characterize suitable conditions for oocyte maturation, fertilization, and culture in the dorcas gazelle. This would, in turn, help in the effort to rescue biomaterials from wildlife for generating offspring. 


\title{
Folliculogenesis/Oogenesis
}

\section{CELLULAR PROLIFERATION IN FETAL OVARIAN FOLLICLES FROM LATE PREGNANT SHEEP FED MAINTENANCE OR RESTRICTED DIETS WITH NORMAL OR ENHANCED SELENIUM CONCENTRATIONS}

\author{
W. J. Arndt, A. Grazul-Bilska, J. S. Caton, E. Borowczyk, P. P. Borowicz, M. A. Ward, D. A. Redmer, \\ L. P. Reynolds, and K. A. Vonnahme \\ Department of Animal and Range Sciences, North Dakota State University, Fargo, ND 58102, USA
}

\begin{abstract}
Hypertrophy and hyperplasia are the major processes for tissue growth and development. The fetal ovaries represent a type of tissue that expresses high cellular proliferation rates. Selenium (Se) is a mineral that has diverse biological functions and affects cellular proliferation in numerous tissues including cancer, digestive tract, and placenta. It has been demonstrated that levels and sources (organic vs. inorganic) of Se may affect tissue growth. This experiment was designed to determine whether maternal consumption of differing levels of energy and Se impacts cell proliferation in fetal ovarian follicles. Sheep $(n=36)$ were fed a maintenance $(\mathrm{M} ; 2.12 \mathrm{Mcal} / \mathrm{kg})$ or energy restricted (ER; $60 \%$ of maintenance; nutrition restriction occurred from Day 50 to Day 135 of pregnancy) diet with high Se (HSe; $81.5 \mu \mathrm{g} / \mathrm{kg}$ body weight) or normal Se (NSe; $7.4 \mu \mathrm{g} / \mathrm{kg}$ body weight) concentration from 21 days before breeding to Day 135 of pregnancy. At slaughter on Day 135 of pregnancy, fetal tissues were collected and fixed in Carnoy's solution. Ovaries ( $n=3-6 /$ treatment group) were weighed, sectioned (one section along the longitudal axis/ovary) and stained for the presence of proliferating cell nuclear antigen (PCNA), a marker for proliferating cells. To determine the proportion of proliferating primordial follicles or the labeling index (percentage of proliferating cells; Jablonka-Shariff et al. 1994 Biol. Reprod. 51, 531) for primary, secondary, and antral follicles, digital images of the tissues were taken and analyzed using a computerized image analysis program (Image-Pro Plus; Media Cybernetics, Inc., Silver Spring, MD, USA). The primordial follicle was considered as proliferating when at least one granulosa cell was PCNA-positive. The number of proliferating and non-proliferating cells was determined for granulosa of primary follicles $(n=225$ total $)$ and for granulosa and theca cells of secondary $(n=198)$ and antral $(n=96)$ follicles, and used to calculate the labeling index. The data were analyzed using the general linear models procedure of SAS (SAS Institute, Inc., Cary, NC, USA). The number of proliferating primordial follicles was decreased $(P<0.05)$ by restricted energy diet and Se treatment ( $11.9 \pm 1.7$ for NSe-M diet vs. $7.2 \pm 1.3$ for HSe-M diet and $8.3 \pm 0.8$ for NSe-ER diet vs. $4.7 \pm 0.8$ for HSe-ER diet). However, energy restriction or Se did not affect labeling index in primary, secondary, and antral follicles. The labeling index was similar for theca and granulosa cells from secondary, or antral follicles. The labeling index was greatest $(P<0.05)$ for antral, less for secondary and least for primary follicles $(24.2 \pm 1.1 \%$ vs. $20.2 \pm 0.7 \%$ vs. $13.3 \pm 0.4 \%)$. These results demonstrate that both level of energy and Se in the maternal diet affects cellular proliferation in primordial but not in primary, secondary, or antral follicles in fetal ovaries. In addition, cellular proliferation increases as fetal follicular development progresses. These data indicate that level of energy and Se in the maternal diet may impact fetal ovarian development during the early stage of folliculogenesis.
\end{abstract}

This work ws supported by USDA grant 2005-35206-15281 and Hatch Project ND01712.

\section{EFFECT OF DIETARY FAT SUPPLEMENTATION ON OOCYTE AND EMBRYO QUALITY AND ON EARLY EMBRYONIC DEVELOPMENT OF SHEEP}

\author{
G. Cancino-Arroyo ${ }^{\mathrm{A}}$, R. Ake-López ${ }^{\mathrm{A}}$, J. Herrera $^{\mathrm{B}}$, F. Centurion $^{\mathrm{A}}$, and A. Ordoñez-León ${ }^{\mathrm{C}}$ \\ ${ }^{\mathrm{A}}$ Universidad Autónoma de Yucatan FMVZ, Mérida, Yucatan, México; ${ }^{\mathrm{B}}$ Universidad Michoacana de Sn Nicolas, Morelia, Michoacan, México; \\ ${ }^{\mathrm{C}}$ Universidad Nacional Autónoma de México, Mexico D.F. México
}

The objective was to evaluate the effect of fat supplementation on oocyte quality and in vitro embryonic development ( $48 \mathrm{~h}$ ). A total of 18 ewes, with a body condition score of 2.5 to 3 points out of 5 , having had three to four lamb births, and at three to four months post-lamb birth, were distributed between an experimental oil group (OG; $n=9)$ that received corn oil ( $4 \%$ of the MS/diet) and a control group (CG; $n=9)$ that didn't receive oil. The two groups were maintained in confinement for 21 days (the duration of the experiment) and fed first with a concentrate diet followed by forage. The animals had access to minerals and water ad libitum. The diets were similar in energy $(10.3 \pm 0.05 \mathrm{MJ} / \mathrm{s} / \mathrm{d})$ and protein $(141.75 \pm 5.7 \mathrm{gPC} / \mathrm{s} / \mathrm{d})$ for both groups. The estrous cycle was synchronized (14 days) with intravaginal sponges ( $40 \mathrm{mg}$ of fluorogestone acetate), inserted 7 days after the beginning of the diets. The end of the diet coincided with the retirement of the sponges. One day before sponge retirement, $75 \mathrm{mg}$ prostaglandin F2 $\alpha$ per sheep was administered, followed by ovarian stimulation with $1000 \mathrm{IU}$ of pregnant mare serum gonadotropin (PMSG). Follicular diameter was determined by ventral laparotomy with the aid of a micrometer. Follicles were classified as small ( 2 to $2.9 \mathrm{~mm})$, medium ( 3 to $4.9 \mathrm{~mm}$ ), and large (>5 mm); oocytes were collected in TCM-199 medium. Oocytes were classified as excellent, good, fair, or low quality and transferred to Petri dishes in drops $(50 \mathrm{~mL})$ of TCM-199. Oocytes were matured and fertilized in vitro and cultured for $48 \mathrm{~h}$. Oocyte quality as well as maturation, fertilization, and cleavage rates were compared by ANOVA. Ewes from the OG group presented a statistically higher proportion of oocytes with excellent quality $(42 \% ; P<0.05)$ than GT ewes $(26 \%)$. The proportion of good quality and fair quality oocytes was similar among groups $(P>0.05)$. A higher proportion of oocytes of low quality was found in the control group than in the OG group ( $40 \% \mathrm{vs.} 18 \%)$; however, there was no significant difference $(P>0.05)$. Higher rates of maturation, fertilization, and early development were found in the OG compared with the CG $(81.8,60.6$, and 36.4 vs. $68.6,42.9$, and 17.1 , respectively); however, the differences were not significant $(P>0.05)$. In conclusion, the addition of $4 \%$ corn oil in the diet improved the quality of the oocytes; however, it had no significant effect on early embryonic development. 


\title{
233 TEMPOROSPATIAL EXPRESSION PROFILES OF GROWTH AND DIFFERENTIATION FACTOR-9 AND BONE MORPHOGENETIC PROTEIN-15 GENES IN THE BOVINE OVARY
}

\author{
M. Hosoe ${ }^{\mathrm{A}}, K_{\text {. Kaneyama }}^{\mathrm{B}}, K_{\text {. Ushizawa }}^{\mathrm{A}}, S$. Akagi $^{\mathrm{C}}$, J. Noguchi $^{\mathrm{A}}$, and T. Takahashi ${ }^{\mathrm{A}}$

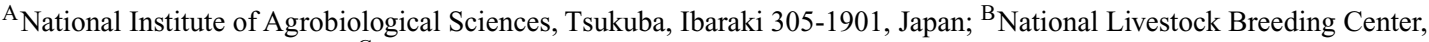 \\ Fukushima 961-1511, Japan; ${ }^{C}$ National Institute of Livestock and Grassland Science, Tsukuba, Ibaraki 305-1901, Japan
}

Bone morphogenetic protein (BMP) family proteins regulate the folliculogenesis and ovulation rate in mammals. Of the members of BMP family, growth and differentiation factor-9 (GDF-9) and BMP-15 are oocyte-derived proteins that play critical roles in follicular development. In the present study, we characterized the temporospatial expression patterns of GDF-9 and BMP-15 in bovine ovaries. Bovine ovaries were obtained from local abattoir. Cumulus-oocyte complexes (COCs) and mural granulosa cells were collected by aspiration of follicles $2-5 \mathrm{~mm}$ in diameter. Follicular aspiration was done the day following slaughter. The COC and mural glanulosa cells were separated and cultured for $20 \mathrm{~h}$ according to the protocol for oocyte maturation. Total RNA was isolated from COCs, cumulus cells, denuded oocytes, and mural granulosa cells and used for PCR. For in situ hybridization, collected ovaries were immediately fixed with $4 \%$ formaldehyde-PBS and embedded in a paraffin block. In situ hybridization was carried out with digoxigenin-labeled probes. In COCs and cumulus cells, the transcripts encoding GDF-9 and BMP-15 were detected immediately after follicular aspiration and still remained at the end of maturation culture. However, in mural granulosa cells, only GDF-9 expression was detected. Germ cell/oocyte markers, ZAR1 and VASA, were not detected in cumulus and mural granulosa cells. Quantitative real-time RT-PCR revealed extensive expressions of both GDF-9 and BMP-15 in oocytes. In situ hybridization study showed that GDF-9 and BMP-15 were co-localized to oocytes in primary, secondary, and antral follicles. The ovular co-expression of GDF-9 and BMP-15 appeared to commence at fetal life. Expression of GDF-9 and BMP-15 was not clear in cumulus cells possibly because of low expression level. In conclusion, our results show that GDF-9 and BMP-15 are co-localized to oocytes from an early stage of folliculogenesis. Since the transcripts encoding GDF-9 and BMP-15 are detected in cumulus cells, it is suggested that the oocyte is not the sole source of them. Additionally, the cumulus and mural granulosa cells are suggested to be functionally differentiated relative to BMP-15 expression.

\section{POTENTIAL SEASONAL INFLUENCE ON THE RECOVERY OF IN VIVO-MATURED CANINE OOCYTES BY FLUSHING FALLOPIAN TUBES}

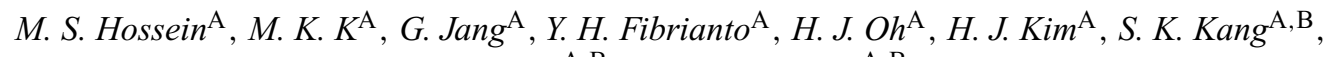 \\ B. C. Lee $\mathrm{A}^{\mathrm{B}}$, and W.S. Hwang $\mathrm{A}, \mathrm{B}$ \\ ${ }^{A}$ Department of Theriogenology and Biotechnology, College of Veterinary Medicine, Seoul, South Korea \\ ${ }^{\mathrm{B}}$ The Xenotransplantation Research Centre, Seoul National University Hospital, Seoul, South Korea
}

Reproductive physiology, especially the gamete physiology of bitches, hinders the success of in vitro maturation procedure. Assisted reproductive techniques (ART) in canine greatly depend on in vivo matured oocytes. The study was designed to evaluate the potential of flushing fallopian tubes to collect in vivo-matured oocytes for ART by laparotomy and to study the seasonal effects and the parity of donor bitches on the success of oocyte retrieval. A total of 124 bitches, 39, 44, 25, and 16 in spring, summer, autumn and winter, respectively, were used. The average temperature $\left({ }^{\circ} \mathrm{F}\right)$ and relative humidity (\%) were 55.29 and $57.17,75.67$, and $72.83,59.65$ and 64.67 , and 33.55 and 58.67 for spring, summer, autumn, and winter, respectively. Eighteen nulliparous and 18 multiparous bitches were used to determine the effect of parity. Vaginal cytology and serum progesterone concentration were checked daily to determine ovulation time. Laparotomy was performed under general anesthesia after $72 \mathrm{~h}$ when progesterone initially reached $4.0 \mathrm{~nm} / \mathrm{mL}$ or more. About $7 \mathrm{~mL}$ of TCM-199 supplemented with HEPES (Gibco, Grand Island, NY, USA) was used to flush each individual fallopian tube. After morphological assessment under a stereomicroscope, the oocytes were denuded in $0.5 \%$ (W/V) hyaluronidase solution (Sigma, St. Louis, MO, USA) and stained with $5 \mu \mathrm{g} / \mathrm{mL}$ bisbenzimide (Sigma). Nuclear status was then observed under ultraviolet light. Based on the nuclear stage, cumulus cell layers, color, and homogeneity of ooplasm, the oocytes were categorized as good, fair, poor, immature, or aged. The good and fair oocytes were considered usable and poor, aged, and immature oocytes were considered unusable for ART. In vivo-matured oocytes can be collected all year around by flushing fallopian tubes. A significantly higher average number of oocytes per bitch were observed during the spring $(11.21 \pm 0.56)$, compared to the other seasons $(7.88 \pm 0.63$ to $9.27 \pm 0.35)(P<0.05)$. A comparatively higher percentage of usable oocytes were collected during autumn. The percentage of oocyte recovery was $74.67,67.51,71.57$, and 62.70 for autumn, spring, summer, and winter, respectively $(P>0.05)$. Both the number and the quality of oocytes were influenced by the parity of the donor bitch. Significantly more oocytes were collected from the multiparous bitches compared to nulliparous bitches (10.4 \pm 0.54 and $7.7 \pm 0.86$, respectively). The percentage of usable oocytes was larger in multiparous bitches compared to nulliparous ( 70.75 and 64.74, respectively) $(P>0.05)$. Collection of in vivo-produced oocytes could be a potential source of matured oocytes for ART in canine.

\section{FOLLICULAR WAVE EMERGENCE AND DEVELOPMENT FOLLOWING ESTRADIOL PLUS PROGESTERONE AT A GROWING OR A STATIC PHASE OF THE DOMINANT FOLLICLE IN CIDR-TREATED, LACTATING DAIRY COWS}

\author{
U. H. Kim ${ }^{\mathrm{A}}$, G. H. Suh ${ }^{\mathrm{B}}$, and I. H. Kim ${ }^{\mathrm{A}}$ \\ ${ }^{\text {A }}$ College of Veterinary Medicine, Chungbuk National University, Cheongju, Chungbuk, Korea; \\ ${ }^{\mathrm{B}}$ National Livestock Research Institute, Cheonan, Chungnam, Korea
}

Treatment with estradiol benzoate (EB), compared to GnRH, in a controlled internal drug release (CIDR)-based timed AI protocol induced relatively asynchronous follicular wave emergence, resulting in ovulation of smaller ovulating follicles (Kim et al. 2005 Theriogenology 63, 260-268). In this 
study, we compared new follicular wave emergence and development following treatment with EB ( $2 \mathrm{mg}$ ) plus progesterone ( $50 \mathrm{mg})$ at a growing (Day 3 after ovulation) or a static (Day 8) phase of the dominant follicle during the first follicular wave in CIDR-treated, lactating dairy cows. Previously synchronized, lactating dairy cows received CIDRs (InterAg, Hamilton, New Zealand; Day 0) 3 days after ovulation and were assigned to two treatment groups and a control group. Cows in the T1 group $(n=11)$ received $2 \mathrm{mg}$ EB (SY Esrone; Samyang, Seoul, Korea) and $50 \mathrm{mg}$ P4 (SY Ovaron; Samyang) i.m., and those in the control group $(n=10)$ received no treatment at that time. Other previously synchronized cows received a CIDR (Day 0) with injections of $2 \mathrm{mg}$ EB plus $50 \mathrm{mg} \mathrm{P4}$ i.m. 8 days after ovulation (T2 group, $n=10$ ). Thereafter, all cows received $\mathrm{PGF}_{2 \alpha}$ (Lutalyse; Pharmacia \& Upjohn, Puurs, Belgium) at the time of CIDR removal on Day 7, and GnRH (Conceral; Dongbang Co., Seoul, Korea) on Day 9. Ovaries of each cow were examined by transrectal ultrasonography (Sonoace 600 with $7.5 \mathrm{MHz}$ linear-array transducer; Medison Co., Ltd, Seoul, Korea) every $24 \mathrm{~h}$ from Days 0 to 9, on Day 11, and on Day 14 of treatment to observe changes in ovarian structures (follicles and corpora lutea (CL)). Data were analyzed by Fisher's exact test, Kruskal-Wallis test, and ANOVA using an SAS program (SAS Institute, Inc., Cary, NC, USA). Follicular wave emergence occurred within 7 days in $3 / 10$ of the control group, $9 / 11$ of the T1 group, and $9 / 10$ of the T2 group $(P<0.05)$. The interval to wave emergence did not differ $(P>0.05)$ among groups $(3.7 \pm 1.2,4.8 \pm 0.5,5.9 \pm 0.3$ days, respectively). While the diameters of dominant follicles on Day 0 were greater $(P<0.01)$ in the T2 group $(14.3 \pm 1.2 \mathrm{~mm})$ than in the control $(9.4 \pm 0.6 \mathrm{~mm})$ and T1 $(10.5 \pm 0.7 \mathrm{~mm})$ group, diameters of preovulatory follicles on Day 9 were significantly smaller $(P<0.01)$ in the T1 $(13.6 \pm 0.7 \mathrm{~mm})$ and T2 $(12.3 \pm 0.5 \mathrm{~mm})$ groups than in the control group $(16.4 \pm 0.8 \mathrm{~mm}$ ). Synchronized ovulation by $40 \mathrm{~h}$ after the GnRH injection (Day 11) occurred in 10/10 of the control group, 10/11 of the T1 group, and 9/10 of the T2 group $(P>0.05)$. In conclusion, administration of $2 \mathrm{mg}$ EB plus $50 \mathrm{mg}$ P4 at both a growing and a static phase of the dominant follicle during the first follicular wave results in synchronous follicular wave emergence and development and also synchronized ovulation in CIDR-treated, lactating dairy cows.

\title{
236 NUCLEAR AND CYTOPLASMIC MODIFICATIONS OF GERMINAL VESICLE BOVINE OOCYTES IN RELATION TO CHROMATIN REMODELING
}

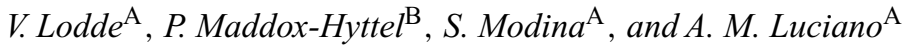 \\ ${ }^{A}$ Institute of Anatomy of Domestic Animals, Histology and Embryology, University of Milan, 20133 Milan, Italy; \\ ${ }^{B}$ Department of Animal and Veterinary Sciences, Royal Veterinary and Agricultural University, 1870 Frederiksberg C, Denmark
}

We previously reported that germinal vesicle (GV) bovine oocytes can be classified on the basis of their chromatin organization and that increased chromatin condensation is accompanied by a major incidence of gap junction-mediated coupling interruption between germ and cumulus cells and by an increase in oocyte developmental competence (Lodde et al. 2005 Reprod. Fertil. Dev. 17(2), 294-295). The aim of this study was to characterize, at the ultrastructural level, both nuclear and cytoplasmic compartments of bovine oocytes classified according to their chromatin configuration because key structural modifications, such as nucleolar inactivation and remodeling of specific ooplasmic structures, take place during the later phases of oocyte growth. Cumulus-oocyte complexes collected from 0.5-2-mm early antral (EA) and 2-6-mm mid-antral (MA) follicles were freed of cumulus cells. Denuded oocytes were stained with Hoechst 33342, classified according to the degree of chromatin condensation, and processed for light microscopy of semi-thin sections (LM; $n=10$ in each class) and transmission electron microscopy (TEM; $n=5$ in each class). Four classes of oocytes were identified by the Hoechst staining: GV0 with filamentous chromatin diffused in the nuclear area, GV1 with few foci of condensed chromatin, GV2 with chromatin further condensed into distinct clumps, and GV3 with chromatin condensed into a single clump. Almost all oocytes collected from EA follicles were classified as GV0. Oocytes of this class were absent in MA follicles, whereas class GV1, GV2, and GV3 oocytes occurred at similar frequency. LM confirmed the chromatin condensation found by the Hoechst staining and revealed that in class GV2 and GV3 oocytes the chromatin was mainly located close to the nucleolus. Ultrastructurally, the nucleolus was fibrillo-granular in GV0 oocytes; the oocytes in the other classes displayed an electron dense fibrillar sphere with the remnant of a fibrillar center on the surface. Organelles were dispersed in the cytoplasm at GV0 while at GV1 and GV2 most organelles were homogenously distributed in the oocyte cortex. At GV3 most organelles were found in clusters in the oocyte cortex. Typical features of completion of the oocyte growth phase, like undulation of the nuclear envelope and reduction of the size of Golgi complex, were found at GV2 and GV3. Moreover, GV3 oocytes presented cortical granules that displayed varying degrees of degeneration. Our findings indicate that the process of chromatin remodeling is strictly related to structural modifications that characterize the later stages of the oocyte growth phase. Because the highest degree of chromatin condensation was combined with degenerative features of cortical granules, we hypothesize that this class of oocytes (GV3) originated from early atretic follicles, as also suggested in other species. The evaluation of oocytes on the basis of chromatin configuration may be useful for the development of new strategies for manipulating fertility in mammals.

This work was supported by a COFIN Grant.

\section{EXPRESSION OF THE FULL-LENGTH AND ALTERNATIVELY SPLICED BOVINE LH RECEPTOR mRNAS IN GRANULOSA CELLS FROM FOLLICLES $\geq 7$ mm IN DIAMETER}

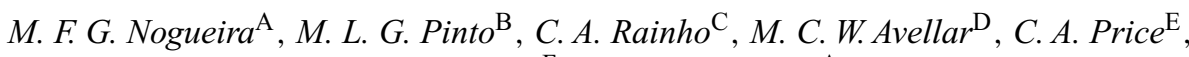 \\ J. Buratini $J r^{\mathrm{F}}$, and C. M. Barros ${ }^{\mathrm{A}}$
}

A Department of Pharmacology, Instituto de Biociências (IB), UNESP, Botucatu, São Paulo, Brazil; B Department of Animal Reproduction, FMVZ, UNESP, Botucatu, São Paulo, Brazil; ${ }^{C}$ Department of Genetics, IB, UNESP, Botucatu, São Paulo, Brazil; ${ }^{D}$ Department of Pharmacology, INFAR, UNIFESP-EPM, São Paulo, Brazil; ${ }^{\mathrm{E}}$ CRRA, Faculté de médecine vétérinaire, Université de Montréal, St-Hyacinthe, Québec, Canadà;

${ }^{\mathrm{F}}$ Department of Phisiology, IB, UNESP, Botucatu, São Paulo, Brazil

The objective of this study was to characterize the pattern of gene expression of LH receptor (LHR) transcripts from bovine antral follicles from 5 to $14 \mathrm{~mm}$ in diameter (theca and granulosa cells, TC and GC, respectively) in crossbreed cattle and from cultured GC. From ovaries collected in abattoir 
(Bos indicus $\times$ B. taurus cattle), antral follicles were dissected, and samples of TC and GC were obtained for total RNA extraction (Trizol protocol). Steroid concentrations in the follicular fluid were determined by RIA. Samples of GC cultured for 6 days (obtained from B. taurus follicles), were treated with FSH ( 0 (control), 1, or $10 \mathrm{ng}$ ) and processed for total RNA extraction. Total RNA ( $1 \mu \mathrm{g})$ was utilized in the RT reaction (SuperScript III; Invitrogen, Brasil Ltd., São Paulo, Brazil). The PCR conditions were 29 cycles of $95^{\circ} \mathrm{C}, 60^{\circ} \mathrm{C}$, and $70^{\circ} \mathrm{C}(1 \mathrm{~min}$ each $)$ for denaturation, annealing, and extension (PTC200 thermocycler; MJ Research, Biozym, Landgraaf, The Netherlands). Gene expression of LHR was measured by semiquantitative RT-PCR with specific primers to amplify the fragment from exon 9 to exon 11 (LHRBC; full-length amplicon with 1240 bp). To investigate a putative site of alternative splicing on exon 3 specific primers were utilized to amplify a fragment containing the exons 2 to 9 (LHRA). As an internal control of the PCR, GAPDH expression was used. After sequencing, four isoforms were detected from the LHRBC fragment- two with deletion of exon 10 (M2 and M4) and two with deletion of part of exon 11 (M3 and M4) - as well as the fragment without deletions (M1). There was no correlation $(P>0.05$; Spearman correlation) between LHRBC isoform expression and steroid hormones or follicular diameter (TC). However, estradiol and progesterone concentrations $(r>0.51$ and $P<0.01)$ and follicular diameter $(r>0.82$ and $P<0.01)$ were correlated with expression of the four LHRBC isoforms (GC). Expression of isoforms from fragment LHRBC was observed in GC from follicles with a diameter of $7 \mathrm{~mm}$. In six follicles with a diameter of $7 \mathrm{~mm}$, only one (16.7\%) expressed LHR in GC, whereas most of the follicles $\geq 8 \mathrm{~mm}$ expressed LHR (87.5\%, 21/24). No LHR isoforms were detected in GC from follicles $(n=7) \leq 6 \mathrm{~mm}$ in diameter. From LHRA fragment amplification, an alternative transcript with a deletion of $75 \mathrm{bp}$, homologous to the rat exon 3, was detected by sequencing. In cultured GC (without FSH treatment), only a weak LHR expression (in vitro control) was observed when compared with the in vivo control (TC sample). The treatment with FSH (1 or $10 \mathrm{ng}$ ) was effective to induce LHR expression in cultured GC, however, with a different pattern of expression (lower M1/M2 ratio) when compared to in vivo GC samples ( $0.8 \pm 0.14$ vs. $3.5 \pm 0.66$; mean \pm SEM, $P<0.01$, unpaired $t$-test). It is concluded that, in $\mathrm{GC}$ from follicles with a diameter $\geq 7 \mathrm{~mm}$, the gene expression of the LHR was positively correlated with follicular diameter and with estradiol and progesterone concentration in the follicular fluid. Treatment with FSH was effective in inducing LHR expression in cultured GC, however, with a different pattern than in the in vivo control. Additionally, sites of alternative splicing were detected in exons 3,10 , and 11 from the bovine LHR gene.

This work was supported by a Fellowship from FAPESP.

\title{
238 THE EFFECT OF TRANSVAGINAL FOLLICULAR ASPIRATION AND THE ROLE OF PROGESTERONE IN FOLLICULAR CYSTS OF DAIRY CATTLE
}

\author{
H. Ortegon ${ }^{\mathrm{A}}$, J. Petrik ${ }^{\mathrm{B}}$, J. S. Walton ${ }^{\mathrm{C}}$, and W. H. Johnson ${ }^{\mathrm{A}}$ \\ ${ }^{\mathrm{A}}$ Department of Population Medicine; ${ }^{\mathrm{B}}$ Department of Biomedical Sciences; ${ }^{\mathrm{C}}$ Department of Animal Sciences, University of Guelph, \\ Guelph, Ontario N1G 2W1 Canada. Email: hernan@uoguelph.ca
}

Ovarian follicular cyst (CYS) is a common condition affecting $5-25 \%$ of postpartum dairy cows, increasing the number of days open, calving intervals, cow costs, and culling rates, and with an effect on fertility rates. Current hormonal treatment has variable responses. Debate exists regarding progesterone (P4) influence on CYS formation and the effect of the aspirated CYS in resumption of cyclicity. The objectives of this descriptive study were to investigate the effect of ultrasound-guided transvaginal follicular aspiration on the resumption of follicular dynamics, to determine the fate of the aspirated CYS, and to find the P4 relationship. Resumption of ovarian cyclicity was determined by ovulation, formation of a corpus luteum (CL), and a P4 rise over $3.2 \mathrm{nmol} / \mathrm{L}$. Ten (10) postpartum (35-60 days in milk (DIM)) lactating Holstein cows with CYS were selected. They were monitored every other day by rectal palpation, ultrasonography, and $\mathrm{P} 4$ concentration for 30 days post-aspiration. Change in size of the aspirated CYS and emergence of a new follicular wave were recorded and images were saved electronically. Results were analyzed using Pearson correlations, linear regression, and one-way ANOVA. Of these cows, 5/10, 3/10, and 1/10 resumed cyclicity after the 1st, 2nd, and 3rd aspirations, respectively. Two (2/10) cows did not resume cyclicity during the observation period. Size of aspirated CYS was $34.6 \pm 0.2 \mathrm{~mm}$ (mean \pm SEM). Seven $(7 / 10)$ cows had intermediate $(0.98 \pm 0.3 \mathrm{nmol} / \mathrm{L})$ and $3 / 10$ cows had high $(8.6 \pm 3.6 \mathrm{nmol} / \mathrm{L}) \mathrm{P} 4$ concentration at aspiration. Mean times from aspiration to emergence of a new follicle, to ovulation, and to detection of a new CL were $3.6 \pm 1.4,10.5 \pm 2.0$, and $15.2 \pm 3.0$ days, respectively. New cysts grew in $4 / 10$ cows after the first aspiration and one aspirated CYS refilled twice. Dominant follicles present at first aspiration (DFPF) had the tendency to undergo cyst turnover (4/9) or to ovulate (2/9) at a larger size $(25.7 \pm 3.2 \mathrm{~mm})$. The CYS size was correlated $(r=0.58 ; P=0.05)$ with circulating $\mathrm{P} 4$ concentration. These data, together with a significant $(P=0.05)$ drop in $\mathrm{P} 4$ concentration following aspiration (from $3.26 \pm 1.5$ to $2.16 \pm 0.1 \mathrm{nmol} / \mathrm{L}$ ), suggest that the CYS may be the source of P4. Persistence of the aspirated cyst was associated $(r=0.87)$ with increased days to ovulation. The significant association $(r=0.96 ; P=0.01)$ between $\mathrm{P} 4$ at ovulation $(2.29 \pm 0.6 \mathrm{nmol} / \mathrm{L})$ and the size of the ovulatory follicle $(18.5 \pm 2.0 \mathrm{~mm})$ may explain the tendency of existing follicles to persist (cyst turnover) or to ovulate at a larger size. In summary, follicular aspiration was successful in inducing resumption of cyclicity and shortening days to ovulation in half of the cases with one aspiration. Persistence of the aspirated CYS increased days to ovulation. P4 may have a follicular origin and an influence on the size of the cyst and the ovulatory follicle. DFPF tend to undergo cyst turnover or to ovulate at a larger size. However, resumption of normal cyclicity may also be influenced by other factors, such as stress, disease, and energy balance.

\section{HORMONE PROFILE AND OVARIAN FOLLICLE DIAMETER IN EARLY POSTPARTUM LACTATING COWS}

\author{
S. J. Park, K. S. Baek, S. B. Park, B. S. Ahn, and H. S. Kim \\ National Lvestock Research Institute, Chunan, Chungnam, Korea
}

The purpose of this study was to assess the ovarian follicle diameter and hormone status during early postpartum in cows. Eighteen Holstein cows, aged 3.5-5.5 years and of 400-600 kg BW with normal calving, were examined every 3rd day starting from Day 15 until Day 33 postpartum. Blood 
samples for hormonal (progesterone (P4) and estradiol-17 $\beta$ (E2)) analyses were withdrawn from the coccygeal vein every 3rd day until the end of the experiment. The ovarian follicular number and size were verified and measured using a sector vaginal transducer ultrasound scanner (6.5 MHz probe, SA 600; Medison Co., Seoul, Korea). The least squares means ( \pm SEM) are presented for each day (GLM of SAS). In postpartum cows, E2 levels were $27.09 \pm 5.22 \mathrm{pg}, 24.50 \pm 5.76 \mathrm{pg}, 26.92 \pm 4.62 \mathrm{pg}, 25.53 \pm 3.74 \mathrm{pg}, 27.47 \pm 5.72 \mathrm{pg}, 26.48 \pm 8.06 \mathrm{pg}$ for Days 15, 18, 21, 24, 27, 30, and 33, respectively, $28.01 \pm 7.52 \mathrm{pg}$; P4 levels were $1.35 \pm 1.14 \mathrm{ng}, 2.41 \pm 1.70 \mathrm{ng}, 2.85 \pm 2.26 \mathrm{ng}, 2.88 \pm 1.90 \mathrm{ng}, 2.91 \pm 1.91 \mathrm{ng}, 3.04 \pm 2.31 \mathrm{ng}$, and $4.04 \pm 3.70 \mathrm{ng}$ for Days 15, 18, 21, 24, 27, 30, and 33, respectively. The ovarian follicle diameter was $12.36 \pm 3.31 \mathrm{~mm}, 11.91 \pm 2.99 \mathrm{~mm}$, $13.09 \pm 3.37 \mathrm{~mm}, 13.55 \pm 2.76 \mathrm{~mm}, 14.55 \pm 3.68 \mathrm{~mm}, 15.64 \pm 5.53 \mathrm{~mm}, 17.45 \pm 3.22 \mathrm{~mm}$ for Days $15,18,21,24,30$, and 33 , respectively. The present results show that mean plasma concentration of P4 and mean follicle diameter gradually increased from Day 15 to Day 33 postpartum. It is concluded that monitoring of $\mathrm{P} 4$ and $\mathrm{E} 2$ during early postpartum period would be useful to predict the ovarian starts for early rebreeding in dairy cows.

\title{
240 RELATIONSHIP BETWEEN PEAK NUMBER OF ANTRAL FOLLICLES AND FOLLICULAR WAVES, HORMONE CONCENTRATIONS, SUPEROVULATORY RESPONSE, AND EMBRYO QUALITY IN BEEF HEIFERS
}

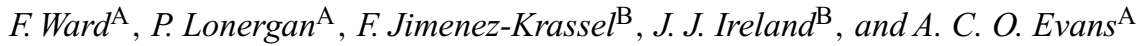 \\ A Department of Animal Science and Conway Institute, University College Dublin, Belfield, Dublin 4, Ireland; \\ ${ }^{\mathrm{B}}$ Molecular Reproductive Endocrinology Laboratory, Department of Animal Science, Michigan State University, \\ East Lansing, MI 48824, USA
}

Recent studies in dairy cattle demonstrate that the number of antral follicles during follicular waves is highly variable among animals, but highly repeatable within individuals, and the numbers of follicles during waves are inversely associated with serum FSH concentrations (Burns et al. 2005 Biol. Reprod. 73, 54-62). The aims of this study were to determine (1) serum FSH and estradiol concentrations during follicular waves in beef heifers categorized according to peak number of antral follicles per wave, and (2) the relationship between this categorization and superovulatory response. Estrous cycles of crossbred beef heifers $(n=90)$ were synchronized with two i.m. injections of PGF2 $\alpha 11$ days apart; animals were scanned daily for 5 days beginning $24 \mathrm{~h}$ after the second PG injection to determine the number of follicles during the first follicle wave in two or three consecutive estrous cycles. Heifers were grouped based on the peak number of follicles $\geq 3 \mathrm{~mm}$ per wave in diameter (Low: $\leq 15$ follicles, $n=12$; High: $\geq 25$ follicles, $n=11)$. Intermediate animals were excluded. Heifers were then re-synchronized and blood samples taken every $8 \mathrm{~h}$ up to Day 7 to characterize FSH and estradiol profiles. Subsequently, animals were superovulated (pFSH, Folltropin ${ }^{\circledR}$; Bioniche Animal Health, Belleville, Ontario, Canada), inseminated, and flushed on Day 7; this procedure was repeated twice. All values for hormone concentrations were aligned relative to the peak FSH value. A mixed model, repeated-measures approach was used to determine if serum FSH and estradiol concentrations were different between groups. Differences in recovery rate (number of oocytes/embryos recovered compared to number of corpora lutea) and proportion of transferable embryos were analyzed using Chi-square analysis. The mean $( \pm \mathrm{SE})$ peak postovulatory FSH concentration was lower $(P \leq 0.03)$ for animals with high numbers of follicles. No differences in estradiol concentrations were observed. The mean superovulatory response (number of corpora lutea) was higher $(P \leq 0.05)$ in the High group than in the low group $(17.6 \pm 3.6$ vs. $8.5 \pm 1.1)$. While there was no difference in the recovery rate of embryos ( 62 vs. $60 \%)$, significantly more $(P \leq 0.05)$ oocytes/embryos $(10.6 \pm 2.7$ vs. $4.7 \pm 0.7)$ and transferable embryos $(5.4 \pm 1.3$ vs. $3.8 \pm 0.8)$ were recovered per animal in the High group. Of the embryos recovered, the proportion of transferable quality was higher for animals in the Low group ( 80 vs. $51 \% ; P \leq 0.05)$. In conclusion, the number of antral follicles during a follicular wave in beef heifers is inversely related to peak postovulatory FSH concentration, but is unrelated to estradiol concentration. In addition, while the superovulatory response and number of transferable embryos were greater in animals with a high number of antral follicles, embryo quality, measured as the proportion of transferable embryos per donor, was reduced. We conclude that cattle with relatively high numbers of follicles per wave respond best to standard superovulation protocols. However, the reason the proportion of high quality embryos is reduced in cattle with high vs. low numbers of follicles per wave is unknown.

Gene Expression

\section{EFFECT OF INTRODUCTION OF THE INVERDALE FECUNDITY (FecX $\left.{ }^{\mathrm{I}}\right)$ GENE INTO A TEXEL $\times$ SCOTTISH HILL EWE (CHEVIOT) FLOCK ON LITTER SIZE, BIRTHWEIGHT, AND LAMB SURVIVAL}

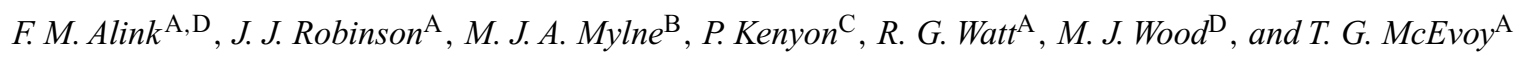

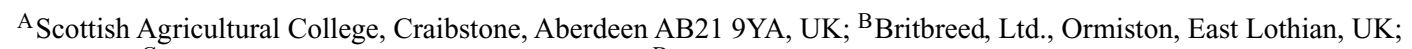 \\ ${ }^{\mathrm{C}}$ Harbro, Ltd., Turriff, Aberdeenshire, UK; ${ }^{\mathrm{D}}$ University of Aberdeen, Foresterhill, Aberdeen, UK
}

The naturally occurring Inverdale fecundity gene $\left(\mathrm{Fec}^{\mathrm{I}}\right)$, first identified in a Romney flock in New Zealand is located on the $\mathrm{X}$-chromosome and is a point mutation of the bone morphogenetic protein-15 (BMP-15) gene. Although homozygous carriers are infertile due to abnormal development (hypoplasia) of the ovaries, heterozygotes exhibit an increase in ovulation rate and numbers of lambs born, thus making the gene a candidate for improving prolificacy of crossbred hill ewes in a stratified hill and lowground sheep industry. Heterozygous Inverdale gene-carrying crossbred (Texel $\times$ Cheviot) ewes $(n=76)$ and noncarrier controls $(n=18)$ were mated by non-carrier Texel rams at approximately 18 months of age. Ultrasound 
pregnancy scanning took place at approximately 70 days of gestation. Lambing records comprised litter size and birthweight data and all losses, including mummified fetuses. Regression analysis tested effects of ewe genotype, pre-mating liveweight, and body condition score on litter size. ANOVA and Kruskal-Wallis tests were used to compare genotype and litter size category effects on dam and offspring data. Mean $( \pm$ SEM) litter size was $2.6 \pm 0.10$ and $1.9 \pm 0.11$ for Fec $\mathrm{X}^{\mathrm{I}}$ carrier and noncarrier ewes $(P<0.001)$. Although carrier ewes had lower pre-mating liveweights $(60 \pm 5.2$ vs. $63 \pm 3.8 \mathrm{~kg} ; P<0.05)$ and body condition scores $(3.1 \pm 0.29$ vs. $3.3 \pm 0.24 ; P<0.01)$ than noncarrier ewes, litter size was not affected by either factor. Ultrasound scanning was a good predictor $\left(r^{2}=0.86 ; P<0.001\right)$ of the eventual lamb crop. Incidences of singletons, twins, triplets and higher multiples were 8, 38, 42 and 12\%, respectively, among FecX ${ }^{\mathrm{I}}$ carrier dams. Corresponding incidences among noncarriers were 17, 78, 5, and $0 \%$. Peri- and neonatal losses increased with litter size. Of lambs from single, twin or triplet births, losses were $20 \%$ and $6 \%$ for FecX $\mathrm{X}^{\mathrm{I}}$ carrier and noncarrier ewe groups, respectively $(P=0.049)$. Of 32 quadruplets (mean live weight $=2.65 \mathrm{~kg}$ ), 9 did not survive; the sole set of quintuplets (mean $=1.52 \mathrm{~kg}$ ) also succumbed. Birth weights of lambs influenced survival $(P<0.001)$; among quadruplets, for example, survivors were $0.5 \mathrm{~kg}$ heavier than counterparts that died. Surviving triplets from FecX ${ }^{\mathrm{I}}$ carrier dams were $0.8 \mathrm{~kg}$ heavier than non-survivors $(3.66 \mathrm{~kg}$ vs. $2.86 \mathrm{~kg})$. Overall, in this study mortality was four times greater among lambs below $3 \mathrm{~kg}$ live weight than among their heavier counterparts. Consequently, further studies are needed to investigate how Inverdale gene-carrying ewes can be managed during the peri-conception period to optimize litter size, placental function, and prenatal growth for maximum lamb survival.

This work was funded by HIE, ANM Group, Britbreed, Ltd., and Harbro Ltd.; F. M. A. is a Genesis Faraday Associate and is sponsored by the SAC Trust, Harbro, Ltd., and Innovis.

\title{
242 POST-TRANSCRIPTIONAL REGULATION OF JY-1 mRNA ABUNDANCE DURING THE BOVINE OOCYTE-TO-EMBRYO TRANSITION
}

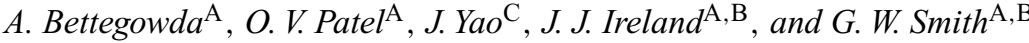 \\ ${ }^{A}$ Department of Animal Science, Michigan State University, East Lansing, MI 48824, USA; ${ }^{B}$ Department of Physiology, \\ Michigan State University, East Lansing, MI 48824, USA; ${ }^{\mathrm{C}}$ Division of Animal and Veterinary Sciences,
} West Virginia University, Morgantown, WV 26506, USA

\begin{abstract}
Oocyte-expressed genes play key roles in folliculogenesis and early embryonic development. The function of JY-1, a novel gene specifically expressed in bovine oocytes and early embryos, is unknown. We previously reported the expression pattern of JY-1 mRNA during meiotic maturation and early embryogenesis. The objective of this study was to elucidate the post-transcriptional regulation of JY-1 mRNA during oocyte maturation and early embryogenesis. For investigation of changes in length of JY-1 transcripts during the oocyte-to-embryo transition, total RNA isolated from germinal vesicle (GV) oocytes, metaphase II (MII) oocytes, and pronucleus (PN) stage embryos (300 oocytes/embryos per time point) was subjected to Northern blot analysis. Three major JY-1 transcripts of different length (approximately $1.8 \mathrm{~kb}, 1.2 \mathrm{~kb}$, and $700 \mathrm{bp}$ ) were detected in GV oocytes. The size of all transcripts was decreased at MII, and PN stages by approximately 100 to $200 \mathrm{bp}$. The intermediate sized transcript was predominant at GV, MII and PN stages. Based on these initial qualitative results focused solely on changes in transcript size, we then conducted more detailed quantitative studies (using real-time PCR) focused on characterization of temporal changes in abundance of polyadenylated versus total JY-1 transcripts during early development. Total RNA samples isolated from GV and MII oocytes and from embryos at PN, 2-cell, 4-cell, 8-cell, 16-cell, morula, and blastocyst stage ( $n=5$ pools of 10 oocytes/embryos per time point) were divided into two equal aliquots. One aliquot was reverse transcribed into cDNA with oligo dT primers for quantification of polyadenylated transcripts and the other aliquot was transcribed with random hexamers $(\mathrm{RH})$ for quantification of total transcripts. Amounts of polyadenylated JY-1 mRNA decreased during meiotic maturation $(P<0.0001)$, were increased $(P<0.05)$ at the PN and 4-cell stages relative to the MII stage, and then decreased to nearly undetectable levels after the 16-cell stage of embryo development. In contrast, amount of total JY-1 transcripts gradually decreased from PN through 16-cell stages to nearly undetectable levels thereafter. To confirm that the up-regulation of polyadenylated JY-1 mRNA in early developing embryos was not due to de novo transcription, alpha-amanitin was used to block the RNA polymerase II enzyme during the window of the first (24-13 h post-fertilization) or the second (33-14 h post-fertilization) embryonic cell cycle, and embryos at the 2-cell and 4-cell stages were collected ( $n=4$ pools of 10 embryos per time point). No significant changes were observed in abundance of JY-1 mRNA in control versus alpha-amanitin treated embryos. We conclude that JY-1 transcripts decrease in length during meiotic maturation and that polyadenylated JY-1 mRNAs detected in early developing embryos are oocyte-derived and not due to de novo transcription in early embryos.
\end{abstract}

This work was supported by the Rackham Foundation and the Michigan Agricultural Experiment Station.

\section{REPROGRAMMING OF FIBROBLASTS TO EXPRESS MALE GERM CELL OR MAST CELL FUNCTIONS USING TESTIS OR MAST CELL-DERIVED CELL EXTRACTS}

\author{
H.-Y.Choi ${ }^{\mathrm{A}}$, B.-W. Kim ${ }^{\mathrm{A}}$, E. - R. Lee ${ }^{\mathrm{A}}, S . \operatorname{Roh}^{\mathrm{B}}$, and S.-G. Cho ${ }^{\mathrm{A}}$ \\ ${ }^{A}$ Department of Animal Biotechnology, Bio/Molecular Informatics Center, BORC/IBST, and RCTCP, Konkuk University, \\ Seoul, 143-101, South Korea; ${ }^{\mathrm{B}}$ College of Dentistry \& Dental Research Institute, \\ Seoul National University, Seoul 110-149, South Korea
}

The concept of epigenetic reprogramming of a somatic nucleus was supported by the birth of cloned animals and the derivation of embryonic stem cells after nuclear transplantation into oocytes. Moreover, recent studies reported that the direct transformation of one differentiated somatic cell type into another is possible and would be advantageous for producing isogenic replacement cells. In this study, we were able to modulate the cell fate of 
fibroblasts by introducing cell extracts derived from a mast cell line, RBL-2H3. NIH-3T3 cells were treated with streptolysin O (SLO; $230 \mathrm{ng} / \mathrm{mL}$ ), which reversibly permeablizes plasma membrane, and incubated for $30 \mathrm{~min}$ with the mast cell- derived cell extracts $(4 \mathrm{mg} / \mathrm{mL})$. After resealing the membrane of the cells, we incubated the cells for 3 weeks and then analyzed them for the expression of mast cell-specific genes such as MAFA (mast cell function-associated antigen) and FceRI (high-affinity IgE receptor). Our results showed that the cell extracts can activate the expression of mast cell-specific genes, implying that cell extracts can provide regulatory components required for reprogramming the cell fate to initiate a transcriptional program specific for the cell type. Moreover, mast cell-specific degranulation and cell morphology changes were observed in cultured mouse fibroblasts. We could detect mast cell-specific functions even after 15 days of incubation. Next, to induce porcine fibroblasts to take on testis sertoli cell-specific properties, we reversibly permeablized porcine primary fibroblasts with SLO $(230 \mathrm{ng} / \mathrm{mL})$ and incubated the cells with porcine testis extracts $(4 \mathrm{mg} / \mathrm{mL})$. As expected, in the reprogrammed primary porcine fibroblasts, the porcine testis extracts activated the expression of porcine testis sertoli cell-specific genes including protamine 1 and 2, SOX9, MIS (mullerian inhibitory substance), preproacrosine (ACR), phosphoglycerate kinase-2 (PGK-2), protein C, and c-kit ligand. The male germ cell functions were sustained for more than 10 days after the reprogramming process. Taken together, our data suggest that testis- or mast cell-derived cell extracts can reprogram fibroblasts to express male germ cell or mast cell functions, respectively, supporting the concept that cell extracts can reprogram the genome activity to activate cell-specific gene expression.

This work was supported by ARPC (Grant no. 204117-03-1-HD110) in Korea, and by Biogreen 21 program (Grant no. 20050401034658).

\title{
244 GATA-1 PLAYS A CRITICAL ROLE IN ENDOTHELIAL CELL-SPECIFIC EXPRESSION OF THE 1.1-kb CD55 PROMOTER REGION
}

\author{
T.-W. Choi, M.-Y. Jeong, B.-W. Kim, J.-Y. Kim, H.-Y. Choi, J.-H. Kim, H. T. Lee, and S.-G. Cho
}

Department of Animal Biotechnology, BMIC, IBST, and RCTCP, Konkuk University, Seoul 143-101, South Korea

\begin{abstract}
The complement system is composed of a complex group of soluble proteins that have important roles in the immune response against foreign cells such as xenografted tissue. Some of the cell surface regulators, also known as membrane complement regulatory proteins (CRPs), are the membrane cofactor protein (CD46), the decay-accelerating factor (CD55), and protectin (CD59). The CD55 is a $70 \mathrm{kDa}$ glycolipid-anchored membrane-bound protein that has regulatory activity by preventing $\mathrm{C} 3$ convertase formation and is reported to be expressed in blood and vascular endothelial cells. Its high expression in leukocytes and endothelium is thought to safeguard against locally augmented C3 activation that can occur with inflammation. In this study, we originally cloned the 1.1-kb promoter region of the CD55 gene and constructed a luciferase reporter plasmid, pGL3-1.1CD55. The pGL3-1.1CD55 plasmid and its control plasmid, pGL3-control (SV40 promoter), were transfected into endothelial (MS-1 and BAEC) and epithelial (HaCaT and HEK293) cells, and the soluble fraction of the cell lysates was assayed for luciferase activity. Its promoter activity was compared with that of the promoters of endothelial-specific genes such as MCP, Flk-1, ICAM-2, and throbomodulin. Luciferase activity in each sample was normalized to the $\beta$-galactosidase activity of the same sample and the data were analyzed by Sigma Plot program $(P<0.01$ versus control). Our results showed that the $1.1 \mathrm{~kb}$ CD55 promoter was the strongest among the endothelial cell-specific promoters. To define the important region for the strong expression, the deletion constructs containing $0.96,0.86$, and $0.74, \mathrm{CD} 55$ promoter regions were prepared. Relative to the activity of the control SV40 promoter, decreased luciferase activity was obtained with these deletion constructs, suggesting that the about $0.2 \mathrm{~kb} 5^{\prime}$-flanking region (between -1125 and -967 ) of the $1.1 \mathrm{~kb}$ CD55 promoter region was important for the strong gene expression. Interestingly, on the $5^{\prime}$-end of the $0.2 \mathrm{~kb}$ region, we could detect two GATA- 1 binding sites (from -1122 to -1118 and from -1111 to -1107 ); the deletion of the sequences between -1125 and -1100 ( $\triangle$ GATA-1) significantly reduced the promoter activity. Furthermore, ectopic expression of GATA-1 dose-dependently induced the $1.1 \mathrm{~kb}$ CD55 promoter activity, but not the $\triangle$ GATA-1 activity, strongly implying that the GATA-1 binding site is important for the strong expression. Finally, we confirmed the binding of GATA-1 on the CD55 promoter region with the electrophoretic mobility shift assay (EMSA) using the 28 base pair oligonucleotide probes corresponding to the GATA-1 binding site and chromatin immunoprecipitation (Ch-IP) using anti-GATA-1 antibody. Taken together, these results strongly suggested that GATA-1 plays a critical role in endothelial cell-specific expression of the $1.1 \mathrm{~kb}$ CD55 promoter region.
\end{abstract}

This work was supported by the Research Project on the Production of Bio-organs, Ministry of Agriculture and Forestry, Republic of Korea, and by the Korea Research Foundation Grant funded by the Korean Government (MOEHRD) (KRF-2005-070-C00095).

\section{EXPRESSION AND FUNCTIONAL ROLE OF CELL DIVISION CYCLE 42 IN MOUSE OOCYTES MATURATION AND PRE-IMPLANTATION DEVELOPMENT}

\author{
X.-S. Cui, X.-Y. Li, and N.-H. Kim \\ Department of Animal Science, Chungbuk National University, Cheongju, Chungbuk, South Korea
}

Cell division cycle 42 (Cdc42), a member of the Rho family of small guanosine triphosphatase (GTPase) proteins, regulates multiple cell functions, including motility, proliferation, apoptosis, and cell morphology. In order to gain insight into the role of Cdc42 in embryo development, we first characterized mRNA and protein levels of $\mathrm{Cdc} 42$ in mouse oocytes and early embryogenesis. We then examined the possible role of the gene in oocyte maturation and pre-implantation development using RNA interference analysis. The relative abundance of Cdc 42 transcripts were measured by real time RT-PCR. After normalization with histone H2a mRNA levels, the mRNA expression of Cdc42 was abundant in immature oocytes and reduced slightly in zygotes and 2- to 8-cell stage embryos. The expression levels were significantly increased during the morula and blastocyst 
stages. Indirect immunocytochemistry showed protein synthesis of Cdc42 in oocytes and embryos of all stages. Introducing small interference RNA (siRNA) of Cdc42 into germinal vesicle stage oocytes or zygotes specifically reduce both mRNA expression and protein synthesis of Cdc42 in metaphase II stage oocytes and early embryos developing in vitro. Meiotic maturation was significantly reduced following siRNA injection into germinal vesicle stage oocytes. It is evident that actin distribution in siRNA treated blastocysts is morphologically abnormal following injection of siRNA for Cdc42. Injection of siRNA into zygotes did not influence cleavage, but significantly decreased in vitro development to morulae and blastocysts. While housekeeping genes such as tissue plasminogen activator were not altered by siRNA, wiskott-aldrich syndrome protein family 1 (WASP1) mRNA was down-regulated in the morula. Interestingly, mRNA of WASP1, tubulin alpha 1 (Tuba1), and actin-related protein $2 / 3$ complex subunit V (Arpc5) increased at the blastocyst stage following siRNA injection. These results suggest that Cdc42 plays an important role during oocyte maturation and early pre-implantation development, likely through linkage with several other genes.

This work was funded by a grant from National Research Laboratory Program in Korea.

\title{
246 EXPRESSION PATTERN OF NANOG AND Par3 GENES IN IN VITRO-DERIVED BOVINE EMBRYOS
}

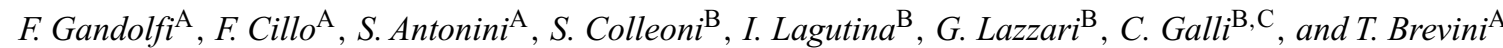 \\ ${ }^{\mathrm{A}}$ Instituto di Anatomia degli Animali Domestici, Milano, Italy; ${ }^{\mathrm{B}}$ Laboratorio di Tecnologie della Riproduzione, CIZ srl, Cremona, Italy; \\ ${ }^{\mathrm{C}}$ Dipartimento Clinico Veterinario, Universita di Bologna, Bologna, Italy
}

Homeobox genes have been demonstrated to be important in patterning and lineage specification during early embryogenesis. Nanog belongs to the family of DNA-binding transcription factors and has been shown to maintain pluripotency of embryonic stem cells, both in murine and human. Par3 plays an essential role in determining cell fate of the early mouse embryo, leading to the generation of the inner cell mass and the trophectoderm. No information is available on these genes in the bovine; therefore, the aim of the present study was to identify and characterize Nanog and Par3 expression in bovine embryos. Oocytes recovered from slaughterhouse ovaries were matured for $22 \mathrm{~h}$, fertilized in vitro and then cultured in mSOFaa medium. RNA was extracted from pools of five oocytes and embryos at different stages of development (2-, 4-, 8-, 16-cell, morula and blastocyst). It was then reverse transcribed, and PCR runs were carried out with primers specifically designed for Nanog and Par3, based on the sequence data bank available. The amplified products were separated on a 2\% TAE agarose gel, purified, sequenced and aligned using Clustal W. Comparison of the bovine Nanog cDNA sequence (EMBL AM039957) with databases revealed a 84\% degree of homology with the human, $97 \%$ with the mouse, and $82 \%$ with the goat genes. IVF bovine embryos express Nanog only upon genome activation, becoming detectable from the 8-cell stage onward indicating that Nanog is zygotically expressed in the bovine similar to what happens in mouse, pig and goat. Bovine Par3 cDNA sequence (EMBL AM039956) shows a high degree of homology with human (83\%), mouse (81\%), and rat (79\%). Also Par3 is expressed only upon the maternal to embryonic transition (MET) at the 8-cell stage. As opposed to the expression patterns of other early embryo genes, like Oct-4 and Zar-1, Nanog and Par3 expression patterns in bovine embryos closely resemble those described in the mouse. Since both are absent in the ooplasm and before MET, they represent useful markers for genome activation.

This work was supported by FIRB RBNE01HPMX, FIRST 2004 and ESF-EuroStells.

\section{MESSENGER RNA EXPRESSION PATTERNS OF DNA AND HISTONE METHYLTRANSFERASES IN PREIMPLANTATION DEVELOPMENT OF IN VIVO-AND IN VITRO-PRODUCED BOVINE EMBRYOS}

\author{
K. Höffmann, H. Niemann, K.-G. Hadeler, D. Herrmann, and C. Wrenzycki \\ Institute for Animal Breeding (FAL), Department of Biotechnology, 31535 Neustadt am Rübenberge, Germany
}

The effects of in vitro production (IVP) and/or somatic nuclear transfer on mRNA expression patterns have mostly been determined in morulae and blastocysts, i.e. after embryonic genome activation. Comparative data regarding mRNA expression patterns throughout the oviductal phase of pre-implantation development are scarce. Here we studied mRNA expression for genes related to DNA methylation and modification of histones which account for the major epigenetic reprogramming during development. Pertubated epigenetic reprogramming of the genome is a likely cause of developmental abnormalities and epigenetic diseases associated with assisted reproduction technologies. The objective of the present study was to compare mRNA expression of DNA methyltransferases Dnmt1, -3a, and -3b and histone methyltransferases SUV39-h1 and G9a between in vivo-derived bovine embryos and their IVP counterparts using a semiquantitative RT-PCR assay (Wrenzycki et al. 2002 Biol. Reprod. 66, 127-134) employing two embryos for each assay. In vivo-derived embryos were collected from 28 superovulated heifers by endoscopic flushing of oviducts (zygotes to 8-cell stages) (Besenfelder et al. 2001 Theriogenology 55, 837-845) or by uterine flushing (16-cell stages to blastocysts). Endoscopic flushing at different time points after AI (Days 1, 1.5, 2, 3, 4, and 4.5) yielded 31 zygotes; 15 two-cell, 5 three-cell, 13 four-cell, 1 five-cell, 2 six-cell, and 11 eight-cell embryos; 4 degenerated embryos; and 18 unfertilized ova. The recovery rate (corpora lutea counted per recovered embryos) was $58 \%$ and $62 \%$ for the endoscopic and uterine flushing, respectively. Differences in the relative abundance of each gene transcript between groups were tested using ANOVA with the main effects being origin (in vivo/in vitro) and developmental stage (zygote to blastocyst) and their interactions followed by multiple pairwise comparisons using a Tukey test $(P<0.05)$. Origin of embryos affected the relative abundance of transcripts for Dnmt1, Dnmt3a, and SUV39-h1, and developmental stage affected the relative abundance of transcripts for Dnmt1, -3a, -3b, SUV39-h1, and G9a. No interactive effects were observed for origin and developmental stage in the relative abundance of all transcripts. The relative abundance of Dnmt 1 transcripts differed significantly between in vivo- and in vitro-produced morulae and blastocysts. For Dnmt3a, mRNA differences were 
determined between in vivo- and in vitro-produced 10-16-cell stages and morulae. Suv39-h1 transcripts differed significantly between in vivo- and in vitro-derived zygotes, 2-cell embryos, 8-cell embryos, 10-16-cell embryos, and blastocysts. The results suggest that IVP alters mRNA expression of genes related to epigenetic modifications very early in development, even before the embryonic genome has been activated.

\title{
248 ALTERED GENE EXPRESSION PROFILES IN PIG scNT-DERIVED UMBILICAL CORD
}

\author{
K. C. Hwang, S. K. Cho, and J. H. Kim \\ Division of Applied Life Science, Gyeongsang National Univeristy, Gwaja, GyeongNam, Republic of Korea
}

\begin{abstract}
Despite numerous studies performed on somatic cell cloned ( $\mathrm{scNT}$ ) animals, the factors behind the anomalies such as overgrowth, placental malformations, and heart failure remain unknown. These observations provide a cautionary note about the safety of current cloning procedures. Ovaries were collected from pubertal gilts at a local slaughterhouse, and oocytes with evenly granulated cytoplasm surrounded by at least three uniform layers of compact cumulus cells were selected and cultured in NCSU-23 medium. A single donor cell was injected into the perivitelline space of each egg and then electrically fused. Approximately $200 \mathrm{scNT}$ embryos were transferred into the oviduct of each recipient. In the study, we generated 58 somatic cell cloned ( $\mathrm{scNT}$ ) piglets. Of these, six scNT piglets displayed severe abnormalities in several organs due to severe disruption of two umbilical cord arteries. Histologically, there was evidence of severe calcification in the kidney and placenta, peliosis in the sinusolidal space of the liver, abnormal stroma cell proliferation in lung, and tubular degeneration as a result of ischemic change due to lung congestion. To determine the genes responsible for disruption of umbilical cord arteries, a pig $13 \mathrm{~K}$ oligo cDNA microarray, developed in-house at GenoCheck Co., Ltd. (Ansan, Korea), was used for gene expression profiling studies. In scNT piglets that have severe disruption in the arteries of umbilical cord, 23 genes were up-regulated, but 127 genes were down-regulated, especially angiogenesis-related genes (VEGFa, PLANH1, CYR61, and SMAD4), cytoskeleton-related genes (14-3-3E, CCT3, DCTN1, KRT7, and MARK4), and hypoxia-related genes (HIG2 and DDIT4). These scNT piglets were chronically exposed to maternal and fetal blood circulation problems and, as a result, they died within the first week of life. The histological findings in this study have not been reported previously in scNT animals and demonstrate a possible correlation between early death of scNT clones and abnormality of two umbilical cord arteries.
\end{abstract}

\section{EXPRESSION OF GROWTH-RELATED IMPRINTED GENES IN DECEASED NEWBORN AND SURVIVING CLONED PIGLETS}

\author{
L. Jiang ${ }^{\mathrm{A}}$, P. Jobst ${ }^{\mathrm{C}}$, D. Ayares ${ }^{\mathrm{C}}$, R. S. Prather ${ }^{\mathrm{B}}$, X. Yang $^{\mathrm{A}}$, and X. C. Tian $^{\mathrm{A}}$ \\ ${ }^{A}$ Department of Animal Science, Center for Regenerative Biology, University of Connecticut, Storrs, CT 06268, USA; \\ ${ }^{B}$ Department of Animal Sciences, University of Missouri-Columbia, Columbia, MO 65201, USA; \\ ${ }^{\mathrm{C}}$ Revivicor Inc., Blacksburg, VA 24060, USA
}

Different from cloned mice and cattle, which are often born with large offspring syndrome (LOS), pigs produced through nuclear transfer have not been found to be overly large compared to their age-matched controls. In our study, significant differences $(P<0.05)$ were found in the body weight of both deceased newborn cloned piglets $(n=9)(1.2 \pm 0.8 \mathrm{vs} .2 .1 \pm 0.2 \mathrm{~kg})$ and cloned pigs at one month of age $(n=7)(6.1 \pm 1.3 \mathrm{vs} .8 .0 \pm 0.8 \mathrm{~kg})$, when compared to their controls ( $n=5$ for each age group). Because imprinted genes are important regulators of fetal growth and are subject to reprogramming during nuclear transfer, we aimed to determine the expression levels of both growth-enhancing and growth-inhibiting imprinted genes in these cloned pigs by real time quantitative reverse transcription polymerase chain reaction (RT-PCR). These genes include: Igf2 and Peg3 (growth-promoting), and Igf2r and Grb10 (growth-inhibiting). Tissues from six major organs were collected from clones and controls of both age groups. SYBR Green I was utilized as a reporter for the PCR reactions and 18S ribosomal RNA was used as the internal reference for quantifying the relative expression level of all genes. With the exception of Igf2, the other three imprinted genes were found to have statistically different levels of expression in certain organs of both groups of pigs when compared to their age-matched controls. However, no strong correlation was found between the levels of gene expression and the low-body-weight phenotype of the cloned pigs. Contrary to the notion that nuclear transfer can reduce the variation in the resulting animals due to their identical genetic make-up, larger variances of gene expression were found in clones of both groups, indicating the randomness of the nuclear reprogramming process. For all four imprinted genes studied, the coefficient of variation (CV) decreased greatly from newborn to one month old in both clones and controls, suggesting a more stringent regulation on imprinted gene expression after birth.

\section{DISTRIBUTION OF HISTONE macroH2A1 IN BOVINE PRE-IMPLANTATION EMBRYOS DERIVED FROM PARTHENOGENETIC ACTIVATION AND IN VITRO FERTILIZATION}

\author{
C. Kim, Y. Ma, C.-C. Chang, T. Rasmussen, X. Yang, and C. Tian \\ Center for Regenerative Biology, University of Connecticut, Storrs, CT 06268, USA
}

It is known that heterochromatin is characterized by the presence of the histone variant of macroH2A1. MacroH2A1 is a core variant histone with a hybrid structure consisting of a domain that resembles a full-length histone H2A1 followed by a large nonhistone domain. We have previously studied the dynamic changes of macroH2A 1 accumulation during the pre-implantation developmental period in the mouse. In the present study, we investigated the distribution of microH2A1 in bovine metaphase II oocytes and pre-implantation embryos at 2-, 4-, 8-, 16-cell, and morula 
stages as well as blastocysts harvested at Days $8,9,10,11,12$, and 13 following activation and in vitro fertilization (IVF). To generate parthenotes, denuded and in vitro-matured oocytes were activated using a combined treatment of calcium ionophore A23187, cycloheximide (CHX), and 6-dimethylaminopurine (6-DMAP). Five oocytes and pre-implantation embryos at each stage of development were used to follow the development expression pattern of microH2A1 by immunocytochemistry. The cross-reactivity of the primary antibody against mouse microH2A 1 was verified by Western blot analysis with bovine fibroblasts. Another staining control included immunostaining with antibody against histone molecules. The stained embryos were observed by laser-scanning confocal microscopy and epiflourescence microscopy. No microH2A1 stain was observed in bovine oocytes or pre-implantation embryos up to the expanded blastocyst stages. In the IVF group, the macroH2A1 was first found in elongated blastocysts (Day 11) after hatching. We observed different expression patterns of macroH2A1 in activated vs. IVF bovine embryos. In the parthenote group, we failed to find robust expression even when embryos were cultured for 13 days. Moreover, the pattern of macroH2A1 expression in bovine embryos was different fromn that in the mouse, in which the onset of macroH2A1 expression occurred by the 16-cell morula stage. These results suggest species differences in the establishment of epigenetic signals.

This work was supported by grants from USDA to X. Y. and X. C. T.

\title{
251 COMPARATIVE ANALYSIS OF GENE EXPRESSION PATTERNS IN PORCINE PRE-IMPLANTATION EMBRYOS DERIVED FROM DIFFERENT ORIGINS
}

\author{
J.-G. Kim, H.-F. Jin, B.-K. Mohana, H.-J. Song, Y.-J. Jeong, S. Balasubramanian, S.-Y. Choe, and G.-J. Rho \\ College of Veterinary Medicine, Gyeongsanga National University, Chinju, GN, Republic of Korea 660-101
}

\begin{abstract}
The amount of information gathered on the kinetics and quantitative profile of gene expression in nuclear transferred (NT) pre-implantation embryos is still scarce and limited to a handful of genes in pig. In the present study, we compared the relative abundance (RA) of six development-related genes of pre-implantation embryos from different origins. Cumulus-oocyte complexes (COCs) were matured, fertilized and cultured by the method of Abeydeera et al. (2000 Theriogenology 54, 787-797). Parthenogenetic (PA) and NT embryos were produced as described by Kim et al. (2005 Mol. Rep. Dev. 70, 308-313). Sets of 10 embryos each at 4-cell, 8-16-cell, morula, and Day 7 blastocyst stages were used to extract total RNA for analyzing the expression pattern of Bax (pro-apoptotic), Bcl-xl (anti-apoptotic), Oct-4 (pluripotent transcription), Stat3 (cytoplasmic transcription), IFN-tau (implantation) and VEGF (vasculogenesis) genes (three replicates) with real-time quantitative PCR (LightCycler ${ }^{\circledR}$; Roche Diagnostics, Mannheim, Germany) following RNA isolation (with Dynabeads ${ }^{\circledR}$ mRNA Direct ${ }^{\mathrm{TM}}$ kit; Dynal, Oslo, Norway) and cDNA amplification (Oligo $(\mathrm{dT})_{12-18}$ primer and Superscript ${ }^{\mathrm{TM}}$ III cDNA synthesis kit; Invitrogen, Carlsbad, CA, USA). The expression of each gene was normalized to Histone H2A expression. Statistically significant $(P<0.05)$ differences in gene expression were analyzed by ANOVA. Oct- 4 , Stat3, Bax, and Bcl-xl were expressed at the 4-cell stage. However, IFN-tau and VEGF were expressed at the morula stage. The expression patterns of all genes throughout the embryonic development in all types of embryos were similar. However, the relative abundance (RA) of Bax in the 8-16 cell stage of NT and PA was significantly $(P<0.05)$ increased as compared to that in IVP counterparts. The RA of Oct-4 and Bcl-xl did not differ throughout all stages in NT and PA embryos. There were no significant differences in all types of embryos with respect to the RA of Oct-4 and Bcl-xl with exception of the blastocysts in NT (significantly decreased, $P<0.01)$. The RA of Stat 3 was significantly $(P<0.05)$ increased in the $8-16$ cell stage (NT and PA) and in blastocysts (NT). Similarly, the RA of IFN-tau was significantly $(P<0.05)$ increased in NT blastocysts. The RA of VEGF was not significantly different in all stages and types of embryos except NT blastocysts (decreased expression, $P<0.01$ ). These results suggest that expression patterns of Bax, Bcl-xl, Oct-4, Stat3, IFN-tau, and VEGF can be used as markers to assess the developmental competence of porcine nuclear transfer embryos.
\end{abstract}

This work was supported by Grant No. 1000520040020000 from Biogreen 21, Republic of Korea.

\section{DIFFERENCES IN GENE EXPRESSION PROFILES IN MOUSE FERTILIZED BLASTOCYSTS AND DIPLOID BLASTOCYST STAGE PARTHENOTES}

\author{
N.-H. Kim, S.-K. Cho, X.-Y. Li, X.-H. Shen, and X.-S. Cui \\ Department of Animal Science, Chungbuk National University, Cheongju, Chungbuk, South Korea
}

Following parthenogenetic activation, in the absence of a male contribution, oocytes progress into early gestation. To gain insight into the role of the paternal genome during pre-implantation development, we used microarray to compare gene expression profiles in pre-implantation embryos following fertilization and parthenogenetic activation. Fertilized embryos and oocytes were collected from superovulated C57BL/6J female mice. The oocytes were activated with $50 \mu \mathrm{M}$ calcium ionophore A23187 for $5 \mathrm{~min}$. After $5 \mathrm{~h}$ of culture in M16 medium with $7.5 \mu \mathrm{g} / \mathrm{mL}$ cytochalasin B, oocytes with one polar body and two pronuclei were used in this experiment. The activated oocytes and zygotes were cultured in M16 to the blatocyst stage. Messenger RNA from 50 blastocysts was extracted by means of the Dynabeads mRNA Direct Kit (Dynal, Oslo, Norway), and then linearly amplified for two rounds using the RiboAmp HS RNA Amplification Kit (Arcturus Bioscience, Inc., Mountain View, CA, USA). A set of cRNA targets from the embryos was assembled into a hybridization reaction on the Applied Biosystems 1700 chemiluminescent microarray analyzer (Jung Hwa Scientific Co., Ltd., Seoul, Korea). Each set was repeated three times. All of the correlation coefficients were above 0.9 for experiment replications. Differences in microarray intensities were normalized and grouped by using the Avadis Prophetic 3.3 version, and categories are based on the PANTHER classification system. According to the cDNA microarray data, we additionally categorized genes into transcription- and developmental process-related genes and compared them in both fertilized and parthenogenetically activated blastocysts. Five transcription-related genes (Goosecoid, transcription factor 1, LIM domain, Spi-C transcription factor, and hypoxia inducible factor 3) and seven developmental process related genes (metaxin 1, serine/threonine kinase 22, stromal antigen, butyrophilin, anti-Mullerian hormone type 2 receptor, prolactin-like protein 
$\mathrm{C} 2$, and otoconin 90) were identified in the fertilized blastocysts compared to the blastocyst-stage parthenotes. In contrast, seven transcription(Amnionless, EHOX-like, calcium signal transducer 2, nuclear receptor 0B, transcription factor CP2, Iroquois related homeobox 3, and zinc finger protein 3) and eight developmental process-related genes (prion protein dublet, X-linked lymphocyte-regulated 3a, muscleblind-like 3, stathmin-like 2, SRY-box-containing gene 7, ephrin B1, muscleblind-like 3, and Iroquois-related homeobox 3) were expressed at a higher level in parthenotes than in fertilized blastocysts. These genes were selected, and their expression levels confirmed, by real-time quantitative RT-PCR. The results indicate that diploid parthenotes at the blastocyst stage may lack or over express genes related to transcription and development processes which possibly result in fetal lethality. Further studies are required to determine whether aberrant gene expression in parthenotes is due to lack of paternal contribution.

This work was funded by a grant from the National Research Laboratory Program in Korea.

\title{
253 GENE SILENCING OF CYCLOOXYGENASE-2 mRNA BY RNA INTERFERENCE IN BOVINE CUMULUS-GRANULOSA CELLS
}

\author{
S.-I. Kobayashi ${ }^{\mathrm{A}}$, M. Sakatani $^{\mathrm{A}}$, S. Kobayashi ${ }^{\mathrm{A}}$, K. Okuda $^{\mathrm{B}}$, and M. Takahashi ${ }^{\mathrm{A}}$ \\ ${ }^{A}$ Department of Animal and Grassland Research, National Agricultural Research Center for Kyushu Okinawa Region, Kumamoto, Japan; \\ ${ }^{B}$ Laboratory of Reproductive Endocrinology, Department of Animal Science, Faculty of Agriculture, \\ Okayama University, Okayama, Japan
}

Recently, interference RNA (RNAi), inducing inhibition of the specific gene expression, attracted a lot of attention. Many researchers have reported that the 21-mer small interference RNA (siRNA) is introduced into target cells and then siRNA can suppress the gene expression. RNAi is a useful tool for functional analysis of specific genes. However, there is little information about RNAi for the analysis of gene function in reproductive physiology in ruminants. Thus, this study was aimed at evaluating RNAi for the analysis of cyclooxygenase-2 (Cox-2) mRNA expression in bovine cumulus-granulosa $(\mathrm{CG})$ cells as well as prostaglandin $\mathrm{F}_{2 \alpha}\left(\mathrm{PGF}_{2 \alpha}\right)$ production. We investigated both the effective concentration of Cox-2 siRNA and the effect of the introduction time of siRNA on Cox-2 mRNA expression. Bovine CG cells were collected at slaughterhouse and cultured in 4-well dishes. After the cells reached confluency, two experiments were conducted. In the present study, Cox-2 siRNA was simply added to culture medium with lipofectamine ${ }^{\mathrm{TM}} 2000$ (Invitrogen Japan K. K., Tokyo, Japan) as the transfection reagent. In experiment 1, the concentration of 0, 100, 250, and $500 \mathrm{pM}$ of Cox-2 siRNA was introduced into the CG cells. After $24 \mathrm{~h}$ of introduction, the amount of mRNA expression for Cox-2 was measured by reverse transcription polymerase chain reaction (RT-PCR) and real-time PCR. In experiment 2, $250 \mathrm{pM}$ siRNA for Cox-2 was introduced into $\mathrm{CG}$ cells for $0,3,6,12$, and $24 \mathrm{~h}$. After culture, the amount of mRNA expression for Cox-2 was measured and the culture medium was collected to determine $\mathrm{PGF}_{2 \alpha}$ concentration by enzyme immunoassay. The Cox- 2 mRNA expression was not affected by $100 \mathrm{pM}$ siRNA introduced into CG cells for $24 \mathrm{~h}$, but 250 and $500 \mathrm{pM}$ Cox- 2 siRNA significantly reduced the Cox $-2 \mathrm{mRNA}$ expression ( $10 \%$ of that of the $0 \mathrm{pM}$ siRNA group). Moreover, the suppressive effect of $250 \mathrm{pM}$ siRNA was observed at $6 \mathrm{~h}$ after introduction $(60 \%$ of that of the $0 \mathrm{pM}$ siRNA group at $0 \mathrm{~h})$ and the reduction of mRNA expression by RNAi became more obvious over $12 \mathrm{~h}(10 \%$ of that of the $0 \mathrm{pM}$ siRNA group at $0 \mathrm{~h})$. On the other hand, the PGF ${ }_{2 \alpha}$ concentration in the culture medium was not significantly different at $12 \mathrm{~h}$ after siRNA introduction, however, the $\mathrm{PGF}_{2 \alpha}$ concentration of the RNAi treatment group at $24 \mathrm{~h}$ was significantly lower than that of the $0 \mathrm{pM}$ siRNA group at the same time point. These results suggest that gene silencing by Cox-2 siRNA is a means of analyzing the function and expression of specific genes in bovine CG cells.

This study was supported by the Japan Society for the Promotion of Science for Young Scientists.

\section{DIFFERENTIAL TRANSCRIPTION AND CYSTOPLASMIC POLYADENYLATION ELEMENTS IN PORCINE GERMINAL VESICLE AND METAPHASE II OOCYTES}

\author{
S. Korte, G. Springer, W. Spollen, R. Patel, K. Whitworth, N. Bivens, L. Forrester, C. Murphy, J. Green, and R. Prather \\ Department of Animal Sciences, University of Missouri-Columbia, Columbia, MO 15201, USA
}

Identification of transcripts produced during porcine oocyte maturation is one of the first steps in defining pathways important to development. Practically, this information will aid in the refinement of in vitro culture processes, allowing for more efficient in vitro embryo production. To this end, mRNA was isolated from 50 in vitro-matured sow metaphase II stage oocytes (Bomed, Inc., Madison, WI, USA) (MIIs), 50 in vitro-matured gilt metaphase II-stage oocytes (MIIg), and 50 gilt germinal vesicle stage oocytes (GVO) by using the Dynabead ${ }^{\circledR}$ system (Dynal, Inc., Lake Success, NY, USA) and amplified by using the SMART system (Clontech, Palo Alto, CA, USA). The PCR products were ligated into a pSport vector and transformed into electro-competent $E$. coli. Colonies were randomly picked and sequenced at the University of Missouri DNA Core. Sequences were clustered with similar sequences derived from a larger expressed sequence tag (EST) project (http://genome.rnet.missouri.edu/Swine/) by using the tlcluster program developed at the University of Iowa. Following clustering, individual clusters in the cDNA libraries were compared by using Fisher's exact test $(P<0.01)$ to determine if they were differentially represented. Two sets of comparisons were performed, one between the MIIs and MIIg libraries, and another between the GVO library and the combination of both metaphase II libraries (MII). The number of clusters per number of clones in the library was 966/1668 (GVO), 458/820 (MIIg), and 158/819 (MIIs). There were 15/419 clusters that were different between the MIIs and MIIg libraries, and 26/1269 that were different between the MII and GVO libraries. Potential cytoplasmic polyadenylation elements (CPEs) identified from the literature were found in the GVO and MII libraries by using a custom pattern-matching program. Of the clusters with differential expression, 4/15 (MIIs vs. MIIg) and 7/26 (MII vs. GVO) contained CPEs. Table 1 contains a partial list of differentially expressed genes and the sequence of their cytoplasmic polyadenylation elements. Many genes were found to be differentially expressed in both (MII vs. GVO and MIIs vs. MIIg) comparisons. Collectively, these findings will facilitate the elucidation of important developmental pathways in swine and other animals. 
Table 1. Comparison of mRNA expression and cytoplasmic polyadenylation elements

\begin{tabular}{lll}
\hline Tissue & Gene & Cytoplasmic polyadenylation element(s) \\
\hline MII $>$ GVO & PCBD2 & TTTAAAA \\
MII $>$ GVO & RBM17 & TTAAAAA, ATAAAAA, ATTAAAA, TTAAAAT \\
MII $>$ GVO & UBE2L & ATTAAAA, TTAAAAT \\
MII $>$ GVO & COX8C & None \\
GVO $>$ MII & FTL & TTAAAAA \\
GVO $>$ MII & CDKL2 & ATAAAAA \\
GVO $>$ MII & CLK2 & ATAAAAA \\
GVO $>$ MII & SLC28A1 & None \\
MIIs $>$ MII & PCBD2 & TTTAAAA \\
MIIs $>$ MII & UBE2L & ATTAAAA, TTAAAAT \\
MIIs $>$ MII & CCNI & None \\
MIIg $>$ MIIs & RBM17 & TTAAAAA, ATAAAAA, ATTAAAA, TTAAAAT \\
MIIg $>$ MIIs & HSPCA & None
\end{tabular}

This work was partially funded by a University of Missouri Life Sciences Mission Enhancement grant and Food for the 21st Century.

\title{
255 GENE EXPRESSION OF Cox 5a, 5b, AND 6b1 AND THEIR ROLES IN PRE-IMPLANTATION OF MOUSE EMBRYOS
}

\author{
S.-E. Lee, X.-Y. Li, X.-S. Cui, and N.-H. Kim \\ Department of Animal Science, Chungbuk National University, Cheongju, Chungbuk, South Korea
}

\begin{abstract}
Despite clear evidence of regulation of mitochondrial respiration by nuclear encoded genes, cytochrome oxidase (Cox), little information is available on their expression and functional roles during early embryonic development. To examine the role of Cox in oocyte maturation and embryogenesis, we first characterized mRNA and protein levels of nuclear encoded genes, Cox 5a, 5b, and 6b1, in mouse oocytes and during early embryogenesis, using real-time RT-PCR and immunocytochemistry. We then examined the possible role of these genes in oocyte maturation and pre-implantation development using RNA interference analysis. The relative abundances of Cox 5a, 5b, and $6 \mathrm{~b} 1$ transcripts was measured by real time RT-PCR. After normalization by comparison to histone H2a mRNA levels, the mRNA expression of Cox $5 \mathrm{a}, 5 \mathrm{~b}$, and $6 \mathrm{~b} 1$ were found to be considerable in mature oocytes and zygotes, but reduced slightly in 2-cell embryos. From the 2-cell to the blastocyst stage, mRNA expression is dependent on the number of blastomeres, as expression increases only gradually with development. Immunocytochemical studies revealed that Cox 5a, 5b, and $6 \mathrm{~b} 1$ proteins were expressed in all blastomeres of the blastocyst. Injection of Cox 5a, 5b, or 6b1 siRNA into GV stage oocytes decreased expression of the target mRNA specifically, while not affecting the expression of mRNAs for the other subunits in mature oocytes. Similarly, each siRNA injection into zygotes specifically reduced target mRNA expression at the 2-cell, morula and blastocyst stages $(P<0.05)$. Silencing of mRNA expression by RNA interference (siRNA) did not inhibit oocyte maturation or developmental events up to the morula and blastocyst stages. The expression level of mtDNA9, as well as overall levels of mitochondrial mRNAs, was not different following injection of siRNA for Cox 5a, 5b, or 6b1. However, it is evident that the number of mitochondria in siRNA treated blastocysts was greatly reduced, and they appeared to be morphologically abnormal. Significantly higher apoptosis and lower cell numbers were observed in siRNA treated blastocysts. Real time RT PCR revealed that silencing of Cox $5 \mathrm{a}, 5 \mathrm{~b}$, and $6 \mathrm{~b} 1$ decreased mRNA and protein levels of E-cadherin. These results suggest that the Cox subunits, Cox 5a, 5b, and $6 \mathrm{~b} 1$, play an important role in mitochondrial function during pre-implantation development.
\end{abstract}

This work was funded by a grant from the National Research Laboratory Program in Korea.

\section{LINES OF PIGS SELECTED FOR COMPONENT TRAITS OF LITTER SIZE EXHIBIT DIFFERENTIAL GENE REGULATION AT THE ONSET OF EMBRYO (TROPHECTODERM) ELONGATION}

\author{
J. R. Miles ${ }^{\mathrm{A}}$, L. A. Blomberg ${ }^{\mathrm{A}}$, B. A. Freking ${ }^{\mathrm{B}}$, and K. A. Zuelke $\mathrm{A}$ \\ AUSDA-ARS, Biotechnology and Germplasm Laboratory, Beltsville, MD 20705, USA; \\ ${ }^{B}$ USDA-ARS, U.S. Meat Animal Research Center, Clay Center, NE 68933, USA
}

Significant embryonic $(\sim 18 \%)$ loss occurs in the pig as the pre-implantation embryo undergoes a dramatic morphological change from ovoid $(8-10 \mathrm{~mm})$ at gestational Day 11 to a long, thin filament $(<150 \mathrm{~mm})$ by Day 12 . Lines of pigs selected for increased uterine capacity have improved embryonic survival, whereas pigs selected for increased ovulation rate have decreased embryo survival. Alterations in the expression of genes that 
play key roles in embryo elongation and maternal recognition of pregnancy may reflect differences in embryo survival observed in these lines of pigs. The objective of the current study was to evaluate the expression level of transcripts involved in developmentally important processes, such as steroidogenesis, cellular differentiation, cell-cell interaction, and immune modulation, at the onset of porcine embryo elongation (gestational Day 11) in control animals and lines selected for increased ovulation rate or uterine capacity. Total RNA was isolated from Day 11 ovoid embryos, and gene expression was measured via real-time PCR. Data were analyzed for analysis of variance using GLM procedures. Expression of steroidogenic acute regulatory protein mRNA was decreased $(P=0.02)$ in embryos from the ovulation rate and uterine capacity lines compared with controls. Similarly, expression of cytochrome $\mathrm{P} 450_{\mathrm{scc}}$ and aromatase mRNA were decreased $(P<0.003)$ in embryos from the ovulation rate and uterine capacity lines, compared with the control line. Interestingly, $17 \alpha$-hydroxylase mRNA expression was decreased $(P=0.0004)$ only in embryos from the ovulation rate line compared with the control and uterine capacity lines. In contrast, expression of cytokeratin- 8 and -18 mRNA were increased $(P<0.02)$ in embryos from the uterine capacity and ovulation rate lines, compared with controls. Expression of galectin $1 \mathrm{mRNA}$ was increased $(P=0.02)$ in embryos from the uterine capacity line, compared with the ovulation rate line. Expression of interleukin-1 receptor type $1 \mathrm{mRNA}$ was increased $(P=0.03)$ in embryos from the uterine capacity line, compared with both the control and the uterine capacity lines. Constitutive expression of $17 \beta$-hydroxysteroid dehydrogenase, stratifin, and interleukin-1 $\beta$ mRNA were observed in embryos from all lines. These results demonstrate altered gene expression in embryos from pigs selected for increased uterine capacity and ovulation rate compared with controls, and may contribute to differences observed in embryo survival between these lines of pigs.

\title{
257 EXPRESSION OF DEVELOPMENTAL GENES IN PORCINE NUCLEAR TRANSFER EMBRYOS RECONSTRUCTED WITH BONE MARROW MESENCHYMAL STEM CELLS
}

\author{
B. Mohana Kumar, H.-F. Jin, J.-G. Kim, S. Balasubramanian, S.-Y. Choe, and G.-J. Rho \\ College of Veterinary Medicine, Gyeongsang National University, Chinju, GN, Republic of Korea 660-101
}

\begin{abstract}
Abnormal gene expression is frequently observed in nuclear transfer (NT) embryos and is one of the suggested causes of the low success rates of this approach. Recent study has suggested that adult stem cells may be better donor cells for NT, as their less differentiated state may ease epigenetic reprogramming by the oocyte (Kato et al. 2004 Biol. Reprod. 70, 415-418). In the present study, we investigated the expression profile of some selected genes involved in the development of the pre-implantation embryos of in vivo- and NT-derived origin using bone marrow mesenchymal stem cells (MSCs) and porcine fetal fibroblasts ( $\mathrm{pFF}$ ) as donors. Isolated population of MSCs from porcine bone marrow were characterized by cell-surface antigen profile $\left(\mathrm{CD} 13^{\text {pos }}, \mathrm{CD} 105^{\mathrm{pos}}, \mathrm{CD} 45^{\text {neg }}\right.$, and $\left.\mathrm{CD} 133^{\text {neg }}\right)$ and by their extensive consistent differentiation to multiple mesenchymal lineages (adipocytic, osteocytic and chondrocytic) under controlled in vitro conditions (Pittenger et al. 1999 Science 284, 143-147). Primary cultures of $\mathrm{pFF}$ from a female fetus at $\sim 30$ days of gestation were established. For NT, donor cells at 3-4 passages were employed. Embryos cloned from MSCs showed enhanced developmental potential compared to $\mathrm{pFF}$ cloned embryos, indicated by higher rates of blastocyst formation $(15.3 \% \pm 4.8$ and $9.0 \% \pm 3.9$, respectively) and total cell number (31.5 \pm 7.2 and $20.5 \pm 5.4$, respectively) in Day 7 blastocysts. Total RNA was extracted from pools (triplicates) of 10 embryos each of 8-cell, morula, and blastocyst stages of in vivo and NT origin using Dynabeads ${ }^{\circledR}$ mRNA DIRECT $^{\mathrm{TM}} \mathrm{kit}^{\mathrm{B}}$ (Dynal, Oslo, Norway). Reverse transcription was performed with a Superscript ${ }^{\mathrm{TM}}$ III cDNA synthesis kit (Invitrogen, Carlsbad, CA, USA). Real-time PCR was performed on a Light cycler ${ }^{\circledR}$ using FastStart DNA Master SYBR Green I (Roche Diagnostics, Mannheim, Germany). The expression profiles of genes involved in transcription (Oct-4, Stat3), DNA methylation (Dnmt1), de novo methylation (Dnmt3a), histone deacetylation (Hdac2), anti-apoptosis $(\mathrm{Bcl}-\mathrm{xL})$, and embryonic growth (Igf2r) were determined. The mRNA of H2a was employed to normalize the levels. Significant differences $(P<0.05)$ in the relative abundance of Stat3, Dnmt1, Dnmt3a, Bcl2, and Igf2r were observed in pFF NT embryos compared with in vivo-produced embryos, whereas embryos derived from MSCs showed expression patterns similar to those of in vivo-produced embryos. However, Oct-4 and Hdac2 revealed similar expression profiles in NT- and in vivo-produced embryos. These results indicate that MSC-derived NT embryos had enhanced embryonic development and their gene expression pattern more closely resembled that of in vivo-produced embryos. Hence, less differentiated MSCs may have a more flexible potential in improving the efficiency of the porcine NT technique.
\end{abstract}

This work was supported by Grant No. R05-2004-000-10702-0 from KOSEF, Republic of Korea.

\section{MESSENGER RNA EXPRESSION PATTERNS OF HISTONE MODIFICATION GENES IN BOVINE EMBRYOS DERIVED FROM DIFFERENT ORIGINS}

\author{
M. Nowak-Imialek ${ }^{\mathrm{A}}$, C. Wrenzycki ${ }^{\mathrm{A}}$, D. Herrmann ${ }^{\mathrm{A}}$, I. Lagutina $^{\mathrm{B}}$, A. Lucas-Hahn $^{\mathrm{A}}$, E. Lemme $^{\mathrm{A}}$, G. Lazzari $^{\mathrm{B}}$, \\ C. Galli ${ }^{\mathrm{B}}$, K.-G. Hadeler ${ }^{\mathrm{A}}$, and H. Niemann ${ }^{\mathrm{A}}$ \\ A Institut for Animal Breeding (FAL), Department of Biotechnology, Neustadt a Rbg, Germany; \\ ${ }^{B}$ Laboratorio di Tecnologie della Riproduzione, CIZ-LTR, Instituto Sperimentale Italiano Lazzaro Spallanzani, Cremona, Italy
}

Epigenetic modifications of the genome, such as covalent modifications of histones, are crucial for transcriptional regulation during development. The N-terminal tails of the histones are subject to post-translational modifications, including acetylation, deacetylation and methylation. histone acetylation loosens chromatin packing and correlates with transcriptional activation. The enzymes Histone acetyltransferases (HATs) transfer acetyl moieties to the lysine residues of histones $\mathrm{H} 2 \mathrm{~A}, \mathrm{H} 2 \mathrm{~B}, \mathrm{H} 3$, and $\mathrm{H} 4$. Histone acetylation is a reversible process which is catalyzed by the histone deacetylase (HDAC) and results in transcriptional repression. Histone methyltransferase (HMT) is responsible for the methylation of arginine in histones 3 and 4, playing an important role in transcriptional activation of genes. In contrast, the H3 Lys 9 methylation is associated with a transcriptionally repressive heterochromatin. The objective of the present study was to determine the effects of different origins of embryos on the relative abundance of transcripts for the histone acetyltransferase 1 (HAT1), histone deacetylase 2 (HDAC2), histone metyltransferases (SUV39H1 
and G9A), and heterochromatin protein 1 (HP1). Messenger RNA expression profiles of these genes were investigated in bovine oocytes and preimplantation embryos up to the blastocyt stage produced either in vitro by two different culture systems, i.e. SOF+BSA or TCM+SERUM, by somatic cloning using adult male and female fibroblasts, parthenogenetic activation, and androgenetic construction, or in vivo, employing semiquantitative reverse transcription-polymerase chain reaction (RT-PCR). Significant differences are described below. HAT1, SUV39H1, G9A, and HP1 mRNA transcripts decreased in enucleated oocytes, compared with immature oocytes. The relative abundance of HAT1 and SUV39H1 transcripts was significantly increased in NT-derived zygotes produced from adult female fibroblasts, compared to their in vitro fertilized and parthenogenetic counterparts. No differences were found in the relative abundances of gene transcripts at the 8-16-cell stage, except for parthenogenetic embryos in which SUV39H1 transcripts were significantly higher than in all other 8-16 cell groups. The relative abundance of SUV39H1, G9A, and HP1 transcripts were significantly higher in NT-derived blastocysts derived from adult male fibroblasts than in their in vivo-generated counterparts. HP1 and G9A transcript levels were significantly increased in NT-derived blastocysts derived from male fibroblasts compared to NT-derived embryos produced from female fibroblasts. The results show that the in vitro environment and the nuclear transfer protocol affect mRNA expression patterns of histone modification genes in pre-implantation bovine embryos.

\title{
259 LOCALIZATION OF LEUKEMIA INHIBITORY FACTOR (LIF) GENE IN BOVINE PLACENTA
}

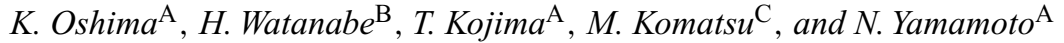 \\ A National Agricultural Research Center for Western Region, Oda, Shimane, Japan; \\ ${ }^{\mathrm{B}}$ Bio-oriented Technology Research Advancement Institution, Tokyo, Japan; \\ ${ }^{\mathrm{C}}$ National Institute of Livestock and Grassland Science, Tsukuba, Ibaraki, Japan
}

Leukemia inhibitory factor (LIF), a member of the group of hemopoietic cytokines, plays a primary role in the control of embryo development and implantation and in the growth of the placenta in humans and mice. The objective of this study was to investigate the localization of LIF gene in bovine placenta tissues during pregnancy using in situ hybridization (ISH). Eleven Japanese Black cows aged between 1.8 and 14.5 years, with normal estrous cycles, were used in this study. They were observed daily for estrous behavior, and the day of estrus was considered as Day 0 . They were artificially inseminated, and their uteri were collected on Days 61 to $63(n=3), 127$ to $142(n=4)$, and 225 to $232(n=4)$ of pregnancy. Pregnancy was confirmed by the presence of a conceptus in the uterus, and the uterus was isolated by dissection, avoiding damage of the uterine artery. The uterus was perfused with 4\% paraformaldehyde (PFA) solution using catheters inserted into the uterine artery, and placental tissues were isolated by dissection. Each tissue was cut into small pieces ( $5 \mathrm{~mm}$ thick) and fixed in $4 \%$ PFA solution for $20-24 \mathrm{~h}$, after which they were embedded in paraffin using routine procedures. Several tissue pieces were collected from each individual cow. Six micrometer-thick sections were cut and placed on MAS coated glass slides (Matsunami Glass, Kishiwada, Japan). The sections were dried in an oven for three days at $40^{\circ} \mathrm{C}$. Anti-sense and sense biotin-labeled oligonucleotide probes for bovine LIF were designed from the sequence information (GenBank accession number D50337). The sense probe was used as the negative control. ISH was carried out using GenPoint System (DakoCytomation, Glostrup, Denmark) according to the manufacturer's instructions. The sections were observed under an Eclipse 800 microscope (Nikon, Tokyo, Japan) to detect the positive signal for LIF gene. Density analysis was performed with Scion Image (Scion Corporation, Frederick, MD, USA). Data were analyzed by one-way ANOVA and Tukey-Kramer's HSD, using 0.05 as a significant level. Leukemia inhibitory factor gene was expressed in stromal cells of the fetal cotyledonary villus and caruncular crypt and in mono- and multi-nuclear epithelium cells of the fetal cotyledonary villus and caruncular crypt. Intensities of LIF gene positive signals in all positive cells at Days 127 to 142 tended to be weak compared with those at other periods. Furthermore, intensities of LIF gene positive signals of multinuclear cells of the villus and crypt demonstrated a tendency to be strong compared with those of other cells. These results suggest that LIF is produced in several cell types in the placenta and may play important roles in pregnancy.

\section{COMPREHENSIVE IDENTIFICATION OF RELATIVE GENES CAUSING ABNORMAL DEVELOPMENT OF BOVINE EMBRYOS PRODUCED BY SOMATIC CELL NUCLEAR TRANSFER}

\author{
J. Park, N. Minami, and H. Imai \\ Koyto University, Kyoto, Japan
}

Developmental failure of a cloned animal using somatic cell nuclear transfer (SCNT) procedures is considered to be the result of abnormal expression of developmentally important genes caused by incomplete reprogramming of the donor cell nuclei. However, there are few reports about stagespecific gene expression during cleavage progression of cloned embryos. The aim of this study was to identify using fluorescein differential display method, the differentially expressed genes in cloned embryos at early developmental stages compared with those produced by in vitro fertilization. Bovine cumulus-oocytes complexes (COCs) were aspirated from follicles (2-8 mm in diameter) of slaughterhouse ovaries and cultured in TCM-199 supplemented with $10 \%$ fetal calf serum (FCS) for $18 \mathrm{~h}$ for somatic cell nuclear transfer (NT) or $24 \mathrm{~h}$ for in vitro fertilization (IVF) at $39^{\circ} \mathrm{C}$. Removal of oocyte nuclei for NT was performed by squeezing out a small amount of the cytoplasm laying beneath the first polar body by means of a glass needle. Donor cells for NT were obtained from skin cells of an adult cow and cultured in DMEM supplemented with $10 \%$ FCS. After the transfer of somatic cell into enucleated oocytes, DC electric pulses at $200 \mathrm{~V} / \mathrm{mm}$ for $2 \times 10 \mu$ s were used for fusion, and the reconstructed embryos were treated with $10 \mu \mathrm{g} / \mathrm{mL}$ cycloheximide for $6 \mathrm{~h}$. The embryos were then cultured for $120 \mathrm{~h}$ (morula stage) or $168 \mathrm{~h}$ (blastocyst stage) in modified SOF medium under $5 \% \mathrm{CO}_{2}, 5 \% \mathrm{O}_{2}$ and $90 \% \mathrm{~N}_{2}$ at $39^{\circ} \mathrm{C}$. Total RNA obtained from NT and IVF embryos were analyzed by differential display RT-PCR (DDRT-PCR) as previously described (Minami et al. 2001 Biol. Reprod. 64, 30-35). We obtained several differences in gene expression patterns between NT and IVF embryos at the morula and blastocyst stage. A total of 52 cDNA fragments were isolated and analyzed. Semiquantitative analysis revealed that some genes (NADH dehydrogenase subunit 1, SR rich protein, KIAA0107, ribosomal protein L19) were highly expressed in IVF embryos compared with NT embryos, whereas other genes (CASK) were highly expressed in NT embryos compared with IVF embryos. These 
results indicate that the differentially expressed genes observed in NT embryos may be representative of marker genes for the production of normal NT offspring and DDRT-PCR procedure is quite useful for identification of several genes that are differentially expressed between NT and IVF embryos.Although the detailed function of the genes and their products remains to be determined, it is likely that the reprogramming mechanisms can be elucidated genetically by the analysis of differentially expressed genes in the future.

\title{
261 IDENTIFICATION OF OOCYTE- AND CUMULUS-DERIVED CO-REGULATED AND DIFFERENTIALLY REGULATED TRANSCRIPTS ASSOCIATED WITH BOVINE MEIOTIC MATURATION
}

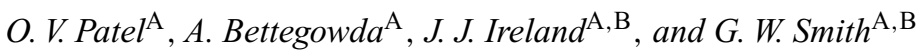 \\ ${ }^{A}$ Department of Animal Science, Michigan State University, East Lansing, MI 48824, USA; \\ ${ }^{B}$ Department of Physiology, Michigan State University, East Lansing, MI 48824, USA
}

\begin{abstract}
Understanding the process of oocyte maturation is critical for efficient application of biotechnologies such as in vitro embryo production and nuclear transfer/cloning. Intercellular communication between the oocyte and the encompassing somatic (cumulus) cells is pivotal for successful growth of ovarian follicles and oocyte maturation. Therefore, we utilized global gene expression profiling to determine changes in the transcriptome of oocytes and their adjacent cumulus cells during meiotic maturation in vitro to identify both co-regulated and differentially regulated transcripts within the two cell compartments of the cumulus oocyte complex (COC). Germinal vesicle (GV) and in vitro matured metaphase II (MII) COC ( $n=5$ pools of 5 COC per group) were denuded and separated into oocytes and cumulus cells. RNA was extracted from the oocytes and cumulus cells and subjected separately to microarray analysis using a bovine cDNA array containing expressed sequence tags (ESTs) representing 15500 unique genes. A combined total of 1045 genes displaying greater mRNA abundance in GV oocytes and associated cumulus cells compared to MII samples were detected $(P<0.05$; false discovery rate $(\mathrm{FDR})=5 \%)$. A combined total of 711 genes displaying greater mRNA abundance in MII oocytes and enclosing cumulus cells compared to GV samples were detected $(P<0.05 ; \mathrm{FDR}=5 \%)$. Fourteen transcripts were identified that were co-regulated and of greater abundance in GV or MII oocytes and in their matching cumulus cells $(P<0.05 ; \mathrm{FDR}=5 \%)$. The co-regulated transcripts identified are implicated in metabolism (e.g. heme oxygenase-2, leukotriene B4 12-hydroxydehydrogenase), signal transduction (e.g. caveolin 1, ring finger protein 31 ), and cell growth (e.g. BTG family member 2, myosin regulatory light chain 2). In contrast, thirteen transcripts differentially regulated in the GV oocyte versus MII cumulus cells were identified $(P<0.05 ; \mathrm{FDR}=5 \%)$. Similarly, nine transcripts differentially regulated in the MII oocyte versus GV cumulus cells were identified $(P<0.05$; FDR $=5 \%)$. Some of the identified differentially regulated transcripts encode for genes associated with the cytoskeleton (e.g. tropomyosin 1), apoptotic activity (e.g. death effector domain containing protein 2) and DNA replication (e.g. epsilon polymerase). The results provide novel insights into the identity of transcripts whose abundance is co-regulated or differentially regulated between the oocyte and cumulus cells during the transition of a COC from the GV to the MII stage. Characterization of the signaling pathways driving changes in transcript abundance for co-regulated and differentially regulated genes in oocytes versus associated cumulus cells may lead to a better understanding of regulation of meiotic maturation and potential cross-talk between germ cells and somatic cells during the oocyte maturation cascade.
\end{abstract}

This work was supported by the Rackham Foundation and the MI Agriculture Experiment Station.

\section{TISSUE-SPECIFIC ANALYSIS OF PRION EXPRESSION IN EARLY BOVINE FETUSES}

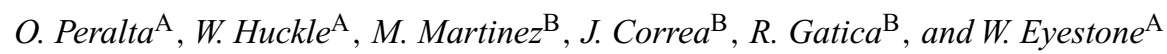 \\ ${ }^{A}$ Virginia-Maryland Regional College of Veterinary Medicine, Blacksburg, VA 24061, USA; \\ ${ }^{\mathrm{B}}$ Austral University, Valdivia, Chile
}

The prion protein $(\mathrm{PrP})$ is best known for its mis-folded, pathogenic isoform, which is widely regarded as the infectious agent in transmissable spongiform encephalopathies. However, the role of normal, cellular PrP, a host-encoded $29-\mathrm{kD}$ glycoprotein tethered to the cell membrane by a phosphatidyl-inositol glycane (GPI) anchor, is poorly understood. PrP binds copper with high affinity, has antioxidant activity and may play a role in cell adhesion and/or signaling. PrP is expressed in mouse embryos on 6.5 days post-coitum in extra-embryonic tissue and at 13.5 days in the central and peripheral nervous system, intestine, and dental lamina. Our previous data revealed PrP gene expression in bovine embryos throughout preimplantation embryo development. As part of a larger effort to map the ontogeny of cellular PrP expression in cattle, we sought here to analyze in early bovine fetuses (1) total PrP gene expression by real-time quantitative PCR (QPCR), and (2) tissue-specific PrP expression by immunohistochemistry. Fetuses were obtained from donor cattle bred by artificial insemination (AI; Day 0$)$ and subjected to mid-ventral laparotomy on Days $32(n=2)$ and $39(n=2)$. Immediately upon recovery, one fetus from each stage was placed in RNAlater for RNA isolation and the other fixed in $10 \%$ formalin for immunohistochemistry. RNA was isolated using an RNeasy ${ }^{\circledR}$ mini kit (Qiagen, Valencia, CA, USA). cDNA was generated by reverse transcription with random hexamer priming and used the $\Delta \Delta \mathrm{cT}$ method for estimation of PrP expression by QPCR. Tissue-specific expression was determined by immunohistochemistry. Formalin-fixed fetuses were embedded in paraffin, sagittally sectioned, dehydrated, and subjected to an unmasking protocol that employed Vectorlab unmasking solution and autoclaving. Tissues were then probed with a primary anti-PrP monoclonal antibody (SAF 32; Cayman Chemical Company, Ann Arbor, MI, USA). Bound primary antibody was detected with a biotinylated horse anti-mouse secondary antibody complexed to horseradish peroxidase using the ABC kit (Vector Laboratories, Burlingame, CA, USA). Probed sections were then counterstained with hematoxolin and eosin. Neighboring sections, processed identically but to which no primary antibody was added, served as controls. PrP gene expression was detected by QPCR at both stages examined and tended to be higher in Day 39 compared to Day 32 fetuses. PrP immunoreactivity was found throughout the central and peripheral nervous systems, ganglia, nerve trunks, and neural cell populations of sensory organs in both Day 32 and Day 39 fetuses. PrP immunolabeling was also observed in the mesonephric kidney, liver, and heart in the Day 39 fetus. 
At both stages, immunoreactivity was most intense in the nervous system. Thus, PrP is expressed in a tissue-specific pattern in early bovine fetuses. Tissue distribution of fetal PrP expression appears similar to that of adult PrP. Moreover, PrP appears to be expressed in a developmentally regulated fashion in some tissues.

\title{
263 USE OF PORCINE PARTHENOTES AND GENE EXPRESSION PROFILING USING MICROARRAYS FOR IDENTIFICATION OF IMPRINTED GENES
}

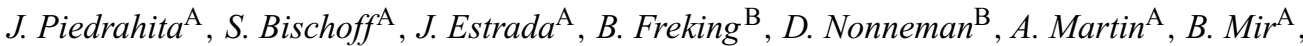 \\ G. Rohrer $^{\mathrm{B}}$, and S. Tsai ${ }^{\mathrm{A}}$ \\ ${ }^{\text {A } M o l e c u l a r ~ B i o m e d i c a l ~ S c i e n c e s, ~ N o r t h ~ C a r o l i n a ~ S t a t e ~ U n i v e r s i t y, ~ R a l e i g h, ~ N C ~ 27606, ~ U S A ; ~}$ \\ B ARS, USDA, U.S. Meat Animal Research Center, Clay Center, NE 68933, USA
}

\begin{abstract}
Genomic imprinting arises from differential epigenetic markings including DNA methylation and histone modifications and results in one allele being expressed in a parent-of-origin specific manner. For further insight into the porcine epigenome, gene expression profiles of parthenogenetic (PRT; two maternally derived chromosome sets) and biparental embryos (BP; one maternal and one paternal set of chromosomes) were compared using microarrays. Comparison of the expression profiles of the two tissue types permits identification of both maternally and paternally imprinted genes and thus the degree of conservation of imprinted genes between swine and other mammalian species. Diploid porcine parthenogenetic fetuses were generated using follicular oocytes (BOMED, Madison, WI, USA). Oocytes with a visible polar body were activated using a single square pulse of direct current of $50 \mathrm{~V} / \mathrm{mm}$ for $100 \mu \mathrm{s}$ and diploidized by culture in $10 \mu \mathrm{g} / \mathrm{mL}$ cycloheximide for $6 \mathrm{~h}$ to limit extrusion of the second polar body. Following culture, BP embryos obtained by natural matings, and PRT embryos, were surgically transferred to oviducts on the first day of estrus. Fetuses recovered at 28-30 days of gestation were dissected to separate viscera including brain, liver, and placenta; the visceral tissues were then flash-frozen in liquid nitrogen. Porcine fibroblast tissue was obtained from the remaining carcass by mincing, trypsinization, and plating cells in $\alpha$-MEM. Total RNA was extracted from frozen tissue or cell culture using RNA Aqueous kit (Ambion, Austin, TX, USA) according to the manufacturer's protocol. Gene expression differences between BP and PRT tissues were determined using the GeneChip ${ }^{\circledR}$ Porcine Genome Array (Affymetrix, Santa Clara, CA) containing 23256 transcripts from Sus scrofa and representing 42 genes known to be imprinted in human and/or mice. Triplicate arrays were utilized for each tissue type, and for PRT versus BP combination. Significant differential gene expression was identified by a linear mixed model analysis using SAS 5.0 (SAS Institute, Cary, NC, USA). Storey's $q$-value method was used to correct for multiple testing at $q \leq 0.05$. The following genes were classified as imprinted on the basis of their expression profiles: In fibroblasts, ARHI, HTR2A, MEST, NDN, NNAT, PEG3, PLAGL1, PEG10, SGCE, SNRPN, and UBE3A; in liver, IGF2, PEG3, PLAGL1, PEG10, and SNRPN; in placenta, HTR2A, IGF2, MEST, NDN, NNAT, PEG3, PLAGL1, PEG10, and SNRPN; and in brain, none. Additionally, several genes not known to be imprinted in humans/mice were highly differentially expressed between the two tissue types. Overall, utilizing the PRT models and gene expression profiles, we have identified thirteen genes where imprinting is conserved between swine and humans/mice, and several candidate genes that represent potentially imprinted genes. Presently, our efforts are focused in the identification of single nucleotide polymorphisms (SNPs) to more carefully evaluate the behavior of these genes in normal and abnormal gestations and to test whether the candidate genes are indeed imprinted.
\end{abstract}

This research was supported by USDA-CSREES grant 524383 to J. P. and B. F.

\section{IDENTIFICATION OF THE PREDOMINANT TRANSCRIPT IN BOVINE OOCYTES, IN VITRO-DERIVED BLASTOCYSTS, AND IN NUCLEAR TRANSFER BLASTOCYSTS}

\author{
R. S. Prather, S. Korte, R. Woods, L. Spate, N. Bivens, L. J. Forrester, G. K. Springer, R. V. Patel, \\ Z. H. Liu, C. N. Murphy, and J. A. Green
}

Department of Animal Sciences, Univeristy of Missouri-Columbia, Columbia, MO 15201, USA

Identification of transcripts produced during bovine embryogenesis is the first step in describing the normal developmental program. To that end, mRNA was isolated from in vitro-matured metaphase II oocytes (MPII), in vitro-produced 2-cell-stage (2-Cell), in vitro-produced precompact morula-stage (PCM), in vitro-produced blastocyst-stage (BL), and in vitro-produced nuclear transfer blastocyst-stage (NTBL) embryos. The mRNA was isolated by using Dynabeads ${ }^{\circledR}$ (Dynal, Inc., Lake Success, NY, USA), and amplified by using the SMART system. PCR products were purified and ligated into pSPORT1 and electroporated into E. coli. Random clones were selected for DNA sequencing. Sequence data were evaluated for quality and clustered by sequence similarity with sequences generated from a larger expressed sequence tag (EST) project (http://genome.rnet.missouri.edu/Bovine/) by using the tlcluster program from the University of Iowa. Sequences over 100 bp in length with average Phred scores of over 20 for the entire sequence were submitted to GenBank (NIH genetic sequence database). Sequences were compared to the bovine TIGR (The Institute for Genomic Research) and human databases to gather annotation. The best comparison is listed below by using the HUGO Gene Nomenclature Committee standards (http://www.gene.ucl.ac.uk/nomenclature/) when possible. The number of unique clusters, i.e. no match in GenBank, was 53,120, 109, 115, and 135, for MPII, 2-Cell, PCM, BL, and NTBL, respectively. The total number of clusters per tissue ranged from 224 to 992 . The percent of clusters (number of clusters per total number of ESTs) per library was $12 \%(224 / 1762), 42 \%$ (746/1771), $48 \%$ (819/1715), 49\% (900/1818) and 53\% (992/1876) for MPII, 2-Cell, PCM, BL, and NTBL, respectively. Either the quality of the MPII library was lower or the complexity of the MPII mRNA was less than mRNA in the other tissues. Examples of mRNA that were in different abundance are shown in Table 1. Clearly, as in other species, there are significant changes in mRNA abundance during early embryogenesis. Furthermore, NTBL embryos, even though they are morphologically similar to BL, possess a population of mRNA that is distinct from that in BL. 
Table 1. Comparison of mRNA Abundance During Bovine Embryogenesis

\begin{tabular}{ll}
\hline Tissue & Genes \\
\hline MPII $>$ 2-Cell & DOC2A, USP22, AZIN1, SFTPB, FTL \\
2-Cell $>$ MPII & RPS18, PRKAR1A, PTTG1, COX7A2L \\
2-Cell $>$ PCM & RPS18, PTTG1, PRKAR1A, KPNA2, CALM1, PSMA7, CKS1B \\
PCM $>$ 2-Cell & RPL15, HNRPAB, RPL36, RPL23, PISRT1, ACTG1 \\
PCM $>$ 2-Cell & RPL15, HNRPAB, RPL36, RPL23, PISRT1, ACTG1 \\
PCM $>$ 2-Cell & RPL15, HNRPAB, RPL36, RPL23, PISRT1, ACTG1 \\
PCM $>$ 2-Cell & RPL15, HNRPAB, RPL36, RPL23, PISRT1, ACTG1 \\
PCM $>$ 2-Cell & RPL15, HNRPAB, RPL36, RPL23, PISRT1, ACTG1 \\
PCM $>$ 2-Cell & RPL15, HNRPAB, RPL36, RPL23, PISRT1, ACTG1 \\
PCM $>$ 2-Cell & RPL15, HNRPAB, RPL36, RPL23, PISRT1, ACTG1 \\
\hline
\end{tabular}

This work was funded by the USDA NRI 2003-35205-12812 and Food for the 21st Century.

\title{
265 DEVELOPMENT AND DYNAMICS OF GENE EXPRESSION OF BOVINE EMBRYOS CULTURED IN VITRO IN THREE DIFFERENT MEDIA
}

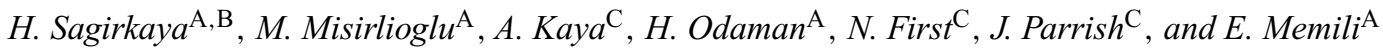 \\ A Department of Animal \& Dairy Sciences, Mississippi State University, Mississippi State, MS 39762, USA; \\ ${ }^{B}$ Department of Reproduction \& Artificial Insemination, Uludag University, Veterinary Faculty, Gorukle-Bursa, Turkey; \\ ${ }^{\mathrm{C}}$ Department of Animal Sciences, University of Wisconsin-Madison, Madison, WI 53706, USA
}

\begin{abstract}
Dramatic reprogramming of gene expression occurs during embryonic genome activation (EGA), an essential event initiating as early as the 1-cell zygotic stage in the bovine and increasing gradually as embryonic development advances. It is this reprogramming of gene expression that sets the stage for later development. Expression of embryonic genes is altered in different culture conditions and this may influence developmental potential both during pre-implantation and during fetal development. The objective of this study was to define some most commonly used embryo culture media (KSOMaa, CR1aa, and SOFaa) based on their ability to support embryonic development to the blastocyst stage, mean cell number, percentages of apoptotic cells, and the expression patterns of a panel of developmentally important genes. Oocytes with several layers of cumulus cells obtained from an abattoir were matured in TCM 199 (supplemented with $0.25 \mathrm{mM}$ pyruvate, $0.5 \mu \mathrm{g} / \mathrm{mL} \mathrm{FSH}, 5 \mu \mathrm{g} / \mathrm{mL} \mathrm{LH}, 100 \mathrm{U} / \mathrm{mL}$ penicillin, $100 \mu \mathrm{g} / \mathrm{mL}$ streptomycin, and $10 \%$ FCS) for $24 \mathrm{~h}$ and in vitro-fertilized (Day 0) using frozen bull semen. Presumptive zygotes were transferred into three different media (KSOMaa, CR1aa, and SOFaa) 16-18 h post-insemination, supplemented with 10\% FCS on Day 4, and cultured until Day 8 at which time they were fixed or frozen for further analysis. Mean cell numbers and percentages of apoptotic cells in blastocysts were determined using terminal deoxynucleotidyl transferase biotin-dUTP nick end labeling (TUNEL). Real-time quantitative PCR was performed to assess gene transcripts of glucose transporter-1 (Glut-1), heat shock protein 70.1 (Hsp70), interferon-tau (IF-tau), insulin-like growth factor II receptor (Igf-2r), desmosomal glycoprotein desmocollin III (DcIII), and DNA methyltransferase 3a (Dnmt3a). Gene expression data were analyzed relative to transcripts of housekeeping gene glyceraldehyde 3-phosphate dehydrogenase (Gapdh). In three separate trials, a total of 538, 518, and 503 oocytes were used for KSOMaa, CR1aa, and SOFaa groups, respectively. Cleavage rates were $79.2 \%, 77.5 \%$, and $80.2 \%$; and rates of development to the blastocyst stage were $22.2 \%, 23.4 \%$, and $32.9 \%$ for KSOMaa, CR1aa, and SOFaa groups, respectively. The blastocyst rate of the SOFaa group was significantly higher than those of the KSOMaa and CR1aa groups $(P<0.05)$. Mean cell numbers were 109.3, 101.0, and 114.0; and the percentages of apoptotic cell numbers per blastocyst were 1.25, 1.91, and 1.87 for KSOMaa, CR1aa, and SOFaa groups, respectively. There was no difference among groups in terms of mean cell numbers and percentages of apoptotic cells per blastocyst. The expressions of Glut-1 and DcIII genes did not differ among the groups. However, expressions of Hsp70, IF-tau, and Dnmt3a genes were all significantly up-regulated in the CR1aa group as compared to the SOFaa and KSOMaa groups $(P<0.05)$. In conclusion, SOFaa supports higher development to the blastocyst stage than KSOMaa and CR1aa, and culture conditions influence gene expression.
\end{abstract}

\section{IDENTIFICATION OF Oct-4 AND Nanog, THE TWO PLURIPOTENCY MARKER GENES IN RABBIT PRE-IMPLANTATION-STAGE EMBRYOS}

\author{
Z. Tancos ${ }^{\mathrm{A}}$, J. Kobolak ${ }^{\mathrm{A}}$, A. Baji Gal ${ }^{\mathrm{A}}$, and A. Dinnyes ${ }^{\mathrm{A}, \mathrm{B}}$ \\ ${ }^{\mathrm{A}}$ Micromanipulation and Genetic Reprogramming Group, Agricultural Biotechnology Center; ${ }^{\mathrm{B}}$ Research Group for Applied \\ Animal Genetics and Biotechnology, Hungarian Academy of Sciences and Szent Istvan \\ University, Godollo, Hungary
}

Several genes, including Oct-4 and Nanog, coordinate the embryogenesis of mammalian embryos. Whereas Oct-4 has an activator effect, the Nanog protein blocks the transcription of several genes in early stages; however, the product of these two genes appears parallel and directs early embryogenesis. The goal of this work was to isolate the Oct-4 and Nanog genes from rabbit, based on the sequences of other species published so far. The sequence of known genes has been analyzed, and primers have been designed based on similarity of sequences. Oocyte-to-blastocyt-stage embryos were collected from superovulated rabbits in RNase-free water. Embryonic mRNA was isolated by using the Dynal mRNA isolation KIT (Dynal, Biotech, Oslo, Norway). Real-time PCR was performed in a ABI PRISM ${ }^{\circledR} 7000$ Sequence Detection System (Applied Biosystems, Foster City, CA, USA). The reaction mixture consisted SYBR ${ }^{\circledR}$ Green PCR Master Mix (Applied Biosystems), $300 \mathrm{nM}$ of each primer, and an 1/8 aliquot 
of the embryo cDNA in a $25-\mu \mathrm{L}$ final volume. The cDNA template was denatured by heating to $95^{\circ} \mathrm{C}$ for 10 min and amplified by 45 cycles of $95^{\circ} \mathrm{C}$ for $15 \mathrm{~s}$ and $60^{\circ} \mathrm{C}$ for $1 \mathrm{~min}$, with a single fluorescence measurement at each cycle. When the reaction was finished, melting curves were plotted to confirm product purity. The reaction mix was electrophoretically separated to facilitate fragment isolation. In the case of Nanog, a 131-bp fragment was cloned. Sequence analysis revealed significant homology with the mouse sequence. Based on this finding, new primers were designed in order to isolate a larger fragment as well as the genomic copy of the gene. In the case of Oct-4, several combinations of primers were tested, because of the rather conservative sequence of the Oct- 4 transcription factor family. As a result, an optimal primer pair was found that yielded a $450 \mathrm{bp}$ fragment, expected according to known sequences. After direct sequencing, there was a high similarity to Oct-4 genes, indicating that the isolated cDNA is probably part of the rabbit Oct-4 cDNA. For further analysis, the cDNA fragments of both genes were isolated (MinElute Gel Extraction Kit; Qiagen, Valencia, CA, USA) and cloned into bacterial plasmids (TOP 10 Cloning KIT; Invitrogen, Carlsbad, CA, USA). At this point, it can be stated that: (1) both genes have been successfully identified in rabbit genome, (2) a method has been developed to detect expression of genes, and (3) gene-specific primers have been produced. Our further goal is to clone the whole coding region of the genes and to identify the sequence. The cloned fragments will be used for in situ hybridization in implanted stage embryo sections.

This research was supported by EU-FP6-MEXT-CT-2003-59582, Wellcome Trust (Grant No. 070246), OTKA T046171, and NKTH BIO-00017/2002.

\title{
267 TARGETED SUPPRESSION OF THE EXPRESSION OF MATERNAL AND EMBRYONIC GENES DURING IN VITRO DEVELOPMENT OF BOVINE EMBRYOS
}

\author{
D. Tesfaye, K. Nganvongpanit, F. Rings, M. Gilles, D. Jennen, M. Hoelker, \\ E. Tholen, and K. Schellander \\ Institute of Animal Science, Animal Breeding and Husbandry Group, University of Bonn, Endenicher \\ Allee 15, 53115 Bonn, Germany
}

\begin{abstract}
Despite enormous advances in the identification and sequencing of developmentally relevant bovine genes, the function of the majority of these transcripts is not yet known. Here we aimed to apply the RNA interference (RNAi) approach to suppress the expression of the maternal transcript c-mos (AY630920) and embryonic transcripts E-cadherin (AY508164) and Oct-4 (AY490804) during in vitro development of bovine embryos using microinjection of sequence-specific double-stranded RNA (dsRNA). For this 435-, 341- and 341-bp-long dsRNA specific to the coding sequences of c-mos, E-cadherin and Oct-4 transcripts, respectively, were synthesized using Promega RiboMax ${ }^{\mathrm{TM}}$ T7 system (Promega, Madison, WI, USA), where sense and antisense strands were transcribed from the target DNA template. Slaughterhouse ovaries were used to aspirate bovine oocytes, which were matured in TCM-199 with $12 \%$ estrus cow serum (ECS), fertilized in Fert-TALP, and cultured in CR1 medium at $39^{\circ} \mathrm{C}$ under humidified atmosphere of 5\% $\mathrm{CO}_{2}$ in air. In Experiment 1, immature oocytes were categorized into three groups, each containing 50-60 oocytes: those injected with c-mos dsRNA, those injected with RNase-free water, and uninjected controls. In Experiment 2, zygotes were categorized into four groups, each containing 50-60 zygotes: those injected with E-cadherin dsRNA, those injected with Oct-4 dsRNA, those injected with RNase-free water, and uninjected controls. Each experiment was repeated four times. The effect of dsRNA on in vitro development of oocytes or embryos was assessed after microinjection during culture. The level of mRNA and protein expression was investigated using real-time PCR and western blot analysis, respectively. Data were analyzed using SAS, version 8 (SAS Institute Inc., Cary, NC, USA). Microinjection of c-mos dsRNA resulted in a $70 \%$ reduction of c-mos transcript abundance after maturation compared to the water-injected and uninjected controls $(P<0.05)$. Similarly, microinjection of E-cadherin and Oct- 4 dsRNA at the zygote stage resulted in $80 \%$ and $60 \%$ reduction in transcript abundance at the blastocyst stage, respectively, compared to the uninjected controls $(P<0.05)$. Decreases in the c-mos $(39 \mathrm{kDa})$ and E-cadherin proteins $(119 \mathrm{kDa})$ were observed in the c-mos and E-cadherin dsRNA-injected groups, respectively, compared to the control. A higher proportion of oocytes (75\%) showed first polar body extrusion after maturation in c-mos dsRNA-injected groups, compared to $52 \%$ in water-injected and $57 \%$ in uninjected controls. Only $22 \%$ from E-cadherin dsRNA- and 24\% from Oct-4 dsRNA-injected zygotes developed to the blastocyst stage compared to 39 and $37 \%$ blastocyst rates in water-injected and uninjected control groups, respectively. In conclusion, injection of sequence-specific dsRNA in bovine oocytes and embryos resulted in suppression of mRNA and their protein products, thereby affecting in vitro development of bovine embryos.
\end{abstract}

\section{BOVINE AND EQUINE RECOMBINANT FOLLICLE-STIMULATING HORMONE PRODUCED IN TWO HETEROLOGOUS HOSTS: ESCHERICHIA COLI AND TRYPANOSOMA BRUCEI}

\author{
M. Wilson ${ }^{\mathrm{A}}$, J. Morris ${ }^{\mathrm{B}}$, and J. Gibbons ${ }^{\mathrm{A}}$ \\ A Animal and Veterinary Sciences Department, Clemson University; \\ ${ }^{\mathrm{B}}$ Genetics and Biochemistry Department Clemson University, Clemson, SC 29631, USA
}

Follicle-stimulating hormone (FSH) is a heterodimeric glycoprotein hormone consisting of $\alpha$ and $\beta$ subunits produced by different genes. Following release from the anterior pituitary, FSH is responsible for development of ovarian follicles from the time of emergence of a follicular wave until divergence of the dominant follicle. Treatment of cattle and horses with recombinant FSH would be valuable for induction of superovulation in an effort to harvest multiple embryos for use in embryo transfer. Glycosylation of the subunits may be essential for proper function and receptor recognition, thereby complicating the production of recombinant FSH. Therefore, a eukaryotic, rather than a prokaryotic, host would be most beneficial for generation of recombinant protein. To explore recombinant protein synthesis, we utilized the ancient protozoan Trypanosoma brucei. Perhaps best known for its parasitic life style, T. brucei has been the focus of extensive biochemical and genetic research, with the nonpathogenic insect stage of the parasite serving as a model eukaryote for the study of many fundamental eukaryotic pathways, including glycosyl phosphatidylinositol (GPI) anchor biosynthesis and RNA editing. The ease of molecular manipulation of this protozoan, and the organism's robust glycosylation machinery, 
has led us to explore the possibility of using the non-pathogenic life stage for recombinant protein production. The objective of this study was to produce recombinant bovine and equine FSH in both prokaryotic and eukaryotic expression systems. Protein has been produced in Escherichia coli using the pQE30 expression system. Subunit genes were ligated into pQE30 and expressed in M15 E. coli cells. These cells were found to produce detectable amounts of the individual subunit protein after a three hour induction. To express recombinant protein in T. brucei, subunit genes were cloned into pLEW2T7(6His), a stably integrated vector designed to allow inducible expression of proteins fused to a C-terminal six-histidine tag. Clones were subsequently transformed into T. brucei and are awaiting protein expression. All protein purification will be performed via nickel affinity chromatography and protein concentration will be measured using radioimmunoassay. Bioactivity will be analyzed using a rat ovarian weight assay prior to use in cattle and horses. The purified protein will be administered as intramuscular injections twice daily for four days beginning on the day of follicular wave emergence. Producing recombinant FSH will be useful for the embryo transfer and food safety industries by increasing consistency of biologic activity, decreasing production costs, and, perhaps most importantly, decreasing the opportunity for zoonotic transmission of prions such as bovine spongiform encephalopathy.

IVF/IVP

\title{
269 EFFECTS OF REPRODUCTIVE STATUS AND OOCYTE QUALITY ON THE DEVELOPMENTAL COMPETENCE OF OOCYTES FOLLOWING IN VITRO PRODUCTION IN DOMESTIC CAT
}

\author{
B. Agung, P. Wongsrikeao, H. Fuchieda, and T. Otoi \\ Laboratory of Animal Reproduction, Department of Veterinary Science, Yamaguchi University, Yamaguchi, Japan 753-1515
}

\begin{abstract}
A recent study showed that the developmental competence of cat oocytes after IVM and IVF was affected by the estrous cycle stage of the donor (Freistedt et al. 2001 Biol. Reprod. 65, 9-13). This study was conducted to examine the effect of the cat reproductive cycle stage and the oocyte quality on the developmental competence of the oocyte following IVF production. Cat ovaries were collected at veterinary clinics and stored at $4^{\circ} \mathrm{C}$ for $24 \mathrm{~h}$. Based on the presence or absence of follicles and corpora lutea, the ovaries were classified into the luteal, follicular, or inactive stage. Cumulus oocyte complexes (COCs) from the different stage ovaries were separated at recovery into three ranks (A, B, and C) according to pigmentation, uniformity and smoothness of ooplasm, and amount of surrounding cumulus cell mass and were cultured separately in $100 \mu \mathrm{L}$ drops of maturation medium (TCM-199), supplemented with $0.4 \%$ bovine serum albumin, $0.1 \mathrm{IU} / \mathrm{mL}$ (human menopausal gonadotropin (HMG), $10 \mathrm{IU} / \mathrm{mL} \mathrm{HCG}, 1 \mu \mathrm{g} / \mathrm{mL} 17 \beta$-estradiol, and $100 \mu \mathrm{g} / \mathrm{mL}$ gentamicin) for $24-\mathrm{h}$ at $38^{\circ} \mathrm{C}, 5 \% \mathrm{CO}_{2}$ in air. After 24 -h in vitro culture, the oocytes were transferred into $100 \mu \mathrm{L}$ sperm microdrops of Brackett-Oliphant medium $\left(2 \times 10^{6}\right.$ sperm $\left./ \mathrm{mL}\right)$ for fertilization and were co-incubated for $12 \mathrm{~h}$. Subsequently, presumptive zygotes were transferred into a modified Earle's balanced salt solution (MK-1) supplemented with $4 \mathrm{mg} / \mathrm{mL}$ BSA and $50 \mu \mathrm{g} / \mathrm{mL}$ gentamicin. Three days after insemination, all embryos were transferred into culture medium of MK-1 supplemented with $5 \%$ (v/v) fetal bovine serum and $50 \mu \mathrm{g} / \mathrm{mL}$ gentamicin. The cleaved embryos were further cultured for 5 days to evaluate their ability to develop to the blastocyst stage. Data were analyzed by ANOVA. The percentages of COCs of A $(35.5 \%, 31.8 \%, 27.7 \%)$, B $(41.5 \%, 39.9 \%, 42.9 \%)$, and C $(23.0 \%, 28.3 \%$, $29.4 \%)$ ranks did not show significant differences $(P>0.05)$ among the reproductive stages of ovaries (luteal, follicular, and inactive, respectively). There were significant differences in the percentages of cleavage $(P<0.05)$ among the $\mathrm{A}, \mathrm{B}$, and $\mathrm{C}$ ranks of oocytes from ovaries classified as luteal and follicular stages $(50 \%, 35 \%, 1.8 \% ; 52 \%, 35 \%, 3.9 \%$, respectively, $P<0.05)$. However, there were no significant differences between the A and B ranks of oocytes obtained from inactive stages of ovaries ( $47 \%$ vs. $44 \%$, respectively; $P>0.05)$ but there was a significant difference for $\mathrm{C}$ rank $(1.9 \%)$. The percentages of blastocyst formation were significantly different $(P<0.05)$ among the ranks of oocytes obtained from luteal, follicular, and inactive stages $(27 \%, 14.6 \%$, and $0 \%$ for $\mathrm{A} ; 24 \%, 11.7 \%$, and $0.6 \%$ for $\mathrm{B} ; 35 \%, 21.6 \%$, and $2.9 \%$ for $\mathrm{C}$ ). However, there were no significant differences $(P>0.05)$ among the reproductive cycle stages of ovaries in the three oocyte ranks with respect to the percentages of cleavage and blastocyst formation. These results indicate that the reproductive stage of donor cat ovaries stored at $4{ }^{\circ} \mathrm{C}$ for $24 \mathrm{~h}$ has no apparent effect on the developmental competence of the oocyte following IVF, but development is affected by oocyte quality.
\end{abstract}

\section{APOPTOSIS AND ONCOSIS ASSESSMENT IN CRYOPRESERVED MOUSE EMBRYOS}

\author{
M. E. O. A. Assumpção, A. R. S. Coutinho, W. B. Feitosa, C. M. Mendes, R. Simões, and J. A. Visintin \\ Department of Animal Reproduction-FMVZ, São Paulo University, São Paulo-São Paulo, Brazil
}

Cryopreservation of mammalian embryos is an important tool for the application of reproductive biotechnology. Recent evidence indicates that apoptosis of cryopreserved embryos may be a negative factor for their viability. The aim of this study was to detect apoptosis and to characterize and quantify the embryonic cell death caused by cryopreservation. Mouse morulae were separated to be subjected to two cryopreservation protocols (slow freezing and vitrification) and a control group (fresh). In the slow-freezing procedure, embryos were exposed to $10 \%$ ethylene glycol (EG) for $10 \mathrm{~min}$. Straws were placed in a methanol bath at $-7^{\circ} \mathrm{C}$ until it reached $-31^{\circ} \mathrm{C}$ and then plunged and stored in liquid nitrogen. The embryos were thawed in air for $10 \mathrm{~s}$ and in a $25^{\circ} \mathrm{C}$ water bath for $20 \mathrm{~s}$. In the vitrification method, embryos were exposed to $10 \%$ and $20 \% \mathrm{EG}$ for $5 \mathrm{~min}$, followed by $40 \% \mathrm{EG}+18 \%$ Ficoll $+10 \%$ sucrose (EFS) for $30 \mathrm{~s}$ and then plunged and stored in liquid nitrogen. These embryos were thawed in a $25^{\circ} \mathrm{C}$ water bath for $20 \mathrm{~s}$. For the cell death evaluation, cell membrane integrity from the fresh and cryopreserved embryos was assessed by Hoechst and propidium iodide (H/PI staining). Morphology and apoptosis were assessed by means of the haematoxylin-eosin staining (HE) and by electron microscopy (MET). To confirm apoptosis, 64 cryopreserved mouse morulae (34 submitted to slow freezing and 30 to vitrification) were used to evaluate Caspase-3 activity. The cryopreserved embryos were divided into experimental and control groups and incubated with Caspase-3 and 
buffer solution, respectively. Afterward, the embryos were incubated with rhodamine and the Caspase activity was determined under a fluorescence microscope. H/PI staining detected more membrane permeability in the vitrification (69.7\%) than in the slow-freezing $(48.4 \%)$ or fresh (13.8\%) groups $(P<0.05$; Wilcoxon's test). Nuclear evaluation by HE revealed that vitrification and slow freezing induced pyknosis and chromatin condensation. HE staining revealed weakly staining cytoplasm and degenerated cells in the vitrification group (indicating oncosis), whereas in the slow-freezing the presence of cytoplasmic condensation and eosinophilic structures indicating apoptosis were observed. MET examination of the ultrastructure confirmed the HE results. The Caspase-3 activity showed a fluorescence increase in both experimental groups compared with the control group. In conclusion, staining with $\mathrm{HE}$ allows detection of oncosis and apoptosis in cryopreserved embryos. Regarding the cryopreservation techniques, both slow freezing and vitrification showed oncosis and apoptosis injuries. However, in this experiment vitrification caused more cellular injuries, with less embryo viability, than slow freezing.

This work was supported by FAPESP 04/01252-4 and CAPES.

\title{
271 USE OF SEXED SEMEN FOR BOVINE IVF IN MEXICO: PRELIMINARY RESULTS
}

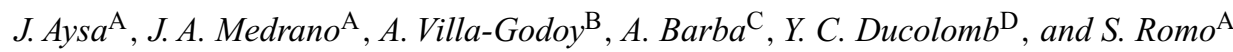

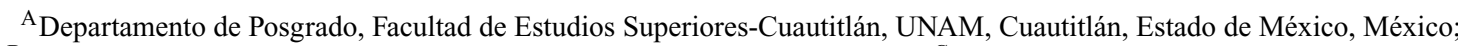

${ }^{B}$ Facultad de Medicina Veterinaria y Zootecnia, UNAM, México D.F. México; ${ }^{C}$ Private Practice, Tizayuca, Hidalgo, México;

Departamento de Ciencias de la Salud, UAM-I, México D.F. México

The use of sexed embryos in dairy cattle is useful for the genetic and economic improvement of production. The aim of this study was to determine differences in in vitro maturation (IVM), in vitro fertilization (IVF), and in vitro development (IVD) with the use of sexed and unsexed sperm. Semen from one Holstein bull was used for the experiment. The semen was sexed and frozen by X-Y, Mexico (subsidiary of X-Y Inc., Fort Collins, CO, USA). Only the $\mathrm{X}$ fraction of spermatozoa was sorted and frozen in $0.25-\mathrm{mL}$ plastic straws with $2.0 \times 10^{6}$ spermatozoa/straw. A modified protocol for IVF was used. A total of 334 ovaries were obtained from a local slaughterhouse and transported to the laboratory in physiological saline $\left(25^{\circ} \mathrm{C}\right)$ From these, 1019 cumulus-oocyte complexes (COCs) were obtained and used for the procedures of IVM, IVF, and IVD. The average number of follicles aspirated per ovary was 3.6, and an average of 3.05 COCs were recovered per ovary. The oocyte recovery rate was $85 \%$. For IVM, COCs were incubated in TCM-199 supplemented with BSA, pyruvate, $\mathrm{FSH}$, and $\mathrm{LH}$ for $24 \mathrm{~h}$. All incubations were performed at $38.5^{\circ} \mathrm{C}$ in a humidified atmosphere of $5 \% \mathrm{CO}_{2}$ in air. After this period, $\mathrm{COCs}$ were placed in fertilization medium (TALP supplemented with BSA, heparin, penicillamine, and hypotaurine). For IVF, oocytes were randomly assigned to two groups: sexed semen (Holstein) or treatment group (TG), and non-sexed semen (Brahman, used as control in our laboratory) or control group (CG). For insemination, frozen-thawed semen from the Holstein and Brahman bulls was washed by centrifugation in two concentration gradients of a silicone solution. In both groups, sperm concentration used for IVF was $1 \times 10^{6}$ spermatozoa $/ \mathrm{mL}$. After insemination, oocytes and semen were co-incubated for $18 \mathrm{~h}$. For IVD, presumptive zygotes were incubated for 7 days in a modified IVD medium (Barc-1) supplemented with BSA. The degree of IVD was evaluated according to the number of divisions of the embryos produced, and the number of embryos that developed to the morula and blastocyst stages. Five replicates were made. The rates of IVM, IVF, and IVD were analyzed by logistic regression. The COCs produced 890 fertilized oocytes. Of these, 442 were from the TG and 448 from the CG. A total of 393 embryos from the TG and 372 from the CG developed in vitro; embryos were evaluated for development on Day 7. A total of 108 morulae (21\%) were produced in the TG and $100(19 \%)$ in the CG, whereas $99(19 \%)$ blastocysts developed in the TG and $105(20 \%)$ in the CG. There were no statistically significant differences between the two groups studied for embryo IVD $(P>0.05)$. It is concluded that IVM, IVF, and IVD procedures used for conventional non-sexed semen can be used for similar results with sperm sexed by flow cytometry. This is the first report of sexed semen use for bovine IVF in Mexico and is a precedent for future investigations on in vitro embryo production in Mexico. More experiments are needed to confirm these preliminary findings.

Sexed semen was provided by Rancho El Nacimiento, Establo 196, Tizayuca, State of Hidalgo, México. Funding for J. A. was provided by CONACYT and UNAM

\section{DEVELOPMENTAL COMPETENCE OF OOCYTES OBTAINED FROM BOS TAURUS AND BOS INDICUS DAIRY COWS RAISED IN A TROPICAL CLIMATE}

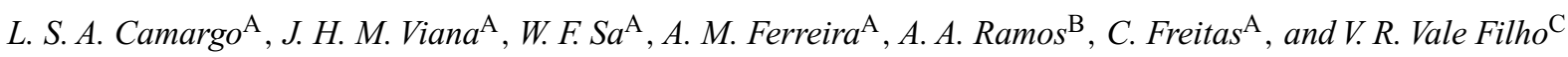 \\ ${ }^{\mathrm{A}}$ Embrapa Dairy Cattle, Juiz de Fora, MG, Brazil; ${ }^{\mathrm{B}}$ Epamig, Juiz de Fora, MG, Brazil; \\ C Veterinary School, UFMG, Belo Horizonte, MG, Brazil
}

The effects of heat stress on Bos taurus reproductive performance in tropical and subtropical regions are well known, and have been associated with lower oocyte developmental capacity. The aim of this study was to evaluate the developmental competence of oocytes from Bos taurus (Holstein) and Bos indicus (Gyr) dairy cows raised in a Brazilian tropical region, located at $21^{\circ} 35^{\prime} \mathrm{S}$ latitude, $43^{\circ} 51^{\prime} \mathrm{W}$ longitude, and 435 meters altitude Cumulus-oocyte complexes (COCs) were recovered by oocyte pickup (OPU) from mature non-lactating Holstein $(n=9)$ and Gyr $(n=13)$ donor cows between the end of spring and the beginning of autumn, with at least two OPU sessions/cow. COCs were in vitro-maturated in TCM-199 (GIBCO, Grand Island, NY, USA) with 10\% inactivated estrus cow serum for $24 \mathrm{~h}$ under $5 \% \mathrm{CO}_{2}$ at $38.5^{\circ} \mathrm{C}$ in air. Bos taurus and Bos indicus semen with similar cleavage rates, previously evaluated by in vitro fertilization with oocytes obtained from slaughterhouse ovaries, were used to reduce bull effect. Holstein and Gyr spermatozoa were obtained through swim-up method and co-incubated with Holstein $(n=390)$ and Gyr $(n=505)$ oocytes, 
respectively, in Fert-TALP medium (Parrish et al. 1988 Biol. Reprod. 38, 1171-1180) supplemented with $10 \mu \mathrm{g} / \mathrm{mL}$ heparin (Sigma-Aldrich, Sao Paulo, Brazil) and $6 \mathrm{mg} / \mathrm{mL}$ fatty acid-free bovine albumin (Sigma) for $18 \mathrm{~h} \mathrm{in} 5 \% \mathrm{CO}_{2}$ at $38.5^{\circ} \mathrm{C}$ in air. Presumptive zygotes were co-cultured with their own cumulus cells in CR2aa medium (Wilkinson et al. 1996 Theriogenology 45, 41-49) supplemented with $10 \%$ fetal calf serum in humid atmosphere of $5 \% \mathrm{CO}_{2}$ at $38.5^{\circ} \mathrm{C}$ in air. On Day 7 to 8 of co-culture, Gyr and Holstein blastocysts were assessed and those classified as grade 1 (IETS Manual) were transferred to synchronized Bos indicus $\times$ Bos taurus crossbred recipients managed under the same nutritional and environmental conditions. Pregnancy diagnosis was performed between 35 and 50 days after estrus. Cleavage, blastocyst, and pregnancy rates were analyzed by chi-square test. Cleavage and blastocyst rates were greater $(P<0.05)$ in Gyr than in Holstein $(66.7 \%$ vs. $53.1 \%$ for cleavage and $19.6 \%$ vs. $10.8 \%$ for blastocyst, respectively), but the pregnancy rate was similar $(P>0.05 ; 44.5 \%$ vs. $60 \%$ for Gyr and Holstein, respectively). These results show that Gyr oocytes obtained in a tropical region have greater developmental capacity than Holstein oocytes, suggesting an interaction between genotype and environment that influences the success of an in vitro embryo production program; nevertheless, the blastocyst viability after transferring to recipients is similar for both breeds.

\title{
273 APOPTOSIS EVALUATION OF IN VITRO-PRODUCED PIG EMBRYOS (PARTIAL RESULT)
}

\author{
A. R. S. Coutinho, M. P. Milazzotto, M. A. Peres, M. G. Marques, A. C. Nicacio, \\ J. A. Visintin, and M. E. O. A. Assumpção \\ Department of Animal Reproduction, FMVZ, São Paulo University, São Paulo, SP, Brazil
}

Apoptosis is a physiological event involved with death and tissue replication, fulfilling an important function of tissue organizations during embryogenesis. This mechanism occurs in in vivo as well as in vitro pre-implantation embryos, but most frequently in the latter. The transcriptional activation of pig embryos occurs at the four-cell stage, which is the longer stage during the pre-implantation period. This stage is characterized by embryonic developmental blockage that decreases the production rates (embryos loss). The aim of this study was to evaluate a correlation between apoptosis mechanism and developmental blockage of IVP porcine embryos. Immature oocytes after IVM/IVF were submitted to IVC in PZM-1 medium containing BSA $3 \mathrm{mg} / \mathrm{mL}$ at $38.5^{\circ} \mathrm{C}, 5 \% \mathrm{CO}_{2}$ in air and high humidified atmosphere. The embryo development was analyzed at $96 \mathrm{~h}$ of cultute (Day 4) in order to verify cleavage rate and blockage (4 cells) and non-blockage ( $\geq 8$ cells) embryo rates. Out of 625 grade I, II, and III oocytes submitted to IVP, $70.3 \pm 5.2 \%(430 / 625)$ cleaved from which $27.1 \pm 10.3 \%(166 / 625)$ were blocked and $43 \pm 10.8 \%(264 / 625)$ were non-blocked. Blocked and non-blocked embryos were assessed to evaluate apoptosis rates. Qualitative assays of embryo cells were achieved with two different DNA stains: YOPRO-1 (Molecular Probe ${ }^{\circledR}$; Invitrogen Brasil, Ltd., Sao Paulo, Brazil), permeable though plasma membrane in the early stage of apoptosis, and TUNEL (Roche ${ }^{\circledR}$; Amersham Biosciences, Sao Paulo, Brazil), which detects DNA fragmentation in the last stages of apoptosis. The embryos were stained with $0.1 \mu \mathrm{M}$ YOPRO- $1 / \mathrm{mL}$ PBS, incubated $15 \mathrm{~min}$ at $38^{\circ} \mathrm{C}, 5 \% \mathrm{CO}_{2}$ in air and high humidified atmosphere, and immediately observed by means of confocal microscopy. For the TUNEL assay, embryos were fixed in $4 \%$ paraformaldehyde solution (w/v) in PBS for $1 \mathrm{~h}$ at room temperature, and incubated in permeabilization solution $[0.5 \%(\mathrm{v} / \mathrm{v})$ Triton X-100, $0.1 \%(\mathrm{w} / \mathrm{v})$ sodium citrate in PBS] for $2 \mathrm{~h}$. For positive control, samples were treated with DNase-I at $37^{\circ} \mathrm{C}$ for $1 \mathrm{~h}$. The negative control and experimental samples were incubated with buffer solution under the same conditions. The positive control and experimental samples were incubated with enzymatic and stain solution (FITC) at $37^{\circ} \mathrm{C}$ for $1 \mathrm{~h}$; the negative control was incubated with only enzymatic solution. The embryos were stained with Hoechst $33342(5 \mu / \mathrm{mL})$ and observed by means of fluorescence microscopy. Blocked embryos showed more apoptosis ( $66 \%$ and $40 \%$ to YOPRO- 1 and TUNEL, respectively) than non-blocked embryos (25\% and $0 \%$ to YOPRO-1 and TUNEL, respectively). In conclusion, the developmentally blocked embryos suffered more apoptosis, although morphologic apoptosis assays (light and electronic microscopic) must be performed to confirm this finding.

This work was supported by FAPESP 04/01252-4

\section{LAPAROSCOPIC OVUM PICK-UP IN SHEEP AND GOATS: EFFECTS OF REPEATED RECOVERIES AND FOLLICULAR DIAMETER}

\author{
A. Gibbons ${ }^{\mathrm{A}}$, F. Pereyra Bonnet ${ }^{\mathrm{A}}$, M. Cueto ${ }^{\mathrm{A}}$, M. $_{\text {Catala }}^{\mathrm{B}}$, and D. Salamone ${ }^{\mathrm{B}}$ \\ A Instituto Nacional de Tecnología Agropecuaria, Estación Experimental Bariloche, CC 277 (8400) Bariloche, Argentina; \\ ${ }^{\mathrm{B}}$ Laboratorio Biotecnología de la Reproducción, Facultad de Agronomía, Universidad de Buenos Aires, Buenos Aires, Argentina
}

Laparoscopic ovum pickup (LOPU) is a modern technique that may be used in programs of sheep and goat in vitro embryo production. The effects of repeated recoveries and follicular diameter on the efficiency of LOPU were evaluated in these species. In six Merino donor sheep and five Criolla goats, ovarian stimulation for follicular development was performed by administering a single dose of $60 \mathrm{mg}$ NIH-FSH-P1 (Folltropin ${ }^{\circledR}$; Bioniche, Canada) and $300 \mathrm{UI}$ of eCG (Novoromon $5000^{\circledR}$; Syntex, Argentina). Every four days, a total of 4 LOPU in sheep and 3 in goats were performed $24 \mathrm{~h}$ after each ovarian stimulation treatment. The intravaginal sponge with $60 \mathrm{mg}$ of medroxyprogesterone (Esponjas Vaginales Syntex ${ }^{\circledR} ;$ Syntex, Argentina) were removed after the last LOPU. Follicular fluid was aspirated under laparoscopic observation according to the follicle diameter with a cannula for intrauterine artificial insemination without a vacuum pump. The number and size of follicles, oocyte quantity and quality and recovery rate were evaluated. A total of 566 follicles were aspirated (14.4 \pm 2.4 follicles/sheep and $14.6 \pm 7.5$ follicles/goat), yielding a total of 263 oocytes $(6.8 \pm 1.5$ oocytes/sheep and $6.6 \pm 2.9$ oocytes/goat) and a recovery rate of $47 \%$ in sheep and $46 \%$ in goats (Table 1$)$. In both species, there were no significant differences in the number of aspirated follicles, recovered oocytes, overall recovery rate, and the recovery rate of good quality oocytes in the repeated LOPU. The oocyte recovery rates in sheep differed significantly according to the follicular size (55\% in large follicles, $36 \%$ in small follicles; $P<0.05)$, whereas in goats recovery rates of oocytes related to follicular size showed no significant differences ( 42 and $54 \%$ for large and small follicles, respectively). Follicular sizes from which oocytes were obtained was not a determining factor in the recovery rate of good quality oocytes. The technique used would allow effective and repeated oocyte recoveries for in vitro embryo production in sheep and goats. 
Table 1. Repeated recoveries by laparoscopic ovum pickup (LOPU) in sheep and goats

\begin{tabular}{lcccccc}
\hline Species & $\begin{array}{c}\text { LOPU } \\
\text { no. }\end{array}$ & $n$ & $\begin{array}{c}\text { Follicles/ } \\
\text { animal } \pm \text { SD }\end{array}$ & $\begin{array}{c}\text { Oocytes/ } \\
\text { animal } \pm \text { SD }\end{array}$ & $\begin{array}{c}\text { Overall oocyte } \\
\text { recovery rate (\%) }\end{array}$ & $\begin{array}{c}\text { Good quality oocyte } \\
\text { recovery rate (\%) }\end{array}$ \\
\hline Sheep & 1 & 6 & $12.3 \pm 3.6$ & $5.6 \pm 2.0$ & 46 & 65 \\
Sheep & 1 & 6 & $12.3 \pm 3.6$ & $5.6 \pm 2.0$ & 46 & 65 \\
Sheep & 1 & 6 & $12.3 \pm 3.6$ & $5.6 \pm 2.0$ & 46 & 65 \\
Sheep & 1 & 6 & $12.3 \pm 3.6$ & $5.6 \pm 2.0$ & 46 & 65 \\
Sheep & 1 & 6 & $12.3 \pm 3.6$ & $5.6 \pm 2.0$ & 46 & 65 \\
Sheep & 1 & 6 & $12.3 \pm 3.6$ & $5.6 \pm 2.0$ & 46 & 65 \\
Sheep & 1 & 6 & $12.3 \pm 3.6$ & $5.6 \pm 2.0$ & 46 & 65 \\
Sheep & 1 & 6 & $12.3 \pm 3.6$ & $5.6 \pm 2.0$ & 46 & 65 \\
\hline
\end{tabular}

Results in the same column are not different $(P>0.05)$.

\title{
275 PENTOXIFYLLINE ADDED TO FREEZING EXTENDER HAS A DELETERIOUS EFFECT ON POST-THAW BOAR SPERM QUALITY AND IN VITRO FERTILIZATION RATE
}

\author{
M. A. Gil, J. Roca, M. Hernandez, C. Cuello, C. Almiñana, J. M. Vazquez, and E. A. Martinez \\ Department of Medicine and Animal Surgery, University of Murcia, E-30071 Murcia, Spain
}

Pentoxifylline, a methylxanthine derivative, is considered to be a hyperactivation and acrosome reaction-improving agent. The purpose of this study was to test how the addition of pentoxifylline to freezing extender influences post-thaw sperm motility and membrane integrity. The ability of thawed spermatozoa to fertilize in vitro-matured oocytes was also assessed. Pooled ejaculate sperm-rich fractions collected from three fertile boars were frozen in $0.5-\mathrm{mL}$ straws after being diluted in lactose/egg yolk/glycerol/Orvus-ES-Paste extender ( 0 pentoxifylline $=$ control) or the same extender supplemented with $2,4,8,16$, and $32 \mathrm{mM}$ pentoxifylline. To evaluate post-thaw sperm survival, the percentage of total motile spermatozoa and rapid progressive spermatozoa (CASA system) and plasma membrane and acrosome integrity (flow cytometry) were assessed. Data from six replicates were analyzed in a split plot design using a PROMIXED model. The addition of $4,8,16$, and $32 \mathrm{mM}$ pentoxifylline to freezing extender significantly decreased progressive and total motility $(P<0.001)$ compared with control $(4.5 / 26.6 \%, 4.5 / 24.5 \%, 2.8 / 20.5 \%, 0.6 / 11.4 \%$, and $13.2 / 49.7 \%$ for the $4,8,16$, and $32 \mathrm{mM}$ pentoxifylline groups and the control group, respectively). The same was observed for sperm viability; the percentage of viable spermatozoa with intact acrosomes was significantly lower $(P<0.001)$ in pentoxifylline-treated groups compared with the control group, chiefly in the $16 \mathrm{~mm}$, and $32 \mathrm{mM}$ pentoxifylline groups $(54,11.6$, and $6.2 \%$ for control, 16 and $32 \mathrm{mM}$, respectively). To evaluate in vitro fertilization parameters, cumulus-oocyte complexes were matured in BSA-free NCSU23 medium with $10 \%$ porcine follicular fluid, $0.1 \%$ cysteine, $10 \mathrm{ng}$ EGF, $10 \mathrm{IU} / \mathrm{mL}$ eCG, and $10 \mathrm{IU} / \mathrm{mL}$ hCG, in a incubator at $39^{\circ} \mathrm{C}$ and $5 \% \mathrm{CO}_{2}$. After $40-44 \mathrm{~h}$ of maturation, oocytes $(n=1067$, in three replicates) were denuded of cumulus cells, washed, transferred to droplets (30 oocytes in $50 \mu \mathrm{L}$ ) of TBM medium supplemented with $2 \mathrm{mM}$ caffeine and $0.2 \%$ BSA, and inseminated (2000 thawed sperm/oocyte). After a co-incubation period of $6 \mathrm{~h}$, oocytes were washed and transferred to droplets $(500 \mu \mathrm{L})$ of NCSU23 with $0.4 \%$ BSA for another $10-14 \mathrm{~h}$, then fixed and stained for $72 \mathrm{~h}$, and examined under a phase-contrast microscope. Data were analyzed as split plot design using a PROMIXED model. The addition of pentoxifylline to the freezing extender reduced significantly $(P<0.001)$ the penetration rate $(51.4,17.5,15.8,17.8,9.5$, and $4.8 \%$ for the $0,2,4,8,16$, and $32 \mathrm{mM}$ pentoxifylline groups, respectively) and the efficiency (monospermic oocytes/total inseminated oocytes) of fertilization $(34.8,14.6,14.7,15.5,7.9$, and $4.8 \%$ for the $0,2,4,8,16$, and $32 \mathrm{mM}$ pentoxifylline groups, respectively) as compared with the control group (the first value in each of these two cases). It is therefore concluded that pentoxifylline added to the freezing extender has a deleterious effect on post-thaw boar sperm quality and on their ability to fertilize the oocytes in vitro.

This work was supported by CICYT (AGL05-0471).

\section{IN VITRO FERTILIZATION PROCEDURES AFFECT THE HEPATIC GENOMIC CYTOSINE METHYLATION LEVEL AND THE PHENOTYPE OF BOVINE FETUSES}

\author{
S. Hiendleder ${ }^{\mathrm{A}}$, C. Mund ${ }^{\mathrm{B}}$, M. Klempt ${ }^{\mathrm{A}}$, H.-D. Reichenbach ${ }^{\mathrm{C}}$, M. Stojkovic ${ }^{\mathrm{A}}$, M. Weppert $^{\mathrm{A}}$, \\ H. Wenigerkind ${ }^{\mathrm{D}}$, M. Elmlinger ${ }^{\mathrm{E}}, F$. Lyko ${ }^{\mathrm{B}}$, and E. Wolf ${ }^{\mathrm{A}, \mathrm{D}}$ \\ ${ }^{\text {A } M o l e c u l a r ~ A n i m a l ~ B r e e d i n g ~ a n d ~ B i o t e c h n o l o g y, ~ G e n e ~ C e n t e r ~ o f ~ t h e ~ L u d w i g-M a x i m i l i a n ~ U n i v e r s i t y, ~ D-81377 ~ M u n i c h, ~ G e r m a n y ; ~}$ \\ ${ }^{B}$ Division of Epigenetics, Deutsches Krebsforschungszentrum (DKFZ), D-69120 Heidelberg, Germany; \\ ${ }^{\mathrm{C}}$ Biotechnology Group, Bavarian State Research Center for Agriculture (LfL), D-85586 Grub, Germany; \\ D Bavarian Research Center for Biology of Reproduction (BFZF), D-85764 Oberschleissheim, Germany; \\ ${ }^{E}$ Pediatric Endocrinology Section, University Hospital Tuebingen, D-72076 Tuebingen, Germany
}

Epigenetic perturbations are assumed to be responsible for phenotypic abnormalities of fetuses and offspring originating from in vitro embryo techniques. We studied 29 viable bovine Day 80 fetuses to assess the effects of different in vitro fertilization (IVF) protocols on hepatic genomic 
cytosine methylation levels and on fetal phenotype. Two groups of IVF-fetuses (IVF1, $n=5$ and IVF2, $n=10$ ) were compared with control fetuses generated by artificial insemination (AI, $n=14$ ). Both IVF protocols were previously employed to generate live offspring but differed with respect to gonadotropins in the oocyte maturation medium $(0.01$ units $/ \mathrm{mL}$ b-FSH and b-LH for IVF1 versus 0.2 units/mL o-FSH for IVF2) and serum concentrations in the embryo culture media (5\% estrous cow serum in IVF1 versus $10 \%$ estrous cow serum in IVF2). Analysis of variance (General Linear Model Procedure, SPSS for Windows version 12.0; SPSS GmbH Software, Munich, Germany) showed that fetus group significantly affected fetus weight and length $(P<0.01)$, heart $(P<0.05)$ and liver $(P<0.01)$ weight, and 5-methylcytosine $(5 \mathrm{mC})$ content of liver DNA $(P<0.001)$. Comparison of group means ( $t$-test) showed that methylation levels in both groups of IVF fetuses differed significantly from AI controls. We observed hepatic DNA hypomethylation $(-15.4 \%$ vs. AI control, $P<0.01)$ in IVF1 fetuses, and hypermethylation $(+11.6 \%$ vs. AI control, $P<0.001)$ in IVF2 fetuses, but only IVF2 fetuses showed phenotypic abnormalities. The IVF2 fetuses were significantly heavier $(18.6 \%, P<0.01)$ and longer $(4.3 \%$, $P<0.05)$ than AI fetuses, with increased heart $(21.8 \%, P<0.05)$ and liver $(25 \%, P<0.001)$ weights, and thus displayed an overgrowth phenotype. A clinical-chemical screen of 18 plasma parameters failed to detect abnormalities in IVF1 fetuses but revealed significantly increased levels of insulin-like growth factor $1(40.8 \%, P<0.001)$ and creatinine $(28.5 \%, P<0.05)$ in IVF2 fetuses. Our data indicate that bovine IVF-procedures can induce protocol-specific and persistent changes in hepatic cytosine methylation level with or without obvious concomitant changes in fetal phenotype. This suggests that epigenetic change after bovine IVF could be more widespread than previously thought and highlights the value of epigenetic diagnostic screening.

\title{
277 FOLLICULAR GROWTH, SUBSEQUENT OVUM PICKUP, AND DOMINANT FOLLICLE REMOVAL IN COWS
}

\author{
K. Imai, M. Tagawa, S. Matoba, M. Narita, and K. Kanayama
}

National Livestock Breeding Center, Nishigo, Fukushima 961-8511, Japan

The present study was designed to assess the recruitment of follicles after ovum pickup (OPU) and dominant follicle (DF) removal on the follicular wave after OPU in Holstein dry cows. Cows were reared under the same feeding and environmental conditions. In Experiment 1, follicle aspiration ( $>2 \mathrm{~mm}$ in diameter) by OPU using a 7.5-MHz linear transducer with needle (COVA needle; Misawa Medical, Tokyo, Japan) connected to an ultrasound scanner (SSD-1200; ALOKA, Tokyo, Japan) was performed in four cows. Then, ovaries were observed after OPU from Day 1 (Day $0=$ the day of OPU) to Day 11 to assess the number of follicles developed. In Experiment 2, two sessions of OPU were performed with a 7 day interval between sessions, with or without dominant follicle removal, to assess the quality of developing follicles and oocytes. In the DF removal group, $>8$ - $\mathrm{mm}$ follicles were aspirated at Day 5 after the first OPU session, and the same cows without DF removal were designated as a control $(n=4$, crossover trial). Oocytes were evaluated by their cumulus cell morphology, cytoplasmic color, and density. To assess the developmental competence of oocytes, Grades 1 and 2 cumulus-oocyte complexes (COCs) were collected, matured, fertilized, and cultured as described by Imai et al. (2002 J. Vet. Med. Sci. 64(10), 887-891). Embryo development was assessed by the cleavage rate on Day 2 and the blastocyst formation rate on Days 7 to 9 (the day of insemination = Day 0). Data were analyzed by ANOVA or Student $t$-test. In Experiment 1, a dominant follicle ( $>8 \mathrm{~mm}$ in diameter) was developed during Days 3 to 5 after OPU in each donor. The mean number of developing follicles ( $>2 \mathrm{~mm}$ in diameter) were increased from Day 1 to Day 9 (Day 1: 7.5 \pm 2.1 , Day 3: $19.0 \pm 1.2$, Day 5: 23.3 \pm 9.0, Day 7: $30.3 \pm 11.0$, Day 9: $42.0 \pm 15.8$ and Day 11: $41.0 \pm 16.7$ (mean \pm SD), $P<0.05$ ). In Experiment 2, there was no difference in the mean number of developing follicles on the day of OPU and collected oocytes between DF removal and control groups (follicles: $47.8 \pm 23.0$ and $39.3 \pm 6.2$; oocytes: $27.0 \pm 11.6$ and $26.5 \pm 5.4$, respectively). The number of Grades 1 and 2 oocytes for the DF removal group was significantly higher $(P<0.05)$ than that for the control $(83.6 \pm 1.5$ and $63.2 \pm 14.2$, respectively), and no significant difference was found within cleavage $(60.0 \pm 37.2,53.6 \pm 23.2)$ and blastocyst rates $(34.1 \pm 33.9,34.4 \pm 16.8)$. These results indicate that populations of follicles were increased till Day 9 after OPU, and the DF removal was effective at increasing oocyte quality in the developing follicles.

\section{EFFECTS OF LEPTIN ON IN VITRO DEVELOPMENT AND GENE EXPRESSION IN PORCINE EMBRYOS}

\author{
Y.-J. Jeong, J.-G. Kim, B. Mohana Kumar, S. Balasubramanian, S.-Y. Choe, and G.-J. Rho \\ College of Veterinary Medicine, Gyeongsang National University, Chinju, Republic of Korea 660-701
}

Recent evidence suggests that leptin is an important signal in female reproduction, including control of ovarian function (Brann et al. 2002 Steroids 67, 95-104). Leptin receptor transcripts are presented in various cell types of humans, rats, and pigs. However, the results have been contradictory on the role of leptin in embryo development. The objective of this study was to determine the effects of different concentrations of leptin during porcine oocyte maturation on subsequent embryo development and expression of genes related to leptin signal transduction or apoptosis. Porcine oocytes were matured for $44 \mathrm{~h}$ in TCM-199 with the addition of leptin at 0 (control), 10, 50, 100, and $200 \mathrm{ng} / \mathrm{mL}$ (leptin 10, 50, 100, and 200 groups, respectively), and subsequently fertilized with frozen thawed semen $\left(1 \times 10^{5} \mathrm{sperm} / \mathrm{mL}\right)$ in mTBM. Presumptive zygotes were then cultured in NCSU-23 medium. Cleavage and blastocyst rates (in seven replicates) were assessed on Days 2 and 7 (the day of IVF was defined as Day 0), respectively. The total cell number in blastocysts was counted and the number sssssssssof apoptotic cells was determined by TUNEL. Expression of genes encoding leptin receptor (LEPR), cytoplasmic transcription factor (STAT3), pro-apoptotic (Bax), and anti-apoptotic (Bc12) regulators in blastocysts were determined by quantitative reverse transcription-polymerase chain reaction (RT-PCR). The percentage of MII oocytes (in four replicates) was significantly $(P<0.05)$ higher in 50 and 100 groups than in the others $[80(216 / 270)$ and $84 \%(236 / 280)$ vs. $70(158 / 224), 74(172 / 230)$, and $75 \%(186 / 248)$ in 
the 0,10 , and 200 groups, respectively]. Oocytes matured in the 50 and 100 groups showed a significantly $(P<0.05)$ greater proportion of cleaved embryos than those in the other groups [82 (368/448) and $84 \%(380 / 451)$ vs. $71(339 / 476), 76(358 / 470)$, and $74 \%(327 / 441)$ in 0,10 , and 200 groups, respectively]. Furthermore, the rate of blastocyst formation at Day 7 was significantly $(P<0.05)$ higher in the leptin 100 group than in the other groups [29\% (131/451) vs. 18 (86/476), 23 (109/470), 20 (90/448), and 22\% (98/441) in control and leptin 10, 50, and 200 groups, respectively] Increased cell number and reduced proportion of apoptotic cells were observed in blastocysts originating from the oocytes matured in the presence of $100 \mathrm{ng} / \mathrm{mL}$ leptin. Depending on the concentrations used in the maturation medium, leptin increased LEPR, STAT3, and Bcl2 mRNA levels and reduced the Bax mRNA level in blastocysts. The results indicate that addition of leptin at $100 \mathrm{ng} / \mathrm{mL}$ during oocyte maturation improved embryonic development with up-regulation of LEPR, STAT3, and Bcl2 genes and suppression of the Bax gene, and thus optimizes the in vitro culture systems for porcine embryos.

This work was supported by Grant No. 1000520040020000 from Biogreen 21, Republic of Korea.

\title{
279 LIVE RAT OFFSPRING FROM CRYOPRESERVED EJACULATED SPERMATOZOA THROUGH IN VITRO FERTILIZATION
}

\author{
N. Kashiwazaki, Y. Seita, M. Shino, S. Hisamatsu, and T. Inomata \\ Graduate School, Azabu University, Sagamihara, Kanagawa, Japan
}

We have previously reported successful cryopreservation of epididymal rat spermatozoa (Nakatsukasa et al. 2001 Reproduction 122, 463). However, the procedure for cryopreservation of rat spermatozoa has a disadvantage; a male has to be euthanized for collection of spermatozoa from its epididymides. Obtaining ejaculated spermatozoa repeatedly from the same male could be useful for cryopreservation of invaluable spermatozoa which carry mutations including transgenes. The objective of the present study was to develop a reliable system for cryopreservation of ejaculated rat spermatozoa and efficient production of offspring from the cryopreserved spermatozoa. Matured Wistar females were mated with three males of the same strain, and killed by cervical dislocation after formation of vaginal plugs. The uteri of mated females were excised and flushed with freezing medium containing $23.0 \%$ egg yolk, $8.0 \%$ lactose, and $0.7 \%$ Equex STM to recover ejaculated spermatozoa. The semen samples were loaded into $0.25-\mathrm{mL}$ straws. The straws were cooled to $5.0^{\circ} \mathrm{C}$ at $0.5^{\circ} \mathrm{C} / \mathrm{min}$ in a programmable freezer and then exposed to liquid nitrogen (LN) vapor at $4 \mathrm{~cm}\left(-150^{\circ} \mathrm{C}\right)$ above the $\mathrm{LN}$ level for $15 \mathrm{~min}$. The straws were then plunged into $\mathrm{LN}$ and stored for at least a week. The straws were thawed in a $37.0^{\circ} \mathrm{C}$ water bath for $10 \mathrm{~min}$. The thawed samples were diluted to $0.5-1.5 \times 10^{6} \mathrm{sperm} / \mathrm{mL}$ into a $200-\mu \mathrm{L}$ droplet of R1ECM and then pre-incubated for $5 \mathrm{~h}$. Ovulated oocytes collected from superovulated females were introduced into the droplet and co-cultured for $10 \mathrm{~h}$ for in vitro fertilization (IVF). The oocytes were denuded and examined for the presence of two pronuclei (2PN) microscopically. The denuded oocytes with $2 \mathrm{PN}$ were transferred into the oviducts of pseudo-pregnant females. The rates of sperm motility at recovery, post-thaw, and the initiation of IVF (after pre-incubation) were $57 \pm 6 \%, 24 \pm 5 \%$, and $18 \pm 3 \%$, respectively. After co-culture, $46(14 \%)$ of the total 329 co-cultured oocytes were confirmed to contain $2 \mathrm{PN}$. A total of the 44 putative zygotes were transferred to five recipients, and a total of 21 live young (48\%) were born from all of the transferred recipients. We were able to produce zygotes and offspring derived from cryopreserved ejaculated spermatozoa of all three males used in the present study. In conclusion, the cryopreservation system for ejaculated rat spermatozoa used in the present study is a workable protocol for banking of valuable genetic resources of laboratory rats. Further studies on the IVF procedure with cryopreserved ejaculated spermatozoa in the rat are needed to improve the fertilization rate.

\section{PORCINE BLASTOCYSTS DERIVED FROM IN VITRO-MATURED OOCYTES INJECTED INTRACYTOPLASMICALLY WITH SPERM FROM TESTICULAR TISSUE XENOGRAFTED INTO NUDE MICE}

\author{
K. Kikuchi ${ }^{\mathrm{A}}, M_{.}$Nakai $^{\mathrm{A}, \mathrm{B}}, N . K_{\text {Kashiwazaki }}^{\mathrm{B}}$, M. Ozawa $^{\mathrm{A}}, N$. Maedomari $^{\mathrm{A}, \mathrm{B}}$, \\ J. Noguchi ${ }^{\mathrm{A}}, K$. Ohnuma $^{\mathrm{A}}$, and H. Kaneko ${ }^{\mathrm{A}}$ \\ ${ }^{A}$ National Institute of Agrobiological Sciences, Tsukuba, Ibaraki 305-8602, Japan; \\ ${ }^{\mathrm{B}}$ Graduate School, Azabu University, Sagamihara, Kanagawa 229-8501, Japan
}

The utilization of spermatogonia from testicular tissue after xenografting into immuno-deficient mice should lead to new insights for the conservation of male gametes. However, successful embryo production using sperm cells from xenografted testicular tissues has been limited to rhesus monkeys (Honaramooz et al. 2004 Biol. Reprod. 70, 1500-1503). In the present study, the objective was to establish this new technology for pig conservation in combination with intracytoplasmic sperm injection. Testes were obtained from male piglets 6 to 15 days old, in which most of the germ cells were gonocytes; these were minced into pieces of approximately $1.5 \times 1.5 \times 1.5 \mathrm{~mm}$. Approximately 20 fragments were transplanted under the back skin of castrated nude mice 5 to 8 weeks old. The testicular grafts were recovered between 125 and 192 days after xenografting, minced in Dulbecco's phosphate-buffered saline, and centrifuged several times, to serve as a sperm suspension. In vitro maturation of the recipient oocytes (Kikuchi et al. 2002 Biol. Reprod. 66, 1033-1041) and injection with an intact spermatozoon, followed by electrical stimulation at $1 \mathrm{~h}$ post-injection (Nakai et al. 2003 Biol. Reprod. 68, 1003-1008), were carried out. The putative zygotes were cultured in vitro for 6 days (Kikuchi et al. 2002), and were then fixed, stained, and assessed for embryonic development and quality. From a total of 27 mice that were xenografted with testicular tissues, spermatids and spermatozoa were obtained in 19 of the mice $(70.4 \%)$. Most of the spermatozoa were matured morphologically, showing faint motility after release into the collection medium. From a total of 253 oocytes (four replications) that were injected with sperm, $63(24.9 \pm 7.1 \%)$ oocytes developed to the blastocyst stage. The average total cell number was $41.9 \pm 3.9$. These values are comparable to those in in vitro fertilization by frozen-thawed spermatozoa, resulting in developmental ability to piglets after embryo transfer ( $25.3 \%$ and 48.7 cells; Kikuchi et al. 2002). These results suggest the possibility of embryo production using porcine spermatozoa that are differentiated from gonocytes within the xenografts. 


\title{
281 ALGINATE-ENCAPSULATED BOVINE EMBRYOS SUPPORT IN VITRO DEVELOPMENT OF A SMALL NUMBER OF EMBRYOS
}

\author{
S. Kobayashi, M. Sakatani, S. Kobayashi, and M. Takahashi \\ National Agricultural Research Center for Kyushu Okinawa Region, Nishigoushi, Kumamoto, Japan
}

Ova are genetic resources that can be obtained from slaughterhouse ovaries or live cows by ovum pickup (OPU). However, the number of oocytes recovered by OPU is low. Previous studies show that embryos cultured in large numbers have better developmental competence than those in small numbers in mice, sheep, and cattle. Therefore, to improve development of small numbers of embryos, co-culture with other types of embryos is an efficient way. However, it is necessary to distinguish the desired embryos from the co-cultured embryos. Recently, encapsulation of embryos using calcium-alginate was reported to be useful for handling and in vivo culture of porcine embryos (Iwamoto et al. 2003 Theriogenology 59, 261). In the present study, we investigated the effect of co-culture of embryos encapsulated with calcium-alginate on development of small numbers of embryos. In vitro-matured and fertilized zygotes from slaughterhouse-derived ovaries were used for the experiment, and data were analyzed by Student $t$-test. Encapsulation was carried out by putting the $1 \%$ sodium alginate solution containing zygotes slowly into $0.1 \%$ calcium chloride solution (microcapsule). We used the microcapsule for the following experiments. In Experiment 1, twenty zygotes were cultured in CR1 aa containing $5 \%$ FCS with a capsule containing 20 zygotes or without (control) a microcapsule. The rate of cleavage (capsule: $80.0 \%$ vs. control: $72.1 \%$ ) and development to blastocyst stage (capsule: $31.7 \%$ vs. control: $33.7 \%$ ) were not significantly different. This result indicates that the microcapsule is not toxic to embryo development. In Experiment 2, five zygotes were co-cultured with 15 zygotes (microcapsule), and culture of five zygotes without capsules served as a control. The rate of cleavage (co-culture: $81.4 \%$ vs. control: $80.0 \%$ ) was not significantly different, but the rate of development to the blastocyst stage was significantly higher $(P<0.05)$ in the co-culture $(47.1 \%)$ than in the control $(30.6 \%)$. This result indicates that co-culture with a microcapsule including zygotes enhances the development of small numbers of embryos. In Experiment 3 , five zygotes derived from a single cow were encapsulated, and four microcapsules from different cows were cultured in the same droplet. The microcapsules could be distinguished by the inclusion of different numbers of glass beads with the zygotes. Culture of five zygotes without capsules was assigned as a control. The rate of cleavage (co-culture: $75.6 \%$ vs. control: $69.6 \%$ ) was not significantly different, but the rate of development to the blastocyst stage was significantly higher $(P<0.05)$ for the co-culture $(30.6 \%)$ than for the control $(17.8 \%)$. These results indicate that co-culture with bovine embryos encapsulated with calcium-alginate may improve development of small numbers of embryos.

\section{IMPROVED QUALITY OF PORCINE BLASTOCYSTS BY AGGREGATION OF EMBRYOS} AT THE 4-8-CELL STAGE

\author{
S.-G. Lee ${ }^{\mathrm{A}, \mathrm{B}}$, C.-H. Park ${ }^{\mathrm{A}, \mathrm{B}}$, D.-H. Choi ${ }^{\mathrm{A}, \mathrm{B}}, H .-Y . S^{\mathrm{A}}{ }^{\mathrm{B}}$, and C.-K. Lee $e^{\mathrm{A}, \mathrm{B}}$ \\ ${ }^{\mathrm{A}}$ School of Agricultural Biotechnology, Seoul National University; \\ ${ }^{B}$ Xenotransplantation Research Center, Seoul National University, Seoul, Korea
}

Use of blastocysts produced in vitro would be an efficient way to generate embryonic stem (ES) cells for the production of transgenic animals and the study of developmental gene regulation. In pigs, the morphology and cell number of in vitro-produced blastocysts are inferior to these parameters in their in vivo counterparts. Therefore, establishment of ES cells from blastocysts produced in vitro might be hindered by poor embryo quality. The objective of this study was to increase the cell number of blastocysts derived by aggregating 4-8-cell stage porcine embryos produced in vitro. Cumulus-oocyte complexes were collected from prepubertal gilt ovaries, and matured in vitro. Embryos at the 4-8-cell stage were produced by culturing embryos for two days after in vitro fertilization (IVF). After removal of the zona pellucida with acid Tyrode's solution, one (1X), two (2X), and three (3X) 4-8-cell stage embryos were aggregated by co-culturing them in aggregation plates followed by culturing to the blastocyst stage. After 7 days, the developmental ability and the number of cells in aggregated embryos were determined by staining with Hoechst 33342 and propidium iodide. The percentage of blastocysts was higher in both $2 \mathrm{X}$ and $3 \mathrm{X}$ aggregated embryos compared to that of $1 \mathrm{X}$ and that of intact controls (Table 1). The cell number of blastocysts also increased in aggregated embryos compared to that of non-aggregated (1X) embryos and controls. This result suggests that aggregation might improve the quality of in vitro-fertilized porcine blastocysts by increasing cell numbers, thus becoming a useful resource for isolation and establishment of porcine ES cells. Further studies are required to investigate the quality of the aggregated embryos in terms of increasing the pluripotent cell population by staining for Oct-4 and to apply improved aggregation methods in nuclear-transferred (NT) porcine embryos.

Table 1. Development, cell number, and ICM ratio of aggregated porcine embryos

\begin{tabular}{lcccccc}
\hline Embryo & $\begin{array}{c}\text { 4-8-cell } \\
\text { stage }\end{array}$ & $\begin{array}{c}\text { Aggregates } \\
(\%)\end{array}$ & $\begin{array}{c}\text { Blastocysts } \\
(\%)\end{array}$ & $\begin{array}{c}\text { Total cells } \\
(\text { mean } \pm \text { SEM) }\end{array}$ & $\begin{array}{c}\text { ICM } \\
\text { cells }\end{array}$ & $\begin{array}{c}\text { ICM ratio } \\
\text { ICM/(ICM+TE) }\end{array}$ \\
\hline 1X IVF & 122 & n.a. & $45(36.9)$ & $38.5 \pm 2.4$ & 7.7 & 0.22 \\
2 X IVF & 182 & $62 / 91(68.1)$ & $45 / 62(72.6)$ & $98.5 \pm 3.6$ & 25.4 & 0.26 \\
3 X IVF & 66 & $20 / 24(83.3)$ & $19 / 20(95.0)$ & $103.5 \pm 1.2$ & 28.3 & 0.27 \\
Control & 30 & n.a. & $18(60.0)$ & $43.5 \pm 3.3$ & 11.5 & 0.26 \\
\hline
\end{tabular}

n.a. $=$ not applicable. 


\title{
283 EFFECTS OF CUMULUS CELL REMOVAL ON IN VITRO OOCYTE MATURATION, FERTILIZATION, AND EMBRYONIC DEVELOPMENT IN PIGS
}

\author{
N. Maedomari ${ }^{\mathrm{A}, \mathrm{B}}, N$. Kashiwazaki $^{\mathrm{A}}$, M. Ozawa $^{\mathrm{B}}$, A. Takizawa $^{\mathrm{A}}$, J. Noguchi $^{\mathrm{B}}$, \\ H. Kankeo ${ }^{\mathrm{B}}$, M. Shino ${ }^{\mathrm{A}}$, and K. Kikuchi ${ }^{\mathrm{B}}$ \\ ${ }^{\text {A }}$ Graduate School of Veterinary Medicine, Azabu University, Sagamihara, Kanagawa 229-8501, Japan; \\ ${ }^{B}$ Genetic Diversity Department, National Institute of Agrobiological Sciences, Tsukuba, Ibaraki 305-8602, Japan
}

It is generally accepted that cumulus cells (CCs) support the nuclear maturation of immature oocytes in mammals. However, the precise mechanism of interaction between cumulus cells and oocytes has not been clarified. Furthermore, the role of cumulus cells in embryonic development has not been reported. In the present study, the effect of denuding cumulus cells from porcine oocytes on oocyte maturation, fertilization, and their subsequent development to the blastocyst stage was examined in vitro. In vitro maturation, fertilization, and culture were carried out as previously reported (Kikuchi et al. 2002 Biol. Reprod. 66, 1033-1041). Porcine cumulus-oocyte complexes (COCs) were collected; some of them were completely denuded of cumulus cells immediately after the collection (DO-0 group). The remaining intact COCs and the DO-0 oocytes were cultured for $24 \mathrm{~h}$ in the presence of dbcAMP and hormones. After the initial culture, some of the intact COCs were denuded either completely (DO-24 group) or partially (H-DO-24 group). Additionally, some of DO-24 oocytes were co-cultured with the cumulus cells removed at $0 \mathrm{~h}$ and pre-cultured for $24 \mathrm{~h}$ (DO-24 + CCs group). The denuded oocytes in each experimental group and intact COCs (control) were further cultured for total $46 \mathrm{~h}$. The remaining oocytes with a first polar body were either examined for the levels of intracellular glutathione (GSH) or fertilized in vitro with frozen-thawed boar spermatozoa. The inseminated oocytes were cultured and examined for their fertilization status after $10 \mathrm{~h}$ and for their developmental competence after 6 days. Data were analyzed by ANOVA, followed by the Duncan's multiple range tests. The maturation rates of all denuded groups were significantly lower $(P<0.05 ; 34.3$ to $45.0 \%)$ than that of the control group $(64.5 \%)$. Intracellular GSH concentrations of all denuded groups were also significantly lower $(P<0.05 ; 4.03$ to $7.00 \mathrm{pmol} /$ oocyte $)$ than that of the control group ( $9.60 \mathrm{pmol} /$ oocyte); however, the GSH level of H-DO-24 oocytes was significantly higher $(P<0.05)$ than the GSH levels in the other denuded groups. Male pronuclear formation rates of completely denuded oocytes (DO-0, DO-24, and DO-24 + CCs groups) were significantly lower $(P<0.05 ; 41.4$ to $59.3 \%)$ than those of the control $(89.4 \%)$ and the H-DO-24 (80.0\%) groups. The blastocyst rate of the control group was significantly higher $(P<0.05 ; 19.9 \%)$ than that of H-DO-24 group (11.6\%), and these rates were significantly higher $(P<0.05)$ than those of the completely denuded groups $(3.0$ to $4.5 \%)$. The results suggest that the presence of cumulus cells during maturation culture improves nuclear maturation of oocytes and plays an important role in embryonic development to the blastocyst stage in vitro.

\section{COMPARATIVE FERTILITY OF FRESHLY-COLLECTED VERSUS FROZEN-THAWED SPERMATOZOA FOR IN VITRO FERTILIZATION IN THE FISHING CAT (PRIONAILURUS VIVERRINUS)}

\author{
${\text { G. } \text { Magarey }^{\mathrm{A}}, \text { J. Herrick }^{\mathrm{A}}, \text { K. Thiangtum }^{\mathrm{B}}, W . \text { Tunwattana }^{\mathrm{C}} \text {, and W. Swanson }}^{\mathrm{A}}$ \\ ${ }^{\mathrm{A}}$ Center for Conservation and Research of Endangered Species, Cincinnati Zoo \& Botanical Garden, Cincinnati, OH 45220, USA; \\ ${ }^{\mathrm{B}}$ Center of Agricultureal Biotechnology, Kasetsart University, Nakhon Pathom, Thailand; \\ ${ }^{\mathrm{C}}$ Khao Kheow Open Zoo, Chonburi, Thailand
}

Wild populations of fishing cats (Prionailurus viverrinus) in Southeast Asia are in decline, primarily due to habitat loss. Because the fishing cat population in North American zoos is small $(n=69)$ and inbred $(F=0.17)$ with relatively low genetic variation $(86 \%)$, infusion of new founder genes from Asia is a conservation priority. Importation of cryopreserved semen for use with IVF and ET may offer one alternative to the international transport of living animals. In this study, our objectives were to (1) compare motility longevity of fresh vs. frozen-thawed fishing cat spermatozoa in two culture media, (2) evaluate ovarian responses to exogenous gonadotropins, and (3) assess development of IVF embryos produced with fresh vs. frozen-thawed spermatozoa. Raw semen was collected via electroejaculation from male fishing cats $(n=4)$, divided into groups, and washed. Two sperm pellets were resuspended in either Ham's F10 medium (HF10; with 5\% FBS) or our feline optimized culture medium (FOCM; with 0.4\% BSA); another pellet was diluted in TEST egg yolk, cooled to $5^{\circ} \mathrm{C}$ over $3 \mathrm{~h}$, glycerated (4\%), and cryopreserved in straws over $\mathrm{LN}_{2}$ vapor. Frozen sperm samples were thawed, washed, and diluted in either HF10 or FOCM. Fresh and frozen-thawed sperm motility (percent motile, rate of forward progress) in each medium $\left(10 \times 10^{6}\right.$ motile sperm $\left./ \mathrm{mL}\right)$ was assessed (at $0,1,3$, and $\left.6 \mathrm{~h}\right)$ in microdrops under oil during culture $\left(38^{\circ} \mathrm{C} ; 6 \% \mathrm{CO}_{2}\right.$ in air). Female fishing cats $(n=10)$ were treated with exogenous gonadotropins ( $150 \mathrm{IU}$ eCG, $100 \mathrm{IU}$ hCG, 85 -h interval) and ovarian follicles were aspirated laparoscopically. Recovered oocytes were inseminated with fresh $\left(2 \times 10^{5}\right.$ motile sperm $\left./ \mathrm{mL}\right)$ or frozen-thawed $\left(5 \times 10^{5} \mathrm{motile}\right.$ sperm $\left./ \mathrm{mL}\right)$ spermatozoa in FOCM microdrops; resulting embryos were either cryopreserved or cultured in FOCM (with 5\% FBS added at $72 \mathrm{~h}$ post-insemination) for 7 days. Sperm motility over time did not differ $(P>0.05)$ between media for either fresh or frozen-thawed samples; however, across media, frozen-thawed sperm motility was lower $(P<0.05)$ and declined faster $(P<0.05)$ compared to fresh spermatozoa. Females produced an average $( \pm$ SEM) of $9.8 \pm 2.9$ mature ovarian follicles, allowing recovery of $7.3 \pm 2.6$ high-quality oocytes per female. Oocyte cleavage percentage at $42 \mathrm{~h}$ p.i. was lower $(P<0.05)$ with frozen-thawed spermatozoa $(38 \%, 11 / 29)$ compared to freshly collected spermatozoa $(68 \%, 17 / 25)$. Overall, 35\% $(6 / 17)$ of cultured embryos developed to blastocysts with no difference $(P>0.05)$ between embryos produced with frozen-thawed (4/11) vs. fresh (2/6) spermatozoa. Although fishing cat sperm motility and fertility appear compromised after cryopreservation, our results demonstrate the ability of frozen-thawed spermatozoa to produce IVF embryos that are capable of developing to blastocyst stage in vitro.

This work was supported by (NIH RR015388). 


\title{
285 VARIATION FACTORS INFLUENCING CLEAVAGE AND EMBRYONIC DEVELOPMENT RATES OF BOVINE OOCYTES COLLECTED ON A FARM IN A COMMERCIAL OPU-IVP SYSTEM
}

\author{
B. Marquant-Le Guienne, F. Aymar, C. Ponsart, C. Guyader-Joly, S. Ponchon, and P. Humblot \\ UNCEIA, Maisons Alfort, France
}

\begin{abstract}
The aim of this work was to identify factors influencing the success rate of each production step through a retrospective study conducted for 362 commercial OPU-IVP sessions performed on a farm. Donor females were stimulated with FSH in five decreasing doses ( $400 \mu \mathrm{g}$ for cows and $250 \mu \mathrm{g}$ for heifers). Collected oocytes were matured for $24 \mathrm{~h}$ in M199 plus fetal calf serum, FSH, epidermal growth factor (EGF), and estradiol. They were then fertilized in TALP with frozen-thawed semen; zygotes were cultured for 6 days on Vero cell monolayers in B2 medium. Embryos were transferred as fresh to recipients on Day 7. The effects of donor characteristics, OPU and IVP conditions on cleavage, development rates, and number of transferred embryos (TE) were analyzed by ANOVA (GLM program in SAS; SAS Institute, Inc., Cary, NC, USA). Effects are mentioned when significant at $P<0.05$. The mean number of collected oocytes per session was $14 ; 11.6$ were selected for IVM, 8.5 cleaved, and 3.9 developed to the blastocyst stage ( $40.4 \%$ of the embryos were Grade $1,35.3 \% \mathrm{G} 2$, and $20.5 \% \mathrm{G} 3$ ). On average, 2.9 embryos were transferred into recipients. The number of transferred embryos was higher when the dominant follicle (DF) was punctureed prior to OPU (see Table 1). This resulted in a better cleavage rate in punctured DF donor females. Higher cleavage rates were observed in infertile females as well as numbers of TE per session. Pregnancy and body condition score (BCS) recorded at OPU only influenced cleavage rates. Embryonic development rates were mainly influenced by donor breed and parity. In the Abundance breed, $87.5 \%$ of the sessions resulted in at least one embryo being transferred compared to $58.6 \%$ in Holsteins. Higher numbers of embryos were transferred per session when donor females were cows rather than heifers. Fertilization conditions (heparin and sperm concentrations) had no effect on cleavage and embryonic development rates. To conclude, cleavage and development rates were mainly influenced by donor characteristics (breed, parity, physiological status). Improvement of results may be achieved by systematic puncture of DF prior to OPU. Cleavage rates were dependent on BCS. To improve management of body condition, BCS variations before OPU could be a new parameter to be followed in donor females.
\end{abstract}

Table 1. Factors affecting steps of the OPU-IVP procedure

\begin{tabular}{llccc}
\hline Factor & & Cleavage (\%) & Blastocysts (\%) & Embryos transferred \\
\hline DF puncture & Yes, $n=94$ & $79.2^{\mathrm{a}}$ & 42.9 & $3.2^{\mathrm{a}}$ \\
& No, $n=168$ & $69.7^{\mathrm{b}}$ & 43.7 & $2.6^{\mathrm{b}}$ \\
Parity & Cow, $n=190$ & 75.2 & 45.6 & $3.1^{\mathrm{a}}$ \\
& Heifer, $n=72$ & 73.3 & 33.8 & $2.4^{\mathrm{b}}$ \\
Fertile & Yes, $n=233$ & $72.5^{\mathrm{a}}$ & 43.7 & 2.7 \\
& No, $n=129$ & $78.9^{\mathrm{b}}$ & 42.6 & 3.4 \\
Pregnant & Yes, $n=199$ & $78.3^{\mathrm{a}}$ & 40.9 & 3.2 \\
& No, $n=160$ & $72.4^{\mathrm{b}}$ & 45.6 & 2.8 \\
BCS at OPU & $\leq 2, n=38$ & $76.6^{\mathrm{a}}$ & 36.2 & 2.2 \\
& $2-3, n=198$ & $70.3^{\mathrm{a}}$ & 46.0 & 2.8 \\
& $\geq 3, n=24$ & $82.6^{\mathrm{b}}$ & 39.3 & 3.7 \\
\hline
\end{tabular}

${ }^{\mathrm{a}, \mathrm{b}}$ Values with different superscripts in a column differ significantly $(P<0.005)$.

\section{INFLUENCE OF REVERSIBLE MEIOSIS INHIBITION ON SWINE EMBRYOS PRODUCED BY IN VITRO FERTILIZATION AND PATHENOGENETIC ACTIVATION}

\author{
M. G. Marques, A. B. Nascimento, V. P. Oliveira, A. R. S. Coutinho, M. E. O. A. Assumpção, and J. A. Visintin \\ Department of Animal Reproduction, FMVZ, São Paulo University, São Paulo, SP, Brazil
}

The present work evaluated the reversible meiosis inhibition effect on the development of swine embryos produced by in vitro fertilization (IVF) or parthenogenetic activation (PA). The efficiency of PZM3 and NCSU23 embryo culture media was also evaluated. Oocytes from ovaries collected at a slaughterhouse were subjected to IVM in two different groups: CHX (cycloheximide $5 \mu \mathrm{M}$ for $10 \mathrm{~h}$ ) and control, both with TCM- $199+3.05 \mathrm{mM}$ glucose $+0.91 \mathrm{mM}$ sodium pyruvate $+10 \%$ porcine follicular fluid $(\mathrm{pFF})+0.57 \mathrm{mM}$ cystein $+10 \mathrm{ng}$ epidermal growth factor $(\mathrm{EGF}) / \mathrm{mL}+10 \mathrm{IU}$ $\mathrm{eCG} / \mathrm{mL}+10 \mathrm{IU} \mathrm{hCG} / \mathrm{mL}$ for the initial $22 \mathrm{~h}$. In the remaining period ( $20 \mathrm{~h}$ for CHX and $22 \mathrm{~h}$ for control), medium without hormones was utilized. After IVM, oocytes were denuded and fertilized for $6 \mathrm{~h}$ (IFV) or the matured oocytes were submitted to activation by electric pulses (PA) (2 DC of $1.5 \mathrm{kV} / \mathrm{cm}$ for $30 \mu \mathrm{s}$ ), incubated for $1 \mathrm{~h}$ in culture medium with $10 \mu \mathrm{M}$ of CHX, and again submitted to the same electric pulses for $60 \mu \mathrm{s}$. Embryo development was evaluated by cleavage rate on Day 3 and blastocyst rate and blastocyst cell number on Day 7 of culture. Cleavage and blastocyst rates were analyzed by the equality-of-two-ratios test and cell number by the Kruskal-Wallis and Mann-Whitney tests $(P<0.05)$. In relation to IVF, the PZM3 medium was more efficient than NCSU23 for cleavage rate in the CHX group (PZM3: 68.4\%, NCSU23: 44.4\%) and had a better blastocyst rate in the control group (PZM3: 13.4\%, NCSU23: 5.6\%). With reference to PA, NCSU23 presented better cleavage and blastocyst rates 
than PZM3 in the CHX group (NCSU23: 89.5\%, PZM3: $78.5 \%$ and NCSU23: $20.4 \%$, PZM3: $13.0 \%$, respectively). In the control group, only the NCSU23 blastocyst rate was higher than that for PZM3 (NCSU23: 22.5\%, PZM3: 10.8\%). No culture medium effect on cell number mean of IVF and PA blastocysts was observed. Maturation block improved cleavage rates in IVF groups cultured with PZM3 (68.4\% and 50.6\%, respectively, for CHX and control) and in PA groups cultured with NCSU23 (89.5\% and $80.3 \%$, respectively, for CHX and control), but no improvement of blastocyst rates in both groups (IVF and PA) was verified. Table 1 below shows that maturation block decreased the IVF and increased the PA blastocyst cell numbers. As older oocytes are more effectively activated, oocytes blocked with $\mathrm{CHX}$ achieved the maturation stage faster than the control group, therefore resulting in high-quality PA blastocysts. In conclusion, PZM3 was more efficient for IVF embryo production in contrast to NCSU23, whereas NCSU23 can be indicated for PA embryo production. Moreover, maturation blockage with CHX influenced blastocyst cell number, decreasing in IVF embryos and increasing in PA embryos.

Table 1. Mean ( \pm SD) of blastocyst cell numbers for IVF or PA groups after in vitro maturation without (control) or with cycloheximide (CHX) and cultured in NCSU23 or PZM3 medium

\begin{tabular}{lcccc}
\hline & PZM3 control (embryo $n)$ & PZM3 CHX (embryo $n)$ & NCSU23 control (embryo $n)$ & NCSU23 CHX (embryo $n$ ) \\
\hline IVF & $46.40 \pm 13.73^{\mathrm{Aa}}(20)$ & $35.73 \pm 15.76^{\mathrm{Ab}}(30)$ & $49.41 \pm 18.42^{\mathrm{Aa}}(22)$ & $34.44 \pm 12.7^{\mathrm{Ab}}(32)$ \\
PA & $31.95 \pm 16.40^{\mathrm{Ba}}(20)$ & $40.92 \pm 16.99^{\mathrm{Ab}}(52)$ & $25.70 \pm 8.08^{\mathrm{Ba}}(46)$ & $34.92 \pm 15.11^{\mathrm{Ab}}(38)$ \\
\hline
\end{tabular}

${ }^{\mathrm{A}, \mathrm{B}}$ Values with different superscripts in the columns or ${ }^{\mathrm{a}, \mathrm{b}}$ in the rows differ significantly $(P<0.05)$.

This work was supported by FAPESP 02/10747-1.

\title{
287 EFFECT OF CYSTEAMINE DURING IN VITRO MATURATION OF OPU DERIVED BOVINE OOCYTES ON FURTHER IN VITRO EMBRYONIC DEVELOPMENT AND PREGNANCY RATE
}

\author{
J. S. Merton, B. Landman, and E. Mullaart
}

HG, R\&D, PO Box 5073, 6802 EB Arrnhem, The Netherlands

Glutathione (GSH) plays an important protective role in relation to reactive oxygen species generated by normal oxidative metabolism in the cell. The presence of cysteamine during in vitro maturation may facilitate the synthesis of GSH by immature oocytes. In a previous study we showed a positive effect of the presence of cysteamine during in vitro maturation of slaughterhouse derived bovine oocytes on subsequent in vitro embryonic development (Merton et al. 2004 Rep. Fert. Dev. 16, 279 abstract). This report shows the results of a field trial with ultrasound guided transvaginal oocyte collection (OPU) derived oocytes, in order to confirm our previous results obtained with slaughterhouse derived oocytes. Immature cumulusoocyte complexes (COCs) were recovered twice weekly by ovum pick-up (OPU) at two collection centres from 11 cows and 147 pregnant heifers. COCs were matured in vitro in TCM199/FCS/LH/FSH supplemented either with or without cysteamine $(0.1 \mathrm{mM})$. Subsequently, matured oocytes were fertilised with frozen-thawed gradient-separated semen and further cultured for 7 days in SOFaaBSA. At Day 7 , Morula grade 1 (IETS) were transferred fresh and early-, mid- and exp-Blast grade 1 and 2 were transferred either fresh or frozen/thawed. The experimental design was a $2 \times 2$ factorial. Results were analysed by Chi-square analyses. The results show that the presence of cysteamine during in vitro maturation significantly affected embryo production from OPU derived COCs (23.4\% and 34.4\% Morula + Blastocyst rate at Day 7 for control and cysteamine, respectively; Table 1). This higher embryo production rate was mainly due to an increased number of Blastocysts. Also the proportion of grade 3 embryos was significantly reduced in the cysteamine group $(P<0.01)$. The number of transferable embryos per session was 1.06 and 1.73 for control and cysteamine, respectively. Pregnancy rate was not significantly affected by the presence of cysteamine during in vitro maturation for both fresh and frozen/thawed embryos (fresh: $40.5 \%$ and $44.8 \%$, frozen/thawed: $44.4 \%$ and $47.2 \%$ for control and cysteamine, respectively). These results show that the presence of cysteamine during in vitro maturation affects further in vitro embryonic development, resulting in a higher embryo production rate. This suggest that an apparently 'simple' extra protection of the oocyte, due to the free radical scavenging potency of GSH, can have an enormous effect (63.2\% relative increase in transferable embryos) on its in vitro developmental potency. The intrinsic quality of the 'extra' produced transferable embryos seems not to be different, since pregnancy rate was not affected.

Table 1. Effect of cysteamine during in vitro maturation of OPU-derived bovine oocytes on subsequent in vitro embryonic development

\begin{tabular}{lcccccc}
\hline Group & \# Sessions & \# Oocytes (\%) & \# Cleavage (\%) & $\begin{array}{c}\text { Embryo production day 7 } \\
\text { \# Mor+Blast (\%) }\end{array}$ & $\begin{array}{c}\text { Transferrable } \\
\text { Total number } \\
\text { (per session) }\end{array}$ & $\begin{array}{r}\text { Relative } \\
\text { increase }\end{array}$ \\
\hline Control & 696 & 5454 & $3296(60.4)^{\mathrm{a}}$ & $1277(23.4)^{\mathrm{a}}$ & $740(1.06)^{\mathrm{a}}$ \\
Cysteamine & 589 & 4557 & $2939(64.5)^{\mathrm{b}}$ & $1569(34.4)^{\mathrm{b}}$ & $1017(1.73)^{\mathrm{b}}$ & $63.2 \%$ \\
\hline
\end{tabular}

${ }^{\mathrm{a}, \mathrm{b}}$ Values in columns with different superscript are significantly different, $P<0.001$. 


\title{
288 EFFECT OF IN VITRO MATURATION PERIOD AND OOCYTE ACTIVATION BY CALCIUM IONOPHORE ON MONOSPERMIC PORCINE EMBRYO RATES
}

\author{
V. P. Oliveira, M. G. Marques, A. B. Nascimento, H. V. A. Caetano, M. E. O. A. Assumpção, and J. A. Visintin \\ Department of Animal Reproduction, FMVZ, São Paulo University, São Paulo, SP, Brazil
}

It has already been reported that polyspermy in prepubertal pig oocytes is higher than in mature oocytes. Oocyte activation by calcium ionophore may help cortical granule exocytose. Normally, pig zygotes stay in maturation medium for $44 \mathrm{~h}$, but at $36 \mathrm{~h}$ oocytes are already at metaphase II stage (MII). Arrest at MII for a longer period may cause deterioration of various cytoskeletal components. The aim of this study was to evaluate the effect of maturation period ( 36 or $44 \mathrm{~h}$ ) and 0 or $50 \mu \mathrm{M}$ calcium ionophore on monospermic pig embryo rates. Prepubertal pig oocytes were matured in TCM-199 with $10 \mathrm{IU}$ eCG $+10 \mathrm{IU} \mathrm{hCG}+50 \mathrm{IU}$ epidermal growth factor (eGF) $+90 \mu \mathrm{L}$ porcine follicular fluid (pFF) for $20 \mathrm{~h}$ and in the same medium without hormones for the last 16 or $24 \mathrm{~h}$. In vitro fertilization was performed in mTBM medium with $1 \times 10^{5}$ spermatozoa/mL in three different periods $(2,4$, and $6 \mathrm{~h}$ ). For nuclear evaluation, the zigotes were stained by Hoechst $3334218 \mathrm{~h}$ after fertilization. Results were analyzed by PRC GLM of SAS (ANOVA and Tukey test) at a 5\% level. Data were expressed as mean \pm standard error of the mean non transformed. Independent variables were maturation period, effect of calcium ionophore, interaction among calcium*maturation period, and manipulations. In non-fertilized oocytes, there was no effect of maturation period, manipulation, or interaction calcium*maturation, but there was effect of calcium ionophore, having $43.5 \pm 2.5$ more non-fertilized oocytes at $36 \mathrm{~h}$ and $43.1 \pm 5.1$ more at $44 \mathrm{~h}$ of maturation $(P<0.01)$. At $36 \mathrm{~h}$ oocytes not activated by calcium ionophore showed $29.7 \pm 4.9$ more monospermic embryos than activated oocytes $(P<0.01)$. However, at $44 \mathrm{~h}$ of maturation, activation with calcium ionophore produced $47.4 \pm 7.2$ more monospermic embryos than non activated oocytes. From this study we conclude that monospermic embryo rates were influenced by maturation period, with the best results at $36 \mathrm{~h}$ in the absence and at $44 \mathrm{~h}$ in the presence of $50 \mu \mathrm{M}$ of calcium ionophore.

This work was supported by CAPES.

\section{IN VITRO PRODUCTION OF OVINE EMBRYOS FROM WOOL AND HAIR BREEDS}

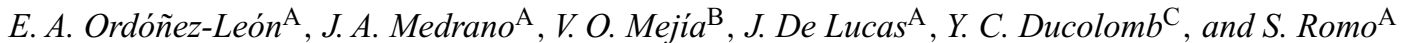

${ }^{\mathrm{A}}$ Departamento de Posgrado, Facultad de Estudios Superiores-Cuautitlán, UNAM, Cuautitlán, Estado de México, México; ${ }^{\mathrm{B}}$ Facultad de Medicina Veterinaria y Zootecnia, UNAM, México D.F. México; ${ }^{\mathrm{C}}$ Departamento de Ciencias de la Salud, UAM-I, México D.F. México

In tropical areas, wool-less or haired breeds of sheep are more prolific than wool breeds. There are no reports about IVF in tropical breeds; therefore, it is not known how these respond under IVF conditions. Developing protocols for in vitro production of embryos in haired breeds could contribute to the preservation and use of their genetic potential in tropical countries where they are economically important. The aim of this study was to determine differences in IVM, IVF, and in vitro (IVD) between wool and hair breeds of sheep. A protocol for IVF (Wani 2002 Small Rum. Res. 44, 89-95) was used in wool $(\mathrm{W})$ and hair $(\mathrm{H})$ breeds. A total of $251 \mathrm{~W}$ and $251 \mathrm{H}$ ewes were used. The ovaries were obtained after slaughter and transported to the laboratory in physiological saline $\left(25^{\circ} \mathrm{C}\right)$. A total of 411 ovaries from $\mathrm{W}$ and 440 from $\mathrm{H}$ ewes were used, and 805 follicles of $\mathrm{W}$ and 790 of $\mathrm{H}$ ewes were aspirated. From these, 663 (82\%) cumulus-oocyte complexes (COCs) of W and 597 (76\%) of $\mathrm{H}$ ewes were obtained and then used for the procedures of IVM, IVF, and IVD. The average number of COCs recovered per ovary was 1.6 for $\mathrm{W}$ and 1.4 for $\mathrm{H}$. The average number of follicles per ovary was 1.9 for W and 1.7 for H. For IVM, COCs were incubated in TCM-199 supplemented with 20\% serum from estrous ewe (SEE) for $24 \mathrm{~h}$. All incubations were performed at $38.5^{\circ} \mathrm{C}$ in a humidified atmosphere of $5 \% \mathrm{CO}_{2}$ in air. After this period, COCs were placed in fertilization medium (TALP supplemented with $200 \mu \mathrm{g} / \mathrm{mL}$ heparin, $3 \mu \mathrm{g} / \mathrm{mL}$ penicillamine, and $1 \mu \mathrm{g} / \mathrm{mL}$ hypotaurine). For insemination, frozen-thawed semen from $\mathrm{H}$ and $\mathrm{W}$ rams was washed by centrifugation in two concentration gradients of a silicone solution. Oocytes and semen from the corresponding breed types were co-incubated for $18 \mathrm{~h}$. For IVD, presumptive zygotes were incubated in SOF medium supplemented with $20 \%$ SEE for 7 days. Eight replicates were made. The rates of IVM, IVF, and IVD were analyzed by logistic regression, using as response variables: IVM, IVF, and IVD results, and as independent variables: breed and replicate. The percentage of recovered oocytes was $82 \%$ for W and $76 \%$ for $\mathrm{H}$. For IVM and IVF, the recovered oocytes produced 535 fertilized oocytes of $\mathrm{W}$ and 446 of $\mathrm{H}$. From these, $81 \%$ of the $\mathrm{W}$ oocytes and $75 \%$ of $\mathrm{H}$ were fertilized. Oocytes from $\mathrm{W}$ showed a higher percentage of IVM and IVF, with a statistically significant difference $(P<0.05)$. The percentage of division was $63 \%$ for $\mathrm{W}(n=419)$ and $52 \%$ for $\mathrm{H}(n=312)$. There were no statistically significant differences between the two groups for embryo IVD $(P>0.05)$. No statistical differences were found between replicates and no interactions were observed for breed $\times$ replicate $(P>0.05)$. It is concluded that IVM, IVF, and IVD procedures used for the development of embryos in $\mathrm{W}$ ewes can be used with similar results in $\mathrm{H}$ ewes. This is the first report of sheep IVF in Mexico that provides relevant information about the procedures of IVM, IVF, and IVD in hair and wool sheep, andsets a precedent for future investigations on in vitro embryo production in haired sheep breeds in Mexico.

Funding for E.A. Ordónez-León was provided by CONACYT and UNAM.

\section{THE FACTORS ON RATES OF ABNORMALITY, DISEASE AND MORTALITY OF CALVES DERIVED FROM IN VITRO EMBRYOS OF KOREAN NATIVE CATTLE}

$$
\text { Y.-S. Park }{ }^{\mathrm{A}} \text {, S.-S. Kim }{ }^{\mathrm{B}} \text {, M.-C. Park }{ }^{\mathrm{C}} \text {, and H.-D. Park }{ }^{\mathrm{C}}
$$

${ }^{A}$ Kyongbuk Livestock Research Institute, Youngju, Kyongbuk, South Korea; ${ }^{B}$ College of Veterinary Medicine, Chungbuk National University, Chenongju, Chungbuk, South Korea; ${ }^{C}$ Division of Life Food and Biotech, Daegu University, Gyeongsan, Kyongbuk, South Korea

In Korea, in vitro production and transfer of bovine embryos has advanced remarkably and applied commercially. However, in vitro-produced embryos result in lower pregnancy and higher abortion rates and in some cases increased rates of abnormality and mortality in calves. The present study 
was conducted to investigate the effects of various factors such as recipient parity, delivery season, offspring number, pregnancy period, delivery type, midwifery type, dystocia and vaccination, on the viability of calves derived from embryos produced in vitro. Korean Native Cow ovaries were obtained from local slaughterhouse and cumulus-oocyte complexes (COCs) were aspirated from 2 to $8 \mathrm{~mm}$ follicles. Selected COCs were matured in TCM-199 supplemented with 10\% fetal calf serum (FBS), $1 \mu \mathrm{ML}$ FSH, $10 \mu \mathrm{ML}$ LH and $1 \mu \mathrm{ML}$ Estradiol-17 $\beta$ for 20-22 h. In vitro-matured oocytes were fertilized using frozen-thawed percoll separated semen (Day 0) in fer-TALP medium for $20 \mathrm{~h}$. The presumptive zygotes were cultured in CR1 aa medium supplemented with $0.3 \%$ BSA (before Day 3) or 10\%FBS (After Day 3). All types of cultures were made in an incubator at $38.5^{\circ}$, $5 \% \mathrm{CO}_{2}$ in air. Statistical analysis was performed using the Chi-square test. Two blastocysts were transferred to the Holstein recipients $(n=1888)$. The parturition was occurred in total 755 recipients. There was no difference in the abnormality of calves among treatments. The incidence of disease was significantly higher in single calf than twin calves (18.4 vs. 6.7\%), in multiparous than nulliparous group (40.0 vs. $9.9 \%)$, in eutocia than dystocia group (20.0 vs. $4.8 \%)$, in spring and winter groups than summer and autumn groups $(20.3,22.7$ vs. $4.3,0.0 \%)$, and in non-vaccinated than vaccinated group $(22.7$ vs. $1.6 \%)$, respectively $(p<0.05)$. The rate of mortality was significantly higher when transferred into nulliparous than multiparous ( 22.3 vs. $0.0 \%)$, when were dystocia than eutocia group ( $71.4 \mathrm{vs.} 14.1 \%)$, when were non-midwifery than midwifery $(45.0$ vs. 13.6$)$, when delayed midwifery than earlier midwifery ( $31.6 \mathrm{vs.} 11.5 \%)$, and when were non-vaccinated than vaccinated group ( $28.0 \mathrm{vs.} 9.8 \%)$, respectively $(P<0.05)$. The present study suggested that the viability of bovine calves derived from in vitro was affected by the recipient parity, parturition treatment technique and vaccination.

This study was supported by the BIO-GREEN 21 PROGRAM.

\title{
291 VARIOUS FACTORS INFLUENCING PREGNANCY RATES AND CALVING CHARACTERISTICS FOLLOWING TRANSFER OF OPU-IVP EMBRYOS PRODUCED IN A COMMERCIAL SYSTEM
}

\author{
C. Ponsart ${ }^{\mathrm{A}}$, F. Aymar ${ }^{\mathrm{A}}$, B. Marquant-Le Guienne ${ }^{\mathrm{A}}$, C. Guyader-Joly ${ }^{\mathrm{B}}$, S. Ponchon ${ }^{\mathrm{B}}$, and P. Humblot ${ }^{\mathrm{A}}$ \\ ${ }^{A}$ UNCEIA, R\&D, Maisons-Alfort, 94704, France; ${ }^{B}$ UNCEIA, Sation FIV, Chateauvillain, 38300, France
}

OPU-IVP is nowadays an assisted reproduction technique in which output for each step is variable. The aim of this work was to identify factors influencing calving rate and calf characteristics through a retrospective study conducted from 356 OPU sessions and 137 pregnancies on a farm. Donor females were stimulated with FSH in five decreasing doses ( $400 \mu \mathrm{g}$ for cows and $250 \mu \mathrm{g}$ for heifers). Collected oocytes were matured for $24 \mathrm{~h}$ in M199 plus fetal calf serum, FSH, epidermal growth factor (EGF), and estradiol. They were then fertilized in TALP with frozen-thawed semen and zygotes were cultured for 6 days on Vero cell monolayers in B2 medium. Embryos were transferred as fresh to recipients on Day 7; 137 pregnancies were followed up to calving. Gestation length, calving conditions and calf characteristics (sex and birth weight assessed by farmers) were recorded and compared to national standards (UPRAs data). The mean number of collected oocytes per session was 14; 11.6 were selected for IVM, 8.5 cleaved, and 3.9 developed to the blastocyst stage ( $40.4 \%$ of the embryos were Grade $1,35.3 \%$ G2, and 20.5\% G3). On average, 2.9 embryos were transferred into recipients, leading to an average of 1.6 pregnancies on Day 35 and 1.4 on Day 90 ( $76 \%$ of recipients were heifers). Pregnancy rates were higher in heifers than in cows $(54.5 \%$ vs. $47.8 \% ; P<0.05)$. Pregnancies led to birth of a healthy calf in $81.9 \%$ of the OPU sessions (1.1 per session), the $18.1 \%$ losses being divided between $4.4 \%$ abortion $(n=6)$ and $13.7 \%$ perinatal mortality $(n=18)$. Gestation length from IVF pregnancies was longer than in national breeds standards (trial $=291$ vs. national $=287$ days). Moreover, $10 \%$ of calvings were induced. Calving conditions were mainly dependent on age of recipients: $70 \%$ of the heifers were assisted compared to $40.9 \%$ of the cows. No effect of gestation length or sex of calf was observed on calving difficulties. Sex ratio did not deviate in calves produced by OPU-IVF $(52.3 \%$ of males and $47.7 \%$ of females). However, it was influenced by embryo quality, with the proportion of males decreasing from $56.3 \%$ to $44.4 \%$ for G1 to G3 embryos. The mean weight of healthy calves issued from IVP embryos averaged $47.1 \mathrm{~kg} \pm 10.1$ and was not significantly higher than the national breed standards. The deviation $(\mathrm{kg})$ from national breeds standards ranged from $-20 \mathrm{~kg}$ to 0 in $68.8 \%(n=90)$, from 1 to $15 \mathrm{~kg}$ in $16.0 \%(n=29)$, and greater than $15 \mathrm{~kg}$ in $9.2 \%(n=12)$ of the calves. Even if the mean deviation was not significant, those 12 calves in the $>15 \mathrm{~kg}$ group should be considered as large. However, they were issued from two different donor females, so that this effect could also be attributed to a mother effect. To conclude, the effect of recipient parity on pregnancy rates was confirmed. The OPU-IVP system used in this trial did not seem to influence significantly sex ratio and weight of calves. Further studies are needed to investigate the sources of variation of gestation length.

\section{EXPRESSION PROFILES OF STRESS AND METABOLIC MARKER GENES DURING IN VITRO PRODUCTION OF BUFFALO (BUBALUS BUBALIS) EMBRYOS}

\author{
R. Rajhans ${ }^{\mathrm{A}}$, G.S. Kumar ${ }^{\mathrm{B}}$, and G. T. Sharma ${ }^{\mathrm{A}}$
}

${ }^{A}$ Reproductive Physiology Laboratory, P\&C Division, Indian Veterinary Research Institute, Izatnagar, Bareilley, Uttar Pradesh, India; ${ }^{B}$ Division of Pathology, Indian Veterinary Research Institute, Izatnagar, Bareilley, Uttar Pradesh, India; ${ }^{C}$ Reproductive Physiology Laboratory, P\&C Division, Indian Veterinary Research Institute, Izatnagar, Bareilley, Uttar Pradesh, India

An increased understanding of the pre-implantation embryo developmental stage, with respect to physiological interaction of embryo with its micromilieu both in vivo and in vitro, is imperative to comprehend the events of pre-implantation development.The objective of the present study was to examine the temporal expression of heat shock protein 70 (Hsp-70) and glucose transporter 1 (Glut1) genes in pre-implantation-stage buffalo embryos produced under the standard in vitro production (IVP) system. Embryos were produced from slaughterhouse ovaries employing standard in vitro embryo production protocol, and presumptive zygotes produced following IVM/IVF were cultured in vitro in mSOF under mineral oil; 
FCS $(10 \%)$ was added at $48 \mathrm{~h}$ post-insemination (hpi).The time series of development at stages being zygote (18-20 hpi), $2-1$ cell ( $48 \mathrm{hpi}), 8-16 \mathrm{cell}$ (94-96 hpi), morula (120-144 hpi), and blastocyst (168-192 hpi), pre-implantation embryos conforming to the above developmental pattern were considered as 'fast-cleaving embryos', and all the embryos that did not conform to the above developmental timing were regarded as 'slow-cleaving embryos'. Pools of immature oocytes (IM, 120), Matured oocytes (MO, 120), 8-16 cell stages ( $8-16,70)$, morula (M, 28), and blastocyst (B, 9) were collected and prepared for total RNA isolation and RT-PCR for the specific transcripts, with $\beta$-actin as loading control. The total RNA content ranged from 2.5 to $5.0 \mathrm{ng}$ per oocyte/embryo. Presence of Hsp 70 and Glut1 gene transcripts was assessed in different stages of buffalo pre-implantation embryos using primers designed from bovine Hsp 70 and Glut1 by using the OLIGO program. RT was standardized using the embryo equivalent of 1-10 oocytes/embryo as the template as described by Arcellana-Panlilio and Schultz 1993 (Methods Enzymol. 225, 303-328) with PCR conditions being $59^{\circ} \mathrm{C}$ and $62^{\circ} \mathrm{C}$ for $45 \mathrm{~s}$ with 39 cycles for Glut1 and Hsp 70 gene transcripts, respectively. Amplicons were subjected to restriction digestion and sequencing (Acc. No. AJ812563, AJ812564). The expression of Hsp 70 throughout pre-implantation development in the fast-cleaving embryos indicated their maternal and zygotic origin, but transcripts of the Hsp 70 gene, represented by a single 488-bp amplicon, were not detected in slow-cleaving embryos, suggesting altered zygotic expression. Glut1 expression was prominent from the $8-16$ cell stage, indicating a metabolic shift from pyruvate to glucose after the pre-compaction stage. For slow-cleaving embryos, transcripts of the Glut 1 gene, represented by a single 327-bp amplicon, were absent during morula- and blastocyst-stage embryos, indicating the poor developmental competence of these embryos, which morphologically appeared normal. These transcription patterns reflect the embryonic response to the in vitro culture conditions and also correlate with the embryo quality and the speed of development of the pre-implantation buffalo (Bubalus bubalis) embryos.

\title{
293 EFFECT OF FOLLICULAR WAVE SYNCHRONIZATION ON IN VITRO EMBRYO PRODUCTION IN COWS
}

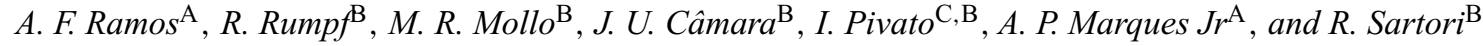 \\ ${ }^{\mathrm{A}}$ Federal University of Minas Gerais, Belo Horizonte, MG, Brazil; ${ }^{\mathrm{B}}$ Embrapa Genetic Resources and Biotechnology, Brasílilia, \\ DF, Brazil; ${ }^{\mathrm{C}}$ Cidasc, Indaial, SC, Brazil
}

In order to achieve the ideal time of ovum pickup (OPU) for in vitro embryo production (IVP) in cows regarding number and quality of oocytes recovered, this study investigated the effect of synchronization of wave emergence with estradiol benzoate (EB) injected 7 days prior to follicular aspiration. In a Latin square design, 12 crossbred beef cows were randomly divided into three groups, with three replicates each. Cows were synchronized with a norgestomet ear implant for 7 days followed by an i.m. prostuglandin $\mathrm{F}_{2 \alpha}$ (PGF2 $\alpha$ ) injection and aspiration of all ovarian follicles larger than $3 \mathrm{~mm}$ in diameter. After that, follicles from cows in group $2 \mathrm{X}$ were aspirated twice a week with 4- and 3-day intervals, and follicles from groups $1 \mathrm{X}$ and $1 \mathrm{X}$-EB were aspirated once a week. Cows from group $1 \mathrm{X}-\mathrm{EB}$ also received an im injection of $2 \mathrm{mg}$ of EB immediately after OPU. Throughout the study cows were kept with an ear norgestomet implant that was replaced every 2 weeks. Ultrasound evaluations of numbers of follicles greater than $3 \mathrm{~mm}$ in diameter and size of the largest follicle at the time of OPU were performed. Recovered oocytes were evaluated for quality, and the viable ones (Grades I, II, and III) were in vitro-fertilized on Day 0. Cleavage rate was evaluated on Day 2 and blastocyst production on Day 7. Continuous variables were compared by ANOVA and binomial data were compared by chi-square. For the $2 \mathrm{X}$ group, only data from the OPU performed 3 days after the last OPU were used for analysis. Results are presented as percentages or mean \pm SEM. Size of the largest follicle was greater $(P<0.05)$ in $1 \mathrm{X}$ coes $(12.9 \pm 0.2 \mathrm{~mm})$ than in $1 \mathrm{X}$-EB cows $(11.1 \pm 0.3 \mathrm{~mm})$, which was greater than in $2 \mathrm{X}(9.6 \pm 0.4 \mathrm{~mm})$ cows. The $1 \mathrm{X}$ cows had more follicles at OPU than $2 \mathrm{X}$ cows $(17.5 \pm 0.7 \mathrm{vs.} 14.1 \pm 0.9)$, whereas the $1 \mathrm{X}$-EB group $(15.9 \pm 0.7)$ was intermediate and not different from the others. There was no difference in the mean number of recovered oocytes among $2 \mathrm{X}(9.6 \pm 0.6), 1 \mathrm{X}(12.7 \pm 0.8)$ and $1 \mathrm{X}$-EB (12.3 \pm 1.0$)$ cows, and the mean number of viable oocytes among groups $(5.8 \pm 0.5,7.3 \pm 0.5$, and $7.0 \pm 0.6)$ for $2 \mathrm{X}, 1 \mathrm{X}$, and $1 \mathrm{X}$-EB cows, respectively). The rate of viable oocytes was also similar among groups [58.8\% (191/325) for 2X, 58.4\% (267/457) for 1X, and 57.2\% (231/404) for 1X-EB cows]. Cleavage $[68.6 \%(131 / 191), 65.2 \%(174 / 267)$, and 68.4\% (158/231)] and blastocyst $[38.7 \%(74 / 191), 43.8 \%(117 / 267)$, and $44.2 \%(102 / 231)]$ rates were also not different among $2 \mathrm{X}, 1 \mathrm{X}$, and $1 \mathrm{X}$-EB groups, respectively. Although the use of $2 \mathrm{mg}$ of EB in association with a norgestomet implant 7 days prior to OPU altered the follicular wave profile, it was not enough to improve number and quality of the oocytes recovered. Moreover, this study failed to demonstrate a positive effect of OPU earlier after wave emergence, when the effect of dominance should be less pronounced, on IVP in cows.

The first author was supported by the fellowship 141077/2004-2 of CNPq, Brazil.

\section{THE EFFECT OF PLASMIN ON THE IN VITRO FERTILIZATION ABILITY OF PORCINE GAMETES}

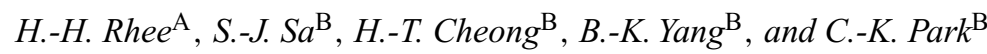 \\ ${ }^{A}$ Hanna Women's Clinic, Seoul, Korea; ${ }^{B}$ College of Animal Resource Science, Kangwon University, Chunchon, Korea
}

Plasminogen activators (PAs) are specific proteolytic enzymes that convert the inactive proenzyme plasminogen to plasmin. The plasmin formed is a nonspecific, potent protease that cleaves blood fibrin clots and several other extracellular proteins. The purposes of the present study were (1) to assess the effect of plamin on sperm viability and acrosome reaction (AR), (2) to examine the effect of plasmin on zona pellucida (ZP) solubility and the binding of sperm to ZP, and (3) to evaluate the effect of plasmin on fertilization responses, including penetration and incidence of polyspermy during in vitro fertilization in the pig. Ejaculated semen was collected from three mature Duroc boars by artificial vagina. The same three boars were used for all experiments. The oocyte maturation medium used was North Carolina State University-23 (NCSU-23) medium supplemented with 10\% $(\mathrm{v} / \mathrm{v})$ porcine follicular fluid $(\mathrm{pFF}), 0.6 \mathrm{mM}$ cysteine, $10 \mathrm{IU} / \mathrm{mL}$ human chorionic gonadotropin (hCG; Sigma-Aldrich Corporation, St. Louis, MO, 
USA), and $10 \mathrm{IU} / \mathrm{mL}$ pregnant mare's serum gonadotropin (PMSG; Sigma). Porcine spermatozoa, which were washed in Dulbecco PBS (Sigma), were resuspended and incubated in fertilization medium (mTBM) containing $0,0.1,1.0,10.0$, or $100.0 \mathrm{ng} / \mathrm{mL}$ plasmin (Sigma). Data were analyzed by ANOVA and Duncan's multiple-range test using the Statistical Analysis System (SAS Institute, Inc., Cary, NC, USA). The present study suggests that sperm viability was not affected by plasmin treatment. Also, addition of plasmin in doses ranging between $0.1 \mathrm{and} 100.0 \mathrm{ng} / \mathrm{mL}$ for 2,4 , or $6 \mathrm{~h}$ to washed boar spermatozoa resulted in enhancement of acrosome reaction (AR), compared with untreated cells. Concentrations of $0 \mathrm{and} 0.1 \mathrm{ng} / \mathrm{mL}$ plasmin ( $83 \pm 15$ and $95 \pm 18$ sperm/oocyte, respectively) had no effect on sperm binding, whereas 1.0 (123 \pm 21 sperm/oocyte), 10.0 (124 \pm 16 sperm/oocyte), and $100 \mathrm{ng} / \mathrm{mL}(124 \pm 15 \mathrm{sperm} /$ oocyte $)$ plasmin increased $(P<0.05)$ sperm binding, compared with the control. The zona pellucida solubility (zona digestion time) was significantly $(P<0.05)$ lower in medium with $1.0(123 \pm 24 \mathrm{~s}), 10.0(99 \pm 15 \mathrm{~s})$, or $100.0 \mathrm{ng} / \mathrm{mL}(95 \pm 19 \mathrm{~s})$ plasmin, compared with control $(176 \pm 27 \mathrm{~s})$. When porcine oocytes and spermatozoa were co-incubated in various concentrations of plasmin for $6 \mathrm{~h}$, the penetration rate was significantly $(P<0.05)$ higher in medium with $1.0 \mathrm{ng} / \mathrm{mL}$ plasmin $(77.5 \pm 3.1 \%)$, compared with control. However, there were no significant differences in the polyspermic rates and mean numbers of sperm (MNS)/oocyte among the groups treated with plasmin and the control group. We found that addition of plasmin to fertilization medium increases the percentage of acrosome-reacted spermatozoa and the sperm-binding ability of the pig ZP. These results suggest that plasmin may play a role in events related to fertilization in the pig.

\title{
295 RELATIONSHIP BETWEEN PLASMINOGEN ACTIVATOR/PLASMIN SYSTEM AND IN VITRO FERTILIZATION ABILITY IN THE PIG
}

\author{
S.-J. Sa, H.-T. Cheong, B.-K. Yang, and C.-K. Park \\ College of Animal Resource Sciences, Kangwon University, Chunchon, Kangwon-do, Korea
}

Plasminogen activators (PAs) are serine proteases, known to be secreted by a large number of cell types, that convert plasminogen into plasmin. The present study was undertaken to identify PAs in porcine gametes and to investigate a possible role of plasminogen in fertilization in vitro in the pig. The oocyte maturation medium used was North Carolina State University-23 (NCSU-23) medium supplemented with 10\% (v/v) porcine follicular fluid (pFF), $0.6 \mathrm{mM}$ cysteine, $10 \mathrm{IU} / \mathrm{mL}$ human chorionic gonadotropin (hCG), and $10 \mathrm{IU} / \mathrm{mL}$ pregnant mare's serum gonadotropin (PMSG). To determine the changes of PA activities in porcine oocytes during maturation, the cumulus-oocyte complexes (COCs) were incubated in NCSU-33 in an atmosphere of $5 \% \mathrm{CO}_{2}$ in air at $39^{\circ} \mathrm{C}$ for 0,24 , or $48 \mathrm{~h}$. On the other hand, to investigate the release of PAs by boar spermatozoa, fresh spermatozoa were pre-incubated in fertilization medium (mTBM) for $0,2,4$, or $6 \mathrm{~h}$. After culture, $40 \mathrm{COCs}, 40$ cumulus-free oocytes, and $40 \times 10^{6}$ spermatozoa were separately put into microtubes containing $20 \mu \mathrm{L}$ of sample buffer $[5.0 \%$ (w:v) sodium dodecyl sulfate (SDS), 20\% (v:v) glycerol, and $0.0025 \%$ (w:v) bromophenol blue in $0.125 \mathrm{M}$ Tris- $\mathrm{HCl}$ buffer] and frozen at $-80^{\circ} \mathrm{C}$ until used for analysis. PA activities in porcine oocytes and spermatozoa were quantified using SDS-PAGE, casein-agar zymography, and densitometry. Data were analyzed by ANOVA and Duncan's multiple-range test using the Statistical Analysis System (SAS Institute, Inc., Cary, NC, USA). In the COCs cultured for 24-18 h, tissue-type plasminogen activator (tPA), urokinase-type PA (uPA), and tPA-PA inhibitor (tPA-PAI) were observed. Also, PA activities increased as duration of culture increased. However, no uPA activity was detected in cumulus-free oocytes. In procine fresh spermatozoa, tPA, uPA, and tPA-PAI were observed. When spermatozoa were incubated for 2, 4, or $6 \mathrm{~h}$ in fertilization medium, the rate of acrosome reaction (AR) in spermatozoa increased as the duration of culture increased, but PA activities decreased gradually. However, PA activities in sperm-conditioned medium increased as duration of culture increased. On the other hand, to determine the effect of plasminogen on fertilization ability of porcine oocyte and spermatozoa, plasminogen $(50 \mu \mathrm{g} / \mathrm{mL}) \mathrm{was}$ added in fertilization medium. Addition of plasminogen to fertilization medium increased $(P<0.05)$ AR in spermatozoa and sperm binding to the zona pellucida (ZP), compared with control group. The ZP solubility (zona digestion time) was higher in medium with than that without plasminogen. When porcine oocytes and spermatozoa were co-incubated in fertilization medium with plasminogen, the polyspermic rate was lower in medium with than that without plasminogen. Also, plasminogen significantly $(P<0.05)$ increased formation rate of the male pronucleus in oocytes penetrated by spermatozoa. These results suggest that supplementing of plasminogen in fertilization medium may play a positive role in improving of fertilization ability in vitro in the pig.

\section{INFLUENCE OF SHORT-TERM HEAT STRESS ON THE BLASTOCYST RATE OF BOVINE EMBRYOS (INDICUS VS. TAURUS) FROM OOCYTES OBTAINED BY OVUM PICKUP}

\author{
E. Sartorelli e Sartorelli ${ }^{\mathrm{A}}$, A. C. Z. Barcelos ${ }^{\mathrm{B}}$, R. A. Satrapa ${ }^{\mathrm{A}}$, D. F. Martins ${ }^{\mathrm{B}}$, M. F. G. Nogueira $^{\mathrm{A}}$, \\ J. R. Potiens ${ }^{\mathrm{C}}$, M. M. Seneda ${ }^{\mathrm{D}}$, and C. M. Barros ${ }^{\mathrm{B}}$ \\ ${ }^{A}$ Faculdade de Medicina Veterinaria e Zootecnia - UNESP, Botucatu, São Paulo, Brasil; ${ }^{B}$ Instituto de Biociencias, UNESP, Botucatu, \\ São Paulo, Brasil; ${ }^{\mathrm{C}}$ Central Bela Vista, Pardinho, São Paulo, Brasil; ${ }^{\mathrm{D} C C A-U E L, ~ L o n d r i n a, ~ P a r a n a ́, ~ B r a s i l ~}$
}

There is evidence that the deleterious effects of heat stress (HS) on fertility are less pronounced in Bos indicus than in B. taurus breeds, due primarily to differences in their thermoregulatory capacity. In the present work, the resistance to heat stress of Nelore embryos $(B$. indicus) was compared to either a breed not adapted (Angus; B. taurus) or adapted to high temperatures (Bonsmara; $5 / 8$ B. indicus $\times 3 / 8$ B. taurus). In Experiments (Exp.) 1 (Nelore vs. Angus) and 2 (Nelore vs. Bonsmara), oocytes obtained by ovum pickup OPU (during autumn) were matured (TCM-199), fertilized, and cultured (SOFaaci) in vitro. Ninety-six hours post-insemination (hpi), embryos with more than 16 cells were randomly allocated in two main groups: Group Control (embryos were maintained at $39^{\circ} \mathrm{C}$ all of the time) and Group HS (embryos were maintained at $41^{\circ} \mathrm{C}$ during $12 \mathrm{~h}$ and afterwards returned to $39^{\circ} \mathrm{C}$ ). Blastocyst rates were determined on the 7th day of culture. In Exp. 1, 294 oocytes from Nelore and 144 from Angus cows had a 
cleavage rate of 67.9 and $59.4 \%$, respectively. Ninety-six-hpi embryos ( $>16$ cells) were distributed in four groups: Nelore Control $(n=97)$, Nelore HS $(n=95)$, Angus Control $(n=34)$ and Angus HS $(n=25)$. The blastocyst rates were 39/97 (40.2\%), 23/95 $(24.2 \%), 19 / 34(55.9 \%)$, and e $8 / 25$ $(32.0 \%)$, respectively. The difference in rate of blatocyst formation caused by heat stress on Nelore (16.0\%) and Angus (23.9\%) was not significantly different $(P<0.05)$, and suggests, from oocytes obtained by OPU, that Nelore embryos may be more tolerant to HS than Angus embryos. However, it is necessary to increase the number of blastocysts per group in order to better characterize the effects of heat stress on these embryos. In Exp. 2, 294 oocytes from Nelore and 101 from Bonsmara cows had a cleavage rate of 41.2 and 51.2\%, respectively. Ninety-six-hpi embryos ( $>16$ cells) were distributed in four groups: Nelore Control $(n=44)$, Nelore HS $(n=49)$, Bonsmara Control $(n=22)$, and Bonsmara HS $(n=22)$. The blastocyst rates were 35/44 (79.5\%), 30/49 (61.2\%), 10/22 (45.5\%), and 6/22 (27.3\%), respectively. In spite of the fact that Bonsmara embryos had a lower blastocyst rate as compared to Nelore, the decline on blastocyst rate caused by HS was very similar in Nelore (18.3\%) and Bonsmara embryos $(18.2 \%)$. Additional OPU are underway to test the hypothesis that thermotolerance of Nelore embryos is similar to that in embryos from a breed adapted to high temperatures (Bonsmara), and superior to embryos from a non adapted breed (Angus).

E. S. S., R. A. S., and M. F. G. N. were supported by a fellowship from FAPESP, and A. C. Z. B. by a fellowship from CAPES of Brazil.

\title{
297 BIRTH OF LIVE RATS THROUGH IN VITRO FERTILIZATION USING CRYOPRESERVED SPERMATOZOA: SPERM FERTILITY IMPROVED BY FREEZING AT $-150^{\circ} \mathrm{C}$
}

\author{
Y. Seita, Y. Okuda, A. Takizawa, S. Hisamatu, T. Inomata, and N. Kashiwazaki \\ Graduate School of Veterinary Medicine, Azabu University, Sagamihara, Kanagawa 229-8501, Japan
}

We previously reported that damages to spermatozoa by cold shock can be avoided by cooling slowly at $0.5^{\circ} \mathrm{C} / \mathrm{min}$ to $5.0^{\circ} \mathrm{C}$ (Seita et al. $2005 \mathrm{Reprod}$. Fertil. Dev. 17, 277-278). The objective of the present study was to develop an in vitro fertilization (IVF) system with frozen-thawed rat spermatozoa for more efficient reproduction of live offspring. We examined the effect of freezing temperatures (cooling $5.0^{\circ} \mathrm{C}$ to pre-plunging) on post-thaw sperm motility, plasma membrane integrity, and fertility in vitro. Epididymal spermatozoa of Wistar rats were collected in $2.0 \mathrm{~mL}$ of freezing medium containing 23\% (v/v) egg yolk, 8.0\% (w/v) lactose monohydrate, and 0.7\% (v/v) Equex STM (Nova Animal Sales, Inc., Scituate, MA, USA) at room temperature. Samples were loaded into $0.25-\mathrm{mL}$ straws and cooled to $5.0^{\circ} \mathrm{C}$ at $0.5^{\circ} \mathrm{C} / \mathrm{min}$ in a programmable freezer. Next, the samples were exposed to liquid nitrogen (LN) vapor at various freezing temperatures $\left(-120^{\circ} \mathrm{C},-150^{\circ} \mathrm{C}\right.$ or $\left.-180^{\circ} \mathrm{C}\right)$ above the $\mathrm{LN}$ level for $15 \mathrm{~min}$ and then plunged into $\mathrm{LN}$. Straws were thawed in a $37^{\circ} \mathrm{C}$ water bath for $10 \mathrm{~min}$. The thawed samples were diluted to $0.5-1.5 \times 10^{6}$ sperm $/ \mathrm{mL}$ in a droplet of $200 \mu \mathrm{L}$ of R1ECM and then pre-incubated for $5 \mathrm{~h}$. Ovulated oocytes were introduced into the droplet and co-cultured for $10 \mathrm{~h}$. The oocytes were denuded, fixed, and/or examined for two pronuclei (2PN) formation microscopically. The denuded oocytes, which were fertilized with spermatozoa frozen at $-150^{\circ} \mathrm{C}$ and were microscopically confirmed to have $2 \mathrm{PN}$ formation, were transferred to pseudo-pregnant recipient females. IVF was also performed by the same method using fresh spermatozoa as the control. Differences in the sperm motility and plasma membrane integrity were analyzed by ANOVA, and the IVF data were analyzed by chi-square test. At $2 \mathrm{~h}$ after thawing the motility of spermatozoa frozen at $-150^{\circ} \mathrm{C}$ was significantly higher than that of spermatozoa frozen at $-180^{\circ} \mathrm{C}(19.8 \%$ and $11.1 \% ; P<0.05)$, although the sperm plasma membrane integrity was not significantly different among different freezing temperatures, $-120^{\circ} \mathrm{C},-150^{\circ} \mathrm{C}$, and $-180^{\circ} \mathrm{C}(18.2 \%, 23.5 \%$, and $17.9 \% ; P>0.05)$. The percentage of oocytes with $2 \mathrm{PN}$ was not significantly different between the $-150^{\circ} \mathrm{C}$ frozen and the control (fresh spermatozoa) groups [59\% (131/221) and $62 \%(155 / 251) ; P>0.05]$, although that of frozen spermatozoa at $-120^{\circ} \mathrm{C}$ and $-180^{\circ} \mathrm{C}[20 \%(38 / 188)$ and $23 \%(35 / 153)]$ were significantly lower than that of frozen spermatozoa at $-150^{\circ} \mathrm{C}(P<0.05)$. A total of 168 putative fertilized zygotes with $2 \mathrm{PN}$ were transferred to eleven recipients, and 87 live young were born. In conclusion, our results indicated that post-thaw motility of cryopreserved rat spermatozoa was improved by using a suitable cooling protocol, and the IVF system used in the present study would effectively produce offspring from the cryopreserved epididymal rat spermatozoa. To our knowledge, this procedure is the first successful production of live offspring from cryopreserved rat spermatozoa through in vitro fertilization.

\section{SPERM CAPACITATED WITH CALCIUM IONOPHORE AS A VECTOR FOR IN VITRO PRODUCTION OF BOVINE TRANSGENIC EMBRYOS}

\author{
R. Simões, M. P. Milazzotto, C. Yamada, W. B. Feitosa, A. R. S. Coutinho, J. A. Visintin, \\ and M. E. O. A. Assumpção
}

São Paulo University, São Paulo, São Paulo, Brazil

Production of transgenic mouse embryos by microinjection is a well established and successful technique. However, when microinjection protocols
were used for bovine, the amount of the oocyte lipid content did not allow the production of bovine transgenic embryos. Sperm-mediated gene
transfer (SMGT) is an alternative for this species because it has lower cost and does not require microinjection handling. One of the procedures to
introduce exogen DNA into oocytes is by means of sperm capacitated with calcium ionophore (CaI). The aim of this work was to evaluate different
CaI concentrations ([CaI]), sperm incubation times with CaI (tCa), and incubation times of sperm capacitated with DNA (tDNA) (EYFP; Clontech,
Palo Alta, CA, USA) to establish a satisfactory method for IVP of bovine transgenic embryos. Slaughterhouse oocytes with compact cumulus and
uniform ooplasm were in vitro maturated in TCM- 199 medium $+10 \% \mathrm{FCS}+\mathrm{FSH}+\mathrm{hCG}+$ estradiol $\left(\mathrm{E}_{2}\right)+$ piruvate + gentamicin under $5 \% \mathrm{CO} \mathrm{C}_{2}$
in air, at $39^{\circ} \mathrm{C}$ and high humidified atmosphere for $24 \mathrm{~h}$. Semen was thawed in a water bath at $37^{\circ} \mathrm{C}$ for $30 \mathrm{~s}$ and separated by Percoll gradient
$(45 / 90 \%)$ at $600 \mathrm{~g}$ for 30 min. After this procedure, sperm cells were washed in TALP-semen medium by centrifugation at $200 \mathrm{~g}$ for $5 \mathrm{~min}$ at room
temperature. Supernatant was removed and capacitation $\left(5 \times 10^{6}\right.$ spermatozoa/group) was induced with CaI $(250 \mathrm{nM}$ or $500 \mathrm{nM}$ for 1 or $5 \mathrm{~min})$.
Capacitated sperm cells were incubated with $500 \mathrm{ng} / \mathrm{mL} \mathrm{DNA} \mathrm{for} 1$ or $2 \mathrm{~h}$. Nontreated spermatozoa were used as control group. Sperm cells $\left(1 \times 10^{5}\right)$ 
were used to inseminate 20 oocytes $/ 90 \mathrm{~mL}$ microdroplets for $18 \mathrm{~h}$. The presumptive zygotes were co-cultured in SOFaa medium with a granulosa cell monolayer under high humidified atmosphere, at $39^{\circ} \mathrm{C}$ and $5 \% \mathrm{CO}_{2}$ in air. Blastocyst rates were analyzed by ANOVA. Independent variables were replicate, $[\mathrm{CaI}], \mathrm{tCa}, \mathrm{tDNA}$, and the double and triple interactions among the last three variables; when appropriate, means were compared by orthogonal contrasts. There was $[\mathrm{CaI}] \times \mathrm{tCa} \times \mathrm{tDNA}$ interaction for blastocyst rate $(P<0.02)$. Treatments with $250 \mathrm{nM}([\mathrm{CaI}]), 5 \mathrm{~min}(\mathrm{tCaI})$, and $1 \mathrm{~h}(\mathrm{tDNA})$ or $500 \mathrm{nM}$ ([CaI]), $1 \mathrm{~min}(\mathrm{tCaI})$, and $1 \mathrm{~h}(\mathrm{tDNA})$ resulted in $36.1 \%$ and $37.4 \%$ blastocyst rates, respectively, similar to the control group $(30.5 \% ; P>0.4)$. These results demonstrated that it is possible to capacitate spermatozoa with CaI to produce transgenic embryos, without alteration of blastocyst rate.

This work was supported by FAPESP 03/08542-5 and 03/07456-8.

\title{
299 CONSEQUENCES OF REPEATED ULTRASOUND-GUIDED FOLLICLE ASPIRATION OF THE OVARIES OF GIR (BOS INDICUS) COWS
}

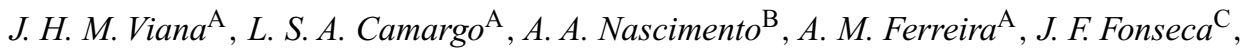 \\ and A. P. Marques Junior ${ }^{\mathrm{D}}$

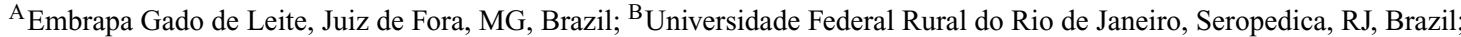 \\ ${ }^{C}$ Embrapa Caprinos, Sobral, CE, Brazil; ${ }^{D}$ Universidade Federal de Minas Gerais, Belo Horizonte, MG, Brazil
}

Ultrasound-guided follicle aspiration has been widely used to recover cumulus-oocyte complexes (COC) for in vitro embryo production in cattle, but the safety of this procedure is still under discussion. The aim of this study was to evaluate the negative effects of repeated follicle aspiration on ovarian structure. Pluriparous, non-lactating Gir breed (Bos indicus) cows $(n=22)$, previously used as COC donors, were evaluated. Follicle aspiration was performed twice a week with a portable ultrasound device, using disposable 19G or 20G needles (Neolus; Terumo Europe, Leuven, Belgium) and a vacuum pressure ranging from 60 to $80 \mathrm{mmHg}$. At the end of the collection period, cows were examined by rectal palpation and ultrasonography to estimate the degree of ovarian adherence and fibrosis. A group of cows $(n=8)$ punctured for different periods was selected for histological evaluation of ovarian lesions. Ovaries were surgically recovered $48 \mathrm{~h}$ after the last aspiration session, fixed by immersion in a solution of $4 \%$ paraformoldehyde and processed for histology. The slides obtained were stained by hematoxylin-eosin and Gomori's trichromic techniques. The association of follicle aspiration and the presence of ovarian abnormalities were estimated by Pearson's correlation method. Results are presented as means \pm SEM. The cows evaluated underwent from 9 to 42 puncture sessions, and yielded from 10 to 719 oocytes. Some degree of adherence and/or fibrosis was detected in $37.5 \%$ of the ovaries. The incidence of lesions was higher in cows undergoing more than 20 OPU sessions $(77.8 \%$ vs. $28.6 \%$; $P<0.05)$. There was a positive correlation between the degree of ovarian adherence and the number of puncture sessions performed $(R=0.31 ;$ $P<0.05)$, but not with the total of oocytes recovered $(R=0.03 ; P>0.05)$. The opposite was observed for ovarian fibrosis, which presented a positive correlation with oocyte yield $(R=0.52 ; P<0.05)$, but not with the number of puncture sessions $(R=0.17 ; P>0.05)$. This difference may be related to the wide range in oocyte yield per donor (means ranging from $1.1 \pm 0.9$ to $38.6 \pm 12.8$ ) and, consequently, in the number of punctures performed in the ovaries. Histological evaluation showed thickening of the tunica albuginea, presence of inflammatory cells infiltration in punctured areas, and increase in the proportion of conjunctive tissue in the ovarian stroma. Punctured follicles showed hemorrhagic clots and variable degrees of disorganization in the follicular wall layers, including dispersion of luteinized cells. These results show that ultrasound-guided follicle aspiration may have negative effects on ovarian structure, depending on the number of sessions performed and oocytes recovered.

This work was sponsored by FAPEMIG and CNPq.

Male Physiology

\section{IMMUNO-EXPRESSION OF POTENTIAL FACTORS REGULATING SERTOLI CELL PROLIFERATION IN THE PREPUBERTAL BOAR}

\author{
J. Baldrighi, W. Averhart, T. Phillips, K. Carnes, R. Hess, and S. Clark \\ University of Illinois, College of Veterinary Medicine, Urbana, IL, USA
}

Today's pork production is very dependent on reproductive efficiency. Any improvements in the production capability (e.g., number of sperm produced) of the animals involved would be invaluable. Many researchers have examined methods to improve oocyte production, but have not focused on the concentration of sperm produced from a single boar used for artificial insemination (AI). The benefit of using AI is that a greater number of females can be bred to a single boar; thus the total amount of sperm per ejaculation is the main factor in the efficiency of AI. An increase in the number of Sertoli cells leads to an increase in testis size and the number of sperm produced because there are a finite number of germ cells that can be supported by the Sertoli cell during spermatogenesis. Therefore, by examining the factors that control the growth and differentiation of Sertoli cells, the amount of sperm per ejaculation in an individual boar can be increased. The purpose of this research is to begin a systematic analysis of cell cycle regulators expressed in the Sertoli cell during testicular development in the pig. By using pigs of different ages, we will establish a baseline of what regulatory factors are present at different time points in the developing Sertoli cell. Identification of certain factors could lead to an increase in sperm production by enabling the growth of Sertoli cells or inhibition of the natural reduction of these cells. Immunohistochemistry (IHC) analysis 
was performed on testis tissues from different males $(n=2)$ at various ages $(3,7,14,25$, and 50 days $)$ prior to puberty. Tissues were fixed in modified Davidson's fixative, embedded in paraffin, and sectioned; slides were processed for IHC. After antigen retrieval, endogenous hydrogen peroxidase was blocked with $0.6 \% \mathrm{H}_{2} \mathrm{O}_{2}$ and sections were incubated overnight at $4{ }^{\circ} \mathrm{C}$ with the appropriate antibody. The following antibodies were used to examine the factors controlling Sertoli cell proliferation: GATA-4 (transcription factor specific for developing and adult pig Sertoli cells), Ki67 (a nuclear protein present in all phases of the cell cycle except G0), cyclin-dependent kinase inhibitor p27 (Kip1), and steroid receptors AR (androgen receptor) and LH2 (estrogen receptor). The slides were then incubated with an appropriate secondary antibody, visualized with DAB chromagen, and counterstained with Mayer's hematoxylin. Immunostaining using p27 (Kip1) revealed no positive staining in any of the days tested, as there is cell division during all of these time points. Protein expression for Ki67 stained mildly after Day 25, suggesting that Sertoli cells became more active at this stage of development. The AR weakly stained and GATA-1 stained intensely at all time points. The data for LH2 were inconclusive and the procedure needs to be performed again. These experiments only give a glimpse of the regulatory factors involved in Sertoli cell proliferation in the developing boar testis. Further studies are being planned to explore other potential factors involved.

\title{
301 NEW APPROACHES TO CONTRACEPTION IN DOGS USING GnRH ANALOGUES, CALCIUM, AND NALOXONE
}

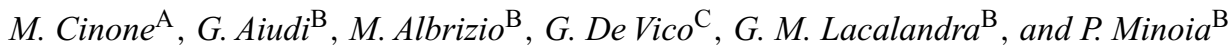 \\ ${ }^{A}$ Department of Animal, Plant and Environmental Sciences, University of Molise, Campobasso, Italy; ${ }^{\mathrm{B}}$ Department of Animal Production, \\ University of Bari, Bari, Italy; ${ }^{\mathrm{C}}$ Department of Veterinary Public Health, University of Messina, Messina, Italy
}

The continuous administration of GnRH leads to the desensitization and down-regulation of GnRH receptors on pituitary gonadotropes (Parker and Schimmer 2001 in Goodman \& Gilman's The Pharmacological Basis of Therapeutics, New York: McGraw-Hill, 1541-1562). In this study, we combined the use of a GnRH analogue with calcium/naloxone association (Sciorsci et al. 2000 Immunopharmacol. Immunotoxicol. 22, 575-626) to obtain a desensitizing effect on LH receptors in an attempt to regulate spermatogenesis in dogs. Eight post-pubertal mixed breed dogs were divided into two groups: (A) four subjects were pharmacologically treated with a 30 day administration of buserelin acetate (Suprefact Aventis Pharma, Italy) $0.3 \mathrm{mg} / \mathrm{each}$ s.c. TID, associated with $0.1-\mathrm{mL} / \mathrm{kg} / \mathrm{bw}$ i.m. injection SID of calcium gluconate $20 \%$ (New ICC, Upjohn, Italy) in which $0.4 \mathrm{mg} / \mathrm{mL}$ of naloxone hydrochloride (Diosynth, The Netherlands) was dissolved; (B) four subjects received placebo (NaCl $0.9 \%$ s.c. TID for 30 days). At the end of the treatment, hemiorchidectomy was performed and 4 months later the second testis was removed. Clinical examination of the genital tract was done by ultrasound monitoring. Before and after pharmacological treatment and until total orchidectomy, semen was collected and evaluated for macroscopic and microscopic parameters. Plasma testosterone concentration was measured twice a week by RIA. Testicular specimens were fixed in formalin solution, embedded in paraffin wax and stained with hematoxylin-eosin. The presence of germ cells and the testicular tubule diameters were analyzed using a computer assisted image analyzer (MONO system, Italy). Data were analyzed by the ANOVA test. In the treated group, all dogs showed a reduction in testicular and prostatic diameters compared to the control group. Transient elevations in testosterone concentrations were observed in response to the early injections and reached baseline levels after 8-10 days. The testosterone decline resulted in lower ejaculate volume and, at the end of treatment, azoospermia was observed. At the end of treatment, testosterone values had progressively regained normal values over 2 months and, when measured immediately before total orchidectomy, sperm concentration and motility had returned to normal. At the histological observation, seminiferous tubules showed the block of spermatogenesis at the level of spermatocytes with a statistically significant cell reduction in spermatides and spermatozoa $(P<0.001)$ whereas the seminiferous tubular morphometry was normal. Total orchidectomy demonstrated that a normal histological testicular structure had been restored. In the control group, no modifications were observed. As observed in a previous study (Aiudi et al. 2005 Reprod. Fert. Dev. 17(2), 280-281), GnRH induced suppression of the reproductive function in male dogs. In this study, we demonstrated that GnRH analogue associated with calcium and naloxone induces the same effect in a shorter time and that this effect is reversible. These devices designed for the shutdown of gonadal steroidogenesis and spermatogenesis would be useful for treating sex hormone-dependent syndromes such as malignant and benign prostatic tumors.

\section{VIABILITY OF BOVINE SPERMATOZOA INCUBATED WITH FOREIGN DNA}

\author{
W. B. Feitosa, M. P. Milazzoto, M. Rovegno, L. F. Martins, J. A. Visintin, and M. E. O. A. Assumpção \\ Department of Animal Reproduction-FMVZ, University of São Paulo, São Paulo-SP, Brazil
}

Several studies have been performed over the years to promote the understanding and improvement of the sperm-mediated gene transfer technique. However, little is known about the effect of exogenous DNA in the sperm cells. The aim of this study was to evaluate the effect of the incubation period and exogenous DNA addition on mitochondrial activity and acrosomal status of bovine spermatozoa. Frozen-thawed semen was separated by Percoll gradient $(45 / 90 \%)$ at $600 \mathrm{~g}$ for $30 \mathrm{~min}$, and the pellet was resuspended and washed by centrifugation in sperm-TALP at $200 \mathrm{~g}$ for $5 \mathrm{~min}$. The spermatozoa were resuspended in fertilization medium (without heparin) at a concentration of $5 \times 10^{6}$ spermatozoa $/ \mathrm{mL}$ and incubated at $39^{\circ} \mathrm{C}$, $5 \% \mathrm{CO}_{2}$ in air with $500 \mathrm{ng} \mathrm{pEYFP-Nuc/mL}$ (Clontech, Mountain View, CA, USA) (DNA group) for 1 and $2 \mathrm{~h}$ or without DNA (control group) for 0,1 and $2 \mathrm{~h}$. JC-1 (Molecular Probes; Invitrogen Brasil, Ltd., Sao Paulo, Brazil) was used to determine mitochondrial potential and fluorsecein isothiogyanate (FITC-PSA; Sigma-Aldrich, Brazil) was used to assess acrosomal status. Two microliters of JC-1 (76.5 $\mu \mathrm{M}$ in DMSO) and $50 \mu \mathrm{L}$ of FITC-PSA $\left(100 \mu \mathrm{g} / \mathrm{mL}\right.$ in DPBS) were added to $150 \mu \mathrm{L}$ of IVF medium $+5 \times 10^{6}$ spermatozoa $/ \mathrm{mL}$; the mixture was incubated at $25^{\circ} \mathrm{C}$ for 10 min. DNA incorporation was evaluated by p-EYFP-Nuc PCR amplification. All treatments were repeated ten times and data were analyzed by ANOVA and Tukey test $(P<0.05)$. The results are described in Table 1. The sperm were classified as: Class 1 (intact acrosome and high mitochondria potential), Class 2 (intact acrosome and low mitochondria potential), Class 3 (reacted acrosome and high mitochondria potential) and Class 4 (reacted 
acrosome and low mitochondria potential). In both groups, the Class 1 sperm decreased over time, whereas an increase in number was verified for Class 2 sperm. There was no significant difference in numbers among the incubation periods in both groups for Class 3 sperm. However, there was an increase in Class 4 sperm number over time, indicating that the main effect of the incubation period was the loss of mitochondria potential. There was no effect of the exogenous DNA addition on sperm viability in relation to the control group, indicating that the exogenous DNA had no effect on mitochondrial activity and acrosomal status of bovine semen.

Table 1. Effect of incubation period and exogenous DNA addition on sperm viability

\begin{tabular}{lccccc}
\hline Sperm & $0(\% \pm$ SEM $)$ & \multicolumn{2}{c}{$1 \mathrm{~h}(\% \pm$ SEM $)$} & \multicolumn{2}{c}{$2 \mathrm{~h}(\% \pm$ SEM $)$} \\
& Control & Control & DNA & Control & DNA \\
\hline Class 1 & $72.7 \pm 9.1^{\mathrm{a}}$ & $55.9 \pm 11.9^{\mathrm{b}}$ & $57.4 \pm 16.6^{\mathrm{b}}$ & $37.5 \pm 13^{\mathrm{c}}$ & $31.4 \pm 15.8^{\mathrm{c}}$ \\
Class 2 & $12.9 \pm 4.4^{\mathrm{a}}$ & $19.3 \pm 4.9^{\mathrm{a}}$ & $20.8 \pm 7.2^{\mathrm{a}}$ & $38.7 \pm 17.9^{\mathrm{b}}$ & $36.1 \pm 15.6^{\mathrm{b}}$ \\
Class 3 & $9.2 \pm 9.1^{\mathrm{a}}$ & $12.4 \pm 7.2^{\mathrm{a}}$ & $11.9 \pm 7.2^{\mathrm{a}}$ & $10.3 \pm 7.6^{\mathrm{a}}$ & $15.5 \pm 9.5^{\mathrm{a}}$ \\
Class 4 & $5.3 \pm 3.4^{\mathrm{a}}$ & $12.3 \pm 6.7^{\mathrm{b}}$ & $9.9 \pm 4^{\mathrm{a}}$ & $13.4 \pm 6.3^{\mathrm{b}}$ & $16.9 \pm 5.9^{\mathrm{b}}$ \\
\hline
\end{tabular}

a,b,c Differences $(P<0.05)$ within each treatment (control or DNA) at 0,1 , and $2 \mathrm{~h}$.

This work was supported by FAPESP 03/10234-7; 03/07456-8.

\title{
303 INDUCED MOTILITY OF MESA/TESE SPERMATOZOA IS ASSOCIATED WITH INCREASED FERTILIZATION AND IMPLANTATION RATES IN HUMAN
}

\author{
H. L. Feng and L. J. Zheng
}

Assisted Reproduction Technology and Solution, New Hyde Park, NY 11040, USA

\begin{abstract}
The key for human IVF using the intracytoplasmic sperm injection (ICSI) procedure is to find moving and living sperm in azoospermic men. The objective of this study was to determine the effect of induced sperm motility on the fertilization and implantation rates in humans. Testicular sperm extraction (TESE) medium containing sperm stimulators and nutritional elements is specially designed for male factor and/or poor quality sperm with lower motility. Men with obstructed or absent ejaculatory ducts were treated by using a microepididymal sperm aspiration (MESA) procedure. Sperm are aspirated directly from the epididymis for use in the ICSI procedure. Men with non-obstructive azoospermia were treated by using the TESE procedure. Small samples of testicular tissue are obtained by needle or microsurgical biopsy and a few sperm are painstakingly dissected out of the tissue for use in the ICSI procedure. Sperm specimens either from epididymis or testis were divided into the two respective groups. In Group I, the specimens were washed in a TESE medium plus $10 \%$ human serum albumin (HSA), and then incubated with TESE medium containing $10 \%$ HSA in a $5 \% \mathrm{CO}_{2}$ incubator to induce sperm motility. In Group II, the specimens were washed in human tubal fluid (HTF) medium plus $10 \% \mathrm{HSA}$, and then incubated with HTF containing $10 \% \mathrm{HSA}$ in a $5 \% \mathrm{CO}_{2}$ incubator. After $1 \mathrm{~h}$ of incubation, the motility of spermatozoa was evaluated under a microscope. The ICSI procedure was performed under an inverted microscope. Fertilized eggs were cultured in HTF medium containing $10 \%$ serum substitute supplement (SSS) until Day 3 for embryo transfer. Patients' pregnancy was followed after embryo transfer. This study demonstrated that incubation of spermatozoa from MESA and TESE procedures in TESE medium, as compared with HTF medium, results in significantly increased sperm progression (MESA sperm: $2-3$ vs. $0-1$; TESE sperm: $1-1.5$ vs. $0-0.1$ ) and motility (MESA sperm: $12.0 \pm 1.8 \%$ vs. $3.3 \pm 0.5 \%$; TESE sperm $4.5 \pm 0.2 \%$ vs. $0.15 \pm 0.02 \%$ ) all respectively. Induced motility of spermatozoa in TESE medium, as compared with HTF medium, is associated with a significantly increased fertilization rate (MESA sperm: $74.5 \pm 3.5 \%$ vs. $60.2 \pm 3.1$; TESE sperm: $73 \pm 4.3 \%$ vs. $48.4 \pm 3.7 \%$ ) and implantation rate (MESA sperm: $29.8 \pm 3.8 \%$ vs. $19.4 \pm 2.7 \%$, for patient age $32 \pm 3.0$, in Group I, $n=13$, and in Group II, $n=12$; TESE sperm: $23.2 \pm 3.3 \%$ vs. $15.4 \pm 2.8 \%$, patient age $33 \pm 2.8 \%$, in Group I, $n=11$, and in Group II, $n=9$ ), and significantly decreased the ICSI performing time ( $30 \pm 10$ vs. $120 \pm 20 \mathrm{~min}$ ), all respectively. TESE medium can significantly increase the motility of spermatozoa from TESE/MESA, because it contains specific sperm stimulators and nutritional elements that directly resulted in the increase of fertilization and implantation rates.
\end{abstract}

\section{TREATMENT OF BOAR SPERM WITH RESPIRATION INHIBITOR MENADIONE: EFFECTS ON MOTILITY, REACTIVE OXYGEN SPECIES (ROS), MITOCHONDRIAL TRANSMEMBRANE POTENTIAL (MMP), AND ATP CONTENT}

\author{
D. Guthrie, J. Long, G. Welch, and N. Grimm
}

Biotechnology \& Germplasm Laboratory, ARS, USDA, Beltsville, MD 20705, USA

Previously we found that live, fresh or thawed boar sperm show little tendency to accumulate ROS spontaneously, but live sperm accumulated ROS during a 30-min incubation with xanthine and xanthine oxidase and showed marked reduction in motility. High mitochondrial transmembrane potential (MMP) is required to drive the F0/F1 ATPase responsible for producing ATP in most cell types, and ATP is required for sperm motility. This experiment was conducted to investigate the effects of menadione (disrupter of electron transport at Complex I) on sperm motility, MMP, and ATP content. The incidence of cells with high MMP was determined by measuring the fluorescence of JC-1 aggregates bound to the inner mitochondrial 
membrane using flow cytometry. Computer-assisted motion analysis was conducted using the IVOS version 12 (Hamilton Thorne Research, Beverly, MA, USA), and ATP (pmoles $/ 10^{6}$ sperm) was determined using the luciferin-luciferase assay. Sperm from six boars were individually Percoll washed to eliminate seminal plasma and incubated at $40 \times 10^{6} / \mathrm{mL}$ with $0,1,10$, or $30 \mu \mathrm{M}$ menadione for $5,30,60$, and 120 min at $38^{\circ} \mathrm{C}$ in a modified Tyrode's medium containing $0.1 \%$ polyvinyl alcohol with no bicarbonate or BSA. The formation of ROS was confirmed by measuring the red fluorescence developed by the oxidation of hydroethidine to ethidium using flow cytometry. Whereas the basal level of ethidium fluorescence in the absence of menadione was low ( $2 \%$ ethidium-positive cells at $5 \mathrm{~min}$ ), 10 and $30 \mu \mathrm{M}$ menadione increased $(P<0.05)$ the percentage of ethidium-positive cells to 47 and $87 \%$, respectively, at $30 \mathrm{~min}$. Sperm motility did not decrease significantly (79-83\%) during the 120 min incubation with no menadione, but menadione caused a significant dose-related decrease $(P<0.05)$ over time, with 10 and $30 \mu \mathrm{M}$ menadione decreasing motility to 60 and $40 \%, 51$ and $7 \%$, and 10 and $1 \%$ at 30,60, and $120 \mathrm{~min}$, respectively. JC- 1 aggregate fluorescence intensity decreased $(P<0.05)$ by $45-60 \%$ in a dose-related fashion at $120 \mathrm{~min}$ compared to the same doses at $5 \mathrm{~min}$. Sperm viability, as measured by number of propidium iodide negative cells, averaged $93.6 \%$ during the incubation and was not significantly affected by treatment. The effect of menadione on ATP content was much less than that on motility or JC-1 fluorescence intensity. Mean ATP content averaged 63 pmoles through $60 \mathrm{~min}$ at all menadione doses; at 120 min only $30 \mu \mathrm{M}$ menadione decreased $(P<0.05)$ ATP to 43 pmoles, compared to all other treatments. Menadione caused an increase in ROS formation and a decline in MMP, which suggested uncoupling of the respiratory chain and oxidative phosphorylation. However, sperm ATP content was not highly correlated with motility. This suggests that ATP content was maintained by the activity of other intermediary metabolism pathways. The reduction in motility may have been due to a ROS induced lesion in ATP utilization or in the contractile apparatus of the cell.

\title{
305 SPERM CHROMATIN STRUCTURE AND DNA METHYLATION BULLS
}

\author{
K. Hengstberger, N. Phutikanit, O. Berking, M. D’Occhio, D. Sester, and B. Carroll \\ University of Queensland, Brisbane, Queensland, Australia
}

\begin{abstract}
Mammalian spermatogenesis is a complex process that involves genome-wide deprogramming and reprogramming of DNA methylation at different stages in order to produce sperm that are capable of fertilization and also TO support ongoing embryo development (Webster et al. 2005 PNAS 102, 4068-4073). Embryonic mortality occurs when there is inappropriate expression of developmentally regulated genes of both maternal and paternal origin. DNA methylation is associated with epigenetic regulation of gene expression and it was recently shown that aberrant DNA methylation causes a change in sperm chromatin structure (Webster et al. 2005). Studies, primarily in man, have indicated that sperm chromatin instability, as determined by the sperm chromatin structure assay (SCSA), does not influence fertilization but is linked with early pregnancy failure (Evenson and Jost 2000 Methods Cell Sci. 22, 169-189). The aim of the present study was to investigate the relationship between chromatin structure and DNA methylation in bull sperm. Chromatin structure was determined using the SCSA that involves the exposure of sperm to a low $\mathrm{pH}(1.2)$ detergent solution followed by the addition of acridine orange (AO). When exposed to a $488 \mathrm{~nm}$ laser, AO fluoresces green when bound to native (double-stranded) DNA and red when bound to denatured (single-stranded) DNA. The SCSA yields a value for the DNA fragmentation index (DFI), and in men a DFI $>27-30 \%$ is associated with early embryonic mortality (Larson-Cook et al. 2003 Fertil. Steril. 80, 895-902). Semen was obtained by electroejaculation from Zebu (Bos indicus) bulls $(n=12)$ in a subtropical environment; a proportion of bulls had a DFI $<15 \%(n=4)$ and the remainder a DFI $>27 \%(n=8)$. The DNA methylation pattern, as determined by the amplified methylation polymorphism (AMP) protocol (Webster et al. 2005), appeared to differ between sperm with a DFI $<15 \%$ and sperm with a DFI $>27 \%$ when assessed by principle coordinate analysis. Also, sperm with a DFI $<15 \%$ had a more consistent methylation pattern compared with apparent differences in methylation among sperm with a DFI $>27 \%$. These preliminary findings could be interpreted to suggest that methylation status can contribute to chromatin structure in sperm of mature bulls. It remains to be determined whether the environment can influence sperm chromatin status in bulls by altering DNA methylation which then impacts on the conformational changes in DNA that occur during spermiogenesis.
\end{abstract}

This work was supported, in part, by Meat and Livestock Australia.

\section{ANALYSIS OF GLOBAL DNA METHYLATION IN BOVINE SPERMATOZOA}

\author{
H. Kato ${ }^{\mathrm{A}}$, M. Kishimoto $^{\mathrm{B}}$, T. Mitani $^{\mathrm{A}}$, K. Matsumoto $^{\mathrm{A}, \mathrm{B}}, K_{\text {. Saeki }}^{\mathrm{A}, \mathrm{B}}$, Y. Hosoi $^{\mathrm{A}, \mathrm{B}}$, and A. Iritani $^{\mathrm{A}, \mathrm{B}}$ \\ ${ }^{A}$ Institute of Advanced Technology, Kinki University, Kainan, Wakayama, 642-0017, Japan; \\ ${ }^{B}$ Department of Biology-Oriented Science and Technology, Kinki University, Uchita, Wakayama, 649-6493, Japan
}

Methylation of cytosine base in the $\mathrm{CpG}$ dinucleotide sequence (DNA methylation) is a major epigenetic modification of the genome and plays an important role in gene expression. Recently, global DNA methylation in genome was studied by using a restriction landmark genomic scanning (RLGS) method and/or a representational difference analysis (RDA) method. However, these methods are complicated and need to use restriction enzymes. Therefore, the information derived from those methods is restricted to the region of the DNA sequence which is able to be cleaved by restriction enzymes. In this study, to establish a simple method to estimate global DNA methylation level in bovine spermatozoa, we tried to develop the DNA methylation analyzing method by using immunostaining of 5-methylcytosine. The immunostaining method for 5-methylcytosine in this study was based on the method developed by Benchaib et al. (2003 Fertil. Steril. 80, 947-952) for human spermatozoa. Because of the species difference, we modified some treatments to apply to bovine spermatozoa. Frozen-thawed bovine spermatozoa were washed by using 30 and $45 \%$ Percoll gradient solutions. After washing, spermatozoa were treated with 0.25 dithicthreitol M (DTT) and $1 \%$ sodium dodecyl sulfate (SDS) at room temperature (RT). Then, treated spermatozoa were spread on a slide glass with Cytospin $4\left(30 \mathrm{~g}, 5 \times 10^{4} \mathrm{cells} / \mathrm{mL}\right)$ and air-dried at RT. Air-dried bovine spermatozoa specimens were fixed in methanol: glacial acetic acid (3:1) solution at RT and treated with $1 \%$ Triton X and $1 \%$ SDS at RT; 
DNA was denatured with $6 \mathrm{~N} \mathrm{HCl}$ at RT. After the denaturation, 5-methylcytosine in sperm DNA was analyzed by immunofluorescence technique with mouse anti 5-methylcytosine antibody and fluorescein isothiocyanate (FITC)-conjugated goat anti mouse IgG antibody. The total sperm DNA was counterstained with propidium iodide (PI). Stained samples were observed with a confocal laser scanning microscope and obtained images were analyzed with fluorescence image analysis software. The area that was clearly stained with PI in each sperm head was designated and measured as the area of total sperm DNA, and the number of the dots that showed FITC fluorescence within the total sperm DNA area was designated and measured as the area of 5-methylcytosine in total sperm DNA. The area measurement was performed with fixed light strength. Three bovine spermatozoa samples derived from different bulls, used daily for calf production by AI, were examined. The ratio of the mean total area of the 5-methylcytosine in sperm DNA to the mean total area of the sperm DNA was $34.1 \%$ in bull A $\left(9.13 \pm 5.66 \mu \mathrm{m}^{2}, 26.75 \pm 5.29 \mu \mathrm{m}^{2}, n=57\right), 45.2 \%$ in bull $\mathrm{B}\left(16.60 \pm 3.79 \mu \mathrm{m}^{2}, 36.74 \pm 5.95 \mu \mathrm{m}^{2}, n=41\right)$ and $43.9 \%$ in bull $\mathrm{C}\left(14.66 \pm 4.27 \mu \mathrm{m}^{2}, 33.45 \pm 7.13 \mu \mathrm{m}^{2}, n=22\right)$. There was significant difference in the ratio between bull $\mathrm{A}$ and bulls $\mathrm{B}$ and $\mathrm{C}(P<0.01)$. More research is required to evaluate the meaning of this individual difference of DNA methylation between bulls.

This work was supported by Wakayama Prefecture Collaboration of Regional Entities for the Advanced of Technological Excellence, Japan, and by a Grant-in-Aid for the 21 st Century Center of Excellence Program of the MEXT, Japan.

\title{
307 EFFECT OF BULL EPIDIDYMIS STORAGE CONDITIONS ON SPERM RESISTANCE AGAINST LIPID PEROXIDATION AND SUBSEQUENT IN VITRO EMBRYO PRODUCTION
}

\author{
M. Nichi ${ }^{\mathrm{A}, \mathrm{B}}$, J. B. P. De Clercq ${ }^{\mathrm{A}}$, I. G. F. Goovaerts ${ }^{\mathrm{A}}$, V. H. Barnabe , and P. E. J. Bols $^{\mathrm{A}}$ \\ ${ }^{A}$ Laboratory for Veterinary Physiology, Department of Veterinary Sciences, University of Antwerp, Wilrijk, Antwerp, Belgium; \\ ${ }^{B}$ Animal Reproduction Department, Faculty of Veterinary Medicine, University of São Paulo, São Paulo, Brazil
}

Sperm recovery from the cauda epididymis can be a usefull tool in case of unexpected death of genetic high-value animals or endangered species or when the collection of sperm by other means becomes impossible. Studies indicate that the lower the temperature of epididymis storage, the better the sperm quality after collection (Kaabi et al. 2003 Theriogeneology 60, 1249-1259). One of the main factors that can negatively affect sperm viability during storage is lipid peroxidation, where sperm membrane resistance against reactive oxygen species (ROS) attacks is an important factor. The objective of this experiment was to study whether the temperature of epididymis storage following slaughter would have an influence on the membrane's resistance against lipid peroxidation and on the sperm cell's fertilizing capacity. Sixteen epididymides (from eight bulls) were collected after slaughter and divided into two groups, one stored at $4^{\circ} \mathrm{C}$ and the other at $37^{\circ} \mathrm{C}$ for $2 \mathrm{~h}$, after which semen was collected from the caudae epididymides. Sperm concentration was measured and an aliquot containing $10^{8}$ sperm cells was submitted to induced lipid peroxidation with ferrous sulfate and ascorbate $\left(37^{\circ} \mathrm{C} ; 2 \mathrm{~h}\right)$. Subsequently, thiobarbituric acid reactive substances (TBARS), as an index of lipid peroxidation, were measured according to a method previously described (Beorlegui et al. 1997 Andrologia 29, 37-42). A second aliquot of the sample was used for fertilization in a routine IVF-IVC set up in duplicate (24-h maturation, SOF culture medium in 5\% $\mathrm{CO}_{2}, 5 \% \mathrm{O}_{2}$, and $90 \% \mathrm{~N}_{2}$ ). In vitro embryo production and level of TBARS were statistically analyzed using SAS (SAS Institute, Inc., Cary, NC, USA). TBARS levels were transformed to logarithm form in order to obey the residue normality being analyzed using PROC GLM. The percentage of blastocysts was analyzed using the Wilcoxon test. When compared to the samples stored at $4^{\circ} \mathrm{C}$, semen of caudae epididymides stored at $37^{\circ} \mathrm{C}$ showed higher levels of TBARS and lower mean blastocyst rates $\left(324.7 \pm 59.6\right.$ and $36.6 \pm 1.6$ vs. $466.9 \pm 67.9 \mathrm{ng}$ of TBARS $/ 10^{8}$ spermatozoa and $28.8 \pm 2.9 \%$, respectively; $\left.P<0.05\right)$. A negative correlation was found between TBARS and blastocyst rates $(R=-0.43)$. The lower quality of sperm collected from epididymides maintained at higher temperatures may be related to a decrease in sperm resistance against lipid peroxidation which would further impair sperm fertilizing capacity. However, further studies are necessary in order to study the effect of temperature on the sperm membrane lipid profile, because the content of polyunsaturated fatty acids may be affected by temperature; this is an important factor relative to sperm membrane lipid peroxidation susceptibility (Ollero et al. 2000 Mol. Reprod. Dev. 55, 326-334). Another important factor is the epididymal environment because interactions between the sperm membrane and its surroundings can play an important role on the membrane's antioxidant protection.

\section{TESTICULAR DEVELOPMENT IN PREPUBERTAL JERSEY BULL CALVES IMMUNIZED AGAINST INHIBIN}

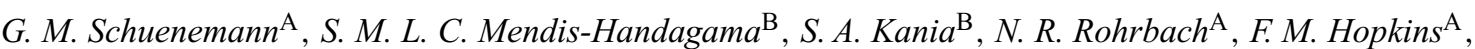 \\ H. Moorehead ${ }^{\mathrm{C}}$, P. Lunn ${ }^{\mathrm{C}}$, H. H. Dowlen ${ }^{\mathrm{C}}$, and F. N. Schrick ${ }^{\mathrm{A}}$
}

A Department of Animal Science, University of Tennessee, Knoxville, TN 37996, USA; ${ }^{\mathrm{B}}$ Department of Comparative Medicine, College of
Veterinary Medicine, Knoxville, TN 37996, USA; ${ }^{C}$ Dairy Research and Education Center, University of Tennessee, Lewisburg, TN 37091 , USA

Previous studies have shown that immunization against inhibin (INH) in bull calves increased subsequent sperm production (Martin et al. 1991 Biol. Reprod. 45, 73; Bame et al. 1996 Biol. Reprod. 54, 328). The objective of the current study was to evaluate the effectiveness of gonadotropin administration at initiation of inhibin immunization in bull calves on testicular morphology. The study was performed using the inhibin peptide (bovine inhibin $\alpha^{1-26}$ ) conjugated to keyhole limpet hemocyanin (KLH). Primary treatments administered to Jersey bull calves (initial immunization at $27 \pm 5$ days of age; Day 1 of the experimental period) consisted of control (KLH, $250 \mu \mathrm{g}, n=9$ ) or immunization (INH; $500 \mu \mathrm{g} \mathrm{INH:250 \mu \textrm {g }}$ $\mathrm{KLH}, n=9$ ) with each emulsified in $2 \mathrm{~mL}$ of Freund's complete adjuvant (FCA). Booster immunizations (identical preparation in FCA) occurred every 21 days with the last administration on Day 84 of the trial. Subsets of calves were randomly assigned within primary treatments (TRT) to receive saline ( $1 \mathrm{~mL}, n=3 / \mathrm{TRT})$, FSH (20 mg, $n=3 / \mathrm{TRT})$, or GnRH $(50 \mu \mathrm{g}, n=3 / \mathrm{TRT})$ every $8 \mathrm{~h}(0600,1400$, and $2200 \mathrm{~h})$ from Day 1 to Day 3 
of the study. Blood samples were obtained daily from Days 0-14 and weekly until testes collection (Day 91) for FSH, LH, testosterone (T), and determination of antibody titers. Body weight and scrotal circumference (SC) were measured at each immunization and immediately before testes removal. The right testis was weighed and used for absolute volume calculation of cell components per testis. Tissue sections were examined using a light microscope $(400 \times)$. For each cell type, absolute volume of Sertoli, Leydig, and germ cells were counted according to the point counting method. Data were analyzed using MIXED procedure of SAS (SaS Institute, Inc., Cary, NC, USA). Antibody titers were increased in INH bulls compared to KLH bulls $(P<0.05)$ during the experimental period. Body weight $(89.8 \pm 14.2 \mathrm{~kg}), \mathrm{SC}(14.6 \pm 1.3 \mathrm{~cm})$, and single testicular weight $(19.2 \pm 6.2 \mathrm{~g})$ recorded at the end of the experimental period did not differ between treatments. Neither serum concentrations of FSH, LH, and T nor population of Leydig and Sertoli cells differed between treatment groups. However, a significant immunization X hormone treatment interaction was noted for germ cell volume per testis $(P<0.008)$. Administration of FSH at the time of initial immunization against inhibin significantly increased germ cell population $\left(1.22 \pm 0.1 \mathrm{~cm}^{3}\right)$ compared to INH-saline bulls $\left(0.64 \pm 0.1 \mathrm{~cm}^{3}\right)$ with INH-GnRH bulls intermediate $\left(0.84 \pm 0.1 \mathrm{~cm}^{3} ; P<0.05\right)$. In contrast, germ cell volume was not increased following hormone administration in KLH bulls. These results suggest that gonadotropin administration at the time of inhibin immunization increases germ cell volume in the testis without altering Sertoli and Leydig cell volume.

\title{
309 THE SPERMATOGENIC CYCLE IN MAMMALIAN TESTIS XENOGRAFTS
}

\author{
W. Zeng ${ }^{\mathrm{A}}$, G. F. Avelar ${ }^{\mathrm{B}}$, R. Rathi ${ }^{\mathrm{A}}$, L. R. Franca ${ }^{\mathrm{B}}$, and I. Dobrinski ${ }^{\mathrm{A}}$ \\ ${ }^{\mathrm{A}}$ University of Pennsylvania, Kennett Square, PA 19348, USA; ${ }^{\mathrm{B}}$ Federal University of Minas Gerais, Belo Horizonte, MG, Brazil
}

Grafting of immature testis tissue from different mammalian donor species into mouse hosts results in production of spermatozoa from the donor species. Xenografting of testis tissue from rhesus monkeys, pigs, and sheep accelerates sperm production. To determine whether this shortened time to sperm production is due to the reduced spermatogenic cycle length, we applied bromodeoxyuridine (BrdU) incorporation to analyze the spermatogenic cycle in porcine and ovine testis xenografts. Testes from 1-2-week-old Yorkshire cross pigs and 1-week-old Suffolk sheep were cut into small fragments (approximately $1 \times 1 \times 2 \mathrm{~mm}$ ) and eight fragments were grafted under the back skin of each castrated male immunodeficient NCR nude recipient mouse ( $n=7$ for pig, $n=5$ for sheep). Mice were given BrdU ( $100 \mathrm{mg} / \mathrm{kg}$ i.p.) at 7 months (porcine tissue) or 6 months (ovine tissue) post-transplantation. Mice carrying porcine tissue were sacrificed $1 \mathrm{~h}, 9$ days or 18 days after BrdU injection. Mice with ovine testicular tissue were sacrificed $1 \mathrm{~h}, 11$ days or 22 days after BrdU injection. Analysis time points were chosen based on the reported length of the spermatogenic cycle in pigs and sheep (approximately 9 days and 11 days, respectively). All eight stages of the spermatogenic cycle were analyzed to identify the most advanced germ cells labeled in each time period after BrdU injection. All seminiferous tubules containing full spermatogenesis were analyzed. Histologically, $51.8 \%$ (range 7 to $98 \%, n=2040$ tubules) of seminiferous tubules from porcine grafts, and $64.4 \%$ (range 2 to $92 \%, n=2903$ tubules) of seminiferous tubules from ovine grafts presented complete spermatogenesis. In porcine grafts, the most advanced germ cells labeled $1 \mathrm{~h}$ after BrdU injection were primary spermatocytes in pre-leptotene/leptotene at stage I of the spermatogenic cycle. At 9 days and 18 days after injection, the most advanced labeled germ cells were primary spermatocytes at pachytene at stage I and elongating spermatids at late stage II, respectively. In ovine grafts, the most advanced labeled germ cells at $1 \mathrm{~h}, 11$ days and 22 days were pre-leptotene/leptotene at stage II, primary spermatocytes at the pachytene at stage I and elongating spermatids at stage II, respectively. These results indicate that each spermatogenic cycle in porcine and ovine testis xenografts lasts around 9 days and 11 days, respectively. Therefore, the length of the spermatogenic cycle is conserved in porcine and ovine testis xenografts and shortened time to sperm production is likely due to accelerated maturation of the testicular somatic components, such as Sertoli cells.

This work was supported by NIH R01 RR17359-01.

\author{
Microstructural Analysis
}

\section{NUCLEAR AND MICROTUBULE REMODELING AND IN VITRO DEVELOPMENT OF NUCLEAR TRANSFERRED CAT OOCYTES WITH SKIN FIBROBLASTS OF DOMESTIC CAT AND LEOPARD CAT}

\author{
X. J. Yin ${ }^{\mathrm{A}}$, E. G. Choi ${ }^{\mathrm{A}}$, S. J. Cho ${ }^{\mathrm{A}}$, J.Y.Jin ${ }^{\mathrm{B}}$, N. H. Kim ${ }^{\mathrm{B}}$, H. S. Lee ${ }^{\mathrm{B}}$, and I. K. Kong ${ }^{\mathrm{A}}$ \\ ${ }^{\text {A }}$ Sunchon National University, Sunchon, JeonNam Province, South Korea; \\ ${ }^{\mathrm{B}}$ Chungbuk National University, Cheongju, Chungbuk Province, South Korea
}

The leopard cat (Prionailurus bengalensis), a member of the felidae family, is a threatened animal in South Korea. In terms of endangered felids, nuclear transfer is a potentially valuable technique for assuring the continuation of species with dwindling numbers. The protocol for nuclear transfer has been described previously (Yin et al. 2005 Reproduction 129, 245-249). In this experiment we evaluated nuclear and microtubule remodeling and the in vitro developmental potential of enucleated cat oocytes reconstructed with nuclei from either domestic cat fibroblasts (DCF) or leopard cat fibroblasts (LCF). Microtubule aster was allocated to decondensed chromatin at $6 \mathrm{~h}$ post-activation following nuclear transfer of fibroblast cells from both DCF and LCF (3/3 in DCF, 2/3 in LCF), suggesting the introduction of a somatic cell centrosome (Kim et al. $1996 \mathrm{Mol}$. Reprod. Dev. 43, 248-255; Park et al. 2004 Mol. Reprod. Dev. 68, 25-34). At $12 \mathrm{~h}$ following nuclear transfer, the nucleus swelled into a large pronucleus-like structure in most reconstructed oocytes (5/9 in DCF and 4/6 in LCF), which showed further enlargement until $18 \mathrm{~h}$ after nuclear transfer (4/6 in DCF, $4 / 6$ in LCF). Two microtubule asters were seen near the swollen nucleus. At $18 \mathrm{~h}$ following nuclear transfer, the mitotic metaphase ( $1 / 6$ in DCF) or two cell divisions (1/6 in DCF, 2/6 in LCF) were observed. The percentage of blastocyst formation from nuclear transfer embryos derived from DCF $(4 / 46,8.6 \%)$ was not significantly different from that for nuclear transfer embryos constructed with LCF (4/52, 7.6\%). Statistical significance 
was established at the $P<0.05$ level by a X2-test (SAS; SAS Institute, Inc., Cary, NC, USA). These results indicate that nuclear and microtubule remodeling processes and in vitro developmental ability are similar in cat oocytes reconstructed with both domestic cat and leopard cat nuclei.

This work was supported by KOSEF (grant \# M10525010001-05N2501-00110).

Oocyte Activation

\title{
311 ULTRASTRUCTURAL ANALYSIS OF OVINE PREANTRAL FOLLICLES CULTURED IN THE PRESENCE OF INDOLE ACETIC ACID, EGF, AND FSH
}

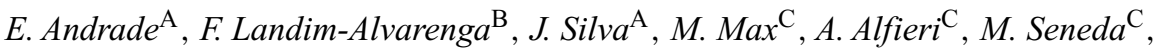 \\ J. Figueiredo ${ }^{\mathrm{A}}$, and R. Toniolli ${ }^{\mathrm{A}}$

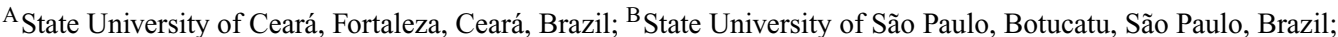 \\ ${ }^{\mathrm{C}}$ State University of Londrina, Londrina, Paraná, Brazil
}

Previous studies from our team demonstrated that ovine primordial follicles are successfully activated in vitro after culturing in medium supplemented with $40 \mathrm{ng} / \mathrm{mL}$ indole acetic acid (IAA); besides that, the addition of IAA and epidermal growth factor (EGF) or EGF and follicle stimulating hormone (FSH) to the culture media were the most effective treatments to sustain the health and viability of activated ovine primordial follicles during in vitro culture. In this work, follicular quality was assessed only by histological studies; there is a great need to evaluate ultrastructural changes occurring in primordial follicles during activation in vitro. The aim of this study was to investigate the ultrastructural characteristics of preantral follicles after culturing of cortical slices in media containing IAA, EGF, and FSH, alone and in combination. Ovaries $(n=8)$ from adult merino ewes were collected at local slaughterhouses. Small pieces of ovarian fragments were removed for transmission electron microscopy (TEM). These pieces of ovarian cortex were cultured in culture dishes containing $1 \mathrm{~mL}$ aliquots of culture medium at $39^{\circ} \mathrm{C}$ with $5 \% \mathrm{CO}_{2}$ in air. The media used were: (T1) Minimum essential medium (MEM) supplemented with ITS (insulin $6.25 \mu \mathrm{g} / \mathrm{mL}$, transferrin $6.25 \mu \mathrm{g} / \mathrm{mL}$ and selenium $6.25 \mathrm{ng} / \mathrm{mL}$ ), $0.23 \mathrm{mM} \mathrm{pyruvate,} 2 \mathrm{mM}$ glutamine, $2 \mathrm{mM}$ hypoxantine, $1.25 \mathrm{mg} / \mathrm{mL}$ bovine serum albumin (BSA), and antibiotics $(100 \mu \mathrm{g} / \mathrm{mL}$ penicillin and $100 \mu \mathrm{g} / \mathrm{mL}$ streptomycim) (MEM+, control medium); (T2) MEM+IAA (40 ng/mL); (T3) MEM+IAA + epidermal growth factor (EGF) (100 ng/mL; Sigma, St. Louis, MO, USA); or (T4) MEM+EGF+FSH (100 ng/mL). After 6 days of culture of cortex tissue fragments in media, ultrastructural analysis was performed on preantral follicles $(n=3$ in each treatment) included in a small cortical fragments. Preantral follicles were classified according to the stage of development as primordial follicles or as developing follicles. Preantral follicles cultured in supplemented media for 6 days were ultrastructurally normal. Their oocytes had an intact nucleus and cytoplasm that contained heterogeneous-sized lipid droplets, and numerous round or elongated mitochondria with intact parallel cristae were observed. Occasionally these organelles were associated with smooth endoplasmic reticulum (SER). Rough endoplasmic reticulum (RER) was rarely found. The cytoplasm of granulosa cells contained a large number of mitochondria and abundant RER. In contrast, follicles cultured in MEM+ (control) had a large number of vacuoles in the oocyte cytoplasm and excessive clustering of the chromatin material in the nucleus, suggesting an initial process of oocyte degeneration. In conclusion, the presence of IAA, EGF, FSH and their combinations helped to maintain ultrastructural integrity of ovine preantral follicles in cortical slices cultured in vitro.

\section{CHEMICAL ACTIVATION OF IN VITRO-MATURED PORCINE OOCYTES}

\section{A. Bali Papp and E. Varga}

University of West Hungary, Mosonmagyaróvár, Hungary

Parthenogenetic oocyte activation is important for nuclear transfer and for the understanding of cell cycle regulation of oocytes. Several chemical agents, including ethanol, cycloheximide, strontium, cytochalasin $\mathrm{B}, 6$ dimethylaminopurine, $\mathrm{CaCl}_{2}$ and ionophore $\mathrm{A} 23187$ can induce mammalian oocyte activation in vitro. The objectives of the present study were: (1) to assess the ability of strontium chloride (S), cytochalasin B (CB), cycloheximide (CX), and 6-dimethylaminopurine (D) to induce activation and parthenogenetic development in porcine oocytes; and (2) to verify whether the combinations of treatments $(\mathrm{SB}$ group $=$ strontium combined with cytochalasin; SX group = strontium combined with cycloheximide, and SD group = strontium combined with 6-dimethylaminopurine) improves activation and parthenogenetic development rates. Oocytes from slaughterhouse ovaries were matured in vitro for $42 \mathrm{~h}$ at $39^{\circ} \mathrm{C}$, in $5 \% \mathrm{CO}_{2}$ in air. The basic medium used for oocyte maturation was TCM- 199 supplemented with $10 \%$ pig follicular fluid, $1.25 \mathrm{mM} \mathrm{L}$-glutamine, $0.9 \mathrm{mM}$ Na-pyruvate, $100 \mu \mathrm{M}$ cysteamine, $0.1 \mathrm{mg} / \mathrm{mL}$ streptomycin sulfate, $10 \mathrm{IU} / \mathrm{mL}$ pregnant mare serum gonadotropin (PMSG), and $10 \mathrm{IU} / \mathrm{mL}$ hCG (Werfft-Chemie GmbH, Vienna, Austria). Denuded MII oocytes were cultured in activation solution for $5 \mathrm{~h}$. Thereafter the oocytes were cultured in NCSU37 for 6 days. At $48 \mathrm{~h}$ and 6 days after activation, oocytes, zygotes were fixed in acetic acid:alcohol $(1 / 3 \mathrm{w} / \mathrm{v})$, then stained with $0.1 \%(\mathrm{w} / \mathrm{v})$ orcein in $45 \%(\mathrm{v} / \mathrm{v})$ acetic acid, and evaluated under a phase contrast microscope. Each experiment was repeated four times. All data were analyzed by ANOVA, followed by Duncan's multiple range test $(P<0.05)$. A total of 2243 oocytes were activated in the different groups. In all groups, more than $45 \%$ of the oocytes were activated. No significant difference was observed in activation rate among SD (346/170, 49.13\%), SX (302/164, 54.3\%), and SB (318/182, 57.23\%) groups. The activation rate for CB was significantly higher $(P<0.05)$ than for $\mathrm{D}$ or $\mathrm{S}(323 / 192,59.44 \pm 6.84 \%$; 366/176, 48.09 $\pm 3.43 \%$; and 319/183, 53.29 $\pm 5.39 \%$, respectively). The blastocyst rate for SX was significantly higher $(P<0.05)$ than that for $\mathrm{D}$, SD, or SB $(8.64 \pm 8.07 \%$; and $0 \pm 0 \%$; $0 \pm 0 \%$; and $1.27 \pm 2.41 \%$, 
respectively). In conclusion, this study suggests that chemical activation procedure is the most effective in strontium chloride combined with cycloheximide. The lowest oocyte fragmentation rates were in SX $(28.40 \pm 1.26 \%)$ and CX $(21.05 \pm 1.12 \%)$

This work was supported by the the Hungarian Scientific T 43131 Research Foundation and the Hungarian Science on Technology Foundation E $14 / 04$.

\title{
313 APOPTOSIS IN PARTHENOGENETIC PIG EMBRYOS PRODUCED BY DIFFERENT METHODS
}

\author{
J. Hyslop and Z. Machaty \\ Purdue University, West Lafayette, IN, USA
}

\begin{abstract}
Apoptosis or programmed cell death is a process during which cells die in a controlled fashion in response to a variety of stimuli. Apoptosis has been demonstrated to occur during pre-implantation development both in vivo and in vitro and it is believed to contribute to early embryonic loss. It is also believed that parthenogenetic embryos generally have a lower developmental potential compared to those derived from fertilization. In the present study we investigated the rate of apoptosis in parthenogenetic pig embryos produced by activating oocytes through various methods. Pig oocytes were collected from slaughterhouse ovaries and matured in vitro. Parthenogenetic development was induced by three different methods. In Group 1, oocytes were activated by two consecutive electrical pulses. In Group 2, oocytes were electroporated and then incubated for $4 \mathrm{~h}$ in $5 \mathrm{mM}$ butyrolactone I, a specific inhibitor of cyclin dependent kinases. In Group 3, electroporated oocytes were incubated for $5 \mathrm{~h}$ in cycloheximide, a protein synthesis inhibitor. Activated oocytes were cultured for 7 days in NCSU-23 medium. At the end of the culture period the embryos were fixed in $4 \%$ paraformaldehyde and permeabilized in $0.1 \%$ Triton X-100 with sodium citrate. They were then incubated in a TUNEL reaction medium that specifically identifies apoptotic nuclei by labeling fragmented DNA with a fluorochrome. Blastocysts produced by in vitro fertilization and DNase I-treated embryos were used as controls. The proportions of apoptotic cells were compared using ANOVA. Forty-three blastocysts were analyzed for apoptotic activity in the electroporation group. These embryos had a blastocyst rate of $33.6 \pm 8.7 \%$, total cell number of $31.9 \pm 13.2$, and an average of $2.7 \pm 2.2$ apoptotic cells per embryo; the rate of apoptosis was $9.1 \pm 7.1 \%$. Twenty-eight blastocysts were used for the TUNEL reaction in the group where activation was induced with the combined treatment of electroporation and butyrolactone (Group 2). On average, the blastocyst rate was $54.5 \pm 6.4 \%$ and blastocysts contained $27.4 \pm 9.6$ cells of which $2.8 \pm 3.9$ were apoptotic; the percentage of apoptosis for this group was $10.0 \pm 12.1 \%$. In the cycloheximide treated group (Group 3), the onset of apoptosis was investigated using 29 blastocysts. The blastocyst rate was $38.2 \pm 15.9 \%$ with an average total cell number of $27.2 \pm 11.4 \%$. The TUNEL assay revealed that the mean number of apoptotic cells per embryo in these blastocysts was $2.1 \pm 1.5$, representing $9.0 \pm 7.6 \%$ apoptotic cells. The blastocysts $(n=14)$ produced by in vitro fertilization had a blastocyst rate of $18.0 \pm 5.1 \%$ and $29.9 \pm 12.0$ cells; $9.2 \pm 8.1 \%$ of these cells $(2.6 \pm 2.2$ cells per embryo) showed signs of apoptosis. All nuclei in the DNase I-treated embryos showed positive signals following the TUNEL reaction. The results confirm previous findings that apoptosis occurs in blastocyst stage embryos. There was no difference in the percentage of apoptotic cells between embryos whose development was triggered by different oocyte activation methods; the rate of apoptosis in parthenogenetic blastocysts was similar to that in blastocysts produced by in vitro fertilization.
\end{abstract}

\section{PARTHENOGENETIC ACTIVATION OF PORCINE OOCYTES AT THE MII STAGE BY Ca-EDTA TREATMENT}

\author{
H. Kawase, H. Imai, and M. Yamada \\ Laboratory of Reproductive Biology, Graduate School of Agriculture, Kyoto University, Kyoto, 606-8501, Japan
}

We previously found that treatment of porcine oocytes at the germinal vesicle (GV) stage with a cell membrane-impermeable metal ion chelator, EDTA saturated with $\mathrm{Ca}^{2+}$ (Ca-EDTA, $1 \mathrm{mM}$ ), induced artificial activation followed by formation of a pronucleus (PN) (Azuma et al. $2001 \mathrm{Biol}$. Reprod. 64, 647-653). In our preliminary experiments, it was found that oocytes at the metaphase of the second meiotic division (MII) are activated by Ca-EDTA treatment, leading to formation of PN and development to the blastocyst stage, and that it takes about $30 \mathrm{~h}$ for the oocytes to form PN by Ca-EDTA treatment. Normally, the optimal time for MII oocytes to be activated by sperm or conventionally used activation stimuli such as $\mathrm{Ca}^{2+}$ ionophore is much shorter than that in Ca-EDTA and prolonged arrest of the oocytes leads to aging. In this study, we examined how MII porcine oocytes were activated by Ca-EDTA treatment. To obtain MII oocytes, immature porcine oocytes enclosed by cumulus cells from ovaries of gilts were cultured in TCM-199 $+\mathrm{NaHCO}_{3}+\mathrm{Na}$ pyruvate + pregnant mare serum conadotropin (PMSG) $+\mathrm{hCG}\left(199\right.$ medium) at $38^{\circ} \mathrm{C}$ under $5 \% \mathrm{CO}_{2}$ in air for $48 \mathrm{~h}$. First, to elucidate an intracellular signaling pathway required for Ca-EDTA induced activation, MII oocytes were cultured for $36 \mathrm{~h}$ in 199 medium containing Ca-EDTA $(1 \mathrm{mM})$ with or without each inhibitor as follows, calphostin C (PKC inhibitor, $1 \mu \mathrm{M})$, U73122 (phospholipase $\mathrm{C}$ (PLC) inhibitor, $10 \mu \mathrm{M}$ ), PD98059 (MAPK inhibitor, $50 \mu \mathrm{M}$ ), and BAPTA-AM (intracellular $\mathrm{Ca}^{2+}$ chelator, $50 \mu \mathrm{M}$ ). Of the oocytes treated with Ca-EDTA, $60 \%$ underwent PN formation, whereas none of oocytes kept in medium alone was activated. Concomitant addition of BAPTA-AM to 199 medium with Ca-EDTA inhibited PN formation, whereas other inhibitors had no effects. These results suggest that a sufficient amount of intracellular calcium ion is necessary for parthenogenetic activation of porcine oocytes by Ca-EDTA, and that the activation may be independent on the mobilization of $\mathrm{Ca}^{2+}$ by PLC activation. Next, we analyzed changes of maturation-promoting factor (MPF) and MAPK activities in the oocytes during $48 \mathrm{~h}$ of culture in 199 medium with or without Ca-EDTA. Both kinases were measured by assays of the phosphorylation of histone H1 and myelin basic protein (MBP), respectively. When MII oocytes were cultured in 199 medium without Ca-EDTA, MPF and MAPK activities in oocytes were high at the onset of culture, and then gradually decreased as the oocytes underwent aging until $48 \mathrm{~h}$ of culture. On the other hand, in the Ca-EDTA treated oocytes, MPF and MAPK activities were maintained at high levels similar to those in oocytes at the onset of culture until $18 \mathrm{~h}$ or $24 \mathrm{~h}$, respectively, and then both activities rapidly decreased. The timing of the rapid reduction of MAPK activity almost coincided with the time when PN formation was started. These results indicate that Ca-EDTA regulates MPF and MAPK activities in porcine oocytes during a prolonged period of culture from the MII stage to induce PN formation in a $\mathrm{Ca}^{2+}$-dependent manner. 


\title{
315 EFFICIENCY OF CHEMICAL OR ELECTRICAL ACTIVATION OF BOVINE OOCYTES
}

\author{
M. P. Milazzotto, W. B. Feitosa, R. Simões, C. M. Mendes, M. E. O. A. Assumpção, and J. A. Visintin \\ Department of Animal Reproduction, University of Sao Paulo, São Paulo, SP, Brazil
}

\begin{abstract}
Activation of in vitro matured oocytes is essential for the success of nuclear transfer embryo production. Oocyte activation is promoted by the release of intracellular calcium and influx of extracellular ions, and can be chemically induced by calcium ionophores such as A23187 (CA) or ionomycin (IO). Electrical stimulation (EL) is an essential stage in nuclear transfer protocols for the fusion of enucleated oocytes with the donor's cell nucleus. Moreover, EL can be used as an alternative method to induce calcium influx through the formation of pores in the plasma membrane. This work aimed to evaluate the effect of electrical pulse vs the use of different calcium ionophores (A23187 or ionomycin) as primary agents of bovine oocyte activation, with or without the addition of BSA, on the rate of blastocyst formation and blastocyst quality. BSA was used to quench the activation process after a 5-min exposure to CA or IO. Cumulus-oocyte complexes were matured in TCM-199 medium with FCS and hormones for $18 \mathrm{~h}$ at $38.5^{\circ} \mathrm{C}$ and $5 \% \mathrm{CO}_{2}$ in air. After removal of cumulus cells, oocytes presenting the first polar body were selected and maintained in SOFaa medium to complete $24 \mathrm{~h}$ of maturation. They were then divided into five treatments groups 1-CA (CA $5 \mathrm{mM}, 5 \mathrm{~min}$ ); 2 -CAB (CA $5 \mathrm{mM}, 5 \mathrm{~min}$; BSA, $5 \mathrm{~min}$ ); 3-IO (IO $5 \mathrm{mM}, 5 \mathrm{~min}$ ); 4-IOB (IO $5 \mathrm{mM}, 5 \mathrm{~min}$; BSA, $5 \mathrm{~min}$ ); and 5-EL (EL $1.5 \mathrm{kV} / \mathrm{cm}, 20 \mu \mathrm{s}, 2$ pulses). After treatments, oocytes were kept in 6-dimethylaminopurine for $3 \mathrm{~h}$ and cultured in SOFaa medium for 7 days at $38.5^{\circ} \mathrm{C}$ and $5 \% \mathrm{CO}_{2}$ in air. Rates of cleavage and blastocyst were evaluated respectively on Days 2 and 7 of culture. To evaluate embryo quality, Hoechst 33342/propidium iodide staining was used. Data were evaluated by ANOVA and submitted to LSD test for embryo rates and $t$-test for embryo quality. Four replicates were carried out with a total of 89 oocytes per treatment. There was a difference $(P<0.05)$ in rate of development to blastocyst between treatments $1-\mathrm{CA}\left(54.4 \%{ }^{\mathrm{a}}\right), 3-\mathrm{IO}\left(51.4 \%{ }^{\mathrm{a}}\right)$, and 5-EL $\left(54.5 \%^{\mathrm{a}}\right)$ compared with 4 -IOB $\left(18.3 \%^{\mathrm{b}}\right)$. Treatment 2 -CAB $\left(39.8 \%{ }^{\mathrm{ab}}\right)$ did not show any difference from the others. There was no difference $(P>0.05)$ among treatments in total number of cells: $1-\mathrm{CA}\left(63.1^{\mathrm{a}}\right), 2-\mathrm{CAB}\left(57.2^{\mathrm{a}}\right), 3-\mathrm{IO}\left(60.9^{\mathrm{a}}\right), 4-\mathrm{IOB}\left(72.4^{\mathrm{a}}\right)$, and 5 -EL $\left(58.4^{\mathrm{a}}\right)$. However, there was a difference $(P<0.01)$ in the percentage of viable cells between treatments $1-\mathrm{CA}\left(49.9 \%^{\mathrm{a}}\right), 2-\mathrm{CAB}\left(45.8 \%{ }^{\mathrm{a}}\right), 3-\mathrm{IO}\left(64.9 \%{ }^{\mathrm{a}}\right)$, and $4-\mathrm{IOB}\left(50.9 \%{ }^{\mathrm{a}}\right)$ in comparison to 5-EL $\left(82.7 \%^{\mathrm{b}}\right)$. In conclusion, BSA, when associated with IO, had a negative effect on embryonic developmental rates. The different calcium ionophores used and the BSA did not improve embryo quality. Although there were no significant differences between electrical and chemical activation on the rate of blastocyst formation, it is important to point out that higher quality embryos were achieved by using electrical activation.
\end{abstract}

This work was supported by FAPESP 03/00156-9.

\section{CHANGES IN MPF ACTIVITY IN PORCINE OOCYTES FOLLOWING ACTIVATION BY VARIOUS METHODS}

\author{
L. Nanassy ${ }^{\mathrm{A}, \mathrm{B}}$, K. Lee ${ }^{\mathrm{B}}$, A. Javor ${ }^{\mathrm{A}}$, and Z. Machaty ${ }^{\mathrm{B}}$ \\ ${ }^{A}$ Department of Animal Breeding and Nutrition, University of Debrecen, Debrecen, Hungary; \\ ${ }^{B}$ Department of Animal Sciences, Purdue University, West Lafayette, IN 47907, USA
}

In mammalian oocytes metaphase II arrest is maintained by high activity of the M-phase promoting factor (MPF), which is a heterodimer of cdc2 and cyclin B. The repetitive increase in the intracellular free calcium concentration at fertilization leads to the inactivation of MPF; low MPF activity will then stimulate the resumption of meiosis and entry into interphase. Most oocyte activation methods are able to trigger only a single calcium transient that may not be sufficient to keep MPF levels low enough to induce pronuclear formation. The objective of this study was to determine the profile of MPF activity after different oocyte activation protocols. Mature pig oocytes from prepubertal gilts were allocated into three groups: (1) oocytes activated by two consecutive electrical pulses; (2) oocytes electroporated and incubated for $4 \mathrm{~h}$ in $100 \mu \mathrm{M}$ butyrolactone I, an inhibitor of cdc2; (3) oocytes electroporated and treated for $5 \mathrm{~h}$ with $10 \mu \mathrm{g} / \mathrm{mL}$ cycloheximide, a protein synthesis blocker. Some oocytes were removed from the medium at 2, 4 , and $6 \mathrm{~h}$ after the electrical pulse and used to determine MPF activity using a cdk1/cdc2 kinase assay kit. The remaining oocytes were placed into PZM-1 medium and cultured for 7 days. Data were compared using ANOVA. After the electrical stimulus the initial activity of MPF $(0.25 \mathrm{pmol} / \mathrm{min} /$ oocyte $)$ dropped in all cases, i.e., the amount of phosphate incorporated into histones decreased. However, in oocytes activated with an electrical pulse only, MPF activity that by had $4 \mathrm{~h}$ dropped to $0.15 \mathrm{pmol} / \mathrm{min} /$ oocyte started to increase and by $6 \mathrm{~h}$ it reached $0.2 \mathrm{pmol} / \mathrm{min} / \mathrm{oocyte}$. In the cycloheximide-treated oocytes this increase was negligible (by $6 \mathrm{~h}$ the activity was $0.12 \mathrm{pmol} / \mathrm{min} / \mathrm{oocyte}$ ) whereas incubation in butyrolactone resulted in a continuous decrease in MPF activity which by $6 \mathrm{~h}$ was as low as $0.07 \mathrm{pmol} / \mathrm{min} /$ oocyte. Developmental data of the activated oocytes are presented in Table 1. The percent of embryos cleaved and the percent of oocytes that formed blastocysts were higher in the cycloheximide-treated group than in controls. In contrast, the total cell number per blastocyst was the highest in oocytes that were activated by electroporation only. Inhibiting cdc2 kinase or protein synthesis helps to maintain low MPF activity after stimulation and results in a high cleavage ratio and blastocyst formation in the pig. Such treatment, however, had a negative effect on total cell number at the blastocyst stage.

Table 1. Developmental data of activated oocytes

\begin{tabular}{lcccc}
\hline Treatment & $n$ & $\begin{array}{c}\text { Cleaved } \\
(\% \pm \text { SEM })\end{array}$ & $\begin{array}{c}\text { Blastocyst } \\
\text { total }(\% \pm \text { SEM })\end{array}$ & $\begin{array}{c}\text { Cell number/ } \\
\text { Blastocyst }(\% \pm \text { SEM })\end{array}$ \\
\hline Electroporation (EP) & 414 & $43.5 \pm 7.82^{\mathrm{a}}$ & $24.3 \pm 4.87^{\mathrm{a}}$ & $40.9 \pm 1.96^{\mathrm{a}}$ \\
EP + Butyrolactone I & 403 & $52.6 \pm 5.65^{\mathrm{ab}}$ & $37.4 \pm 5.79^{\mathrm{ab}}$ & $34.3 \pm 1.22^{\mathrm{b}}$ \\
Ep + Cyclohexamide & 410 & $63.5 \pm 4.77^{\mathrm{b}}$ & $45.9 \pm 4.83^{\mathrm{b}}$ & $36.9 \pm 1.08^{\mathrm{b}}$ \\
\hline
\end{tabular}

${ }^{\mathrm{a}, \mathrm{b}}$ Different supercripts in a column indicate significant difference $(P<0.005)$ 


\title{
317 EFFECT OF REACTIVE OXYGEN SPECIES (ROS) ON ACTIVATION OF BOVINE OOCYTES MATURED IN VITRO
}

\author{
J. I. Park ${ }^{\mathrm{A}}$, Y. Jang ${ }^{\mathrm{A}}$, and E. S. Lee $\mathrm{B}$ \\ ${ }^{\mathrm{A}}$ College of Veterinary Medicine, Konkuk University, Seoul, 143-101, South Korea; \\ ${ }^{B}$ Department of Veterinary Medicine, Kangwon National University, Chunchon, 200-101, South Korea
}

\begin{abstract}
Vitamin $\mathrm{E}$ is an antioxidant that protects embryos from oxidative stress and supports embryonic development in vitro. This study was carried out to assess the effect of reactive oxygen species (ROS) by measuring the effects of hydrogen peroxide $\left(\mathrm{H}_{2} \mathrm{O}_{2}\right)$ and vitamin $\mathrm{E}$ (VitE) on chemical activation of in vitro-matured oocytes. Bovine ovaries were collected from slaughtered cows at a local abattoir. Oocytes were aspirated from follicles 3-8 mm in diameter and transferred to maturation medium: tissue culture medium (TCM)-199 supplemented with $10 \%$ (v/v) fetal calf serum, $100 \mathrm{mg} / \mathrm{L}$-cysteinr, $44 \mathrm{mg} / \mathrm{L}$ sodium pyruvate, gonadotropins (each $250 \mathrm{IU}$ of eCG and $\mathrm{hCG} / \mathrm{mL}$ ), and $10 \mathrm{ng} / \mathrm{mL}$ epidermal growth factor. Oocytes were cultured at $38.9^{\circ} \mathrm{C}$ in $5 \% \mathrm{CO}_{2}$ in humidified air. After $22 \mathrm{~h}$ of culture, oocytes with polar bodies were selected and submitted to activation treatments. Oocytes were exposed to calcium ionomycin $(5 \mu \mathrm{M}$ for $5 \mathrm{~min})$ followed by incubation with 6-DMAP $(2 \mathrm{mM})$ for $3.5 \mathrm{~h}$ in medium supplemented with or without VitE $(100 \mu \mathrm{m})$. Routine in vitro fertilization (IVF) was also performed for $18 \mathrm{~h}$ as control treatment with or without VitE. After activation, oocytes were cultured in mSOF medium containing $0.8 \% \mathrm{BSA}$ at $38.9^{\circ} \mathrm{C}$ in $5 \% \mathrm{CO}_{2}, 5 \% \mathrm{O}_{2}$ in humidified air for $16-18 \mathrm{~h}$. $\mathrm{H}_{2} \mathrm{O}_{2}$ content was measured and rate of development was monitored. For detection of $\mathrm{H}_{2} \mathrm{O}_{2}$, individual oocytes were stained with $2^{\prime}, 7^{\prime}$-dichlorofluorescein diacetate (DCFDA) and observed under fluorescence microscope (Hashimoto et al. $2000 \mathrm{Mol}$. Reprod. Dev. 56, 520-526). Levels of $\mathrm{H}_{2} \mathrm{O}_{2}$ were analyzed by counting the number of black-white pixels of TIFF images (ImageJ ver. 1.32; http://rsb.info.nih.gov/ij) after conversion of fluorescence image. Data were analyzed using Student's $t$-test and chi-square test. The $\mathrm{H}_{2} \mathrm{O}_{2}$ contents were significantly higher $(P<0.05)$ in the activation group without VitE (218 pixels, $n=49)$ than IVF without VitE (210 pixels, $n=50)$. Levels of $\mathrm{H}_{2} \mathrm{O}_{2}$ in oocytes activated with VitE were also higher $(P<0.05)$ than in the IVF group with VitE (216 pixels, $n=50$ vs. 210 pixels, $n=51$ ). Between groups with or without VitE, the $\mathrm{H}_{2} \mathrm{O}_{2}$ contents were not different in both activation and IVF. The cleavage rate to the 2-cell stage and development rate to blastocysts after activation with VitE $(77.8 \%$ and $15.1 \%$, $n=72)$ were significantly higher $(P<0.05)$ than in those without VitE $(63.3 \%$ and $10.2 \%, n=60)$. The results of the present study demonstrate that the chemical activation increased accumulation of $\mathrm{H}_{2} \mathrm{O}_{2}$ in oocytes compared to the IVF condition. Although ROS level did not affect chemical activation, vitamin $\mathrm{E}$ added in the activation medium promoted further development of activated embryos.
\end{abstract}

This work was supported by a grant from the BioGreen 21 Program (20050301-034-443-026-03-00), Rural Development Administration, Korea .

\section{INHIBITION OF FIRST POLAR BODY EXTRUSION BY CYTOCHALASIN B DURING IN VITRO MATURATION OF PORCINE OOCYTES LEADS TO RE-ARRANGEMENT OF THE SEGREGATED CHROMOSOMES AND IMPROVEMENT IN THE QUALITY OF THE PARTHENOGENETIC BLASTOCYSTS}

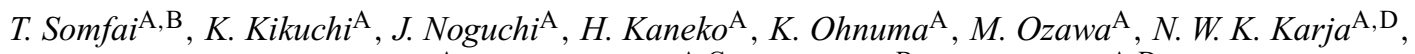

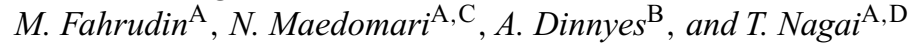 \\ ${ }^{\text {A }}$ Genetic Diversity Department, National Institute of Agrobiological Sciences, Tsukuba, Ibaraki, Japan; \\ ${ }^{B}$ Micromanipulation and Genetic Reprogramming Group, Institute of Animal Biology, Agricultural Biotechnology Center, Gödöllö, Hungary; \\ ${ }^{\mathrm{C}}$ Graduate School, Azabu University, Sagamihara, Kanagawa, Japan; \\ D Department of Research Planning and Coordination, National Institute of Livestock and Grassland Science, Tsukuba, Ibaraki, Japan
}

Diploid parthenotes are usually obtained by the inhibition of second polar body (PB2) extrusion after activation of metaphase II (MII) oocytes. However, diploid embryos can be generated by the inhibition of the first polar body (PB1) extrusion as well, using cytochalasin B (CB) during in vitro maturation prior the activation procedure. A higher percentage of mouse embryos generated by the activation of MII oocytes and the inhibition of PB2 extrusion were proven to be homozygous than for parthenotes obtained by the latter method (Kubiak et al. 1991 Development 111, 763-769). The aim of the present study was to examine if such difference has any effect on the development of parthenogenetic embryos in vitro. Nuclear progression and in vitro embryonic development after parthenogenetic activation of porcine oocytes exposed to CB during in vitro maturation (IVM) was investigated in the present study. The tendency of nuclear maturation was similar in oocytes matured in the presence of $1 \mu \mathrm{g} / \mathrm{mL} \mathrm{CB}$ (IVM-CB group) and control oocytes matured without $\mathrm{CB}$ after $37 \mathrm{~h}$ of IVM; at this time the frequency of oocytes that had reached/or passed through anaphase-I stage did not differ significantly $(P<0.05)$ between the IVM-CB and the control groups $(61.3 \%$ and $69.9 \%$, respectively $)$, however, no polar body extrusion was observed in the IVM-CB group and the two lumps of homologue chromosomes remained in the oocyte and turned into two irregular sets of condensed chromosomes. By $41 \mathrm{~h}$ of IVM, the double sets of chromosomes re-united in $89.5 \%$ of IVM-CB oocytes and formed a single large metaphase plate, whereas $68.8 \%$ of the control oocytes had reached metaphase-II stage (MII) by this time. When IVM-CB oocytes were electrically $(1.5 \mathrm{kV} / \mathrm{cm}$ for $100 \mu \mathrm{s})$ activated and subsequently cultured without CB, 39\% of the oocytes extruded a polar body (PB) and $82.9 \%$ of them had a female pronucleus. When those oocytes with PB were cultured, the blastocyst rate of the cleaved embryos did not differ $(P<0.05)$ from those of the control that were stimulated at MII and subsequently treated with CB (43.3\% and 48.2\%, respectively). The number of blastomeres in Day 6 blastocysts was significantly higher $(P<0.05)$ in the IVM-CB derived embryos than in those in the control group ( 47.8 and 40.7 , respectively); moreover, the ratio of dead blastomeres (dead cells : live cells) was higher $(P<0.05)$ in the control than in the IVM-CB blastocysts $(0.047$ and 0.031 , respectively). A possible explanation for this result might be a lower frequency of homozygous genes in IVM-CB parthenotes, in which segregation of sister chromatids were promoted instead of segregation of homologous chromosomes to obtain diploid embryos. In such embryos the expression of recessive lethal, sublethal and subvital genes might have a lower probability. 
Oocyte Maturation

\title{
319 OXYGEN CONSUMPTION OF BOVINE CUMULUS CELLS AND OOCYTES CULTURED IN DIFFERENT CULTURE SYSTEMS FOR OOCYTE MATURATION
}

\author{
H. Abe ${ }^{\mathrm{A}}, H . S h i k u^{\mathrm{B}}, S$. Aoyagi $^{\mathrm{C}}, T$. Matsue $^{\mathrm{B}}$, and H.Hoshi ${ }^{\mathrm{D}}$ \\ ${ }^{\text {A }}$ Tohoku University Biomedical Engineering Research Organization, Sendai, Japan; ${ }^{\mathrm{B}}$ Graduate School of Environmental Studies, Tohoku \\ University, Sendai, Japan; ${ }^{\mathrm{C}}$ Hokuto Denko Corporation, Tokyo, Japan; ${ }^{\mathrm{D}}$ Research Institute for the Functional Peptides, Yamagata, Japan
}

Oxygen consumption is a ubiquitous parameter that can provide valuable information on metabolic mechanisms and on oocyte and embryo quality. Recently, we succeeded in non-invasively and quantitatively determining oxygen consumption of individual bovine embryos by scanning electrochemical microscopy (SECM). The aim of this study was to assess by SECM the oxygen consumption of bovine cumulus cells and oocytes cultured in serum-free and serum-supplemented media for oocyte maturation. Bovine cumulus-oocyte complexes (COCs) were obtained from ovarian follicles 2-6 mm in diameter. COCs were cultured in IVMD101 medium for serum-free culture and HPM199 medium supplemented with 5\% calf serum (HPM199+CS) for serum-supplemented culture in a humidified atmosphere of $5 \% \mathrm{CO}_{2}$ in air $\left(20 \% \mathrm{O}_{2}\right)$ at $38.5^{\circ} \mathrm{C}$ for $24 \mathrm{~h}$. Oxygen consumption by single COCs was non-invasively quantified by a SECM measuring system (Abe et al. $2004 \mathrm{~J}$. Mamm. Ova Res. 21, 22). After the measurements, COCs were treated with $0.5 \%$ pronase to completely remove the cumulus cells. The oxygen consumption of single denuded oocyte was measured by SECM. Some COCs and oocytes were prepared for transmission electron microscopy. Oxygen consumption has been monitored in COCs and oocytes cultured in IVMD101 and HPM199+CS media for oocyte maturation (Table 1). Oxygen consumption rates of the immature COCs and denuded oocytes (immediately upon recovery from ovary: control) were 6.91 and $0.70\left(\times 10^{-14} \mathrm{~mol} \mathrm{~s}^{-1}\right)$, respectively. In serum-free culture (IVMD101), an increase in oxygen consumption rate was found in oocytes, whereas the oxygen consumption of COCs decreased during oocyte maturation. On the other hand, the oxygen consumption of COCs and oocytes cultured in serum-supplemented medium (HPM199+CS) were not change compared with that of controls. Electron microscopic study demonstrated that the mitochondria moved from a peripheral location in the ooplasm to an even spatial distribution in the oocytes cultured in IVMD101 medium, whereas many of the mitochondria in oocytes cultured in HPM199+CS were distributed in the peripheral region of the ooplasm after oocyte maturation. These results suggest that the respiration activity of bovine cumulus cells and oocytes changed during oocyte maturation, and the respiration activity and ultrastructural features of oocytes may affect the culture conditions. The SECM procedures may provide valuable information on oocyte quality and culture conditions for oocyte maturation.

Table 1. Oxygen consumption rates $\left(F \times 10^{-14} \mathrm{~mol} \mathrm{~s}^{-1}\right)$ of the bovine COCs and oocytes in oocyte maturation cultures

\begin{tabular}{lcc}
\hline Medium & COC $(n)$ & Oocyte $(n)$ \\
\hline IVMD101 & $2.98 \pm 0.29(35)^{\mathrm{a}}$ & $1.05 \pm 0.04(29)^{\mathrm{a}}$ \\
IVMD101 & $2.98 \pm 0.29(35)^{\mathrm{a}}$ & $1.05 \pm 0.04(29)^{\mathrm{a}}$ \\
IVMD101 & $2.98 \pm 0.29(35)^{\mathrm{a}}$ & $1.05 \pm 0.04(29)^{\mathrm{a}}$ \\
HPM199+CS & $5.25 \pm 1.01(20)^{\mathrm{b}}$ & $0.63 \pm 0.07(19)^{\mathrm{b}}$ \\
\hline
\end{tabular}

${ }^{\mathrm{a}, \mathrm{b}}$ Values with different superscripts in each column differ significantly $(P<0.05)$.

\section{MPF ACTIVITY AND DEVELOPMENTAL COMPETENCE IN DIFFERENT SIZES OF PREPUBERTAL GOAT OOCYTES}

\author{
B. Anguita, A. R. Jimenez-Macedo, D. Izquierdo, and M. T. Paramio \\ Facultat de Veterinaria, Universitat Autonoma de Barcelona, Barcelona, Spain
}

Developmental competence of prepubertal goat oocytes recovered from a slaughterhouse is low, probably due to an incomplete cytoplasmic maturation. Regulation of cytoplasmic maturation is still unknown, although maturation-promoting factor (MPF) is suggested to play an important role in this process. To better understand the role of MPF in cytoplasmic maturation, we have studied MPF kinase activity in oocytes with different developmental competence. Ovaries were obtained from a slaughterhouse, and oocytes were recovered by slicing and were selected according to morphological criteria. Some oocytes were denuded and classified in diameter groups $(<110 \mu \mathrm{m}, 110-125 \mu \mathrm{m}, 125-135 \mu \mathrm{m}$, and $>135 \mu \mathrm{m})$, placed in lysis buffer ( $50 \mathrm{mM}$ Tris- $\mathrm{HCl}$ (pH 7.5), $0.5 \mathrm{M} \mathrm{NaCl}, 5 \mathrm{mM}$ EDTA, $0.01 \%$ Brij35, $1 \mathrm{mM}$ phenyl methyl sultonyl fluoride (PMSF), $0.05 \mathrm{mg} / \mathrm{mL}$ leupeptin, $50 \mathrm{mM}$ 2-mercaptoethanol, $25 \mathrm{mM} \alpha$-glycerophosphate, $1 \mathrm{mM} \mathrm{Na}$-orthovanadate) and frozen in liquid $\mathrm{N}_{2}$. Cell extracts were stored at $-80^{\circ} \mathrm{C}$ until use. The rest of oocytes were matured in vitro in medium TCM199 supplemented with hormones, $10 \%$ (DBS), and $400 \mu \mathrm{M}$ cysteamine, for $27 \mathrm{~h}$ in $5 \% \mathrm{CO}_{2}$ in air and $38.5^{\circ} \mathrm{C}$. After IVM, a sample of oocytes were also denuded, classified by diameters, and frozen as described above. The rest of oocytes were used for IVF in $\mathrm{mDM}$ with spermatozoa capacitated with heparin and ionomicin. After $24 \mathrm{~h}$, presumptive zygotes were cultured for 7 days in medium $\mathrm{SOF}$ in $5 \% \mathrm{CO}_{2}, 5 \% \mathrm{O}_{2}$, and $90 \% \mathrm{~N}_{2}$ at $38.5^{\circ} \mathrm{C}$. At $48 \mathrm{~h}$ post-insemination, we added $0.1 \mu \mathrm{L}$ FBS per embryo. Embryonic development was evaluated with Hoechst staining after IVC. MPF kinase activity was detected using the MESACUP cdc2 kinase assay kit (MBL Woburn, MA, USA). Briefly, the oocyte extract corresponding to 10 oocytes was mixed with $10 \times$ reaction buffer (25 mM HEPES buffer (pH 7.5), 
$10 \mathrm{mM} \mathrm{MgCl}_{2}$ ) and $10 \%$ biotinylated MV peptide (SLYSSPGGAYC). We added $0.1 \mathrm{mM}$ ATP to start the reaction. The mixture was incubated at $30^{\circ} \mathrm{C}$ for $30 \mathrm{~min}$. The reaction was finished by adding $200 \mu \mathrm{L}$ of PBS containing $50 \mathrm{mM}$ EGTA. The phosphorylated MV peptide was detected by specific antibody using an ELISA procedure, and the OD was measured at $492 \mathrm{~nm}$. Fisher's exact test was used to analyze IVC results, and ANOVA to analyze cdc2 kinase activity results. We considered differences statistically significant when $P<0.05$. Results are shown in Table 1 . We observed that embryonic cleavage and blastocyst rate increased with oocyte diameter. The MPF activity detected was higher in the largest oocytes after IVM. As a consequence, we could establish that oocytes with a higher MPF activity are more capable of maintaining embryonic development until the blastocyst stage, which may indicate the important role that MPF plays in cytoplasmic maturation.

Table 1. Cleavage, blastocyst rate, and MPF kinase activity in different sizes of prepubertal goat oocytes

\begin{tabular}{lccccc}
\hline $\begin{array}{l}\text { Diameter } \\
(\mu \mathrm{m})\end{array}$ & $\begin{array}{c}\text { Embryonic development } \\
\text { number }\end{array}$ & Cleavage (\%) & Blastocysts (\%) & \multicolumn{2}{c}{$\begin{array}{c}\text { MPF kinase activity (OD at } 492 \mathrm{~nm}) \\
0 \text { hours }\end{array}$} \\
\hline$<110$ & 30 & $0^{\mathrm{a}}$ & $0^{\mathrm{a}}$ & $0.013 \pm 0.010$ & $0.013 \pm 0.008^{\mathrm{a}}$ \\
$110-125$ & 74 & $16.21^{\mathrm{b}}$ & $0^{\mathrm{a}}$ & $0.015 \pm 0.011$ & $0.189 \pm 0.093^{\mathrm{a}}$ \\
$125-135$ & 152 & $40.13^{\mathrm{c}}$ & $1.97^{\mathrm{a}}$ & $0.045 \pm 0.037$ & $0.409 \pm 0.33^{\mathrm{ab}}$ \\
$>135$ & 72 & $61.11^{\mathrm{d}}$ & $12.5^{\mathrm{b}}$ & $0.036 \pm 0.022$ & $0.669 \pm 0.244^{\mathrm{b}}$ \\
\hline
\end{tabular}

${ }^{\mathrm{a}-\mathrm{d}}$ Values with different superscripts within a column differ significantly $(P<0.05)$.

\title{
321 OOCYTE DIAMETER INFLUENCES THE MEIOTIC RESUMPTION AND PROGRESSION INDUCED BY OKADAIC ACID IN DOG
}

\author{
F. Ariu, L. Bogliolo, I. Rosati, M. T. Zedda, S. Pau, and S. Ledda \\ Obstetrics Section of the Institute of General Pathology, Pathological Anatomy and Veterinary Obstetrics-Surgery Clinic, \\ University of Veterinary Medicine, Sassari, Sardegna, Italy
}

\begin{abstract}
The acquisition of meiotic competence, in the bitch as in many other mammalian species, is related to the oocyte diameter. This study was designed to determine the effect of okadaic acid (OA), a potent inhibitor of seronine/threonine 1 and $2 \mathrm{~A}$ phosphatases, on meiotic resumption and progression in canine oocytes with different diameters. In two experiments, healthy cumulus-oocytes complexes were collected from ovaries of bitches at various stages of the estrous cycle and divided, by diameters, into three treatment groups for in vitro maturation: $<110 \mu \mathrm{m}, 110-120 \mu \mathrm{m}$, and $>120 \mu \mathrm{m}$. In Experiment 1, oocytes were pre-incubated for $1 \mathrm{~h}$ in TCM- $199+20 \%$ estrous canine serum (SCE) + cysteamine + OA $(0.5 \mu \mathrm{M})$. Then, oocytes were cultured for $48 \mathrm{~h}$ in the same medium without $\mathrm{OA}$ at $38.5^{\circ} \mathrm{C}, 5 \% \mathrm{CO}_{2}$ in air. As a control group, oocytes were matured in vitro under the same conditions but without pre-incubation with OA. In Experiment 2, to determine if the effect of OA is mediated by cumulus cells, $>120 \mu \mathrm{m}$ oocytes were denuded from cumulus cells, incubated with or without OA, and cultured in vitro as previously described. At $48 \mathrm{~h}$, all oocytes were stained and fixed with glycerol-Hoechst 33342 to assess the stage of meiotic maturation. In Experiment 1, OA induced a significantly higher incidence of meiotic resumption in oocytes $<110 \mu \mathrm{m}(16 / 108,14.8 \% ; P<0.05)$ and $110-120 \mu \mathrm{m}(70 / 130,53.8 \% ; P<0.01)$ as compared to that of oocytes in the $<110 \mu \mathrm{m}$ and $110-120 \mu \mathrm{m}$ control groups $(2 / 58,3.4 \% ; 24 / 82,29.3 \%)$. The percentage of oocytes in the $110-120 \mu \mathrm{m}$ OA group that underwent in vitro maturation to metaphase II (MII) was significantly higher than in the $110-120 \mu \mathrm{m}$ control group $(18 / 130,13.8 \%$ vs. $4 / 82,4.9 \%$, respectively; $P<0.05)$. In contrast, smaller oocytes $(<110 \mu \mathrm{m})$ did not develop to MII with or whitout OA. Meiotic resumption rate of $>120 \mu \mathrm{m}$ OA group $(64 / 78,82.0 \%)$ was similar to the $>120 \mu \mathrm{m}$ control group $(56 / 72,77.8 \%)$, but a significantly higher proportion of the oocytes pre-incubated with OA progressed to MII than did the control oocytes $(40 / 78,51.3 \%$ vs. $12 / 72,16.7 \%$, respectively; $P<0.01)$. Low rates of meiotic resumption were observed in denuded $>120-\mu \mathrm{m}$ oocytes with $(7 / 63,11.1 \%)$ or without $\mathrm{OA}(7 / 55,12.7 \%)$ and none of them progressed to MII. In conclusion, the results of the present study indicate that treatment of fully grown $(>120 \mu \mathrm{m})$ oocytes with okadaic acid at the onset of in vitro maturation can result in a higher frequency of meiotic maturation than previously reported. Also, we determined that the beneficial effect of okadaic acid was mediated by cumulus cells.
\end{abstract}

\section{LEPTIN TREATMENT DURING BOVINE OOCYTE MATURATION AFFECTS mRNA LEVELS OF APOPTOSIS-RELATED GENES}

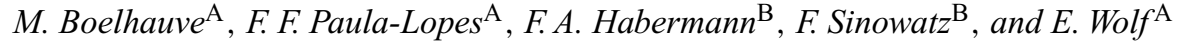 \\ ${ }^{\mathrm{A}}$ Institute of Molecular Animal Breeding and Biotechnology, Munich, Germany; \\ ${ }^{B}$ Institute of Veterinary Anatomy, Histology and Embryology, Munich, Germany
}

The series of events associated with oocyte growth and maturation determines its ability to undergo successful fertilization, cleavage, and embryonic development. Among the molecules involved in these events, leptin has been identified as a modulator of oocyte function. The latest studies have focused on long-term effects of leptin during maturation of bovine oocytes on apoptosis and gene expression in in vitro-produced blastocysts. Briefly, blastocysts originating from leptin-treated oocytes exhibited decreased transcript levels of BCL2 associated X-protein $(B A X)$, but increased mRNA concentrations for leptin receptor $(L E P R)$, signal transducer and activator of transcription 3 (STAT3), and baculoviral inhibitor of apoptosis protein repeat-containing 4 (BIRC4, also known as XIAP) (Boelhauve et al. 2005 Biol. Reprod. 73, 737-744). In the present study, we analyzed single oocytes and their surrounding cumulus cells matured in the presence of 0,1 , and $10 \mathrm{ng} / \mathrm{mL}$ leptin. Transcript levels of LEPR, STAT3, BAX, BIRC4, 
FASLG (encoding Fas ligand), and FAS (encoding Fas receptor) were determined by reverse transcriptase-quantitative PCR (RT-qPCR) analysis of matured oocytes and cumulus cells. Four IVM replicates with four individual samples were collected $22 \mathrm{~h}$ after the start of IVM. Total RNA was isolated using a modified TriZol protocol, and reverse transcribed using the enzyme Superscript II-RT and random hexamer primers. Quantitative PCR was conducted with SYBR-Green as a double-stranded DNA-specific fluorescent dye in an ABI 7000 SDS apparatus. Treatment of COCs with 1 or $10 \mathrm{ng} / \mathrm{mL}$ leptin increased transcript levels of $L E P R$ (2-fold; $P<0.001$ ), STAT3 (2-fold; $P<0.001$ ), BAX $(2$-fold; $P<0.001)$ and FAS (2-fold; $P<0.001$ ) in cumulus cells. Interestingly, the transcript level of the well-known inhibitor of apoptosis, BIRC4, was increased about 4-fold $(P<0.001)$. In oocytes, leptin treatment increased the mRNA abundance of $S T A T 3(10 \mathrm{ng} / \mathrm{mL} ; P<0.05), F A S(1 \mathrm{ng} / \mathrm{mL} ; P<0.05 \mathrm{and} 10 \mathrm{ng} / \mathrm{mL} ;$ $P<0.001)$, and FASLG $(10 \mathrm{ng} / \mathrm{mL} ; P<0.05)$. In conclusion, physiological doses of leptin during maturation of COCs have effects on the expression of developmentally important and apoptosis-associated genes in the oocyte and surrounding cumulus cells. The higher level of BIRC4 mRNA in leptin-treated cumulus cells was associated with a reduced proportion of cumulus cell apoptosis, which might explain the positive and long-lasting effects of leptin on the developmental competence of bovine oocytes.

\title{
323 INTERACTIONS OF OXYGEN TENSION, GLUCOSE CONCENTRATION, AND HEAT SHOCK ON MATURATION OF BOVINE OOCYTES
}

\author{
L. A. de Castro e Paula and P. J. Hansen \\ University of Florida, Gainesville, FL 32611, USA
}

In many cell types, oxygen tension and glucose concentration affect the magnitude of heat shock effects. Experiments were performed to (1) develop oocyte maturation protocols under high $(20.95 \%)$ and low $(5 \%)$ oxygen tensions, and (2) test the hypothesis that low oxygen tension and high glucose concentration reduce the magnitude of the deleterious effects of heat shock on in vitro maturation of bovine oocytes. In Experiment 1 , oocytes were matured in tissue culture medium-199 (TCM-199) or synthetic oviduct fluid (SOF) containing 5.6 or 20 mM glucose, under either high or low oxygen. Fertilization was performed under high oxygen and embryos were cultured in KSOM-BE2 under low oxygen. Data were analyzed by least squares analysis of variance using the GLM procedure of SAS (SAS Institute, Inc., Cary, NC, USA), with maturation medium, glucose concentration, and oxygen tension as main effects. Oxygen concentration affected percent blastocyst formation when TCM-199 was used (greater for high oxygen than low oxygen) but not when SOF was used (medium $\times$ oxygen, $P<0.05$ ). Similarly, glucose had no effect on percent blastocyst formation under high oxygen but high glucose was superior under low oxygen (glucose $\times$ oxygen, $P<0.05$ ). Therefore, SOF with either 5.6 or $20 \mathrm{mM}$ glucose was used to study the effect of heat shock on maturation under high and low oxygen in Experiment 2 . Maturation was at $38.5^{\circ} \mathrm{C}$ for $22 \mathrm{~h}$ (control) or $41^{\circ} \mathrm{C}$ for $12 \mathrm{~h}$ and $38.5^{\circ} \mathrm{C}$ for $10 \mathrm{~h}$ (heat shock). Fertilization was performed at $38.5^{\circ} \mathrm{C}$ under high oxygen and embryos were cultured in KSOM-BE2 under low oxygen at $38.5^{\circ} \mathrm{C}$. Data were analyzed as described above with glucose concentration, temperature and oxygen tension as main effects. The experiment was replicated nine times with a total of 3215 embryos. Heat shock decreased the cleavage rate slightly regardless of the maturation conditions $(69 \pm 1 \%$ vs. $66 \pm 1 \%, P<0.01)$. For oocytes matured in $5.6 \mathrm{mM}$ glucose, percent blastocyst formation was greater for high oxygen while there was no effect of oxygen tension for oocytes matured in $20 \mathrm{mM}$ glucose (glucose $\times$ oxygen, $P<0.01$ ). Heat shock decreased the percent blastocyst formation under all maturation conditions except for the group matured under high oxygen and high glucose (temperature $\times$ oxygen $\times$ medium, $P=0.07$; temperature $\times$ oxygen for oocytes under $5 \%$ oxygen; $P<0.05$ ). The percent blastocyst formation for control and heat-shocked oocytes was $25.9 \%$ vs. $22.5 \%$ (low oxygen- $-6.6 \mathrm{mM}$ glucose), $41.6 \%$ vs. $34.9 \%$ (low- $20 \mathrm{mM}$ ), $41.7 \%$ vs. $35.0 \%$ (high$5.6 \mathrm{mM})$, and $37.6 \%$ vs. $37.5 \%$ (high-20 mM) (SEM $=2.0 \%)$. In conclusion, heat shock during maturation under an oxygen tension $(5 \%$ oxygen) that approaches physiological conditions reduces the percent blastocyst formation. Moreover, a high glucose concentration protects oocytes from heat shock under high oxygen.

This work was supported by USDA NRICGP 2002-35203-12664 and CAPES \#134202-9.

\section{FUNCTIONAL ANALYSIS USING SHORT-INTERFERING (si)RNA OF TRAM-6, A NOVEL TRANSCRIPT ASSOCIATED WITH OOCYTES MATURATION IN CATTLE}

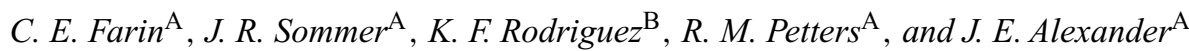 \\ ${ }^{A}$ Department of Animal Science, North Carolina State University, Raleigh, NC 27606, USA; \\ ${ }^{B}$ National Institutes of Environmental and Health Sciences, Research Triangle Park, NC 27709, USA
}

Maturation of cumulus-oocyte complexes (COCs) with gonadotropins requires transcription of new mRNAs. When COCs are cultured with FSH and the transcriptional inhibitor DRB, cumulus expansion and germinal vesicle breakdown (GVBD) are arrested. Differential mRNA display was used to identify a novel transcript associated with maturation of COCs (TRAM-6) that is expressed during early maturation but not when maturation is inhibited by DRB. The objective of this study was to use siRNAs targeted against TRAM-6 mRNA to assess its functional role in oocyte maturation. Exp. 1: Pools of $60-10$ bovine COCs $(n=5 / \mathrm{trt})$ were randomly assigned to culture in maturation medium consisting of $0.5 \mathrm{~mL}$ TCM-199 with $2.5 \mu \mathrm{g}$ FSH, $0.5 \mu \mathrm{g}$ estradiol, and $10 \%$ estrous cow serum with or without DRB $(120 \mu \mathrm{M})$ or with increasing doses of siTRAM-6 $(25,50$, or $100 \mathrm{nM})$. Exp. 2: COC pools $(n=4 / \mathrm{trt})$ were cultured in maturation medium with one of the following treatments: control, $120 \mu \mathrm{M} \mathrm{DRB}, 100 \mathrm{nM}$ non-specific siRNA (siNS), or $100 \mathrm{nM}$ siTRAM-6. After $4 \mathrm{~h}$ of culture, COC pools were used to assess levels of mRNA for TRAM-6,VEGF (vascular endothelial growth factor), and GAPD (glyceraldehyde-3-phosphate dehydrogenase) by semiquantitative RT-PCR. Exp. 3: COCs were cultured in maturation medium for either $8 \mathrm{~h}(n=9$ pools $/ \mathrm{trt})$ or $20 \mathrm{~h}(n=7$ pools $/ \mathrm{trt})$ with one of the following treatments: control, $120 \mu \mathrm{M} \mathrm{DRB}, 100 \mathrm{nM} \mathrm{siNS}$, or $100 \mathrm{nM}$ siTRAM-6. Cumulus expansion was graded every $4 \mathrm{~h}$. At the end of culture, a subset of COCs from each treatment was used to assess oocyte meiotic stage. Remaining COCs were used to assess TRAM-6,VEGF, and GAPD mRNA. Data were analyzed using ANOVA and Duncan's test. A 
$4 \mathrm{~h}$ of culture, relative expression of TRAM-6:GAPD mRNA (least squares means \pm SEM) was decreased in the DRB and the $100 \mathrm{nM}$ siTRAM-6 treatments but was unaffected by $25 \mathrm{nM}$ TRAM-6, $50 \mathrm{nM}$ siTRAM-6, or $100 \mathrm{nM}$ siNS (Exp. 1: $100 \pm 13 \%^{\mathrm{a}}, 17 \pm 13 \%{ }^{\mathrm{b}}, 101 \pm 13 \%{ }^{\mathrm{a}}, 60 \pm 13 \%{ }^{\mathrm{ab}}$ and $48 \pm 13 \%{ }^{\mathrm{b}}$ for control, DRB, $25 \mathrm{nM}$ siTRAM-6, $50 \mathrm{nM}$ siTRAM-6, and $100 \mathrm{nM}$ siTRAM-6, respectively; ${ }^{\text {abc }} P<0.05 ;$ Exp. $2: 100 \pm 10 \%{ }^{\mathrm{a}}$, $14 \pm 10 \%{ }^{\mathrm{b}}, 106 \pm 10 \%^{\mathrm{a}}$ and $68 \pm 10 \%^{\mathrm{c}}$ for control, DRB, $100 \mathrm{nM}$ siNS, and $100 \mathrm{nM}$ siTRAM- 6 , respectively; ${ }^{\text {abc }} P<0.05$ ). There was no effect of treatment on expression of $V E G F$ and GAPD mRNA, both of which are present in COCs at the start of culture and are unrelated to TRAM-6 mRNA. At $8 \mathrm{~h}$, GVBD was inhibited by treatment with DRB and siTRAM- $6\left(68 \pm 8 \%^{\mathrm{a}}, 3 \pm 8 \%{ }^{\mathrm{b}}, 72 \pm 8 \%^{\mathrm{a}}\right.$, and $30 \pm 8 \%^{\mathrm{c}}$ for control, DRB, siNSs and siTRAM-6, respectively; ${ }^{\text {abc }} P<0.05$ ). At $20 \mathrm{~h}$, the incidence of metaphase II (MII) oocytes was decreased in the DRB and siTRAM-6 groups $\left(96 \pm 8 \%^{\mathrm{a}}, 9 \pm 8 \%^{\mathrm{b}}, 94 \pm 8 \%\right.$ a , and $56 \pm 8 \%^{\mathrm{c}}$ for control, DRB, siNS, and siTRAM-6, respectively; ${ }^{\text {abc }} P<0.05$ ). Cumulus expansion was inhibited $(P<0.05)$ by DRB but was not affected by any other treatment. In summary, TRAM-6 siRNA decreased the expression of TRAM-6 mRNA in bovine COCs at $4 \mathrm{~h}$ of culture as well as the proportions of oocytes in GVBD at $8 \mathrm{~h}$ and in MII at $20 \mathrm{~h}$ of culture, but did not affect cumulus expansion. In conclusion, these data are consistent with the identification of a novel mRNA transcript, TRAM- 6 , that has a functional role in regulating meiotic maturation in bovine COCs.

This work was supported by USDA Grant \#2002-35205-12810.

\title{
325 ACTIVITY OF MATURATION-PROMOTING FACTOR (MPF) AND MITOGEN-ACTIVATED PROTEIN KINASES DURING IN VITRO MATURATION OF BUFFALO OOCYTES (BUBALUS BUBALIS)
}

\author{
B. Gasparrini ${ }^{\mathrm{A}}$, G. Leoni $^{\mathrm{B}}$, L. Boccia $^{\mathrm{A}}, M$. Galiotto $^{\mathrm{B}}, S$. Ledda $^{\mathrm{B}}$, and L. Zicarelli ${ }^{\mathrm{A}}$ \\ ${ }^{A}$ Discizia, Federico II University, Naples, Italy; \\ ${ }^{B}$ Department of Animal Biology, University of Sassari, Sassari, Italy
}

The maturation promoting factor (MPF) and mitogen-activated protein kinases (MAPK) are the key regulators of both meiotic and mitotic cell cycles. The absence of data on the activity of the major cell cycle kinases in buffalo oocytes during meiotic progression provided the bases for this study. More specifically we assayed the MPF and MAP kinase activity of buffalo oocytes during meiosis. Abattoir-derived cumulus-oocyte complexes (COCs) with a compact, non-atretic cumulus and a homogeneous cytoplasm were utilized for the study. The COCs $(n=293$, over four replicates) were matured in vitro in TCM-199 supplemented with $10 \%$ fetal calf serum (FCS), $0.2 \mathrm{mM}$ sodium pyruvate, $0.5 \mu \mathrm{g} / \mathrm{mL} \mathrm{FSH}, 5 \mu \mathrm{g} / \mathrm{mL} \mathrm{LH}, 1 \mu \mathrm{g} / \mathrm{mL}$ $17 \beta$-estradiol, $50 \mu \mathrm{M}$ of cysteamine, and $50 \mu \mathrm{g} / \mathrm{mL}$ kanamycin (B199). In vitro maturation (IVM) was carried out at $38.5^{\circ} \mathrm{C}$ under a controlled gas atmosphere of $5 \% \mathrm{CO}_{2}$ in humidified air. At scale times during the culture $(0,3,6,9,12,15,18,21,24 \mathrm{~h})$ groups of oocytes were stained with Hoechst 33342 to assess chromatin configuration and stored according to the maturation stage (GV, GVDB, MI, and MII) at $-80^{\circ} \mathrm{C}$ pending protein analysis. SDS-polyacrylamide gel electrophoresis wase performed using Laemmli discontinuous buffer system (Laemmli 1970 Nature 227, 680) with a $12 \%$ running gel. Groups of oocytes were analyzed for MPF activity $(n=65)$ by histone H1 kinase activity (Naito and Toyoda $1991 \mathrm{~J}$. Reprod. Fertil. 93, 467-473) and for MAPK activity $(n=48)$ by myelin basic protein assays (Chesnel et al. 1995 Biol. Reprod. 52, 895-902). The activity of both MPF and MAP kinases was quantified by measuring the density of the bands on the autoradiographic film with a densitometer. Differences in the levels of the kinases among groups were analyzed by ANOVA. It was assumed that the value of MPF and MAPK was $100 \%$ in metaphase II (MII) stage oocytes. The lowest levels of MPF and MAPK activities were found in the oocytes at the GV (0-6h post-IVM: $40 \%$ and $17.2 \%$, respectively) and at the GVBD (6-9 h post-IVM: $41.2 \%$ and $18 \%$ ) stages. The activities increased at metaphase I (MI) stage (9-15 h post-IVM) and at MII (21-24 post-IVM). Interestingly, although similar levels of MAP kinases were found at MI and MII stages (95.1\% vs. $100 \%)$, MPF levels were significantly lower $(P<0.01)$ at the MI stage compared to those detected at MII $(82.8 \% \mathrm{vs} .100 \%)$. The fluctuations of the MPF levels in buffalo appear different compared to those observed in other species; in particular, no differences were recorded between the GV and the GVBD stages whereas a significant increase of the MPF levels was found at MII compared to the MI stage. It seems that MPF and MAPK could differently guide meiotic resumption and progression to the MII arrest in this species. To our knowledge, this is the first report on biochemical analysis of the cell cycle regulation in buffalo oocytes.

\section{EVALUATION OF CULTURE DURATION AND PARTIAL CUMULUS CELL REMOVAL DURING CANINE OOCYTE MATURATION}

\author{
C. Hanna, C. Long, M. Westhusin, D. Kraemer \\ Department of Veterinary Physiology and Pharmacology, Texas A\&M University, College Station, TX 77843, USA
}

\begin{abstract}
The objectives of this study were to determine whether the percentage of canine oocytes that resume meiosis during in vitro maturation could be increased by either increasing culture duration or by removing approximately one-half of the cumulus cells $24 \mathrm{~h}$ after oocytes were placed into culture. Canine female reproductive tracts were collected from a local clinic and ovaries were minced in warm TL-HEPES. Oocytes with a consistently dark ooplasm and at least two layers of cumulus cells were selected, cultured in a basic canine oocyte in vitro maturation medium consisting of TCM-199 with Earl's salts, $2.92 \mathrm{mM}$ Ca-lactate, $20 \mathrm{mM}$ pyruvic acid, $4.43 \mathrm{mM}$ HEPES, $10 \%$ fetal calf serum, $1 \%$ Penicillin/Streptomycin (GibcoBRL, Grand Island, NY, USA), and $5 \mu \mathrm{g} / \mathrm{mL}$ porcine somatotropin, and incubated at $38.5^{\circ} \mathrm{C}$ in $5 \% \mathrm{CO}_{2}$ in humidified air. Treatment groups were randomly assigned and oocytes were cultured for 60,84 , or $132 \mathrm{~h}$ (Basic). From each of these groups, one-half of the oocytes were pipetted through a fine bore pipette to partially remove the cumulus cells $24 \mathrm{~h}$ after the start of culture (Basic-1/2). At the end of culture, all oocytes were denuded and the nuclear status was observed with Hoechst 33342 under ultraviolet fluorescence. All data were analyzed by ANOVA with $P<0.05$. Since the canine oocyte is ovulated at the germinal vesicle $(\mathrm{GV})$ stage of meiosis and requires up to five days to mature in the oviduct, it was hypothesized that an increased
\end{abstract}


culture time would allow for more oocytes to undergo nuclear maturation to metaphase II (MII). It was also hypothesized that partial removal of cumulus cells would decrease the cumulus cell component in the ooplasm that sustains meiotic arrest, allowing for more oocytes to resume meiosis $(\mathrm{RM}=$ germinal vesicle breakdown to MII). Results within each treatment group indicate that there is no significant difference between culture duration and the percent of oocytes that mature to MII. Additionally, there was no significance in the percent of oocytes that resumed meiosis after partial cumulus cell removal. Taken together, these data suggest that neither treatment is effective in canine in vitro maturation systems, given the current maturation culture conditions.

Table 1. Nuclear status* of oocytes for three time periods with or without partial cumulus cell removal

\begin{tabular}{lcccccc}
\hline Treatment & \# Hours & GV $(\%)$ & RM $(\%)$ & MII (\%) & Deg (\%) & Total oocytes \\
\hline Basic & 60 & 16 & 15 & 6 & 70 & 213 \\
& 84 & 30 & 9 & 2 & 61 & 299 \\
& 132 & 31 & 18 & 12 & 51 & 157 \\
Basic-1/2 & 60 & 23 & 11 & 2 & 66 & 461 \\
& 84 & 42 & 8 & 1 & 53 & 243 \\
& 132 & 29 & 9 & 6 & 59 & 101 \\
\hline
\end{tabular}

* $\mathrm{GV}=$ germinal vesicle stage; $\mathrm{RM}=$ resumed meiosis; $\mathrm{MII}=$ metaphase $\mathrm{II} ; \mathrm{Deg}=$ degerate

\title{
327 OOCYTE-SECRETED FACTORS DIRECTLY AFFECT OOCYTE DEVELOPMENTAL COMPETENCE DURING IN VITRO MATURATION OF THE BOVINE CUMULUS-OOCYTE COMPLEX
}

\author{
T. S. Hussein, R. B. Gilchrist, and J. G. Thompson \\ Research Centre for Reproductive Health, Department of Obstetrics and Gynaecology, University of Adelaide, Adelaide, \\ South Australia, Australia
}

Paracrine factors secreted by the oocyte (oocyte-secreted factors, OSFs) regulate a broad range of cumulus cell functions including proliferation, differentiation, and apoptosis. The capacity of oocytes to regulate their own microenvironment by OSFs may in turn contribute to oocyte developmental competence. The aim of this study was to determine if OSFs have a direct influence on bovine oocyte developmental competence during in vitro maturation (IVM). Cumulus-oocyte complexes (COCs) were obtained by aspiration of $>3-\mathrm{mm}$ follicles from abattoir-derived ovaries. IVM was conducted in Bovine VitroMat (Cook Australia, Eight Mile Plains, Brisbane, Australia) supplemented with $0.1 \mathrm{IU} / \mathrm{mL}$ rhFSH for $24 \mathrm{~h}$ under $6 \%$ $\mathrm{CO}_{2}$ in air at $38.5^{\circ} \mathrm{C}$. In the first experiment, $\mathrm{COCs}$ were co-cultured with denuded oocytes (DOs, $5 / \mathrm{COC}$ in $10 \mu \mathrm{L}$ ) beginning at either 0 or 9-h of IVM. To generate the 9-h DO group, COCs were first cultured intact for 9-h and then denuded. In the second experiment, specific OSFs, recombinant bone morphogenetic protein-15 (BMP-15) and growth differentiation factor 9 (GDF-9), were prepared as partially purified supernatants of transfected $293 \mathrm{H}$ cells, and used as $10 \% \mathrm{v} / \mathrm{v}$ supplements in Bovine VitroMat. Treatments were: (1) control (no supplement), (2) BMP-15, (3) GDF-9, (4) BMP-15 and GDF-9, and (5) untransfected 293H control. Following maturation, in vitro production of embryos was performed using the Bovine Vitro system (Cook Australia) and blastocysts were examined on Day 8 for development. Developmental data were arcsinetransformed and analyzed by ANOVA, followed by Tukey's test. Cell numbers were analyzed by ANOVA. Co-culturing intact COCs with DOs from 0 or $9 \mathrm{~h}$ did not affect cleavage rate, but increased $(P<0.001)$ the proportion of cleaved embryos that reached the blastocyst stage post-insemination $(50.6 \pm 1.9$ and $61.3 \pm 1.9 \%$, respectively), compared to COCs cultured alone $(40.7 \pm 1.4 \%)$. Therefore, paracrine factors secreted by DOs increased the developmental competence of oocytes matured as COCs. OSFs also improved embryo quality, as co-culture of COCs with DOs $(0$ or $9 \mathrm{~h})$ significantly increased total cell ( $156.1 \pm 1.3$ and $159.1 \pm 1.3$, respectively) and trophectoderm (105.7 \pm 1.3 and $109.8 \pm 0.4$, respectively) numbers, compared to control COCs (total $=148 \pm 1.2$, trophectoderm $=98.2 \pm 0.8, P<0.001)$. BMP-15 alone or with GDF-9 also significantly $(P<0.001)$ increased the proportion of oocytes that reached the blastocyst stage post insemination $(57.5 \pm 2.4 \%$ and $55.1 \pm 4.5 \%$, respectively), compared to control $(41.0 \pm 0.9 \%)$ and $293 \mathrm{H}$-treated $(27.1 \pm 3.1 \%)$ COCs. GDF-9 also increased blastocyst yield $(49.5 \pm 3.9 \%)$ but not significantly. These results are the first to demonstrate that OSFs, and particularly BMP-15 and GDF-9, directly affect bovine oocyte developmental competence. These results have far-reaching implications for improving the efficiency of IVM in domestic species and human infertility treatment, and support the role of OSF production by oocytes as a diagnostic marker for developmental competence.

\section{EPIDERMAL GROWTH FACTOR ENHANCES MEIOTIC MATURATION OF CANINE OOCYTES IN THE PRESENCE OF BSA}

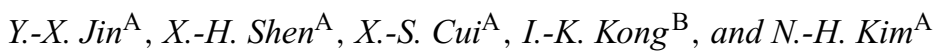 \\ ${ }^{A}$ Department of Animal Sciences, Chungbuk National University, Cheongju, Chungbuk, South Korea; \\ ${ }^{B}$ Department of Animal Sciences and Technology, Sunchon National University, Suncheon, Chonnam, South Korea
}

Despite many attempts to improve the in vitro maturation (IVM) of canine oocytes using various culture conditions, the efficiency of canine IVM remains very low compared with that of other domestic animals. In the present study we examined the effect of (1) ovarian estrous stage on oocyte quality, and (2) epidermal growth factor (EGF) in the presence and absence of macromolecules on the IVM of canine oocytes. Reproductive tracts from normal, client-owned bitches aged approximately 7 months were collected after routine ovariohysterectomy at private veterinary clinics. The 
ovaries were removed, washed free of blood with PBS, and repeatedly cut with a scalpel blade at room temperature in HEPES-buffered Tyrodes medium containing $0.1 \%(\mathrm{w} / \mathrm{v})$ polyvinyl alcohol. Each group of 10 cumulus-oocyte complexes (COCs) was matured in North Carolina State University (NCSU)-17 medium supplemented with $1 \mu \mathrm{g} / \mathrm{mL} \mathrm{FSH}, 10 \mathrm{IU} / \mathrm{mL} \mathrm{hCG}$, and $1 \mu \mathrm{g} / \mathrm{mL} \beta$-estradiol under paraffin oil for 48 or $72 \mathrm{~h}$ at $37^{\circ} \mathrm{C}$. Follicular ovaries contained the largest number of oocytes (more than 300 ), and the number of oocytes $\geq 100 \mu \mathrm{m}$ in diameter was also significantly greater in follicular ovaries than in ovaries at other stages $(P<0.001)$. Luteal ovaries had the smallest number of both total and large oocytes. After $72 \mathrm{~h}$ of culture, significantly more oocytes recovered from follicular ovaries were in GVBD than oocytes from anestrous or luteal ovaries. After $72 \mathrm{~h}$ of culture, oocytes recovered from follicular ovaries had a significantly lower percentage reaching the GV stage (59.7 $\pm 5.2 \%)$ and more in GVBD $(40.3 \pm 3.1 \%)$ compared with both anestrous (GV, 75.1 $\pm 5.2 \%$; GVBD, $24.9 \pm 3.1 \%)$ and luteal (GV, 79.8 $\pm 5.2 \%$; GVBD, $22.0 \pm 3.1 \%)$ ovaries $(P<0.05)$. However, maturation to MI/MII was not statistically different between the stages of the estrous cycle. BSA or FBS supplementation did not improve meiotic maturation when compared to PVA supplementation. In the presence of $100 \mathrm{nM}$ epidermal growth factor (EGF), either FBS or BSA enhanced the proportion of oocytes in GVBD/MI (59.7 $\pm 2.2 \%$ [FBS] and $51.7 \pm 2.2 \%$ [BSA] vs. $36.1 \pm 2.2 \%$ [PVA], $P<0.05)$. In addition, $0.4 \%$ BSA significantly increased the percentage of oocytes in MII $(18.5 \pm 0.8 \%)$ compared to $10 \%$ FBS $(7.1 \pm 0.8 \% ; P<0.05)$. These results suggest that the estrous cycle of bitches influences the meiotic maturation of oocytes cultured in vitro, and EGF increases the meiotic maturation of canine oocytes in the presence of BSA in vitro.

This work was funded by a grant from the Ministry of Science and Technology of Korea and the National Research Laboratory Program.

\title{
329 SUPPRESSION OF BOVINE OOCYTE IVM BY SERUM AND FSH DEPRIVATION OR BY $\alpha$-AMANITIN: EFFECT ON FERTILIZATION AND EMBRYO DEVELOPMENT
}

\author{
K. Kananen-Anttila ${ }^{\mathrm{A}}$, M. Eronen ${ }^{\mathrm{A}}$, J. Matilainen $^{\mathrm{A}}$, M. Kallio $^{\mathrm{B}}$, J. Peippo $^{\mathrm{C}}$, and M. Halmekytö ${ }^{\mathrm{A}}$
}

${ }^{A}$ University of Kuopio, Institute of Applied Biotechnology, FIN-10211 Kuopio, Finland; ${ }^{B}$ VTT Biotechnology, FIN-10521 Turku, Finland;

${ }^{\mathrm{C}}$ MTT Agrifood Research Finland, Animal Production Research, FIN-11600 Jokioinen, Finland

We have studied the effect of suppressed IVM on the developmental competence of bovine oocytes, aiming at elucidating the importance of cytoplasmic maturation in fertilization and embryo development. Six replicates of abattoir-derived oocytes were randomly divided into three IVM groups. Control ( $n=950)$ : TCM-199 with glutamax-I (Gibco, Grand Island, NY, USA), $0.25 \mathrm{mM}$ Na-pyruvate, $100 \mathrm{IU} \mathrm{mL}^{-1}$ penicillin and $100 \mu \mathrm{g} \mathrm{mL}^{-1}$ streptomycin, $50 \mathrm{ng} \mathrm{mL}^{-1} \mathrm{FSH}$, and $10 \%$ fetal bovine serum (FBS) (Gibco); Serum+FSH-free $(n=944)$ : same as control but without FSH and FBS; $\alpha$-amanitin $(n=977)$ : same as control but with $10 \mu \mathrm{g} \mathrm{mL}^{-1} \alpha$-amanitin. Nuclear maturation of oocytes was studied $24 \mathrm{~h}$ after the onset of IVM, the formation of sperm aster structure 10 hours post-insemination (hpi) and the formation of pronuclei $20 \mathrm{hpi}$. Sperm aster was visualized with $\beta$-tubulin antibody (modified from Navara et al. 1999 Dev. Biol. 162, 29-40). Presumptive zygotes were cultured until Day 7 in modified SOFaaci $+4 \mathrm{mg} \mathrm{mL}^{-1}$ fatty acid-free BSA in $5 \% \mathrm{O}_{2}$. Cumulus cell expansion was seen only in the control group. The results of nuclear maturation, fertilization, and embryo development are summarized in Table 1. Serum and FSH deprivation did not have a statistically significant effect on the parameters studied (vs. control). $\alpha$-amanitin exposure during IVM reduced nuclear maturation, fertilization, and Day 3 embryo cleavage vs. control, and resulted in total blockage of Day 7 blastocyst development. The treatment groups had significantly smaller mean diameters of male pronuclei (control: $14 \pm 0.6 \mu \mathrm{m}$; serum+FSH-free: $12 \pm 0.5 \mu \mathrm{m}, P<0.05 ; \alpha$-amanitin: $10 \pm 0.6 \mu \mathrm{m}, P<0.001$ ) and sperm asters (control: $86 \pm 4 \mu \mathrm{m}$; serum+FSH-free: $82 \pm 4 \mu \mathrm{m}, P<0.01 ; \alpha$-amanitin: $49 \pm 7 \mu \mathrm{m}, P<0.001)$ (nonparametric Kruskall Wallis and Mann-Whitney $\mathrm{U}$ tests) vs. control group. Despite reduction in pronucleus and sperm aster diameter, serum and FSH deprivation during IVM did not affect in vitro developmental competence of bovine oocytes, suggesting a need for re-evaluation of the components of IVM. $\alpha$-Amanitin exposure in IVM disturbed nuclear maturation, fertilization, and embryo development, indicating the essence of early transcription.

Table 1. Average percentages $\pm(n)$ for nuclear maturation, fertilization (min two pronuclei), embryo cleavage, and blastocyst development

\begin{tabular}{lccccc}
\hline Treatment & Nuclear maturation & Fertilized oocytes & Day 3 cleagave & Day 7 blastocysts & Grade I blastocysts \\
\hline Control $(n)$ & $81 \pm 4^{\mathrm{a}}(111)$ & $95 \pm 3^{\mathrm{a}}(117)$ & $75 \pm 4^{\mathrm{a}}(722)$ & $30 \pm 4^{\mathrm{a}}(676)$ & $56 \pm 6^{\mathrm{a}}(202)$ \\
Serum+FSH-free $(n)$ & $73 \pm 6^{\mathrm{a}}(98)$ & $81 \pm 8^{\mathrm{a}}(124)$ & $78 \pm 2^{\mathrm{a}}(722)$ & $23 \pm 3^{\mathrm{a}}(676)$ & $45 \pm 3^{\mathrm{a}}(145)$ \\
$\alpha$-amanitin $(n)$ & $32 \pm 5^{\mathrm{d}}(109)$ & $55 \pm 7^{\mathrm{c}}(124)$ & $50 \pm 3^{\mathrm{d}}(744)$ & $0(593)$ & $0(0)$ \\
\hline
\end{tabular}

${ }^{\mathrm{a}-\mathrm{d}}$ Values with different superscripts within a column are significantly different $\left(P<0.01\right.$ for $^{\mathrm{a}-\mathrm{c}}, P<0.001$ for ${ }^{\mathrm{a}-\mathrm{d}}$ vs. control; univariate ANOVA and Dunett $t$-test).

\section{THE PROTEIN EXPRESSION PROFILE (SECRETOME) OF INDIVIDUAL CUMULUS-OOCYTE COMPLEXES DURING IVM IN THE MOUSE IS AFFECTED BY FSH}

\author{
M. G. Katz-Jaffe, C. Sheehan, W. B. Schoolcraft, and D. K. Gardner \\ Colorado Center for Reproductive Medicine, Englewood, CO 80110, USA
}

Studies of the protein expression profile into the surrounding medium (secretome) of in vitro-matured cumulus-oocyte complexes (COCs) have the potential to elucidate biochemical pathways involved in oogenesis, including the complex dialogue between the oocyte and its supporting cells. 
The understanding of these processes should assist in improving IVM success and fertility outcome, as early embryo development reflects the quality of the oocyte and its cumulus cells. Through the analysis of the individual COC secretome, we have investigated the effects of adding follicle stimulating hormone (FSH) to a defined maturation medium during IVM. COCs were collected from 3-week-old female mice $(\mathrm{C} 57 \mathrm{BL} / 6 \times \mathrm{CBA})$ $48 \mathrm{~h}$ post-pregnant mare serum gonadotropin (PMSG) (5/iu) injection. Individual COCs were cultured in $5-\mu \mathrm{L}$ drops of a defined maturation medium $(0.25 \mathrm{mg} / \mathrm{mL}$ recombinant albumin) with the addition of $0,2,20$, or $200 \mathrm{ng} / \mathrm{mL} \mathrm{FSH}$, under oil for $17 \mathrm{~h}$. Oocytes were denuded and maturity recorded Each microdrop of media ( $n=8$ oocytes per group) was collected, processed through an optimized series of buffers and washes prior to analysis by time-of-flight mass spectrometry (TOF-MS). Differential protein expression profiles were obtained from the secretome of individual COCs producing MII oocytes after maturation in differing doses of FSH. Statistical analysis revealed significant differences observed across 10 proteins/biomarkers with mass-to-charge $(\mathrm{m} / \mathrm{z})$ ratios ranging from 2.7 to $6 \mathrm{kDa}$ (Mann-Whitney non-parametric test; $P<0.05$ ). In addition, hierarchical and horizontal clustering analysis identified unique clusters of both up-regulated and down-regulated proteins/biomarkers within the $\mathrm{m} / \mathrm{z}$ range of 2 to $18 \mathrm{kDa}$ in the $2 \mathrm{ng} / \mathrm{mL}$ FSH group. Several of the individual COCs from the $20 \mathrm{ng} / \mathrm{mL}$ FSH group were also clustered alongside the $2 \mathrm{ng} / \mathrm{mL}$ group with similar protein expression profiles. In contrast, COCs cultured in the presence of $0 \mathrm{ng} / \mathrm{mL}$ and $200 \mathrm{ng} / \mathrm{mL} \mathrm{FSH}$ were observed to cluster as a separate branch with distinctly different protein expression profiles. This study has determined for the first time the secretome profiles of individual COCs after IVM. These data have shown that the FSH dose in a defined maturation medium affects the secretome of an individual COC. Further investigation is currently underway to characterize these protein differences. The development of this proteomics approach will assist in revealing the intricate cellular function of an individual COC and elucidate critical pathways involved in mammalian oocyte maturation.

\title{
331 DEVELOPMENT OF CANINE SYNTHETIC OVIDUCT FLUID (cSOF) MEDIUM AND EFFECT OF THE CSOF IN IN VITRO NUCLEAR MATURATION OF CANINE OOCYTES
}

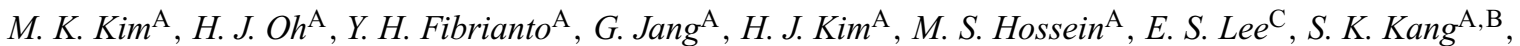 \\ B. C. Lee $e^{\mathrm{A}, \mathrm{B}}$, and W.S. Hwang ${ }^{\mathrm{A}, \mathrm{B}}$
}

A Department of Theriogenology and Biotechnology, College of Veterinary Medicine, Seoul National University, Kwanak-gu, Seoul, Korea;

${ }^{\mathrm{B}}$ The Xenotransplantation Research Center, Seoul National University Hospital, Seoul, Korea;

${ }^{\mathrm{C}}$ Department of Theriogenology, Kangwon National University, Chuncheon, Kangwon-do, Korea

A bitch ovulates a primary oocyte that undergoes both maturation and fertilization within the oviduct fluid for 3 days. In an attempt to define the physiologically appropriate conditions for oocyte maturation in the bitch, in vitro conditions based upon the oviductal environment need to be established. The present study was conducted to develop canine synthetic oviduct fluid (cSOF) by investigating the composition of canine oviduct fluid, follicular fluid, and bursa fluid. The bursa and oviduct fluid were collected at Days 1 and 3 of ovulation, respectively. Before ovulation, follicles were punched and the fluid was collected by aspiration. Biochemical parameters ( $\mathrm{Ca}, \mathrm{P}, \mathrm{Mg}$, albumin, total protein, and glucose) were measured using a chromatographic enzymic method. Quantitative determination of electrolytes $(\mathrm{Na}, \mathrm{Cl}, \mathrm{K})$ concentration in the follicular, bursa, and oviductal fluids was performed using an Electrolyte 5 Analyzer (Nora Biomedical, Waltham, MA, USA). The concentrations of sodium, potassium, and chloride were similar among oviduct (153.5, 5.2, and $121.5 \mathrm{mmol} / \mathrm{L}$, respectively), bursa $(149.5,4.3$, and $123 \mathrm{mmol} / \mathrm{L}$, respectively), and follicular (147, 4.2, and $120.5 \mathrm{mmol} / \mathrm{L}$, respectively) fluids. Glucose concentration was different in oviduct, bursa, and follicular fluids $(1.09,3.75$, and $3.94 \mathrm{mmol} / \mathrm{L}$, respectively). Total protein and magnesium concentrations were not different among the three fluids, but phosphorus concentration differed in oviduct, bursa, and follicular fluids $(0.001,0.044$, and $0.024 \mathrm{~g} / \mathrm{L}$, respectively). The oviduct fluid showed lower concentrations of albumin and calcium $(0.001 \mathrm{~g} / \mathrm{L}$ and $1.372 \mathrm{mmol} / \mathrm{L}$, respectively) compared to bursa $(0.023 \mathrm{~g} / \mathrm{L}$ and $2.532 \mathrm{mmol} / \mathrm{L}$, respectively) or follicular fluid $(0.025 \mathrm{~g} / \mathrm{L}$ and $2.632 \mathrm{mmol} / \mathrm{L}$, respectively). The $\mathrm{CSOF} 1$ and $\mathrm{CSOF} 2$ were developed on the basis of the oviduct and follicular fluids, respectively. Canine oocytes were recovered by slicing ovaries collected after ovariohysterectomy in bitches at follicular stages, and in vitro nuclear maturation of canine oocytes cultured in $\mathrm{CSOF} 1$ or cSOF2 were compared to that of intra-oviduct (in vivo) culture. For in vivo culture, the canine oocytes were transferred and cultured in intra-oviduct for $72 \mathrm{~h}$ and were recovered by intra-oviduct flushing using surgical methods. For in vitro culture, canine oocytes were cultured in cSOF1, cSOF2, or TCM-199 (control) for $72 \mathrm{~h}$ at $38.5^{\circ} \mathrm{C}$ in a humidified atmosphere of $5 \% \mathrm{CO}_{2}$ in air. The experiment was replicated three times and statistical analysis was carried out by ANOVA with GLMs in the statistical analysis system program (SAS Institute, Inc., Cary, NC, USA). Nuclear maturation of canine oocytes to MII was not different in cSOF1, cSOF2, or intra-oviduct $(2.5 \%, 2.5 \%$, and $2.2 \%$, respectively) compared to the control $(1.6 \%)$. However, the degeneration rates were significantly higher in oocytes cultured in intra-oviduct (48.2\%) compared to control, $\mathrm{CSOF} 1$, and $\mathrm{cSOF} 2(1.6 \%, 6.8 \%$, and $7.5 \%$, respectively). In conclusion, the present study analyzed the components of the oviduct, bursa, and follicular fluids and developed two canine synthetic oviduct fluids (cSOF1 and 2). In addition, the present study demonstrated that cSOFs can be used for in vitro maturation of canine oocytes.

\section{EMBRYONIC DEVELOPMENT AFTER SOMATIC CELL NUCLEAR TRANSFER OF PORCINE OOCYTES MEIOTICALLY INHIBITED WITH ROSCOVITINE}

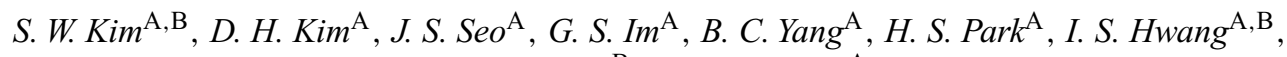 \\ H. T. Cheong ${ }^{\mathrm{B}}$, and B. S. Yang ${ }^{\mathrm{A}}$ \\ A Animal Biotechnology Division, National Livestock Research Institute, Suwon, South Korea \\ ${ }^{\mathrm{B}}$ College of Animal Resources, Kangwon National University, Chuncheon, South Korea
}

Numerous factors affect on the developmental competence of cloned embryos, and one of the factors might be the disturbed synchronization of nuclear and cytoplasm maturation. Roscovitine, a purine known to specifically inhibit M-phase promoting factor (MPF) kinase activity by blocking 
the ATP in numerous cell systems, has been successfully used in maintaining porcine oocytes at GV stage without affecting their developmental potential. However, developmental ability of roscovitine treated porcine oocytes after nuclear transfer has not been evaluated. The purpose of this study was to examine the development of nuclear transferred porcine embryos after meiotic inhibition with roscovitine (ROS). Cumulus-oocyte complexes (COCs) were collected from antral follicles of slaughtered prepubertal gilts. COCs were cultured in pre-maturation medium (TCM-199 containing $50 \mu \mathrm{M}$ Roscovitine) for $24 \mathrm{~h}$, and then further cultured in conventional maturation medium for $44 \mathrm{~h}$. A control group was cultured in the maturation medium for $44 \mathrm{~h}$. Matured oocytes were enucleated and a porcine fetus cell was inserted into each enucleated oocyte. Couplets were simultaneously fused and activated with electric pulse of two $1.2 \mathrm{kV} / \mathrm{cm}$ for $30 \mu \mathrm{s}$. Nuclear transferred (NT) embryos were cultured in PZM-1 medium for 6 days (five replicates). Apoptotic cell death was analyzed by using a TUNEL assay and total cell number was examined by Hoechest 33342 counterstaining. At $3 \mathrm{~h}$ after fusion, NT embryos were fixed for microfilament staining. Data were analyzed by ANOVA and Student's $t$-test. The rates of fusion, cleavage, and blastocyst formation of the ROS-treated group (85, 68, and 18\%, respectively) after nuclear transfer did not differ from control $(78,76$, and $16 \%$, respectively). The cell number in blastocysts of the ROS-treated group (30.8 \pm 10.6$)$ was significantly lower than that of the control $(42.3 \pm 13.7)(P<0.01)$, but the mean proportion of apoptotic cells was not different between the two groups $(6.9 \pm 7.1$ and $4.8 \pm 4.9 \%$ for control and ROS group, respectively). Recovery of microfilaments after fusion was delayed in NT embryos derived from ROS-treated oocytes. This study demonstrated that porcine oocytes pre-cultured for $24 \mathrm{~h}$ in presence of roscovitine can be developed to blastocysts after somatic cell nuclear transfer. This could provide flexibility for studying porcine oocyte development and embryo cloning.

\title{
333 IN VIVO PREMATURATION AFFECTS PROTIEN SYNTHESIS AT THE BEGINNING OF IN VITRO MATURATION
}

\author{
H. M. Knijn, P. L. A. M. Vos, S. J. Dieleman, and P. J. M. Hendriksen \\ Faculty of Veterinary Medicine, Department of Farm Animal Health, Utrecht, The Netherlands
}

The cumulus-oocyte complex (COC) from a preovulatory follicle is better equipped than a COC from a small healthy follicle to develop into a blastocyst. This might be due to the presence of mRNAs and proteins in the oocyte and/or cumulus cells from which the expression is increased or induced during the prematuration period. There are several reports that beginning atresia enhances in vitro oocyte competence and the processes of atresia show similarities with prematuration on an ultrastructural level. Therefore, we investigated the effect of in vivo prematuration and atresia on the protein synthesis in bovine oocytes collected from preovulatory and small follicles during the first period of IVM. To study the follicular population of an undisturbed cycle we synchronized 14 cows with a norgestomet ear implant. We performed progesterone and LH analysis (Hendriksen et al. 2003 Biol. Reprod. 69, 2036-2044) to determine the start of luteolysis and the LH surge. At 48-62 h after the start of luteolysis, 10 cows were ovariectomized (OVX) and were considered to be shortly before the LH surge. At 1.5-3 days after the LH surge, at the beginning of a new cycle, four cows were OVX. From the ovaries collected before the LH surge the preovulatory follicle (POF) and all follicles $\geq 3 \mathrm{~mm}$ were dissected. From the ovaries collected at the start of a new cycle all follicles $\geq 3 \mathrm{~mm}$ were dissected. All follicles were classified for degree of atresia as non-atretic (NA), light atretic (LA), and atretic (A) by the proportion of apoptotic granulosa cells and levels of estradiol and progesterone, and of small IGF-binding proteins (IGFBPs) in the follicular fluid (Hendriksen et al. 2003). The COCs were individually incubated for $4 \mathrm{~h}$ in maturation medium containing ${ }^{35} \mathrm{~S}$-labeled methionine/cysteine (Amersham-Pharmacia, Sweden). After separation of oocyte and cumulus cells, the proteins produced in the oocyte were analyzed by 1-D electrophoresis (SDS-PAGE, ExcelGel-12.5\%, Amersham-Pharmacia). Gels were dried and digitized images were obtained by use of a Phosphor Imaging System (Molecular Imager, Bio-Rad, Hercules, CA, USA). Twenty-eight different proteins were distinguished. To compare different oocytes, the ${ }^{35} \mathrm{~S}$ incorporation per protein band was calculated as proportion of the ${ }^{35} \mathrm{~S}$ incorporation of all bands of the oocyte. The total amount of newly synthetized proteins per oocyte was normalized to the oocyte on the gel with the highest incorporation of ${ }^{35} \mathrm{~S}$. In all POF oocytes $(n=7)$, the total amount of newly synthetized protein was lower than that in the oocytes from the NA and LA follicles (Table 1). This suggests that already during in vivo prematuration new proteins were synthesized in POFs and therefore less protein synthesis occurs during maturation. We can speculate that oocytes from small follicles have to synthesize many proteins during the beginning of IVM to make up for what is normally produced during the prematuration period. In atretic oocytes fewer proteins were synthesized, which suggests that these oocytes have either undergone prematuration-like processes or are less viable. Nevertheless, some proteins remain to be synthesized in POFs, such as, for example, protein number ' $p$ 23'.

Table 1. New proteins at the onset of IVM in oocytes from small follicles and POFs

\begin{tabular}{|c|c|c|c|c|}
\hline Protein number & $\mathrm{NA}(n=33)$ & LA $(n=24)$ & $\mathrm{A}(n=45)$ & POF $(n=7)$ \\
\hline $\mathrm{P} 4 \mathrm{a}$ & $7.9 \pm 7.9 \%{ }^{\mathrm{a}}$ & $0.8 \pm 0.6 \%{ }^{\mathrm{a}}$ & $1.5 \pm 0.8 \%{ }^{b}$ & $1.4 \pm 1.1 \%{ }^{\mathrm{ab}}$ \\
\hline $\mathrm{P} 4 \mathrm{a}$ & $7.9 \pm 7.9 \% \mathrm{o}^{\mathrm{a}}$ & $0.8 \pm 0.6 \%{ }^{\mathrm{a}}$ & $1.5 \pm 0.8 \% \mathrm{o}^{\mathrm{b}}$ & $1.4 \pm 1.1 \% \mathrm{o}^{\mathrm{ab}}$ \\
\hline $\mathrm{P} 4 \mathrm{a}$ & $7.9 \pm 7.9 \% \%^{\mathrm{a}}$ & $0.8 \pm 0.6 \% \%^{\mathrm{a}}$ & $1.5 \pm 0.8 \% \%^{\mathrm{b}}$ & $1.4 \pm 1.1 \% \%^{\mathrm{ab}}$ \\
\hline $\mathrm{P} 4 \mathrm{a}$ & $7.9 \pm 7.9 \% \%^{\mathrm{a}}$ & $0.8 \pm 0.6 \% \%^{\mathrm{a}}$ & $1.5 \pm 0.8 \% \%^{\mathrm{b}}$ & $1.4 \pm 1.1 \% \%^{\mathrm{ab}}$ \\
\hline $\mathrm{P} 4 \mathrm{a}$ & $7.9 \pm 7.9 \% \%^{\mathrm{a}}$ & $0.8 \pm 0.6 \%{ }^{\mathrm{a}}$ & $1.5 \pm 0.8 \%$ b & $1.4 \pm 1.1 \% \%^{\mathrm{ab}}$ \\
\hline $\mathrm{P} 4 \mathrm{a}$ & $7.9 \pm 7.9 \% \%^{\mathrm{a}}$ & $0.8 \pm 0.6 \%{ }^{\mathrm{a}}$ & $1.5 \pm 0.8 \%{ }^{\mathrm{b}}$ & $1.4 \pm 1.1 \% \%^{\mathrm{ab}}$ \\
\hline $\mathrm{P} 4 \mathrm{a}$ & $7.9 \pm 7.9 \% \%^{\mathrm{a}}$ & $0.8 \pm 0.6 \%{ }^{\mathrm{a}}$ & $1.5 \pm 0.8 \%{ }^{\mathrm{b}}$ & $1.4 \pm 1.1 \%^{\mathrm{ab}}$ \\
\hline $\mathrm{P} 4 \mathrm{a}$ & $7.9 \pm 7.9 \% \%^{\mathrm{a}}$ & $0.8 \pm 0.6 \% \%^{\mathrm{a}}$ & $1.5 \pm 0.8 \% \%^{\mathrm{b}}$ & $1.4 \pm 1.1 \% \%^{\mathrm{ab}}$ \\
\hline
\end{tabular}

${ }^{\mathrm{a}-\mathrm{c}}$ Means $( \pm \mathrm{SD})$ with different superscripts within a row differ significantly $(P<0.05$, one-way ANOVA, Bonfferoni). 


\title{
334 EFFECT OF INSULIN ON IN VITRO MATURATION OF CANINE OOCYTES
}

\author{
H. S. Lee, Y. I. Seo, X. J. Yin, S. G. Cho, I. H. Bae, D. H. Oh, and I. K. Kong \\ Sunchon National University, Sunchon, JeonNam Province, South Korea
}

In spite of our increased knowledge of in vitro oocyte maturation techniques, the success rate of obtaining mature canine oocytes in vitro remains very low compared with that for other domestic animals. The inefficient rate of meiotic resumption of canine oocytes is probably due to both the unique reproductive cycle and inappropriate in vitro maturation (IVM) medium. In an unpublished experiment, we found that the concentration of insulin was higher in estrus bitch serum (EBS; $8833 \mathrm{pg} / \mathrm{mL}$ ) than in dog follicular fluid (DFF; preovulatory follicle, $122 \mathrm{pg} / \mathrm{mL}$ ), which implies its possible role in the acquisition of oocyte competence. Therefore, in the present study we investigated the effects of supplementing the IVM medium with insulin on the incidence of maturation to metaphase II. Ovaries were collected from various stages of the estrous cycle by ovariohysterectomy, and oocytes with two or more intact cumulus layers and with a diameter $>110 \mu \mathrm{m}$ were selected and used for IVM. Oocytes were cultured in modified synthetic oviduct fluid (2004 Reprod. Nutr. Dev. 44, 105-109) supplemented with 10\% EBS, $20 \mu \mathrm{g} / \mathrm{mL}$ estradiol, and different concentrations of insulin $(0,10,100$, or $1000 \mathrm{ng} / \mathrm{mL}$ ) at $38.5^{\circ} \mathrm{C}, 5 \% \mathrm{CO}_{2}$ in air. After $72 \mathrm{~h}$, cumulus cells were removed from around oocytes using a small glass pipette. Denuded oocytes were fixed in $3.7 \%$ paraformaldehyde supplemented with $10 \mu \mathrm{g} / \mathrm{mL}$ Hoechst 33342 at room temperature for $40 \mathrm{~min}$. Nuclear status was observed under UV light using a fluorescence microscope. The percentage of oocytes at the metaphase II stage was not different among the four groups- $6.8,1.8,5.4$, and $2.1 \%$ in the control, 10,100 , and $1000 \mathrm{ng} / \mathrm{mL}$ insulin groups, respectively. The incidence of oocytes with pronuclear-like structures or cleaving beyond the two-cell stage was not significant higher in the 10 and $100 \mathrm{ng} / \mathrm{mL}$ insulin treatment groups than in the control and $1000 \mathrm{ng} / \mathrm{mL}$ insulin groups20.0 and $19.6 \%$ vs. 6.8 and $6.4 \%$, respectively. These results indicate that the addition of insulin to the in vitro maturation medium of dog oocytes had no effect on the incidence of meiotic maturation to metaphase II, nor did it affect the frequency of occurrence of spontaneous oocyte activation.

\section{DEVELOPMENTAL ABILITY OF PIG OOCYTES TREATED WITH EGF DURING} IN VITRO MATURATION

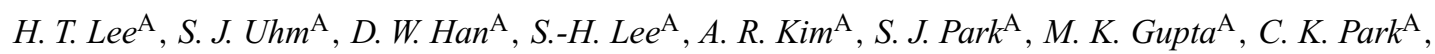 \\ H. M. Chung ${ }^{\mathrm{B}}$, Y. B. Kim ${ }^{\mathrm{A}}$, and K.S. Chung ${ }^{\mathrm{A}}$
}

${ }^{A}$ Department of Animal Science, Konkuk University, Seoul, Korea; ${ }^{B}$ College of Medicine, Pochon CHA University, Seoul, Korea

Epidermal growth factor (EGF) is a major follicular factor affecting maturation of oocyte in many species. The insulin-like growth factor II (IGF2) gene is an imprinted gene in embryonic development that functions primarily as a regulator of cell growth and differentiation. Thus, this study examined the maturation and developmental ability of in vitro-fertilized (IVF) pig immature oocytes cultured in maturation medium supplemented with EGF. The blastocysts derived from these oocytes were further examined for the expression level of IGF2 as a cell survival activity. Pig immature oocytes were cultured in TCM-199 medium (with no supplement) with or without $10 \mathrm{ng} / \mathrm{mL}$ EGF for $42-14 \mathrm{~h}$, and then matured oocytes were coincubated with $5 \times 10^{5}$ sperm $/ \mathrm{mL}$ in modified Tris-buffered medium containing $1 \mathrm{mM}$ caffeine sodium benzonate and $0.1 \%$ bovine serum albumin (BSA) for $6 \mathrm{~h}$ for IVF. Subsequently, embryos were cultured in $50 \mu \mathrm{L}$ of NCSU-13 containing $0.4 \%$ BSA for 7 days at $39^{\circ} \mathrm{C}$ in a humidified atmosphere of $5 \% \mathrm{CO}_{2}$ in air. Total cell numbers in blastocysts were examined by fluorescence staining with Hoescht 33342, and the expression level of IGF2 was analyzed with a fluorescence-monitored quantitative real-time reverse transcriptase-polymerase chain reaction method. We found that pig oocytes matured with EGF showed significant improvement of their development ability (Table 1). Presence of EGF in TCM-199 medium significantly increased $(P<0.05)$ the rates of maturation, sperm penetration, male pronucleus (MPN) formation, cleavage, and blastocyst formation. Furthermore, blastocysts derived from oocytes cultured with EGF were 24-fold higher in the relative expression level of IGF2 than those without EGF. Therefore, these data suggest that pig oocytes matured in medium supplemented with EGF increases the developmental ability and cell viability during cell divisions following IVF. In conclusion, EGF may increase cytoplasmic as well as nuclear maturation of pig immature oocytes.

Table 1. Improvement of developmental ability of pig oocytes matured with EGF

\begin{tabular}{|c|c|c|c|c|c|c|c|}
\hline \multirow[t]{2}{*}{ Treatment } & \multicolumn{2}{|c|}{$\%$ of maturation $(n)$ after } & \multicolumn{2}{|c|}{$\%$ of fertilization $(n)$} & \multicolumn{2}{|c|}{$\%$ of culture $(n)$} & \multirow{2}{*}{$\begin{array}{l}\text { Total cell no./ } \\
\text { blastocyst }(n)\end{array}$} \\
\hline & $24 \mathrm{~h}$ & $44 \mathrm{~h}$ & sperm penetration & MPN formation & cleavage & blastocyst & \\
\hline Control & $4.3(185)$ & 26.7 (196) & $7.8(190)$ & $22.2(15)$ & $24.6(256)$ & $1.2(256)$ & $27.3 \pm 11.5(3)$ \\
\hline EGF & $12.4 *(201)$ & $83.3 *(241)$ & $83.2 *(188)$ & $62.8 *(158)$ & $47.6 *(250)$ & $5.2 *(250)$ & $41.0 \pm 12.9 *(10)$ \\
\hline
\end{tabular}

*Significantly different from other values within the column $(P<0.05$, chi-square). Experiments were repeated three times.

This work was supported by the Research Project on the Production of Bio-organs, Ministry of Agriculture and Forestry, Republic of Korea.

\section{SHEEP OOCYTES MATURED IN A SIMPLEX MEDIUM (HECM-1), AN ANSWER TO IN VITRO FERTILIZATION}

\author{
C. Navarro-Maldonado, Y. Ducolomb-Ramirez, A. Galindo-Rodriguez, and A. Rosado-Garcia \\ Universidad Autonoma Metropolitana-Iztapalapa, Mexico City, Iztapalapa, Mexico
}

In vitro maturation and in vitro fertilization (IVM and IVF) of mammalian oocytes still show unsatisfactory results when applied to the study of embryo development. This is probably due to inadequate information about the use of media components and supplements for oocyte maturation 
and to a discrepancy between results obtained by focusing strictly on oocyte maturation and those that are focused on IVF. A conventional medium that provides adequate results in studies of oocyte maturation (TCM-199) contains hypoxanthine, phosphate ions, and glucose, all known to inhibit embryo development in vitro in some species. In contrast, it has been shown that a simpler medium (HECM-9) increases embryo development in bovine although its use for oocyte maturation has not been defined. This medium contains taurine (an amino acid that reduces intracellular peroxide content) and is supplemented with polyvinyl alcohol (PVA) instead of using protein components, making it a simple defined medium that reduces variability in embryo development. Adding sodium panthothenate to media also confers cell protection against reactive oxygen species. Finally, supplements such as epidermal growth factor (EGF) increase the number of oocytes that complete maturation (Metaphase II, MII) and facilitate embryo development. An adequate combination of our knowledge about in vitro maturation and fertilization of oocytes, together with the requirements for embryo development, is important for the preparation of culture media to study regulatory mechanisms for embryo development and to increase the number of viable and normal term individuals. In this study we compared the effects of HECM- 9 (containing panthothenate) vs. TCM-199 (both media supplemented with PVA, EGF, and FSH/LH) on the integrated processes involving IVM and IVF. No significant differences were found between the results obtained with these media in relation to oocyte maturation (65\% MII for HECM-9 vs. $71 \%$ for TCM-199); however, those oocytes matured in HECM-9 showed a highly significant difference in in vitro fertilization using a conventional IVF medium (SOFm) (25\% in HECM-9 vs. 6\% in TCM-199). Maturation results were relatively low but in accordance with those reported by other groups, whereas IVF results are lower than those reported in the literature, perhaps because we have been using frozen and thawed samples and do not have complete control over the sperm quality. At present, we are extending our investigation using fresh semen samples.

\title{
337 EFFECTS OF CANINE SYNTHETIC OVIDUCT FLUID MEDIUM SUPPLEMENTED WITH THE VARIOUS ENERGY SUBSTRATES ON IN VITRO MATURATION OF CANINE OOCYTES
}

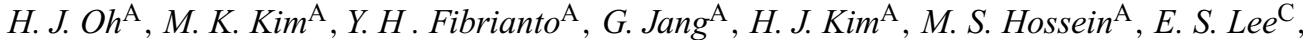 \\ S. K. Kang ${ }^{\mathrm{A}, \mathrm{B}}$, B. C. Lee $\mathrm{A}^{\mathrm{A}, \mathrm{B}}$, and W. S. Hwang $\mathrm{A}, \mathrm{B}$
}

${ }^{A}$ Department of Theriogenology and Biotechnology, College of Veterinary Medicine, Seoul National University, Seoul, Korea;

${ }^{\mathrm{B}}$ The Xenotransplantation Research Center, Seoul National University Hospital, Seoul, Korea;

${ }^{\mathrm{C}}$ Department of Veterinary Medicine, College of Animal Resources Sciences, Kangwon National University, Kangwon-do, Korea

In most mammals, maturation occurs within the ovarian follicle, and preovulatory oocytes are ovulated and ready for fertilization within the oviduct. In contrast, bitch ovulate primary oocytes, over a three day period, undergo both maturation and fertilization within the oviduct. The present study was conducted to evaluate the effects of canine synthetic oviduct fluid (cSOF) supplemented with the various energy substrates on in vitro maturation of canine oocytes. Oocytes were recovered by mincing ovaries collected after ovariohysterectomy in bitches at the follicular stage. Only oocytes with more than two layers of cumulus cells and with homogeneous cytoplasm $>100 \mathrm{~mm}$ in diameter were selected. Then, oocytes cultured in tissue culture medium (TCM)-199 (control) or cSOF supplemented with various concentrations of glucose $(0,1.11,3.89$, or $5.56 \mathrm{mM}$, Exp. 1) or fructose $(0,1.11,3.89$, or $5.56 \mathrm{mM}$, Exp. 1), pyruvate $(0,0.1,0.25$, or $0.5 \mathrm{mM}$, Exp. 2$)$ or lactate $(0,0.5,1.0$, or $5.0 \mathrm{mM}$, Exp. 3$)$. In Exp. 4, the combined effects of glucose $(1.11 \mathrm{mM})$, pyruvate $(0.5 \mathrm{mM})$ and lactate $(5.0 \mathrm{mM})$ on nuclear maturation of canine oocytes were investigated. A total of 2990 canine oocytes from 205 ovaries were used for experiments with replication at least three times. The oocytes were cultured for $72 \mathrm{~h}$ at $38.5^{\circ} \mathrm{C}$ in a humidified atmosphere of $5 \% \mathrm{CO}_{2}$ in air. After $72 \mathrm{~h}$, the oocytes were stained with $1.9 \mu \mathrm{g} / \mathrm{mL}$ Hoechst 33342 in glycerol and then evaluated under UV light to determine the stage of meiosis as follows: germinal vesicle (GV), germinal vesicle breakdown (GVBD), metaphase I (MI), metaphase II (MII) with first polar body. The results of Exp. 1 showed that maturation of canine oocytes to MII was significantly higher $(P<0.05)$ in medium supplemented with $1.11 \mathrm{mM}$ glucose $(4.8 \%)$ than for the control $(1.8 \%)$ and the other glucose-supplemented groups $(0$ to $1.8 \%)$. In Exp. 2, oocytes cultured in cSOF supplemented with $0.5 \mathrm{mM}$ pyruvate showed a significantly higher $(P<0.05)$ maturation rate to MII $(6.3 \%)$ than did the other pyruvate-supplemented $(0,0.8$, or $2.5 \%)$ groups or the control $(2.4 \%)$. In Exp. 3 , more oocytes were matured to the MII stage in cSOF supplemented with $5.0 \mathrm{mM}$ lactate (7.3\%) than were the other lactate-supplemented groups (0 to 2.4\%) or the control (2.5\%). Results of Exp. 4 showed more oocytes progressed to MII in cSOF supplemented with $0.5 \mathrm{mM}$ pyruvate $(8.2 \%), 1.11 \mathrm{mM}$ glucose $+0.5 \mathrm{mM}$ pyruvate $(7.4 \%)$, or $1.11 \mathrm{mM}$ glucose $+0.5 \mathrm{mM}$ pyruvate $0.5+5.0 \mathrm{mM}$ lactate $(7.3 \%)$ than did the other combination groups (2.2 to $5.2 \%)$. In conclusion, the present study demonstrated that supplementing cSOF with $1.11 \mathrm{mM}$ glucose, $0.5 \mathrm{mM}$ pyruvate, or $5.0 \mathrm{mM}$ lactate significantly increased the maturation of canine oocytes to MII, and the combined supplementation of $1.11 \mathrm{mM}$ glucose, $0.5 \mathrm{mM}$ pyruvate, and $5.0 \mathrm{mM}$ lactate further promoted oocyte nuclear maturation compared to $1.11 \mathrm{mM}$ glucose alone and the control.

This study was supported by grants from the Korean MOST (Top Scientist Fellowship) and MAF (Biogreen 21 \#20050301-034-443-026-01-00).

\section{EFFECTS OF CUMULUS CELLS AND FOLLICULAR FLUID ON PLASMINOGEN ACTIVATOR ACTIVITY AND OOCYTE MATURATION IN VITRO IN THE PIG}

\author{
C.-K. Park, J.-Y. An, S.-J. Sa, H.-T. Cheong, B.-K. Yang, and C.-I. Kim \\ College of Animal Resource Science, Kangwon University, Chunchon, Korea
}

Plasminogen activators (PAs) are serine proteases, known to be secreted by a large number of cell type. PAs are reported to play a role in variety of physiologic processes, including fibrinolysis, ovulation, mammary involution, implantation, and fertilization. The present study investigated the effects of cumulus cells and porcine follicular fluid (pFF) on PA activity and oocyte maturation in vitro in the pig. Porcine oocytes were harvested from slaughterhouse ovaries, selected, and matured in modified North Carolina State University-23 (NCSU-23) media. After culture, cumulus-oocyte complexes (COCs) and denuded oocytes (DOs) were separately put into microtubes containing $20 \mu \mathrm{L}$ of sample buffer [5.0\% (w:v) sodium dodecyl 
sulfate, $20 \%$ (v:v) glycerol, and $0.0025 \%(\mathrm{w}: \mathrm{v})$ bromophenol blue in $0.125 \mathrm{M}$ Tris-HCl buffer] and frozen at $-80^{\circ} \mathrm{C}$ until used for zymographic analysis. Differences in data were evaluated by Duncan's multiple-range test using the General Linear Models procedure in the Statistical Analysis System (SAS Institue, Inc., Cary, NC, USA). To determine the effect of porcine follicular fluid (pFF) on PA activity in porcine oocytes during maturation, the COCs and DOs were incubated in NCSU-23 medium with or without $10 \%$ (v/v) pFF for 0,24 , or $48 \mathrm{~h}$. In the presence of cumulus cells, the proportions of oocytes matured to metaphase-II stage were significantly $(P<0.05)$ higher in medium with pFF than without $\mathrm{pFF}(69.8 \%$ vs. $37.7 \%$, respectively). When COCs and DOs were cultured in the presence of pFF, tissue-type PA (tPA), urokinase-type PA (uPA), and tPA-PA inhibitor (tPA-PAI) were observed in COCs, and PA activities were higher at $48 \mathrm{~h}$ than $24 \mathrm{~h}$. However, no PA activity was detected in DOs. Under the same conditions, when COCs and DOs were cultured in the absence of pFF, tPA and tPA-PAI were observed in COCs, and PA activities were increased as duration of culture increased. However, no PA activity was detected in DOs. When porcine oocytes were cultured in the presence of $\mathrm{pFF}$, the activities of tPA-PAI, tPA, and uPA were observed in conditioned medium with COCs and DOs cultured for $24 \mathrm{~h}$ and $48 \mathrm{~h}$. In the absence of pFF, PA activities were observed only in conditioned medium with COCs, and no PA activities were detected in conditioned medium with DOs. On the other hand, three plasminogen-dependent lytic bands (tPA-PAI, tPA, and uPA) were observed in pFF cultures. Particularly uPA activity was higher than the other kinds of PA activity. When oocytes and cumulus cells were separated from porcine COCs at $0 \mathrm{~h}$ of cultrue, tPA-PAI, tPA, and uPA were detected in cumulus cells at 48-h culture, but no PA activities were in DOs. The presence of pFF and cumulus cells in maturation medium stimulated not only nuclear and cytoplasmic maturation in porcine COCs, but also PA production by cumulus cells and COCs. It is possible that PAs produced by cumulus cells migrated through the gap junction between oocyte and cumulus cells. These results suggest that porcine oocytes have no ability to produce PA themselves.

\title{
339 DIFFERENTIAL MECHANISM OF LEPTIN ACTION IMPROVING NUCLEAR MATURATION AND DEVELOPMENTAL COMPETENCE OF BOVINE OOCYTES
}

\author{
F. F. Paula-Lopes ${ }^{\mathrm{A}}$, M. Boelhauve ${ }^{\mathrm{A}}$, F. Habermann ${ }^{\mathrm{B}}$, F. Sinowatz $^{\mathrm{B}}$, and E. Wolf ${ }^{\mathrm{A}}$ \\ ${ }^{A}$ Department of Molecular Animal Breeding and Biotechnology, Ludwig-Maximilian University, Munich, Germany; \\ ${ }^{B}$ Department of Veterinary Anatomy, Histology and Embryology, Ludwig-Maximilian University, Munich, Germany
}

Leptin is a pleiotrophic peptide that has been implicated in the events associated with oocyte maturation and acquisition of developmental competence. Previous studies indicated that leptin supplementation during oocyte maturation has long-term effects, increasing blastocyst development and reducing the proportion of TUNEL-positive cells per blastocyst. Moreover, blastocysts derived from leptin-treated oocytes showed increased BIRC4 and reduced $B A X$ expression (Boelhauve et al. 2005 Biol. Reprod. 73, 737-744). The objective of the current study was to determine the mechanism of leptin action during bovine oocyte maturation. In the first series of experiments cumulus-oocyte complexes (COCs) were matured in serum-free medium containing 0,1 , or $10 \mathrm{ng} / \mathrm{mL}$ leptin or $10 \%$ estrous cow serum (ECS) as positive control. Leptin reduced the proportion of cumulus cells undergoing cell death through apoptosis as determined by TUNEL staining $(6.8 \pm 0.4,1.8 \pm 0.4,1.5 \pm 0.4$, and $6.3 \pm 0.5 \%$ for 0,1 , or $10 \mathrm{ng} / \mathrm{mL}$ leptin or $10 \% \mathrm{ECS}$, respectively; $P<0.0001$ ), but had no effect on the frequency of apoptotic oocytes. Nuclear maturation was also enhanced by leptin. The proportion of oocytes with extruded polar bodies $(64.0 \pm 2.9,75.7 \pm 2.9,73.8 \pm 2.9$, and $63.3 \pm 2.9 \%$ for 0,1, or $10 \mathrm{ng} / \mathrm{mL}$ leptin or $10 \%$ ECS; $P<0.05)$ and the proportion of DAPI-stained metaphase II oocytes $(72.9 \pm 2.9,90.5 \pm 2.9,85.8 \pm 2.9$, and $82.0 \pm 2.9 \%$ for 0,1, or $10 \mathrm{ng} / \mathrm{mL}$ leptin or $10 \% \mathrm{ECS} ; P<0.05)$ were increased by 1 and $10 \mathrm{ng} / \mathrm{mL}$ leptin. There was no effect of ECS in any of the parameters examined. A second series of experiments tested whether the maturation-promoting activity of leptin was mediated by cumulus cells. Denuded oocytes (DO) and COCs were matured in serum-free medium containing 0 or $10 \mathrm{ng} / \mathrm{mL}$ leptin. The percentage of oocytes with extruded polar bodies (COCs: $59.1 \pm 3.7 \%$ vs. $75.7 \pm 3.7 \%$, and DO: $45.5 \pm 3.7 \%$ vs. $54.8 \pm 3.7 \%$ for 0 or $10 \mathrm{ng} / \mathrm{mL}$ leptin; $P<0.01$ ) was increased by leptin regardless of cumulus cells. Even though leptin did not affect cleavage rate, it increased blastocyst development. The proportion of COCs that developed to the blastocyst stage increased from $22.3 \pm 4.6 \%$ in the control group to $35.2 \pm 4.1 \%$ in the leptin-treated group. On the other hand, DO matured without cumulus cells did not acquire developmental competency, and this was not reversed by leptin supplementation $(1.0 \pm 5.0 \% \mathrm{vs} .1 .0 \pm 4.7 \%$ for 0 or $10 \mathrm{ng} / \mathrm{mL}$ leptin; $P<0.05$; leptin $\times$ oocyte interaction $P<0.05$ ). In conclusion, leptin enhanced oocyte maturation by acting directly in the oocyte and indirectly through cumulus cells. This suggests that leptin modulates the release of cumulus-derived factors secreted in the oocyte through gap junction coupling and in the extracellular environment.

\section{IN VITRO MATURATION OF PREPUBERTAL BOVINE OOCYTES ON A GRANULOSA CELL MONOLAYER FROM ADULT ANIMALS IMPROVES THEIR DEVELOPMENTAL COMPETENCE}

\author{
S. Ponebsek, C. Wrenzycki, K.-G. Hadeler, D. Herrmann, K. Korsawe, and H. Niemann \\ Institute for Animal Breeding (FAL), Department of Biotechnology, Mariensee, Neustadt, Germany
}

Oocytes from prepubertal calves have a decreased developmental competence compared with oocytes from adult animals. The goal of this study was to improve the developmental competence of juvenile oocytes by maturation on granulosa cell (GC) monolayers from adult animals. Oocytes were recovered by ovum pickup (OPU) from 48 Holstein Friesian calves at 7-8 months of age and 18 adult cows. Animals received intramuscular injections of $60 \mathrm{mg}$ FSH $48 \mathrm{~h}$ prior to each OPU session. Follicles were punctured twice per week in six consecutive OPU sessions. Cumulus oocyte complexes (COCs) recovered from calves were divided into three quality groups (classes I-III) and were then randomly distributed into three maturation groups: $\mathrm{COCs}$ were matured for $24 \mathrm{~h}$ on either $\mathrm{GC}$ or fibroblasts or without co-culture. Cow oocytes were matured without co-culture. TCM-199 supplemented with BSA $(0.1 \%)$, hCG $(5 \mathrm{IU} / \mathrm{mL})$, and eCG $(10 \mathrm{IU} / \mathrm{mL})$ served as the medium in all groups. After maturation, all COCs were fertilized in vitro; after $18 \mathrm{~h}$, presumptive zygotes were cultured in SOF+BSA for 8 days $\left(37^{\circ} \mathrm{C}, 5 \% \mathrm{CO}_{2}\right)$. On Day 3 , cleavage rates and on Day 8, blastocyst rates were determined. The relative mRNA abundance of the following transcripts, critically involved in early embryonic 
development was determined: growth differentiation factor-9 (GDF-9), heat shock protein 70 (Hsp-70), and glucose transporter-3 (Glut-3). Single immature and matured oocytes (for GDF-9 and Hsp-70) and 8-16-cell embryos and expanded blastocysts (for Hsp-70 and Glut-3) from calves and cows were examined by semiquantitative RT-PCR. Cleavage and blastocyst rates were similar in oocytes derived from cows and calves matured on GC ( $74.3 \%$ vs. $70.0 \%$ and $22.3 \%$ vs. $22.3 \%$, respectively), but were significantly higher $(P<0.05$; one way ANOVA, Student-Newman-Keuls Method) than in the group without co-culture on fibroblasts (55.2\% vs. $53.6 \%$ and $11.7 \%$ vs. $5.5 \%$, respectively). GDF- 9 expression was similar in immature calf and cow oocytes. After maturation, a significant decrease in GDF-9 expression was observed in calf oocytes. Matured cow oocytes showed a significantly higher mRNA abundance of GDF-9 than matured calf oocytes. The relative abundance of Hsp-70 was decreased in matured oocytes of all groups. Expanded blastocysts derived from adult oocytes expressed Hsp-70 significantly higher than blastocysts derived from oocytes of the control calves. The relative abundance of Glut-3 mRNA was similar in 8-16 cell embryos and expanded blastocysts in all groups. Overall, mRNA expression pattern for Hsp-70 and Glut-3 in blastocysts from GC matured oocytes were similar to that of cow blastocysts. Results indicate that maturation of juvenile calf oocytes on granulosa cells from adult animals improves their developmental competence. These findings provide clues toward identification of factors critically involved in acquiring full developmental capacity at puberty.

\title{
341 OXYGEN TENSION AND EGF AFFECT METABOLISM OF IN VITRO-MATURED CUMULUS-ENCLOSED MOUSE OOCYTES
}

\author{
K. A. Preis ${ }^{\mathrm{A}, \mathrm{B}}$, G. E. Seidel Jr${ }^{\mathrm{B}}$, and D. K. Gardner $\mathrm{A}, \mathrm{B}$ \\ ${ }^{A}$ Colorado Center for Reproductive Medicine, Englewood, CO 80110, USA; \\ ${ }^{B}$ Animal Reproduction and Biotechnology Laboratory, Colorado State University, Fort Collins, CO 80521, USA
}

In vitro maturation of immature oocytes results in limited success in both clinical and research laboratories. Although reduced oxygen concentration is beneficial to embryo development, the optimal concentration for oocyte maturation has yet to be determined. The objective of this study was to determine whether oxygen tension $\left(20 \%\right.$ or $\left.5 \% \mathrm{O}_{2}\right)$ affects oocyte physiology. Additionally, the effect of epidermal growth factor (EGF) in maturation medium on oocyte metabolic activity and subsequent embryo development was determined. Cumulus-oocyte complexes (COCs; $n=231)$ were collected from 28-day-old unprimed $\mathrm{F} 1(\mathrm{C} 57 \mathrm{BL} / 6 \times \mathrm{CBA} / \mathrm{ca})$ mice. COCs were individually matured in defined medium at $37^{\circ} \mathrm{C}$ in $6 \% \mathrm{CO}_{2}$ in one of four groups (Table 1). For the metabolism study, COCs were further divided into two groups: individual maturation in a $2-\mu \mathrm{L}$ drop of medium for $16 \mathrm{~h}(n=131)$; or individual maturation in $5-\mu \mathrm{L}$ for $12 \mathrm{~h}$ and then placed in a $0.5-\mu \mathrm{L}$ drop of medium for $4 \mathrm{~h}(n=100)$, the time of greatest metabolic activity of the COC. At $17 \mathrm{~h}$ of maturation, COCs were individually fertilized, and zygotes were individually cultured until $96 \mathrm{~h}$, at which time blastocyst development was assessed. Metabolic profiles were analyzed by ANOVA, and blastocyst rates were analyzed by Fisher's exact test. Maturation rates and blastocyst development were not different between groups. However, at $12-16 \mathrm{~h}$ of maturation, metabolism of COCs was affected by both oxygen tension and EGF (Table 1). Concerning metabolism over the entire course of maturation, glucose uptake and lactate production were higher in COCs in $5 \% \mathrm{O}_{2}+100 \mathrm{ng}$ EGF $(P<0.05)$ than in the remaining three groups. There was no difference between $5 \% \mathrm{O}_{2}$ and $20 \% \mathrm{O}_{2}+100 \mathrm{ng}$ EGF, but $20 \% \mathrm{O}_{2}$ caused less glucose uptake and lactate production than did the other three treatment groups $(P<0.05)$. Results of this study are the first to show that oxygen tension alters COC metabolism: COCs matured under $5 \% \mathrm{O}_{2}$ were more active metabolically than COCs matured under $20 \% \mathrm{O}_{2}$. The effect of oxygen tension is to some extent moderated by the presence of EGF, as metabolic activity of COCs matured under $20 \% \mathrm{O}_{2}+100 \mathrm{ng}$ EGF was closer to that of COCs matured under $5 \% \mathrm{O}_{2}$ conditions. Although blastocyst rates were similar across the four groups, embryos derived from oocytes matured in different oxygen tensions may exhibit different developmental potential. In conclusion, results of this study have implications for the improvement of maturation conditions in both clinical and research laboratories.

Table 1. Carbohydrate metabolism of individual COCs at $12-16 \mathrm{~h}$ of maturation

\begin{tabular}{lcccc}
\hline & $20 \% \mathrm{O}_{2}$ & $20 \% \mathrm{O}_{2}+100 \mathrm{ng}$ EGF & $5 \% \mathrm{O}_{2}$ & $5 \% \mathrm{O}_{2}+100 \mathrm{ng}$ EGF \\
\hline Glucose uptake & $21 \pm 2 \mathrm{pmol} / \mathrm{h}^{\mathrm{a}}$ & $36 \pm 3 \mathrm{pmol} / \mathrm{h}^{\mathrm{b}}$ & $50 \pm 3 \mathrm{pmol} / \mathrm{h}^{\mathrm{c}}$ & $53 \pm 4 \mathrm{pmol} / \mathrm{h}^{\mathrm{c}}$ \\
Lactate production & $13 \pm 2 \mathrm{pmol} / \mathrm{h}^{\mathrm{a}}$ & $43 \pm 4 \mathrm{pmol} / \mathrm{h}^{\mathrm{b}}$ & $56 \pm 4 \mathrm{pmol} / \mathrm{h}^{\mathrm{b}}$ & $61 \pm 5 \mathrm{pmol} / \mathrm{h}^{\mathrm{b}}$ \\
\hline
\end{tabular}

${ }^{\mathrm{abc}}$ Values within rows with different superscripts $\operatorname{differ}(P<0.05)$. Values are means $\pm \mathrm{SEM}$.

\section{DEVELOPMENT OF PIG OOCYTES MATURED AND FERTILIZED IN CHEMICALLY DEFINED MEDIUM CONTAINING PVA, PVP, AND PFF}

\author{
H.-B. Seok and I.-D. Kim \\ Department of Animal Science, Dankook University, Cheonan, 330-114, South Korea
}

This study was conducted to develop a serum-free, defined medium for IVM of pig oocytes. TCM-199 with supplemented with polyvinyl alcohol (PVA), polyvinylpyrrolidone (PVP), and porcine follicular fluid (pFF) were used as basal medium. The effects of the these additives on the rates of maturity and development during in vitro fertilization and in vitro culture were examined and subsequently considered as possibile substitutes for bovine serum albumin (BSA). In an experiment that contained nine replicates, pig oocytes from abattoir-derived ovaries were matured in TCM-199 containing pyruvic acid, gentamycin, L-cysteine, $\beta$-estradiol, and FSH, and supplemented with $0.1 \%$ PVA, $0.1 \%$ PVP, $10 \%$ pFF, or $0.1 \%$ BSA (control) for $44 \mathrm{~h}$ at $39^{\circ} \mathrm{C}, 5 \% \mathrm{CO}_{2}$. Fertilized oocytes were cultured in glucose-free NCSU 23 supplemented with $5 \mathrm{mM}$ sodium pyruvate, $0.5 \mathrm{mM}$ sodium lactate, and the same indicated supplements for 2 days. Maturation, fertilization, and morula and blastocyst formation were examined at 
$44 \mathrm{~h}$ after IVM and at 12, 48, and $120 \mathrm{~h}$ after IVF, respectively. Morphology of oocytes and cleaved cells was desplayed by staining with Hoechst 33342. Maturation rates of pig oocytes in IVM media containing PVA $(82.4 \%)$, pFF $(89.4 \%)$, or BSA $(90.0 \%)$ were significantly higher $(P<0.05)$ than that with PVP (78.6\%). Cleavage rates after IVF with PVP $(64 \%)$ was significantly lower $(P<0.05)$ than those in PVA $(73 \%)$, pFF $(77 \%)$, or BSA (73\%) supplements. In vitro development rates to morulae and blastocysts with PVP $(54 \%)$ were also significantly lower $(P<0.05)$ than those with the supplements of PVA (63\%), pFF (69\%), or BSA (65\%). In comparison of maturation and fertilization rates of pig oocytes in each of the supplements, the maturity rates with PVA $(82.4 \%)$, pFF $(89.4 \%)$, or BSA $(90.0 \%)$ were significantly lower $(P<0.05)$ than that with PVP $(72.4 \%)$ and the fertilization rates with pFF $(87.1 \%)$ or BSA $(89.1 \%)$ were significantly higher $(P<0.05)$ than those with PVA $(78.0 \%)$ or PVP $(70.6 \%)$ It may be concluded that PVA and pFF can be substituted for BSA in medium for culturing pig oocytes, and that PVP would not be an acceptable substitute for BSA.

\title{
343 EFFECTS OF EPIDERMAL GROWTH FACTOR SUPPLEMENTATION ON IN VITRO MATURATION AND GENE EXPRESSION OF CANINE OOCYTES
}

\author{
H.-J. Song, S.-H. Lee, G.-H. Maeng, J.-G. Kim, S. Balasubramanian, S.-Y. Choe, and G.-J. Rho \\ College of Veterinary Medicine, Gyeongsang National University, Chinju, Republic of Korea, 660-701
}

Despite many efforts to improve canine in vitro maturation (IVM), the efficiency is still low compared to that of other mammalian species (Marie et al. 2004). Epidermal growth factor (EGF) has stimulatory effects on the resumption of oocyte maturation and cumulus expansion in vitro and on prei-mplantation embryonic development in mammals by either an autocrine or a paracrine pathway, or a combination of both systems (Paria et al. 2001 PNAS 98, 1047-1052). The present study investigated the effects of EGF supplementation on in vitro maturation and gene expression of canine oocytes. Oocytes were recovered by slicing ovaries recovered from 40 bitches after ovariohysterectomy at random stages of the estrous cycle. Cumulus-oocyte complexes (COCs) were matured in TCM-199 containing 10\% FBS, $1 \mu \mathrm{g} / \mathrm{mL}$ FSH and LH, and EGF $(0$, 10 , or $30 \mathrm{ng} / \mathrm{mL})$ for 48 or $72 \mathrm{~h}$ at $39^{\circ} \mathrm{C}$ in a humidified atmosphere of $5 \% \mathrm{CO}_{2}$ in air. In Experiment $\mathrm{I}(n=2520$ oocytes $)$, the nuclear maturation status was assessed by fluorescence microscopy after bisbenzimide (Hoechst 33342) staining $(10 \mu \mathrm{g} / \mathrm{mL})$ at 0,48 , and $72 \mathrm{~h}$ of incubation In Experiment II ( $n=90$ oocytes), expression of transcripts such as EGF receptor $(E G F R)$, luteinizing hormone receptor (LHR), and gap junction protein (GJA5) were determined in 10 intact COCs each at 0,48 , and $72 \mathrm{~h}$, respectively, by reverse transcription-polymerase chain reaction (RT-PCR). At $0 \mathrm{~h} 10-20 \%$ of the oocytes had undergone resumption of meiosis (GVBD MII). After $48 \mathrm{~h}$ of IVM, rate of meiotic resumption for 0,10 , and $30 \mathrm{ng} / \mathrm{mL}$ EGF were 28,35 , and $30 \%$, respectively. At $72 \mathrm{~h}$ of IVM, oocytes in the $10 \mathrm{ng} / \mathrm{mL}$ EGF group had resumed meiosis at a higher frequency $(55 \% ; P<0.05)$ than in the $30 \mathrm{ng} / \mathrm{mL}$ EGF or the control group ( 39 and $42 \%$, respectively). At $72 \mathrm{~h}$ of IVM, the frequency of maturation to the MII stage was significantly higher in the $10 \mathrm{ng} / \mathrm{mL}$ EGF group (9.6\%) than in the $30 \mathrm{ng} / \mathrm{mL}$ EGF or the control group (4.2 and $3.3 \%$, respectively). The expression of $E G F R$ was significantly higher $(P<0.05)$ in 0 h oocytes than in the $48-$ or 72 -h oocytes. Further $E G F R$ expression levels were decreased in the presence of EGF in a dose dependent manner. Transcripts for $L H R$ were detected at all maturation intervals and its expression patterns were not altered by supplementation with $10 \mathrm{ng} / \mathrm{mL}$ EGF. Expression of $G J A 5$ was observed only after $48 \mathrm{~h}$ of IVM, and levels of expression were similar in oocytes supplemented with both 10 and $30 \mathrm{ng} / \mathrm{mL}$ EGF. In summary, our results indicate that supplementation of canine IVM medium with $10 \mathrm{ng} / \mathrm{mL}$ EGF had a positive influence on the progression of maturation to MII at $72 \mathrm{~h}$. The effect may not be related to the alteration of mRNA expression of genes analyzed in the present study, due to the complex patterns regulating meiotic arrest in canine oocytes.

This work was supported by Grant no. 204119-03-1-LG000 from ARPC, Republic of Korea.

\section{PENTOSE PHOSPHATE PATHWAY ACTIVITY CONTROLS NUCLEAR MATURATION OF PORCINE OOCYTES}

\author{
L. Tubman ${ }^{\mathrm{A}}$, A. Peter $^{\mathrm{B}}$, and R. Krisher ${ }^{\mathrm{C}}$ \\ ${ }^{\mathrm{A}}$ School of Veterinary Medicine, Purdue University; ${ }^{\mathrm{B}}$ Department of Clinical Sciences, Purdue University; \\ ${ }^{\mathrm{C}}$ Department of Animal Sciences, Purdue University, West Lafayette, IN 47907, USA
}

Diphenyleneiodonium (DPI), an inhibitor of the pentose phosphate pathway (PPP), arrests nuclear maturation of porcine oocytes. This inhibition is reversed using products or cofactors of PPP such as nicotinamide adenine dinucleotide phosphate (NADP), phosphoribose diphosphate (PRPP), and ribose-5-phosphate (R5P). The objective of this study was to determine the relationship between DPI-mediated meiotic inhibition, reversal of this inhibition, and metabolism of in vitro-matured (IVM) porcine oocytes. Oocytes were aspirated, searched, and selected in the presence of DPI, with the exception of control oocytes. Oocytes were then matured in one of five treatments for $40 \mathrm{~h}$ in $7 \% \mathrm{CO}_{2}$ in air at $39^{\circ} \mathrm{C}$ in defined Purdue Porcine Medium for maturation (PPMmat). Treatments included control, $50 \mathrm{nM}$ DPI (DPI), DPI $+5 \mathrm{mM}$ NADP (NADP), DPI $+12.5 \mathrm{mM}$ PRPP (PRPP), and DPI + $10 \mathrm{mM}$ R5P (R5P). Following IVM, oocytes were denuded by vortexing. Glycolysis and PPP activities were measured in $4 \mu \mathrm{L}$ hanging drops containing labeled glucose $\left(0.0125 \mathrm{mM} \mathrm{5-}{ }^{3} \mathrm{H}\right.$ glucose and $0.482 \mathrm{mM} 1-{ }^{14} \mathrm{C}$ glucose, respectively) for $3 \mathrm{~h}$ in $6 \% \mathrm{CO}_{2}$. Oocytes were then individually fixed in a 3:2:1 solution of ethanol:acetic acid:chloroform and stained with aceto-orcein for determination of meiotic stage (germinal vesicle $=1$ through metaphase $\mathrm{II}=7$ ). Data were analyzed using one-way ANOVA. The use of DPI inhibited PPP and nuclear maturation; additionally glycolysis was decreased by DPI compared to control. Addition of NADP and PRPP increased both metabolic pathways and nuclear 
maturation compared to DPI. R5P restored glycolysis and nuclear maturation to control levels, and PPP to above the control level. There were no significant differences among meiotic stages relative to glycolytic activity. PPP activity was significantly different (values with different superscripts; $P<0.05$ ) among oocytes of different meiotic stages (germinal vesicle $=0.24 \pm 0.03^{\text {ad }}$, germinal vesicle breakdown $=0.40 \pm 0.05^{\text {bcde }}$, condensed chromatin $=0.44 \pm 0.05^{\text {bcd }}$, metaphase $\mathrm{I}=0.45 \pm 0.12^{\text {abcd }}$, anaphase $=0.76 \pm 0.50^{\text {abcde }}$, telophase $=0.92 \pm 0.17^{\text {be }}$, metaphase $\mathrm{II}=0.74 \pm 0.08^{\text {be }}$ ). Percentages of oocytes reaching MII were 43.48 (control), 2.08 (DPI), 28.30 (NADP), 18.18 (PRPP), and 46.94 (R5P). These results demonstrate that the PPP is a critical control mechanism for nuclear maturation of porcine oocytes, as inhibition of this metabolic pathway resulted in arrest of nuclear maturation. Addition of PPP cofactors or end products to the arresting medium led to reversal of inhibition as demonstrated by restoration of PPP activity resulting in nuclear maturation.

Table 1. Meiotic stage, glycolysis, and pentose phosphate pathway activity after in vitro maturation of porcine oocytes

\begin{tabular}{lcccc}
\hline Treatment & $n$ & Meiosis & Glycolysis* & PPP* $^{*}$ \\
\hline Control & 39 & $5.18 \pm 0.31^{\mathrm{a}}$ & $0.66 \pm 0.09^{\mathrm{ac}}$ & $0.37 \pm 0.05^{\mathrm{a}}$ \\
DPI & 36 & $1.92 \pm 0.22^{\mathrm{b}}$ & $0.43 \pm 0.08^{\mathrm{b}}$ & $0.22 \pm 0.02^{\mathrm{b}}$ \\
NADP & 42 & $4.02 \pm 0.32^{\mathrm{c}}$ & $0.50 \pm 0.07^{\mathrm{c}}$ & $0.46 \pm 0.04^{\mathrm{ac}}$ \\
PRPP & 32 & $4.38 \pm 0.38^{\mathrm{c}}$ & $0.52 \pm 0.08^{\mathrm{c}}$ & $0.68 \pm 0.11^{\mathrm{c}}$ \\
R5P & 45 & $5.44 \pm 0.28^{\mathrm{a}}$ & $0.75 \pm 0.06^{\mathrm{a}}$ & $0.94 \pm 0.11^{\mathrm{d}}$ \\
${ }^{*}$ pmol/oocyte/3h. & & \\
${ }^{\mathrm{a}-\mathrm{c}}$ Values with different superscripts within columns are significantly different $(P<0.05)$.
\end{tabular}

\title{
345 INFLUENCE OF DIFFERENT MATURATION MEDIA ON IN VITRO SWINE EMBRYO DEVELOPMENT
}

\author{
J. A. Visintin, A. S. Lima, A. B. Nascimento, A. C. Nicacio, M. G. Marques, and M. E. O. A. Assumpção \\ Department of Animal Reproduction-FMVZ, University of São Paulo, São Paulo-SP, Brazil
}

The aim of this work was to study the influence of maturation media NCSU23, Whitten, and TCM199 on in vitro development of swine embryos. Oocytes from slaughtered gilt ovaries were in vitro-matured (IVM) with NCSU23 $(n=148)$, Whitten $(n=151)$, and TCM199 $(n=170)$ media for $44 \mathrm{~h}$. After IVM, all oocytes were fertilized in mTBM medium for $6 \mathrm{~h}$ and cultured in NCSU23 with $0.4 \%$ BSA for 5 days. After Day 5 , all embryos were cultured in NCSU23 supplemented with $20 \%$ FCS for 4 days. Cleavage, blastocyst, and hatching rates were evaluated. Cleavage rates were $29 \%$ for NCSU23, 35.8\% for Whitten, and 42.3\% for TCM199 maturation media, with no significant difference among media $(P>0.05)$. Blastocyst rates were similar $(P>0.05), 18.9 \%, 21.8 \%$, and 20\%, respectively, for NCSU23, Whitten and TCM199 maturation media. Hatching rates were $16.1 \%$, $34.3 \%$, and $32.3 \%$, respectively, for NCSU23, Whitten, and TCM199 maturation media, with no significant differences $(P>0.05)$. In conclusion, all three maturation media supported IVF, allowing embryo development from cleavage until blastocyst hatching.

\section{BENEFICIAL EFFECTS OF ACETYL-L-CARNITINE TREATMENT DURING IVM ON POST-FERTILIZATION DEVELOPMENT OF BOVINE OOCYTES IN VITRO}

\author{
T. Yamada, H. Imai, and M. Yamada
}

Laboratory of Reproductive Biology, Graduate School of Agriculture, Kyoto University, Kyoto, 606-1501, Japan.

The lower competence of in vitro-matured oocytes for post-fertilization development is attributed to the lack of physiological factors in in vitro maturation (IVM) that regulate maturation events which occur exclusively in the cytoplasm of oocytes. It has been found recently that mitochondrial function plays an important role in regulation of oocyte developmental competence via metabolic regulation of energy production. Acetyl-Lcarnitine (ALC) is known to enhance fatty acid oxidation and energy production in the mitochondria, and to exert enhancing effects on cellular proliferation and survival. In this experiment, we examined the effects of ALC on IVM and post-fertilization development of bovine oocytes. Cumulus-oocyte complexs (COCs) were aspirated from $2-5 \mathrm{~mm}$ follicles of ovaries from a slaughterhouse. COCs were cultured in IVM medium $(\mathrm{mSOFaa}+$ estradiol $+\mathrm{hCG}+\mathrm{BSA})$ with or without ALC $(10 \mathrm{mM})$ for $24 \mathrm{~h}$ at $39^{\circ} \mathrm{C}$ under $5 \% \mathrm{CO}_{2}$ in air, and then fertilized according to the conventional method. After $6 \mathrm{~h}$ of insemination, presumptive zygotes were freed from cumulus cells by repeated pipetting and cultured in mSOFaa with $1 \% \mathrm{FCS}$ at $39^{\circ} \mathrm{C}$ under $5 \% \mathrm{CO}_{2}, 5 \% \mathrm{O}_{2}$, and $90 \% \mathrm{~N}_{2}$. At $48 \mathrm{~h}$ post-fertilization, the rates of cleaved embryos were assessed. The cleaved embryos were transferred to mSOFaa with $5 \% \mathrm{FCS}$ and cultured for additional 6 days at $39^{\circ} \mathrm{C}$ under $5 \% \mathrm{CO}_{2}, 5 \% \mathrm{O}_{2}$, and $90 \% \mathrm{~N}_{2}$. The percentages of embryos developing to the blastcyst stage were assessed on Days 6, 7, and 8 (fertilization = Day 0), and the data were analyzed for statistically significant differences with the $t$-test. For examinination of mitochondrial organization in oocytes at different maturation stages, oocytes were stained for active mitochondria with MitoRed $\left(1 \mu \mathrm{M}\right.$ in IVM medium for $2 \mathrm{~h}$ at $\left.37^{\circ}\right)$. When COCs were matured in medium without (control) or with ALC, although the rates of post-fertilization cleavage of oocytes were $60 \%$ to $70 \%$ despite the presence or absence of ALC, ALC significantly $(P<0.05)$ increased the rates of cleaved embryos forming blastcysts on Days 6, 7, and $8(30 \%, 36 \%, 40 \%)$ compared with those in the control (13\%, $21 \%$, $34 \%$ ). We next examined effects of ALC treatment during IVM on active mitochondria distribution in oocytes. In $75 \%$ of immature oocytes, active 
mitochondria localized in the periphery of the oocytes (peripheral type). After $24 \mathrm{~h}$ of IVM without ALC, while $17 \%$ of oocytes remained in a peripheral type, $44 \%$ showed some migration of active mitochondria toward the center of the oocytes (semiperipheral type) and $39 \%$ presented a diffused distribution of active mitochondria in the whole oocyte cytoplasm (diffused type). On the other hand, in ALC treated oocytes, $60 \%$ of the oocytes presented a diffused type, $25 \%$ exhibited a semiperipheral type, and $15 \%$ had still maintained a peripheral distribution. These results provide the first evidence that ALC treatment during IVM of bovine oocytes enhances their post-fertilization development to the blastcyst stage and enhances the frequency of oocytes that exhibit an extensive relocation (diffused type) of active mitochondria to the inner oocyte cytoplasm.

Sexing

\title{
347 ARTIFICIAL INSEMINATION OF LACTATING HOLSTEIN COWS WITH SEXED SPERM
}

\author{
E. Crichton ${ }^{\mathrm{A}}$, S. Huffman ${ }^{\mathrm{A}}$, K. McSweeney ${ }^{\mathrm{B}}$, and J. Schenk ${ }^{\mathrm{A}}$ \\ ${ }^{\mathrm{A}} \mathrm{XY}$, Inc., Fort Collins, CO 80524, USA; ${ }^{\mathrm{B}}$ Bovine Reproductive Specialists, Loveland, CO 80537, USA
}

The recommended use of sexed sperm has been limited to heifers. The objective of this field trial was to determine pregnancy rates in lactating dairy cows following artificial insemination of 2 or $10 \times 10^{6}$ sexed or $10 \times 10^{6}$ unsexed frozen/thawed sperm. Sperm were separated into X chromosomebearing populations at rates of 3000-5000/s, on the basis of DNA content, with a flow cytometer/sperm sorter operating at $40 \mathrm{psi}$ and using $150 \mathrm{~mW}$ of laser intensity. Sperm were sorted, collected and cryopreserved in a Tris-based medium. Holstein cows, averaging 2.4 lactations (range: 1-9), were presynchronized with prostaglandin $\mathrm{F}_{2 \alpha}(\mathrm{PGF})_{2 \alpha} ;(25 \mathrm{mg})$ i.m. at $40-58$ days in milk (DIM) (average, 44 days). A second dose of $\mathrm{PGF}_{2 \alpha}(25 \mathrm{mg})$ i.m. was administered 14 days later. All cows were screened with ultrasound 14 days later, and those with normal ovarian and uterine status were used for this study. Qualifying cows received $100 \mu$ g gonadotropin-releasing hormone (GnRH) i.m. (average, $72 \mathrm{DIM})$ and $25 \mathrm{mg}$ PGF $2 \alpha$ i.m. 7 days later, and then $100 \mu \mathrm{g}$ GnRH i.m. 2 days after the prostaglandin. Cows $(n=172)$ were fixed-timed inseminated $16-19 \mathrm{~h}$ after the last GnRH treatment, balanced over three sperm insemination treatments, two Holstein bulls, and two inseminators. Both 32-day pregnancy and 60-day fetal sex were determined using ultrasound. Cows carrying fetuses identified as dying at the 32-day ultrasound check were considered not pregnant. Data were subjected to ANOVA. Effects of sperm treatment, bulls, inseminators, and subsequent interactions on pregnancy rate were not significant $(P>0.05)$ The 32-day pregnancy rate and $95 \%$ confidence interval for $10 \times 10^{6}$ unsexed, $10 \times 10^{6}$ sexed, and $2 \times 10^{6}$ sexed inseminates were 55.6 (42.5-68.6), 43.9 (31.6-57.0), and 40.4\% (28.4-53.6), respectively. Additionally, DIM or cow parity did not affect pregnancy rate $(P>0.05)$. Fetal death $(<32$ days) for unsexed inseminates was $5.5 \%$ (two pregnancies) compared to $9.5 \%$ (four pregnancies) for pooled sexed inseminates $(P>0.05)$. There were four confirmed pregnancies of sexed sperm that also aborted between Days 32-60. Fetal sex ratio was significantly altered $(P<0.05)$ for sexed ( $93 \%$ female fetuses) compared to unsexed (52\% female fetuses) inseminates. Pregnancy rate in these selected lactating cows inseminated with sexed sperm was not significantly lower $(P>0.05)$ than that for unsexed sperm. However, these results should be viewed cautiously due to the limited numbers of cows used. Successful use of sexed sperm in dairy cows, which approached an elective waiting period of 80 DIM, was achieved with cows that were selected for reproductive status prior to insemination.

\section{DEVELOPMENT OF A REAL-TIME POLYMERASE CHAIN REACTION ASSAY FOR QUANTIFICATION OF Y-CHROMOSOME-BEARING SPERM IN CATTLE}

\author{
A. S. Maschari, A. Bettegowda, G. W. Smith, and J. R. Pursley \\ Department of Animal Science, Michigan State University, East Lansing, MI 48824, USA
}

Recent data from our laboratory indicate that increasing the time from AI until ovulation in cattle affects the sex ratio of offspring in favor of females (Pursley et al. 1998 J. Dairy Sci. 81, 2139-2144, and unpublished data). One possible explanation for this phenomenon is that the population of Xand Y-chromosome-bearing sperm (X-sperm, Y-sperm) deviate over time to the detriment of Y-sperm. Thus, at the time of ovulation, fewer Y-sperm would constitute the population available for fertilization. Co-culture of sperm and oviductal cells is one way to test whether a greater percentage of Y-sperm detaches from oviductal cells following prolonged incubation. In order to test this, a reliable high-throughput method to determine numbers of Y-sperm is required. The objective of this study was to develop and validate a quantitative PCR (q-PCR)-based assay to accurately determine numbers of Y-sperm in samples from cattle. Primers were designed to amplify a 57-base pair (bp) portion of the SRY gene (for quantification of Y-sperm) and a 21-bp section of autosomal DNA from the 1.715 satellite (for estimation of total sperm numbers). Standard curves were developed from plasmids containing cloned fragments of the SRY gene and the 1.715 satellite region from which primers were designed. Average $\mathrm{R}^{2}$ of 0.9977 and 0.9886 were obtained for the 1.715 satellite and SRY standard curves. Assay of fixed amounts of male genomic DNA revealed between and within assay coefficients of variation of $2.17 \%$ and $1.54 \%$ for the 1.715 satellite and $8.79 \%$ and $9.08 \%$ for the SRY assay, respectively. Specificity of the assay for the SRY gene was verified using female and male genomic DNA. Similar copy numbers per unit DNA were obtained for the 1.715 satellite sequence in both male and female DNA samples, but the SRY sequence was undetectable in female DNA samples. Samples of separated semen with known amounts of X- and Y-sperm (donated by XY, Inc., Fort Collins, CO, USA) were used to determine accuracy of the q-PCR assay in quantification of numbers of Y-sperm. DNA was extracted from sorted semen samples containing $16.8 \%, 32 \%, 66.5 \%$, and $79 \%$ Y-sperm and copies of SRY and 1.715 satellite DNA determined in duplicate samples. Percent recovery of genomic DNA from sorted semen samples was calculated using 1.715 satellite primers and was used to adjust expected number of SRY copies for each sample of sorted semen analyzed. Differences in observed copies of SRY gene versus expected copies of SRY gene were determined by Student's $t$-test. No differences in observed versus expected copies of the SRY gene were detected for each sample $(P>0.05)$. In conclusion, an assay that accurately quantifies numbers of Y-sperm was developed and validated. This assay will be utilized in future studies to determine if the proportions of Y-sperm released from cultured oviductal cells deviate over time. 


\title{
349 IMPROVING THE FUNCTIONALITY OF STORED FLOW CYTOMETRICALLY SEX-SORTED BOAR SPERMATOZOA: SEDIMENTATION VS. CENTRIFUGATION
}

\author{
I. Parrilla, J. M. Vazquez, M. A. Gil, E. M. Garcia, I. Caballero, J. Roca, and E. A. Martinez \\ Department of Animal Medicine \& Surgery, Faculty of Veterinary, University of Murcia, Murcia, Spain
}

\begin{abstract}
Centrifugation has been the routine procedure used to concentrate the highly diluted spermatozoa obtained after sorting. However, this harmful procedure has a detrimental effect on the lifespan of the spermatozoa, particularly when a short-term storage period is required to perform the inseminations in the farms. The aim of the study was to evaluate the procedure of concentration by sedimentation of the flow cytometrically sexsorted boar spermatozoa after a 24 -h storage period, in terms of sperm functionality and recovery efficiency. Spermatozoa were sex-sorted by flow cytometry (Johnson and Welch 1999 Theriogenology 52,1323-1342) and collected in 50-mL tubes containing $5 \mathrm{~mL}$ of Beltsville thawing solution (BTS) with $10 \%$ seminal plasma (SP). The collected samples were split into two aliquots. The first aliquot was concentrated by centrifugation $(800 \mathrm{~g} / 5 \mathrm{~min})$ just after sorting and stored at $17^{\circ} \mathrm{C}$ for $24 \mathrm{~h}$ at a concentration of $6 \times 10^{6} \mathrm{sperm} / \mathrm{mL}$. The second aliquot was directly stored $\left(0.5 \times 10^{6}\right.$ sperm $/ \mathrm{mL}$ ) at $17^{\circ} \mathrm{C}$ for $24 \mathrm{~h}$. In this case, after storage the supernatant was discarded and the pellet obtained by sedimentation was adjusted to $6 \times 10^{6} \mathrm{sperm} / \mathrm{mL}$. For assessment of the sperm functionality after the storage, motility and viability of the spermatozoa were analyzed using a CASA system (Sperm Class Analyzer; Microptic, Barcelona, Spain) and a EPICS XL flow cytometer (Coulter Corporation, Inc., Miami, FL, USA) after the sperm were stained with propidium iodide and SYBR-14, respectively. For assessment of the recovery efficiency, the total number of spermatozoa in each sample was counted. The results are expressed as percentages of five replicates. Statistical analysis was performed using a $\chi^{2}$ test. Percentages of motile and viable spermatozoa just after sorting were $80 \%$ and $83.3 \%$, respectively. After storage at $17^{\circ} \mathrm{C}$ for $24 \mathrm{~h}$, the percentage of viable spermatozoa was significantly higher $(P<0.05)$ in settled spermatozoa $(82.4 \%)$ compared to centrifuged spermatozoa $(63.3 \%)$. Similarly, the percentage of motile spermatozoa was significantly higher $(P<0.05)$ in settled spermatozoa $(78.3 \%)$ compared to centrifuged spermatozoa $(58.3 \%)$, as was the percentage of progressive motility $(63.6 \%$ vs. $35.5 \%$, respectively; $P<0.05)$. Interestingly, the percentage of cell recovery was significantly higher $(P<0.05)$ after sedimentation $(80 \%$ of the sorted population) than after centrifugation $(60.3 \%$ of the sorted population). The results show that sedimentation is more beneficial than centrifugation when short-term storage of sex-sorted boar sperm is required.
\end{abstract}

This work was supported by CDTI and Fundación Seneca.

\section{COMPARATIVE TIME-LAPSE STUDIES ON PORCINE EMBRYOS FERTILIZED IN VITRO WITH FLOW CYTOMETRICALLY SEX SORTED SPERMATOZOA}

\author{
D. Rath and S. Schulze \\ Institute for Animal Breeding, Mariensee, Neustadt, Germany, LS
}

\begin{abstract}
The objective of the present time-lapse studies was to compare developmental characteristics of porcine embryos after in vitro fertilization with flow cytometrically sexed spermatozoa during an in vitro culture period. Immature oocytes were matured $(n=851)$ and fertilized in vitro using 50 spermatozoa of either sex per oocyte. Additionally, in vivo-matured oocytes $(n=700)$ were derived from hormonally stimulated (eCG/hCG) prepuberal gilts, which were slaughtered $38 \mathrm{~h}$ after hCG treatment. Potential zygotes were introduced into the time-lapse system $(50 \mu \mathrm{L}$-microdrops, NCSU-23) $18 \mathrm{~h}$ after the onset of in vitro fertilization. The onset and duration of cell cycles and blastomere rotation as well as collapses and reexpansion of cytoplasm were investigated. At the end of the culture period $(168 \mathrm{~h})$, embryos were labeled with Hoechst 33342 to identify the number of cell nuclei. The time-lapse system consisted of an incubation chamber installed on an inverted phase-contrast microscope and gassed with $5 \%$ $\mathrm{CO}_{2}$ in maximally humidified air. Computer controlled positioning of the chamber allowed the capture and storage of digital images of individual embryos every 30 min over a 7-day period. Converted time values were tested by ANOVA or ANOVA on ranks. In total, 700 in vivo-matured oocytes were fertilized in vitro (Y-sperm: 342 ; X-sperm: 358 ). Cleavage rates were $45.6 \%$ for male and $40.2 \%$ for female embryos. Out of these, $45.5 \%$ developed to male and $62.5 \%$ to female blastocysts, respectively. The onset and duration of cell cycles differed significantly at the 2-cell and morula stages $(P<0.01)$. The onset and number of rotations as well as collapses and re-expansion of cytoplasm were not different. Mean cell numbers of blastocysts were equal for both sexes (male: 35.2; female: 38.8). In parallel, 851 in vitro-matured oocytes were fertilized in vitro (Y-sperm: 431; X-sperm: 422). Cleavage rates were $45.5 \%$ for male and $49.3 \%$ for female embryos. Out of these, $54.1 \%$ developed to male and $56.7 \%$ to female blastocysts, respectively. The onset and duration of cell cycles and rotations as well as collapses were not significantly different between sexes. Mean cell numbers of blastocysts were equal for both sexes (male: 29.5; female: 27.7). Comparison between male embryos derived from in vivo (VV) or in vitro (VT)-matured oocytes showed a delay of the onset of the second cell cycle for VV $(P<0.001)$ and at the blastocyst stage for VT $(P<0.001)$. Accordingly, in these stages the duration of the cell cycle was shortened $(P<0.001)$. The onset and duration of rotations as well as collapses and re-expansion of cytoplasm were not different. Similar data were obtained for the female embryos. The data suggest slight sex related differences (onset of cell cycles), but these might be masked in embryos produced from in vitro-matured oocytes due to their higher individual variability. The experiment also shows the advantages of a time-lapse system to identify dynamic cell processes. It might also be a useful tool to precisely correlate cell cycle events to activation of certain marker genes.
\end{abstract}

\section{INSEMINATION OF HOLSTEIN HEIFERS WITH SEXED SPERM PROCESSED WITH OR WITHOUT EGG YOLK IN THE GLYCEROL-CONTAINING FREEZING MEDIUM}

\author{
J. Schenk and E. Crichton \\ XY, Inc., Fort Collins, CO 80524, USA
}

Preparation of media for use in processing sorted sperm is laborious and expensive. The objective was to determine whether pregnancy rates in heifers following $\mathrm{AI}$ of frozen/thawed $\mathrm{X}$ chromosome-bearing sperm were equivalent for sperm processed for cryopreservation using 
glycerol-containing medium with or without egg yolk. X chromosome-bearing sperm from each of two Holstein bulls were isolated on the basis of DNA content using a flow cytometer. Sorted sperm were collected in $50-\mathrm{mL}$ plastic tubes containing $2-\mathrm{mL}$ of $20 \%$ egg yolk-TRIS extender without glycerol until each tube contained $12.4 \mathrm{~mL}$ and approximately 12 million sperm. Sorted sperm were cooled $\left(5^{\circ} \mathrm{C}\right)$ over 90 min before the addition of $12 \%$ glycerol-containing TIS medium with or without $20 \%$ egg yolk, as two equal volumes $(6.5 \mathrm{~mL})$ at 15 -min intervals. Tubes containing $25 \mathrm{~mL}$ were centrifuged at $850 \mathrm{~g}$ for $20 \mathrm{~min}\left(5^{\circ} \mathrm{C}\right)$. After the supernatant was removed, sperm pellets within treatments and bulls were pooled. Sperm concentration was adjusted to $10 \times 10^{6} / \mathrm{mL}$ with medium containing $6 \%$ glycerol and $20 \%$ egg yolk-TRIS. Sorted sperm were packaged and frozen in $0.25 \mathrm{~mL}$-coded straws on freezing racks in $\mathrm{LN}_{2}$ vapor. Straws from each bull-treatment combination were equally packaged in goblets to assure random use. Post-thaw sperm progressive motility, determined with time-lapse photography ('track motility'), was $44 \%$ for non-egg yolk and $43 \%$ for $20 \%$ egg yolk-glycerol-containing media. Nonsynchronized Holstein heifers $(n=119)$ were inseminated 4,12 , or $24 \mathrm{~h}$ after observed standing estrus. Sperm were deposited into the uterine body anterior to the internal cervical os. Approximately 1 month after insemination, pregnancy was determined using ultrasound. Data were subjected to ANOVA. The pregnancy rate and $95 \%$ confidence interval for sorted sperm processed with non-egg yolk-glycerol-containing medium was $56 \%(49-73 ; n=59)$, and $55 \%(38-62 ; n=60)$ for the $20 \%$ egg yolk-glycerol-containing medium $(P>0.1)$. Bull or inseminator effects on pregnancy rates were not significant $(P>0.01)$. Pregnancy rates as a function of time of insemination at 4,12 , or $24 \mathrm{~h}$ relative to observed estrus were $62 \% n=65 ; 48 \% n=48$; and $50 \% n=6$, respectively $(P>0.1)$. Even though the scope of the field trial was limited, omission of egg yolk from the glycerol-containing medium did not adversely affect pregnancy rates. Preparation of non-egg yolk-glycerol-containing medium greatly reduces associated time, labor, and expense. This study also resulted in quite acceptable pregnancy rates with insemination doses of $2 \times 10^{6}$ total sorted frozen/thawed sperm.

\title{
352 IMPROVING THE EFFICIENCY OF LAPAROSCOPIC INTRAOVIDUCTAL INSEMINATION WITH SEX-SORTED BOAR SPERMATOZOA
}

\author{
J. M. Vazquez, E. A. Martinez, I. Parrilla, C. Cuello, M. A. Gil, E. Garcia, I. Caballero, C. Almiñana, \\ J. Roca, and J. L. Vazquez \\ Department of Animal Medicine and Surgery, Faculty of Veterinary, University of Murcia, Murcia, Spain
}

The insemination of a low number of sex-sorted spermatozoa is a critical issue that must be solved in order to enable the commercial application of this technology in pigs. A new procedure for laparoscopic intraoviductal insemination in sows has recently been reported (Vazquez et al. 2005 Reprod. Dom. Animals 40, 375 abst.). To improve the efficiency of this technique, this experiment was designed to determine the influence of insemination time, relative to the time of ovulation, on the number and quality of zygotes recovered after laparoscopic insemination of sows with sex-sorted spermatozoa. Spermatozoa were stained with Hoechst 33342 and sexed using the EPICS Altra flow sorter (Coulter Corporation, Miami, FL, USA) modified to operate at $42 \mathrm{psi}$ for spermatozoa. Sorted spermatozoa were collected in tubes containing $1.5 \mathrm{~mL}$ of TEST-yolk ( $2 \%)$-seminal plasma ( $10 \%)$. Post-weaning crossbred sows $(n=212$; parity $2-4)$ were hormonally treated with eCG (Folligon; Intervet, Boxmeer, The Netherlands) and hCG (Veterin Corion, Divasa, Spain) and their ovaries were examined using transrectal ultrasonography at intervals of $4 \mathrm{~h}$ from $30 \mathrm{~h}$ after hCG injection to the laparoscopic insemination. Sows were allotted into three groups according to their ovarian status at insemination: preovulatory (P; $n=131$ ), ovulating ( $\mathrm{O} ; n=43)$, and ovulated ( $\mathrm{CL} ; n=38)$ follicles. Follicle status was reconfirmed at insemination by direct observation using the laparoscope. Sows were inseminated in both oviducts with 0.3 million sex-sorted spermatozoa in $0.1 \mathrm{~mL}$ of extender. Eighteen hours later, putative zygotes were collected by washing the oviducts after laparotomy, fixed, stained with lacmoid, and examined by phase-contrast microscopy. Penetration rates were evaluated as numbers of monospermic and polyspermic oocytes per oocytes collected. Monospermic rates were evaluated as numbers of monospermic oocytes per oocytes penetrated. Data were analyzed by ANOVA. The number of putative zygotes collected were 2825,957 , and 736 for $\mathrm{P}, \mathrm{O}$, and $\mathrm{CL}$ groups, respectively. Penetration rates were not different $(P>0.05)$ among groups $(90.4 \%, 94.5 \%$, and $93.7 \%$ for $\mathrm{P}, \mathrm{O}$, and $\mathrm{CL}$, respectively). However, the monospermic rate was significantly higher $(P<0.05)$ in the $\mathrm{P}$ group $(97.4 \%)$ when compared to the $\mathrm{O}$ or $\mathrm{CL}$ groups $(66.7 \%$ and $5.1 \%$ for $\mathrm{O}$ and CL, respectively). Moreover, percentages of sows with six or more zygotes, potentially able to carry the pregnancy to term, were $90.8 \%$ and $46.5 \%$ for $\mathrm{P}$ and $\mathrm{O}$ groups, respectively $(P<0.05)$. No sow of the $\mathrm{CL}$ group presented six or more zygotes. In conclusion, laparoscopic insemination should be performed only in sows with preovulatory follicles when sex sorted spermatozoa are inseminated using this technology.

This work was supported by CDTI and Fundacion Seneca.

\section{IN VITRO FERTILIZATION WITH FLOW-SORTED BUFFALO SPERM}

\author{
M. Zhang ${ }^{\mathrm{A}, \mathrm{B}}, X . W$. Liang $^{\mathrm{A}, \mathrm{B}}, Y . Q . L u^{\mathrm{A}, \mathrm{B}}$, and $K . H . L u^{\mathrm{A}, \mathrm{B}}$ \\ ${ }^{A}$ Guangxi Key Laboratory for Subtropical Bio-Resource Conservation and Utilization; ${ }^{B}$ Animal Reproduction Institute, Guangxi University, \\ Nanning, Guangxi 530005, China
}

Flow cytometrically sorted X and Y sperm have been successfully used for IVF and the production of offspring in cattle and sheep (Maxwell et al. 2004 Anim. Reprod. Sci. 82-83, 79-95). The objective of this study was to test the feasibility of flow sorted buffalo sperm used in IVF systems and to establish a suitable IVF system for sorted buffalo sperm. Oocytes were aspirated from 2-6 mm follicles on the buffalo ovaries from a slaughterhouse and matured for $22-24 \mathrm{~h}$ in a $1-\mathrm{mL}$ dish containing TCM199 $+10 \%$ OCS $+3 \%$ BFF (bovine folliciular fluid) + hormones at $38.5^{\circ} \mathrm{C}, 5 \% \mathrm{CO}_{2}$ in air with maximum humidity. Semen was sorted by a flow-sorter (Clontech, Mountain View, CA, USA) into X- and Y-chromosome bearing sperm at $90 \%$ accuracy and stored at $4^{\circ} \mathrm{C}$ for later use with IVF. Sorted sperm were prepared for IVF by centrifugation $(700 \mathrm{~g}, 20 \mathrm{~min})$ through a Percoll gradient (90\%:45\%), and washed (centrifugation at $700 \mathrm{~g}, 5 \mathrm{~min}$ ) in mTALP-BSA. For IVF, groups of 10-15 oocytes were transferred to $40-\mu \mathrm{L}$ drops of mTALP-BSA and incubated with motile sperm at a concentration of $2 \times 10^{6}$ sperm mL ${ }^{-1}$ in each fertilization drop for $8-10 \mathrm{~h}$ under mineral oil. 
Presumptive zygotes were cultured until Day 8 in $25-\mu \mathrm{L}$ drops of TCM-199 supplemented with $0.33 \mathrm{mM}$ pyruvate and $10 \%$ NCS with granulosa cells at $38.5^{\circ} \mathrm{C}$ under $5 \% \mathrm{CO}_{2}$ in air. Cleavage and blastocyst rates per oocyte insemination were recorded on Day 2 and Days 6-8 after insemination, respectively. Data were analyzed by ANOVA procedures with replicates and treatments in the model. There were significant differences in cleavage rate $(42.23 \%$ vs. $52.28 \%)$ and blastocyst rate $(20.62 \%$ vs. $27.67 \%)$ between sorted and unsorted sperm, respectively (Table 1$)$. There were no significant differences in the proportions of blastocyst development rates on Days 6, 7, or 8 after insemination with sorted and unsorted sperm. The results indicate that sorted buffalo sperm from two bulls have been successfully used in an IVF system to produce sex-controlled embryos.

Table 1. Cleavage and blastocyst rates with different sperm types

\begin{tabular}{lcccccc}
\hline $\begin{array}{l}\text { Sperm } \\
\text { type }\end{array}$ & $\begin{array}{c}\text { Oocytes } \\
(n)\end{array}$ & $\begin{array}{c}\text { Cleaved } \\
(\%)\end{array}$ & $\begin{array}{c}\text { Blastocysts/ } \\
\text { oocyte }\end{array}$ & Day 6 & Doy 7 & Day 8 \\
\hline Sorted & 1020 & $42.23 \pm 2.60^{\mathrm{a}}$ & $20.62 \pm 2.90^{\mathrm{a}}$ & $42.93 \pm 6.69$ & $39.71 \pm 4.58$ & $16.48 \pm 4.14$ \\
Unsorted & 780 & $52.28 \pm 2.98^{\mathrm{b}}$ & $27.67 \pm 0.98^{\mathrm{b}}$ & $42.93 \pm 6.69$ & $37.68 \pm 2.98$ & $16.77 \pm 7.04$ \\
\hline
\end{tabular}

${ }^{a b}$ Values with different superscripts in the same column are significantly different $(P<0.01)$.

This research was supported by grants from the National Natural Science Foundation of China (30360073) and the Guangxi Department of Science and Technology (0330004-13). The authors (M. Z. and X.W. L.) contributed equally to this work.

Sperm Injection

\title{
354 POOR EMBRYO DEVELOPMENT AFTER ICSI WITH DOMESTIC CAT TESTICULAR SPERM IS OVERCOME BY CENTROSOME AND MIDPIECE REPLACEMENT
}

\author{
P. Comizzoli, D. Wildt, and B. Pukazhenthi
}

Department of Reproductive Science, Smithsonian's National Zoological Park, Washington, DC 20008, USA

Testicular sperm from the cat can be used via intracytoplasmic sperm injections (ICSI) to produce embryos in vitro, but the proportion of morulae and blastocysts is less than that obtained using ejaculated sperm. Compromised embryo development has been linked to the inability of the cat testicular sperm centrosome to form a normal sperm aster during the first cell cycle post-ICSI. The aim of the present study was to improve embryo development after ICSI with a testicular spermatozoon by centrosome/midpiece replacement from an ejaculated spermatozoon. Sperm suspensions used for ICSI (fresh testicular sperm from one adult testis vs. frozen-thawed sperm from one ejaculate in each replicate; four replicates) were sonicated for $3 \mathrm{~s}$ at setting 3 using a 60 Sonic Dismembrator (Fisher Scientific, Pittsburgh, PA, USA) to separate heads from centrosome/midpieces. Control immunostaining with monoclonal centrin antibodies revealed that centrosomes were attached to midpieces and were separated from sperm heads after sonication. A single sperm head (testicular, $\mathrm{T}_{h}$; ejaculated, $\mathrm{E}_{\mathrm{h}}$ ) was carefully positioned proximal to a single centrosome/midpiece (testicular, $\mathrm{T}_{\mathrm{c}}$; ejaculated, $\mathrm{E}_{\mathrm{c}}$ ) before injection into an oocyte with a visible polar body (in each replicate, $n=18$ oocytes injected with each $\mathrm{T}_{\mathrm{h}} \mathrm{E}_{\mathrm{c}}, \mathrm{E}_{\mathrm{h}} \mathrm{E}_{\mathrm{c}}, \mathrm{E}_{\mathrm{h}} \mathrm{T}_{\mathrm{c}}$, or $\mathrm{T}_{\mathrm{h}} \mathrm{T}_{\mathrm{c}}$ combination; four replicates). Injected oocytes then were activated with $7 \%$ ethanol and cultured in vitro in $\mathrm{Ham}$ 's $\mathrm{F} 10\left(38.5^{\circ} \mathrm{C}, 5 \% \mathrm{CO}_{2}\right.$ in air). Percentages of first cleavages were recorded from 20 to $32 \mathrm{~h}$ post-activation (hpa). Nuclear status of uncleaved oocytes was evaluated at $48 \mathrm{hpa}$, and embryo development was assessed after 7 days of in vitro culture. Values were expressed as mean \pm standard deviation and analyzed by ANOVA. None of the uncleaved oocytes were activated. Percentages of cleaved oocytes (relative to the total number of injected oocytes) were not different (range, 61-65\%; $P>0.05)$ among combinations of sperm heads and centrosome/midpieces. The mean times of the first cleavage, however, were earlier $(P<0.05)$ after combinations with ejaculated centrosome/midpieces $\left(\mathrm{T}_{\mathrm{h}} \mathrm{E}_{\mathrm{c}}, 25.1 \pm 1.1 \mathrm{~h} ; \mathrm{E}_{\mathrm{h}} \mathrm{E}_{\mathrm{c}}, 25.0 \pm 0.9 \mathrm{~h}\right)$ than with testicular centrosome/midpieces $\left(\mathrm{E}_{\mathrm{h}} \mathrm{T}_{\mathrm{c}}, 29.2 \pm 0.8 \mathrm{~h} ; \mathrm{T}_{\mathrm{h}} \mathrm{T}_{\mathrm{c}}, 29.3 \pm 1.2 \mathrm{~h}\right)$. Percentages of morulae produced were higher $(P<0.05)$ after combinations with ejaculated centrosome/midpieces $\left(\mathrm{T}_{\mathrm{h}} \mathrm{E}_{\mathrm{c}}, 15.3 \pm 2.7 \% ; \mathrm{E}_{\mathrm{h}} \mathrm{E}_{\mathrm{c}}, 16.7 \pm 2.1 \%\right)$ than with testicular centrosome/midpieces $\left(\mathrm{E}_{\mathrm{h}} \mathrm{T}_{\mathrm{c}}, 8.3 \pm 3.1 \% ; \mathrm{T}_{\mathrm{h}} \mathrm{T}_{\mathrm{c}}, 6.9 \pm 2.2 \%\right)$ Likewise, percentages of blastocysts were higher $(P<0.05)$ after combinations with ejaculated centrosome/midpieces $\left(\mathrm{T}_{\mathrm{h}} \mathrm{E}_{\mathrm{c}}, 12.5 \pm 3.0 \% ; \mathrm{E}_{\mathrm{h}} \mathrm{E}_{\mathrm{c}}\right.$, $13.9 \pm 2.8 \%)$ than with testicular centrosome/midpieces $\left(\mathrm{E}_{\mathrm{h}} \mathrm{T}_{\mathrm{c}}, 5.6 \pm 3.3 \% ; \mathrm{T}_{\mathrm{h}} \mathrm{T}_{\mathrm{c}}, 6.9 \pm 2.9 \%\right)$. These results demonstrated that: (1) kinetics of the first cell cycle and the success of embryonic development were determined by centrosome/midpiece source; and (2) poor developmental potential of domestic cat testicular sperm could be circumvented by centrosome/midpiece replacement from an ejaculated spermatozoon.

\section{IMPROVEMENT OF CUMULUS-FREE IN VITRO MATURATION AND ITS APPLICATION TO MICROINSEMINATION WITH PRIMARY SPERMATOCYTES IN MICE}

\author{
H. Miki ${ }^{\mathrm{A}, \mathrm{B}}, N$. Ogonuki $^{\mathrm{B}}, K$. Inoue $^{\mathrm{B}}, T . B a b a^{\mathrm{A}}$, and A. Ogura ${ }^{\mathrm{B}}$ \\ ${ }^{A}$ Graduate School of Life and Environmental Science, University of Tsukuba, Tsukuba Science City, Ibaraki, Japan; \\ ${ }^{B}$ RIKEN Bioresource Center, Tsukuba Science City, Ibaraki, Japan
}

Manipulation of immature oocytes requires removal of cumulus cells, which may compromise ooplasmic maturation. This problem can be overcome by cytoplasm exchange at the MII stage, but this requires a second micromanipulative step. This study was undertaken to determine whether cumulus-free IVM can be improved by modifying IVM medium. We assessed the quality of oocytes by several cytochemical parameters, and by the 
subsequent development of embryos constructed by IVF, parthenogenetic activation, or microinsemination using primary spermatocytes. GV-stage oocytes were collected from superovulated B6D2F1 females. After freeing the oocytes from cumulus cells, they were subjected to IVM in either $\alpha$ MEM or TYH media, or a 1:1 mixture of the two (termed TaM). IVM was performed under chemically semidefined conditions by using BSA as the only protein source. Oocytes that reached the MII stage were analyzed for distribution of mitochondria, activity of the catalytic p34cdc $2 \mathrm{kinase}$ subunit of the maturation promoting factor (MPF), and chromosomal alignment within the MII spindle. Microinsemination with primary spermatocytes was performed in oocytes at the MI stage as described previously (Ogura et al. 1998 PNAS 95, 5611-5615), but without MII chromosome exchange. TYH and $\alpha$ MEM media had opposing effects on several parameters. TYH resulted in a better maturation rate $(181 / 196,92.3 \%)$ than $\alpha \mathrm{MEM}(184 / 257$, 71.6\%). In contrast, $\alpha$ MEM supported better embryo development to the morula/blastocyst stage than TYH following IVF (93.3\% vs. $76.5 \%$ ) or parthenogenetic activation ( $82.4 \%$ vs. $60.4 \%)$. Mitochondrial distribution in MII oocytes was diffuse in $\alpha$ MEM, but aggregated in TYH. MPF activity in MII oocytes was significantly higher in TYH than in $\alpha$ MEM $(P<0.05)$. Oocytes derived from TaM had intermediate characteristics between TYHand $\alpha$ MEM-oocytes and essentially resembled in vivo-matured oocytes, with the mitochondrial distribution pattern being most typically intermediate By using TaM, 23.8\% of GV oocytes developed into full-term fetuses following IVF and embryo transfer (compared to TYH: $6.3 \%$, and $\alpha$ MEM: $18.2 \%$ ). This IVM system was then applied to oocytes to be injected with spermatocytes when in the MI stage. Approximately $60 \%$ of the oocytes survived injection. After 127 two-cell embryos were transfered, two normal-looking offspring were obtained. This is the first successful birth of pups derived from fertilization with spermatocytes by single injection, without serial nuclear transfer. Chromosomal analysis at MII revealed that the low developmental efficiency after embryo transfer could be attributed mainly to premature sister chromatid separation, as reported previously (Ogura et al. 1998). These results demonstrate that optimization of IVM media can improve the quality of cumulus-free IVM oocytes in mice, although it cannot overcome defects in the kinetics of injected spermatocyte chromosomes.

\title{
356 BLASTOCYST DEVELOPMENT FROM DOMESTIC CAT OOCYTES INJECTED WITH DEHYDRATED SPERMATOZOA
}

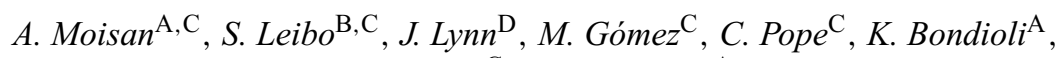 \\ B. Dresser ${ }^{\mathrm{C}}$, and R. Godke $\mathrm{A}^{\mathrm{A}}$
}

${ }^{A}$ Reproductive Biotechnology Center, Department of Animal Sciences, Louisiana State University Agricultural Center, Baton Rouge, LA 70803, USA;

${ }^{B}$ Department of Biological Sciences, University of New Orleans, New Orleans, LA 70148, USA;

${ }^{\mathrm{C}}$ Audubon Nature Institute Center for Research of Endangered Species, New Orleans, LA 70178, USA;

${ }^{D}$ Department of Biological Sciences, Louisiana State University, Baton Rouge, LA 70803, USA

Live offspring have been produced by intracytoplasmic sperm injection (ICSI) of dehydrated spermatozoa into mouse and rabbit oocytes (Wakayama and Yanagimachi 1998 Nature Biotechnol. 16, 639; Liu et al. 2004 Biol. Reprod. 70, 1776). The objective of the present study was to determine the capability of dehydrated domestic cat spermatozoa to fertilize oocytes after ICSI. Spermatozoa were collected from three toms by ejaculation into an artificial vagina. Freshly collected samples were layered under $1 \mathrm{~mL}$ of Tyrode's salt solution supplemented with HEPES, BSA, glutamine, pyruvate, lactate, and $50 \mathrm{mM}$ ethyleneglyeotetraacetic acid (EGTA) $\mathrm{pH}=8.2-8.4$, and allowed to swim-up during a 12 -min incubation at $38^{\circ} \mathrm{C}$. From the upper $600 \mu \mathrm{L}$ of the sperm suspension, four $100-\mu \mathrm{L}$ aliquots were collected and placed into 2-mL glass ampoules, plunged into liquid nitrogen $\left(\mathrm{LN}_{2}\right)$, freeze dried at $-43^{\circ} \mathrm{C}$ to $-45^{\circ} \mathrm{C}$ and 44 to $76 \times 10^{-3} \mathrm{mbar}$ pressure, and stored at $4^{\circ} \mathrm{C}$. Four to twelve $10-\mu \mathrm{L}$ aliquots of the same suspension were placed onto glass slides, air-dried for $30 \mathrm{~min}$, and stored in a desiccator at room temperature. Domestic cat oocytes were collected from minced ovaries and allowed to mature in vitro for 22 to $24 \mathrm{~h}$. After maturation, oocytes were either fertilized in vitro (IVF; $n=36$ ), or sperm injected (ICSI) with fresh or refrigerated $(n=74)$, freeze-dried $(n=45)$, or air-dried $(n=45)$ spermatozoa. After ICSI or IVF, oocytes were cultured in a three-step-sequential medium (Gómez et al. 2004 Cloning Stem Cells 6, 247) for up to 8 days. Cleavage and development to the blastocyst stage was assessed on Days 2 and 8 of culture, respectively. Cleavage rates after IVF (56\%), ICSI with freeze-dried (60\%), or ICSI with fresh spermatozoa $(59 \%)$ were higher than those obtained after ICSI with air-dried spermatozoa $(35 \% ; P<0.05)$. Blastocyst development after ICSI treatments was obtained only with fresh spermatozoa $(9 \%)$, and was lower than that obtained after IVF $(25 \% ; P<0.05)$. Recently, one hatching blastocyst was produced when oocytes $(n=18)$ were exposed to calcium ionophore $2 \mathrm{~h}$ after ICSI with freeze-dried sperm. This is the first report that domestic cat embryos can be produced in vitro by injecting oocytes with dried spermatozoa.

\section{FERTILIZATION AND EMBRYONIC DEVELOPMENT OF PORCINE OOCYTES FOLLOWING INTRACYTOPLASMIC SPERM HEAD INJECTION ENHANCED BY ELECTRIC STIMULATION TO OOCYTES BUT NOT BY PRE-TREATMENT OF SPERMATOZOA WITH DITHIOTHREITOL}

\author{
M. Nakai ${ }^{\mathrm{A}, \mathrm{B}}, N_{\text {. Kashiwazaki }}^{\mathrm{A}}$, A. Takizawa $^{\mathrm{A}}$, N. Maedomari $^{\mathrm{A}}$, M. Ozawa $^{\mathrm{B}}$, J. Noguchi $^{\mathrm{B}}$, H. Kaneko $^{\mathrm{B}}$, \\ M. Shino ${ }^{\mathrm{A}}$, and K. Kikuchi ${ }^{\mathrm{B}}$ \\ ${ }^{A}$ Azabu University, Sagamihara, Kanagawa, 229-1501, Japan; \\ ${ }^{B}$ Genetic Diversity Department, National Institute of Agrobiological Sciences, Tsukuba, Ibaraki, 305-8602, Japan
}

Failure of sperm nuclear decondensation has been reported after injection into oocytes in pigs (Kren et al. 2003 J. Reprod. Dev. 49, 271-273). We examined the effects of pretreatment of spermatozoa with Triton X-100 (TX-100) and dithiothreitol (DTT) and electric stimulation of oocytes after injection on sperm decondensation, male pronuclear formation, and in vitro development to the blastocyst stage. We performed three replicates in each experimental group, with a total of about 70 oocytes per group. In Experiment 1, spermatozoa were pretreated with $1 \%$ TX-100 and 5 mM 
DTT (T+D), and injected into IVM oocytes that were collected from crossbred gilts. Electric stimulation (1.5 kV/cm, $20 \mu \mathrm{s} ;$ Nakai et al. 2003 Biol. Reprod. 68, 1003-1008) was applied $1 \mathrm{~h}$ to the oocytes after the injection (the stimulated group) or was not applied (the nonstimulated group). Some of the oocytes in each group were evaluated for morphological changes of sperm nuclei at hourly intervals until $10 \mathrm{~h}$ post-injection. Of nonstimulated oocytes, those injected with untreated spermatozoa showed a delayed peak in nuclear decondensation $(39.4$ to $44.1 \%$, 3-6h after the injection) compared to that of oocytes injected with $\mathrm{T}+\mathrm{D}$ treated spermatozoa $(57.0$ to $52.6 \%, 1-1 \mathrm{~h})$. The rate of male pronuclear formation increased after $4 \mathrm{~h}$ post-stimulation (by 40 to $60 \%$ ) when the injected oocytes were stimulated, whether or not spermatozoa were pretreated. In nonstimulated oocytes, the rate of male pronuclear formation stayed at the basal level (less than $20 \%$ ) throughout the culture period regardless of sperm treatments. Thus, the $\mathrm{T}+\mathrm{D}$ treatment of spermatozoa did not affect decondensation and pronuclear formation. In Experiment 2 , the effects of electric stimulation and sperm treatments with $\mathrm{T}+\mathrm{D}$ on the rate of blastocyst formation and the mean numbers of cells per blastocyst were evaluated. Oocytes that were stimulated after injection of either T+D-treated or untreated spermatozoa showed significantly higher percentages of blastocyst formation ( $24.8 \%$ and $27.1 \%$, respectively) than did nonstimulated oocytes $(1.1 \%$ and $4.1 \%$ for T+D-treated and untreated, respectively; $P<0.01)$. The rate of blastocyst formation was not different between the $\mathrm{T}+\mathrm{D}$-treated and the untreated groups. The mean number of cells per blastocyst was not different among all groups (14.0-29.4). In conclusion, the pretreatment of sperm with TX-100 and DTT shifted the timing of sperm nuclear decondensation forward. However, pronuclear formation and development to the blastocyst stage in vitro were not improved by the sperm treatment. Electric stimulation to the injected oocytes enhances in vitro development to the blastocyst stage in pigs.

\title{
358 MICROINSEMINATION USING MALE GERM CELLS FROM EPIDIDYMIDES AND TESTES STORED IN FREEZERS WITHOUT CRYOPROTECTANT
}

\author{
N. Ogonuki ${ }^{\mathrm{A}}, K$. Mochida $^{\mathrm{A}}, A$. Shinmen $^{\mathrm{A}}, M$. Ohkawa $^{\mathrm{A}}, H . M_{i k i}^{\mathrm{A}}, K$. Inoue $^{\mathrm{A}}, M$. Fray $^{\mathrm{B}}, K_{\text {Moriwaki }}{ }^{\mathrm{A}}$, \\ Y. Obata ${ }^{\mathrm{A}}$, and A. Ogura $\mathrm{A}$ \\ A RIKEN Bioresource Center, Tsukuba, Ibaraki, Japan; \\ ${ }^{B}$ MRC, Harwell, UK
}

\begin{abstract}
Cryopreservation of male germ cells is a strategy for the conservation of species and strains valuable to biomedical researchers. However, to minimize damage that may occur during freezing and thawing, complex cryopreservation protocols that have been optimized for the stage and species of the male germ cell are usually employed. This study was undertaken to see whether mouse male germ cells could be safely cryopreserved for later use by freezing the whole epididymides and testes without cryoprotectant. Furthermore, we examined whether frozen male germ cells maintained their fertilization ability after international transportation on dry ice. Epididymides and testes were collected from sexually mature male ICR and $\mathrm{C} 57 \mathrm{BL} / 6 \mathrm{Cr}$ mice and placed in polypropylene cryotubes. The cryotubes were frozen at $-80^{\circ} \mathrm{C}$ with or without a freezing container, or were plunged directly into liquid nitrogen $\left(\mathrm{LN}_{2}\right)$. They were stored at $-80^{\circ} \mathrm{C}$ or in $\mathrm{LN}_{2}$ from between one week and one year. Epididymides and testes were thawed by placing the cryotubes in a water bath at room temperature. B6D2F1 and $\mathrm{C} 57 \mathrm{BL} / 6 \mathrm{Cr}$ oocytes were microinseminated with either epididymal and testicular spermatozoa or round spermatids. After embryo transfer into pseudopregnant females, normal pups were obtained irrespective of the method of cryopreservation and cell type used. However, their birth rates (2-33\%) were lower than those of our conventional microinsemination using fresh sperm or spermatids (20-60\%). For transportation experiments, testes were collected from C57BL/6J mice and placed in a cryotube. The cryotubes were frozen at $-80^{\circ} \mathrm{C}$ in a freezing container. On the day of transportation, the cryotubes were placed in a polystyrene foam case filled with dry ice and were transported from Harwell (UK) to Tsukuba (Japan) by air and land. After three days, the samples were delivered to the recipient facility and were stored at $-80^{\circ} \mathrm{C}$ until use (about 1 month). After thawing and collection of spermatogenic cells, C57BL/6J oocytes were microinseminated with either testicular spermatozoa or elongated spermatids. After embryo transfer, 24 (34\% per transfer) and 8 (16\%) offspring, respectively, were obtained from the two groups. These results indicate that mouse male germ cells retain their nuclear integrity even after freezing epididymides or testes in freezers without cryoprotectant. Since this cryopreservation technique is very simple and allows storage at $-80^{\circ} \mathrm{C}$ for at least several months, it may enable transportation of mouse male germ cells internationally on dry ice, even when the senders are not specialized in cryopreservation
\end{abstract}

\section{ICSI AND ACTIVATION OF OOCYTES RECOVERED FROM TRANSITIONAL AND CYCLING MARES}

\author{
T. Suh, S. Purcell, and G. Seidel Jr \\ Animal Reproduction and Biotechnology Laboratory, Colorado State University, Fort Collins, CO 80523, USA
}

Ovarian follicular development in mares during the transitional period before the breeding season leads to an accumulation of antral follicles of various sizes. The quality of oocytes at this stage may be compromized until the first seasonal ovulation. In this study, we evaluated the developmental competence of oocytes recovered from transitional and cyclic mares, and the effect of zygote activation after intracytoplasmic sperm injection (ICSI). A $2 \times 2 \times 2$ factorial experiment consisting of oocytes from transitional and cyclic mares, two follicle sizes (10 to 20 and $20+$ mm), and two treatments (control and activated) was conducted. Follicular oocytes of 14 mares were aspirated in March and April (transitional) and May to July (cyclic) five times per each period at 10-day intervals, without use of hCG. Oocytes aspirated from mares were matured in vitro in a defined medium similar to SOF plus FSH, LH, epidermal growth factor (EGF), insulin-like growth factor (IGF), estradiol ( $\left.\mathrm{E}_{2}\right)$, prostaglandin $\left(\mathrm{P}_{4}\right)$ and $10 \% \mathrm{FCS}$, for $30 \pm 1 \mathrm{~h}$ under $5 \% \mathrm{CO}_{2}$ in air at $38.5^{\circ} \mathrm{C}$; oocytes with a first polar body were used for ICSI. Motile sperm from frozen-thawed semen were used for sperm injection with a piezo-driven pipet. For activation after ICSI, presumptive zygotes were cultured in G1.3 containing $0.02 \mu \mathrm{M}$ phorbol 12 -myristate 13 -acetate (PMA) for $2 \mathrm{~h}$, and then in $2 \mathrm{mM}$ 6-dimethylaminopurine (6-DMAP) for $3 \mathrm{~h}$ under $6 \% \mathrm{CO}_{2}$ in air at $38.5^{\circ} \mathrm{C}$. Zygotes were cultured in $50 \mu \mathrm{L}$ drops of DMEM/F12 containing $10 \% \mathrm{FCS}$ for 9 days at $38.5^{\circ} \mathrm{C}$ in $5 \% \mathrm{CO}_{2} / 5 \% \mathrm{O}_{2} / 90 \% \mathrm{~N}_{2}$. Medium was replaced every 3 days. Cleavage and blastocyst rates were calculated based on non-degenerating injected oocytes. Data were analyzed by Fisher's exact test. A total of 115 and 78 oocytes 
were recovered from cyclic and transitional mares. Average maturation rates to $\mathrm{MII}$ in the respective groups were 76.5 and $65.4 \%$, respectively $(P<0.07)$, and those of 10 to 20 and $20+\mathrm{mm}$ follicle groups were 70.6 and $80.0 \%$, respectively $(P>0.05)$. The average cleavage rate in cyclic mares was higher than in transitional mares, and that of the activated group averaged over follicle sizes was higher than that of controls $(P<0.05$; Table 1); those of 10 to 20 and $20+\mathrm{mm}$ follicle groups were not different $(P<0.05$; Table 1$)$. Blastocyst rates per oocyte within main effects were not different $(P<0.05$; Table 1). Oocytes from transitional mares had lower cleavage rates than those of cyclic mares, but blastocyst development was similar. Activation of zygotes clearly improved cleavage rates of in vivo-derived immature equine oocytes after ICSI.

Table 1. Main effect means of responses after ICSI

\begin{tabular}{llcccc}
\hline Main effect & Treatment & No. oocytes injected & No. degenerated after ICSI & Cleaved (\%) & Blastocysts (\%) \\
\hline Mares & Cyclic & 86 & 5 & $59.3^{\mathrm{a}}$ & 18.5 \\
& Transitional & 51 & 8 & $41.9^{\mathrm{b}}$ & 16.3 \\
Follicle size (mm) & $10-10$ & 113 & $13^{\mathrm{a}}$ & 51.0 & 16.0 \\
\multirow{3}{*}{ Activation } & $\leq 20$ & 69 & $0^{\mathrm{b}}$ & 62.5 & 25.0 \\
& Control & 68 & 7 & $40.3^{\mathrm{a}}$ & 12.9 \\
& PMA & 6 & $66.1^{\mathrm{b}}$ & 22.6 \\
\hline
\end{tabular}

${ }^{\mathrm{ab}}$ Means within columns within main effects without common superscripts differ $(P<0.05)$.

\title{
Superovulation
}

\section{THE EFFECTS OF PROGESTERONE AND PRESENCE OF CORPUS LUTEUM ON SUPEROVULATION IN BEEF CATTLE (BOS TAURUS)}

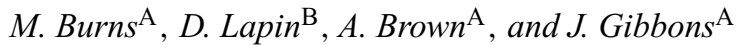

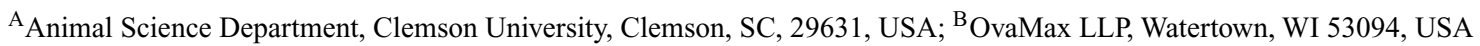

Bovine embryo transfer is a multi-million dollar industry that relies on hyperstimulation of cattle ovaries with exogenous follicle stimulating hormone (FSH). This process is variable among donors, breeds, and embryo transfer organizations. Historically, donor cattle are administered FSH (IM; $\sim 15$ to $50 \mathrm{mg}$ ) for 4 days and a luteolytic dose of prostaglandin F2 $\alpha$ (PGF2 $\alpha$; IM; $25 \mathrm{mg}$ twice daily) on Day 3 . The presence of a functional corpus luteum (CL) is crucial for this protocol; however, there are embryo donor cattle that do not exhibit progesterone indicative of a functional CL. Experiment 1 was performed to determine whether the number of embryos and ovulations that could be generated from donors without a functional CL might be increased by progesterone supplementation via a vaginal insert (CIDR). Experiment 2 was performed to determine an optimal FSH (Sioux Biochemical, Inc., Sioux Center, IA, USA) dosage (high dose $=30$ units, $n=9$; low dose $=20$ units, $n=7$ ) for cattle without a functional CL. Embryos were collected nonsurgically by uterine lavage and were evaluated morphologically according to the IETS system. Progesterone concentrations were determined using an RIA on venous blood samples $(10 \mathrm{~mL})$. Ovulations (as evident by resulting CL) were counted using transrectal ultrasonography. Embryo, ovulation, and progesterone data were analyzed using ANOVA with SAS (SAS Institute, Inc., Cary, NC, USA), to evaluate the main effects of group and time (progesterone concentrations only). In Experiment 1, there was no difference in the number of high-quality embryos that were recovered from hyperstimulated (IM; 35 units) Angus-cross cattle that either had a functional CL ( $n=12$ ) or a CIDR insert $(n=12)$ following PGF $2 \alpha$ injection. Progesterone concentrations were similar until Day 3 (Day $0=$ injection of PGF2 $\alpha$ and CIDR insertion). On Day 3, the CL group had higher $(P<0.05)$ progesterone concentrations $(3.5 \pm 0.4 \mathrm{ng} / \mathrm{mL})$ than the CIDR group $(1.5 \pm 0.1 \mathrm{ng} / \mathrm{mL})$. Further, there was no difference between the average number of ovulations for the cows with a CL (22.5 \pm 2.4$)$ or cows without a CL (22.8 \pm 1.5$)$. In Experiment 2, there was no difference in the number of high-quality embryos recovered from cattle in the high-dose group $(8.1 \pm 0.4)$ compared to cattle in the low dose group (5.7 \pm 1.3$)$. There were no differences during the treatment period in progesterone concentrations between high- and low-dose groups. Although there was no statistical difference in the number of ovulations between cattle in the high dose group (20.2 \pm 2.5$)$ compared to cattle in the low dose group (15.6 \pm 0.9 ), the higher FSH dose did yield over 30\% more ovulations. Embryo collection from cattle that do not show signs of a $\mathrm{CL}$ can increase the number of offspring and embryos, and the genetic progress of otherwise unavailable cattle, thus benefiting the beef and beef cattle industries.

\section{OVIDUCT CILIARY CURRENTS IN BOVINE AND BUFFALO AT DAY 1 AFTER OVULATION}

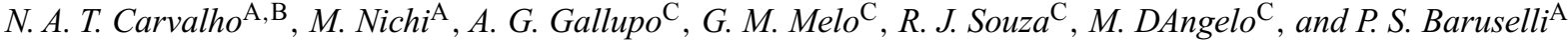 \\ AFMVZ, USP, Sao Paulo, Sao Paulo, Brazil; ${ }^{\mathrm{B} A P T A-V a l e ~ d o ~ R i b e i r a, ~ R e g i s t r o, ~ S a o ~ P a u l o, ~ B r a z i l ; ~}$ \\ ${ }^{\mathrm{C}}$ Instituto Biolõgico, Sao Paulo, Brazil
}

Buffalo present a low embryo recovery rate after superovulation, due to failure of oocyte entry into the oviduct. The aim of this study was to evaluate the effect of the estradiol-17 $\beta$ on oviduct ciliary currents in bovine and buffalo at Day 1 after ovulation. Six Nelore cows and six Murrah buffalo cows were synchronized with an intravaginal progesterone device (DIB ${ }^{\circledR}$; Syntex, Argentina) plus $2 \mathrm{mg}$ of estradiol benzoate intramuscular (i.m.) on Day 0 (D0). After eight days (D8) the device was removed and the animals received prostaglandin F2 $\alpha$ analogue $\left(0.15 \mathrm{mg}\right.$ i.m.; Prolise ${ }^{\circledR}$; Tecnopec, Sao Paulo, Brazil) plus 400 IU of eCG (i.m.; Novormon ${ }^{\circledR}$; Syntex). On D9, ovulation was induced with GnRH (25 $\mu$ g i.m.; Gestran Plus ${ }^{\circledR}$; Tecnopec). 
After $48 \mathrm{~h}$ the animals were slaughtered and the oviducts were removed and rinsed in a solution of Dulbeccos's phosphate-buffered saline with $1 \%$ gentamicin (DPBS + gentamicin). The oviduct was lavaged with Hank's balanced salt solution supplemented with 25 mM HEPES (HBSS), and the mesosalpinx and fimbria were removed. The oviduct was then carefully incised along its entire length. After the luminal surface was exposed, the oviduct was secured with the luminal surface exposed in a $150 \times 20$-mm dish containing a layer of paraffin. The oviduct was then completely submerged in $100 \mathrm{~mL}$ HBSS (Group $1-\mathrm{G} 1, n=5$ bovine; G2, $n=7$ buffalo) or $100 \mathrm{~mL}$ HBSS with $10 \mathrm{ng} / \mathrm{mL}$ estradiol-17 $\beta$ (G3, $n=6$ bovine; $\mathrm{G} 4, n=4$ buffalo; factorial $2 \times 2$ ) that had been prewarmed to $38.5^{\circ} \mathrm{C}$. The dish containing the oviduct was incubated for $30 \mathrm{~min}$ at $38.5^{\circ} \mathrm{C}$ to allow equilibration of temperature and culture medium. After equilibration, the oviduct in the culture dish was placed on a stereomicroscope (SMZ-1T) and the luminal epithelium was observed $(\times 30)$. To assess ciliary beat, 450 black microspheres $\left(10 \mu \mathrm{m}\right.$; Polybead ${ }^{\circledR}$; Polysciences, Inc., Warrington, PA, USA) were applied to the luminal surface of the oviduct. The microspheres were applied dropwise $(10 \mu \mathrm{L})$ to one portion of the infundibulum and the area of application was immediately observed to determine the direction of microsphere motion over approximately 1 min. The direction of motion was graduated in scale $(0=$ none, $1=$ uterine, $2=$ ovarian-uterine, and $3=$ ovarian). Two-way analyses of variance was used to compare the effects of species (bovine $\times$ buffalo) and treatments (without estradiol $\times$ with estradiol) and the species-by-treatment interaction. The data were analyzed by General Linear Models procedure of SAS/STAT (SAS Institute, Inc., Cary, NC, USA) and the direction of motion was compared by the $t$ test's LSD. There was no effect of species and treatment, and no species-by-treatment interaction. The direction of motion of microspheres was consistently toward the uterus (pro-uterine) for G1 $(0.80 \pm 0.20), \mathrm{G} 2(1.00 \pm 0.00), \mathrm{G} 3(1.00 \pm 0.00)$, and G4 $(1.00 \pm 0.33 ; P>0.05)$. Analyzing the main effects, bovine presented score $(0.94 \pm 0.05)$ similar to that of buffalo $(1.00 \pm 0.17 ; P>0.05)$. Animals treated without estradiol $(0.92 \pm 0.08)$ showed the same score as animals treated with estradiol $(1.00 \pm 0.12)$. In conclusion, the presence of estradiol-17 $\beta$ in culture medium had no effect on the oviduct ciliary currents in bovine and buffalo.

This work was supported by FAPESP (02/03245-1; 03/00798-1), SPVS, Syntex, Cultilab, and Tecnopec.

\title{
362 EFFECT OF TIME OF REMOVAL OF A PROGESTERONE RELEASING DEVICE AND GnRH TREATMENT ON THE INTERVAL TO AND DISTRIBUTION OF OVULATIONS IN SUPERSTIMULATED BEEF COWS
}

\author{
P. Chesta ${ }^{\mathrm{A}}$, D. Maraña ${ }^{\mathrm{A}}, L \cdot$ Peres $^{\mathrm{A}}$, and G. A. Bo $\mathrm{A}^{\mathrm{A}, \mathrm{B}}$ \\ A Instituto de Reproducción Animal Córdoba (IRAC), JL de Cabrera 106, X5000GVD Córdoba, Argentina; \\ ${ }^{\mathrm{B}}$ Universidad Católica de Córdoba, Cordoba, Argentina
}

An experiment was designed to evaluate the effect of the time of removal of an intravaginal progesterone (P4) releasing device and GnRH treatment on the interval to and the distribution of ovulations in beef cows superstimulated with Folltropin-V (Bioniche Animal Health, Belleville, Ontario, Canada). Red Angus donors (22 cows and 12 heifers) with a body condition score between 2.5 to 3.5 out of 5 were blocked by parity and randomly assigned to one of four treatment groups in a $2 \times 2$ factorial design. On Day 0, all donors received an i.m. DIB vaginal device (Syntex, Argentina) plus $2.5 \mathrm{mg}$ estradiol-17 $\beta$ and $50 \mathrm{mg}$ P4 (Laboritorio Rio de Janeiro, Argentina). Superstimulatory treatments were initiated on Day 4, with a total dose of $320 \mathrm{mg}$ (cows) or $200 \mathrm{mg}$ (heifers) NIH-FSH-P1 (Folltropin-V; Bioniche Animal Health, Inc., Belleville, Ontario, Canada) in twice daily i.m. injections over 4 days. All cows received PGF treatment $(150 \mu \mathrm{D}(+)$ cloprostenol: Ciclase; Syntex $)$ in the morning and afternoon of Day 6 and were randomly divided to have DIB devices removed at the time of the second PGF treatment (Day 6.5) or $12 \mathrm{~h}$ later (Day 7). On Day 8, donors were further subdivided to receive GnRH (0.050 mg Lecirelina, Gonasyn; Syntex) or no further treatment, and all cows were fixed-time AI either 60 and $72 \mathrm{~h}$ after the first PGF injection $(0 \mathrm{~h})$. All cows and heifers were examined by ultrasonography every $8 \mathrm{~h}$ for a period of $120 \mathrm{~h}$ commencing at the time of DIB removal, to determine the time and distribution of ovulations. For each cow, mean time of ovulation was used for the statistical analysis. The distributions of ovulations were compared among groups by Levene's test, and then by ANOVA (equal variances) or Friedman two-way nonparametric ANOVA (unequal variances). No significant differences were detected between cows and heifers $(P>0.13)$ for the parameters evaluated, and data were combined. There was no effect $(P>0.22)$ of treatment on the number of ovulations (GnRH: $12.6 \pm 1.6$ vs. no GnRH: $15.1 \pm 1.1$ and Day 6.5: $13.7 \pm 1.3$ vs. Day 7: $14.3 \pm 1.5$ ), or numbers of transferable embryos (GnRH: $5.1 \pm 1.1$ vs. no GnRH: $3.1 \pm 0.8$ and Day $6.5: 3.5 \pm 0.8$ vs. Day 7: $4.81 \pm 0.1)$. However, there was a significant effect of GnRH treatment and a GnRH-by-time-of-DIB-removal interaction on the distribution of ovulations $(P<0.05$; Table 1$)$. The administration of GnRH in a superstimulatory protocol using $\mathrm{P} 4$ releasing devices results in earlier and more synchronous ovulations than when GnRH is not used.

Table 1. Distribution of ovulations (hours after the first PGF) in superstimulated Red Angus donors treated with progesterone-releasing devices (DIB) for 6.5 or 7 days with and without GnRH treatment on Day 8

\begin{tabular}{llrllr}
\hline Day of DIB removal & GnRH & $n$ & Mean & Range & Variance \\
\hline 6.5 & No & 8 & 81.3 & $69-96$ & $72.8^{\mathrm{b}}$ \\
6.5 & Yes & 10 & 77.4 & $67-83$ & $22.5^{\mathrm{ab}}$ \\
7 & No & 8 & 86.3 & $73-96$ & $58.6^{\mathrm{b}}$ \\
7 & Yes & 8 & 79.7 & $74-82$ & $7.1^{\mathrm{a}}$ \\
Main effects & No & 16 & $83.8^{\mathrm{a}}$ & $69-96$ & $67.9^{\mathrm{a}}$ \\
& Yes & 18 & $78.4^{\mathrm{b}}$ & $67-83$ & $16.2^{\mathrm{b}}$ \\
6.5 & & 18 & 79.1 & $67-96$ & $45.8^{\mathrm{b}}$ \\
7 & & 16 & 83.0 & $73-96$ & 42.2 \\
\hline
\end{tabular}

${ }^{\mathrm{ab}}$ Means or variances with different subscripts within the column differ $(P<0.05)$. 


\title{
363 EFFECT OF OESTRADIOL VALERATE OR BENZOATE ON INDUCTION OF A NEW FOLLICULAR WAVE EMERGENCE IN BOS INDICUS COWS AND HEIFERS TREATED WITH NORGESTOMET AURICULAR IMPLANT
}

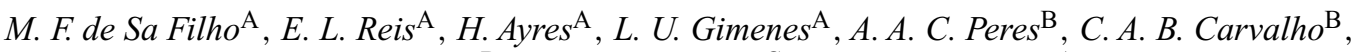 \\ J. B. Carvalho $^{\mathrm{B}}$, C. A. S. C. Araujo ${ }^{\mathrm{C}}$, and P. S. Baruselli ${ }^{\mathrm{A}}$ \\ ${ }^{A}$ Departamento de Reproduão Animal, FMVZ, USP, São Paulo, SP, Brazil; \\ ${ }^{B}$ APTA, Polo Regional do Vale do Paraiba, Pindamonhangaba, SP, Brazil; \\ ${ }^{\mathrm{C}}$ Universidade Paulista, São Paulo, SP, Brazil
}

\begin{abstract}
Estradiol given during progestin-treatment promotes emergence of a new follicular wave in cattle. The interval from estradiol treatment to follicular wave emergence is dependent on the estradiol preparation, the dose, and possibly the animal category (cows or heifers). Therefore, this experiment aimed to compare the effects of either estradiol benzoate (EB) or estradiol valerate (EV) on the synchrony of ovarian follicular wave emergence in Bos indicus (Nelore) heifers and cows treated with a Norgestomet implant (Crestar ${ }^{\circledR}$; Intervet, Boxmeer, The Netherlands; factorial $3 \times 2$ design). Lactating cows $(n=30 ; 70-90$ days postpartum) and cycling heifers $(n=29)$ were homogeneously assigned, according to the presence of corpora lutea, to six experimental groups (G2EB, G2.5EV, and G5EV for each animal category). At random stages of the estrous cycle (Day 0), the females received an auricular implant and were assigned to receive $2 \mathrm{mg}$ of EB (Farmavet, São Paulo, Brazil; 10 cows and 10 heifers; G2EB), 2.5 mg of EV plus $1.5 \mathrm{mg}$ of Norgestomet ( 10 cows and 10 heifers; G2.5EV; half of a recommended dose), or $5 \mathrm{mg}$ of EV plus $3 \mathrm{mg}$ of Norgestomet ( 10 cows and 10 heifers; G5EV, full recommended dose) i.m. Transrectal ultrasonography was performed once daily from Day 0 to confirm the follicular dominance (new growing dominant follicle $>8 \mathrm{~mm}$ in diameter). The day of wave emergence was retrospectively defined as the day when the dominant follicle was first detected at a diameter of $3 \mathrm{~mm}$. Two-way analysis of variance was used to compare the effects of treatments (G2EB, G2.5EV, and G5EV), category (cows and heifers), and the treatments-by-category interaction on the interval from beginning of the treatment to wave emergence. The LSD was used to locate differences among groups and the Bartlett's test revealed heterogeneity of variance. Statistical analysis was conducted by SAS for Windows (SAS Institute, Inc., Cary, NC, USA). There was a significant effect of treatment and category $(P<0.001)$, but there were no treatments-by-category interactions $(P>0.05)$. A new follicular wave was induced in all animals after treatment with EB or EV. The heifers given G2EB $\left(2.50 \pm 0.19^{\mathrm{a}} ; 2\right.$ to $3^{\mathrm{x}}$ days $)$ had a reduced $(P<0.01)$ and least variable interval $(P<0.05)$ than those given $\mathrm{G} 2.5 \mathrm{EV}\left(4.20 \pm 0.27^{\mathrm{b}} ; 3\right.$ to $6^{\mathrm{xy}}$ days) or G5EV $\left(6.10 \pm 0.57^{\mathrm{c}} ; 3\right.$ to $8^{\mathrm{y}}$ days $)$. The cows given G5EV $\left(4.00 \pm 0.52^{\mathrm{b}} ; 2\right.$ to $6^{\mathrm{y}}$ days $)$ had the longest $(P=0.05)$ and more variable interval $(P<0.001)$ than those given G2EB $\left(2.50 \pm 0.22^{\mathrm{a}} ; 2\right.$ to $4^{\mathrm{x}}$ days $)$, but were similar to those given G2.5EV $\left(3.10 \pm 0.41^{\mathrm{ab}} ; 2\right.$ to $6^{\mathrm{xy}}$ days $)$. The effect of EB was similar in heifers and cows $(P>0.05)$; however, the EV or $2.5 \mathrm{EV}$ promoted a longer emergence wave interval in the heifers than in the cows $(P<0.05)$. Analyzing the main effects, cows and heifers given G2EB $\left(2.50 \pm 0.15^{\mathrm{a}} ; 2\right.$ to $4^{\mathrm{x}}$ days $)$ had a smaller and less variable interval than those given G2.5EV $\left(3.65 \pm 0.27^{\mathrm{b}} ; 2\right.$ to $\left.6^{\mathrm{y}}\right)$ or G5EV $\left(5.05 \pm 0.44^{\mathrm{c}} ; 2\right.$ to $\left.8^{\mathrm{z}}\right)$. In conclusion, the EV treatment, in both doses $(5$ or $2.5 \mathrm{mg})$, resulted in a delayed and more variable interval to emergence of a new follicular wave than $2 \mathrm{mg}$ of EB in Bos indicus cows and heifers treated with a Norgestomet auricular implant.
\end{abstract}

This work was supported by Intervet, Brazil.

\section{DISTRIBUTION OF OVULATION AND SUBSEQUENT EMBRYO PRODUCTION USING LUTROPIN AND ESTRADIOL-17 $\beta$ FOR TIMED AI OF SUPERSTIMULATED BEEF FEMALES}

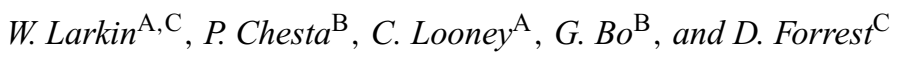 \\ ${ }^{\text {A }}$ Ovagenix, LP, Navasota, TX 77868, USA; \\ ${ }^{B}$ Instituto de Reproduccion Animal Cordoba, Cordoba, Argentina; \\ ${ }^{\mathrm{C}}$ Department of Animal Science, Texas A\&M University, College Station, TX 77843, USA
}

\begin{abstract}
This experiment was designed to compare timing of ovulation and embryo production between traditional superstimulatory and AI methods and the use of additional treatments of Estradiol-17 $\beta$ and pLH (Lutropin; Bioniche Animal Health, Belleville, Ontario, Canada) to facilitate timed AI in beef cows. A total of 31 mature cross-bred beef cows were randomly assigned three treatments. On Day 0 , experimental cows were selected upon transrectal ultrasound evaluation for corpus leutum $(>10 \mathrm{~mm}$ ) prior to CIDR insertion plus $2.5 \mathrm{mg}$ estradiol-17 $\beta$ and $50 \mathrm{mg}$ Progesterone i.m. Superstimulatory treatments with Folltropin-V (Bioniche Animal Health) began on Day 4 for 3.5 days (236 mg NIH-FSH-P10) in decreasing dosages (7 injections at 0700 and 1900). All cows received prostaglandin F (PGF) treatments of $625 \mathrm{mcg}$ D-cloprostenol i.m. (Estrumate; Schering-Plough, Union, NJ, USA) in both AM and PM on Day 6, and CIDRs were removed in AM of Day 7, followed by the final injection of Folltropin. In addition, Heat Watch patches (Heat Watch; Cow Chips, Denver, CO, USA) were applied to optimize heat detection. Donors selected as control treatment were $\mathrm{AI}$ at 12 and $24 \mathrm{~h}$ post-onset of estrus (first mount) with frozen semen from the same bull. Donors selected on the Estradiol-17及 treatment received $1 \mathrm{mg}$ i.m. $12 \mathrm{~h}$ after CIDR removal and timed AI at 24 and $36 \mathrm{~h}$. Donors selected for Lutropin treatment received $12.5 \mathrm{mg}(5 \mathrm{cc} \mathrm{i.m}$.) $24 \mathrm{~h}$ after CIDR removal and were timed AI at 12 and $24 \mathrm{~h}$. All AI procedures used 2 units from the same bull. All cows were examined by ultrasonography every $8 \mathrm{~h}$ beginning $24 \mathrm{~h}$ post-CIDR removal and ending at $60 \mathrm{~h}$ to determine the time and distribution of ovulation. Ovulation was determined by the disappearance of follicles $(>12 \mathrm{~mm})$ from the prior examination. All AI and ultrasound procedures were performed by the same technician. There were no differences detected between the three treatments as evaluated by all the variables we studied (Table 1). However, donors treated with Lutropin tended to produced more viable embryos per collection and had a tighter degree of distribution of ovulations. Donors treated with Estradiol- $17 \beta$ tended to have fewer viable embryos. More research is needed to determine if donors can be timed AI without regard to estrus.
\end{abstract}


Table 1. Mean onset of estrus, distribution of ovulation, and embryo production in superstimulated beef donors treated with Estradiol-17 $\beta$, and Lutropin

\begin{tabular}{lcccrr}
\hline Protocol (no. of cows) & Estrus post-CIDR removal (h) & Ovulation time $(\mathrm{h})$ & No. of ovulations & Total ova & Viable embryos \\
\hline Control (10) & 25.1 & 58.5 & 28.5 & 11.0 & 7.8 \\
Estradiol (11) & 22.0 & 57.5 & 23.9 & 6.0 & 3.5 \\
LH (10) & 26.3 & 57.3 & 21.4 & 11.6 & 9.6 \\
\hline
\end{tabular}

*Means not different $(P>0.1)$

\title{
365 THE EFFECT OF ADMINISTERING PROGESTERONE AND ESTRADIOL PRIOR TO eFSH ON THE SUPEROVULATORY RESPONSE OF MARES
}

\author{
N. Logan, P. McCue, M. Alonso, and E. Squires \\ Animal Reproduction and Biotechnology Laboratory, Colorado State University, Fort Collins, CO 80523, USA
}

eFSH has been used to induce multiple ovulation in cycling mares. However, the response to eFSH is variable. Generally, eFSH is initiated 5 to 7 days after ovulation at a time when the follicular population on the ovaries may be variable. The objective of this study was to determine whether administration of progesterone and estradiol for 10 days prior to eFSH (Bioniche Animal Health, Athens, GA) would enhance the response to eFSH administration. Thirty normal cycling mares were assigned to 1 of 2 groups. Group 1: Control eFSH treatment - mares were examined daily with ultrasonography beginning 5 days after ovulation. Once the follicles in these mares reached 20 to $25 \mathrm{~mm}$ in size, eFSH treatment (12.5 mg, i.m.) was administered twice a day. Cloprostenol (Schering-Plough, Union, NJ, USA) treatment $(250 \mu \mathrm{g})$ was administered on the second day of eFSH treatment. eFSH treatment continued until the majority of the cohort of follicles reached $\geq 35 \mathrm{~mm}$. Treatment was stopped and after approximately $36 \mathrm{~h} \mathrm{hCG} \mathrm{(2500} \mathrm{IU,} \mathrm{i.v.;} \mathrm{Chorulon;} \mathrm{Intervet,} \mathrm{Millsboro,} \mathrm{MD,} \mathrm{USA)} \mathrm{was} \mathrm{administered.} \mathrm{Group} \mathrm{2:} \mathrm{Injectable} \mathrm{progesterone}+$ estradiol (150 mg of progesterone and $10 \mathrm{mg}$ of estradiol; $\mathrm{P}+\mathrm{E}$ ) treatment was initiated in diestrus ( 5 to 7 days post-ovulation) for 8 mares and in early estrus for 7 mares in this group. Injectable progesterone was continued for 10 days and Cloprostenol $(250 \mu \mathrm{g})$ was administered on Day 10 . Mares were then examined daily with ultrasonography and, once they had acquired 20- to $25-\mathrm{mm}$ follicles, eFSH treatment was initiated. Twice-daily injections of eFSH $(12.5 \mathrm{mg}$, i.m.) were continued until a majority of the cohort of follicles was $\geq 35 \mathrm{~mm}$. hCG was administered approximately $36 \mathrm{~h}$ later. All mares were inseminated with 1 billion progressively motile spermatozoa from one of two stallions on the day of hCG administration and on the following day with cooled semen (1 billion progressively motile spermatozoa) from the same stallion. Data were analyzed by $t$-test and Fisher's Exact Test. The number of days of eFSH treatment was similar for the P+E (2) vs. the control (1) group ( $4.2 \pm 2.0$ vs. $4.9 \pm 1.3$ days, respectively). However, the number of ovulations induced in response to eFSH was greater for mares in the eFSH control (1) group (5.6 \pm 2.0$)$ than for those in the $\mathrm{P}+\mathrm{E}(2)$ group $(3.0 \pm 1.9)$. Embryo recovery per flush was also greater for eFSH control (1) mares (2.7) vs. P+E (2) mares (1.1). Embryo quality was excellent or good for all embryos in both groups. Seventy-three percent of the mares (11 of 15) in both groups gave at least one embryo at each recovery attempt. However, more mares in the eFSH control (1) group gave two or more embryos $(60 \%)$ compared to those in the $\mathrm{P}+\mathrm{E}(2)$ group $(20 \%)$. In summary, treatment of mares with $\mathrm{P}+\mathrm{E}$ prior to $\mathrm{eFSH}$ treatment resulted in fewer ovulations, fewer embryos recovered, and fewer mares providing $\geq 2$ embryos. Thus, there was no advantage in pretreating mares with $\mathrm{P}+\mathrm{E}$ prior to $\mathrm{eFSH}$ treatment.

\section{THE BOVINE MODEL FOR REPRODUCTIVE AGING: SUPEROVULATORY RESPONSE}

\author{
P. S. Malhi ${ }^{\mathrm{A}}$, G. P. Adams ${ }^{\mathrm{A}}$, R. J. Mapletoft ${ }^{\mathrm{B}}$, and J. Singh ${ }^{\mathrm{A}}$ \\ ${ }^{A}$ Department of Veterinary Biomedical Sciences, University of Saskatchewan; \\ ${ }^{B}$ Department of Large Animal Clinical Sciences, University of Saskatchewan, Saskatoon, Saskatchewan, Canada
}

Two experiments were done to test the hypotheses that aging in cattle is associated with a reduction in the number of follicles recruited into an ovarian follicular wave, and a reduction in the ovarian response following gonadotropin treatment for superovulation. Old cows (13 to 16 years old; $n=6$ to 9 ) and their young daughters ( 3 to 6 years old; $n=7$ to 9 ) were treated to induce an ovarian superstimulatory response twice in each of two consecutive years (i.e. 31 and 33 superstimulatory cycles, respectively). In Experiment 1, follicular wave emergence was synchronized using a single treatment of estradiol $(5 \mathrm{mg})$ combined with progesterone $(100 \mathrm{mg})$ at the time of CIDR-B (Bioniche Animal Health, Belleville, Ontario, Canada) insertion. Superstimulatory treatment consisted of a total dose of $50 \mathrm{mg}$ NIH-FSH-P1 (Folltropin-V; Bioniche Animal Health) per $100 \mathrm{~kg}$ body weight divided into twice daily intramuscular injections over 4 days. CIDR devices were removed at the time of the last FSH treatment and $500 \mu \mathrm{g}$ cloprostenol (Estrumate; Schering-Plough Animal Health, Montreal, Quebec, Canada) was administered twice along with last two FSH treatments to induce luteolysis. Ovulation was induced using $25 \mathrm{mg}$ porcine LH (Lutropin-V; Bioniche Animal Health) administered $24 \mathrm{~h}$ after the last FSH treatment. Ovulations were detected by ultrasonography at 24, 48, and $72 \mathrm{~h}$ after LH treatment. In Experiment 2, follicular wave emergence was synchronized with ultrasound-guided follicle ablation in the presence of a functional corpus luteum. Superstimulatory treatment was given as in Experiment 1. Ultrasound-guided follicle aspirations were performed 48 to $72 \mathrm{~h}$ after the last FSH for the purpose of another experiment. In both experiments, the ovarian follicular response was monitored daily by ultrasonography. There were no differences $(P>0.3)$ in mean body weight 
and dose of FSH treatment between old cows and their daughters. Fewer $2-5$-mm follicles $(P<0.01)$ were detected at the expected time of wave emergence in old cows than in their daughters. In Experiment 1, the difference in mean number of ovulations in old and young cows ( $32 \pm 4$ vs. $40 \pm 3)$ did not reach significance $(P=0.11)$, but fewer follicles $\geq 6 \mathrm{~mm}(P<0.01)$ were detected on the day before ovulation in old cows than in their daughters. The number of follicles $\geq 6 \mathrm{~mm}$ detected just before ovulation (Experiment 1 ) or follicular aspiration (Experiment 2 ) in successive superstimulatory cycles was correlated within animals $(r>0.8 ; P<0.0001)$. Similarly, the number of ovulations in successive superstimulatory cycles (Experiment 1$)$ was correlated within animals $(r=0.6 ; P=0.04)$. In conclusion, aging was associated with fewer $2-1 \mathrm{~mm}$ follicles at the time of wave emergence, a lower follicular response, and a tendency for a lower ovulatory response subsequent to gonadotropin treatment. The follicular and ovulatory response to superstimulatory treatment was repeatable within individuals.

\title{
367 INFLUENCE OF SUPERESTIMULATION AND HORMONAL DEPRIVATION PROTOCOLS ON IN VITRO PRODUCTION OF NELORE EMBRYOS (BOS TAURUS INDICUS)

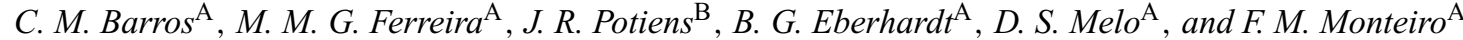 \\ ${ }^{A}$ Instituto de Biociencias - UNESP, Botucatu, Sao Paulo, Brazil; \\ ${ }^{\mathrm{B}}$ Central Bela Vista, Pardinho, Sao Paulo, Brazil
}

There are indications in the literature that delaying the period between ovarian superestimulation and ovum pickup (OPU) will induce follicles to a condition of initial atresia, which could be beneficial to oocyte development. Blondin et al. (2002 Biol. Reprod. 66, 38-43) superstimulated (FSH) Holstein heifers and delayed OPU for $48 \mathrm{~h}$, i.e. they induced initial atresia in these follicles deprived of FSH (starvation) for $48 \mathrm{~h}$. Additionally, $6 \mathrm{~h}$ before OPU, LH was administered to accelerate follicular maturation. This protocol yielded a surprisingly high blastocyst rate $(80.4 \pm 9.4 \%)$. In the present work, Blondin's protocol was simultaneously compared to other protocols used for OPU and in vitro production of embryos (IVP), in Nelore cattle. Nelore cows $(n=18)$ were randomly allocated in three groups: Group 1 (just OPU), Group 2 (superestimulation and OPU), and Group 3 (superestimulation associated with FSH deprivation and OPU). Three OPU were performed, and the animals were switched to a different group each time in such a way that at the end of the experiment all cows received the three protocols. At a random stage of the estrous cycle (D2), follicles $\geq 6 \mathrm{~mm}$ were aspirated to induce a new follicular wave 2 days afterward (D0). In Group 1, OPU was performed on D2 and oocytes were processed to IVP. In Group 2, starting on D0, cows were superestimulated (pFSH, Folltropin ${ }^{\circledR}, 30 \mathrm{mg}$ administered daily, IM, during 3 consecutive days; Bioniche Animal Health, Belleville, Ontario, Canada), and $6 \mathrm{~h}$ after the last FSH dose they received exogenous LH (12.5 mg, Im, Lutropin ${ }^{\circledR}$, D3; Bioniche Animal Health). OPU was performed $6 \mathrm{~h}$ after LH administration, i.e. $12 \mathrm{~h}$ after the last dose of FSH. Animals in Group 3 received the same treatment as in Group 2, except that LH was administered $36 \mathrm{~h}$ after the last dose of FSH, and OPU occurred $6 \mathrm{~h}$ later. Therefore, in this Group, follicles were deprived of FSH during $48 \mathrm{~h}$. Both cleavage and blastocyst rates were similar $(P>0.05$, logistic regression) among oocytes from Groups 1,2 , and 3, respectively: $77.4 \%(144 / 185)$ and $42.70 \%(79 / 185) ; 75.54 \%(105 / 139)$ and $31.65 \%(44 / 139) ; 63.52 \%(101 / 159)$ and $33.33 \%(53 / 159)$. However, hatched blastocyst rate was higher $(P<0.01)$ in Group $1(30.27 \%, 56 / 185)$ when compared to Group $2(11.51 \%, 16 / 139)$ or $3(15.72 \%, 25 / 159)$. It is concluded that, contrary to previous work on European breeds (taurus), ovarian superestimulation associated with deprivation of FSH and OPU (Group 3) do not increase IVP of Nelore embryos (indicus). Additionally, the highest hatched blastocyst rates were observed in oocytes from non superstimulated cows (Group 1).

\section{OOCYTE ASPIRATION FROM TRANSITIONAL AND CYCLING MARES TREATED WITH EFSH}

\author{
S. H. Purcell, T. K. Suh, and G. E. Seidel Jr \\ Animal Reproduction and Biotechnology Laboratory, Colorado State University, Fort Collins, CO 80523, USA
}

\begin{abstract}
A major obstacle in studying equine oocyte maturation is a source of oocytes. The aim of this study was to determine if oocyte recovery following transvaginal ultrasound-guided aspirations could be improved by using eFSH. Fourteen mares ranging from 3 to 16 years of age were used as oocyte donors during the transitional (March to April) and cycling (May to June) seasons. Each follicle was aspirated with a 12-gauge double-lumen needle using a pressure of $150 \mathrm{mmHg}$ and then flushed eight times using PBS supplemented with $10 \mathrm{U} / \mathrm{mL}$ heparin and $0.05 \%$ BSA. Large follicles $(>20 \mathrm{~mm})$ were aspirated first, followed by small follicles $(10-20 \mathrm{~mm})$. The first aspiration in each season was used to deplete the follicular pool of all follicles $>10 \mathrm{~mm}$; these data were not considered. Following this initial aspiration, mares were subjected to four rounds of aspirations every 10 to 11 days. In each round during the transitional season, half the mares received $12.5 \mathrm{mg}$ eFSH (Bioniche Animal Health, Belleville, Ontario, Canada) once daily for four days prior to aspiration. For each round during the cycling season, half the mares received 12.5 mg eFSH twice daily for three days prior to aspiration. In each replicate, mares were alternated from treated to control groups during both seasons. Oocyte recovery and superstimulatory responses were analyzed using the GLM procedure of SAS (SAS Institute, Inc., Cary, NC, USA). During both the transitional and the cycling season, treatment with eFSH did not have a significant effect $(P>0.1)$ on the number of small or large follicles present at the time of aspiration (Table 1). For both seasons and size classes, the number of follicles varied by mare $(P<0.02)$. Oocyte recovery from small follicles during the transitional season improved during the later two rounds of aspiration $(0.58$ vs. $1.49 ; P<0.01)$ and was also affected by mare $(P=0.06)$. During the cycling season, recovery of oocytes from both small and large follicles varied by mare $(P<0.05)$. Treatment with eFSH increased the number of oocytes recovered from large follicles in cycling mares $(P<0.05)$. This study indicates that responses to short-term eFSH treatment for the purpose of oocyte recovery vary greatly by mare. While treatment with eFSH did not increase the number of follicles available for aspiration $(P>0.1)$, it did increase the number of oocytes recovered from large follicles during the cycling season.
\end{abstract}


Table 1. Oocyte recovery and superstimulatory response of mares treated with eFSH

\begin{tabular}{lccccr}
\hline Group & No. aspirations & \multicolumn{2}{c}{ No. oocytes recovered per mare (\% per follicle) } & \multicolumn{2}{c}{ No. follicles per mare } \\
& & Small & Large & Small & Large \\
\hline Transitional control & 28 & $1.15(23)$ & $0.12(7)$ & 5.85 & 1.58 \\
Transitional eFSH & 28 & $0.93(19)$ & $0.22(6)$ & 6.26 & 1.15 \\
Cycling control & 28 & $1.18(22)$ & $0.18^{\mathrm{a}}(6)$ & 5.83 & 2.07 \\
Cycling eFSH & 28 & $1.37(20)$ & $0.46^{\mathrm{b}}(18)$ & 6.25 & 2.68 \\
SE for each group & - & 0.21 & 0.10 & 0.57 & 0.27 \\
\hline
\end{tabular}

${ }^{a b}$ Values with different superscripts differ $(P<0.05)$.

\title{
369 ASSESSMENT OF SEMEN QUALITY FOR PREDICTING RECOVERY OF VIABLE EMBRYOS IN SUPEROVULATED CATTLE
}

\author{
F. N. Schrick ${ }^{\mathrm{A}}$, A. M. Saxton ${ }^{\mathrm{A}}$, and B. K. Stroud ${ }^{\mathrm{B}}$ \\ ${ }^{A}$ Department of Animal Science, University of Tennessee, Knoxville, TN 38237, USA; \\ ${ }^{\mathrm{B}}$ Stround Veterinary Embryo Services, Inc., Weatherford, TX 76086, USA
}

Several studies have illustrated a relationship between male fertility and subsequent pregnancy rates following natural service and artificial insemination. However, few studies have evaluated the relationship of semen quality to recovery of viable embryos within a commercial embryo transfer setting. The objective of the current study was to retrospectively evaluate the relationship of semen quality, determined immediately before insemination, and subsequent embryo recovery from superovulated cattle. All donor animals were superovulated and then inseminated (same technician throughout the study) with frozen-thawed semen at 12 and $20 \mathrm{~h}$ after onset of estrus with one straw of semen per insemination. Since data were collected from a commercial setting, several different bulls $(n=277)$ and breeds $(n=33)$ were included within the analyses; however, the same semen (bull and collection date of semen) was used for each insemination for that respective flush. Immediately following thawing of semen and before insemination, $5 \mu \mathrm{L}$ of semen was obtained from each straw and evaluated by the same individual for concentration, motility, rate of forward movement, direction of movement, and morphology using a phase contrast microscope. Approximately 7 days later, ova/embryos were recovered and defined as unfertilized (not cleaved), degenerate, or viable. Semen was assessed and utilized in 742 recoveries resulting in the evaluation of 9732 ova/embryos. Chi-square analyses were used to explore relationships among semen and fertilization/embryo variables. A complex model addressing combinations of semen variables was developed with logistic regression. Total ova/embryos recovered had a significant impact on embryo data and was included in the model during statistical analyses with the significance level set at $P<0.05$. Analyses were conducted only on uterine recoveries in which ova/embryos were collected. Effects of bull and breed were significant on embryo data evaluated but at a very low level of relevance $(F<2.0)$. Total ova/embryos collected per recovery highly influenced the percentage of cleaved embryos $(P<0.0001)$. Results indicate that all semen parameters evaluated affected various aspects of embryo development (percent fertilized, viable, or degenerate). In particular, semen morphology was more associated with quality of embryos recovered than the other semen factors measured. Semen classified as excellent-to-good morphology resulted in 59\% Quality 1 embryos (percent of fertilized embryos) whereas semen rated fair was intermediate $(53.5 \%)$ and semen rated poor produced only $29.5 \%$ Quality 1 embryos $(P<0.05)$. In conclusion, semen evaluation within a commercial embryo transfer setting provides some expectation of resulting embryo quality.

Tissue Culture

\section{HISTONE PHOSPHORYLATION PATTERNS AND CHROMOSOMAL STABILITY OF CULTURED BOVINE FIBROBLASTS}

\author{
A. Giraldo ${ }^{\mathrm{A}}, J$. Lynn $^{\mathrm{B}}$, R. Godke ${ }^{\mathrm{A}}, J_{\text {J Jenkins }}{ }^{\mathrm{C}}$, and K. Bondioli ${ }^{\mathrm{A}}$ \\ ${ }^{A}$ Department of Animal Sciences, Louisiana State Agricultural Center, Baton Rouge, LA 70803, USA; \\ ${ }^{B}$ Department of Biological Sciences, Louisiana State University, Baton Rouge, LA 70803, USA; \\ ${ }^{\mathrm{C}}$ USGS, National Wetlands Research Center, Lafayette, LA 70506, USA
}

The low percentage of cloned offspring produced by nuclear transfer has been attributed to a variety of factors, including aneuploidy of the donor cells. Previous reports indicate that cultured bovine fibroblasts have a significantly higher level of aneuploidy in late passages than in early passages (Giraldo et al. 2005 J. Reprod. Fert. Dev. 17, 167). Phosphorylation of histone H3 at Serine 10 (Ser10) has been shown to be involved in chromosome compaction during cell division. Abnormal phosphorylation of this histone residue during metaphase could lead to abnormal chromosome segregation and extensive chromosome loss during mitosis. Suboptimal culture conditions may lead to abnormal histone H3 phosphorylation (HP) patterns, ultimately inducing missegregation and loss of chromosomes. The objective of the present study was to determine if the high percentage of aneuploid bovine fibroblast cells observed after long-term culture is associated with an abnormal HP pattern. Four bovine fibroblast cell lines were established from 40- to 60-day fetuses. Cells were cultured in DMEM supplemented with 10\% fetal bovine serum and 1\% penicillin and streptomycin in 5\% $\mathrm{CO}_{2}$ at $37^{\circ} \mathrm{C}$ and passaged at confluence. Relative levels of $\mathrm{HP}$ were determined in three different replicates at population doublings (PD) 2, 10, and 20. Cells were fixed and incubated with an anti-phosphorylated histone H3 (Ser10) antibody, labeled with a secondary antibody, counterstained 
with propidium iodide, and analyzed for HP fluorescence by flow cytometry. The number of chromosomes was also determined in counts of 800 metaphases. Differences in aneuploidy and HP fluorescence of cells in metaphase among PD were analyzed by $\chi^{2}$ and two-way ANOVA, respectively $(P<0.05)$. The percentages of aneuploid cells in each of the cell lines increased progressively with duration of culture and were elevated from the start. Multinucleated cells were frequently observed after prolonged time in culture in all of the cell lines. The mean phosphorylated histone levels (relative fluorescence intensity) in cells during metaphase were 180.0, 131.5, 174.7, and 157.6 in PD 2; 170.4, 105.72, 145.8, and 152.7 in PD 10; and 274.0, 251.6, 191.4, and 308.3 in PD 20 for the four cell lines, respectively. No difference in HP levels was observed between PD 2 and PD 10. The average of HP during metaphase for the cell lines increased significantly from 160.9 at early passage to 256.3 at late passage (see Table 1). The increase in levels of HP occurred concurrently with the high percentage of aneuploid cells after extended time in culture. These data are consistent with the hypothesis that aneuploid cells observed after long in vitro culture are associated with abnormal HP patterns.

This study was supported by Grants in Aid of Research Program (GIAR) from Sigma Xi to A.G.

Table 1. Relative HP fluorescence and aneuploidy at different PD of fetal bovine fibroblasts

\begin{tabular}{lccc}
\hline Parameter & PD 2 & PD 10 & PD 20 \\
\hline Aneuploid cells (\%) \pm SEM & $42.4 \pm 2.8^{\mathrm{a}}$ & $59.1 \pm 1.6^{\mathrm{b}}$ & $75.8 \pm 4.3^{\mathrm{c}}$ \\
Mean HP fluorescence \pm SEM & $160.9 \pm 14.8^{\mathrm{a}}$ & $143.6 \pm 15.1^{\mathrm{a}}$ & $256.4 \pm 18.2^{\mathrm{b}}$ \\
\hline
\end{tabular}

${ }^{\mathrm{a}-\mathrm{c}}$ Different superscripts within rows indicate statistical significance $(P<0.05)$.

\title{
371 EVALUATION OF ENRICHMENT STRATEGIES FOR PORCINE SPERMATOGONIA BY EXPRESSION OF PROTEIN GENE PRODUCT 9.5, A SPERMATOGONIA-SPECIFIC MARKER IN THE PIG TESTIS
}

\author{
J. Luo, S. Megee, R. Rathi, and I. Dobrinski
}

Center for Animal Transgenesis and Germ Cell Research, Department of Clinical Studies, New Bolton Center, School of Veterinary Medicine, University of Pennsylvania, Kennett Square, PA 19348, USA

Transplantation of genetically altered male germ cells is under investigation as a novel route to generate transgenic animal models. Identification and isolation of spermatogonial stem cells are a prerequisite for this strategy. The objectives of this study were to validate a marker for identification of undifferentiated porcine spermatogonia, and to use this marker to develop a practical enrichment strategy for spermatogonia from pig testis. We established that expression of protein gene product (PGP) 9.5 is a spermatogonia-specific marker in porcine testis through analysis of its expression pattern in testis cells, by comparison with the expression of the cell-type specific proteins GATA-4 (expressed in Sertoli cells) or PLZF (expressed in undifferentiated mouse spermatogonia) in seminiferous tubules at different ages, and by comparison of expression levels of PGP 9.5 and the germ cell-specific protein VASA in different cell fractions after differential plating. Using expression of PGP 9.5 as a marker, we characterized enrichment of porcine spermatogonia from two-week-old ( $2 \mathrm{wo}$ ) and 10-week-old (10wo) pigs by immunofluorescence either after differential plating only or after velocity sedimentation at unit gravity followed by differential plating. After differential plating with overnight culture to deplete testicular somatic cells that firmly attach to culture dishes, spermatogonia (mean \pm SEM per 1000 cells) were 5 -fold enriched $(P<0.05)$ in cells remaining in suspension (fraction I) (2wo: $54.0 \pm 9.1 ; 10 \mathrm{wo}: 162.7 \pm 30.5)$ and in populations slightly attached to the culture plate (fraction II) (2wo: $92.7 \pm 8$; 10wo: $159.5 \pm 22.5$ ) compared to the initial samples ( 2 wo: $12.3 \pm 2.7 ; 10$ wo: $27.2 \pm 2.9$ ). Slightly attached spermatogonia appear to be superior for future experiments due to higher viability $(>90 \%)$ than spermatogonia remaining in suspension $(\sim 50 \%)$. Cell populations containing up to $70 \%$ spermatogonia with good viability $(>80 \%)$ were achieved by velocity sedimentation isolation followed by differential plating. These results indicate that expression of PGP 9.5 is a useful marker for identification of undifferentiated porcine germ cells. Simple differential adhesion culture of testis cells harvested from pre-pubertal boars can supply cell populations enriched in spermatogonia for subsequent genetic manipulation and transplantation.

This work was supported by 1 R01 RR17359-01.

Transgenesis

\section{LASER-ASSISTED LENTIVIRAL TRANSGENESIS IN BOVINE EMBRYOS}

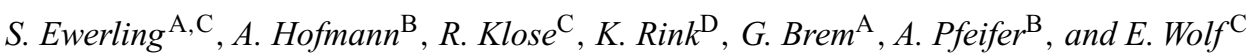 \\ A apoGene $\mathrm{GmbH} \& \mathrm{Co} . \mathrm{KG}$, Freising, Germany; \\ ${ }^{\mathrm{B}}$ Department of Pharmacy, Institute for Pharmacology, Center for Drug Research, LMU, Munich, Germany; \\ ${ }^{\mathrm{C}}$ Department of Molecular Animal Breeding and Biotechnology, LMU, Munich, Germany; \\ D OCTAX Microscience GmbH, Herborn, Germany
}

Lentiviral vectors have been shown to be a powerful tool to create transgenic livestock (Hofmann et al. 2003 EMBO Rep. 4, 1054-1060; Hofmann et al. 2004 Biol. Reprod. 71, 405-409). Due to their inability to pass the zona pellucida (ZP) by themselves, viral particles were delivered by subzonal 
microinjection of oocytes or zygotes. Here we show that an artificial opening of the ZP, generated by an infrared $1.48 \mu \mathrm{m}$ microsurgical diode laser (OCTAX Laser Shot ${ }^{\mathrm{TM}}$, kindly provided by MTG, Altdorf, Germany) and subsequent culture of oocytes in virus suspension, was sufficient to allow virus integration and expression of eGFP. The ZP of denuded in vitro-matured bovine oocytes was microdrilled with 2 to 4 laser shots of 3.0-ms pulse length each to create an opening of at least $40 \mu \mathrm{m}$. Oocytes were transferred to microdroplets ( $20 \mu \mathrm{L}$ of Fert Talp) containing the virus suspension (lentiviral vector containing the eGFP reporter gene under the control of the human phosphoglycerate-kinase 1 promoter). After incubation for 2 or $4 \mathrm{~h}\left(5 \% \mathrm{CO}_{2}\right.$, maximum humidity, $\left.39^{\circ} \mathrm{C}\right)$, oocytes were washed thoroughly in fresh droplets of medium to remove excessive virus. Sperm was added $\left(0.5 \times 10^{6} / \mathrm{mL}\right)$ for $8 \mathrm{~h}$. In vitro culture of embryos $\left(5 \% \mathrm{CO}_{2}, 5 \% \mathrm{O}_{2}\right.$, maximum humidity, $\left.39^{\circ} \mathrm{C}\right)$ was continued in culture medium (SOF) under oil for up to 8 days. Cleavage rate and number of blastocysts were determined on Days 2 and 8, respectively. Expression of eGFP was analyzed on Day 8 by fluorescence microscopy using an eGFP-specific filter with UV light source under an inverted microscope. In total, 267 oocytes were microdrilled. The range of optimal virus concentration was analyzed by titration. Seven different concentrations in a range of $1.25 \times 10^{6}$ to $5 \times 10^{7} \mathrm{IU} / \mathrm{mL}$ were used at an incubation time of $4 \mathrm{~h}$. In one experiment using a high virus concentration, the incubation time was reduced to $2 \mathrm{~h}$. Control groups were treated equally except for addition of virus. All experiments resulted in blastocysts showing eGFP expression in both the trophoblast and the inner cell mass. In four experiments we used aliquots of the same virus preparation $\left(1.25 \times 10^{7} \mathrm{IU} / \mathrm{mL}\right)$ for infection. The cleavage rate ranged from 50 to $70 \%$, and blastocyst rate from 15 to $35 \%$. In three experiments showing good to average embryo development, 25 to $83 \%$ of the resulting blastocysts expressed eGFP, indicating efficient lentiviral transduction of oocytes following laser-assisted zona microdrilling. Studies directly comparing zona microdrilling and subzonal injection with regard to the efficacy of lentiviral transgenesis and the incidence of polyspermy are underway.

\title{
373 SPERM TREATMENT AFFECTS THE EFFICIENCY OF PORCINE TRANSGENIC EMBRYO PRODUCTION BY ICSI
}

\author{
F. A. García-Vázquez ${ }^{\mathrm{A}}$, Al. Gutiérrez-Adán ${ }^{\mathrm{B}}$, and J. Gadea ${ }^{\mathrm{A}}$ \\ ${ }^{A}$ Department Physiology, Faculty of Veterinary Science, University of Murcia, Murcia, Spain; \\ ${ }^{B}$ Department of Animal Reproduction, INIA, Madrid, Spain. Email: jgadea@um.es
}

Sperm mediated gene transfer (SMGT) is an interesting tool for animal transgenesis, consisting of the use of sperm cells as a vector for transmitting exogenous DNA into eggs at the moment of fertilization. A degradation of sperm membrane followed by incubation with DNA and fertilization using intracytoplasmic sperm injection (ICSI) proved to be efficient in mice (Perry et al. 1999 Science 284, 1180-1183; Moreira et al. 2004 Biol. Reprod. 71, 1943-1947) and rats (Kato et al. 2004 Mol. Reprod. Dev. 69, 153-158). In this study, we evaluated the effect of the sperm treatment (a quick freezing-thawing process for disrupting sperm membranes) on further transgenic expression and the embryo development of injected porcine oocytes. Ejaculated sperm cells from five fertile mature boars were used as vectors for transferring plasmid DNA (GFP: green fluorescent protein) into matured porcine in vitro oocytes by ICSI. Semen was recovered and immediately diluted 1:10 in SFM (swine fertilize medium) at $37^{\circ} \mathrm{C}$ and later centrifuged $\left(800 \mathrm{~g}, 10 \mathrm{~min}, 25^{\circ} \mathrm{C}\right)$, discarding the seminal plasma to avoid a detrimental effect on DNA binding to cells without further preparation (control), or after they had been subjected to membrane disruption by a quick freeze-thawing process (FT). Linealized plasmid DNA $(5.4 \mathrm{~kb})$ was added $(1 \times 108 \mathrm{sperm} / \mathrm{mL}+5 \mathrm{mg} \mathrm{DNA} / \mathrm{mL})$ and incubated at $16^{\circ} \mathrm{C}$ for $30 \mathrm{~min} .5 \mathrm{~min}$ before ICSI, sperm were pre-warmed at $37^{\circ} \mathrm{C}$. Denuded oocytes were washed twice in DPBS medium supplemented with $10 \%$ FCS (fetal calf serum) and transferred to ICSI drops. Injected oocytes were kept in TALP medium (Rath et al. $1999 \mathrm{~J}$. Anim. Sci. 77, 3346-3352) for $18 \mathrm{~h}$, and then transferred to NCSU-23 medium for further embryo culture. Embryos were examined for cleavage rate at $48 \mathrm{~h}$ following injection, and for embryo development at $144 \mathrm{~h}$. GFP expression in embryos was examined under fluorescent light using a fluorescence inverted microscope. In this preliminary study, 105 and 101 oocytes were injected for control and FT groups, respectively. The cleavage rate was similar between groups (control: 49/105 (46.7\%) vs. FT: 39/101 (38.6\%); $P=0.25)$. However, the blastocyst formation rate was lower in the FT than in control group (control: $13 / 105(12.4 \%)$ vs. FT: $4 / 101(4.0 \%)$; $P=0.03)$. In relation to transgenic expression, the FT group showed a significantly higher number of transgenic embryos (control: $50.98 \%$ vs. FT: 75.51\%; $P=0.01$ ). These results confirmed that treatment of the sperm prior to the ICSI could affect the efficiency of the production of transgenic embryos. The disruption of sperm membrane increases the DNA binding and the possibilities of carrying the DNA into the oocyte, and freezing-thawing technique is simple and effective to achieve this purpose. However, the freezing process could damage the sperm nucleus structure and decrease the viability of the embryo produced. Nevertheless, the efficiency of the transgenesis expression was very high (more than $75 \%$ of the embryos were obtained), and it should be a useful tool to produce transgenic pigs.

This work was supported by AGL2003-03144.

\section{PRODUCTION OF TRANSGENIC CLONED PIGLETS BY USING PORCINE FETAL FIBROBLASTS OVEREXPRESSING ENDOTHELIAL NITRIC OXIDE SYNTHASE}

\author{
Y. H. Hao, H. Y. Yong, C. N. Murphy, D. Wax, M. Samuel, A. Rieke, E. M. Price, R. M. McAllister, \\ J. R. Turk, E. B. Rucker, M. H. Laughlin, and R. S. Prather
}

Department of Animal Sciences, Univeristy of Missouri-Columbia, Columbia, MO 65201, USA

Vascular function, vascular structure, and homeostasis are thought to be regulated in part by endothelial nitric oxide synthase (eNOS). In addition, eNOS plays an important role in modulating metabolism of skeletal and cardiac muscle in health and disease. The pig is an optimal model for human diseases because of the large number of important similarities between the genomic, metabolism, and cardiovascular systems of pigs and humans. The main goal of this study was to produce pigs that carry an endogenous eNOS gene driven by a Tie-2 promoter and tagged with a V5 His tag so that a better understanding of cardiovascular regulation by eNOS can be gained. Nuclear transfer was conducted to create these animals, 
and the effects of two different oocyte activation treatments and two different culture systems were examined. Mature oocytes were purchased from BoMed, Inc. (Maidson, WI, USA) and enucleated by micromanipulation. Donor cells were injected into the perivitelline space and electrically fused to the recipient oocyte. Treatment 1 consisted of electrical fusion/activation in $1 \mathrm{mM}$ calcium in mannitol; Treatment 2 consisted of electrical fusion in $0.1 \mathrm{mM}$ calcium in mannitol, followed by chemical activation in $200 \mu \mathrm{M}$ thimerosal for $10 \mathrm{~min}$ followed by $8 \mathrm{mM}$ DTT for $30 \mathrm{~min}$. The fusion rate $(71 \%$ vs. $82 \%)$ was lower $(P<0.0001)$ in Treatment 2 than in Treatment 1 . For those oocytes that fused, the cleavage rates $(5.9 \%$ vs. $0.4 \%)$ at 14-18 h were higher $(P<0.0001)$ for Treatment 2 . Fused oocytes were cultured for $12-18 \mathrm{~h}$ in $5 \% \mathrm{O}_{2}, 5 \% \mathrm{CO}_{2}, 90 \% \mathrm{~N}_{2}\left(\right.$ low oxygen) or $5 \% \mathrm{CO}_{2}$ in air (normal oxygen), and were then surgically transferred to the oviducts of gilts that exhibited estrus on the day of fusion or the day of transfer. In February four cloned transgenic piglets were born (birth weights were 495, 595, 965, and $685 \mathrm{~g}$ ). Two were from Treatment 1 and low oxygen, and the other two were from Treatment 2 and normal oxygen, and all are currently healthy. PCR, RT-PCR, and Western blotting analysis confirmed that the pigs were transgenic, made message, and made the fusion protein. Immunohistochemistry confirmed that the fusion protein localized in the endothelial cells of the placental vasculature from the conceptuses as did the endogenous eNOS. In addition to the transgenic males, four wild-type females were born (birth weights were 585 (this one died on Day 4) 445, 930, and $585 \mathrm{~g}$ ). All four were from Treatment 2 and low oxygen, and three are healthy. In conclusion, viable piglets were produced from both Treatments 1 and 2, as well as in both high and low oxygen conditions. Thus, both activation conditions and culture systems are compatible with development to term. These pigs will serve as the founders for a colony of miniature pigs that will help to elucidate the function of eNOS in regulating muscle metabolism and the cardiorespiratory system.

This work was funded by a grant from the NIH RR18276 and Food for the 21st Century.

\title{
375 PRODUCTION OF GERMLINE TRANSGENIC CHICKENS USING MURINE LEUKEMIA VIRUS-BASED RETROVIRUS VECTOR SYSTEM
}

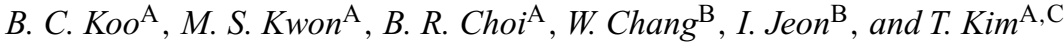 \\ ${ }^{A}$ Dept. of Physiology, Catholic Univ. of Daegu School of Medicine, Daegu, Korea; \\ ${ }^{B}$ National Livestock Research Institute, RDA, Suwon, Kyonggi-Do, Korea; \\ ${ }^{\mathrm{C}}$ Research Center for Transgenic Cloned Pigs, Chungnam National University, Daejeon, Korea
}

We report here successful generation of germline transgenic chickens expressing the enhanced green fluorescence protein (EGFP) gene throughout whole bodies. The founder chickens were produced by injecting replication-defective recombinant retroviruses encapsulated with the vesicular stomatitis virus G glycoprotein (VSV-G)-pseudotyped retrovirus vector system beneath the blastoderm of non-incubated chicken embryos (stage X). Of 129 injected eggs, 13 chickens hatched. These 13 chickens were analyzed at 6-16 weeks post-hatching and all were found to emit green fluorescence in at least one part of the body. One cock of the 13 Go chimeric founder cockerels was mated with several non-transgenic hens, and one of 102 G1 siblings was found to emit a green fluorescent signal in its whole body. Successful germline transmission of the EGFP transgene was further confirmed in the G2 generation chickens: Crossing of the G1 cock and several non-transgenic hens resulted in about 50\% (18/37) of the G2 cockerels being transgenic. The results of this study would be very helpful in establishing a useful transgenic chicken model system for generation of transgenic chickens as bioreactors producing therapeutic proteins, as well as for studies on embryo development.

\section{VECTOR-MEDIATED GENE TRANSFER INTO RHESUS MACAQUE EMBRYOS

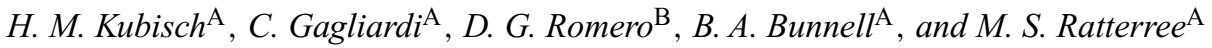 \\ ${ }^{A}$ Divisions of Veterinary Medicine and Comparative Pathology, Tulane National Primate Research Center, Covington, LA 70433, USA;

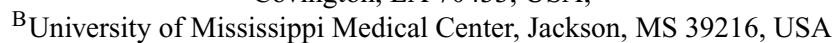

\begin{abstract}
A series of experiments was performed to assess the suitability of various viral vectors for transformation of rhesus macaque (Macaca mulatta) embryos. Viral vectors included the adenovirus-associated virus (AAV) containing a CMV-EGFP transgene and a lentivirus (FUGW) carrying the green fluorescent protein (GFP) gene from either the jellyfish or a humanized version from renilla linked to an ubiquitin promoter. Embryos were generated by in vitro fertilization of oocytes retrieved by laparoscopy from superovulated females. Resulting zygotes were injected under the zona pellucida (ZP) with varying viral concentrations. Embryos were subsequently cultured for 5 days and thereafter analyzed by epifluorescence microscopy. A total of 62 zygotes were injected with one of three vectors AAV2 (25), AAV2.1 (15), or AAV2.7 (22). Of these 22, 9 and 16, respectively, reached the blastocyst and morula stage. There was no difference in the percentage of embryos expressing GFP between vectors $(24,53.2$, and $36.4 \%$, respectively). However, all of the positive embryos proved to be mosaics. In a second set of experiments, FUGW was injected under the ZP of 155 embryos. Of these, 76 received virus carrying renilla GFP, while the remaining 79 were injected with virus carrying the jellyfish GFP. Following injection with renilla, 52 reached the blastocyst/morula stage on Day 7, while 43 containing jellyfish GFP proceeded to this stage. Expression of jellyfish GFP could be seen in $65 \%$ of the embryos of which $35.6 \%$ were mosaics, whereas renilla GFP was found in only $15.8 \%$ of the embryos, although none of these were mosaic. To determine whether the mosaic expression was caused by transgene silencing, three mosaic embryos were dissociated on Day 3 and 10; 10 and 8 blastomeres, respectively, were obtained. Analysis by PCR showed all but one blastomere carrying the vector. Similarly, the presence of the vector was identified by PCR in 17 of 19 non-expressing embryos injected with renilla. These results show that AAV and lentivirus can transform rhesus embryos, which can subsequently continue in development. However, identification of positive embryos by epifluorescence alone may not be sufficient.
\end{abstract}




\title{
377 DEVELOPMENT OF A VIBRATORY MICROINJECTION SYSTEM TO INCREASE THE PRODUCTION RATE OF TRANSGENIC ANIMALS
}

\author{
F. Miyawaki ${ }^{\mathrm{A}}$, J. Hasegawa $^{\mathrm{B}}$, T. Kikuchi $^{\mathrm{A}}$, M. Tsuchiya $^{\mathrm{A}}$, Y. Saito $^{\mathrm{C}}$, H. Suzuki $^{\mathrm{C}}$, T. Ippongi $^{\mathrm{B}}$, \\ K. Kobayashi ${ }^{\mathrm{B}}$, and S. Itohara ${ }^{\mathrm{C}}$ \\ ${ }^{\mathrm{A}}$ Tokyo Denki University, Saitama, Japan; ${ }^{\mathrm{B}}$ Takushoku University, Tokyo, Japan; ${ }^{\mathrm{C}} \mathrm{RIKEN}$, Saitama, Japan
}

\begin{abstract}
Pronuclear injection is the standard method to deliver genes to zygotes, especially of medium- to large-size animals, but the production rate of transgenic animals is still low. To increase it, we developed a vibratory microinjection system (VMS) that vibrates a micropipette (Femtotip ${ }^{\circledR}$; Eppendorf Co., Ltd., Tokyo, Japan) longitudinally to facilitate its penetration of the zona pellucida and nuclear membrane. The VMS can provide a wide range of frequencies and amplitudes of vibration, and is different from the piezo impact micromanipulator that uses an internal force resulting from a rapid deformation of piezoelectric elements. $P$ Vibratory microinjection (VM) at a frequency of $5 \mathrm{kHz}$ and an amplitude of $0.5 \mu \mathrm{m}$, which was not at the tip of micropipettet but at the vibrator's level, was compared with ordinary microinjection (OM). No fragmentation of the Venus gene encoding a variant of yellow fluorescent protein was confirmed after it had been exposed to this vibration for $1 \mathrm{~h}$. A total of 910 eggs obtained from 36 BDF-1 mice were used. This experiment consisted of 15 sets of injections. In each set, the same number $(\leq 40)$ of eggs was allotted to the VM and OM groups. The eggs in each group were injected with the Venus gene using a single micropipette. The two groups were treated simultaneously with two sets of injection apparatuses. Embryonic development was assessed after 4-day culture. The following results were achieved: (1) VM made the micropipette penetrate zona pellucida with much less cellular deformation; the deformation rates were $28.7 \pm 7.2 \%$ for VM and $35.4 \pm 7.3 \%$ for OM $(P<0.0001)$. (2) Ninety-six of 455 embryos $(21.1 \%)$ in the VM group developed to the blastocyst stage, whereas only 41 of 455 eggs $(9.0 \%)$ in the OM group did $(P<0.0001)$. The numbers of Venus-expressed blastocysts were 29 for the VM group and 19 for the OM group $(P=0.14)$. However, the death rates in VM and OM groups were $39.1 \%$ and $32.7 \%$, respectively $(P=0.045)$. This higher death rate in the VM group might have been due to transverse vibration at the tip of micropipette because it was bent approximately $20^{\circ}$. (3) Two of 15 micropipettes in the VM group pulled out a part of the nuclear DNA and/or cytoplasm before finishing the given number of injections while 9 of 15 micropipettes in the OM group did $(P=0.0063)$. We conclude that the VMS achieved zygote injection with significantly less cellular deformation and also resulted in significantly better embryonic development, suggesting anticipated improvement in the production of transgenic animals. The VMS can also cut the cost of micropipettes and save the trouble of frequent replacement of micropipettes
\end{abstract}

\section{EFFECT OF TRANSGENE CONCENTRATION AND FLANKING MATRIX ATTACHMENT REGIONS ON THE EFFICIENCY OF ICSI-MEDIATED TRANSGENIC MOUSE PRODUCTION}

\author{
P. N. Moreira ${ }^{\mathrm{A}}$, J. Pozueta ${ }^{\mathrm{B}, \mathrm{C}}$, F. Valdivieso $^{\mathrm{C}}$, L. Montoliu $^{\mathrm{B}}$, and A. Gutiérrez-Adán ${ }^{\mathrm{A}}$ \\ ${ }^{A}$ Department of Animal Reproduction, INIA, Madrid, Spain; ${ }^{B}$ Department of Molecular and Cellular Biology, CNB-CSIC, Madrid, Spain; \\ ${ }^{\mathrm{C}}$ Departamento de Biologia Molecular, Centro de Biologia Molecular Severo Ochoa, CSIC-UAM, Madrid, Spain
}

The efficiency of ICSI-mediated transgenesis can be influenced by many different factors. In this study, we have assessed the impact of transgene concentration and of the presence of transgene-flanking matrix attachment regions (MARs) on the efficiency of this procedure. Presented data include assays with three DNA constructs; a plasmid EGFP of $5.4 \mathrm{~kb}$ (pEGFP, Clontech Laboratories, Inc., Palo Alto, CA, USA); this plasmid EGFP construct flanked with two MAR elements (M-EGFP-M; $2.3 \mathrm{~kb}$ of the human $\beta$-interferon domain boundaries) (Gutiérrez-Adán et al. 2000 Transgenic Res. 9, 81-89); and a yeast artificial chromosome (YAC) of $520 \mathrm{~kb}$. ICSI-mediated transgenesis was done in the B6D2 hybrid mouse strain as previously described (Moreira et al. 2004 Biol. Reprod. 71, 1943-1947), using two concentrations (1 and $15 \mathrm{ng} / \mu \mathrm{L}$ ) of the M-EGFP-M transgene, three concentrations $(1,6$, and $15 \mathrm{ng} / \mu \mathrm{L})$ of the pEGFP transgene, and three concentrations $(2,3.6$, and $5 \mathrm{ng} / \mu \mathrm{L})$ of the YAC construct. Embryos were cultured in vitro until the 2-cell stage and transferred into Day 1 pseudopregnant females. Live offspring or Day 14 fetuses were analyzed by PCR for the detection of the genomic presence of the integrated transgene. The proportion of transgenics (PT) obtained with 2,3.6, and $5 \mathrm{ng} / \mu \mathrm{L}$ of the YAC construct was 10,21 , and $13 \%$ respectively. A Z-test analysis of the data generated with this construct indicated that the number of transgenic offspring, when expressed as a proportion of injected ova (efficiency rate of the procedure, ER), increased with DNA concentration up to a threshold of $3.6 \mathrm{ng} / \mu \mathrm{L}$, and suggested that higher concentrations of this transgene (and maybe of co-purifying contaminants) decreased the ER of the procedure. Interestingly, although much higher transgene concentrations were used for the pEGFP construct, this threshold was not reached. With an increase in transgene concentration (from 1 to $15 \mathrm{ng} / \mu \mathrm{L}$ ), the PT increased (from 39 to $70 \%$ ), as well as the ER of the procedure (from 3 to $7 \%$ ). In addition, independently of the transgene concentration tested, when M-EGFP-M was used, the ER of the procedure and the PT observed were similar to the ones obtained with pEGFP. These results indicate that, regardless of the importance of other factors such as transgene size, purity, and copy number, the concentration to be used on ICSI-mediated transgenesis should be optimized for each construct, in order to maximize the PT among the liveborn offspring and the overall efficiency of the procedure. Our results also indicate that transgene-flanking MARs do not have a significant impact on the efficiency of this transgenesis method.

\section{LACTATION INDUCTION IN PREPUBERTAL BULLS AND HEIFERS AS A TOOL FOR PREDICTING MAMMARY SPECIFIC TRANSGENE EXPRESSION IN CATTLE}

\author{
A. Powell ${ }^{\mathrm{A}}$, D. Kerr ${ }^{\mathrm{B}}$, and R. Wall $\mathrm{A}$ \\ ${ }^{A}$ Agricultural Research Service, United States Department of Agriculture, Beltsville, MD 20705, USA; \\ ${ }^{B}$ Department of Animal Sciences, University of Vermont, Burlington, VT 05401, USA
}

The bovine's long generation interval results in a lapse, from the time of birth, of two to three years before mammary-specific transgenes can be assessed in genetically engineered animals. This experiment was conducted in an attempt to reduce that waiting period by up to two years. 
Lactation was induced in prepubertal bull and heifer calves, 3 to 8 mo of age, as a means of predicting transgene behavior during subsequent normal lactations in the heifers and daughters of bulls. Transgenic animals tested were either founder animals, produced by somatic cell nuclear transfer, or G1 offspring of founder bulls. The transgene consists of a lactation specific sequence encoding lysostaphin, an antimicrobial protein targeted against Staphylococcus aureus, a mastitis-causing pathogen. Estrogen, progesterone, and dexamethasone were administered as previously described (Ball et al. 2000 J. Dairy Sci. 83, 2459) to nine heifers (transgenics = 5) ranging in weight from 90 to $165 \mathrm{~kg}$. Eight bull calves (transgenics $=7$ ) weighing from 81 to $178 \mathrm{~kg}$ received additional estrogen and progesterone injections as well as reserpine prior to dexamethasone treatment. Animals were hand-milked twice daily for 4 to 7 days. All nine heifers responded to the milk induction scheme, yielding between $19 \mathrm{~mL}$ and $4.5 \mathrm{~L}$. Milk volume from the three responding males $(100 \mu \mathrm{L}$ to $2.5 \mathrm{~mL})$ was significantly less than that harvested from females $(P=0.025)$. Only bull calves over $150 \mathrm{~kg}$ had a positive response. Transgenic females produce less milk then non-transgenics $(313 \pm 494$ vs. $2276 \pm 552 \mathrm{~mL}$, respectively; $P=0.033)$. Most importantly, there was no detectable difference between the concentration of lysostaphin in milk from induction $(8.1 \pm 2.7 \mu \mathrm{g} / \mathrm{mL})$ and natural lactations $(3.5 \pm 2.6 \mu \mathrm{g} / \mathrm{mL})$ in the four transgenic heifers tested $(P=0.229)$. The result was the same when lysostaphin was analyzed as a percentage of total protein $(P=0.427)$. Induction of a $\mathrm{G} 1$ heifer and a bull calf from the same founder bull produced similar lysostaphin concentrations in their milk (5.6 \pm 0.9 and $5.2 \pm 0.5 \mu \mathrm{g} / \mathrm{mL}$; respectively). $\beta$-lactoglobulin concentration was also similar during induced and natural lactation $(P=0.165)$ for all animals studied. However, total protein was greater in induced milk samples compared to natural lactation samples $(28.4 \pm 1.7$ vs. $21.2 \pm 1.7 \mathrm{mg} / \mathrm{mL} ; P=0.007)$ as was lactoferrin $(707 \pm 51 \mathrm{vs.} 213 \pm 51 \mu \mathrm{g} / \mathrm{mL} ; P<0.001)$. Conversely, compared to that in induced milk samples, lactose was more concentrated in the natural lactation samples $(34.6 \pm 2.5 \mathrm{vs} .46 .0 \pm 2.1 \mathrm{~g} / \mathrm{L})$. In this study transgene expression was detected in milk from induced lactations and its concentration in those samples was generally predictive of product concentration in the natural lactation milk. The induction protocol was effective in male $(>150 \mathrm{~kg})$ and female calves.

\title{
380 COMPARISON OF TRANSGENE EXPRESSIONS BY ICSI AND PRONUCLEAR MICROINJECTION IN MURINE AND PORCINE EMBRYOS
}

\author{
H. Saito, H.-O. Kawano, M. Kurome, R. Tomii, S. Ueno, K. Hiruma, and H. Nagashima \\ Laboratory of Developmental Engineering, Department of Life Science, School of Agriculture, \\ Meiji University, Kawasaki, Kanagawa, Japan
}

Intracytoplasmic sperm injection (ICSI) of DNA-binding sperm produces transgenic offspring as effectively as pronuclear microinjection (PNM). A significant difference in these two methods is that DNA is introduced into MII oocytes during ICSI, which is likely to allow earlier gene integration compared to PNM. This leads us to hypothesize that ICSI reduces the chance of development of a mosaic embryo, a mixture of transgene-positive and -negative cells. To test this hypothesis, we compared expression patterns of the green flourescent protein (GFP) gene introduced by ICSI and PNM into murine and porcine oocytes. For ICSI, 2 to $5 \times 10^{5} / \mu \mathrm{L}$ of sperm frozen-thawed in CZB (for mice) or NIM (for pigs) were co-incubated with $2.5 \mathrm{ng} / \mu \mathrm{L}$ of transgene fragments (CAG-EGFP; $3 \mathrm{~kb}$ ) for $5 \mathrm{~min}$. Murine sperm were microinjected into in vivo-matured oocytes, and porcine sperm into in vitro-matured oocytes. PNM was performed by microinjection of several picoliters of the transgene fragments $(10 \mathrm{ng} / \mu \mathrm{L})$ into pronuclei of in vivo-fertilized oocytes for mice and in vitro-matured and -fertilized oocytes for pigs. ICSI and PNM embryos were cultured in vitro to the morula stage and treated with $0.5 \%$ pronase to remove the zona pellucida. These morulae were disassembled into individual blastomeres by pipetting into PBS containing $100 \mu \mathrm{M}$ EDTA and examined for GFP expression under fluorescence microscopy. As shown in Table 1, the rate of mosaicism in GFPexpressing embryos was significantly lower for ICSI than for PNM $(P<0.01)$. In addition, GFP-expressing ICSI embryos were likely to contain high percentages, 81 to $100 \%$, of GFP-positive cells, whereas GFP-expressing PNM embryos were significantly less likely to contain such high percentages of GFP-positive cells $(P<0.01)$. From these results, we conclude that transgenesis by ICSI was less likely to produce mosaic embryos, and that produced transgenic embryos contained higher proportions of transgene-positive cells, although genomic integration remains to be determined.

Table 1. Transgene expression by ICSI and pronuclear microinjection in murine and porcine embryos

\begin{tabular}{llcc}
\hline Animal & Transgenic method & Rate of mosaicism (\%) & $\begin{array}{c}\text { Rate of embryos containing 81-100\% } \\
\text { of GFP-positive cells (\%) }\end{array}$ \\
\hline Mouse & ICSI & $5 / 20(25.0)^{\mathrm{a}}$ & $15 / 20(75.0)^{\mathrm{a}}$ \\
& PNM & $15 / 19(78.9)^{\mathrm{b}}$ & $7 / 19(36.8)^{\mathrm{b}}$ \\
Pig & ICSI & $15 / 39(38.5)^{\mathrm{a}}$ & $32 / 39(82.1)^{\mathrm{a}}$ \\
& PNM & $11 / 12(91.7)^{\mathrm{b}}$ & $1 / 12(8.3)^{\mathrm{b}}$ \\
\hline
\end{tabular}

${ }^{\mathrm{a}, \mathrm{b}}$ Values with different subscripts within a column are significantly different $(P<0.01)$

This work was supported by PROBRAIN.

\section{PRODUCTION OF A TRANSGENIC PIGLET BY A NEW SPERM INJECTION TECHNIQUE}

\author{
H. Y. Yong, C. Murphy, A. Rieke, L. Lai, Y. Hao, R. Li, D. Wax, M. Samuel, S. Korte, \\ K. Whitworth, and R. Prather \\ Department of Animal Science, University of Missouri-Columbia, Columbia, MO 65211, USA
}

The technique for intracytoplasmic sperm injection (ICSI) has, until now, focused on scoring the tail of the sperm prior to catching and aspiration into the injection pipette. This is in spite of the fact that damage to the head would more closely simulate what occurs during normal fertilization. In 
addition, to aid in visualizing the injection process so that a reduced volume can be injected, the oocyte is generally centrifuged to clear a portion of the cytoplasm. Thus, with conventional ICSI, the sperm are immobilized with polyvinylpyrrolidone, repeatedly frozen and thawed, treated with DTT or Triton X-100, and severed between the head and tail; the oocyte is centrifuged or activated. All of the above treatments are designed to compensate for the intrinsic defects in conventional ICSI. Our objective was to use a modified ICSI procedure whereby aggressively motile sperm were captured onto the broken tip of an injection pipette and then injected into noncentrifuged oocytes. Damage to the head of the sperm occurred on the pipette or while pushed through the zona pellucida. These procedures are based on the work of Yong et al. 2003 Hum. Reprod. 18, 2390, where they achieved an improvement in development in vitro as compared to conventional methods. Ovaries were collected from prepubertal gilts, and oocytes were aspirated and matured in vitro. Sperm were collected from a transgenic boar carrying the green fluorescent protein (GFP) and frozen. After thawing, aggressively motile sperm were captured and injected through the zona pellucida and into the cytoplasm of the in vitro-matured oocytes. A total of 452 injected oocytes (43-171 oocytes per recipient) were surgically transferred into the oviduct of six surrogate gilts. Two gilts (33\%) became pregnant. One gave birth to a healthy male piglet. GFP expression was observed in the nose and hooves by direct epifluorescent examination of the newborn piglet. This pattern of GFP expression is identical to that in non-ICSI-derived GFP pigs in this line. This result showed for the first time that this new sperm injection technique could be used for production of a viable transgenic piglet using in vitro-matured oocytes and frozen-thawed sperm.

\title{
Ultrasonography
}

\section{ULTRASONOGRAPHIC EVALUATION OF AMNIOTIC SAC SIZE DURING EARLY PREGNANCY IN COWS}

\author{
M. Panarace, C. Garnil, G. Jauregui, J. Lagioia, M. Marfil, and M. Medina \\ Goyaike S.A.A.C.I Y F, Carmen de Areco CP6725, Buenos Aires, Argentina
}

In ruminants, the embryonic vesicle is composed of the yolk and the allantoic and amniotic sacs. In cattle, the allantoic sac represents the largest fluid accumulation in the uterus from early pregnancy onward, but is irregular in shape as it conforms to the uterine horns. It is, therefore, difficult to ultrasonographically measure the allantoic compartment. Conversely, the amniotic sac remains ellipsoidal in shape and can be evaluated more easily. The aim of this study was to characterize the development of the amniotic sac in relation to embryo development. Thirteen multiparous, nonlactating Aberdeen Angus cows were examined weekly from 30 days to 72 days of gestation. Epidural anesthesia was induced ( $6 \mathrm{~mL}$ of $2 \%$ lidocaine) to obtain light relaxation of the rectum and prevent straining. Measurements were taken by ultrasonography (Toshiba Nemio 20, Tokyo, Japan) using a $5-10 \mathrm{MHz}$ intraoperative finger probe from 30 days to 58 days of pregnancy, and a 3-6 MHz linear-array probe thereafter. Length (D1) was registered in a longitudinal section of the amniotic sac, whereas width (D2) and height (D3) were measured in a cross section. Volume $\left(\mathrm{cm}^{3}\right)$ was calculated with the formula $4 \div 3 \times \mathrm{Pi} \times(\mathrm{D} 1 \div 2) \times(\mathrm{D} 2 \div 2) \times(\mathrm{D} 3 \div 2) \div 1000$ and mean amniotic diameter $(\mathrm{MAD})$ was calculated as the average of the three measurements (Hellman et al. 1969 Obstet. Gynecol. 103, 789). Crown rump length (CRL) was measured in a longitudinal section of the embryo when it was in a neutral position. Results show that volume, MAD, and the CRL increase progressively during the first trimester (Table 1). In the first 50 days of gestation, the difference (MAD-CRL) changed from a negative to a positive value, probably as a reflection of increasing embryonic production of amniotic fluid (Wallenburg et al. 1988 Med. 5, 191). From 58 days of gestation onward, the difference became progressively more negative due to a faster relative growth rate of the embryo than of the amniotic sac. To conclude, the present table may be useful in distinguishing between normal and abnormal early pregnancy, including embryonic growth retardation and hydrops amnios (Wintour 1986 et al. Aust. Vet. J. 216 , 221).

Table 1. Mean amniotic diameter (MAD), crown rump length (CRL), and the difference (MAD-CRL) during pregnancy in cattle (mean $\pm \mathrm{SD} ; \boldsymbol{n}=13$ )

\begin{tabular}{lccrc}
\hline Days of gestation & Volume $\left(\mathrm{cm}^{3}\right)$ & MAD $(\mathrm{mm})$ & CRL $(\mathrm{mm})$ & MAD-CRL $(\mathrm{mm})$ \\
\hline 30 & $0.2 \pm 0.1$ & $7.1 \pm 1.4$ & $9.3 \pm 1.5$ & $-1.3 \pm 1.2$ \\
36 & $1.1 \pm 0.4$ & $13.2 \pm 1.4$ & $15.6 \pm 1.3$ & $-1.4 \pm 1.1$ \\
43 & $7.7 \pm 3.8$ & $24.4 \pm 3.9$ & $25.1 \pm 1.9$ & $-1.7 \pm 3.2$ \\
50 & $26.7 \pm 7.4$ & $37.9 \pm 3.2$ & $36.9 \pm 2.4$ & $1.0 \pm 2.1$ \\
58 & $62.8 \pm 14.7$ & $50.8 \pm 3.6$ & $61.2 \pm 3.5$ & $-10.4 \pm 4.6$ \\
64 & $119.9 \pm 26.1$ & $63.8 \pm 4.6$ & $80.7 \pm 4.7$ & $-16.9 \pm 5.4$ \\
72 & $217.5 \pm 52.3$ & $76.9 \pm 5.9$ & $100.5 \pm 4.6$ & $-13.6 \pm 6.4$ \\
\hline
\end{tabular}

